\title{
Quantification of Black Carbon Emissions from Gas Flaring and Standardization of the Sky-LOSA Measurement Technique
}

\author{
by
}

\author{
Bradley M. Conrad
}

A thesis submitted to The Faculty of Graduate Studies and Research in partial fulfilment of the degree requirements of

\author{
Doctor of Philosophy \\ in
}

Mechanical Engineering

\author{
Ottawa-Carleton Institute for \\ Mechanical and Aerospace Engineering \\ Department of Mechanical and Aerospace Engineering \\ Carleton University \\ Ottawa, Ontario, Canada \\ September 2020
}

Copyright (C) 2020

Bradley Conrad 


\section{Abstract}

This thesis details the deployment and refinement of an emergent optical diagnostic for soot/black carbon (BC) emissions from gas flaring alongside investigations into optical properties of flare BC. Research efforts were first focussed on the field-deployment of the existing sky-LOSA (line-of-sight attenuation using skylight) technique to measure BC emissions from gas flares. Fourteen measurements from nine flares revealed BC emissions spanning more than four orders of magnitude, highlighting the disproportionate emissions contributions of individual "super-emitting" flares. BC yields measured at four flares varied with flare gas energy content, permitting extension of a laboratory-based emission factor model to consider field data for actual in-field flares.

Available gas flare simulation data were subsequently leveraged to perform numerical simulations of radiative transfer through realistic flare plumes to quantify previously ignored radiative effects in the sky-LOSA algorithm. Refractive index gradientdriven beam steering was found to be negligible through cooled flare plumes. By contrast, multiple scattering was observed to significantly affect inscattering within the radiative transfer theory for sky-LOSA. These data revealed a simple model to correct for multiple scattering effects in the sky-LOSA algorithm with negligible impact on measurement uncertainties as evidenced by case study analyses.

Laboratory studies of flare BC were performed in parallel to address a lack of data for flare-relevant BC mass-normalized absorption cross-section (MAC). BC MAC was quantified for myriad flare gas compositions/conditions and varied with numerous flare metrics. A phenomenological model for BC MAC was developed using a novel scaling 
parameter thought to capture the in-flame time-temperature history of $\mathrm{BC}$ particulate. The new model reconciled anomalous field data and suggested that flare BC MAC might be $>1.3-2.0$ times larger than other sources.

The final focus of this thesis was the completion of a general uncertainty analysis (GUA) to support standardized setup and measurement protocols for sky-LOSA. Uncertainties over all practical measurement conditions were computed in a variancereduced Monte Carlo framework. GUA data were compiled and presented in a new opensource software tool to allow sky-LOSA users to consistently obtain optimal measurement data for arbitrary measurement conditions, enabling broader deployment of sky-LOSA to quantify and reduce flare $\mathrm{BC}$ emissions. 


\section{Acknowledgements}

First and foremost, I would like to express my tremendous appreciation to my thesis supervisor, Professor Johnson. You are truly a great mentor and have provided such an abundance of opportunities through the years. Thank you for your patience, unwavering support, and dedication to making our efforts as impactful and meaningful as possible. In addition to the amazing academic experiences, there are many life lessons I have learnt along the way. My favourites? "Life's too short for white wine" and (while sharing a trailer in the Ecuadorean jungle) "you can survive anything for a week."

To all my EERL colleagues, thank you for making this journey so much fun. I owe extra debts of gratitude to Darcy and Melina as the flare pit-masters and to those involved in field measurements. Also, I would like to specially thank Dave and Brian for the many brain-picking sessions and their valuable insights on any and all topics.

My grandparents, my sister, and especially my Mom and Dad, thank you for the continuous love and support in so many ways, I am so grateful to have you all. To my wife, Brit, what can I say? The countless challenges that you have helped me through, the off-hours work that you have put up with, and everything you have done to keep me functioning; I've said it many times, but I am truly so very lucky. This is as much yours as it is mine. It's high time for the next chapter!

To Grandpa Hadden. With our early morning math lessons and your endless enjoyment of everything learning, you prepared me for this path since I was a wee bairn. This is for you - how I'd love to chat with you about this. 


\section{Table of Contents}

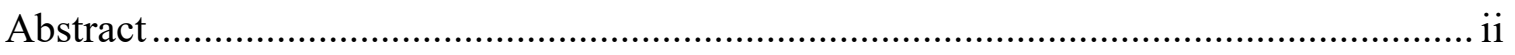

Acknowledgements............................................................................................. iv

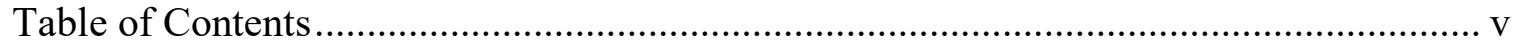

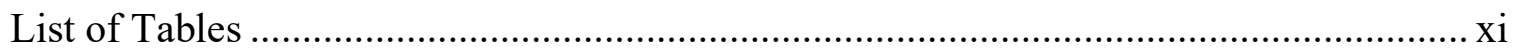

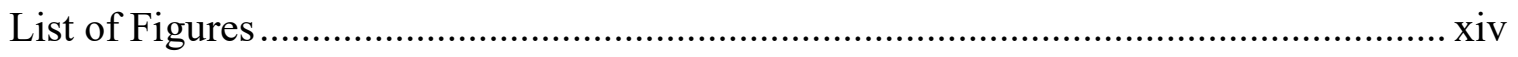

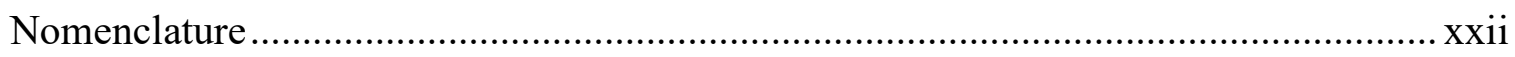

Preface

Student Statement Regarding Student Involvement ………...................................... 35

Supervisor Statement Regarding Student Involvement ............................................ 35

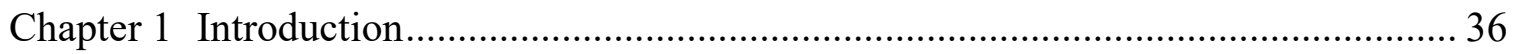

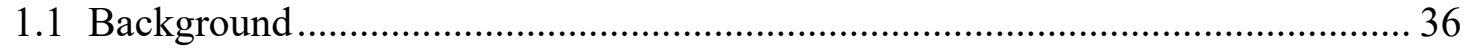

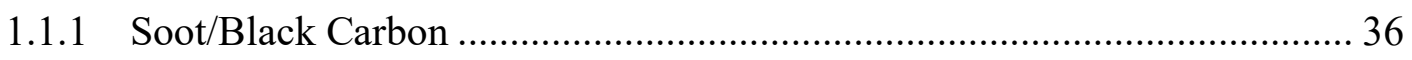

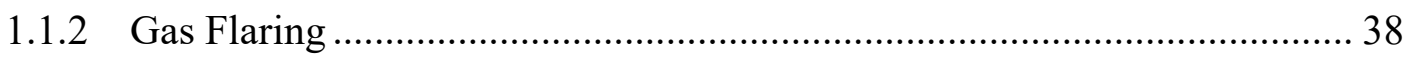

1.1.3 Black Carbon Emissions from Gas Flaring ................................................. 40

1.1.4 Regulatory Framework and Impact of Presented Work ................................ 44

1.1.5 A Note on Black Carbon Terminology ...................................................... 46

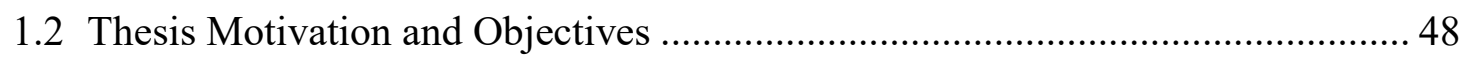

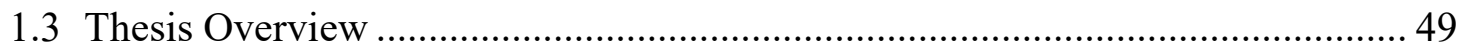

Chapter 2 Field Measurements of Black Carbon Yields from Gas Flaring...................... 54

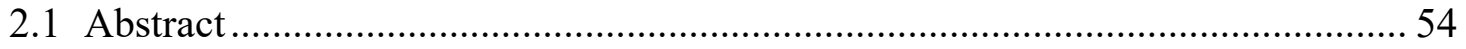

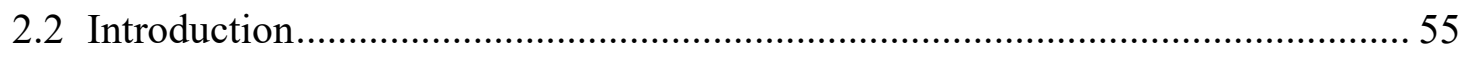

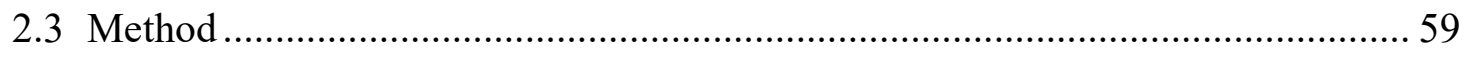

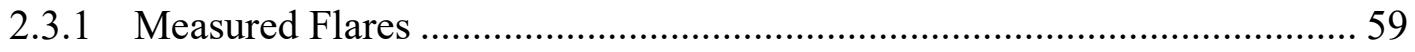

2.3.2 Black Carbon Emission Rate - Sky-LOSA Theory ............................................... 61

2.3.3 Black Carbon Emission Rate - Sky-LOSA Implementation .......................... 63

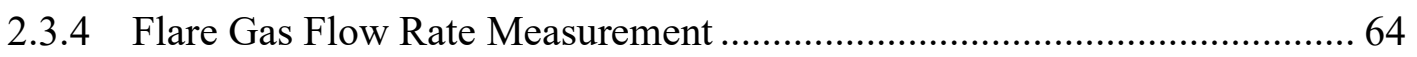

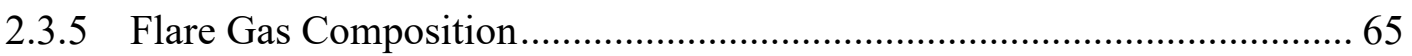

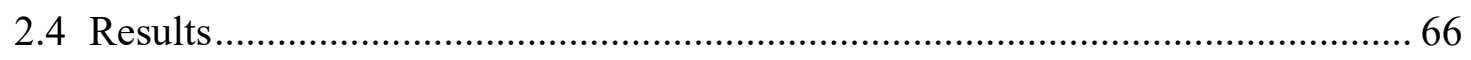

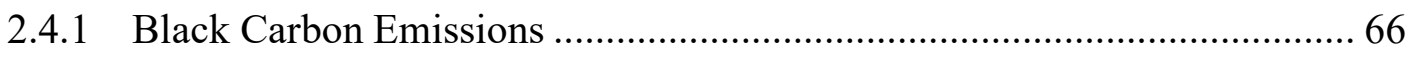

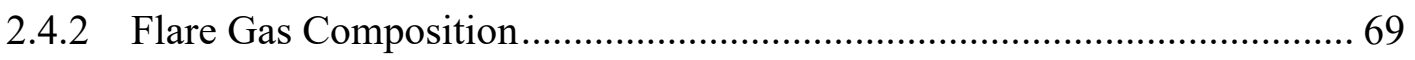

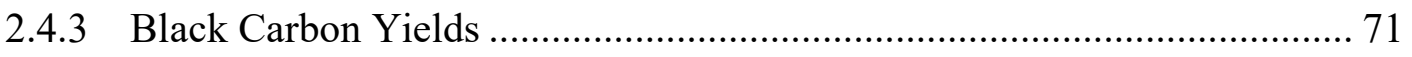




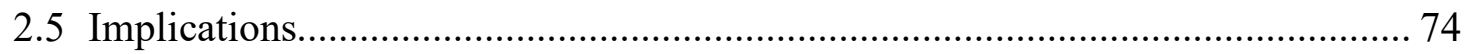

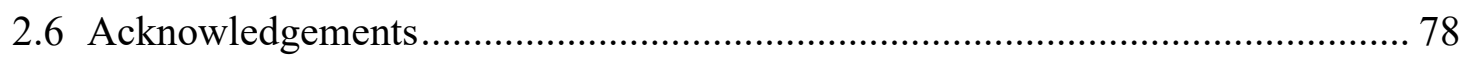

Chapter 3 Beam Steering Effects on Remote Optical Measurements of Pollutant Emissions in Heated Plumes and Flares .................................................... 79

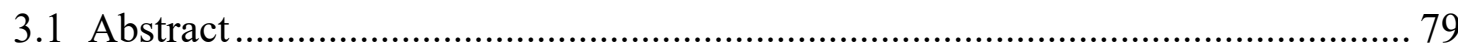

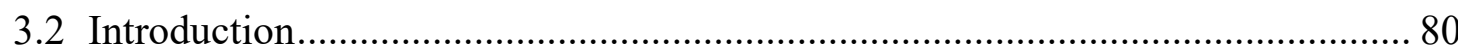

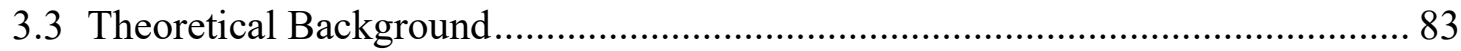

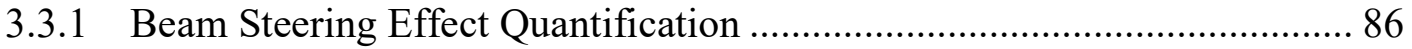

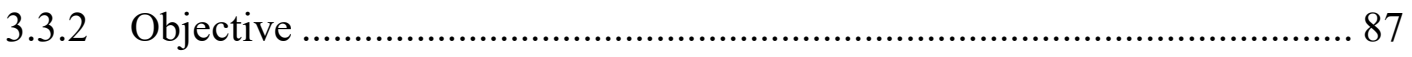

3.4 Methodology

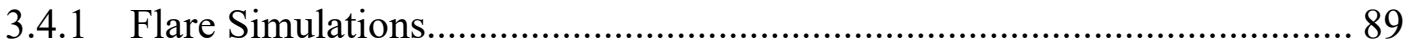

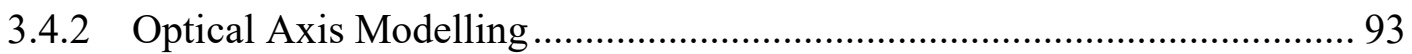

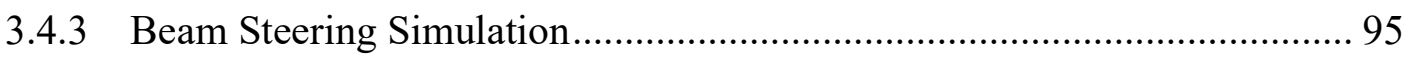

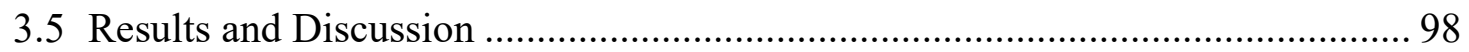

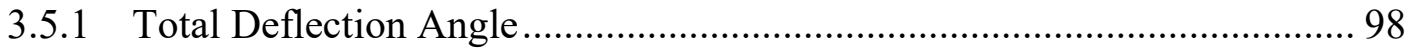

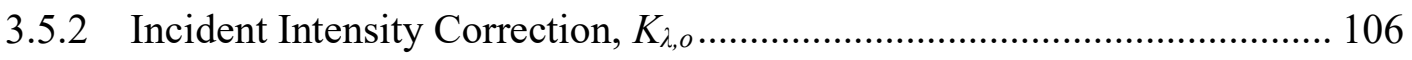

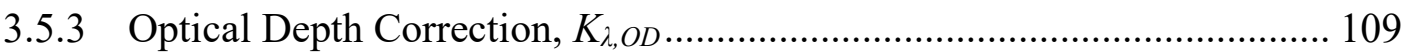

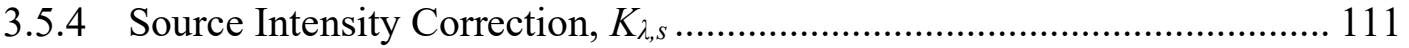

3.5.5 Further Considerations - Beam Steering by Fine-Scale Turbulence ........ 113

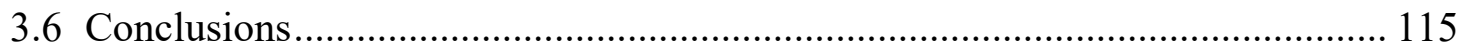

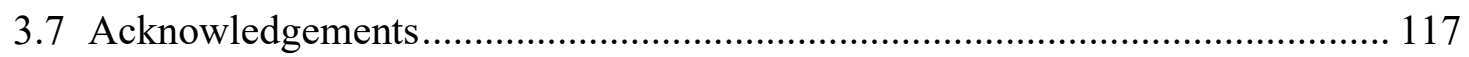

Chapter 4 The Effect of Multiple Scattering on Optical Measurement of Soot

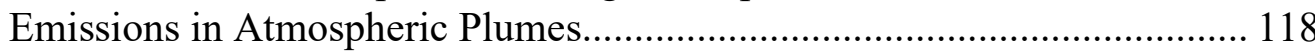

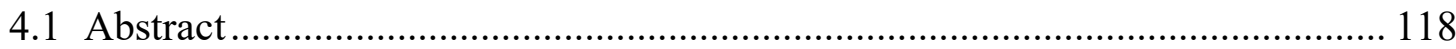

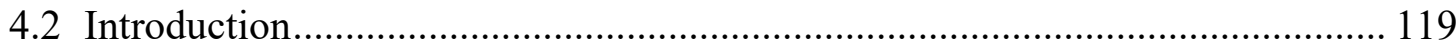

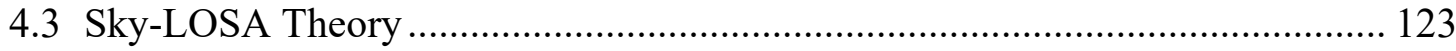

4.3.1 Multiple Scattering ...................................................................... 128

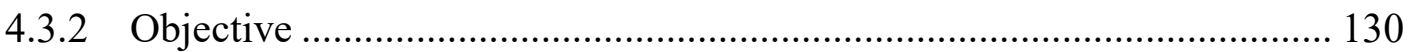

4.4 Methodology

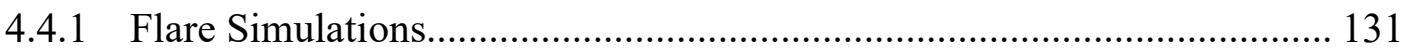

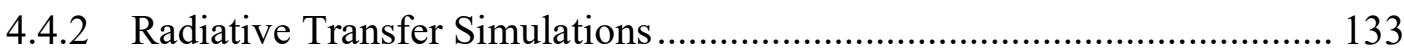

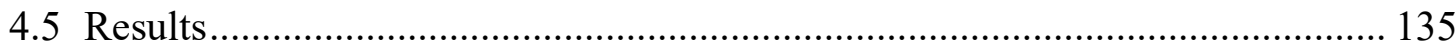

4.5.1 MS Correction Parameter Model ....................................................... 135

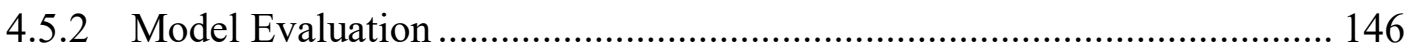

4.5.2.1 Consideration of Residual Errors When Using the MS Correction Model with Sky-LOSA .................................................................. 150

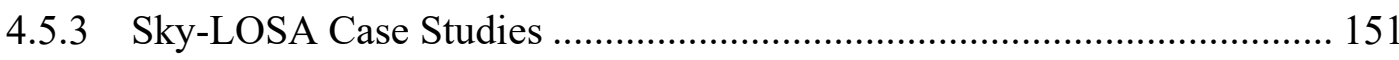




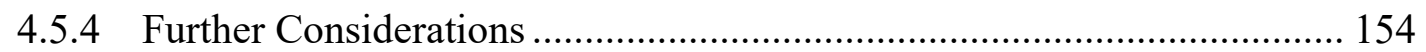

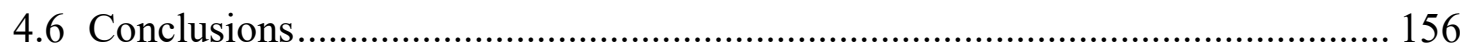

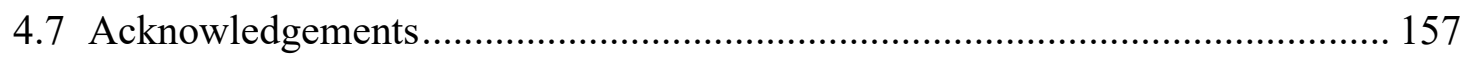

Chapter 5 Mass Absorption Cross-Section of Flare-Generated Black Carbon: Variability, Predictive Model, and Implications.......................................... 158

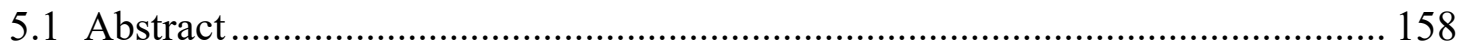

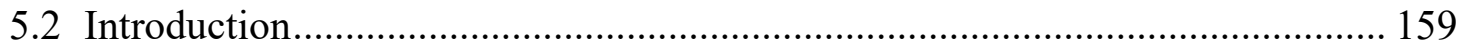

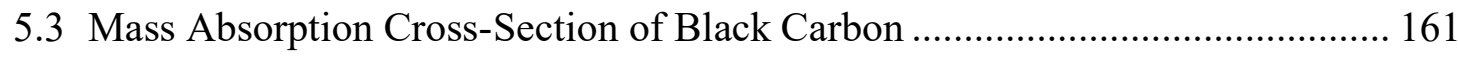

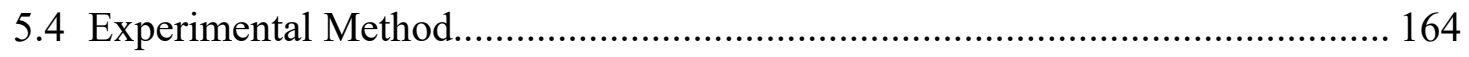

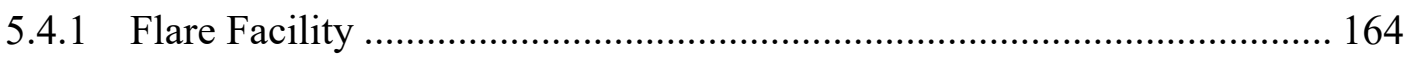

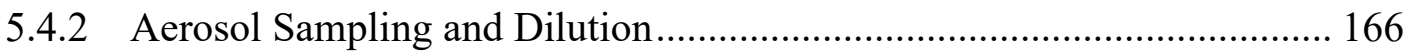

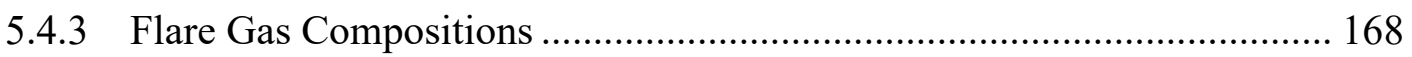

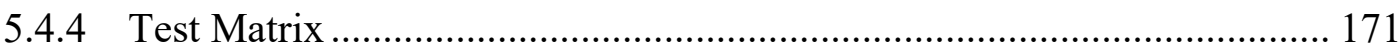

5.4.5 MAC Determination and Data Reduction ............................................. 172

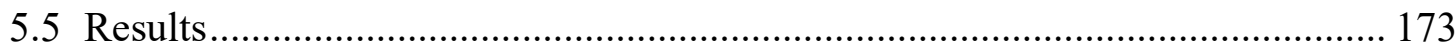

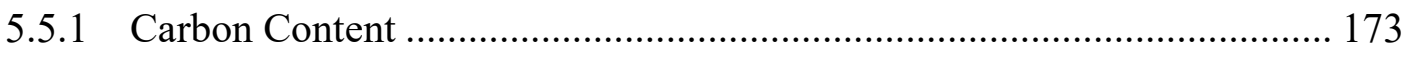

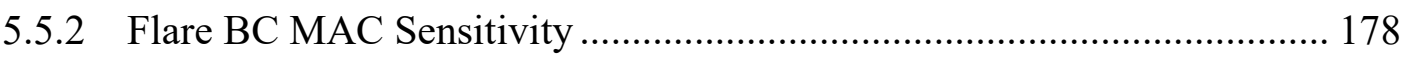

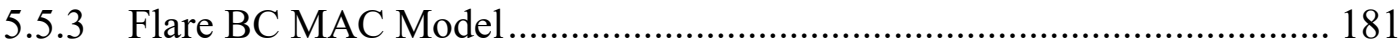

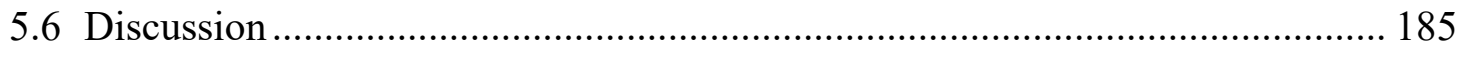

5.6.1 Potential Mechanism of BC MAC Variability via Changes in

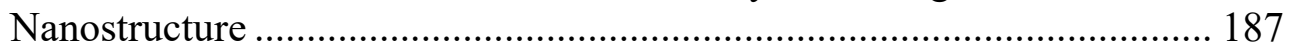

5.6.2 Limits to the Phenomenological Model ................................................... 190

5.6.3 Implications for Climate Forcing of Flare BC ……................................. 192

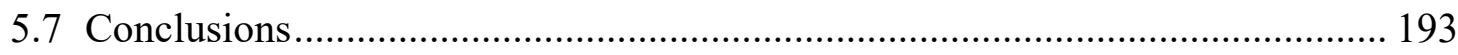

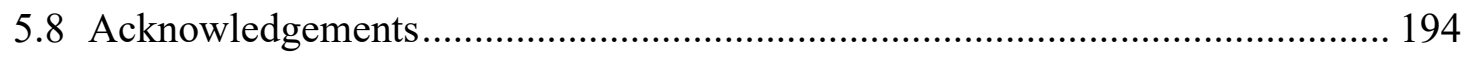

Chapter 6 An Uncertainty-Based Protocol for the Setup and Measurement of Soot/Black Carbon Emissions from Gas Flares using Sky-LOSA ................ 195

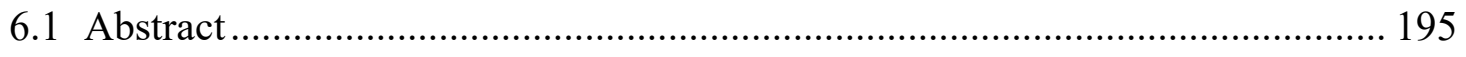

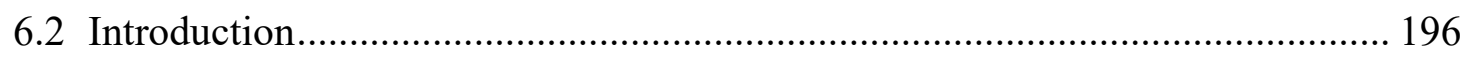

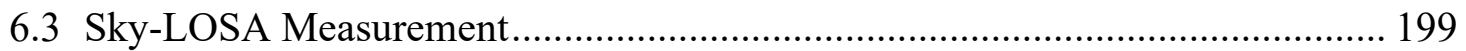

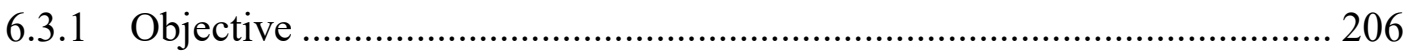

6.4 General Uncertainty Analysis Methodology .................................................... 206

6.4.1 Special Considerations for MC Analysis ………….................................. 207

6.4.1.1 Expansion of the Scattering Phase Function …………………...... 207

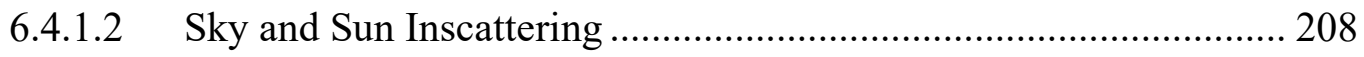

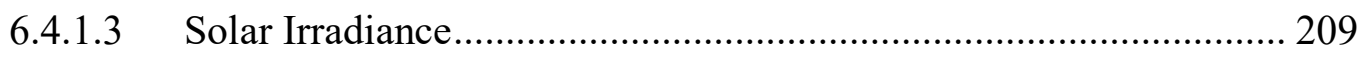

6.4.1.4 Truncation of the Expanded SPF ................................................. 211 


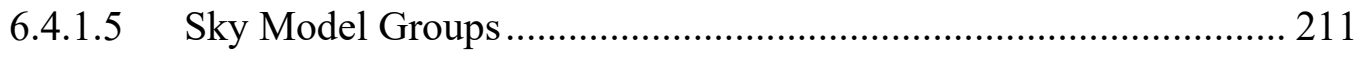

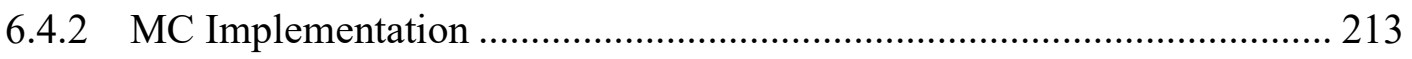

6.4.2.1 Relative Uncertainty Metric ………………….......................... 216

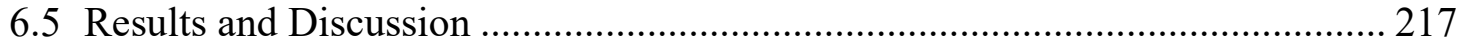

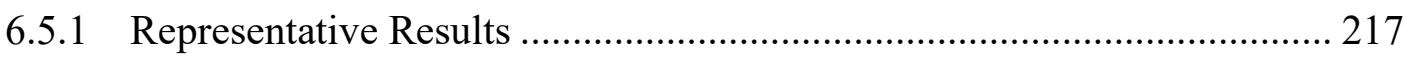

6.5.1.1 Camera Pointing Heuristics ......................................................... 223

6.5.1.2 Further Camera Heuristics ............................................................... 226

6.5.2 Open-source Software Tool for Simpler Sky-LOSA Setup -

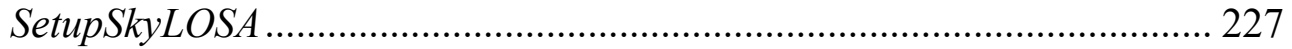

6.5.3 Case Study - Atasta Facility ............................................................. 231

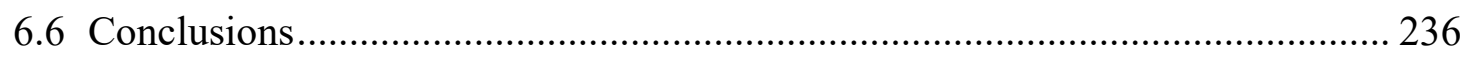

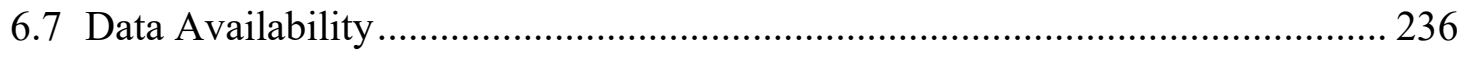

6.8 Acknowledgements.................................................................................... 237

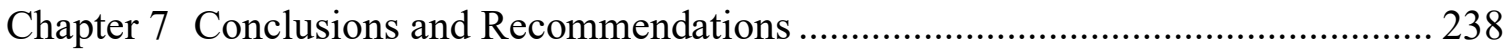

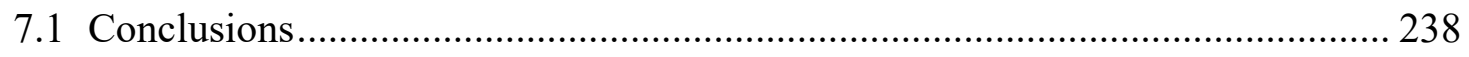

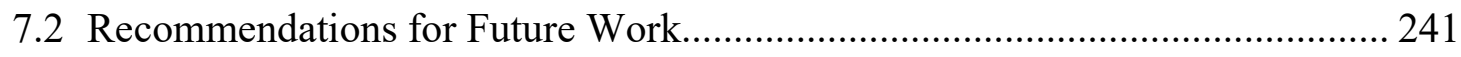

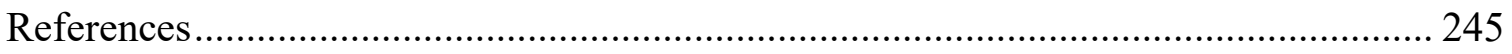

Appendix A Supplemental Information for Field Measurements of Black Carbon Yields from Gas Flaring ...................................................................... 263

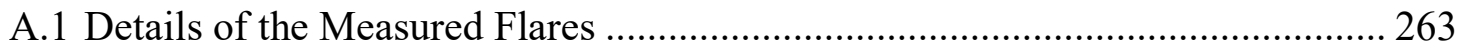

A.1.1 Measured Flares in Veracruz, Mexico ……………………………….... 263

A.1.2 Measured Flares in Orellana, Ecuador ...................................................... 268

A.2 Sky-LOSA Measurement of BC Emission Rate ………................................... 270

A.2.1 Sky-LOSA Data Acquired in Veracruz, Mexico....................................... 271

A.2.2 Sky-LOSA Data Acquired in Orellana, Ecuador ....................................... 271

A.3 Sky-LOSA Emission Rate Uncertainty Calculations ....................................... 272

A.3.1 CM-LHS Monte Carlo Method ......................................................... 272

A.3.2 Assessment of Uncertainty Associated with Sky Reconstructions ........... 273

A.3.3 Derivation of Time-Averaged BC Emission Rate Probability

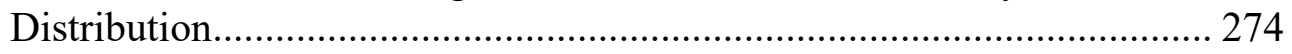

A.3.4 Handling of Non-Physical Results during MC Draws ............................. 275

A.3.5 Black Carbon Emission Rate Distributions and Observations .................. 278

A.4 Flare Gas Composition Measurements ……………........................................ 280

A.5 Calculation of Flare Gas Flow Rate and Uncertainty ...................................... 282

A.5.1 Optical Velocity Measurement......................................................... 282

A.5.2 Tracer-Dilution Measurement .............................................................. 284

A.6 Calculation of Black Carbon Yield................................................................. 287 
A.7 Estimation of a Representative Global Flare Gas Heating Value 288

Appendix B Supplemental Information for Beam Steering Effects on Remote Optical Measurements of Pollutant Emissions in Heated Plumes and Flares....... 290

B.1 Computation of the Complex Refractive Index for Multi-Component GasPhase Plumes 290

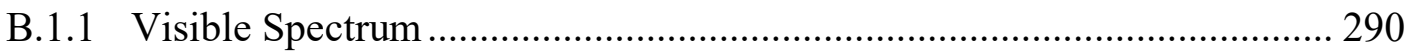

B.1.2 Infrared Spectrum........................................................................... 291

B.2 The Effect of Soot Particulate on Beam Steering in Flare Plumes .................... 293

B.3 Source Intensity Correction for Inscattering Sources .................................... 296

B.4 The Effect of Sub-Grid Turbulence ............................................................... 298

Appendix C Supplemental Information for The Effect of Multiple Scattering on Optical Measurement of Soot Emissions in Atmospheric Plumes............ 303

C.1 Simplification of Radiative Transfer using the Idealized Transmittance ........... 303

C.2 Prior Distributions of Fundamental Soot Properties ........................................ 304

C.3 Inscattering of Polarized Light........................................................................ 305

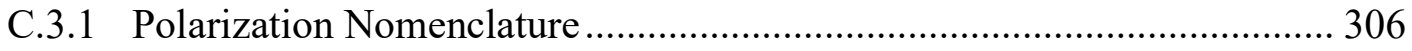

C.3.2 Stokes Mueller Formalism and the Stokes Vector ................................. 308

C.3.3 Intensity of Skylight Single-Scattered by Soot ..................................... 309

C.3.4 Intensity of Skylight Multiple-Scattered by Soot................................... 316

C.4 Reverse Monte Carlo "Ray Tracing" Procedure .............................................. 316

C.5 Forward Propagation of Light along rMCRT-Computed Paths ........................ 319

C.5.1 Skylight Component............................................................................. 321

C.5.2 Sunlight Component.............................................................................. 322

C.6 Solution of Multiple Scattering-Corrected Transmittance Equation .................. 324

C.7 Supplementary Data: Raw Errors in MS Correction Model.............................. 326

Appendix D Supplemental Information for Mass Absorption Cross-Section of FlareGenerated Black Carbon: Variability, Predictive Model, and Implications ......................................................................... 327

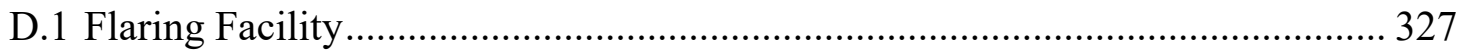

D.2 Light Absorption by Black Carbon Aggregates................................................. 329

D.3 Derivation of Reduced Flare Gas Compositions for Experimental Work .......... 335

D.4 Consideration of Uncertainty via Monte Carlo Analysis................................. 339

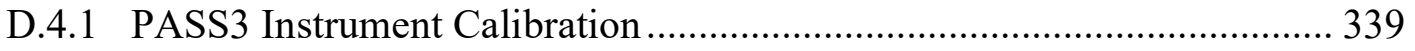

D.4.1.1 Employed Calibrations ........................................................ 343

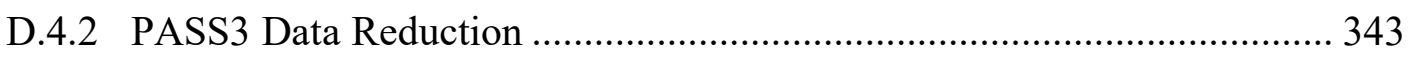

D.4.2.1 Optical Fouling Correction ....................................................... 343

D.4.2.2 Monte Carlo Computation of Absorption Coefficients .................. 347 
D.4.3 OCEC Instrument Calibration ................................................................. 348

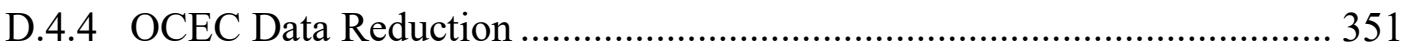

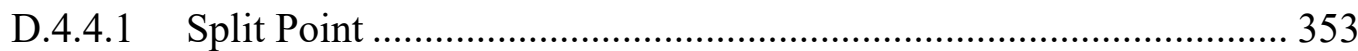

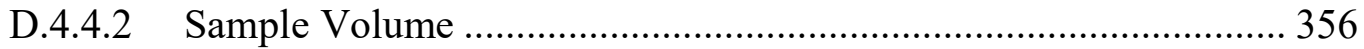

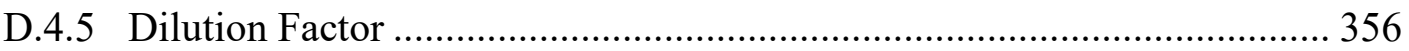

D.4.6 Mass Absorption Cross-Section Calculation and Analysis ....................... 358

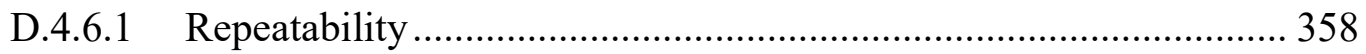

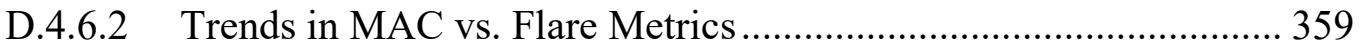

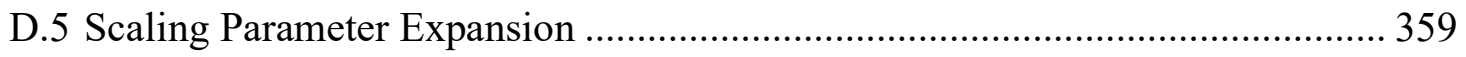

D.5.1 Model-Inferred Flare Flow Rate from MAC Measurements of Weyant

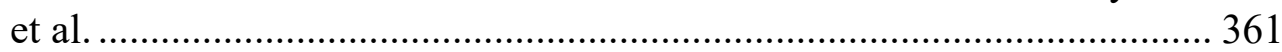




\section{List of Tables}

Table 2.1: Summary of flares measured at OG sites in Veracruz, Mexico and Orellana, Ecuador. Operating conditions of each flare are shown alongside the flare type and key design characteristics.

Table 2.2: Summary of flare gas compositions of the measured flares by chemical group with key flare gas properties relevant to $\mathrm{BC}$ emissions from turbulent diffusion flames. Also shown are mean associated gas compositions from oil and bitumen batteries in Alberta's upstream OG industry (Johnson and Coderre 2012) and an assumed mean associated gas composition from the Bakken region (Wocken et al. 2013)

Table 3.1: Summary of simulation parameters showing (a) flare stack geometry, (b) flare gas flow metrics, and (c) crosswind conditions for the four simulations (I-IV). Flare stack diameters are those of 4" schedule 40 piping commonly used in industry, and flare stack height is typical of flares measured by the authors in Alberta, Canada. Gas was flared at a constant rate across simulations to yield a typical buoyancy-driven flare. Crosswind velocities were selected based on the presented statistics of hourly winds in Edmonton, Alberta from 1992-2016.

Table 4.1: Summary of the sky-LOSA-computed soot emission rates from four previously studied gas flares (Chapter 2, Conrad and Johnson 2017). Results using the single-scattering assumption (i.e., without MS correction) are compared with those computed using the present MS correction model without and with the consideration of residual errors using the error model of Eq. (4.13).

Table 5.1: Summary of flare gas compositions in volume fraction (\%) studied in this experiment and their molecular weight (MW), volumetric higher heating value $\left(\mathrm{HHV}_{\mathrm{V}}\right)$, and molar carbon-hydrogen ratio (CHR). Bold values indicate the inclusion of alkenes (ethylene and propylene).

Table 5.2: Test matrix of the current study indicating the 35 measurements performed. Analysis of experimental repeatability was executed for the AB-M composition at 156 SLPM using the $50.8 \mathrm{~mm}$ diameter stack where a total of six measurements were performed.

Table 5.3: Empirical fit coefficients for the wavelength-dependent BC MAC models plotted in Figure 5.6a, at diagnostic wavelengths of the PASS3 instrument.

Table 6.1: Sky model "groups" derived to propagate error in the CIE sky models through the sky-LOSA algorithm computing soot mass column density.

Table 6.2: Summary of independent, MC-randomized, and pre-computed variables in the GUA.

Table 6.3: Summary of constraints regarding camera pointing relative to the horizon and sun as a function of plume transmittance $\left(\tau_{o b s}\right)$. Compliance with these heuristics ensures that uncertainty in sky-LOSA-computed soot mass column density is low $\left(C V_{95} \leq 17 \%\right)$, regardless of solar zenith angle $\left(Z_{s}\right)$. (N.C. $=$ no constraint). 
Table A.1: The dimensions of flare lines feeding the measured flares in Orellana, Ecuador.

Table A.2: Summary of acquired sky-LOSA image data in Veracruz, Mexico (images were acquired on a cropped sensor to maximize measurement duration).

Table A.3: Summary of acquired sky-LOSA image data in Orellana, Ecuador (all images employed the full-sensor of the sCMOS sky-LOSA camera)....................... 272

Table A.4: Summary of flare-specific mean BC emission rate measurements. .............. 278

Table A.5: Semi-detailed chemical composition (\% Volume) of combined flare gas from all lines feeding each measured flare. (LDL = Lower Detection Limit).......... 281

Table A.6: Summary of input parameter distributions for the Monte Carlo method used to compute the flare gas flow rate and associated uncertainty using optical velocity measurements.

Table A.7: Summary of input parameter distributions for the Monte Carlo method used to compute the flare gas flow rate and associated uncertainty using the tracerdilution technique.

Table A.8: Mean and 95\% confidence intervals for the mean $\mathrm{BC}$ emission rate, mean standard volumetric flow rate, and mean $\mathrm{BC}$ yield of the flares in Orellana, Ecuador measured during the 2015 measurement campaign.

Table A.9: Country-specific flared volumes and representative heating values for Canada, USA, Russia and Ecuador. Flared volumes are sourced from 2012 satellite imagery data analyzed by Elvidge et al. (2015).

Table C.1: Prior distributions of fundamental soot properties derived by Johnson et al. (2013).

Table D.1: Original reported flare gas compositions and CUFF surrogates shown as volume fraction in $\%$. NR $=$ not resolved by analysis or reported by source.

Table D.2: Input parameter distributions for the Monte Carlo analysis of PASS3 calibration uncertainty. Also shown are the sources of the modelled distributions and the means of implementation within the Monte Carlo method.

Table D.3: Typical uncertainties in the calibration of the PASS3 photodiodes and microphone. Repeatability of the calibration procedures are shown alongside the total uncertainty for the PASS3 measurement of absorption.

Table D.4: Input parameter distributions for the Monte Carlo analysis of PASS3 calibration uncertainty. Also shown are the sources of the modelled distributions and the means of implementation within the Monte Carlo method.

Table D.5: Typical uncertainties in the PASS3-measurement of spectral absorption coefficient at standard conditions............................................................................ 348

Table D.6: Typical uncertainty in the calibration of the OCEC instrument. ................... 351

Table D.7: Median uncertainties in OCEC-measured carbon mass. ............................... 356

Table D.8: Typical (median) uncertainties in the calculation of MAC at the

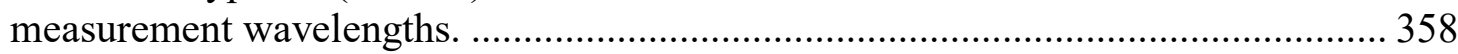

Table D.9: Sensitivity of observed variation in flare BC MAC to specific flare metrics. Trends with fuel chemistry metrics computed at the nominal flaring condition of 
156 SLPM from $50.8 \mathrm{~mm}$ burner, while trends with geometry and flow metrics computed using tests with the AB-M composition. p-values represent the statistical significance of a linear model versus a constant model. Bolded values represent statistically significant trends $(\mathrm{p}<0.05)$.

Table D.10: Summary of data necessary for the estimate of flare gas flow rate for the average MAC results of Weyant et al.'s (2016) study in the Bakken region of North

Dakota. 


\section{List of Figures}

Figure 2.1: Absolute mean BC emission rates measured using sky-LOSA in g/s. Gray bars represent previous measurements in Uzbekistan (Johnson et al. 2011) and Poza Rica, Mexico (Johnson et al. 2013) while the fourteen coloured bars represent measurements in Veracruz, Mexico in 2012 (cyan) and Orellana, Ecuador in 2014 (red) and 2015 (green). Note the logarithmic scale on the vertical axis which is necessary to capture the large variation of emission rates among flares.

Figure 2.2: A $5 \mathrm{~s}$ subset of measured instantaneous BC emission rate (calculated at $50 \mathrm{~Hz}$ ) from Flare $\mathrm{O} 3$ in 2015 showing high variability. The blue symbols represent the instantaneous mean and the shaded region represents the $95 \%$ confidence interval computed using the MC methodology.

Figure 2.3: Mean and 95\% confidence interval of measured BC yields on a mass-pervolume basis shown as a function of volumetric higher heating value. Included are the predictive emission factors using the heating value scaling procedure of CAPP (2007), the correlation developed by McEwen and Johnson (2012), and the emission factor currently used in the GAINS model (Stohl et al. 2013); also shown is the estimated range of yield measurements in the Bakken region by Weyant et al. (2016) based on the span of reported measurements converted from mass-per-mass of hydrocarbons to mass-per-volume of flare gas using the cited range of gas compositions (Wocken et al. 2013).

Figure 3.1: A simplified (idealized straight-path) schematic of radiative effects along an optical line-of-sight axis and entering an optical detector including outscatter of LOS light, absorption of LOS light, inscatter of both skylight and sunlight, and source thermal emission.

Figure 3.2: Orthographic visualization of sample simulation data for each of the four simulation cases, showing the column density of $\mathrm{CO}_{2}$. The red cross in the plume of simulation I represents the optical axis used in Figure 3.5.

Figure 3.3: Summary of Monte Carlo-randomized sky-LOSA camera positioning. The horizontal distance between the camera and flare stack follows a uniform distribution $(\sim \mathcal{U}(25 \mathrm{~m}, 200 \mathrm{~m}))$ and the camera's azimuthal pointing is constrained to be $\pm 30^{\circ}$ from perpendicular to the freestream wind $\left(\sim \mathcal{U}\left(-30^{\circ}, 30^{\circ}\right)\right)$. Blue dots in the image show the $10^{4}$ random camera positions used in the present analysis.

Figure 3.4: Exaggerated example of beam steering through a two-dimensional inhomogeneous scalar field of refractive index. Light propagates towards the plume from pointing $s_{c}$ with an intensity of $I_{\lambda, c}^{o}$ and a direction that makes an angle of $\theta_{\lambda}^{y}$ to the optical axis. As the light traverses through gradients in refractive index that are oblique to its local propagation direction, the path of light is steered towards regions of higher refractive index with an incremental change in its propagation direction related to the perpendicular component of the local gradient. Ultimately, the light enters the camera with a transmitted intensity of $I_{\lambda, c}^{t}$

Figure 3.5: Spectrally resolved total deflection angles for an example optical axis through a plume from simulation I. (a) In the visible spectrum, total deflection 
angle is a smooth function of wavelength. (b-d) In the IR, total defection angle includes substantial spectral dispersion in the vicinity of line transitions. However, when finite spectral resolution of practical detectors is considered through an instrument lineshape (ILS) function, the high-frequency component is smoothed and a low-frequency continuum-like component, which is fairly consistent across the studied absorption bands, dominates. Red crosses indicate wavelengths selected for subsequent analysis.

Figure 3.6: Distributions of total deflection angle of light traversing turbulent flare plumes. (a) Total deflection angle for different turbulent plume conditions (different crosswinds); (b) Total deflection angle over all plume conditions at different wavelengths corresponding to red crosses in Figure 3.5; (c) Probability distributions of deflection angle derived for a subset of the plotted results $\left(\theta_{\lambda}>\right.$ $25 \mu \mathrm{rad}$ ) to yield a conservatively high (on average) model that ignores the distribution mode corresponding to negligibly steered light near the edges of the plume.

Figure 3.7: Logarithmic frequency of non-dimensional correction parameters for steered light through turbulent flare plumes. (a) Absolute value of the deviation of the incident intensity correction $\left(K_{\lambda, o}\right)$ from unity (grey curves) compared with example shot noise limits for a "low-noise" sky-LOSA camera (coloured curves). (b) Absolute value of the deviation of the optical depth correction $\left(K_{\lambda, O D}\right)$ from unity. (c) Absolute value of the deviation of the source intensity correction $\left(K_{\lambda, s}\right)$ from unity compared with instrument noise for commercially available mid-wave hyperspectral imagers (Harley and Gross 2011; Gross et al. 2010b).

Figure 4.1: (a) Schematic of a plume measurement using the sky-LOSA technique beneath the hemispherical sky dome, (b) schematic of light transmission, absorption, outscattering, and inscattering of sky- and sunlight along the optical axis of the camera.

Figure 4.2: Spherical coordinate system for the development of the probabilistic definition of the scattering phase function and "energy distribution function". In an rMCRT analysis, where a MC packet is released from the detector, the angle $\theta$ represents the included angle between the detector LOS and the exiting/outgoing vector (after $n_{s} \geq 1$ total scattering events) and the azimuth $\phi$ represents the latter with respect to the vertical plane. These angles also represent the entry angle of a $\mathrm{MC}$ packet from the sky or sun relative to the detector LOS in the forward direction.

Figure 4.3: (a) Multiple-scattering energy distribution function $\left(p_{m}^{\omega}(\theta, \phi ; b)\right)$ plotted on a logarithmic scale, showing a strong bias toward forward scattering $\left(\theta=0^{\circ}\right)$. Labelled darker regions correspond to a local reduction in the function due to the presence of the plume where, from these directions, light must traverse a larger optical depth along the plume prior to scattering into the optical axis. (b) Singlescattering energy distribution function $\left(p_{I}^{\omega}(\theta ; b)\right.$, computed with RDG-PFA for the selected soot properties), $p_{m}^{\omega}(\theta, \phi ; b)$, and $p_{m}^{\omega}(\theta ; b)$ plotted against scattering angle. As expected, $p_{m}^{\omega}(\theta ; b)$ is upper-bounded by $p_{1}^{\omega}(\theta ; b)$ illustrating the upward bias due to use of the 1SA... 
Figure 4.4: Comparison of $p_{l}^{\omega}(\theta ; b)$ and $p_{m}^{\omega}(\theta ; b)$ for example plume transmittances with variable soot properties. These data show that the difference between $p_{l}^{\omega}(\theta ; b)$ and $p_{m}^{\omega}(\theta ; b)$ is much more sensitive to plume transmittance than soot properties and that there is a high degree of linearity between the two functions.

Figure 4.5: Monte Carlo-computed multiple scattering correction plotted as a function of idealized transmittance (representing plume loading) and coloured according to the soot population's single-scattering albedo. The correction parameter tends to increase with idealized transmittance and approaches unity as the plume becomes optically thin. A simple MS correction model $\left(K_{m}=\left(\tau^{*}\right)^{1 / 2}\right)$ is overlaid in green. ... 145

Figure 4.6: Bias (a) and precision (b) error in soot mass column density computed using the MS correction parameter against the ground truth from LES simulations for each of the $15 \mathrm{CIE}$ sky models as a function of solar position $\left(\alpha_{s}\right.$ and $\left.Z_{s}\right)$ as indicated in the upper left-most panels. These data are used to derive biascorrection $\left(f\left(\alpha_{s}, Z_{s}, a\right)\right)$ and precision error $\left(\sigma\left(\alpha_{s}, Z_{s}, a\right)\right)$ functions for the sky-LOSA error model (Eq. (4.13))

Figure 5.1: Schematic of the Carleton University Flare Facility (CUFF). Flare gas mixtures including $\mathrm{C} 1-\mathrm{C} 7$ alkanes, $\mathrm{C} 2$ and $\mathrm{C} 3$ alkenes, nitrogen, and carbon dioxide are mixed to desired volume fractions and sent to the flare stack. Combustion products and diluent air are captured in a $3.1 \mathrm{~m}$ diameter hexagonal hood and exhausted. Metered volumes of diluted combustion products are sampled from the duct (with optional further dilution) into the CUFF instrument suite.

Figure 5.2: Schematic of the aerosol sampling and dilution tunnel. Combustion products are drawn into the tunnel with a metered rate of filtered dilution air (as necessary). Combustion products fully mix with metered dilution air prior to sampling by instrumentation, which returns sampled volumes to the downstream side of the dilution tunnel, just upstream of the mass flow controller (MFC) controlling total flow rate. Red stars in the figure correspond to sample locations for the quantification of dilution in the sampling tunnel.

Figure 5.3: Studied flare gas compositions shown in the $\mathrm{HHV}_{\mathrm{V}}-\mathrm{CHR}$ domain. Fuels from Ecuador are observed to have a modestly larger CHR than other fieldmeasured compositions due to the high-resolution analysis performed. Experimental composition (X-CP at top right, and $\mathrm{X}-\mathrm{C} 2$ ) deviate largely from fieldobserved compositions, offering an extreme perspective on the effect of fuel composition on flare BC MAC.

Figure 5.4: (a) Elemental carbon to total carbon (EC/TC) ratio in the flare combustion products where shaded area indicates previous estimates from McEwen \& Johnson based on LII and gravimetric measurements (McEwen and Johnson 2012). (b) Raw concentrations of EC and $\mathrm{OC}$ in the collected product plume showing consistent trends of EC concentrations with flare gas heating value. Arrows identify the condition noted in text with higher relative OC likely attributable to the onset of flame liftoff.

Figure 5.5: Sensitivity of BC MAC measurements to flare gas composition and flaring conditions. a) Sensitivity to composition, evaluated for the 15 compositions at the nominal average condition (50.8 $\mathrm{mm}$ burner and $156 \mathrm{SLPM}$ ) shown as a function 
of volumetric higher heating value. b) Sensitivity to flaring conditions, evaluated using the "median" Alberta composition (AB-M). Data at 96 and 216 SLPM in b) are slightly shifted $( \pm 4$ SLPM) for visibility purposes.

Figure 5.6: Proposed predictive correlation for wavelength dependent BC MAC. (a) Wavelength specific power-law predictive models (see Table 5.3) and corresponding 95\% confidence intervals (dark shading) and prediction intervals (light shading). (b) Generalized model for BC MAC at varying wavelength.

Figure 5.7: Absorption by $\mathrm{BC}$ calculated using Mie theory for a range of monomer diameters and refractive indices typical of combustion-generated BC. (a) Volumenormalized absorption cross-section at $550 \mathrm{~nm}$ vs. monomer diameter, coloured by absorption refractive index function and (b) AÅE vs. monomer diameter, coloured by absolute refractive index.

Figure 6.1: Sample sky-LOSA image of the flare and atmospheric plume, which is under slight crosswind in this example. A control surface $(C)$ is shown in blue, which is defined by its constant radius $(r)$ and the angle $\phi$. At each point on the control surface, the mass column density $\left(\rho_{s}^{\prime}(r, \phi)\right.$, not shown) is computed via careful consideration of radiative effects along the lines-of-sight (perpendicular to the image plane) that compose the control surface. Additionally, the path-averaged normal plume velocity $\left(u_{n}(r, \phi)\right)$ is computed via image correlation velocimetry. The instantaneous mass emission rate is computed by integrating the product of these over the control surface defined by $r d \phi$.

Figure 6.2: Schematic of a sky-LOSA measurement under the hemispherical sky dome showing the camera's optical axis relative to the horizon $(\beta)$, sun $\left(\alpha_{s}, \theta_{s}, Z_{s}\right)$, and example sky element $(\alpha, \theta, Z)$ (taken from Chapter 4, Conrad et al. 2020b).

Figure 6.3: Example MC results at solar noon on the summer solstice in Fort St. John, British Columbia - solar zenith $\left(Z_{s}\right)$ of $32.8^{\circ}$ - for a plume of $90 \%$ observed plume transmittance $\left(\tau_{o b s}\right)$. Subplots (a)-(d) show the relative uncertainty $\left(C V_{95}\right)$ of soot mass column density as a function of relative solar azimuth $\left(\alpha_{s}\right)$ and camera inclination $(\beta)$ for sky groups $\mathrm{A}-\mathrm{D}$, respectively. Contour lines in subplot (d) show the scattering angle of sunlight into the sky-LOSA camera $\left(\theta_{s}\right)$.

Figure 6.4: Central tendency (median; left logarithmic axis) and relative uncertainty $\left(C V_{95}\right.$; right linear axis) of the "energy distribution function" $(E D F=\omega(b) p(\theta, b) / 4 \pi)$ that dictates the fraction of incident light energy scattered through angle $\theta$ by soot. The magnitude and uncertainty in the EDF are much larger in the forward scattering direction (small $\theta$ ). As the scattering direction exceeds $\sim 90^{\circ}$ however, the relative uncertainty in the EDF approaches a constant minimum, implying that the relative uncertainty in $S_{1, \text { sun }}$ is minimized for $\theta_{s} \sim 90^{\circ}$. The red line shows that relative uncertainty is within $1 \%$ of the minimum if $\theta_{s}>60^{\circ}$.

Figure 6.5: Percentage relative uncertainty in soot mass column density for a relative solar azimuth $\left(\alpha_{s}\right)$ of $60^{\circ}$ averaged over all solar zenith angles $\left(Z_{s}\right)$ as a function of camera inclination angle $(\beta)$. Data are plotted for each sky group for observed transmittances $\left(\tau_{o b s}\right)$ of 0.25 to 0.95 in subplots (a)-(e). For sky groups A and B (representing overcast and partly cloudy skies), uncertainty can be slightly reduced by ensuring the camera inclination angle exceeds approximately $15^{\circ}$. For sky 
groups $\mathrm{C}$ and $\mathrm{D}$ (representing clear skies), minimal uncertainty is achieved at camera inclination of $9.75-12.50^{\circ}$, and uncertainty can drastically increase for camera inclination increase beyond $20^{\circ}$ - however, the effect becomes muted for more optically thick plumes (lower $\tau_{o b s}$ ). These observations support the general heuristic for clear skies that the camera inclination angle should kept below $\sim 20^{\circ}$, especially for lightly and moderately sooting flares.

Figure 6.6: Flowchart of the main procedure of the SetupSkyLOSA software tool. The user provides the location, Gregorian date, time zone, and local time, which are used to compute the corresponding solar position using the Solar Position Algorithm of the National Renewable Energy Laboratory (NREL, Reda and Andreas, 2008). Then, with data on ambient conditions and observed plume transmittance, the software tool plots the desired statistic of soot mass column density over the $\alpha_{N}-\beta$ domain. Additional utilities include the overlay of the image sensor and optimal positioning relative to the wind on the plotted statistic, in addition to the "maximizer" and "positioner" utilities, which are not shown in the figure. The latter two utilities permit a user to identify acceptable camera positions/pointings over a measurement period and output this data in. $\mathrm{kml}$ format for use with mapping software such as Google Earth.

Figure 6.7: (a) Location of the Atasta Gas Processing and Transport Centre in the Mexican state of Campeche, $35 \mathrm{~km}$ West of Ciudad (Cd) del Carmen and approximately $5 \mathrm{~km}$ South of the Bay of Campeche. (b) Location of the flare that is the focus of the case study located in the Northwest corner of the site amongst other flaring infrastructure.

Figure 6.8: $(\mathrm{a}-\mathrm{d})$ Relative uncertainty $\left(\mathrm{CV}_{95}\right)$ in soot mass column density as a function of camera inclination $(\beta)$ and pointing $\left(\alpha_{s, N}\right)$, maximized over the measurement day for sky groups $\mathrm{A}-\mathrm{D}$, respectively, given an observed plume transmittance $\left(\tau_{o b s}\right)$ of 0.90. Overlaid in the figures is the path of the sun over the day (which approximately reaches $Z_{s}=0^{\circ}$ at 13:05 Central Daylight Time) in addition to a contour of $C V_{95}=16.5 \%$. (e) Permissible regions for sky-LOSA camera positioning relative to the studied flare stack for sky groups $A-D$. The black line shows the intersection of these regions which is a $\sim 600 \mathrm{~m}^{2}$ that the sky-LOSA camera can be positioned to minimize measurement uncertainty, regardless of sky conditions.

Figure A.1: The locations of measured flares in Veracruz, Mexico. (a) The location of Coatzacoalcos and Minatitlán within the state of Veracruz and (b) The location of measured flares with respect to Coatzacoalcos and Minatitlán.

Figure A.2: Sample colour images of the five measured flares in Veracruz, Mexico. (a) Flare V1 (pit flare) showing how visible plume appeared upon arrival; (b) Flare V1 shown as large, 24 hour duration flaring event was beginning (flame is visible in bottom right of image); (c) Flare V2 (enclosed steam-assisted ground flare); (d) Flare V3 (pit flare); (e) Flare V4 (steam-assisted vertical stack flare); and (f) Flare V5 (pit flare)

Figure A.3: Sample images of Flare V1 at various times during 24-hour duration large-volume flaring event. (a) Broadband image of the flare shortly after start of the large flaring event, taken from approximately $158 \mathrm{~m} \mathrm{NE}$ of the flare pit. The 
sun is behind the plume in the top-left corner of the image and is completely blocked by the plume. Given that the optical density of the visibly accessible plume region from this location was sufficient to fully attenuate direct solar radiation, skyLOSA measurements were unfortunately not possible. (b-d) Grayscale images taken from a different set-up location ( $281 \mathrm{~m} \mathrm{SE}$ of the flare pit) approximately 22 hours later as the large-scale flaring event ended. Three independent sky-LOSA measurements were completed over an eleven-minute period and sample images $(b-d)$ are selected from each of these measurements. From these images, it is apparent that the black carbon emissions were rapidly dropping alongside a fieldobserved decrease in audible flare gas flow rate and visible flame size.

Figure A.4: (a) The location of Ecuador's capital, Quito, and Coca within Ecuador. (b) The location of measurements sites with respect to Coca.

Figure A.5: Sample colour images of the measured flares in Petroamazonas' Auca field near Coca, Ecuador. (a) Flare O1 (measured flare is on the right); (b) Flare $\mathrm{O} 2$ (measured flare is on the right); (c) Flare O3; and (d) Flare O4.

Figure A.6: Image showing the nozzle arrangement at the base of Flare $\mathrm{O} 1$ used to induce partial premixing of air into the flare gas prior to combustion.

Figure A.7: (a) Mean BC emission rate data over $1 \mathrm{~s}$ intervals (mean from MC results) for Flare O3 (2015) over the ten-minute analysis period alongside, (b) a $5 \mathrm{~s}$ subset of these data plotted at the data acquisition rate $(50 \mathrm{~Hz})$ with grey shading showing the $95 \%$ CIs computed using the described MC methodology. The stochastic nature of the instantaneous $\mathrm{BC}$ emission rate is clearly visible, where oscillations in the data indicate a highly turbulent atmospheric plume.

Figure A.8: Cumulative distribution function of instantaneous $\mathrm{BC}$ emission rates from each of the measured flares in the current data set. Except for Flare V2 (green), all flares exhibited a positive skewness (as quantified in Table A.4). The measured instantaneous BC emission rates of Flare O2 in 2015 (shown in red) were highly non-stationary as well as strongly-skewed such that $10 \%$ of the instantaneous data were responsible for $56 \%$ of total emissions during the measurement.

Figure B.1: Example results of total deflection angle at $15.456 \mu \mathrm{m}$ for lines-of-sight intersecting the flare plume from simulation I. a) Nominal total deflection angle (in mrad) while ignoring the presence of soot particulate and b) the change in the total deflection angle (in $\mu \mathrm{rad}$ ) due to the inclusion of soot particulate, where soot particulate is modelled by scaling carbon dioxide concentrations to yield a simulation-maximum in-flame soot volume fraction of $10 \mathrm{ppm}$. This example shows the negligible effect of soot particulate on beam steering through flare plumes.

Figure B.2: MC-derived cumulative distribution of the total deflection angle (a) and average total deflection angle (b) for added turbulence at listed values of grid resolution and turbulence intensity. Cumulative distribution of the OD correction parameter (c) and average of the parameter's deviation from unity (d) for added turbulence as in $(a, b)$. Vertical red lines $(a, c)$ correspond to the nominal result on the coarse, raw computational grid and vertical blue lines $(b, d)$ correspond to the required grid resolution to resolve turbulence of $4 \mathrm{~mm}$ in scale. 
Figure C.1: Coordinate system used to describe the polarization state of light. Propagation of light is in the $n$-direction $(n=v \times h=a \times b$, out of the page) $) \ldots \ldots . . .306$

Figure C.2: Definition of the scattering plane, scattering angle $(\theta)$, and polarization coordinate system for a scattering event with incident and outgoing vectors indicated by subscripts " $i$ " and "o", respectively.

Figure C.3: Simplified schematic of single-soot scattered skylight using the Rayleigh sky model. Solar radiation propagates along $n_{0}$ and is scattered by atmospheric aerosol through angle $\chi$ in the direction of $n_{l}$. Diffusely scattered solar radiation (skylight) travels a distance of $c$ towards the plume and is scattered by soot through angle $\theta$ in the direction $n_{2}$, towards the detector. Also shown in the figure are the scattering planes and v-axes for the scattering events. The angle $\gamma_{2,0}$ is the angle of rotation necessary to change the reference of the skylight's Stokes vector (i.e., from $s_{1,0}$ to $s_{1,2}$ ).

Figure C.4: Flowchart describing the reverse Monte Carlo "ray tracing" (rMCRT) procedure. For an a given detector position, line-of-sight, and plume, the rMCRT procedure provides a statistically representative set of paths that ambient light might travel into the detector.

Figure C.5: Notation for the forward modelling of sunlight and skylight along a "reversed" rMCRT-computed path. Sub- and superscripts "n" are omitted for readability....

Figure C.6: (a) Idealized transmittance as a function of the observed transmittance for a range of 1SA-estimated inscattering source terms with MS correction using the present simple model $K_{m}=\left(\tau^{*}\right)^{1 / 2}$. (b) Same as (a) but assuming $K_{m}=1$ as in skyLOSA analyses to date (Chapter 2, Conrad and Johnson 2017; Johnson et al. 2013).

Figure D.1: Carleton University Flare Facility (CUFF). $(a, b)$ An operational flare of $\sim 1.5 \mathrm{~m}$ in length located in the recessed flooring of the facility (outer concentric settling screen removed for visibility). (c) Broader perspective of the facility showing the $40 \mathrm{~cm}$ diameter insulated duct used to transfer emissions to the diagnostic instruments and exhaust combustion products from the laboratory. (d) Instrumentation suite for the calculation BC MAC (PASS3 and OCEC instruments) showing the location of the sampling tunnel and a high-level plumbing schematic

Figure D.2: Refractive indices of light-absorbing carbon (as summarized in Table 4 of Bond and Bergstrom (2006) with respect to the inequality in Eq. (D.5). When the inequality is satisfied (green region), an independent increase in the imaginary refractive index (consistent with graphitization) yields simultaneous increases in the absorption refractive index function and absolute refractive index, which is suggested to occur by the observations of the current study.

Figure D.3: Schematic of the optical path through the PASS3 measurement cell. Laser power is attenuated at the window, through the BC-laden cell, and at the prism prior to detection by the photodiodes. Attenuation at the mirror and within the integrating sphere are constant over time and the photodiodes are calibrated considering this attenuation. 
Figure D.4: An example AVEC plot showing laser attenuation at $632.8 \mathrm{~nm}$ against cumulative evolved carbon from the quartz filter. Colouring of the points represents the instantaneous measurement of front oven (filter) temperature. The inflection in the curve (shown in the green circle) indicates the split point of the analysis. . 


\section{Nomenclature}

\section{Acronym Description}

1SA Single-scattering assumption

AÅE Absorption Ångström exponent

AFE Absorption forcing efficiency

AVEC Attenuation vs. evolved carbon

BCEI Black carbon emissions inventory (Canada)

CAPP Canadian Association of Petroleum Producers

CCAC Climate and Clean Air Coalition (United Nations Environment

Programme)

CDF Cumulative distribution function

CEA Combustion equilibrium with applications

CHR Carbon-hydrogen ratio

CI Confidence interval

CIE Commission International de l'Eclairage

CM-LHS Combined multiple-Latin hypercube sampled

CMOS Complementary metal-oxide-semiconductor

CUFF Carleton University flare facility

$\mathrm{CV}$ Coefficient of variation

DRF Direct radiative forcing

$\mathrm{E}$

$\mathrm{ECCC}$

Enclosed

EDF Energy distribution function

EPA Environmental Protection Agency (United States)

ET Extra-terrestrial

GAINS

GEV

GGFR

GUA

$\mathrm{HHV}_{\mathrm{V}}$

Greenhouse gas

Generalized extreme value

Global Gas Flaring Reduction partnership (World Bank)

General uncertainty analysis

Volumetric higher heating value

High resolution transmission spectroscopic database

Inverse cumulative distribution function

ICDF

$\mathrm{ICV}$

Image correlation velocimetry

ILS

Instrument lineshape function

IPCC

IPIECA

IR Infrared

LDL Lower Detection Limit

LES Large-eddy simulation

LII Laser-induced incandescence

LOESS Locally estimated scatterplot smoothing

LOS Line-of-sight
First use:

Page

51

164

37

174

45

57

44

279

360

169

152

43

64

63

164

216

37

60

45

219

42

209

58

274

44

53

170

91

274

61

100

57

41

91

281

89

42

147

61 


\begin{tabular}{|c|c|c|}
\hline Acronym & Description & $\begin{array}{c}\text { First use: } \\
\text { Page }\end{array}$ \\
\hline LOSA & Line-of-sight attenuation & 59 \\
\hline MAC & Mass-normalized absorption cross-section & 49 \\
\hline $\mathrm{MC}$ & Monte Carlo & 51 \\
\hline MCRT & Monte Carlo ray tracing & 118 \\
\hline MFC & Mass flow controller & 166 \\
\hline MFM & Mass flow meter & 356 \\
\hline MS & Multiple scattering & 51 \\
\hline MW & Molecular weight & 169 \\
\hline NASA & National Aeronautics and Space Administration (United States) & 360 \\
\hline NDIR & Non-dispersive infrared & 348 \\
\hline $\mathrm{NIOSH}$ & National Institute of Occupational Safety and Health (United States) & 352 \\
\hline NOAA & National Oceanic and Atmospheric Administration (United States) & 40 \\
\hline NREL & National Renewable Energy Laboratory (United States) & 228 \\
\hline NSERC & Natural Sciences and Engineering Research Council (Canada) & 78 \\
\hline OCEC & Organic carbon/elemental carbon & 172 \\
\hline OD & Optical depth & 109 \\
\hline OG & Oil and gas & 38 \\
\hline OP-FTIR & Open-path Fourier transform infrared & 80 \\
\hline PASS3 & Three-wavelength photoacoustic soot spectrometer & 172 \\
\hline PEMEX & Petróleos Mexicanos (Mexican Petroleum) & 263 \\
\hline PM & Particulate Matter & 36 \\
\hline $\mathrm{PM}_{2.5}$ & Particulate matter less than $2.5 \mu \mathrm{m}$ in size & 55 \\
\hline $\mathrm{PP}$ & Partially premixed & 60 \\
\hline RDG & Rayleigh-Debye-Gans (as in RDG approximation) & 308 \\
\hline $\mathrm{RDG}^{-}(\mathrm{P}) \mathrm{FA}$ & $\begin{array}{l}\text { Rayleigh-Debye-Gans light scattering theory for (polydisperse) fractal } \\
\text { aggregates }\end{array}$ & 61 \\
\hline rMCRT & Reverse Monte Carlo ray tracing & 133 \\
\hline RTE & Radiative transfer equation & 83 \\
\hline SA & Steam-assisted & 60 \\
\hline Sky-LOSA & Line-of-sight attenuation using skylight & 43 \\
\hline SLPM & Standard litres per minute & 164 \\
\hline SNR & Signal-to-noise ratio & 112 \\
\hline SPA & Solar position algorithm & 228 \\
\hline SPF & Scattering phase function & 207 \\
\hline SVF & Soot volume fraction & 293 \\
\hline TLPM & Thermal laser power meter & 340 \\
\hline $\mathrm{UCF}$ & Uintah computational framework & 89 \\
\hline UNECE & United Nations Economic Commission for Europe & 44 \\
\hline UOG & Upstream oil and gas & 39 \\
\hline
\end{tabular}

\begin{tabular}{clc}
\hline $\begin{array}{c}\text { Chemical } \\
\text { Symbols }\end{array}$ & Description & $\begin{array}{c}\text { First use: } \\
\text { Page }\end{array}$ \\
\hline $\mathrm{BC}$ & Black carbon & 36 \\
$\mathrm{CH}_{4}$ & Methane & 58
\end{tabular}




\begin{tabular}{|c|c|c|c|}
\hline $\begin{array}{l}\text { Chemical } \\
\text { Symbols }\end{array}$ & Description & & $\begin{array}{c}\text { First use: } \\
\text { Page }\end{array}$ \\
\hline $\mathrm{C}_{2} \mathrm{H}_{6}$ & Ethane & & 90 \\
\hline $\mathrm{C}_{3} \mathrm{H}_{8}$ & Propane & & 90 \\
\hline $\mathrm{C}_{4} \mathrm{H}_{10}$ & Butane & & 166 \\
\hline $\mathrm{CO}$ & Carbon monoxide & & 91 \\
\hline $\mathrm{CO}_{2}$ & Carbon dioxide & & 55 \\
\hline $\mathrm{EC}$ & Elemental carbon & & 172 \\
\hline $\mathrm{H}_{2}$ & Hydrogen & & 66 \\
\hline $\mathrm{H}_{2} \mathrm{O}$ & Water & & 91 \\
\hline $\mathrm{H}_{2} \mathrm{~S}$ & Hydrogen sulphide & & 58 \\
\hline $\mathrm{He}$ & Helium & & 66 \\
\hline $\mathrm{MnO}_{2}$ & Manganese oxide & & 348 \\
\hline $\mathrm{N}_{2}$ & Nitrogen & & 58 \\
\hline $\mathrm{NO}_{2}$ & Nitrogen dioxide & & 341 \\
\hline $\mathrm{O}_{2}$ & Oxygen & & 91 \\
\hline $\mathrm{OC}$ & Organic carbon & & 172 \\
\hline $\mathrm{PC}$ & Pyrolyzed carbon & & 351 \\
\hline $\mathrm{TC}$ & Total Carbon & & 175 \\
\hline $\begin{array}{c}\text { Latin } \\
\text { Symbol }\end{array}$ & Description & Units & $\begin{array}{c}\text { First use: } \\
\text { Page }\end{array}$ \\
\hline \multirow[t]{2}{*}{$a$} & $\begin{array}{l}\text { Chapter 3: Shape parameter of the type II extreme } \\
\text { value distribution }\end{array}$ & {$[-]$} & 105 \\
\hline & Chapter 4 and 6: CIE sky model index & {$[-]$} & 129 \\
\hline$a_{\lambda}, b_{\lambda}, c_{\lambda}$ & Spectral coefficients for soot/BC MAC model & [var.] & 181 \\
\hline $\boldsymbol{a}, \boldsymbol{b}, \boldsymbol{h}, \boldsymbol{v}$ & Polarization axes & {$[-]$} & 306 \\
\hline$A \AA ̊ E$ & Absorption Ångström exponent & {$[-]$} & 164 \\
\hline$b$ & $\begin{array}{l}\text { Scale parameter of the type II extreme value } \\
\text { distribution }\end{array}$ & {$[-]$} & 105 \\
\hline $\boldsymbol{b}$ & $\begin{array}{l}\text { Fundamental properties of soot/BC contributing to } \\
\text { aggregate optical properties }\end{array}$ & [var.] & 129 \\
\hline$B$ & Radiant intensity of inscattered skylight & {$\left[\mathrm{W} \mathrm{sr}{ }^{-1}\right]$} & 62 \\
\hline$c$ & Speed of light in vacuum, $c=299792458 \mathrm{~m} \mathrm{~s}^{-1}$ & {$\left[\mathrm{~m} \mathrm{~s}^{-1}\right]$} & 292 \\
\hline$c_{\lambda}$ & $\begin{array}{l}\text { Spectrally dependent curved path of beam-steered } \\
\text { light }\end{array}$ & {$[-]$} & 85 \\
\hline \multirow[t]{2}{*}{ C } & $\begin{array}{l}\text { Chapter 2: Radiant intensity of inscattered } \\
\text { sunlight }\end{array}$ & {$\left[\mathrm{W} \mathrm{sr}^{-1}\right]$} & 62 \\
\hline & Chapter 6: Control surface & {$[-]$} & 200 \\
\hline CHR & Carbon-hydrogen ratio & {$[-]$} & 170 \\
\hline$C V_{95}$ & $\begin{array}{l}\text { Estimator of coefficient of variation for soot/BC } \\
\text { mass column density using } 95 \% \mathrm{CI}\end{array}$ & {$[-]$} & 217 \\
\hline$d_{p}$ & $\begin{array}{l}\text { Soot/black carbon primary particle/monomer } \\
\text { diameter }\end{array}$ & {$[\mathrm{m}]$} & 187 \\
\hline$d_{s}$ & Flare stack diameter & {$[\mathrm{m}]$} & 359 \\
\hline$D_{f}$ & Fractal (Hausdorff) dimension & {$[-]$} & 305 \\
\hline
\end{tabular}




\begin{tabular}{|c|c|c|c|}
\hline $\begin{array}{l}\text { Latin } \\
\text { Symbol }\end{array}$ & Description & Units & $\begin{array}{l}\text { First use: } \\
\text { Page }\end{array}$ \\
\hline$D_{h}$ & Diffuse (skylight) horizontal irradiance & {$\left[\mathrm{W} \mathrm{m}^{-2}\right]$} & 209 \\
\hline$E_{S}$ & Ground-level solar normal irradiance & {$\left[\mathrm{W} \mathrm{m}^{-2}\right]$} & 63 \\
\hline$E_{s h, o}$ & Extra-terrestrial solar horizontal irradiance & {$\left[\mathrm{W} \mathrm{m}^{-2}\right]$} & 209 \\
\hline$E\left(m_{\lambda}\right)$ & $\begin{array}{l}\text { Absorption function of the spectral complex } \\
\text { refractive index }\end{array}$ & {$[-]$} & 62 \\
\hline $\boldsymbol{E}$ & Electric field vector & {$\left[\mathrm{V} \mathrm{m}^{-1}\right]$} & 306 \\
\hline$E C / T C$ & Elemental-to-total carbon ratio & {$[-]$} & 175 \\
\hline$E D F$ & Energy distribution function & {$\left[\mathrm{sr}^{-1}\right]$} & 219 \\
\hline$E F$ & Soot/BC emission factor & {$\left[\mathrm{g} \mathrm{m}^{-3}\right]$} & 57 \\
\hline$f$ & $\begin{array}{l}\text { Chapter 6: Camera lens focal length } \\
\text { Appendix D: Dilution factor of aerosol sampling } \\
\text { and dilution tunnel }\end{array}$ & {$[\mathrm{mm}]$} & $\begin{array}{l}226 \\
357\end{array}$ \\
\hline$f_{s}$ & Stoichiometric mixture fraction & {$[-]$} & 359 \\
\hline$f(\cdot)$ & $\begin{array}{l}\text { Section 4.5.1: Probability density function } \\
\text { Section 4.5.2.1: Bias-correction function }\end{array}$ & $\begin{array}{c}{[\text { var. }]} \\
{[-]}\end{array}$ & $\begin{array}{l}135 \\
150\end{array}$ \\
\hline$f_{\text {pol }}(\cdot)$ & Polarization correction function & {$[-]$} & 129 \\
\hline$F\left(m_{\lambda}\right)$ & $\begin{array}{l}\text { Scattering function of the spectral complex } \\
\text { refractive index }\end{array}$ & {$[-]$} & 305 \\
\hline$F r_{f}$ & Fire Froude number & {$[-]$} & 360 \\
\hline $\mathcal{F}^{-1}(q)$ & Inverse cumulative distribution function & {$[-]$} & 217 \\
\hline$g$ & $\begin{array}{l}\text { Chapter 4: Asymmetry parameter of the } \\
\text { scattering phase function }\end{array}$ & {$[-]$} & 144 \\
\hline & $\begin{array}{l}\text { Appendix D: Gravitational constant, } g=9.81 \\
\mathrm{~m} \mathrm{~s}^{-2}\end{array}$ & {$\left[\mathrm{~m} \mathrm{~s}^{-2}\right]$} & 361 \\
\hline $\boldsymbol{h}_{x, y}$ & $\begin{array}{l}\text { Horizontal polarization axes (for incident direction } \\
x \text { relative to outgoing direction } y \text { ) }\end{array}$ & {$[\mathrm{m}]$} & 307 \\
\hline$h(\cdot)$ & Dummy function (integration by substitution) & {$\left[\mathrm{g} \mathrm{m}^{-2}\right]$} & 303 \\
\hline$H$ & Horizontal stand-off distance from flare stack & {$[\mathrm{m}]$} & 226 \\
\hline$H(x)$ & Heaviside (unit) step function & {$[-]$} & 344 \\
\hline$H H V_{V}$ & Volumetric higher heating value & {$\left[\mathrm{MJ} \mathrm{m}^{-3}\right]$} & 57 \\
\hline$I^{o}$ & Incident intensity & {$\left[\mathrm{W} \mathrm{m}{ }^{-2} \mathrm{sr}^{-1}\right]$} & 124 \\
\hline$I^{t}$ & Transmitted intensity & {$\left[\mathrm{W} \mathrm{m}{ }^{-2} \mathrm{sr}^{-1}\right]$} & 124 \\
\hline$I_{\text {LOS }}^{o}$ & LOS incident intensity & {$\left[\mathrm{W} \mathrm{m}{ }^{-2} \mathrm{sr}^{-1}\right]$} & 62 \\
\hline$I_{\text {sky }}$ & Directionally dependent diffuse skylight intensity & {$\left[\mathrm{W} \mathrm{m}{ }^{-2} \mathrm{sr}^{-1}\right]$} & 63 \\
\hline$I_{\text {sky }}^{s, n}$ & Skylight source intensity for the $n^{\text {th }}$ photon packet & {$\left[\mathrm{W} \mathrm{m}^{-2} \mathrm{sr}^{-1}\right]$} & 322 \\
\hline$I_{\text {sun }}^{\text {s,n }}$ & Sunlight source intensity for the $n^{\text {th }}$ photon packet & {$\left[\mathrm{W} \mathrm{m}{ }^{-2} \mathrm{sr}^{-1}\right]$} & 324 \\
\hline$I_{d}$ & Intensity incident upon detector & {$\left[\mathrm{W} \mathrm{m}{ }^{-2} \mathrm{sr}^{-1}\right]$} & 315 \\
\hline \multirow[t]{2}{*}{$I_{x}$} & $\begin{array}{l}\text { Section C.3.2: Intensity relative to polarization } \\
\text { axis, } x \in\{a, b, h, v\}\end{array}$ & {$\left[\mathrm{W} \mathrm{m}{ }^{-2} \mathrm{sr}^{-1}\right]$} & 308 \\
\hline & $\begin{array}{l}\text { Section C.3.3: Intensity of light from sun to } \\
\text { detector: extra-terrestrial solar radiation }(x=0) \text {, } \\
\text { skylight }(x=1) \text {, single-soot-scattered skylight } \\
(x=2)\end{array}$ & {$\left[\mathrm{W} \mathrm{m}{ }^{-2} \mathrm{sr}^{-1}\right]$} & 310 \\
\hline$\overline{I^{s}}$ & LOS path-averaged source intensity & {$\left[\mathrm{W} \mathrm{m}{ }^{-2} \mathrm{sr}^{-1}\right]$} & 125 \\
\hline$\overline{I_{1}^{S}}$ & LOS path-averaged source intensity using 1SA & {$\left[\mathrm{W} \mathrm{m}^{-2} \mathrm{sr}^{-1}\right]$} & 128 \\
\hline
\end{tabular}




\begin{tabular}{|c|c|c|c|}
\hline $\begin{array}{c}\text { Latin } \\
\text { Symbol }\end{array}$ & Description & Units & $\begin{array}{c}\text { First use: } \\
\text { Page }\end{array}$ \\
\hline$\overline{I_{m}^{s}}$ & $\begin{array}{l}\text { LOS path-averaged source intensity under MS } \\
\text { conditions }\end{array}$ & {$\left[\mathrm{W} \mathrm{m}{ }^{-2} \mathrm{sr}^{-1}\right]$} & 130 \\
\hline$\overline{I_{m, s k y}^{s}}$ & $\begin{array}{l}\text { Skylight component of the LOS path-averaged } \\
\text { source intensity under MS conditions }\end{array}$ & {$\left[\mathrm{W} \mathrm{m}{ }^{-2} \mathrm{sr}^{-1}\right]$} & 322 \\
\hline$\overline{I_{m, s u n}^{S}}$ & $\begin{array}{l}\text { Sunlight component of the LOS path-averaged } \\
\text { source intensity under MS conditions }\end{array}$ & {$\left[\mathrm{W} \mathrm{m}{ }^{-2} \mathrm{sr}^{-1}\right]$} & 324 \\
\hline$I_{\lambda}$ & Spectral intensity & {$\left[\mathrm{W} \mathrm{m} \mathrm{m}^{-2} \mu \mathrm{m}^{-1} \mathrm{sr}^{-1}\right]$} & 344 \\
\hline $\bar{I}_{\lambda}^{c}$ & $\begin{array}{l}\text { Path-averaged incident spectral intensity on } \\
\text { PASS3 sample }\end{array}$ & {$\left[\mathrm{W} \mathrm{m} \mathrm{m}^{-2} \mu \mathrm{m}^{-1} \mathrm{sr}^{-1}\right]$} & 344 \\
\hline$I_{\lambda}^{o}$ & Incident spectral intensity & {$\left[\mathrm{W} \mathrm{m} \mathrm{m}^{-2} \mu \mathrm{m}^{-1} \mathrm{sr}^{-1}\right]$} & 86 \\
\hline$I_{\lambda}^{s}$ & Source spectral intensity & {$\left[\mathrm{W} \mathrm{m} \mathrm{m}^{-2} \mu \mathrm{m}^{-1} \mathrm{sr}^{-1}\right]$} & 84 \\
\hline$I_{\lambda, c}^{o}$ & Incident Spectral intensity (curved path) & {$\left[\mathrm{W} \mathrm{m} \mathrm{m}^{-2} \mu \mathrm{m}^{-1} \mathrm{sr}^{-1}\right]$} & 85 \\
\hline $\bar{I}_{\lambda, c}^{s}$ & $\begin{array}{l}\text { Path-averaged source spectral intensity (curved } \\
\text { path) }\end{array}$ & {$\left[\mathrm{W} \mathrm{m} \mathrm{m}^{-2} \mu \mathrm{m}^{-1} \mathrm{sr}^{-1}\right]$} & 297 \\
\hline$I_{\lambda, c}^{t}$ & Transmitted spectral intensity (curved path) & {$\left[\mathrm{W} \mathrm{m} \mathrm{m}^{-2} \mu \mathrm{m}^{-1} \mathrm{sr}^{-1}\right]$} & 85 \\
\hline$I_{\lambda, \ell}^{o}$ & Incident spectral intensity (linear path) & {$\left[\mathrm{W} \mathrm{m} \mathrm{m}^{-2} \mu \mathrm{m}^{-1} \mathrm{sr}^{-1}\right]$} & 83 \\
\hline $\bar{I}_{\lambda, \ell}^{s}$ & $\begin{array}{l}\text { Path-averaged source spectral intensity (linear } \\
\text { path) }\end{array}$ & {$\left[\mathrm{W} \mathrm{m} \mathrm{m}^{-2} \mu \mathrm{m}^{-1} \mathrm{sr}^{-1}\right]$} & 297 \\
\hline$I_{\lambda, \ell}^{t}$ & Transmitted spectral intensity (linear path) & {$\left[\mathrm{W} \mathrm{m}{ }^{-2} \mu \mathrm{m}^{-1} \mathrm{sr}^{-1}\right]$} & 83 \\
\hline$I_{\lambda, P D}^{c}$ & Spectral intensity incident on PASS3 photodiodes & {$\left[\mathrm{W} \mathrm{m} \mathrm{m}^{-2} \mu \mathrm{m}^{-1} \mathrm{sr}^{-1}\right]$} & 345 \\
\hline$I_{\lambda, P D, i}^{c, n}$ & $\begin{array}{l}\text { Spectral intensity incident on PASS3 photodiodes } \\
\text { during the zeroing period before }(i=1) \text { or after } \\
(i=2) \text { the } \mathrm{n}^{\text {th }} \text { measurement }\end{array}$ & {$\left[\mathrm{W} \mathrm{m}{ }^{-2} \mu \mathrm{m}^{-1} \mathrm{sr}^{-1}\right]$} & 346 \\
\hline$I_{\lambda, s k y}$ & Spectral intensity of skylight & {$\left[\mathrm{W} \mathrm{m} \mathrm{m}^{-2} \mu \mathrm{m}^{-1} \mathrm{sr}^{-1}\right]$} & 107 \\
\hline$I_{b \lambda}$ & Blackbody-emitted spectral intensity & {$\left[\mathrm{W} \mathrm{m} \mathrm{m}^{-2} \mu \mathrm{m}^{-1} \mathrm{sr}^{-1}\right]$} & 112 \\
\hline$I_{T}$ & Turbulence intensity of temperature field & {$[-]$} & 299 \\
\hline$J^{s, m}$ & Mass-normalized source radiant intensity & {$\left[\mathrm{W} \mathrm{sr}{ }^{-1} \mathrm{~g}^{-1}\right]$} & 125 \\
\hline$\overline{J^{s, m}}$ & $\begin{array}{l}\text { LOS path-averaged, mass-normalized source } \\
\text { radiant intensity }\end{array}$ & {$\left[\mathrm{W} \mathrm{sr} \mathrm{g}^{-1} \mathrm{~g}^{-1}\right]$} & 125 \\
\hline$k_{f}$ & Fractal prefactor & {$[-]$} & 305 \\
\hline$K$ & Ratio of $\mathrm{C} 6$ to $\mathrm{C} 6+\mathrm{C} 7$ hydrocarbon species & {$[-]$} & 336 \\
\hline$K_{\lambda, F}$ & PASS3 fouling correction parameter & {$[-]$} & 345 \\
\hline$K_{\lambda, o}$ & $\begin{array}{l}\text { Spectral incident intensity correction for beam } \\
\text { steering }\end{array}$ & {$[-]$} & 86 \\
\hline$K_{\lambda, O D}$ & $\begin{array}{l}\text { Spectral optical density correction for beam } \\
\text { steering }\end{array}$ & {$[-]$} & 87 \\
\hline$K_{\lambda, s}$ & $\begin{array}{l}\text { Spectral source intensity correction for beam } \\
\text { steering }\end{array}$ & {$[-]$} & 87 \\
\hline$K_{m}$ & Multiple scattering correction parameter & {$[-]$} & 130 \\
\hline$\ell$ & Linear/line path through plume & {$[-]$} & 83 \\
\hline$l$ & $\begin{array}{l}\text { Index indicating order of Legendre polynomial or } \\
\text { associated coefficient }\end{array}$ & {$[-]$} & 208 \\
\hline$L_{f}$ & Flame length & {$[\mathrm{m}]$} & 226 \\
\hline$L_{v}$ & Visible/vertical flame length & {$[\mathrm{m}]$} & 181 \\
\hline$L(\boldsymbol{b})$ & $\begin{array}{l}\text { Cut-off index for Fourier-Legendre-expanded } \\
\text { scattering phase function }\end{array}$ & {$[-]$} & 208 \\
\hline
\end{tabular}




\begin{tabular}{|c|c|c|c|}
\hline $\begin{array}{l}\text { Latin } \\
\text { Symbol }\end{array}$ & Description & Units & $\begin{array}{l}\text { First use: } \\
\text { Page }\end{array}$ \\
\hline$m$ & Relative air mass & {$[-]$} & 209 \\
\hline$m_{\lambda}$ & Spectral complex refractive index & {$[-]$} & 62 \\
\hline$m_{C}$ & Mass of carbon & {$[\mathrm{g}]$} & 172 \\
\hline$m_{E C}$ & Mass of elemental carbon & {$[\mathrm{g}]$} & 358 \\
\hline$m_{i j}$ & 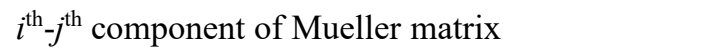 & {$[-]$} & 308 \\
\hline$\dot{m}_{B C}, \dot{m}_{s}$ & Mass emission rate of soot/BC & {$\left[\mathrm{g} \mathrm{s}^{-1}\right]$} & 61 \\
\hline $\mathbf{M}$ & Mueller matrix & {$[-]$} & 308 \\
\hline$\overline{\mathbf{M}}$ & Normalized Mueller matrix & {$[-]$} & 312 \\
\hline $\mathbf{M}_{R A Y}$ & Mueller matrix for Rayleigh (small) particles & {$[-]$} & 312 \\
\hline $\mathbf{M}_{R D G}$ & Mueller matrix under RDG-PFA & {$[-]$} & 314 \\
\hline$M A C_{B C, \lambda}$ & $\begin{array}{l}\text { Spectral mass-normalized absorption cross-section } \\
\text { of soot/BC }\end{array}$ & {$\left[\mathrm{m}^{2} \mathrm{~g}^{-1}\right]$} & 164 \\
\hline$M A C_{B C, \lambda_{o}}$ & $\begin{array}{l}\text { Mass-normalized absorption cross-section of } \\
\text { soot/BC at reference wavelength }\end{array}$ & {$\left[\mathrm{m}^{2} \mathrm{~g}^{-1}\right]$} & 164 \\
\hline$M A C_{B C, 550}$ & $\begin{array}{l}\text { Mass-normalized absorption cross-section of soot/ } \\
\mathrm{BC} \text { at } 550 \mathrm{~nm}\end{array}$ & {$\left[\mathrm{~m}^{2} \mathrm{~g}^{-1}\right]$} & 184 \\
\hline$n$ & $\begin{array}{l}\text { Power-law exponent for mean turbulent velocity } \\
\text { profile in circular duct }\end{array}$ & {$[-]$} & 282 \\
\hline$n_{s}$ & Number of scattering events & {$[-]$} & 135 \\
\hline$\dot{n}_{i}$ & Molar flow rate of species/mixture $i$ & {$\left[\mathrm{kmol} \mathrm{s}^{-1}\right]$} & 285 \\
\hline $\boldsymbol{n}$ & $\begin{array}{l}\text { Chapter } 4 \text { and 6: Normal (unit) vector } \\
\text { Appendix C: Propagation direction of polarized } \\
\text { light }\end{array}$ & $\begin{array}{l}{[-]} \\
{[-]}\end{array}$ & $\begin{array}{l}126 \\
306\end{array}$ \\
\hline $\boldsymbol{n}_{\lambda}$ & $\begin{array}{l}\text { Spectral (unit) vector normal to local light } \\
\text { propagation direction }\end{array}$ & {$[-]$} & 96 \\
\hline $\boldsymbol{n}_{i}$ & Propagation direction of incoming polarized light & {$[-]$} & 306 \\
\hline $\boldsymbol{n}_{o}$ & Propagation direction of outgoing polarized light & {$[-]$} & 306 \\
\hline$N$ & $\begin{array}{l}\text { Number of spherical monomers in soot/BC } \\
\text { aggregate }\end{array}$ & {$[-]$} & 207 \\
\hline$N_{g}$ & Geometric mean (aggregate size) & {$[-]$} & 305 \\
\hline$O D$ & Optical depth & {$[-]$} & 154 \\
\hline$O D_{\lambda}$ & $\begin{array}{l}\text { Total spectral optical depth of PASS } 3 \text { prism and } \\
\text { window }\end{array}$ & {$[-]$} & 345 \\
\hline$O D_{\lambda, p}$ & Spectral optical depth of PASS3 prism & {$[-]$} & 344 \\
\hline$O D_{\lambda, w}$ & Spectral optical depth of PASS3 window & {$[-]$} & 344 \\
\hline$p(\cdot)$ & Scattering phase function & {$\left[\mathrm{sr}^{-1}\right]$} & 129 \\
\hline$p_{\lambda}(\cdot)$ & Spectral scattering phase function & {$\left[\mathrm{sr}^{-1}\right]$} & 296 \\
\hline$p^{\omega}(\cdot)$ & Energy distribution function & {$\left[\mathrm{sr}^{-1}\right]$} & 137 \\
\hline$p_{1}^{\omega}(\cdot)$ & Energy distribution function using the 1SA & {$\left[\mathrm{sr}^{-1}\right]$} & 138 \\
\hline$p_{m}^{\omega}(\cdot)$ & Energy distribution function under MS conditions & {$\left[\mathrm{sr}^{-1}\right]$} & 138 \\
\hline$p_{R A Y}(\cdot)$ & $\begin{array}{l}\text { Scattering phase function for Rayleigh (small) } \\
\text { particles }\end{array}$ & {$\left[\mathrm{sr}^{-1}\right]$} & 312 \\
\hline$P\left(n_{s}\right)$ & Probability of $n_{s}$ scattering events & {$[-]$} & 137 \\
\hline$P$ & Pressure of gaseous mixture & {$[\mathrm{Pa}]$} & 291 \\
\hline $\bar{P}$ & Time-averaged PASS3 cell pressure & {$[\mathrm{Pa}]$} & 341 \\
\hline
\end{tabular}




\begin{tabular}{|c|c|c|c|}
\hline $\begin{array}{l}\text { Latin } \\
\text { Symbol }\end{array}$ & Description & Units & $\begin{array}{l}\text { First use: } \\
\text { Page }\end{array}$ \\
\hline$P_{\lambda, L}$ & Spectral laser power incident on PASS3 sample & [W] & 339 \\
\hline$\overline{P_{\lambda, P D}}$ & $\begin{array}{l}\text { Time-averaged PASS3 photodiode-measured laser } \\
\text { power }\end{array}$ & [W] & 341 \\
\hline$P_{a}$ & PASS3-measured acoustic power & [W] & 339 \\
\hline$P_{l}(\cdot)$ & $1^{\text {th }}$-order Legendre polynomial & {$[-]$} & 208 \\
\hline$P_{m}$ & Measured pressure & {$[\mathrm{Pa}]$} & 283 \\
\hline$P_{o}$ & Standard pressure & {$[\mathrm{Pa}]$} & 283 \\
\hline$q$ & Quantile & {$[-]$} & 275 \\
\hline $\boldsymbol{q}_{d}$ & Stokes vector at ground-based detector & {$\left[\mathrm{W} \mathrm{m}{ }^{-2} \mathrm{sr}^{-1}\right]$} & 315 \\
\hline $\boldsymbol{q}_{x, y}$ & $\begin{array}{l}\text { Stokes vector (for incident direction } x \text { relative to } \\
\text { outgoing direction } y \text { ) }\end{array}$ & {$\left[\mathrm{W} \mathrm{m} \mathrm{m}^{-2} \mathrm{sr}^{-1}\right]$} & 308 \\
\hline $\boldsymbol{q}_{x, y}^{n}$ & $\begin{array}{l}\text { Stokes vector (for incident direction } x \text { relative to } \\
\text { outgoing direction } y \text { ) for the } n^{\text {th }} \text { photon packet } \\
\text { (forward propagation of MCRT analysis) }\end{array}$ & {$\left[\mathrm{W} \mathrm{m} \mathrm{m}^{-2} \mathrm{sr}^{-1}\right]$} & 321 \\
\hline $\boldsymbol{q}_{x, y}^{j, n}$ & $\begin{array}{l}\text { Stokes vector (for incident direction } x \text { relative to } \\
\text { outgoing direction } y \text { ) for the } n^{\text {th }} \text { photon packet } \\
\text { entering at the } j^{\text {th }} \text { scattering event (forward } \\
\text { propagation of MCRT analysis) }\end{array}$ & {$\left[\mathrm{W} \mathrm{m} \mathrm{m}^{-2} \mathrm{sr}^{-1}\right]$} & 323 \\
\hline$\dot{Q}_{t h}$ & Theoretical heat release rate of flame & {$[\mathrm{W}]$} & 181 \\
\hline \multirow[t]{2}{*}{$r$} & $\begin{array}{l}\text { Chapter 6: Radius of control surface in image } \\
\text { plane }\end{array}$ & {$[\mathrm{m}]$} & 200 \\
\hline & $\begin{array}{l}\text { Appendix A: Radial coordinate of cylindrical } \\
\text { coordinate system }\end{array}$ & {$[\mathrm{m}]$} & 282 \\
\hline $\boldsymbol{r}$ & Position vector & {$[\mathrm{m}]$} & 85 \\
\hline $\boldsymbol{r}_{k}$ & $\begin{array}{l}\text { Position vector for the } k^{\text {th }} \text { scattering event } \\
\text { (rMCRT) }\end{array}$ & {$[\mathrm{m}]$} & 316 \\
\hline $\boldsymbol{r}_{k}^{n}$ & $\begin{array}{l}\text { Position vector for the } k^{\text {th }} \text { scattering event of the } n^{\text {th }} \\
\text { photon packet (forward propagation of MCRT } \\
\text { analysis) }\end{array}$ & {$[\mathrm{m}]$} & 320 \\
\hline$R$ & Actual internal radius of duct & {$[\mathrm{m}]$} & 282 \\
\hline$R^{2}$ & Coefficient of determination & {$[-]$} & 73 \\
\hline$R_{g}$ & Radius of gyration of soot/BC aggregate & {$[\mathrm{m}]$} & 329 \\
\hline$R_{L L}$ & Lorentz-Lorenz molar refractivity & {$\left[\mathrm{m}^{3} \mathrm{kmol}^{-1}\right]$} & 290 \\
\hline$R_{o}$ & Nominal internal radius of duct & {$[\mathrm{m}]$} & 283 \\
\hline$R_{u}$ & Universal gas constant, $R_{u}=8314.5 \mathrm{~J} \mathrm{kmol}^{-1} \mathrm{~K}^{-1}$ & {$\left[\mathrm{~J} \mathrm{kmol}^{-1} \mathrm{~K}^{-1}\right]$} & 285 \\
\hline $\mathbf{R}$ & Polarization rotation matrix & {$[-]$} & 314 \\
\hline$s$ & Optical axis pointing & {$[-]$} & 106 \\
\hline $\boldsymbol{s}_{c}$ & Final pointing of optical axis of curved path & {$[-]$} & 107 \\
\hline $\boldsymbol{s}_{k}$ & $\begin{array}{l}\text { Propagation direction of light after the } k^{\text {th }} \\
\text { scattering event (rMCRT) }\end{array}$ & {$[-]$} & 316 \\
\hline $\boldsymbol{s}_{k}^{n}$ & $\begin{array}{l}\text { Propagation direction of light after the } k^{\text {th }} \\
\text { scattering event for the } n^{\text {th }} \text { photon packet (forward } \\
\text { propagation of MCRT analysis) }\end{array}$ & {$[-]$} & 320 \\
\hline $\boldsymbol{s}_{\ell}$ & Pointing of linear path/optical axis & {$[-]$} & 106 \\
\hline$S$ & Inscattering correction & {$[-]$} & 126 \\
\hline$S_{1}$ & Inscattering correction using the $1 \mathrm{SA}$ & {$[-]$} & 129 \\
\hline
\end{tabular}




\begin{tabular}{|c|c|c|c|}
\hline $\begin{array}{c}\text { Latin } \\
\text { Symbol }\end{array}$ & Description & Units & $\begin{array}{l}\text { First use: } \\
\text { Page }\end{array}$ \\
\hline$S_{1, \text { sky }}$ & $\begin{array}{l}\text { Skylight component of the inscattering correction } \\
\text { using 1SA }\end{array}$ & {$[-]$} & 205 \\
\hline$S_{1, \text { sun }}$ & $\begin{array}{l}\text { Sunlight component of the inscattering correction } \\
\text { using } 1 \text { SA }\end{array}$ & {$[-]$} & 205 \\
\hline$S_{i l}$ & Intensity of the $l^{\text {th }}$ transition of gas species $i$ & {$\left[\mathrm{~m} \mathrm{kmol}^{-1}\right]$} & 292 \\
\hline$S_{m}$ & Inscattering correction under MS conditions & {$[-]$} & 130 \\
\hline$T$ & $\begin{array}{l}\text { Chapter 6: Turbidity factor } \\
\text { Elsewhere: Temperature }\end{array}$ & $\begin{array}{c}{[-]} \\
{\left[\mathrm{K},{ }^{\circ} \mathrm{C}\right]}\end{array}$ & $\begin{array}{l}210 \\
112\end{array}$ \\
\hline$T_{a d}$ & Adiabatic flame temperature & {$\left[\mathrm{K},{ }^{\circ} \mathrm{C}\right]$} & 177 \\
\hline$T_{e}$ & Flare gas temperature at the stack exit & {$\left[\mathrm{K},{ }^{\circ} \mathrm{C}\right]$} & 361 \\
\hline$T_{m}$ & Measured temperature & {$\left[\mathrm{K},{ }^{\circ} \mathrm{C}\right]$} & 283 \\
\hline$T_{o}$ & Standard temperature & {$\left[\mathrm{K},{ }^{\circ} \mathrm{C}\right]$} & 283 \\
\hline$T_{o, M F C}$ & Standard temperature of MFC & {$\left[\mathrm{K},{ }^{\circ} \mathrm{C}\right]$} & 285 \\
\hline$T_{r m s}^{\prime}$ & $\begin{array}{l}\text { Fluctuating (root-mean-squared) component of } \\
\text { temperature }\end{array}$ & {$\left[\mathrm{K},{ }^{\circ} \mathrm{C}\right]$} & 299 \\
\hline$T_{\infty}$ & Ambient temperature & {$\left[\mathrm{K},{ }^{\circ} \mathrm{C}\right]$} & 361 \\
\hline $\bar{T}$ & Appendix B: Mean component of temperature & {$\left[\mathrm{K},{ }^{\circ} \mathrm{C}\right]$} & 299 \\
\hline & $\begin{array}{l}\text { Appendix D: Time-averaged PASS3 cell } \\
\text { temperature }\end{array}$ & {$\left[\mathrm{K},{ }^{\circ} \mathrm{C}\right]$} & 341 \\
\hline$u$ & Radially varying flow velocity in circular duct & {$\left[\mathrm{m} \mathrm{s}^{-1}\right]$} & 282 \\
\hline$u_{C L}$ & Centreline flow velocity in circular duct & {$\left[\mathrm{m} \mathrm{s}^{-1}\right]$} & 282 \\
\hline$u_{e}$ & Flare stack exit velocity & {$\left[\mathrm{m} \mathrm{s}^{-1}\right]$} & 359 \\
\hline$u_{m}$ & $\begin{array}{l}\text { Measured flow velocity (overbar implies time- } \\
\text { averaged) }\end{array}$ & {$\left[\mathrm{m} \mathrm{s}^{-1}\right]$} & 283 \\
\hline$u_{n}$ & $\begin{array}{l}\text { Component of LOS mass concentration-weighted, } \\
\text { path-averaged, two-dimensional plume velocity } \\
\text { normal to control surface }\end{array}$ & {$\left[\mathrm{m} \mathrm{s}^{-1}\right]$} & 62 \\
\hline$u_{\infty}$ & Freestream wind velocity & {$\left[\mathrm{m} \mathrm{s}^{-1}\right]$} & 90 \\
\hline $\boldsymbol{u}$ & $\begin{array}{l}\text { LOS mass concentration-weighted, path-averaged, } \\
\text { two-dimensional plume velocity vector in the } \\
\text { image plane }\end{array}$ & {$\left[\mathrm{m} \mathrm{s}^{-1}\right]$} & 126 \\
\hline $\boldsymbol{u}_{3 D}$ & Plume velocity vector & {$\left[\mathrm{m} \mathrm{s}^{-1}\right]$} & 200 \\
\hline $\mathcal{u}(a, b)$ & Uniform distribution from $a$ to $b$ & [var.] & 93 \\
\hline $\boldsymbol{v}_{x, y}$ & $\begin{array}{l}\text { Vertical polarization axes (for incident direction } x \\
\text { relative to outgoing direction } y \text { ) }\end{array}$ & {$[\mathrm{m}]$} & 307 \\
\hline$V_{o}$ & Standard volume & {$\left[\mathrm{m}^{3}\right]$} & 172 \\
\hline$\dot{V}$ & Volumetric flowrate & {$\left[\mathrm{m}^{3} \mathrm{~s}^{-1}, \mathrm{SLPM}\right]$} & 282 \\
\hline$\dot{V}_{i n j}$ & $\begin{array}{l}\text { Standard volumetric flowrate of injected tracer gas } \\
\text { (overbar implies time-averaged) }\end{array}$ & {$[\mathrm{SLPM}]$} & 285 \\
\hline$\dot{V}_{o}$ & $\begin{array}{l}\text { Standard volumetric flowrate (overbar implies } \\
\text { time-averaged) }\end{array}$ & {$\left[\mathrm{m}^{3} \mathrm{~s}^{-1}, \mathrm{SLPM}\right]$} & 283 \\
\hline$V A C_{B C, 550}$ & $\begin{array}{l}\text { Volume-normalized absorption cross-section of } \\
\mathrm{BC} \text { at } 550 \mathrm{~nm}\end{array}$ & {$\left[\mu \mathrm{m}^{-1}\right]$} & 188 \\
\hline$w$ & Substitution variable (integration by substitution) & {$[-]$} & 304 \\
\hline$x$ & Size parameter of spherical particle & {$[-]$} & 331 \\
\hline$x, y, z$ & Components of the cartesian coordinate system & {$[\mathrm{m}]$} & 83 \\
\hline
\end{tabular}




\begin{tabular}{|c|c|c|c|}
\hline $\begin{array}{c}\text { Latin } \\
\text { Symbol }\end{array}$ & Description & Units & $\begin{array}{c}\text { First use: } \\
\text { Page }\end{array}$ \\
\hline$x, y_{\lambda}, z_{\lambda}$ & $\begin{array}{l}\text { Components of the spectral path of beam steered } \\
\text { light }\end{array}$ & {$[\mathrm{m}]$} & 110 \\
\hline$x, y_{\ell}, z_{\ell}$ & Components of the LOS path & {$[\mathrm{m}]$} & 110 \\
\hline$X_{i}$ & $\begin{array}{l}\text { Volume fraction of species } i \text { or volume fraction at } \\
\text { location } i \text { (overbar implies time-averaged) }\end{array}$ & {$[-]$} & 285 \\
\hline$Y_{B C}$ & Black carbon yield & {$\left[\mathrm{g} \mathrm{m}^{-3}\right]$} & 71 \\
\hline$Z$ & Zenith angle of sky element & {$\left[\mathrm{rad},{ }^{\circ}\right]$} & 123 \\
\hline$Z_{d}$ & Zenith angle of LOS/optical axis of detector & {$\left[\mathrm{rad},{ }^{\circ}\right]$} & 310 \\
\hline$Z_{s}$ & Zenith angle of sun & {$\left[\mathrm{rad},{ }^{\circ}\right]$} & 123 \\
\hline $\begin{array}{c}\text { Greek } \\
\text { Symbol }\end{array}$ & Description & Units & $\begin{array}{c}\text { First use: } \\
\text { Page }\end{array}$ \\
\hline$\alpha$ & Relative bearing of sky element & {$\left[\mathrm{rad},{ }^{\circ}\right]$} & 123 \\
\hline$\alpha_{\lambda}$ & Spectral absorption coefficient & {$\left[\mathrm{m}^{-1}\right]$} & 339 \\
\hline$\overline{\alpha_{\lambda}}$ & $\begin{array}{l}\text { Time-averaged spectral absorption coefficient } \\
\text { through PASS } 3 \text { cell }\end{array}$ & {$\left[\mathrm{m}^{-1}\right]$} & 341 \\
\hline$\overline{\alpha_{\lambda, o}}$ & $\begin{array}{l}\text { Time-averaged spectral absorption coefficient } \\
\text { through PASS } 3 \text { cell at standard conditions }\end{array}$ & {$\left[\mathrm{m}^{-1}\right]$} & 173 \\
\hline$\alpha_{N}$ & $\begin{array}{l}\text { Absolute bearing/pointing of camera relative to } \\
\text { North }\end{array}$ & {$\left[\mathrm{rad},{ }^{\circ}\right]$} & 230 \\
\hline$\alpha_{s}$ & Relative bearing of sun & {$\left[\mathrm{rad},{ }^{\circ}\right]$} & 123 \\
\hline$\alpha_{s N}$ & Absolute bearing of sun relative to North & {$\left[\mathrm{rad},{ }^{\circ}\right]$} & 228 \\
\hline$\beta$ & Camera inclination angle & {$\left[\mathrm{rad},{ }^{\circ}\right]$} & 123 \\
\hline$\beta_{i}$ & Coefficients of linear regression, $i \in\{0,1\}$ & [var.] & 180 \\
\hline$\gamma_{i+2, i}$ & $\begin{array}{l}\text { Rotation angle for polarized light referenced from } i \\
\text { to } i+2\end{array}$ & {$\left[\mathrm{rad},{ }^{\circ}\right]$} & 314 \\
\hline$\gamma_{i+2, i}^{n}$ & $\begin{array}{l}\text { Rotation angle for polarized light referenced from } i \\
\text { to } i+2 \text { for the } n^{\text {th }} \text { photon packet }\end{array}$ & {$\left[\mathrm{rad},{ }^{\circ}\right]$} & 321 \\
\hline$\gamma_{i l}$ & Half-width of the $l^{\text {th }}$ transition of gas species $i$ & {$\left[\mathrm{rad} \mathrm{s}^{-1}\right]$} & 292 \\
\hline$\Gamma$ & $\begin{array}{l}\text { Volumetric flowrate conversion factor, } \Gamma= \\
60,000 \mathrm{~L} \mathrm{~s} \mathrm{~min} \mathrm{~m}^{-1} \mathrm{~m}^{-3}\end{array}$ & {$\left[\mathrm{~L} \mathrm{~s} \mathrm{~min} \min ^{-1} \mathrm{~m}^{-3}\right]$} & 286 \\
\hline$\delta$ & Centreline deviation parameter & {$[-]$} & 282 \\
\hline$\hat{\delta}$ & Polarization scaling variable & {$[-]$} & 313 \\
\hline$\delta(\cdot)$ & Degree of linear polarization function & {$[-]$} & 312 \\
\hline$\Delta \theta_{\lambda, s}$ & $\begin{array}{l}\text { Change in magnitude of spectral beam steering } \\
\text { due to the presence of soot }\end{array}$ & {$\left[\mathrm{rad},{ }^{\circ}\right]$} & 294 \\
\hline$\Delta T$ & Adiabatic flame temperature rise & {$\left[\mathrm{K},{ }^{\circ} \mathrm{C}\right]$} & 361 \\
\hline$\varepsilon$ & Fouling parameter & {$[-]$} & 283 \\
\hline$\epsilon_{\rho}$ & Relative random error in mass column density & {$[-]$} & 150 \\
\hline$\zeta_{\lambda}$ & $\begin{array}{l}\text { Fraction of fouling in PASS3 that occurs on the } \\
\text { prism }\end{array}$ & {$[-]$} & 345 \\
\hline$\zeta(\cdot)$ & $\begin{array}{l}\text { Function for the } m_{33} \text { and } m_{44} \text { components of the } \\
\text { normalized Mueller matrix }\end{array}$ & {$[-]$} & 312 \\
\hline$\eta$ & Statistic of soot mass column density & [var.] & 228 \\
\hline$\eta_{\lambda}$ & Spectral real component of the refractive index & {$[-]$} & 96 \\
\hline
\end{tabular}




\begin{tabular}{|c|c|c|c|}
\hline $\begin{array}{c}\text { Greek } \\
\text { Symbol }\end{array}$ & Description & Units & $\begin{array}{l}\text { First use: } \\
\text { Page }\end{array}$ \\
\hline \multirow[t]{2}{*}{$\theta$} & $\begin{array}{l}\text { Appendix A: Angular coordinate of cylindrical } \\
\text { coordinate system }\end{array}$ & {$\left[\mathrm{rad},{ }^{\circ}\right]$} & 282 \\
\hline & Elsewhere: Scattering angle & {$\left[\mathrm{rad},{ }^{\circ}\right]$} & 62 \\
\hline$\theta_{\lambda}$ & Spectral total beam steering angle & {$\left[\mathrm{rad},{ }^{\circ}\right]$} & 97 \\
\hline$\theta_{\lambda}^{y}, \theta_{\lambda}^{z}$ & $\begin{array}{l}\text { Spectral total beam steering angle in } x-y \text { and } x-z \\
\text { planes }\end{array}$ & {$\left[\mathrm{rad},{ }^{\circ}\right]$} & 97 \\
\hline$\theta_{\lambda, s}$ & $\begin{array}{l}\text { Spectral total beam steering angle under the } \\
\text { presence of soot }\end{array}$ & {$\left[\mathrm{rad},{ }^{\circ}\right]$} & 294 \\
\hline$\theta_{k}$ & $\begin{array}{l}\text { Scattering angle for the } k^{\text {th }} \text { scattering event } \\
\text { (rMCRT) }\end{array}$ & {$\left[\mathrm{rad},{ }^{\circ}\right]$} & 316 \\
\hline$\theta_{k}^{n}$ & $\begin{array}{l}\text { Scattering angle for the } k^{\text {th }} \text { scattering event of the } \\
n^{\text {th }} \text { photon packet (forward propagation of MCRT } \\
\text { analysis) }\end{array}$ & {$\left[\mathrm{rad},{ }^{\circ}\right]$} & 320 \\
\hline$\theta_{n}$ & $\begin{array}{l}\text { Scattering angle relative to LOS after } n \text { scattering } \\
\text { events (rMCRT) }\end{array}$ & {$\left[\mathrm{rad},{ }^{\circ}\right]$} & 138 \\
\hline$\theta_{s}$ & Scattering angle of sunlight into LOS & {$\left[\mathrm{rad},{ }^{\circ}\right]$} & 123 \\
\hline$\theta_{s, j}^{n}$ & $\begin{array}{l}\text { Scattering angle of sunlight at the } j^{\text {th }} \text { scattering } \\
\text { event of the } n^{\text {th }} \text { photon packet (forward } \\
\text { propagation of MCRT analysis) }\end{array}$ & {$\left[\mathrm{rad},{ }^{\circ}\right]$} & 323 \\
\hline$\kappa_{\lambda}$ & $\begin{array}{l}\text { Spectral imaginary component of the refractive } \\
\text { index }\end{array}$ & {$[-]$} & 187 \\
\hline$\lambda$ & Wavelength of light & {$[\mu \mathrm{m}, \mathrm{nm}]$} & 61 \\
\hline$\lambda_{o}$ & Reference wavelength of light & {$[\mu \mathrm{m}]$} & 164 \\
\hline$\Lambda$ & MAC scaling parameter & {$[-]$} & 181 \\
\hline$\mu$ & Location parameter of the GEV distribution & [var.] & 275 \\
\hline$\mu^{*}$ & Ideal extinction through clean atmosphere & {$[-]$} & 210 \\
\hline$\mu_{\lambda}$ & Spectral extinction coefficient & {$\left[\mathrm{m}^{-1}\right]$} & 84 \\
\hline$\mu_{\lambda, L}$ & Spectral extinction coefficient along PASS 3 cell & {$\left[\mathrm{m}^{-1}\right]$} & 344 \\
\hline \multirow[t]{2}{*}{$\xi$} & $\begin{array}{l}\text { Chapter 3: Randomized standard uniform } \\
\text { variable }\end{array}$ & {$[-]$} & 105 \\
\hline & $\begin{array}{l}\text { Appendix A: Shape parameter of the GEV } \\
\text { distribution }\end{array}$ & {$[-]$} & 275 \\
\hline$\xi_{\theta}$ & Randomized scattering angle & {$\left[\mathrm{rad},{ }^{\circ}\right]$} & 317 \\
\hline$\xi_{\theta_{\lambda}}$ & Randomized beam steering deflection angle & {$\left[\mathrm{rad},{ }^{\circ}\right]$} & 105 \\
\hline$\xi_{\tau}$ & Randomized propagation transmittance & {$[-]$} & 317 \\
\hline$\xi_{\phi}$ & Randomized scattering azimuth & {$\left[\mathrm{rad},{ }^{\circ}\right]$} & 317 \\
\hline$\xi_{E D}$ & $\begin{array}{l}\text { Randomized scalar multiplier to the sun } \\
\text { component of inscattering }\end{array}$ & {$[-]$} & 210 \\
\hline$\xi_{\mathcal{N}}$ & Randomized standard normal variable & {$[-]$} & 299 \\
\hline$\varrho_{B C}, \varrho_{s}$ & Material density of soot/BC & {$\left[\mathrm{g} \mathrm{m}^{-3}, \mathrm{~kg} \mathrm{~m}^{-3}\right]$} & 61 \\
\hline$\varrho_{e}$ & Density of flare gas at stack exit & {$\left[\mathrm{g} \mathrm{m}^{-3}\right]$} & 360 \\
\hline$\varrho_{o}$ & Density of flare gas at standard conditions & {$\left[\mathrm{g} \mathrm{m}^{-3}\right]$} & 361 \\
\hline$\varrho_{\infty}$ & Ambient density of air & {$\left[\mathrm{g} \mathrm{m}^{-3}\right]$} & 360 \\
\hline$\rho_{E C}$ & Mass concentration of elemental carbon & {$\left[\mathrm{g} \mathrm{m}^{-3}\right]$} & 358 \\
\hline$\rho_{s}$ & Mass concentration of soot/BC & {$\left[\mathrm{g} \mathrm{m}^{-3}\right]$} & 125 \\
\hline$\rho_{s}^{\prime}$ & Mass column density of soot/BC & {$\left[\mathrm{g} \mathrm{m}^{-2}\right]$} & 126 \\
\hline
\end{tabular}




\begin{tabular}{|c|c|c|c|}
\hline $\begin{array}{c}\text { Greek } \\
\text { Symbol }\end{array}$ & Description & Units & $\begin{array}{c}\text { First use: } \\
\text { Page }\end{array}$ \\
\hline$\widetilde{\rho_{s}^{\prime}}$ & Estimated mass column density of soot/BC & {$\left[\mathrm{g} \mathrm{m}^{-2}\right]$} & 147 \\
\hline$\rho_{s a}$ & Ratio of scattering-to-absorption cross-sections & {$[-]$} & 61 \\
\hline \multirow[t]{2}{*}{$\sigma$} & $\begin{array}{l}\text { Chapter } 5 \text { and Appendix D: Stefan-Boltzmann } \\
\text { constant, } \sigma=5.67 \times 10^{-8} \mathrm{~W} \mathrm{~m}^{-2} \mathrm{~K}^{-4}\end{array}$ & {$\left[\mathrm{~W} \mathrm{~m}^{-2} \mathrm{~K}^{-4}\right]$} & 181 \\
\hline & $\begin{array}{l}\text { Appendix A: Scale parameter of the GEV } \\
\text { distribution }\end{array}$ & [var.] & 275 \\
\hline$\sigma(\cdot)$ & Precision error function & {$[-]$} & 150 \\
\hline$\sigma_{e}$ & Extinction cross-section & {$\left[\mathrm{m}^{2}\right]$} & 62 \\
\hline$\sigma_{e}^{m}$ & Mass-normalized extinction cross-section & {$\left[\mathrm{m}^{2} \mathrm{~g}^{-1}\right]$} & 62 \\
\hline$\sigma_{g}$ & Geometric standard deviation (aggregate size) & {$[-]$} & 305 \\
\hline$\sigma_{s}^{\prime}$ & Differential (directional) scattering cross-section & {$\left[\mathrm{m}^{2} \mathrm{sr}^{-1}\right]$} & 62 \\
\hline$\tau$ & $\begin{array}{l}\text { Plume transmittance along path of light } \\
\text { propagation }\end{array}$ & {$[-]$} & 317 \\
\hline$\tau^{*}$ & $\begin{array}{l}\text { Idealized plume transmittance along LOS/optical } \\
\text { axis }\end{array}$ & {$[-]$} & 62 \\
\hline$\tau_{\lambda, \ell}$ & Spectral idealized transmittance along linear path & {$[-]$} & 297 \\
\hline$\tau_{o b s}$ & Observed transmittance & {$[-]$} & 62 \\
\hline \multirow[t]{3}{*}{$\phi$} & $\begin{array}{l}\text { Chapter 3: Azimuth angle of spherical coordinate } \\
\text { system }\end{array}$ & {$\left[\mathrm{rad},{ }^{\circ}\right]$} & 107 \\
\hline & Chapter 4: Scattering azimuth & {$\left[\mathrm{rad},{ }^{\circ}\right]$} & 135 \\
\hline & $\begin{array}{l}\text { Chapter 6: Angular coordinate of two- } \\
\text { dimensional polar coordinate system }\end{array}$ & {$\left[\mathrm{rad},{ }^{\circ}\right]$} & 201 \\
\hline$\phi(\cdot)$ & Complex Lorentzian lineshape & {$\left[\mathrm{s} \mathrm{rad}^{-1}\right]$} & 292 \\
\hline$\phi_{n}$ & $\begin{array}{l}\text { Scattering azimuth after } n \text { scattering events } \\
\text { (rMCRT) }\end{array}$ & {$\left[\mathrm{rad},{ }^{\circ}\right]$} & 138 \\
\hline$\Phi_{l}$ & $1^{\text {th }}$-order soot coefficient & {$[-]$} & 208 \\
\hline$\chi$ & $\begin{array}{l}\text { Scattering angle of solar radiation by } \\
\text { sky/atmospheric particles }\end{array}$ & {$\left[\mathrm{rad},{ }^{\circ}\right]$} & 310 \\
\hline $\bar{\chi}$ & Electric susceptibility of gas mixture & {$[-]$} & 292 \\
\hline$\chi_{i}$ & Electric susceptibility of species $i$ & {$[-]$} & 292 \\
\hline$\psi$ & Polarization angle & {$\left[\mathrm{rad},{ }^{\circ}\right]$} & 306 \\
\hline$\Psi_{l, s k y}$ & $1^{\text {th }}$-order sky coefficient & {$[-]$} & 208 \\
\hline$\Psi_{l, \text { sun }}$ & $1^{\text {th }}$-order sun coefficient & {$[-]$} & 208 \\
\hline \multirow[t]{2}{*}{$\omega$} & Single-scattering albedo & {$[-]$} & 129 \\
\hline & Angular frequency of light, $\omega=2 \pi c / \lambda$ & {$\left[\mathrm{rad} \mathrm{s}^{-1}\right]$} & 292 \\
\hline$\omega_{\lambda}$ & Spectral single-scattering albedo & {$[-]$} & 296 \\
\hline$\omega_{\text {atm }}$ & Single-scattering albedo of atmospheric particles & {$[-]$} & 312 \\
\hline$\omega_{o, i l}$ & $\begin{array}{l}\text { Centre frequency of the } l^{\text {th }} \text { transition of gas species } \\
i\end{array}$ & {$\left[\mathrm{rad} \mathrm{s}^{-1}\right]$} & 292 \\
\hline$\Omega$ & $\begin{array}{l}\text { Propagation direction of light along a LOS into a } \\
\text { detector }\end{array}$ & {$[-]$} & 129 \\
\hline$\Omega_{s}$ & Propagation direction of sunlight & {$[-]$} & 129 \\
\hline$\Omega_{\text {sky }}$ & Propagation direction of skylight & {$[-]$} & 129 \\
\hline
\end{tabular}




\section{Preface}

This integrated article thesis is composed of five articles that have been published or submitted to scholarly journals:

Conrad, B.M. and Johnson, M.R. (2017). Field Measurements of Black Carbon Yields from Gas Flaring. Env. Sci. Tech., 51(3):1893-1900 (doi:

10.1021/acs.est.6b03690).

Conrad, B.M., Thornock, J.N. and Johnson, M.R. (2020). Beam Steering Effects on Remote Optical Measurements of Pollutant Emissions in Heated Plumes and Flares. J. Quant. Spect. Rad. Tran., 254 (doi: 10.1016/j.jqsrt.2020.107191).

Conrad, B.M., Thornock, J.N. and Johnson, M.R. (2020). The Effect of Multiple Scattering on Optical Measurement of Soot Emissions in Atmospheric Plumes. $J$. Quant. Spect. Rad. Tran., 254 (doi: 10.1016/j.jqsrt.2020.107220).

Conrad, B.M. and Johnson, M.R. (2019). Mass Absorption Cross-Section of FlareGenerated Black Carbon: Variability, Predictive Model, and Implications. Carbon, 149:760-771 (doi: 10.1016/j.carbon.2019.04.086).

Conrad, B.M. and Johnson, M.R. (2020). An Uncertainty-Based Protocol for the Setup and Measurement of Soot/Black Carbon Emissions from Gas Flares using Sky-LOSA. Atm. Meas. Tech. [under review] (doi: 10.5194/amt-2020-255).

All five articles are included in their entirety with published supplements provided in the appendices of the thesis. The only noteworthy changes to the articles are adjustments to the nomenclature and minor edits to provide additional clarity and context as suggested by the thesis examiners and to ensure formatting and notational consistency throughout the thesis.

The first article was published in the journal Environmental Science and Technology; its copyright is held by the publisher, the American Chemical Society. The second, third, and fourth articles were published in the Journal of Quantitative 
Spectroscopy and Radiative Transfer or Carbon; as open-access articles in journals published by Elsevier, the copyrights are maintained by the authors. The fifth article is presently under peer-review in the journal Atmospheric Measurement Techniques; the authors maintain copyright during review and once published.

All five articles provide targeted literature reviews and only a brief introductory chapter is included to place this thesis within the current literature and regulatory environment. Detailed accounts of experimental procedures are also provided within each article and their associated supplements and are considered sufficient to allow readers to test the validity of research outcomes. For this reason, the thesis does not contain a "Methods Chapter" as permitted by $\S 12.4 . \mathrm{B}$ of the 2020-2021 Carleton University Graduate Calendar.

The preferred method of citing work from this thesis is to directly reference the published articles when possible, citing this thesis only when an appropriate citation in the included articles cannot be found. 


\section{Student Statement Regarding Student Involvement}

I, Bradley Conrad, was fully involved and responsible for setting up equipment, obtaining data, analyzing results, and writing the first drafts of the material presented in the accompanying thesis.

Bradley Mark Conrad

\section{Supervisor Statement Regarding Student Involvement}

The student, Bradley Conrad, was fully involved and responsible for setting up equipment, obtaining data, analyzing results, and writing the first drafts of the material presented in the accompanying thesis.

Matthew Ronald Johnson 


\section{Chapter 1 Introduction}

\subsection{Background}

\subsubsection{Soot/Black Carbon}

Soot particulate matter (PM) is a product of incomplete combustion that is commonly emitted directly to atmosphere. Frequently referred to as black carbon (BC) when discussed in the context of light absorption (see also Section 1.1.5), soot/BC particles are composed of spherical nanoscale carbon monomers (typically 10 to $50 \mathrm{~nm}$ in diameter (e.g., Kazemimanesh et al. 2019)) that aggregate within hydrocarbon flames under diffusion-limited conditions (Cai and Sorensen 1994; Witten and Sander 1983). Emitted $\mathrm{BC}$ particulate is generally mass fractal-like in morphology with mean radii of gyration much less than $1 \mu \mathrm{m}$ for light alkane fuels (Crosland et al. 2015) and potentially approaching $1 \mu \mathrm{m}$ for more conjugated alkyne and aromatic fuels (e.g., Köylü and Faeth 1992). In-flame formation of soot/BC is quite complex, with competing mechanisms that cause the growth and oxidization of BC aggregates (Glassman 1989). The same physicochemical processes driving soot/BC formation also impact the microstructure of BC monomers. Spherical monomers generally have an onion-like structure of concentric graphene layers, with very little contaminant species, usually hydrogen and oxygen (Andreae and Gelencsér 2006). The highly conjugated graphitic structure results in the delocalization of electrons within the $\pi$-bonds between graphene layers, which results in broadband light absorption that is characteristic and defining of combustion-generated $\mathrm{BC}$ (Andreae and Gelencsér 2006; Bond and Bergstrom 2006). 
While $\mathrm{BC}$ is thought to have important implications for local public health (Cushing et al. 2020; Luben et al. 2017; Li et al. 2016), current research is mostly focussed on its substantial effects on the climate, which are due to its strong broadband absorption. BC influences Earth's climate system through direct and indirect mechanisms. Estimates of direct radiative forcing (DRF) by $\mathrm{BC}$, which identifies the change in Earth's radiation budget at the top of the atmosphere relative to the pre-industrial era due to the presence of a species, are large but are highly uncertain (e.g., $90 \%$ confidence interval of approximately $\pm 85 \%$ (Bond et al. 2013)). Indeed, in the context of DRF, BC has been suggested by some to be the second most important anthropogenic pollutant after carbon dioxide (Bond et al. 2013; Ramanathan and Carmichael 2008) with an effect similar to methane (IPCC 2013). Other, indirect forcing mechanisms like aerosol-cloud interactions and the snow/ice-albedo feedback (reduction of snow and ice albedo by ground-deposited BC) are smaller in magnitude than direct mechanisms and can induce both positive and negative changes to Earth's radiation budget (Bond et al. 2013).

Globally averaged DRF is a function of four variables: the pollutant's global emissions, optical properties, "absorption forcing efficiency" (AFE, forcing per aerosol absorption optical depth), and lifetime in the atmosphere (e.g., Schulz et al. 2006). Relative to other climate-important pollutants, $\mathrm{BC}$ experiences a relatively short atmospheric lifetime. Due to effective dry and wet deposition mechanisms, the lifetime of a $\mathrm{BC}$ particle is typically on the order of days or weeks (Textor et al. 2006) as compared to tens or hundreds and even thousands of years for other important climate forcers like methane and carbon dioxide, respectively (IPCC 2013). BC is thus classified as a "short lived climate pollutant/forcer". This short-lived nature hampers the ability to accurately constrain the 
globally averaged atmospheric loading (and, hence, aerosol absorption optical depth) of BC. Coupled with a factor-of-three diversity in BC's globally averaged AFE, uncertainties in BC DRF are exceedingly large relative to longer-lived pollutants. However, a positive consequence of $\mathrm{BC}$ 's short atmospheric lifetime is that efforts to reduce $\mathrm{BC}$ emissions yield climate benefits that can be observed on quite short timescales, perhaps delaying Earth's crossing of critical temperature thresholds (Bowerman et al. 2013). Thus, there is a significant opportunity to reduce the warming of Earth's climate through the regulation and mitigation of $\mathrm{BC}$ emission sources.

Accurate knowledge of the global emissions and optical properties of $\mathrm{BC}$ is required to better constrain BC DRF, inform regulations, and optimally direct mitigation efforts. One challenge, however, is that there are myriad sources of $\mathrm{BC}$ emissions across the globe that emit $\mathrm{BC}$-containing PM in different quantities and with varying particle properties. Globally significant sources include wildfires; biomass burning for residential cooking and heating; planned and unplanned agricultural burning; power generation; transportation; and other industrial sources including gas flaring (Stohl et al. 2013; U.S. EPA 2012). BC emissions from some sources are better characterized than others and one particularly poorly characterized source of atmospheric BC with notable climate impacts is gas flaring.

\subsubsection{Gas Flaring}

Gas flaring is a standard process in the oil and gas (OG) industry where extraneous or unwanted gases are disposed of by means of open combustion. Such gases at OG facilities can result from emergency overpressure events, maintenance work, and standard operating conditions including disposal of associated gas produced from oil wells. Because most 
flare gas is methane-rich, and methane has a strong global warming potential (34/96 on a 100-/20-year time horizon (Gasser et al. 2017)), flaring reduces carbon dioxide-equivalent emissions relative to direct venting of these gases to atmosphere (Calel and Mahdavi 2020). Thus, disposal of extraneous gases via flaring is generally preferable to venting; however, the optimal solution in the climate context is to capture and forward extraneous gases directly to market or to downstream processing facilities (Tyner and Johnson 2018). Unfortunately, the decision to flare is often based on economic arguments, since gaseous product is much less valuable than produced oil and developing the infrastructure necessary to accommodate extraneous gas-phase product is not economically feasible.

In the upstream OG (UOG) industry gas flares are generally simple in construction, typically composed of vertically oriented pipe stacks creating large-scale, buoyancydriven, turbulent diffusion flames. Flared gases are generally mixtures of light alkanes that can be diluted with hydrogen, helium, nitrogen, carbon dioxide, and/or hydrogen sulphide (e.g., Chapter 2, Conrad and Johnson 2017; Johnson and Coderre 2012). In some instances, unsaturated hydrocarbons like alkenes, naphthenes, and aromatics can also be present (Chapter 2, Conrad and Johnson 2017). Flares in the midstream and downstream OG sectors, as well as industrial flares, can have more complex designs, perhaps integrating emissions control systems such as steam- or air-assist that suppress visible PM emissions (U.S. EPA 2018). This is sometimes necessary since, in these OG sectors, emergency events can require the flaring of pure unsaturated hydrocarbons (such as ethylene in the manufacture of polyethylene), which produce particulate emissions more efficiently than typical alkane-based compositions observed in the upstream sector (Glassman 1989). 
While some jurisdictions require producers to log and report flaring activity by gas volume, global estimates of gas flaring are generally performed by remote sensing methods. Estimates based on satellite imagery reported by the United States National Oceanic and Atmospheric Administration (NOAA) suggest that globally flared volumes were approximately 151 billion $\mathrm{m}^{3}$ in 2017 (NOAA 2020), which is almost nine times larger than Canada's residential natural gas demand in the same year (Statistics Canada 2020). Typically, $\sim 90 \%$ of flared volumes are from UOG activities (Elvidge et al. 2015, 2018). Satellite data also show that, when parsed by flare, global volumes in the UOG industry follow a classic highly skewed (fat-tail) distribution. For example, the latest publicly available satellite data from 2017 (NOAA 2020) suggest that UOG flare-specific volumes span almost five orders of magnitude and just $10 \%$ of global flares are responsible for $\sim 76 \%$ of total volume (Gini coefficient of $\sim 82 \%$ (Gini 1912)). This highlights an opportunity; that is, regulatory and mitigation efforts on a small fraction of the UOG industry can yield disproportionate reductions in flared gas volumes.

\subsubsection{Black Carbon Emissions from Gas Flaring}

Gas flaring is a globally important source of atmospheric soot/BC concentrations. Although estimates of $\mathrm{BC}$ emissions suggest that flaring has a relatively small contribution to global $\mathrm{BC}$ emissions (on the order of a few percent), the locations of flaring activities can accentuate the climate effect of BC emissions (e.g., Stohl et al. 2013). For instance, during the winter months, the Arctic air mass pushes southward (Law and Stohl 2007; Stohl 2006), encompassing regions of severe flaring activity such as Russia's Ural Federal District (Huang et al. 2015). Specifically, low-level BC emissions from this region are efficiently transported over the Arctic (Stohl 2006) and these emissions are likely to 
strongly influence the sensitive Arctic climate through the snow/ice-albedo feedback of ground-deposited particulate (e.g., Flanner et al. 2007; Clarke and Noone 1985). Indeed, modelling efforts have suggested that flaring is the most important anthropogenic source of BC north of $66^{\circ} \mathrm{N}$ (Stohl et al. 2013) and shipborne measurements in Russia's Kara Sea have specifically identified flaring in Russia's Komi Republic and Yamal-Nenets and Khanty-Mansi Autonomous Okrugs as important sources of Arctic BC (Popovicheva et al. 2017).

In addition to the location of flaring sources, the efficiency with which a flare emits soot/BC is required to characterize climate impacts of flaring; importantly, this "sooting propensity" is known to be sensitive to both fuel composition and flame aerodynamics. Studying laboratory-scale flares representative of Alberta's upstream OG industry, McEwen and Johnson (2012) observed high correlation between BC yield (mass of BC emitted per quantity of flare gas) and fuel energy content (i.e., gas heating value). These observations were replicated in the field by Conrad and Johnson (Chapter 2, 2017). McEwen and Johnson (2012), Mehr (2020), and earlier studies (e.g., Becker and Liang 1982) also observed variability in BC production as a function of flame aerodynamics specifically the Froude number, which compares flare gas momentum and flame buoyancy.

Although laboratory and field observations imply that flare $\mathrm{BC}$ emission are sensitive to fuel composition and flame aerodynamics, flare $\mathrm{BC}$ emission factor models that quantify $\mathrm{BC}$ yield for the purposes of emissions inventories, regulation, and climate modelling are generally overly simple. Industry (International Petroleum Industry Environmental Conservation Association, IPIECA 2014) and organizations at the governmental (U.S. EPA 2012) and intergovernmental (Raga et al. 2018) level have all 
identified the need to improve the present understanding of $\mathrm{BC}$ emissions from flares and similar poorly characterized sources. Despite the present knowledge base regarding the variability of $\mathrm{BC}$ emission with quantifiable flare metrics, single-valued $\mathrm{BC}$ emission factors are generally used in inventory and modelling efforts (e.g., Amann et al. 2011; CAPP 2007). Although this is partly due to the lack of available flared volume and composition data from producers, improvements to emission factor models are limited by the challenge of scale. That is, most studies of soot/BC within or emissions from flare-like flames are performed on small-scale laboratory flares combusting fuels much heavier than field-measured flare gas compositions (Chapter 5, Conrad and Johnson 2019a), and are therefore not fully representative of in-field flares. In the laboratory, there are numerous techniques to quantify local soot/BC concentrations or emissions and particulate properties. Among others, these include gravimetric techniques (e.g., Choi et al. 1995), electron microscopy (e.g., Köylü and Faeth 1994), laser-induced incandescence (LII; e.g., Will et al. 1996), elastic light scattering (e.g., Oltmann et al. 2010) and/or extinction (e.g., Iyer et al. 2007), photoacoustic spectrometry techniques (Arnott et al. 1999), thermaloptical approaches (e.g., Chow et al. 1993), and, sometimes, a combination of techniques (e.g., Crosland et al. 2013; De Iuliis et al. 1998). Unfortunately, it is challenging to implement and/or scale these approaches to measure $\mathrm{BC}$ emissions from field-operational flares and alternative approaches are thus required.

The current measurement standard for BC emissions from gas flares is the United States Environmental Protection Agency's (EPA's) Method 9 - Visual Determination of the Opacity of Emissions from Stationary Sources (U.S. EPA 1974). As the title implies, Method 9 requires an observer to determine plume opacity based on visual observations of 
the flare plume. While observers are trained to control variables that can bias their observations, there are other uncontrollable factors affecting the ability of a human observer to accurately assess plume opacity. For example, the physiology of the eye suggests that it is a poor detector for this purpose since it nonlinearly detects intensity (Stevens 1957) and shows variable spectral sensitivity (Commission Internationale de l'Eclairage, CIE 1990). More fundamentally, plume opacity is a poor surrogate for absolute or relative $\mathrm{BC}$ emissions since the link between opacity and $\mathrm{BC}$ quantity is ill-defined for broadband observations. This subjective opacity-based measurement standard was borne out of a lack of quantitative measurement techniques for flare $\mathrm{BC}$, which are needed to improve flare BC emission factor models.

It is only recently that quantitative field measurements of flare $\mathrm{BC}$ emissions have been made possible, using either of two different approaches. Firstly, an imaging technique known as sky-LOSA (line-of-sight attenuation using skylight) has been developed to directly measure time-resolved absolute $\mathrm{BC}$ emission rates (in units of mass per time) from targeted flares in the field. Proof-of-concept laboratory (Johnson et al. 2010) and field experiments (Johnson et al. 2011) contributed to the generalization of the method summarized by Johnson et al. (2013). More recently, aircraft-based sampling techniques combining LII-based point-measurements of soot/BC concentration and gas-phase concentrations in atmospheric plumes have been used to estimate BC yield for targeted flares (as in Gvakharia et al. (2017) and Weyant et al. (2016)) or on a basin level (as in Schwarz et al. (2015)). While useful in their ability to assess multiple flares in a short period of time, aircraft-based measurements are expected to underestimate BC yields due to their inherently short measurement periods of positively skewed instantaneous emissions 
(Chapter 2, Conrad and Johnson 2017) and their methodology that requires the assumption of perfect correlation between particulate and gas-phase products (Seymour 2019). By contrast, sky-LOSA measurements of $\mathrm{BC}$ emissions are insensitive to the limiting assumptions used by aircraft techniques and can be performed over longer periods (up to and exceeding 10 minutes) enabling robust calculations of time-averaged emissions. Moreover, when combined with parallel on-site measurement of flare gas flow rate and extractive gas samples for compositional analysis, sky-LOSA can be used to directly compute $\mathrm{BC}$ yields. Importantly, the availability of flare-specific flow rate and composition data also support the development of parametric flare $\mathrm{BC}$ emission factor models (Chapter 2, Conrad and Johnson 2017).

\subsubsection{Regulatory Framework and Impact of Presented Work}

Despite the well-known climate and public health impacts of soot/BC and the global scale

of gas flaring, regulations of $\mathrm{BC}$ flare emissions are immature. International efforts to reduce flaring and its impacts have been led by the World Bank's Global Gas Flaring Reduction partnership (GGFR), the United Nations Environment Programme's Climate and Clean Air Coalition (CCAC), and the United Nation's Economic Commission for Europe (UNECE). The GGFR is a public-private partnership with the aim to "increase use of natural gas associated with oil production by helping remove technical and regulatory barriers to flaring reduction, conducting research, disseminating best practices, and developing country-specific gas flare reduction programs." Notably, the GGFR has introduced the Zero Routine Flaring by 2030 initiative, of which there are 32 endorsing countries and 38 endorsing oil companies (World Bank 2015). This initiative seeks to abolish flaring during normal production operations by on-site use, re-injection, or sale of 
flare gases. The CCAC's Measuring \& Minimizing Mineral Methane Emissions and Reducing Black Carbon through Flaring initiative (CCAC 2020) similarly seeks to "promote significant reductions in ... black carbon emissions from the oil and gas sector" primarily through technology demonstrations and the development of "best practices and policies for black carbon mitigation." Lastly, in 2012, the UNECE amended its 1999 Protocol to Abate Acidification, Eutrophication, and Ground-Level Ozone (commonly referred to as the Gothenburg Protocol) under the Convention on Long-Range Transboundary Air Pollution to include BC (United Nations Economic Commission for Europe 2012). Ratified by 23 parties, including Canada and the United States, this legally binding instrument includes the requirement to report emissions of $\mathrm{BC}$ where data are available.

To meet requirements under the Gothenburg protocol, Canada has recently developed a Black Carbon Emissions Inventory (BCEI) that parses estimates of BC emissions by source categories and sectors/subsectors (Environment and Climate Change Canada, ECCC 2020). The BCEI presently includes data of Canadian flare BC emissions from 2013-2018 estimated using industry-reported flare volumes coupled with an emission factor model derived by Johnson and Tyner (2019) using data from McEwen and Johnson (2012) and Conrad and Johnson (Chapter 2, 2017). Over these six years, upstream flare BC emissions were generally between 800-1100 tonnes (ECCC 2020); however, with recently introduced regulations on methane emissions from the Canadian OG industry, flaring activity in Canada is expected to increase (Tyner and Johnson 2018).

Within Canada, provincial regulations on flaring are generally designed to ensure flaring activities are performed while considering the "protection of human health, public 
safety, property and the environment and to prevent fire or explosion" (Saskatchewan Ministry of Energy and Resources 2020) using the framework of the Clean Air Strategic Alliance (Clean Air Strategic Alliance 2004). Directives are generally focussed on providing limitations on flaring by volume, with only minor guidance on "smoke emissions". For instance, without further definition, Alberta's Directive 060 (Alberta Energy Regulator 2020) states that "[r]outine gas combustion must not result in continuous or repeat black smoke emissions" and "[b]lack smoke from nonroutine or emergency flaring must not exceed an average of 40 per cent opacity over six consecutive minutes ..."; the directive also provides a means for the public to raise concerns with the Alberta Energy Regulator. This current regulatory framework, which is focussed on the aesthetic notion of plume opacity, could be vastly improved by the introduction of directives requiring quantitative measurements of $\mathrm{BC}$ emissions using a technique like sky-LOSA or, at a minimum, by implementation of improved emission factor models derived from field data.

\subsubsection{A Note on Black Carbon Terminology}

One issue that obstructs the development of robust $\mathrm{BC}$ emissions data for flaring and other sources is the lack of a consistent definition of $\mathrm{BC}$. This is partly due to the breadth of $\mathrm{BC}$ related research that covers freshly emitted to highly aged and even surface-deposited particulate. In an attempt to standardize a definition for BC, Petzold et al. (2013a) have recommended terminologies for light absorbing carbonaceous PM based on measurement techniques and physicochemical properties such as composition, microstructure, morphology, thermal stability, and light absorption. In their paper "Recommendations for Reporting 'Black Carbon' Measurements” (Petzold et al. 2013a), PM exhibiting BC-like 
properties are recommended to be reported as "equivalent BC" (consistent with earlier suggestions by Andreae and Gelencsér (2006)) when using light absorption-based methods like sky-LOSA. They note that:

" $[B C]$ is a useful qualitative description when referring to lightabsorbing carbonaceous substances in atmospheric aerosol; however, for quantitative applications the term requires clarification of the underlying determination."

That is, when reporting quantitative measurements of $\mathrm{BC}$ derived from light absorption measurements, the assumed optical properties used to transform light absorption to an equivalent $\mathrm{BC}$ mass must be explicitly stated.

Despite Andreae and Gelencsér's (2006) and Petzold et al.'s (2013a) recommendations, the term "equivalent $\mathrm{BC}$ " has not been widely adopted in the literature. Although the physicochemical properties of BC listed by Petzold et al. (2013a) are generally agreed upon, Bond et al. (2013) state that:

"In measurement and modeling studies, the term 'black carbon' frequently has not been limited to material with these properties, causing a lack of comparability among results."

This tends to be an issue in the analysis of aged particulate and biomass burning emissions where complex mixtures of PM with condensable organic species can drastically change particulate properties (e.g., You et al. 2016; Bond et al. 2006).

There are myriad data available that permit classification of PM from flares using Petzold et al.'s (2013a) framework. For example, Popovicheva et al. (2019) have shown 
that flare-emitted PM is effectively pure carbon with a graphitic microstructure dominated by $\mathrm{sp}^{2}$-bonds and very minor quantities of oxidized or metal-contaminated particles. Studying PM from methane flames, Coderre et al. (2011) showed that the absorption function of the complex refractive index is approximately constant over the visible spectrum, leading to the expected inverse-wavelength dependence of light absorption for BC. These observations are consistent with what would be termed soot carbon or BC (Petzold et al. 2013a; Andreae and Gelencsér 2006). In sum, the current literature shows that the properties of PM from flare-like flames are unambiguous (e.g., Kazemimanesh et al. 2019; Popovicheva et al. 2019; Coderre et al. 2011) and consistently satisfy the physicochemical properties of BC tabulated by Petzold et al. (2013a). Based on these observations, sky-LOSA-measured particulate from flares is convincingly BC. In this thesis however, for consistency with the literature, the term $\mathrm{BC}$ is used without the "equivalent" prefix. Nevertheless, the absorption-based measurements of soot/BC in this thesis are operationally defined in that they are specific to the assumed optical properties used in sky-LOSA, as first defined by Johnson et al. (2013).

\subsection{Thesis Motivation and Objectives}

The research presented in this thesis was motivated by the lack of available data regarding $\mathrm{BC}$ emissions from gas flares that hampers the development of emission factor models, inventories, and efforts to regulate and mitigate flare $\mathrm{BC}$ emissions. Research efforts were therefore focussed to further develop the knowledge base of flare-emitted $\mathrm{BC}$ using the sky-LOSA measurement technique and laboratory studies.

The first objective was the field-deployment of sky-LOSA to measure BC emissions from flares to expand the existing dataset of absolute $\mathrm{BC}$ emissions and perform 
the first sky-LOSA-measurements of BC yield from flares under field conditions. The second objective was to refine the sky-LOSA algorithm through the detailed consideration of previously ignored radiative transfer effects, specifically beam steering and multiple scattering. The third objective was to perform experimental studies to quantify wavelength dependent light absorption (mass-normalized absorption cross-section, MAC) of BC emitted by large laboratory-scale flares combusting fuels representative of upstream oil and gas flaring and to compare results with data from heavier fuels and laboratory burners and engines that dominate the literature. The fourth and final objective was to complete a general uncertainty analysis of the sky-LOSA technique over all practical measurement conditions with the aim of developing a robust setup and measurement protocol to simplify deployment, minimize uncertainties, and ultimately enable consideration of sky-LOSA as a flare BC measurement standard by an authority such as the U.S. EPA.

\subsection{Thesis Overview}

This thesis is written in the integrated article format and comprises five separate articles four of which have been published in peer-reviewed academic journals and a fifth that at the time of writing is under peer-review.

Chapter 2 presents the first article, "Field Measurements of Black Carbon Yields from Gas Flaring", which was published in Environmental Science and Technology in 2017 and details initial efforts to expand the knowledge base of $\mathrm{BC}$ emission rates from in-field gas flares using the sky-LOSA measurement technique. Data from fourteen sky-LOSA measurements of nine flares during three measurement campaigns - Mexico in 2012 (data acquired prior to this thesis work) and Ecuador in 2014 and 2015 - were analyzed to yield quantitative time-resolved $\mathrm{BC}$ emission rate data. During the latter measurement 
campaign, additional equipment to simultaneously measure flare gas flow rate and extract gas samples for compositional analyses during sky-LOSA data acquisition were brought to the field to enable the first four in situ measurements of $\mathrm{BC}$ yield from field-operational gas flares using sky-LOSA. These new data provide evidence of the extreme variability of $\mathrm{BC}$ emissions from flare-to-flare but also temporally for individual flares. The observed four order of magnitude variation in absolute, time-averaged $\mathrm{BC}$ emission rate highlights how emissions from individual "super-emitter" flares can disproportionately contribute to global emissions. Furthermore, consistent with previous laboratory studies, the fieldmeasured $\mathrm{BC}$ yields showed strong correlation with flare gas heating value, enabling an extended emission factor model for flare $\mathrm{BC}$ that now encompasses actual gas compositions in industry. Considering the likely range of global flare gas heating values from limited available data, this model suggests that worldwide flare $\mathrm{BC}$ emissions presently computed using single-valued emission factors may be significantly underestimated.

Following field measurements, the proven utility of sky-LOSA in enabling flarespecific $\mathrm{BC}$ yield data revealed the need to refine the sky-LOSA theory and computational algorithm and standardize the method for increased accessibility to other users. Chapter 3 presents the second article, "Beam Steering Effects on Remote Optical Measurements of Pollutant Emissions in Heated Plumes and Flares", which was published in the Journal of Quantitative Spectroscopy and Radiative Transfer in 2020, which studies the commonly ignored effect of refractive index gradient-driven beam steering through heated plumes. Using available data from large-eddy-simulated gas flares (performed by a co-author at The University of Utah) as an extreme example, beam steering along realistic optical axes 
intersecting the heated plumes were quantified in a Monte Carlo (MC) framework. Within the visible spectrum and three greenhouse gas-relevant infrared bands, three parameters required to correct for beam steering in the radiative transfer equation were found to be insignificant relative to typical instrument noise of optical detectors, even under imposed conditions of extreme turbulence. These novel observations provide quantitative knowledge of beam steering for remote optical measurements of pollutant emissions, which, typically, have been neglected a priori or otherwise argued as unimportant through qualitative reasoning. The third article (presented in Chapter 4), "The Effect of Multiple Scattering on Optical Measurement of Soot Emissions in Atmospheric Plumes", also published in the Journal of Quantitative Spectroscopy and Radiative Transfer in 2020, describes an analysis of multiple scattering (MS) effects by soot/BC within turbulent atmospheric flare plumes. Using the same large-eddy simulation data, this article addresses the complex radiative transfer within soot-laden air plumes, where inscattering of ambient radiation must be considered using the simplifying single-scattering assumption (1SA) that is known to bias sky-LOSA-computed emissions high. Like the second article, radiative transfer simulations along realistic optical axes through the simulated flare plumes enabled quantification of a correction parameter to the radiative transfer equation, which here corrects for error in 1SA-estimated inscattering. A reverse/backwards MC "ray tracing" algorithm was developed and showed that this MS correction parameter was a strong function of plume transmittance and could be captured by a simple phenomenological model. Subsequent forward propagation of ambient radiation along pre-computed "ray paths" was then used to characterize bias and precision error in the new model as a function of ambient conditions. This new model was implemented on previously acquired sky- 
LOSA data (presented in the first article) and showed that negative bias due to use of the 1SA can be on the order of one-quarter of typical sky-LOSA measurement uncertainties. This bias, however, is easily corrected using the novel MS correction model and impacts on overall measurement uncertainty were found to be negligible.

The fourth paper (presented in Chapter 5) discusses laboratory measurements of flare BC MAC. This paper, "Mass Absorption Cross-section of Flare-generated Black Carbon: Variability, Predictive Model, and Implications" was published in Carbon in 2019 placing it, chronologically, between the first and second papers presented in this thesis. Although substantial data regarding BC MAC exist in the literature, there is significant variability among the highly uncertain data. Furthermore, existing measurement data were mostly from small-scale flames generally burning heavier fuels than typical flare gas; thus, the applicability of the current knowledge base to flare $\mathrm{BC}$ was questionable. Indeed, the only existing data for flare BC MAC were secondary results from an aircraft study of flare plumes (Weyant et al. 2016), where measurements indicated anomalously large values of MAC that the authors could not reconcile. This fourth paper presents the first quantitative measurements of flare $\mathrm{BC}$ MAC from large-scale laboratory flames burning fuels representative of global gas flaring. Backed by a comprehensive MC-based uncertainty analysis, $\mathrm{BC}$ MAC for these flames was observed to be larger than generally accepted values and statistically sensitive to numerous metrics describing flare gas composition and operational conditions. A novel non-dimensional MAC scaling parameter was derived that captures the observed variability in flare BC MAC permitting a phenomenological model that suggests MAC scales with the in-flame time-temperature history of flare BC. Moreover, the derived model reconciled data from different sources and thus closed an 
important gap in the literature. From a climate impact perspective, the new model suggests that flare BC MAC may be $>1.3-2.0$ times larger than other BC sources, implying an underestimation of DRF by flare-generated BC in current climate modelling.

The fifth and final paper, "An Uncertainty-based Protocol for the Setup and Measurement of Soot/Black Carbon Emissions from Gas Flares using sky-LOSA", is under review in Atmospheric Measurement Techniques and is presented in Chapter 6. This paper addresses an impediment to the broad use of sky-LOSA caused by the complexity of its deployment, where decisions during setup and data acquisition can have profound, nonlinear impacts on measurement uncertainties. Drawing upon the results of the second and third papers, a comprehensive, variance-reduced, MC-based general uncertainty analysis (GUA) was implemented for the sky-LOSA technique. The GUA, which effectively performed $66 \times 10^{6} \mathrm{MC}$ analyses over a five-dimensional domain of measurement conditions, was enabled through the reformulation of the sky-LOSA algorithm to independently pre-compute the contributions of $\mathrm{BC}$ properties and ambient conditions to measurement uncertainties. GUA data are presented in an open-source software tool providing data acquisition constraints to a sky-LOSA user on a case-by-case basis. This tool is a powerful means to ensure optimal sky-LOSA data are consistently acquired, regardless of ambient conditions or a user's experience. Furthermore, by removing challenging setup and data acquisition decisions, the presented software tool is expected to increase the accessibility of the sky-LOSA technique and, ultimately, the availability of field measurement data for flare $\mathrm{BC}$ emissions.

Finally, Chapter 7 summarizes key findings from these research efforts and discusses potential avenues for extension of this work through future studies. 


\section{Chapter 2 Field Measurements of Black Carbon Yields from Gas Flaring}

This chapter has been peer-reviewed and was published in the journal Environmental Science and Technology. Only minor clarifications and formatting edits have been made for consistency throughout the thesis. It should be cited as:

Conrad, B.M. and Johnson, M.R. (2017). Field Measurements of Black Carbon Yields from Gas Flaring. Env. Sci. Tech., 51(3):1893-1900 (doi:

$\underline{10.1021 / \text { acs.est.6b03690). }}$.

This article is included in its entirety as Chapter 2 with the corresponding supplemental material included as Appendix A. The article was co-authored by the thesis author, Mr. Conrad, and his thesis supervisor, Prof. Johnson. Mr. Conrad prepared equipment for field measurements in Ecuador, which were performed with Mr. Johnson and colleagues. Field measurements in Mexico were executed prior to this thesis. Mr. Conrad developed the software and performed the formal analysis. Mr. Conrad wrote the first draft of the manuscript; editing and revision of the manuscript were conducted jointly.

\subsection{Abstract}

Black carbon $(\mathrm{BC})$ emissions from gas flaring in the oil and gas industry are postulated to have critical impacts on climate and public health but actual emission rates remain poorly characterized. This paper presents in situ field measurements of $\mathrm{BC}$ emission rates and flare gas volume-specific BC yields for a diverse range of flares. Measurements were performed during a series of field campaigns in Mexico and Ecuador using the sky-LOSA optical measurement technique, in concert with comprehensive Monte Carlo-based uncertainty analyses. Parallel on-site measurements of flare gas flow rate and composition were successfully performed at a subset of locations enabling direct measurements of fuelspecific BC yields from flares under field conditions. Quantified BC emission rates from individual flares spanned more than 4 orders of magnitude (up to $53.7 \mathrm{~g} / \mathrm{s}$ ). In addition, 
emissions during one notable 24 -hour flaring event (during which the plume transmittance dropped to zero) would have been even larger than this maximum rate, which was measured as this event was ending. This highlights the likely importance of superemitters to global emission inventories. Flare gas volume-specific BC yields were shown to be strongly correlated with flare gas heating value. A newly derived correlation fitting current field data and previous lab data suggests that, in the context of recent studies investigating transport of flare-generated $\mathrm{BC}$ in the Arctic and globally, impacts of flaring in the energy industry may in fact be underestimated.

\subsection{Introduction}

Flaring is a common practice within the oil and gas (OG) industry of combusting extraneous gases in an open diffusion flame, usually at the exit of a vertically raised flare stack or within a refractory-lined pit. Flaring is generally preferred over venting to reduce equivalent greenhouse gas emissions and health and safety risks to the local population. However, the combustion of flared gas produces atmospheric pollutants including soot, which is a form of particulate matter of less than $2.5 \mu \mathrm{m}$ in diameter $\left(\mathrm{PM}_{2.5}\right)$ consisting of mass-fractal-like aggregates of nano-scale spherules. Black carbon (BC), the carbonaceous component of soot, is an important atmospheric pollutant with critical impacts on local public health and the global environment. Specifically, recent research (Basagaña et al. 2015; Kim et al. 2015; Grahame et al. 2014) has indicated that BC is a key component contributing to the adverse health effects associated with $\mathrm{PM}_{2.5}$, while $\mathrm{BC}$ has also been identified as likely the second-most important atmospheric direct radiative forcer (after carbon dioxide $\left(\mathrm{CO}_{2}\right)$ (Bond et al. 2013; Jacobson 2010)). 
Satellite imagery of flaring activity by the National Oceanic and Atmospheric Administration (NOAA) suggests that the global volume of flared gas exceeds 140 billion $\mathrm{m}^{3}$ annually, approximately $90 \%$ of which is associated with upstream OG production (Elvidge et al. 2015). The sheer magnitude of global gas flaring causes concern for the climate effects of its associated pollutants, especially $\mathrm{BC}$ and $\mathrm{CO}_{2}$. Effects of $\mathrm{BC}$ are amplified at higher latitudes, where deposition of $\mathrm{BC}$ reduces surface albedo and enhances the melting of snow and ice (Jacobson 2010). Stohl et al. (2013) have suggested that $66 \%$ of source $\mathrm{BC}$ concentrations north of the Arctic circle are from gas flaring, and model data suggest that observed $\mathrm{BC}$ concentrations are due in large part to the transport of flare-generated BC from western Siberia (Stohl et al. 2013; Stohl 2006). Many have thus suggested that gas flaring, especially in the upstream OG industry, is a critical source of $\mathrm{BC}$ emissions, despite its relatively small contribution to global emissions (Stohl et al. 2013; U.S. EPA 2012). Moreover, the atmospheric lifetime of BC is very short relative to $\mathrm{CO}_{2}$, such that the mitigation of $\mathrm{BC}$ emissions could enable climatic benefits on a much shorter timescale than current $\mathrm{CO}_{2}$-reduction efforts (IPIECA 2014; Bond et al. 2013; U.S. EPA 2012; Kopp and Mauzerall 2010), particularly in the developing world (Kopp and Mauzerall 2010).

Unfortunately, $\mathrm{BC}$ emissions from gas flaring in the $\mathrm{OG}$ industry are poorly characterized and highly uncertain (IPIECA 2014; Stohl et al. 2013; McEwen and Johnson 2012; U.S. EPA 2012). BC emissions from in-field gas flares have been historically difficult to measure; as such, published emission factors relating $\mathrm{BC}$ emission to a quantity of flared gas are necessarily derived from a very limited number of small-scale laboratory and controlled field experiments (McEwen and Johnson 2012). Two oft-cited emission 
factors in the literature are those of the Canadian Association of Petroleum Producers (CAPP, 2007) and McEwen and Johnson (2012). CAPP proposed a $\mathrm{PM}_{2.5}$ emission factor of $2.5632 \mathrm{~g} / \mathrm{m}^{3}$ (significant figures as reported) based on a U.S. Environmental Protection Agency (EPA) factor for landfill gas flares attributed to filterable PM measurements conveyed in a confidential report (U.S. EPA 1991). CAPP adjusted the EPA factor to apply to a higher energy content gas stream, where a value of $45 \mathrm{MJ} / \mathrm{m}^{3}$ was used to represent the volumetric higher heating value $\left(\mathrm{HHV}_{\mathrm{V}}\right)$ of associated gas typical of the upstream $\mathrm{OG}$ industry. Although not specified, CAPP presumably assumed a linear relationship with $\mathrm{HHV}_{\mathrm{V}}$ to scale up the EPA landfill gas emission factor of $\sim 0.85 \mathrm{~g} / \mathrm{m}^{3}\left(53 \times 10^{-6} \mathrm{lb} / \mathrm{ft}^{3}\right)$ (U.S. EPA 1991) starting from an approximate $\mathrm{HHV}_{\mathrm{V}}$ of $15 \mathrm{MJ} / \mathrm{m}^{3}$ for landfill gas (McEwen and Johnson 2012). This scaling procedure implies the following BC emission factor (EF) relation: $E F\left[\mathrm{~g} / \mathrm{m}^{3}\right]=0.05696 H H V_{V}\left[\mathrm{MJ} / \mathrm{m}^{3}\right]$ at standard conditions of $1 \mathrm{~atm}$ and $15^{\circ} \mathrm{C}$ as used by CAPP (2007) and the Intergovernmental Panel on Climate Change (IPCC, 2006), with the inherent assumption that within the accuracy of this factor, flare-generated PM is predominantly BC. This is consistent with recent field data (Weyant et al. 2016) suggesting that the presence of non-BC aerosols in a flare plume is "not statistically different from zero," based on absorption measurements downstream of the flame. McEwen and Johnson (2012) performed BC yield measurements from lab-scale flares in quiescent laboratory conditions with alkane-based compositions representative of associated gas in Alberta's upstream OG industry. Based on the expected reduction in sooting propensity under crosswind conditions, they proposed a "worst-case" emission factor as a function of $\mathrm{HHV}_{\mathrm{V}}$ via a linear fit to their data: $E F\left[\mathrm{~g} / \mathrm{m}^{3}\right]=0.0548 H H V_{V}[\mathrm{MJ} /$ $\left.\mathrm{m}^{3}\right]-1.98$, which has been adjusted for the above noted standard conditions. Finally, the 
BC emission factor used by Stohl et al. (2013) in the GAINS (greenhouse gas - air pollution interactions and synergies) model (Amann et al. 2011) is $1.6 \mathrm{~g} / \mathrm{m}^{3}$, derived with data from CAPP (2007), the U.S. EPA (1995), and Johnson et al. (2011).

Recently, aircraft sampling techniques have been used to estimate $\mathrm{BC}$ emissions from flares in the Bakken formation of North Dakota (Weyant et al. 2016; Schwarz et al. 2015). Weyant et al. (2016) derived BC emissions factors starting from recorded concentrations of $\mathrm{BC}, \mathrm{CO}_{2}$, and methane $\left(\mathrm{CH}_{4}\right)$ during 1-2 s transects of atmospheric flare plumes. An estimated flare gas composition $\left(\mathrm{C} 1-\mathrm{C} 15, \mathrm{CO}_{2}\right.$, nitrogen $\left(\mathrm{N}_{2}\right)$, and other trace species) based on measurements of $\mathrm{C} 1$ to $\mathrm{C} 4$, hydrogen sulphide $\left(\mathrm{H}_{2} \mathrm{~S}\right)$, plus combined diluent species from seven sites in the Bakken region (Wocken et al. 2013) was subsequently used to derive hydrocarbon-mass-based $\mathrm{BC}$ emission factors for the flares. The average emission factors of the 26 measured flares spanned two orders of magnitude from 0.0023 to $0.33 \mathrm{~g} / \mathrm{kg}$ hydrocarbons and the average emission factor for all flares was $0.14 \mathrm{~g} / \mathrm{kg}$ hydrocarbons. The authors suggested an upper bound for the average emission factor of their measured flares of $0.31 \mathrm{~g} / \mathrm{kg}$ hydrocarbons based on non-selective absorption measurements of sampled aerosols (although a contradictory value of $3.1 \mathrm{~g} / \mathrm{kg}$ hydrocarbons appears in their abstract, which is presumably a typographical error). Unfortunately, the measured flares were all non-visibly smoking and represent a subset of flares with presumably small relative contributions to global $\mathrm{BC}$ emissions from flaring. It was also noted that "two flares that were resampled on different days did not maintain consistent $\mathrm{BC}$ emission factors," with a provided example varying by a factor of twelve (Weyant et al. 2016). This could point to large variability in the operating conditions of the measured flares and/or could suggest that longer sampling durations (either acquired 
continuously or as an ensemble of many measurements) are needed to account for the inherently turbulent soot formation and emission process.

This paper presents direct field measurements of $\mathrm{BC}$ emission rates from a variety of flares in Ecuador's Orellana province and the state of Veracruz, Mexico. BC emission rates were measured within precisely calculated uncertainties using the recently developed sky-LOSA measurement technique (Johnson et al. 2010, 2011, 2013) (line-of-sight attenuation using skylight). The flares in Ecuador were measured during two separate field campaigns, first in June 2014, and subsequently in October 2015. During the latter campaign, parallel measurements of flare gas flow rates in the lines feeding the flares were performed, and extractive samples were drawn for detailed compositional analysis. These data allowed the first direct measurements of fuel-mass-specific BC yields from in-field associated gas flares. Results are compared with the limited available data and emission factor models in the literature, enabling fresh insights into the potential significance of global $\mathrm{BC}$ emissions from flaring, the strong influence of flare gas chemistry on relative emission rates, and the importance of absolute emission rate data for identifying reduction opportunities and quantifying mitigation efforts.

\subsection{Method}

\subsubsection{Measured Flares}

Fourteen independent measurements were performed on nine flares located at sites under

the jurisdiction of Petróleos Mexicanos in Veracruz, Mexico and Petroamazonas in Orellana, Ecuador. The measured flares in Veracruz included three pit flares, a steamassisted stack flare, and a steam-assisted enclosed ground flare, and were associated with refinery and petrochemical operations. All four measured flares in Orellana were vertical 
stack flares and were associated with upstream oil production activities. Table 2.1 summarizes the types of the measured flares, operating conditions, engineered emissions reduction techniques employed, and the naming convention used in this manuscript. Additional details and photographs of the flares are provided in Section A.1. The flares in Orellana were all simple pipe flares fed by one or more lines connected into the base of each flare stack. As noted in Section A.1.2, Flare O1 employed a partial-premixing technique at its base in which the input flare line terminated in a vertical-axis nozzle situated below a vertically offset flare stack. It was presumed that this design was intended to reduce flare-generated $\mathrm{BC}$ by entraining ambient air at the base of the stack to mix with the flare gas prior to combustion.

Table 2.1: Summary of flares measured at $O G$ sites in Veracruz, Mexico and Orellana, Ecuador. Operating conditions of each flare are shown alongside the flare type and key design characteristics.

\begin{tabular}{|c|c|c|c|c|}
\hline Flare & Type $^{\mathbf{a}}$ & $\begin{array}{c}\text { Nominal } \\
\text { Stack } \\
\text { Diameter }\end{array}$ & $\begin{array}{c}\text { Simultaneous } \\
\text { Flow \& } \\
\text { Composition? }\end{array}$ & Operating Conditions \\
\hline V1 & Pit & n/a & N & $\sim 24-h o u r$ event \\
\hline V2 & Ground (SA, E) & n/a & N & Steady \\
\hline V3 & Pit & n/a & N & Steady \\
\hline V4 & Stack (SA) & $36^{\prime \prime}$ & N & Unsteady/control issue \\
\hline V5 & Pit & n/a & N & Steady \\
\hline O1 & Stack (PP) & $6 "$ & Y (2015) & Steady \\
\hline O2 & Stack & $12^{\prime \prime}$ & Y (2015) & Steady \\
\hline O3 & Stack & $6^{\prime \prime}$ & Y (2015) & Steady \\
\hline O4 & Stack & $12^{\prime \prime}$ & Y (2015) & \\
\hline
\end{tabular}

a $\mathrm{SA}=$ Steam-assisted; $\mathrm{E}=$ Enclosed; $\mathrm{PP}=$ Partially premixed via an air entrainment nozzle arrangement at the base of the flare stack

b Measured using sky-LOSA images.

c One of two lines feeding the flare had variable/oscillating flow, but the flame appeared qualitatively steady.

As more fully detailed in Section A.1, apparent operating conditions and measured flare flow rates where available, were reasonably steady for all flares during measurements 
with the notable exceptions of Flare V1 and Flare V4. Flare V1 experienced a very significant, large-volume, 24 -hour flaring event (see Section A.1.1) during the field campaign and three independent $\mathrm{BC}$ emission rate measurements were successfully performed on this flare as the event was ending and particulate emissions were in decline. Flare V4 was experiencing an apparent steam control issue that caused slow oscillations in the size of the flame.

\subsubsection{Black Carbon Emission Rate - Sky-LOSA Theory}

$\mathrm{BC}$ emission rates were calculated using sky-LOSA, an imaging technique developed by Johnson et al. $(2010,2011,2013)$ that enables the quantification of the instantaneous BC emission rate from the atmospheric plume of an in situ gas flare. This is accomplished through the analysis of high framerate grayscale image data (over a narrow spectral bandwidth) along an artificial control surface transecting the plume in the image plane. Rayleigh-Debye-Gans theory for Polydisperse Fractal Agglomerates (RDG-PFA, rigorously described by Sorensen (2001)) is used to compute line-of-sight (LOS) integrated $\mathrm{BC}$ volume fractions along the control surface, which are coupled with velocity measurements using Image Correlation Velocimetry (ICV) to compute the time-resolved $\mathrm{BC}$ emission rate. Referring to Johnson et al. (2013) for a complete development of skyLOSA theory, the mass emission rate can be derived via the combination of RDG-PFA and Beer-Lambert law, giving:

$$
\dot{m}_{B C}=\frac{\varrho_{B C} \lambda}{6 \pi\left(1+\rho_{s a}\right) E\left(m_{\lambda}\right)} \int u_{n}\left[\left(-\ln \tau^{*}\right)\right] d s=\left(\sigma_{e}^{m}\right)^{-1} \int u_{n}\left[\left(-\ln \tau^{*}\right)\right] d s
$$

where $\varrho_{B C}$ is the material density of BC (i.e., the primary particle density as opposed to the bulk density) $\left[\mathrm{kg} \mathrm{m}^{-3}\right], \lambda$ is the measurement wavelength $[\mathrm{m}], \rho_{s a}$ is the ratio of aggregate- 
average scattering and absorption cross-sections $[-], E\left(m_{\lambda}\right)$ is the absorption index of refraction function for flame-generated $\mathrm{BC}[-], m_{\lambda}$ is the spectral complex refractive index of $\mathrm{BC}[-], u_{n}$ is the volume fraction-weighted particle velocity normal to the control surface along the $\operatorname{LOS}\left[\mathrm{m} \mathrm{s}^{-1}\right.$, and $\tau^{*}$ is the LOS-transmittance that would be observed absent of inscattering effects from ambient radiation (i.e. the idealized transmittance presented by Johnson et al. (2013)) [-]. As shown in Eq. (2.1), the reciprocal of terms outside the integral represents the mass extinction cross-section of the average particle at the measurement wavelength $\left(\sigma_{e}^{m}\right)$ along the LOS axis $\left[\mathrm{m}^{2} \mathrm{~g}^{-1}\right]$ calculated considering polydispersity.

For a sky-LOSA measurement subject to inscattering of hemispherical skylight and solar radiation, the idealized transmittance can be derived through consideration of a control volume spanning the LOS axis (Johnson et al. 2013). Solution of the resulting differential equation yields:

$$
\tau^{*}=\left(\tau_{o b s}-\frac{(B+C)}{\sigma_{e} I_{L O S}^{o}}\right)\left(1-\frac{(B+C)}{\sigma_{e} I_{L O S}^{o}}\right)^{-1}
$$

where $\tau_{o b s}$ is the field-measured/observed transmittance of the plume (including inscatter components) [-], $I_{\text {LOS }}^{o}$ is the unobstructed LOS skylight intensity $\left[\mathrm{W} \mathrm{m}^{-2} \mathrm{sr}^{-1}\right], \sigma_{e}$ is the extinction cross-section of the average aggregate $\left[\mathrm{m}^{2}\right], B=\int_{2 \pi} I_{s k y} \sigma_{s}^{\prime}(\theta) d \Omega$ quantifies the radiant intensity of inscattered hemispheric skylight by the average aggregate $\left[\mathrm{W} \mathrm{sr}^{-1}\right.$ ], $C=E_{S} \sigma_{s}^{\prime}\left(\theta_{S}\right)$ quantifies the radiant intensity of inscattered solar radiation by the average aggregate $\left[\mathrm{W} \mathrm{sr}^{-1}\right], \sigma_{S}^{\prime}(\theta)$ is the angular differential scattering cross-section of the average particle (including $v v$ - and $h h$-polarization components) $\left[\mathrm{m}^{2} \mathrm{sr}^{-1}\right], \theta$ is the scattering angle of incident radiation relative to the $\operatorname{LOS}[\mathrm{rad}]$, and $\theta_{s}$ is the scattering angle of incident 
solar radiation relative to the LOS [rad]. $I_{s k y}$ represents the directionally varying skylight intensity $\left[\mathrm{W} \mathrm{m}^{-2} \mathrm{sr}^{-1}\right]$ and $E_{S}$ represents the solar radiance at ground level $\left[\mathrm{W} \mathrm{m}{ }^{-2}\right]$.

$\mathrm{BC}$ from turbulent diffusion flames, such as gas flares, are known to exist as massfractal-like aggregates of constant size nano-scale spherules (Faeth and Köylü 1995; Köylü et al. 1995; Köylü and Faeth 1992). Consequently, parameters based on the average particle (calculated using RDG-PFA) are evaluated over a polydisperse fractal aggregate size distribution.

\subsubsection{Black Carbon Emission Rate - Sky-LOSA Implementation}

The present sky-LOSA field measurements of $\mathrm{BC}$ emission rate were implemented as in Johnson et al. (2013), apart from the following hardware upgrades and algorithmic enhancements implemented for the measurements in Ecuador. High framerate grayscale image data for sky-LOSA analysis were acquired in-field using the scientific-CMOS (complementary metal-oxide-semiconductor) sky-LOSA camera (pco, Edge 5.5). During previous measurements (Johnson et al. 2013) and the present measurements in Mexico, image data were written directly to the field-computer's RAM at approximately $0.5 \mathrm{~GB} / \mathrm{s}$, which limited full-sensor acquisition times to less than one minute. For the present measurements in Ecuador however, field-hardware was upgraded to include 1 TB of flash storage enabling multiple ten-minute acquisitions before offloading was necessary. This upgrade enabled the calculation of mean $\mathrm{BC}$ emission rates over significantly longer sampling times to better capture the non-stationary behaviour of emissions and provide robust time-averaged emissions data. Upgraded software (LaVision, DaVis v.8.2.3) used to obtain the volume fraction-weighted mean velocity along the LOS-axis allowed more rigorous estimation of velocity field uncertainty based on correlation statistics between the 
first image in the ICV pair and the second image de-warped using the computed velocity field (Wieneke 2015). This additional functionality provides a more robust estimation of uncertainty in the $\mathrm{BC}$ emission rate associated with the determination of plume velocity. Both a standard and a Combined Multiple Latin Hypercube Sampled (CM-LHS) Monte Carlo (MC) method as described by Nakayama (2011) (used where applicable to reduce computation time) were used to rigorously calculate experimental uncertainties. As further detailed in Section A.3, MC calculations used probability distributions (Johnson et al. 2013) of physical, morphological, and optical properties of flame-generated BC aggregates as well as uncertainties in plume velocity and background sky reconstruction to compute uncertainties in $\mathrm{BC}$ emission rate, such that the coefficient of variation for all computed percentiles within the $95 \%$ equal tail confidence interval were less than $0.34 \%$.

\subsubsection{Flare Gas Flow Rate Measurement}

Simultaneous measurements of flare gas flow rate were performed during the 2015 measurements in Ecuador's Orellana province to enable calculation of $\mathrm{BC}$ yields for the measured flares. Standard conditions of $1 \mathrm{~atm}$ and $15^{\circ} \mathrm{C}$ are adopted in the present study in accordance with the IPCC (2006) and CAPP (2007); hereinafter, units of cubic metres $\left[\mathrm{m}^{3}\right]$ are defined to be at these conditions. Existing emission factors in the literature have been adjusted to these standard conditions for comparison with current data.

Two complementary flare gas flow rate measurement techniques were used: a tracer-dilution technique and an optical velocity measurement technique. The tracerdilution technique employed a mass flow controller (Brooks, SLAMF50S) to inject negligibly small volumes of a stable tracer (acetylene) into the turbulent flare lines at a known rate. At a position sufficiently downstream of the injection point, a cavity ring- 
down instrument (Los Gatos Research, 915-0043) was used to continuously sample and measure tracer gas concentration in the fully mixed flare gas-tracer gas mixture. Molar continuity (assuming tracer stability) was used to compute flare gas standard volumetric flow rate with knowledge of flare and tracer gas properties. Injection and sampling were performed via 1/4"-diameter stainless steel insertion probes; each $1 / 4$ " probe had two orthogonal rows of fifteen, 1/32"-diameter holes drilled along its length that spanned the flare line diameter. The probes were positioned so that the rows of holes were aligned at $45^{\circ}$ to the bulk flow direction to promote rapid mixing of the injected tracer or to ensure representative sampling.

For the optical velocity measurement technique, an optical flow meter (OFM, Photon Control, Focus 2.0) was used to measure the flare gas velocity at the centre of the flare line. With knowledge of flare gas properties, dimensions of the flare line, and an assumed axisymmetric velocity profile, the flow rate of flare gas was calculated. In some instances, OFM measurements of raw velocity were corroborated using an insertion vane wheel anemometer (Höntzsch, ZS18GE-mc40). To support flow rate calculations using the optical velocity measurement technique, flare gas pressure and temperature data were acquired via an absolute digital pressure gauge (Additel, ADT 681) and a 4-wire Pt100 platinum resistance thermometer (Höntzsch, TF500).

\subsubsection{Flare Gas Composition}

To enable direct calculation of BC yields and to provide insight into their sensitivity to fuel composition and chemistry, extractive gas samples were acquired from relevant flare lines for off-site analysis. Highly-resolved gas chromatographic analyses (test method: CAN/CGSB-3.0 No. 14.3-99 (Standards Council of Canada 1999)) were contracted to 
obtain volume fractions of alkanes to $\mathrm{C} 15$; alkenes to $\mathrm{C} 10$; cycloalkanes and aromatics to C12; other combustibles (hydrogen $\left(\mathrm{H}_{2}\right)$ and $\mathrm{H}_{2} \mathrm{~S}$ ); and diluents (helium $(\mathrm{He}), \mathrm{CO}_{2}$ and $\mathrm{N}_{2}$ ). Full composition details are available in Section A.4.

\subsection{Results}

\subsubsection{Black Carbon Emissions}

Figure 2.1 shows the time-averaged $\mathrm{BC}$ emission rates of all in-field flares measured using sky-LOSA to date. Included are measurements in Uzbekistan (Johnson et al. 2011); Poza Rica, Mexico (Johnson et al. 2013); and the fourteen measurements of the nine flares constituting the current dataset. Field-measured time-averaged $\mathrm{BC}$ emission rates in the current dataset ranged from $0.003 \mathrm{~g} / \mathrm{s}$ to $53.7 \mathrm{~g} / \mathrm{s}$ and include the lightest and heaviest sooting flares that have been measured using sky-LOSA. 


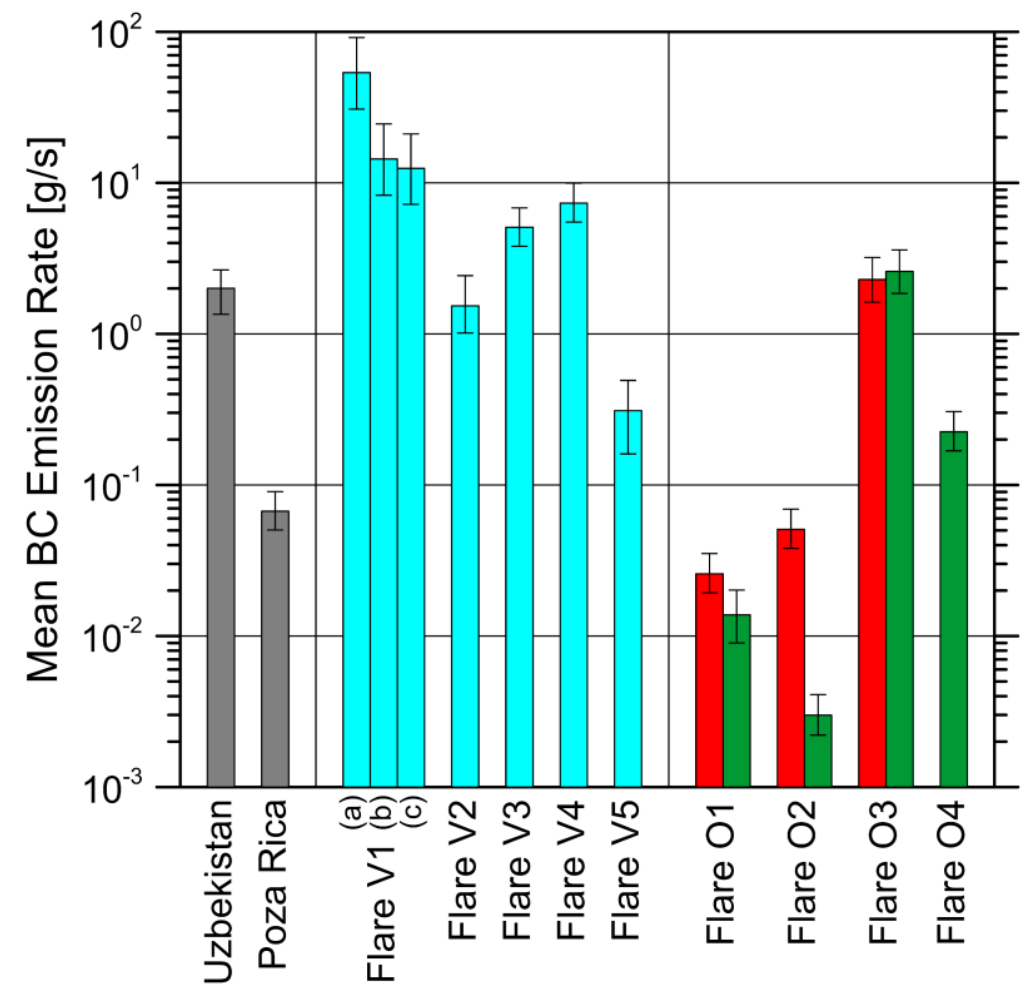

Figure 2.1: Absolute mean BC emission rates measured using sky-LOSA in $\mathrm{g} / \mathrm{s}$. Gray bars represent previous measurements in Uzbekistan (Johnson et al. 2011) and Poza Rica, Mexico (Johnson et al. 2013) while the fourteen coloured bars represent measurements in Veracruz, Mexico in 2012 (cyan) and Orellana, Ecuador in 2014

(red) and 2015 (green). Note the logarithmic scale on the vertical axis which is necessary to capture the large variation of emission rates among flares.

As discussed in Section A.3.5, the time-resolved BC emission rate data for each flare were highly stochastic. All but one flare were statistically trend non-stationary (at a 5\% significance level using the Kwiatkowski-Phillips-Schmidt-Shin test (Kwiatkowski et al. 1992)) and exhibited positive skewness (with a mean and maximum sample skewness of 1.04 and 3.21). An example of this stochastic nature is shown in Figure 2.2, where a $5 \mathrm{~s}$ subset of instantaneous BC emission rate data from Flare O3 measured in 2015 is plotted along with $95 \%$ confidence intervals computed using the described MC methodology. As further discussed in Section A.3.5, in the most extreme case (Flare $\mathrm{O} 2$ as measured in 2015), the inherent variability of the $\mathrm{BC}$ emission rate was to the extent that $10 \%$ of the 
instantaneous data were responsible for $56 \%$ of total emissions. These observations imply that $\mathrm{BC}$ emissions from flares are heavily weighted by intermittent bursts of optically dense structures within the plume, which suggests that long-duration measurements are necessary to obtain robust average emission metrics and future measurements should strive to maximize measurement duration. Coupled with the more than four order of magnitude variation in mean $\mathrm{BC}$ emission rates among the measured flares, this highlights how aggregate emissions can be dominated by outliers and underscores the importance of quantitative measurements in identifying mitigation opportunities. Indeed, action to reduce or eliminate a flare like $\mathrm{V} 1$ (emitting BC continuously at the maximum measured rate) would have equivalent $\mathrm{BC}$ reductions to mitigating almost 18,000 flares similar to $\mathrm{O} 2$ (as measured in 2015). Alternately stated, Flare V1 operating at the maximum measured rate for one day (which as noted in Section A.1.1 is likely much lower than the peak emission rate during the $\sim 24$-hour flaring event) produces equivalent $\mathrm{BC}$ emissions to more than 49 flares similar to O2 (as measured in 2015) operating continuously for a year. 


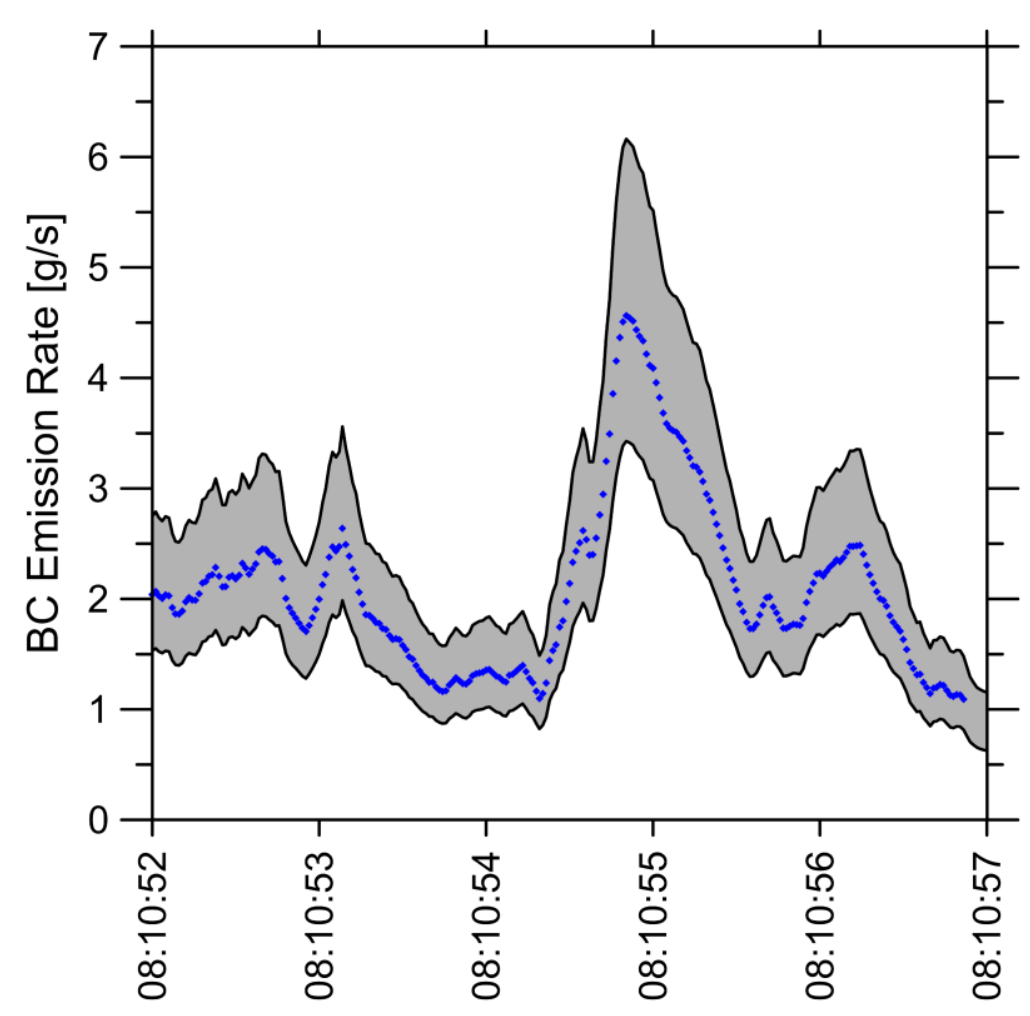

Figure 2.2: A 5 s subset of measured instantaneous BC emission rate (calculated at $50 \mathrm{~Hz}$ ) from Flare $\mathrm{O3}$ in 2015 showing high variability. The blue symbols represent the instantaneous mean and the shaded region represents the $95 \%$ confidence interval computed using the MC methodology.

\subsubsection{Flare Gas Composition}

Table 2.2 shows the measured associated gas compositions by chemical group and key properties of flared volumes relevant to the propensity of $\mathrm{BC}$ emissions from turbulent diffusion flames - refer to Section A.4 for a detailed summary of flare gas composition. For context, Table 2.2 includes data representative of associated gas compositions at oil and bitumen batteries in Alberta (derived from 60,000+ gas samples (Johnson and Coderre 2012)) and an assumed mean composition from the Bakken region (Wocken et al. 2013) used in Weyant et al.'s (2016) analysis. As noted in the footnotes of Table 2.2 and further discussed in Section A.1.2, downstream of the sampling point, Flare O1 incorporated an air entrainment nozzle at the base of the flare stack such that the degree of partial premixing of flare gas and air within the flare stack was unknown. 
Table 2.2: Summary of flare gas compositions of the measured flares by chemical group with key flare gas properties relevant to $\mathrm{BC}$ emissions from turbulent diffusion flames. Also shown are mean associated gas compositions from oil and bitumen batteries in Alberta's upstream OG industry (Johnson and Coderre 2012) and an assumed mean associated gas composition from the Bakken region (Wocken et al. 2013).

\begin{tabular}{|c|c|c|c|c|c|c|}
\hline Composition & Flare O1 $^{\mathrm{a}}$ & Flare O2 & Flare O3 & Flare O4 & Alberta $^{\mathbf{b}}$ & Bakken $^{\mathbf{c}}$ \\
\hline Diluent $-\mathrm{CO}_{2}$ & 12.64 & 9.19 & 5.69 & 6.30 & 1.41 & 0.57 \\
\hline Diluent $-\mathrm{N}_{2}$ & 8.17 & 5.74 & 3.35 & 4.11 & 3.35 & 5.21 \\
\hline Alkanes & 78.10 & 84.46 & 89.80 & 88.79 & 94.85 & 94.19 \\
\hline Cycloalkanes & 0.57 & 0.46 & 1.05 & 0.68 & - & - \\
\hline Alkenes and Cycloalkenes & 0.46 & 0.08 & 0.04 & 0.06 & - & - \\
\hline Aromatics & 0.02 & 0.02 & 0.05 & 0.02 & - & - \\
\hline Other Combustibles & 0.01 & 0.03 & 0 & 0.04 & - & - \\
\hline Property & & & & & & \\
\hline Molecular Weight, $[\mathrm{kg} / \mathrm{kmol}]$ & 31.57 & 27.73 & 36.61 & 30.33 & 19.21 & 25.19 \\
\hline Carbon-to-Hydrogen Ratio $[-]$ & 0.361 & 0.336 & 0.366 & 0.344 & 0.273 & 0.310 \\
\hline $\begin{array}{c}\text { Fraction of Hydrocarbons that were } \\
\text { Unsaturated, }\left[\%{ }_{\mathrm{v} / \mathrm{v}}\right]\end{array}$ & 0.61 & 0.12 & 0.10 & 0.10 & - & - \\
\hline Higher heating value, $\left[\mathrm{MJ} / \mathrm{m}^{3}\right]$ & 51.80 & 48.87 & 71.29 & 57.77 & 38.236 & 55.11 \\
\hline
\end{tabular}

${ }^{a}$ Measured in the line feeding Flare O1. As noted earlier, Flare O1 employed an air entrainment system at the base of the flare stack such that an unquantified amount of air would have been mixed into the flare gas prior to combustion at the flare exit.

${ }^{\mathrm{b}}$ From Johnson and Coderre (2012)

${ }^{\mathrm{c}}$ From Wocken et al. (2013)

While the associated gases in Ecuador's Amazon basin contained heavier alkane mixtures (resulting in a higher energy content and a greater carbon-to-hydrogen ratio) than associated gases in Alberta and the Bakken region, they were also significantly more diluted with $\mathrm{CO}_{2}$ and $\mathrm{N}_{2}$. The current data illustrate that flare gas composition can vary significantly within small geographic areas, which was also observed in Alberta and the Bakken region, where total diluent fractions as high as $29.1 \%$ and $19.8 \%$ were observed, respectively, despite averages of less than $6 \%$. As the literature has shown that $\mathrm{BC}$ emissions from diffusion flames are most sensitive to dilution (Glassman 1998; Gülder and Snelling 1993), aeration (Gülder 1995), energy content of the fuel (McEwen and Johnson 2012), and the relative fraction of unsaturated hydrocarbons (often quantified using the 
fuel's carbon-to-hydrogen ratio) (U.S. EPA 1995), it is imperative that future fieldmeasured $\mathrm{BC}$ emission factors are interpreted in the context of matching flare gas composition data. Moreover, highly detailed compositional analyses are required to resolve unsaturated components, which can strongly influence sooting propensity; unfortunately, although these compounds have been observed in small amounts in crude oil across the globe, petroleum geochemists do not commonly analyze for such compounds (Curiale and Frolov 1998).

\subsubsection{Black Carbon Yields}

Field-measured $\mathrm{BC}$ yields $\left(Y_{B C}\right)$ in Ecuador are shown in Figure 2.3 as a function of $\mathrm{HHV}_{\mathrm{V}}$ with $95 \%$ confidence intervals computed using the MC methodology detailed in Section A.6. A potential range of $\mathrm{BC}$ yields from the measurements of Weyant et al. (2016) in the Bakken region is shown as a shaded area on the graph, where data have been converted from a mass of hydrocarbons basis to a volume of flare gas basis using the cited range of flare gas compositions of seven flares in the Bakken (Wocken et al. 2013), and are plotted spanning the range of heating values in these same samples. The measured $\mathrm{BC}$ yields in

Ecuador spanned more than two orders of magnitude from 0.03 to $3.85 \mathrm{~g} / \mathrm{m}^{3}$ (refer to Section A.6 for site-specific results) with an average of $1.83 \mathrm{~g} / \mathrm{m}^{3}$. The heaviest-sooting flare was $\sim 27$ times greater than the estimated average for the Bakken region (Weyant et al. 2016), while only the lightest-sooting flare was within the range of those same measurements. 

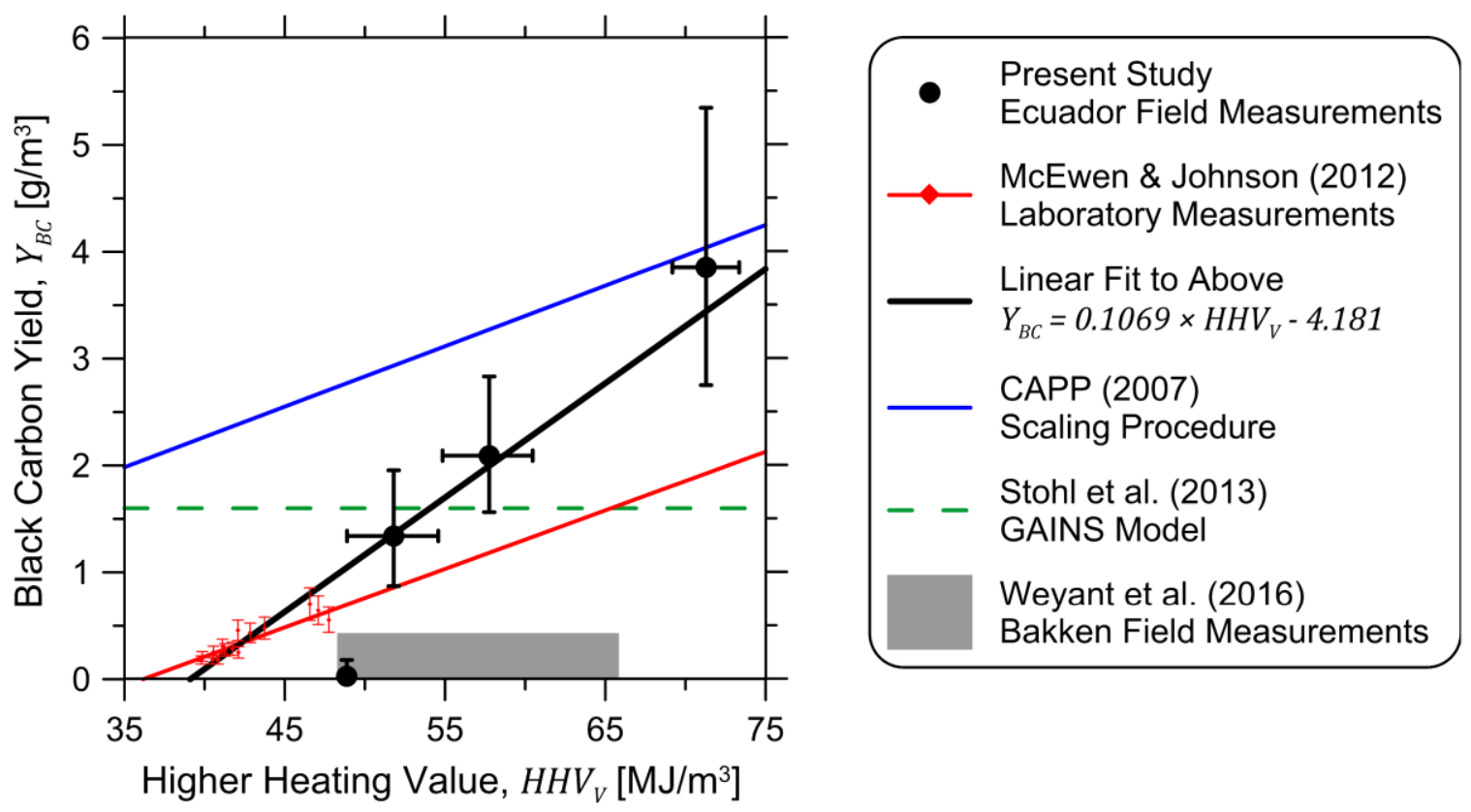

Figure 2.3: Mean and 95\% confidence interval of measured BC yields on a massper-volume basis shown as a function of volumetric higher heating value. Included are the predictive emission factors using the heating value scaling procedure of CAPP (2007), the correlation developed by McEwen and Johnson (2012), and the emission factor currently used in the GAINS model (Stohl et al. 2013); also shown is the estimated range of yield measurements in the Bakken region by Weyant et al. (2016) based on the span of reported measurements converted from mass-per-mass of hydrocarbons to mass-per-volume of flare gas using the cited range of gas compositions (Wocken et al. 2013).

Overlaid in Figure 2.3 are a prediction of BC yield as a function of $\mathrm{HHV}_{\mathrm{V}}$ using the simple scaling procedure implemented by CAPP (2007), the experimental data and empirical correlation derived by McEwen and Johnson (2012), and the current GAINS model emission factor (Stohl et al. 2013). The CAPP scaling procedure tends to overestimate the measured yields, although the heaviest sooting flare (O3) approached the CAPP prediction. Given that the CAPP factor is ultimately extrapolated from a single measurement on a (potentially enclosed) landfill gas flare at $\sim 15 \mathrm{MJ} / \mathrm{m}^{3}$, it is not surprising that the prediction fails at much higher heating values. Alternatively, McEwen and Johnson's correlation, derived using the data points reproduced in red on the figure, 
underestimated the $\mathrm{BC}$ yield for three of the measured flares at greater $\mathrm{HHV}_{\mathrm{V}}$. Although $\mathrm{HHV}_{\mathrm{V}}$ has been observed in laboratory experiments (Corbin 2014) to dominate other effects by order(s) of magnitude, the sooting propensity of turbulent diffusion flames is known to be sensitive to ambient conditions, aerodynamic parameters, and other chemistry metrics, many of which happen to have a positive correlation with $\mathrm{HHV}_{\mathrm{V}}$. Consequently, this presentation of the results is not intended to suggest that $\mathrm{BC}$ emission factor is solely a function of flare gas energy content, but instead highlights the utility of this parametrization as a starting point. Within this context, recognizing the pragmatic need for measurement-backed models, the black line shows an empirical fit to available laboratory (McEwen and Johnson 2012) and field data for black carbon yields from flares as a function of $\mathrm{HHV}_{\mathrm{V}}$. The coefficient of determination $\left(\mathrm{R}^{2}\right)$ for this line is 0.896 . Interestingly, if published associated gas compositions from nearby Alberta (Johnson and Coderre 2012) were representative of flares measured by Weyant et al. (2016), their data would align quite well with this model as well as the data of McEwen and Johnson (2012), perhaps suggesting that the assumed composition of Weyant et al.'s (2016) over-estimates the actual heating value of their measured flares. Moreover, as further discussed in Section A.6, Flare $\mathrm{O} 2$ (corresponding to the lowest data point included in this correlation), might be reasonably considered an outlier relative to other flares, due to its highly non-stationary nature linked with variable flow in one of its supply lines that resulted in large gaps of nondetectable emissions. Without this point included, both the slope and $\mathrm{R}^{2}$-value of the fit would increase $\left(Y_{B C}=0.1106 \times H H V_{V}-4.296, \mathrm{R}^{2}=0.966\right)$. 


\subsection{Implications}

To date, sixteen absolute $\mathrm{BC}$ emission rate measurements have been performed on eleven flares using sky-LOSA, with the results spanning more than four orders of magnitude. At least one flare has also been observed however, with plume $\mathrm{BC}$ concentrations sufficiently high such that direct solar radiation could not penetrate the plume. This suggests that $\mathrm{BC}$ emissions from flaring span an even larger range due to the presence of super-emitters. The existence of such drastic flaring events supports the notion that super-emitters must be targeted when performing measurements to support the improved estimation of emissions inventories and enable effective mitigation efforts. It also begs the question of the global distribution of absolute emission rate, where the relative contribution to global $\mathrm{BC}$ emissions from non- or lightly-visibly sooting flares might be considered negligible. It is therefore important to consider absolute emission rates in addition to flare-gas-specific yields (measured in conjunction with parallel flare gas flow rate and composition data) when directing mitigation actions to optimize emissions reduction efforts. Consistent with the literature noting the non-linear intensity perception (Stevens 1957) and non-uniform spectral sensitivity (CIE 1990) of the human eye, field experience has shown that qualitative visual assessment of emissions is a poor surrogate for quantified emission rates.

The current dataset also demonstrates that flare gas compositions can be quite diverse even in small geographic regions and this diversity can have significant impact on $\mathrm{BC}$ yield. This unfortunately complicates efforts to predict regional or global emissions without considering compositional variations in flare gas.

As of 2013, the GAINS model estimates global BC emissions from gas flaring by coupling calculated flared volumes using satellite imagery from NOAA (including work 
by Elvidge et al. (2007)) and a BC emission factor of $1.6 \mathrm{~g} / \mathrm{m}^{3}$ (Stohl et al. 2013), which is a factor of three greater than McEwen and Johnson's (2012) proposed emission factor for typical associated gas compositions in Alberta. Stohl et al. (2013) contend however that "even when using the emission factor from McEwen and Johnson, gas flaring remains the second largest source of $\mathrm{BC}$ emissions north of $60^{\circ} \mathrm{N}$ and the most important anthropogenic source." Stohl et al. (2013) then suggest that the correlation of McEwen and Johnson likely underestimates equivalent emissions from in-field flares, presumably due to the difficulty in recreating complex compositions containing the heavier alkanes and unsaturated hydrocarbons observed in the OG industry within the laboratory. In contrast, Weyant et al. (2016) note that if the average BC yield from their measurements in the Bakken region is spatially and temporally representative of global flaring (the authors do however emphasize that their measurements were limited to non-visibly sooting flares) then the GAINS model emission factor is more than an order of magnitude high. However, if a similar approach is taken, then the present field measured $\mathrm{BC}$ yields would suggest that the emission factor used within the GAINS model is $\sim 10 \%$ low. This highlights the limited utility of a simple mean BC yield derived from small datasets in small geographic regions.

Recently, Huang et al. (2015) performed independent simulations of anthropogenic $\mathrm{BC}$ transport from Russia to the Arctic. In place of the GAINS value, an emission factor for gas flaring was estimated by extrapolating the relation of McEwen and Johnson (2012) to a representative $\mathrm{HHV}_{\mathrm{V}}$ derived from an example associated gas composition from a Russian oil field by Filippov (2013). Flare gas composition was computed as a weighted sum of flared gases from three stages of oil separation. Although additional data were available for oil and gas producing sites (with significantly lower heating values), Huang 
et al. (2015) argued that the oil field composition was a better representation for the OG industry in Russia as it has been noted elsewhere (PFC Energy 2007) that most associated gas in Russia has a methane content less than $50 \%$. A median $\mathrm{HHV}_{\mathrm{V}}$ of $75.5 \mathrm{MJ} / \mathrm{m}^{3}$ was calculated, yielding an emission factor of $2.27 \mathrm{~g} / \mathrm{m}^{3}$ and applied to an estimated 35.6 billion $\mathrm{m}^{3}$ flared in Russia per year (using NOAA data from 2010). Huang et al. (2015) concluded that gas flaring is responsible for $36.2 \%$ of Russia's anthropogenic $\mathrm{BC}$ emissions, the largest contributor by more than $10 \%$. However, it has been observed in the present study that McEwen and Johnson's emission factor relation is low at greater $\mathrm{HHV}_{\mathrm{V}}$ and if the assumed gas composition for Russia (with a recomputed mean $\mathrm{HHV}_{\mathrm{V}}$ of $71.5 \mathrm{MJ} / \mathrm{m}^{3} \pm 2.5 \%$ at standard conditions as defined) were in line with the trend observed in the current study, it is possible that a more appropriate emission factor using the derived linear fit to the current field measurements and lab data of McEwen and Johnson (2012) would be approximately $3.46 \mathrm{~g} / \mathrm{m}^{3}$. Thus re-considering the work of Huang et al. (2015) in the context of the present field measurements on larger heating value flares would suggest that flaring-related BC emissions from Russia could be on the order of $123 \mathrm{Gg} /$ year or as much as $\sim 46 \%$ of Russia's anthropogenic BC emissions.

Although the authors are not aware of a reliable source for a globally representative heating value for flared gas, as an initial starting point for discussion (see Section A.7 for calculation details), if one were to use satellite derived flaring data for Canada, USA, Russia, and Ecuador (Elvidge et al. 2015) to weight available flare gas composition data (Huang et al. 2015; Wocken et al. 2013; Johnson and Coderre 2012) for each country, and assuming an arithmetic mean of these data for other countries in the world, a representative global flare gas heating value could be $\sim 60.03 \mathrm{MJ} / \mathrm{m}^{3}$. Based on the proposed $\mathrm{BC}$ yield 
model in Figure 2.3, this would imply a global mean BC emission factor of $2.24 \mathrm{~g} / \mathrm{m}^{3}(40 \%$ higher than the emission factor used in the GAINS model). Alternatively, if the volumeweighted average value of $67.23 \mathrm{MJ} / \mathrm{m}^{3}$ for the four countries with available data were considered globally relevant, then the proposed BC model would imply a global mean BC emission factor of $3.01 \mathrm{~g} / \mathrm{m}^{3}$, suggesting the GAINS model emission factor could be low by almost a factor of two. While these estimates are subject to significant uncertainty, they stress the importance of accurate $\mathrm{BC}$ emission factor data in characterizing the scale of global $\mathrm{BC}$ emissions from flaring. Ultimately, accurate prediction of global flare $\mathrm{BC}$ emissions will require significantly augmented field measurement data (specifically including simultaneous measurements of flare gas composition and flow rates) coupled with comprehensive models building on the simple correlation presented here.

Most importantly, the presented direct field measurement data have provided evidence of the extreme variability in emission rates among flares (see Figure 2.1), the potential for individual flares to be very significant single sources of $\mathrm{BC}$, and the strong correlation of volume-specific BC yields with flare gas heating value. Further field measurements are certainly desired to improve upon the presented emission factor relation by introducing additional flare design, operation, and chemistry metrics, where the demonstrated implications justify the need to overcome continued challenges of gaining necessary site access to quantify flare gas flow rate and composition in conjunction with parallel BC emission rate measurements. Nevertheless, if the current results are interpreted in the context of recent efforts to track impacts of flare-generated $\mathrm{BC}$ in the Arctic and globally (Huang et al. 2015; Stohl et al. 2013), it could be the case that the significant 
global public health and climatic impacts of $\mathrm{BC}$ emissions from gas flaring in the $\mathrm{OG}$ industry are in fact underestimated.

\subsection{Acknowledgements}

This work was supported by the World Bank Global Gas Flaring Reduction Partnership (Project Manager Francisco Sucre), the United Nations Environment Programme's Climate and Clean Air Coalition (Project Manager Joseph Odhiambo), Natural Resources Canada (Project Manager Michael Layer), the Petroleum Technology Alliance of Canada (Grant \#09-9185-50), and the Natural Sciences and Engineering Research Council of Canada (NSERC, Grant \#261966, 446199, and 479641). We are especially thankful for the assistance received during field measurements in Ecuador from Petroamazonas personnel including David Neira, Javier Villacis, and David Herrera, as well as from Melina Jefferson and Darcy Corbin (Carleton University). Measurements in Mexico were possible through on the ground support and assistance of Robin Devillers (National Research Council of Canada), Michael Layer, David Picard (Clearstone Engineering Ltd.), Javier Bocanegra (Pemex), and Jorge Plauchú (independent consulting engineer). Finally, we are indebted to Berend van den Berg (Power Latinamerica Inc.) and Kevin Thomson (National Research Council of Canada) for their support in making these field measurements possible. 


\section{Chapter 3 Beam Steering Effects on Remote Optical Measurements of Pollutant Emissions in Heated Plumes and Flares}

This chapter has been peer-reviewed and was published in the Journal of Quantitative Spectroscopy and Radiative Transfer. Only minor clarifications and formatting edits have been made for consistency throughout the thesis. It should be cited as:

Conrad, B.M., Thornock, J.N. and Johnson, M.R. (2020). Beam Steering Effects on Remote Optical Measurements of Pollutant Emissions in Heated Plumes and Flares. J. Quant. Spect. Rad. Tran., 254:1-12 (doi: 10.1016/j.jqsrt.2020.107191).

This article is included in its entirety as Chapter 3 with the corresponding supplemental material included as Appendix B. The article was co-authored by the thesis author, Mr. Conrad, Prof. Thornock (The University of Utah), and Mr. Conrad's thesis supervisor, Prof. Johnson. Mr. Conrad and Prof. Johnson conceptualized the research. Prof. Thornock executed the Large-Eddy Flare Simulations. Mr. Conrad developed the methodology and software and performed the formal analysis. Mr. Conrad wrote the first draft of the manuscript; editing and revision of the manuscript were conducted jointly.

\subsection{Abstract}

Remote optical measurement techniques are valuable tools for the quantification of combustion-generated, climate-forcing emissions. Leveraging radiometric observations along a detector's line-of-sight, these techniques resolve column density information from which pollutant loading and emission rates can be deduced for an in situ atmospheric plume of a targeted source. One commonly neglected source of uncertainty in such measurements is beam steering - the deflection of light as it traverses the plume due to composition- and temperature-driven gradients in the real refractive index field of the plume. In this work, three correction parameters were derived from the radiative transfer equation to enable 
consideration of beam steering effects on these measurement techniques. A Monte Carlo procedure was performed to derive realistic optical axes through plumes of large-eddysimulated gas flares, considered to be an extreme case of beam steering due to elevated temperature and composition gradients near the flame. Deflections of light due to beam steering were quantified at wavelengths in the visible spectrum and within three diagnosticrelevant infrared absorption bands for methane and carbon dioxide. A conservative, empirical model for the degree of beam steering was derived. Moreover, from these data, correction parameters required to account for the impact of beam steering on perceived incident intensity, optical depth, and source intensity were found to be negligible at all studied wavelengths relative to typical instrument noise. Thus, this work demonstrates that even for the extreme case of a turbulent heated flare plume, beam steering has negligible impact on the ability to quantify pollutant loading and emissions.

\subsection{Introduction}

Remote optical measurement of species concentrations and pollutant emissions in industrial plumes is a rapidly evolving research area with innumerable practical applications. The wide range of emergent measurement diagnostics include techniques based on emission/absorption spectroscopy (e.g., open-path Fourier Transform Infrared (OP-FTIR) imaging (Harley et al. 2014; Gross et al. 2010a), laser-based approaches to detect absorption (Edner et al. 1987), stimulated incandescence (Crosland et al. 2015), and scattering (De Iuliis et al. 1998; Dalzell et al. 1970)) and techniques leveraging light transmission (e.g., line-of-sight attenuation (LOSA, Snelling et al. 1999; Greenberg and $\mathrm{Ku}$ 1997), solar occultation flux (Mellqvist et al. 2010), and differential optical absorption spectroscopy (Platt and Stutz 2008)). A common, albeit oft-neglected, challenge in each 
of these approaches is the refraction of light through the medium being measured. This is especially true when measurements are made on heated plumes or any plume with strong spatial inhomogeneities in composition, which can promote light refraction, or "beam steering", due to temperature- and composition-driven gradients in the refractive index (e.g., Weinberg 1963). Beam steering can lead to a loss of spatial resolution of an imaging detector, changing path lengths and optical depth through the plume, and fluctuations in observed light intensity. This challenge is amplified by turbulence typical in plumes of many industrial systems, where the magnitude of these effects would be expected to vary both spatially and temporally. The beam steering effect unfortunately cannot be wholly considered within data analysis since it requires prior knowledge of plume temperature and composition. In most applications the magnitude and impact of beam steering is thus unknown, and the existing literature on the matter is limited.

Thomson et al. (2008) addressed beam steering in their laboratory-based LOSA measurements of soot loading in a laminar ethylene-air diffusion flame at $10 \mathrm{~atm}$. The authors observed a high level of beam steering-driven artefacts in their imaging data that they were able to remove through an alternative optical arrangement that favoured a diffuse rather than collimated light source. While the refractive index gradients at $10 \mathrm{~atm}$ and hence the observed beam steering effects would be much larger than those expected at atmospheric conditions (e.g., Zerbs et al. 2009; Weinberg 1996), these results highlight the potential importance of this effect. Unfortunately, the current dearth of quantitative analysis of beam steering in combustion plumes means researchers generally can only acknowledge the existence of beam steering effects and use qualitative reasoning to argue that they are unimportant in their study (e.g., Crosland et al. 2011). 
Perhaps the most challenging example of optical measurements through heated plumes is the remote measurement of gas flares in the oil and gas sector. Flaring is the common industrial process of burning waste flammable gases in an open-atmosphere turbulent flame (McEwen and Johnson 2012). Optical measurements made near the flame tip can be subject to extreme composition and temperature gradients (Poudenx et al. 2004; Johnson and Kostiuk 2002; Everest et al. 1995), beyond those expected for typical stack or tailpipe emissions measurements, where combustion products are generally well-mixed and lower exhaust temperatures are desired for reasons of thermal efficiency (Moran and Shapiro 2006). Optical measurements of flare-generated pollutant emissions include plume transmittance within the visible spectrum for soot/black carbon particulate (Chapter 2, Conrad and Johnson 2017; Johnson et al. 2010, 2011, 2013) and OP-FTIR measurements for targeted gas species (Savary et al. 2011) and/or overall combustion efficiency (Zeng et al. 2016). As with all remote optical measurement techniques, the effect of beam steering on the measurement of flare plumes is unknown.

This work presents a quantitative assessment of beam steering effects in remote optical measurements of combustion-generated pollutants in atmospheric plumes. Highfidelity large-eddy simulations were first performed to yield spatially resolved plume temperature and composition data for the extreme case of turbulent gas flares. Next, linesof-sight representative of optical imaging measurements were modelled, along which spatially and spectrally resolved refractive index data were computed. Analyses of refractive index data along these lines-of-sight were then performed to quantify the effect of beam steering under a Monte Carlo framework. This analysis was repeated at numerous wavelengths within the visible spectrum and within typical diagnostic absorption bands for 
methane $(3.3 \mu \mathrm{m})$ and carbon dioxide $(4.3 \mu \mathrm{m}$ and $15 \mu \mathrm{m})$, the two most important greenhouse gases (IPCC 2013). Section 3.3 provides the theoretical background required to quantify the beam steering effect, Section 3.4 provides a comprehensive summary of simulation activities, and Section 3.5 presents results from the simulations and discusses the implications for remote optical measurement techniques.

\subsection{Theoretical Background}

Remote optical measurement of combustion-generated pollutant emissions can be used to obtain absolute emission rates and relative pollutant loading within plumes. Generally, such techniques leverage radiative transfer along the lines-of-sight corresponding to a detector's optical axis to quantify the column density of pollutants, spatially resolved in the case of imaging detectors. These techniques can largely be categorized into transmissive or emissive measurements and can be further demarcated by the spectrum of the detector and the targeted pollutant.

The theoretical development for these techniques begins with the radiative transfer equation (RTE). Consider a straight line-of-sight (LOS) passing through an arbitrary, heated, atmospheric plume as shown in Figure 3.1. In this figure, and to enable the subsequent analysis, it is initially assumed that light follows a straight path through the plume, thus ignoring refractive index gradients; the subscript " $\ell$ " is therefore used to identify this straight-path, or line, assumption. In the figure, red arrows signify spectral intensity $\left[\mathrm{W} \mathrm{m}^{-2} \mu \mathrm{m}^{-1} \mathrm{sr}^{-1}\right.$ ] entering the plume (incident intensity, $I_{\lambda, \ell}^{o}$ at $x=0$ ) and exiting the plume towards the detector (transmitted intensity, $I_{\lambda, \ell}^{t}$ at $x=L$ ). Green arrows represent the scattering of light out of the optical axis and green asterisks represent the local absorption of light. Radiative sources are represented by red asterisks (thermal 
emission) and pale blue arrows (inscattering). Given a specific measurement wavelength $(\lambda[\mu \mathrm{m}])$, the RTE provides a complete mathematical description of these phenomena, assuming local thermodynamic equilibrium (e.g., Modest 2013):

$$
I_{\lambda, \ell}^{t}=I_{\lambda, \ell}^{o} \exp \left(-\int_{0}^{\infty} \mu_{\lambda}(x) d x\right)+\int_{0}^{\infty} \mu_{\lambda}(x) I_{\lambda}^{s}(x) \exp \left(-\int_{x}^{\infty} \mu_{\lambda}\left(x^{\prime}\right) d x^{\prime}\right) d x
$$

where $\mu_{\lambda}(x)$ is the local spectral extinction coefficient summed over all gas and particulate plume components $\left[\mathrm{m}^{-1}\right]$ and $I_{\lambda}^{S}(x)$ is the local spectral radiative source in units of spectral intensity.

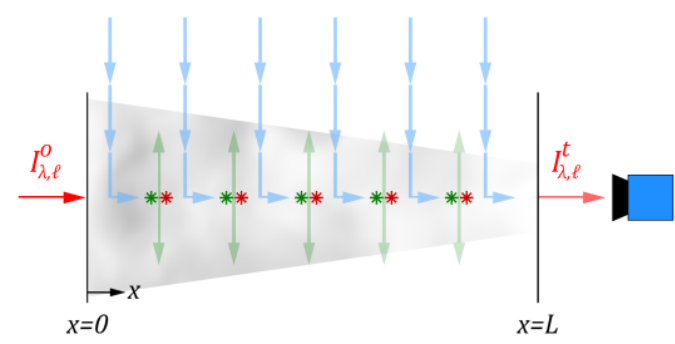

$$
\begin{aligned}
& \longrightarrow \text { Optical Axis / Line-of-Sight (LOS) Light } \\
& \longrightarrow \text { Outscattering of LOS Light } \\
& \longrightarrow \text { Inscattered Skylight and Sunlight } \\
& * * \text { Absorption \& Emission }
\end{aligned}
$$

Figure 3.1: A simplified (idealized straight-path) schematic of radiative effects along an optical line-of-sight axis and entering an optical detector including outscatter of LOS light, absorption of LOS light, inscatter of both skylight and sunlight, and source thermal emission.

The first term in Eq. (3.1) combines the incident light intensity with the typical exponential term representing attenuation of light through the entire plume, via BeerLambert-Bouguer law (e.g., Howell et al. 2016). The second term represents an intensity source via thermal emission and/or inscattering (Modest 2013) at position $x$, which is then 
attenuated through the remainder of the plume and atmosphere towards the detector (hence, integration over $\left.x^{\prime} \in[x, \infty]\right)$.

From this starting point, remote optical measurements may employ different assumptions and/or modelling techniques to extrapolate pollutant column density from radiometric observations. A common challenge, however, is the validity of the assumption that light travels along straight paths. In reality, gradients in the temperature and composition of diluted gas-phase combustion products in the plume yield corresponding gradients in the refractive index. Much like at the interface of two media, refractive index gradients in a continuous medium cause the refraction of light (e.g., Weinberg 1963). In the context of remote optical measurements, this suggests that detected light has not travelled along a straight path as assumed, but rather that it is "steered" through the plume. To accommodate beam steering, a more general form of the RTE considering an arbitrary path of light through the plume is required:

$$
I_{\lambda, c}^{t}=I_{\lambda, c}^{o} \exp \left(-\int_{c_{\lambda}} \mu_{\lambda}(\boldsymbol{r}) d s\right)+\int_{c_{\lambda}} \mu_{\lambda}(\boldsymbol{r}) I_{\lambda}^{S}(\boldsymbol{r}) \exp \left(-\int_{c_{\lambda}^{\prime}(\boldsymbol{r})} \mu_{\lambda}(\boldsymbol{r}) d s^{\prime}\right) d s
$$

where the subscripts " $c$ " and " $c_{\lambda}$ " are used to denote a curved path, which is a function of wavelength. It is important to note that the incident, source, and transmitted intensities are all sensitive to the specific path through the plume. Although Eq. (3.1) is desired to yield the straight-path Beer law transmittance - and infer column density - it is Eq. (3.2) that dictates the actual physics. Thus, the inference of pollutant loading from observed spectral intensity data is indeed complicated by the beam steering phenomenon. 


\subsubsection{Beam Steering Effect Quantification}

To glean quantitative insight into the beam steering effect, the physical meaning of the intensities in the above RTE formulations are compared in the context of a measurement technique. This analysis yields three correction parameters that may be required within a technique's algorithm.

First, consider transmittance-based measurements of a plume where an active or passive light source is leveraged to obtain pollutant column density. If the light source is not uniform and diffuse, the incident spectral intensity $\left(I_{\lambda}^{o}\right)$ may be direction-dependent, such that $I_{\lambda, \ell}^{o}$ is inherently not equivalent to $I_{\lambda, c}^{o}$ under beam steering conditions. In other words, incident light that traverses a turbulent plume and ultimately reaches a detector may originate from a ray that is oblique to the axis of the detector. This beam deflection suggests the need for a dimensionless incident intensity correction $\left(K_{\lambda, o}\right)$ :

$$
K_{\lambda, o}=\frac{I_{\lambda, c}^{o}}{I_{\lambda, \ell}^{o}}
$$

This correction parameter will be a function of the gradient in background intensity and the difference between the curved and linear paths. For the purposes of the incident intensity correction, the latter can be characterized by the angle (hereinafter referred to as the total deflection angle) between the propagation direction of incident light along the curved path and the propagation direction into the detector - i.e., the total angle that light is steered through as it propagates into the detector.

Two other corrections result from the change in the path through the plume due to beam steering, rather than the total deflection angle. The first characterizes the effective change in optical depth through the plume due to deviation from the straight line, which 
would limit the ability to accurately quantify the straight-line column density of a pollutant. This necessitates a dimensionless optical depth correction $\left(K_{\lambda, O D}\right)$ :

$$
K_{\lambda, O D}=\frac{\int_{c_{\lambda}} \mu_{\lambda}(\boldsymbol{r}) d s}{\int_{0}^{\infty} \mu_{\lambda}(x) d x}
$$

Lastly, the change in path can also impact the radiative source term that results from thermal emission and/or inscattering. Consider a radiative emission source as an example and assume that the real and imaginary components of the refractive index are both negatively correlated with gas temperature. As discussed in Section 3.4.3, light is steered towards regions of higher real refractive index, which here corresponds to lower gas temperatures (hence, lower emitted energy). Under a fixed composition constraint, this suggests that for this example the effect of beam steering on an emission source term is to reduce the source intensity. More generally, beam steering effects on source intensity would also be confounded by an associated change in the local attenuation (absorption) coefficient that scales the radiative source. This suggests a need for a dimensionless source intensity correction $\left(K_{\lambda, s}\right)$ :

$$
K_{\lambda, s}=\frac{\int_{c_{\lambda}} \mu_{\lambda}(\boldsymbol{r}) I_{\lambda}^{S}(\boldsymbol{r}) \exp \left(-\int_{c_{\lambda}^{\prime}(\boldsymbol{r})} \mu_{\lambda}(\boldsymbol{r}) d s^{\prime}\right) d s}{\int_{0}^{\infty} \mu_{\lambda}(x) I_{\lambda}^{s}(x) \exp \left(-\int_{x}^{\infty} \mu_{\lambda}\left(x^{\prime}\right) d x^{\prime}\right) d x}
$$

which is the ratio of the path-integrated source intensity for the curve to the line.

\subsubsection{Objective}

To date, limited knowledge of beam steering dictates that remote optical diagnostics inherently assume that the three correction parameters are equivalent to unity. The objective of this work is to quantify these correction parameters at typical diagnostic 
wavelengths for climate forcing dominant pollutants from combustion sources. Through simulation of the extreme case of a turbulent flare plume, reasonable bounds of the correction parameters are estimated and, as necessary, empirical models are developed for use in remote optical diagnostic algorithms.

\subsection{Methodology}

As noted in Section 3.2, prior knowledge of plume geometry (spatial distribution of optically relevant species) is required to quantify beam steering through a plume. Since these data are unavailable in practice, it is necessary to leverage simulation data to obtain statistics and derive empirical models for the above-defined correction parameters. Highfidelity flare simulations were performed for flare conditions typical of Canada's upstream oil and gas industry to yield a broadly representative collection of flare plume geometry and spatially resolved spectral refractive index (refer to Section 3.4.1). To consider reasonably representative optical axes intersecting the simulated plumes, typical optics employed in a LOSA technique to measure soot emissions from flares (sky-LOSA Chapter 2, Conrad and Johnson 2017; Johnson et al. 2010, 2011, 2013) were selected to model optical equipment and its positioning relative to an atmospheric plume. A pinhole model of the sky-LOSA camera was derived and coupled with typical camera positioning constraints and optics in a Monte Carlo (MC) framework. In this MC analysis, $10^{4}$ sets of camera position and optics were randomly selected, enabling a representative collection of realistic imaging data (refer to Section 3.4.2). Next, using the known intersection of these optical axes with the simulation domain, beam steering over randomly selected control surfaces traversing the plumes were quantified (refer to Section 3.4.3). 


\subsubsection{Flare Simulations}

High-fidelity large-eddy simulations (LESs) of typical upstream gas flares were provided by The University of Utah and executed within the Uintah Computational Framework (UCF) Software Suite (e.g., Parker 2006; Parker et al. 2006; Davison De St. Germain et al. 2000). The massively parallel ARCHES component of UCF was employed, which solves the "conservative, finite volume, compressible, low-Mach formulation of the NavierStokes equation with a pressure projection that includes the effect of variable density, reaction, and heat transfer modes in the gas phase including radiation" (Guilkey et al. 2017) with large-eddy simulation closure.

Key flare simulation parameters are summarized in Table 3.1 and were selected to yield data representative of flaring activities in Alberta, Canada's upstream oil and gas industry. To this end, simulated flare stacks were designed to be $10 \mathrm{~m}$ in height and composed of vertically oriented $4 "$ schedule 40 steel piping (Table 3.1a). Flare gas flow rate was selected to yield an exit velocity of $4.0 \mathrm{~m} \mathrm{~s}^{-1}$ and, to enable a rate-controlled constrained equilibrium mechanism for combustion chemistry, flare gas was composed of an alkane-based natural gas mixture (Table 3.1b). Corresponding Reynolds and flare gas Froude numbers were approximately 26,840 and 0.0268 , which suggests that the simulated flares - under quiescent conditions - would be buoyancy-driven with shear-dominated structures in the base of the flame (Delichatsios 1993). While flared gases can have nonnegligible quantities of heavier alkanes and even some unsaturated species (Chapter 2, Conrad and Johnson 2017; Chapter 5, Conrad and Johnson 2019a), compositions tend to be heavily dominated by methane, as observed in Alberta (Johnson and Coderre 2012). Moreover, because adiabatic flame temperatures of hydrocarbon mixtures typical of common flare gases around the world are very similar (e.g., within $55.4 \mathrm{~K}$ or $2.43 \%$ for 
measured upstream flare gas compositions (Chapter 5, Conrad and Johnson 2019a), any variation in the influence of buoyancy forces on the structure of the hot plume is expected to be negligible.

Table 3.1: Summary of simulation parameters showing (a) flare stack geometry, (b) flare gas flow metrics, and (c) crosswind conditions for the four simulations (I-IV). Flare stack diameters are those of 4 " schedule 40 piping commonly used in industry, and flare stack height is typical of flares measured by the authors in Alberta, Canada. Gas was flared at a constant rate across simulations to yield a typical buoyancy-driven flare. Crosswind velocities were selected based on the presented statistics of hourly winds in Edmonton, Alberta from 1992-2016.

\begin{tabular}{|c|c|}
\hline \multicolumn{2}{|c|}{ (a) Stack Geometry } \\
\hline Internal diameter [mm] & 102.3 \\
\hline External diameter [mm] & 114.3 \\
\hline Stack height $[\mathrm{m}]$ & 10.0 \\
\hline
\end{tabular}

\begin{tabular}{|c|c|}
\hline \multicolumn{2}{|c|}{ (b) Flow Metrics } \\
\hline Mass flow rate $\left[\mathrm{g} \mathrm{s}^{-1}\right]$ & 21.538 \\
\hline Flare gas composition & Natural Gas $^{\mathrm{a}}$ \\
\hline Stack exit velocity $\left[\mathrm{m} \mathrm{s}^{-1}\right]$ & 4.0 \\
\hline
\end{tabular}

\begin{tabular}{|c|c|c|c|c|}
\hline (c) Crosswind Conditions & Sim. I & Sim. II & Sim. III & Sim. IV \\
\hline Velocity, $u_{\infty}\left[\mathrm{m} \mathrm{s}^{-1}\right]$ & 1.11 & 1.94 & 3.06 & 7.78 \\
\hline Momentum flux ratio $[-]$ & 7.58 & 2.48 & 1.00 & 0.15 \\
\hline Velocity statistic $^{\mathrm{b}}$ & $10^{\text {th }}$ percentile & Mode & Median & $95^{\text {th }}$ percentile \\
\hline
\end{tabular}

Composition by volume: $90.53 \% \mathrm{CH}_{4}, 3.73 \% \mathrm{C}_{2} \mathrm{H}_{6}, 0.34 \% \mathrm{C}_{3} \mathrm{H}_{8}, 1.54 \% \mathrm{CO}_{2}$, and $3.86 \% \mathrm{~N}_{2}$.

b Statistic used to define magnitude of crosswind velocity - corresponding to data at the Edmonton International Airport from 1992-2016 (Environment and Climate Change Canada 2016).

Four simulations with freestream crosswind velocity $\left(u_{\infty}\right)$ ranging from 1.11$7.78 \mathrm{~m} \mathrm{~s}^{-1}$ were performed, yielding exit-to-crosswind momentum flux ratios of 7.58-0.15. Sample visualizations from each simulation are shown in Figure 3.2. Crosswind velocities were selected using available ambient measurements by the Government of Canada of hourly wind data at the Edmonton international airport from 1992-2016 (Environment and Climate Change Canada 2016), providing four representative velocities as listed in Table 3.1c: the $10^{\text {th }}$ percentile (simulation I), mode (simulation II), median (simulation III), and $95^{\text {th }}$ percentile (simulation IV). Crosswind was implemented as a uniform inlet within the simulations. Although flare flames should be well within the planetary boundary layer, this simplified crossflow is not of concern for the present work since the structure of the flame and plume is most-significantly influenced by interactions between the wind and 
flare stack and buoyancy of the plume, rather than the large-scale turbulence in the crossflow itself (Cameron et al. 2011; Johnson et al. 2001; Johnson and Kostiuk 2000; Gollahalli et al. 1975). At higher wind speeds, the plume structures closely resemble a von Kármán vortex street as in the visualization of simulation IV in Figure 3.2.

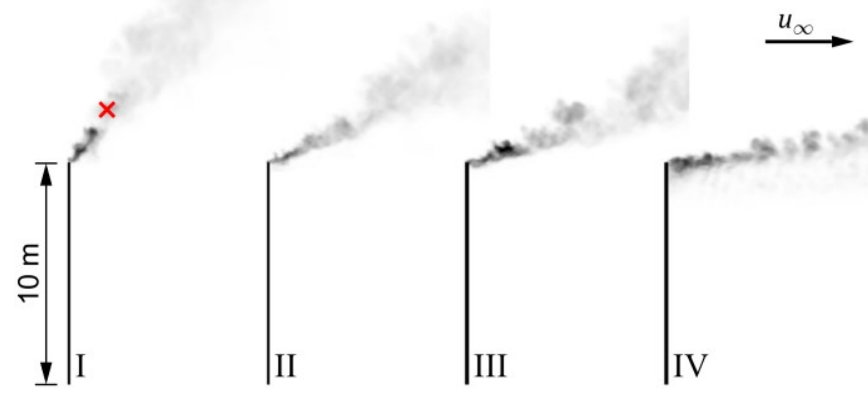

Figure 3.2: Orthographic visualization of sample simulation data for each of the four simulation cases, showing the column density of $\mathrm{CO}_{2}$. The red cross in the plume of simulation I represents the optical axis used in Figure 3.5.

Thirty instantaneous results from each of the above-described simulations were selected for the beam steering analysis and MC simulation. Each flare simulation reported spatially resolved ( $0.05 \mathrm{~m}$ resolution structured grid) gas temperature and composition data including volume fractions of methane $\left(\mathrm{CH}_{4}\right)$, water $\left(\mathrm{H}_{2} \mathrm{O}\right)$, carbon monoxide $(\mathrm{CO})$, and carbon dioxide $\left(\mathrm{CO}_{2}\right)$, with the balance composition being assumed as two-element air with $78.99 \%$ nitrogen $\left(\mathrm{N}_{2}\right)$ and $21.01 \%$ oxygen $\left(\mathrm{O}_{2}\right)$ by volume. Spectrally resolved refractive index data over the simulation domain were computed using one of two techniques dependent on the measurement wavelength as summarized in Section B.1. For the visible spectrum, the dispersion relations of Gardiner et al. (1981) were employed, using the Lorentz-Lorenz mixing rule for mixture refractivity. For the infrared (IR) spectrum, refractivity data were computed as in the models of Mathar $(2004,2007)$, using IR line transitions from HITRAN (High resolution transmission spectroscopic database, Gordon 
et al. 2017) with 10 added pseudo-oscillators for each reported gas species (Jhanwar and Meath 1982; Margoliash and Meath 1978).

Atmospheric plumes of combustion-generated pollutants generally contain fine particulate matter like soot. The presence of soot will impact radiative transfer through the plume but can also affect beam steering due to its impact on the refractive index of the medium. However, this effect is predicted to be negligibly small in the case of flare plumes as elaborated in Section B.1. Using the Maxwell Garnett effective medium approximation (Maxwell Garnett 1904), dispersion relations for the refractive index of soot Chang and Charalampopoulos (1990), and conservatively high estimates of soot volume fraction in flare-like flames (e.g., Crosland et al. 2013, 2015; Köhler et al. 2011; Thomson et al. 2008), this analysis shows that the effect of soot on beam steering is negligible for flare plumes and combustion plumes in general. Specifically, this analysis identifies that the effect of soot is likely more than four orders of magnitude less than the nominal effect when soot is ignored. This justifies excluding effects of fine soot particulate on the refractive index of the medium.

The beam steering phenomenon is also sensitive to fluid pressure. For example, under isothermal conditions, the refractive index, its gradient, and therefore the magnitude of beam steering are linearly proportional to pressure (Weinberg 1963). Thus, beam steering becomes more pronounced at higher pressures (Zerbs et al. 2009; Thomson et al. 2008; Weinberg 1996) and the present study only applies to plumes at or near atmospheric pressure. Importantly, for typical deflagration flames (i.e., far from detonation conditions), slight pressure variations due to gas expansion at the flame front are "...very small and quite trivial from the refractive index point of view..." (Weinberg 1963). Thus, for beam 
steering through the plumes of the studied atmospheric pressure flames, where any pressure variations would be far less than those at the flame front, the effect of pressure can be ignored.

\subsubsection{Optical Axis Modelling}

To quantify the effect of beam steering in remote optical diagnostics, realistic optical axes through the simulated plumes were necessary. With the specific simulation of gas flares in mind, the sky-LOSA measurement technique for soot emissions from gas flaring was selected as a basis to derive realistic optical axes. A MC approach was employed to broadly cover typical positioning and selection of optics for the sky-LOSA camera.

Positioning of the optics relative to the flare stack was first specified while considering typical standoff distances and optical limitations. While the vertical distance between the camera (located at ground-level) and flare stack tip was fixed at $10 \mathrm{~m}$, horizontal distances from the camera to the stack were randomly selected following a uniform distribution from $25 \mathrm{~m}$ to $200 \mathrm{~m}$ (i.e., $\sim \mathcal{U}(25 \mathrm{~m}, 200 \mathrm{~m})$ ), yielding a range of camera-stack tip inclination angles of 2.86-21.80 . In sky-LOSA, where imaging data are leveraged to compute two-dimensional plume velocity within the image plane, best practices suggest that the camera should be positioned such that its axis is approximately orthogonal to the direction of plume propagation (crosswind). Since this can be challenging to precisely achieve in the field (due to site access limitations and variable winds), the modelled camera in this work was randomly positioned obliquely from the ideal axis following a uniform distribution $\left(\sim \mathcal{U}\left(-30^{\circ}, 30^{\circ}\right)\right)$. Figure 3.3 summarizes these

critical dimensions regarding the MC-randomized camera positioning alongside $10^{4}$ randomized camera positions studied in this work (shown in blue). 


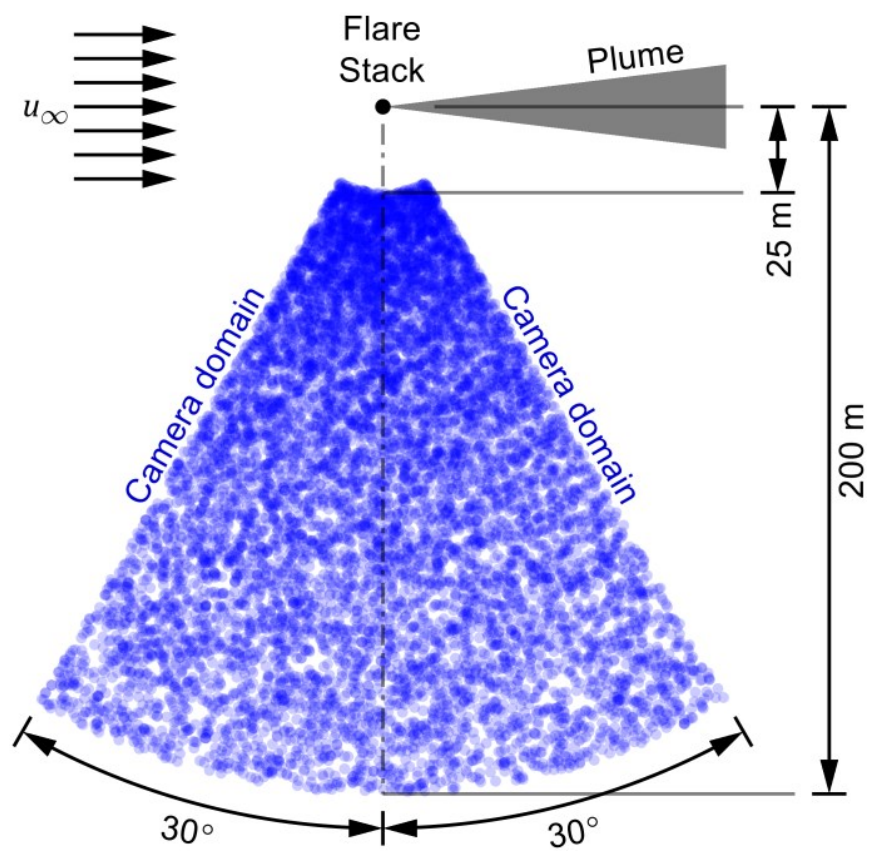

Figure 3.3: Summary of Monte Carlo-randomized sky-LOSA camera positioning. The horizontal distance between the camera and flare stack follows a uniform distribution $(\sim \mathcal{U}(25 \mathrm{~m}, 200 \mathrm{~m}))$ and the camera's azimuthal pointing is constrained to $b e \pm 30^{\circ}$ from perpendicular to the freestream wind $\left(\sim \mathcal{U}\left(-30^{\circ}, 30^{\circ}\right)\right)$. Blue dots in the image show the $10^{4}$ random camera positions used in the present analysis.

Spatially resolved optical axes of the sky-LOSA camera (pco model Edge 5.5, with a sensor of $2560 \times 2160 \mathrm{px}$ and pixel pitch of $6.5 \mu \mathrm{m}$ ) were modelled using a projective pinhole (camera obscura) analogy where, for simplicity, pixel skewness and sensor distortion were assumed to be negligible. Each random camera position was randomly coupled to one of the four simulation datasets allowing for the selection of optics and extrinsic characteristics of the camera (yaw and pitch) to be deterministically obtained considering practical constraints. The optics were chosen as the commercially standard prime lens with the largest focal length that yielded a flame length (maximum over the entire simulation dataset) of less than $600 \mathrm{px}$. Camera extrinsics were then defined such that the flame and plume within the image plane (propagating up and to the right due to jet 
momentum, buoyancy, and crosswind) were no less than 150 and 200 px from the left and bottom edges of the image, respectively.

With the set of $10^{4}$ camera positions, optics, and extrinsics constrained, realistic control surfaces, over which integration of column density and plume velocity would yield mass flux, were then selected under the MC approach. The radius of the control surface (measured within the image plane from the flare stack tip) was randomly selected between

a minimum radius corresponding to $50 \mathrm{px}$ from the edge of the flame envelope to a maximum radius of $50 \mathrm{px}$ from the edge of the image. This procedure yielded a range of control surface radii of 623-2310 px, which tends to bound those employed in sky-LOSA analyses to date (Chapter 2, Conrad and Johnson 2017; Johnson et al. 2013).

\subsubsection{Beam Steering Simulation}

With spatially resolved refractive index data, beam steering of light through the simulation domain was modelled using a simplified form of the governing differential equations for light propagation in continuous media. The simplified governing equations are briefly summarized for completeness, leveraging the exaggerated example of beam steering through a two-dimensional scalar field of refractive index shown in Figure 3.4. Here, a beam of light of intensity $I_{\lambda, c}^{o}$, incident from the left-hand side of the figure, travels towards the heated plume of combustion products. Spatial gradients in the refractive index yield corresponding spatial gradients in the propagation velocity of the beam of light. Whether using Fermat's principle of least time and calculus of variations (Moore 1975) or using energy and momentum conservation considerations (Weinberg 1963), the physics dictate that the beam of light is steered toward regions of higher refractive index (lower propagation velocity) following the governing differential equation: 


$$
\frac{\frac{\partial^{2} y_{\lambda}}{\partial x^{2}}}{\left(1+\left(\frac{\partial y_{\lambda}}{\partial x}\right)^{2}\right)^{3 / 2}}=\frac{\nabla \eta_{\lambda}}{\eta_{\lambda}} \cdot \boldsymbol{n}_{\lambda}
$$

where $\eta_{\lambda}$ is the local, real, spectral refractive index [-] (dimensionless); $\boldsymbol{n}_{\lambda}$ is the unit vector normal to the local direction of propagation; and all variables inherently vary with location in the $x-y$ domain. This differential equation yields the curved path (in red) visible in Figure 3.4 that ultimately enters the detector with a transmitted intensity of $I_{\lambda, c}^{t}$.

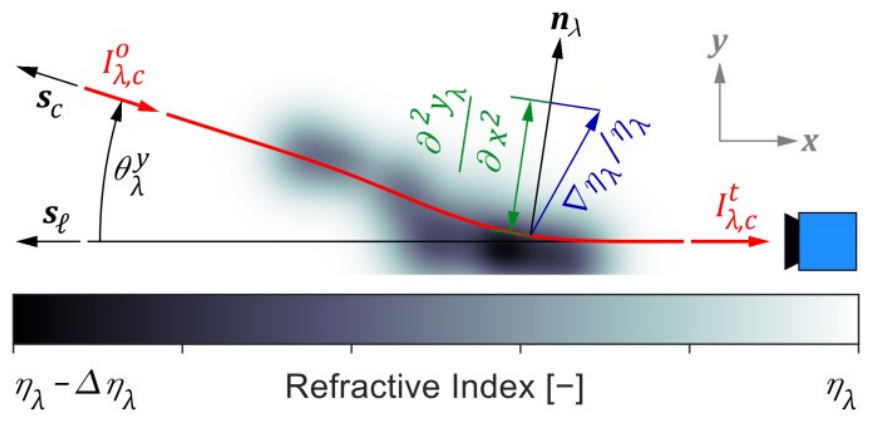

Figure 3.4: Exaggerated example of beam steering through a two-dimensional inhomogeneous scalar field of refractive index. Light propagates towards the plume from pointing $s_{c}$ with an intensity of $I_{\lambda, c}^{o}$ and a direction that makes an angle of $\theta_{\lambda}^{y}$ to the optical axis. As the light traverses through gradients in refractive index that are oblique to its local propagation direction, the path of light is steered towards regions of higher refractive index with an incremental change in its propagation direction related to the perpendicular component of the local gradient. Ultimately, the light enters the camera with a transmitted intensity of $I_{\lambda, c}^{t}$.

For many combustion applications - particularly those at atmospheric pressure local deflections $\left(\partial y_{\lambda} / \partial x\right)$ are typically much smaller than unity (Weinberg 1963), such that the left-hand side of Eq. (3.6) can be simplified to just $\partial^{2} y_{\lambda} / \partial x^{2}$. Furthermore, although $\boldsymbol{n}_{\lambda}$ is necessarily dependent upon the local direction of propagation, in the smallangle limit the direction of the normal vector $\boldsymbol{n}_{\lambda}$ might be reasonably fixed. Then, if arbitrary cartesian axes are aligned with the pixel's optical axis, such that the optical axis is collinear with the $x$-direction (as in the figure), then the normal vector $\boldsymbol{n}_{\lambda}$ is equivalent 
to the $y$-direction at all values of $x$. These assumptions yield the small-angle limit of Eq. (3.6) (Weinberg 1963):

$$
\frac{\partial^{2} y_{\lambda}}{\partial x^{2}}=\frac{1}{\eta_{\lambda}} \frac{\partial \eta_{\lambda}}{\partial y}
$$

Strictly speaking, integration of Eq. (3.6) along the curved path of the beam (i.e., " $c_{\lambda}$ ") is necessary to derive the path of light as it traverses the plume. In the small-angle limit however, the total deflection due to beam steering can be estimated by instead integrating Eq. (3.7) along the $x$-dimension only (i.e., the linear path, " $\ell$ "). For modelling of beam steering in the present work, deflections are calculated by leveraging this smallangle limit and the reciprocity relation of radiative transfer. The total (and directional) deflection of light as it is steered into the detector is computed in the reverse direction. That is, total deflection in the $x-y$ plane (shown as $\theta_{\lambda}^{y}$ in Figure 3.4) and the $x-z$ plane (equivalently, $\theta_{\lambda}^{z}$ ) can be estimated by integrating Eq. (3.7) in the opposite direction of light propagation (from the detector to the source). Additionally, the total deflection angle $\left(\theta_{\lambda}\right)$ can be obtained by summation of the $y$ - and $z$-deflections in quadrature as shown in Eq. (3.8).

$$
\begin{aligned}
& \theta_{\lambda}^{y}=\int_{L}^{0} \frac{1}{\eta_{\lambda}} \frac{\partial \eta_{\lambda}}{\partial y} d x \\
& \theta_{\lambda}^{z}=\int_{L}^{0} \frac{1}{\eta_{\lambda}} \frac{\partial \eta_{\lambda}}{\partial z} d x \\
& \theta_{\lambda}=\sqrt{\left(\theta_{\lambda}^{y}\right)^{2}+\left(\theta_{\lambda}^{z}\right)^{2}}
\end{aligned}
$$




\subsection{Results and Discussion}

Beam steering-related correction parameters were quantified using a MC method in which deflection angles $\theta_{\lambda}^{y}, \theta_{\lambda}^{z}$, and $\theta_{\lambda}$ were computed via Eq. (3.8) for each of the $10^{4}$ simulated control surfaces. In the visible spectrum, where refractive indices were trivially computed using molar refractivities of constituent gases, this procedure was repeated for all 30 available timesteps in each flare simulation. In the IR spectrum however, where refractive indices must be computed by summation of line transitions, the computational burden precluded repeating this procedure over all timesteps and results were therefore obtained for a single timestep in each flare simulation. With an average of approximately 3344 optical axes per control surface, these calculations comprised more than $10^{7.5}$ individual conditions at each considered wavelength in the IR and more than $10^{9}$ individual conditions at each considered wavelength in the visible spectrum.

\subsubsection{Total Deflection Angle}

Figure 3.5 shows total deflection angles for selected spectra for an example optical axis through the plume of simulation I, corresponding to the red cross in Figure 3.2. The spectrally resolved deflection angles at $1 \AA$ resolution are shown in orange. In the visible spectrum (Figure 3.5a), the deflection angle is a smooth function of measurement wavelength since the refractive index is itself a relatively smooth function of temperature and composition in this spectrum, where absorption is negligible. By contrast, there is notable spectral dispersion in the vicinity of line transitions in the IR spectra (Figure $3.5 \mathrm{~b}-\mathrm{d})$, consistent with concomitant variability in the real and imaginary components of the refractive index (e.g., Mathar 2004). Here, there appears to be a continuum-like lowfrequency component coupled with higher-frequency components of the deflection angle 
with respect to wavelength. Within methane's $3.3 \mu \mathrm{m}$ band (degenerate asymmetric stretch), noting the scale of the vertical axis in Figure 3.5b, the spectral dispersion is small relative to the continuum (i.e., $\pm 0.14 \mu \mathrm{rad}$ or $0.065 \%$ of a mean deflection angle of $216.4 \mu \mathrm{rad}$ ). By contrast, the spectral dispersion within carbon dioxide's 4.3 (asymmetric stretch) and $15 \mu \mathrm{m}$ (degenerate bending) bands is substantial, with both high- and lowfrequency variations that appear to yield variability on the order of $50 \%$ of the mean. The scale of the spectral dispersion in the studied carbon dioxide bands is a function of the intensity of nearby rovibrational transitions in addition to the abundance of carbon dioxide in the plume, relative to methane. For flares operating at high combustion efficiency (i.e., efficient destruction of flare gas), it is therefore unsurprising that dispersion in the studied bands of carbon dioxide tend to be larger. 

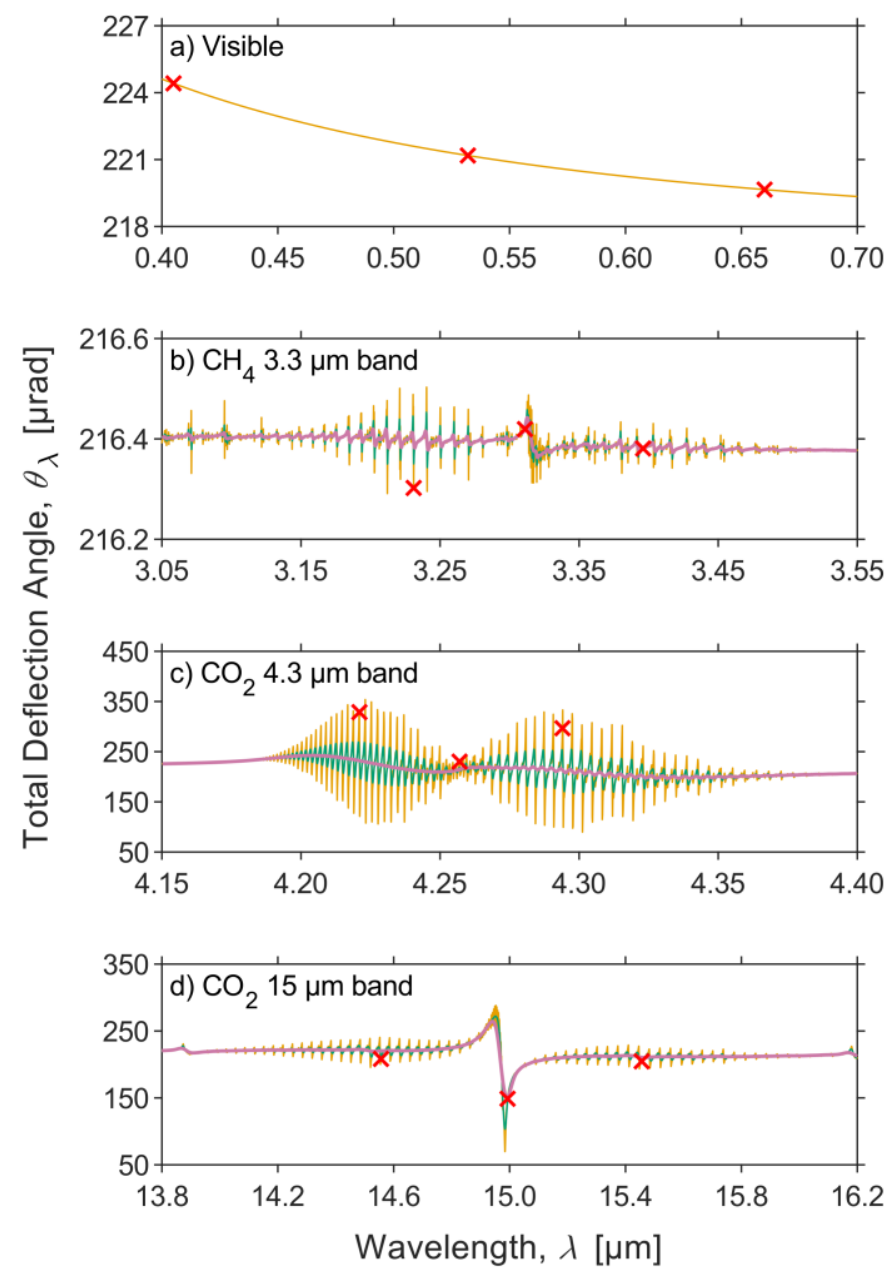

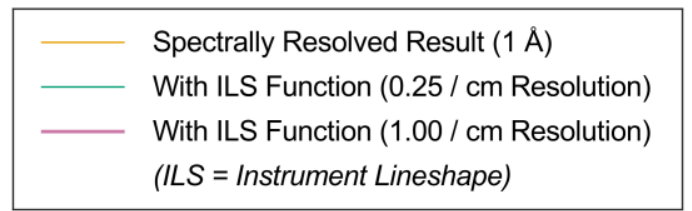

Figure 3.5: Spectrally resolved total deflection angles for an example optical axis through a plume from simulation $I$. (a) In the visible spectrum, total deflection angle is a smooth function of wavelength. (b-d) In the IR, total defection angle includes substantial spectral dispersion in the vicinity of line transitions. However, when finite spectral resolution of practical detectors is considered through an instrument lineshape (ILS) function, the high-frequency component is smoothed and a low-frequency continuum-like component, which is fairly consistent across the studied absorption bands, dominates. Red crosses indicate wavelengths selected for subsequent analysis.

These observations suggest a potential need to quantify beam steering effects with high resolution over the selected spectra to capture the spectrally dependent effect of beam steering. However, in practice, an optical detector will have some finite spectral resolution 
that will tend to smooth the higher-frequency dispersion in beam deflection. To assess the impact of this smoothing effect, consider one commercially available hyperspectral imager the Hyper-Cam manufactured by Telops. Gross et al. (2010b) experimentally derived an instrument line shape (ILS) function for this midwave instrument at its nominal maximum spectral resolution of $0.25 \mathrm{~cm}^{-1}$. If this ILS function is assumed to be linear shift invariant in the spectral domain and is independently applied to the beam steering components $\left(\theta_{\lambda}^{y}\right.$ and $\theta_{\lambda}^{z}$ ) of the example data, the spectral dispersion in the results is significantly dampened (turquoise lines in Figure $3.5 \mathrm{~b}-\mathrm{d}$ ). Furthermore, if the ILS function is expanded to a modest $1 \mathrm{~cm}^{-1}$ resolution, spectral dispersion is dampened (purple lines in Figure 3.5b-d) to the point where the beam steering effect can reasonably be considered as smooth over the selected spectra. That is, although the beam steering effect can vary greatly at specific wavelengths, the continuum-like, low-frequency effects are more practically important; this allows for the analysis of the beam steering effect through the study of few wavelengths over a given band, making the present work computationally feasible. Subsequent analyses thus consider 12 wavelengths: three wavelengths in the visible and three in each of the studied IR bands. Specifically, these were $0.405,0.532$, and $0.660 \mu \mathrm{m}$ in the visible, corresponding to common diagnostic wavelengths; $3.231,3.311$, and $3.396 \mu \mathrm{m}(=3020 \pm$ $\left.75 \mathrm{~cm}^{-1}\right)$ in methane's $3.3 \mu \mathrm{m}$ band; $4.221,4.257$, and $4.294 \mu \mathrm{m}\left(=2349 \pm 20 \mathrm{~cm}^{-1}\right)$ in carbon dioxide's $4.3 \mu \mathrm{m}$ band; and 14.556, 14.993, and $15.456 \mu \mathrm{m}\left(=667 \pm 20 \mathrm{~cm}^{-1}\right)$ in carbon dioxide's $15 \mu \mathrm{m}$ band. These 12 wavelengths correspond to the red crosses in Figure 3.5 and, for the case of the dispersive IR results, include wavelengths yielding steered angles less and greater than the continuum in this example data. 
To glean insight into the effect of plume geometry on the total deflection angle due to beam steering, simulation-specific MC results at $0.405 \mu \mathrm{m}$ were compared. Figure 3.6a shows logarithmic histogram data corresponding to all $\sim 10^{9}$ simulations at $0.405 \mu \mathrm{m}$, parsed by simulation number. Peak steered angles ranged from 1.13 to $1.54 \mathrm{mrad}$ and generally increased with crosswind velocity, most likely due to enhanced turbulence on the leeward side of the flare stack with wind, which could amplify gradients in temperature and species concentration within the plume. Each distribution appears to be bi- or multimodal; one mode peaks near 100-200 $\mu \mathrm{rad}$, and one mode towards zero $\left(<10^{-6} \mu \mathrm{rad}\right)$; the latter of which is due to negligible beam steering at the edges of the plumes and beyond. Interestingly, a third mode near $50 \mu \mathrm{rad}$ is observed in simulation IV. This is likely a consequence of the well-known fuel stripping mechanism in flaring, where the low pressure region on the leeward side of the stack results in the ejection of fuel from the flame (e.g., Johnson and Kostiuk 2002; Johnson et al. 2001) causing a region of cool, unburnt fuel to persist below the heated plume. In the context of beam steering through a gaseous flare plume, this suggests a collection of optical axes with steered angles that are reduced relative to those typical of the heated portion of the plume. Subsequent analyses consolidate data from all flare simulations to yield results broadly relevant to all conditions. 

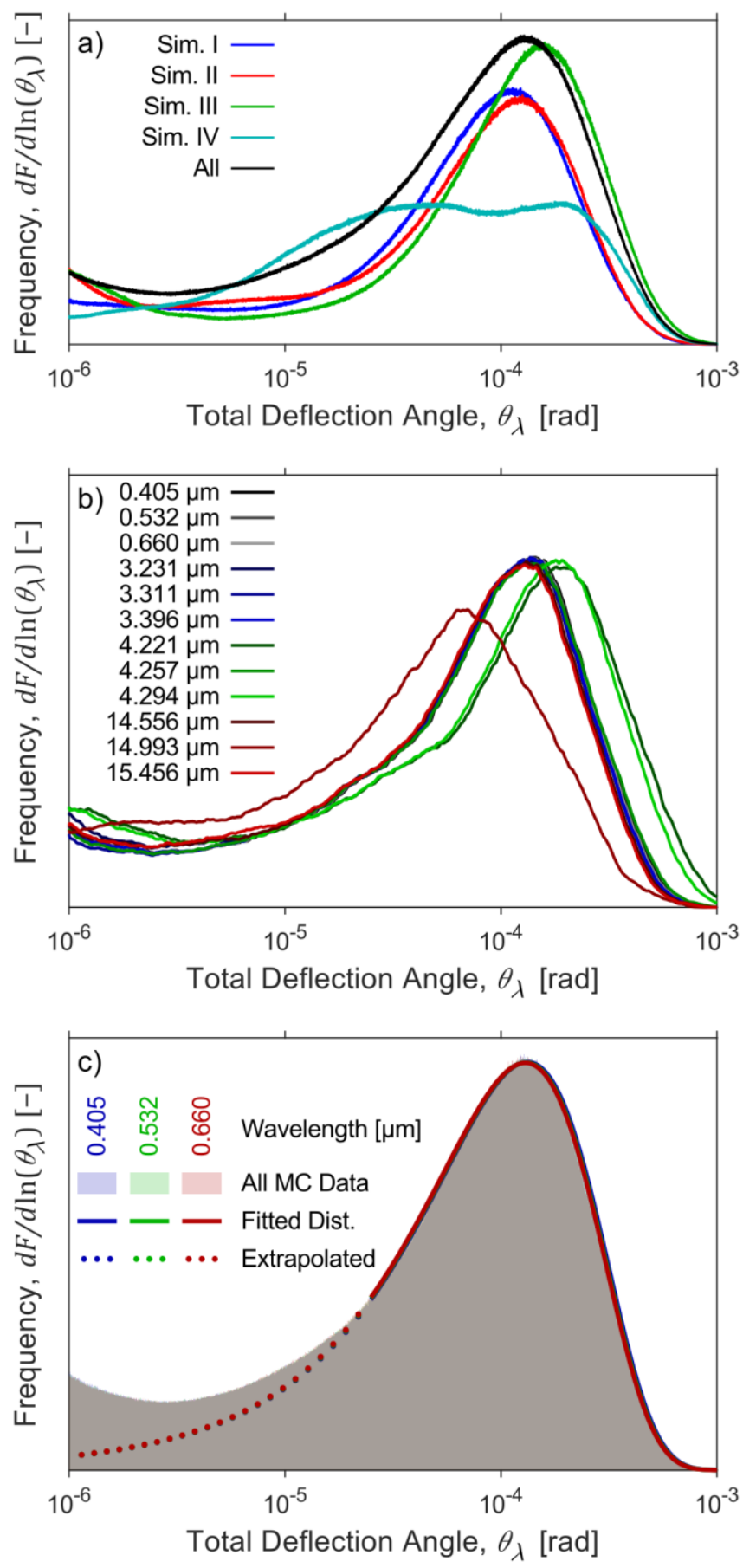

Figure 3.6: Distributions of total deflection angle of light traversing turbulent flare plumes. (a) Total deflection angle for different turbulent plume conditions (different crosswinds); (b) Total deflection angle over all plume conditions at different wavelengths corresponding to red crosses in Figure 3.5; (c) Probability distributions of deflection angle derived for a subset of the plotted results $\left(\theta_{\lambda}>25 \mu \mathrm{rad}\right)$ to yield a conservatively high (on average) model that ignores the distribution mode corresponding to negligibly steered light near the edges of the plume. 
Returning to Figure 3.5, the example data suggest that the spectral variability in beam deflection could be significant at high spectral resolutions, especially within key IR measurement bands. To assess whether the relative shape of the plots in Figure 3.5 persists when considering different plumes and optical axes, the logarithmic histograms of beam steering data for the 12 selected wavelengths, at the selected timestep for analysis of IR data, are compared in Figure 3.6b. Data for nine of these wavelengths follow a similar distribution, representing cases where the deflection angles at high-resolution were within $5.5 \%$ of the continuum-like component in the example dataset of Figure 3.5 (quantified using a linear fit for each analyzed spectrum). The remaining data correspond to the higher dispersion points at 4.221, 4.294, and $14.993 \mu \mathrm{m}$ in Figure 3.5c,d; the former two represent two increased deflections in the R and P branch of carbon dioxide's $4.3 \mu \mathrm{m}$ band and the latter corresponds to reduced deflection in the Q branch of carbon dioxide's $15 \mu \mathrm{m}$ band. The persistence of these results across multiple turbulent plumes and optical axes suggests, at least superficially and over the simulated temperatures and compositions, that the relative deflection between two wavelengths might be reasonably considered as constant, such that there are specific wavelengths that have fixed deflection angle biases about the continuum deflection. Furthermore, the nine histograms that are in close agreement imply that visible spectrum data can be a useful surrogate for the continuum effect.

Leveraging the notion that the continuum-like effect of beam steering might be reasonably modelled with visible spectrum results, the logarithmic histogram of total deflection angle for each considered visible wavelength over all $\sim 10^{9}$ optical axes and all flare simulations are plotted in Figure 3.6c. Histograms are coloured by the measurement wavelength and overlaid atop one another, where it is apparent that there is significant 
overlap/consistency in the results. As in Figure 3.6a, the resulting distribution is bimodal with one mode close to zero, representing negligible beam steering towards and beyond the plume edges. Noting that this would bias central tendency statistics low, probability distributions of the deflection angle at each wavelength were derived for a subset of the plotted results that would yield a conservatively high (on average) model. Specifically, fitted distributions were derived from the set of simulation results with $\theta_{\lambda}>25 \mu \mathrm{rad}$, providing a good model of the leading (righthand) edge of the histogram - as shown via the solid lines in the figure. Candidate distributions were fit to the scaled empirical cumulative distributions of the data; at each wavelength it was discovered that the twoparameter type II extreme value (Fréchet) distribution best-described the dataset (Kolmogorov-Smirnov statistics all $<0.0025$ ). Noting that refractivities are higher towards the ultraviolet, the distribution fitted to the $0.405 \mu \mathrm{m}$ data was selected as a conservative model for the continuum component of the total deflection angle from beam steering through flare plumes, where:

$$
\left(Y=-\ln \theta_{\lambda}\right) \sim \mathrm{EV}_{\mathrm{II}}(\underbrace{9.250}_{a}, \underbrace{9.030}_{b})
$$

Reversing the log-transformation enables an inverse cumulative distribution capable of providing randomized deflection angles $\left(\xi_{\theta_{\lambda}}[\mathrm{rad}]\right)$ for MC simulations:

$$
\xi_{\theta_{\lambda}}=\exp \left(-b(-\ln \xi)^{-1 / a}\right)
$$

where $\xi \sim \mathcal{U}(0,1)$. 


\subsubsection{Incident Intensity Correction, $K_{\lambda, o}$}

Detected light that has been steered through a turbulent plume may be subject to erroneous interpretation of incident intensity $\left(I_{\lambda}^{o}\right)$. If the incident intensity varies with pointing $(s)$, such that $I_{\lambda}^{o}=I_{\lambda}^{o}(\boldsymbol{s})$, then the incident intensity for a curved path $\left(I_{\lambda, c}^{o}\right)$ may differ from that of the linear path $\left(I_{\lambda, \ell}^{o}\right)$ where the pointing $\boldsymbol{s}$ is collinear with the optical axis (i.e., $\boldsymbol{s}=$ $\boldsymbol{s}_{\ell}$, as shown in Figure 3.4). Qualitatively, this observation implies that uniform, diffuse background light sources are ideal for transmissive measurement techniques and efforts should be made to avoid high contrast backgrounds.

For field measurements of emission sources from raised stacks, the plume background is generally skylight. For measurements in the IR, the portion of detected light from the background (first term in Eq. (3.2)) is likely to be overshadowed by the emission source term, such that incident intensity can be reasonably ignored in the RTE and an incident intensity correction is not required. By contrast however, this correction can be necessary for visible spectrum measurements with a skylight background. As an example, the above-mentioned sky-LOSA measurement technique is used to probe the potential importance of this correction parameter.

Sky-LOSA requires images of a flare plume with a skylight background, acquired at $0.531 \mu \mathrm{m}$ (Johnson et al. 2013). The potential for error in the incident intensity due to beam steering is a function of the total deflection angle and gradient in the skylight intensity distribution at the optical axis; the latter of which is dictated by the optical axis' pointing relative to the horizon and sun. In the field, the sky-LOSA camera is positioned according to numerous heuristics but is generally pointed far from the solar position and at inclination angles consistent with the present MC simulations. Models of skylight intensity 
distribution, such as those by the Commission Internationale de l'Eclairage (CIE, 2003), suggest that skylight intensity/luminance can be reasonably defined as a function of a sky region's angle from the horizon and angle from the sun. As shown in Figures 2 and 3 of the CIE standard (CIE 2003), gradients in skylight intensity tend to be largest near the sun and slightly above the horizon. This latter case suggests that the sky-LOSA camera is typically pointed in a non-ideal direction in the context of the beam steering effect on incident intensity. Hence, the incident intensity correction for sky-LOSA is proposed to be a reasonable estimate of the upper bound on the importance of this effect.

To quantify the incident intensity correction factor for sky-LOSA, a MC analysis was executed. For an arbitrary optical axis pointing in the direction $\boldsymbol{s}_{\ell}$, a beam steered pointing $\boldsymbol{s}_{c}$ was derived by first randomly obtaining a total deflection angle $\left(\theta_{\lambda}=\right.$ $\operatorname{acos}\left(\boldsymbol{s}_{\ell} \cdot \boldsymbol{s}_{c}\right)$ ) following the distribution in Eq. (3.9) (yielding conservatively high total deflections at the diagnostic wavelength of $0.531 \mu \mathrm{m})$. Next, component deflections in the $x-y$ and $x-z$ planes $\left(\theta_{\lambda}^{y}\right.$ and $\theta_{\lambda}^{z}$; which sum in quadrature to yield $\theta_{\lambda}$ as per Eq. (3.8)) that define the pointing of incident light along the curved path $\left(\boldsymbol{s}_{c}\right)$ were obtained. This was done by randomly selecting the angle $\phi \sim \mathcal{U}\left(0,360^{\circ}\right)$, where $\phi=\operatorname{atan}_{2}\left(\theta_{\lambda}^{y}, \theta_{\lambda}^{z}\right)$ and $\operatorname{atan}_{2}$ represents the four-quadrant arctangent. Thus, for a given skylight intensity distribution and solar position (which influences the former), MC-randomized values of $K_{\lambda, o}=I_{\lambda, s k y}\left(\boldsymbol{s}_{c}\right) / I_{\lambda, s k y}\left(\boldsymbol{s}_{\ell}\right)$ at the sky-LOSA measurement wavelength were calculated.

The above MC procedure was executed for realistic camera positions, solar positions, and skylight intensity distributions consistent with 14 previously published skyLOSA measurements (Chapter 2, Conrad and Johnson 2017). Results are summarized in Figure 3.7a. In the figure, grey lines represent the logarithmic frequency of the incident 
intensity correction parameter's absolute deviation from unity, where the modes of the computed distributions vary from $10^{-5.00}$ to $10^{-3.81}$ and the $99.99^{\text {th }}$ percentiles are all less than $10^{-2.77}$.
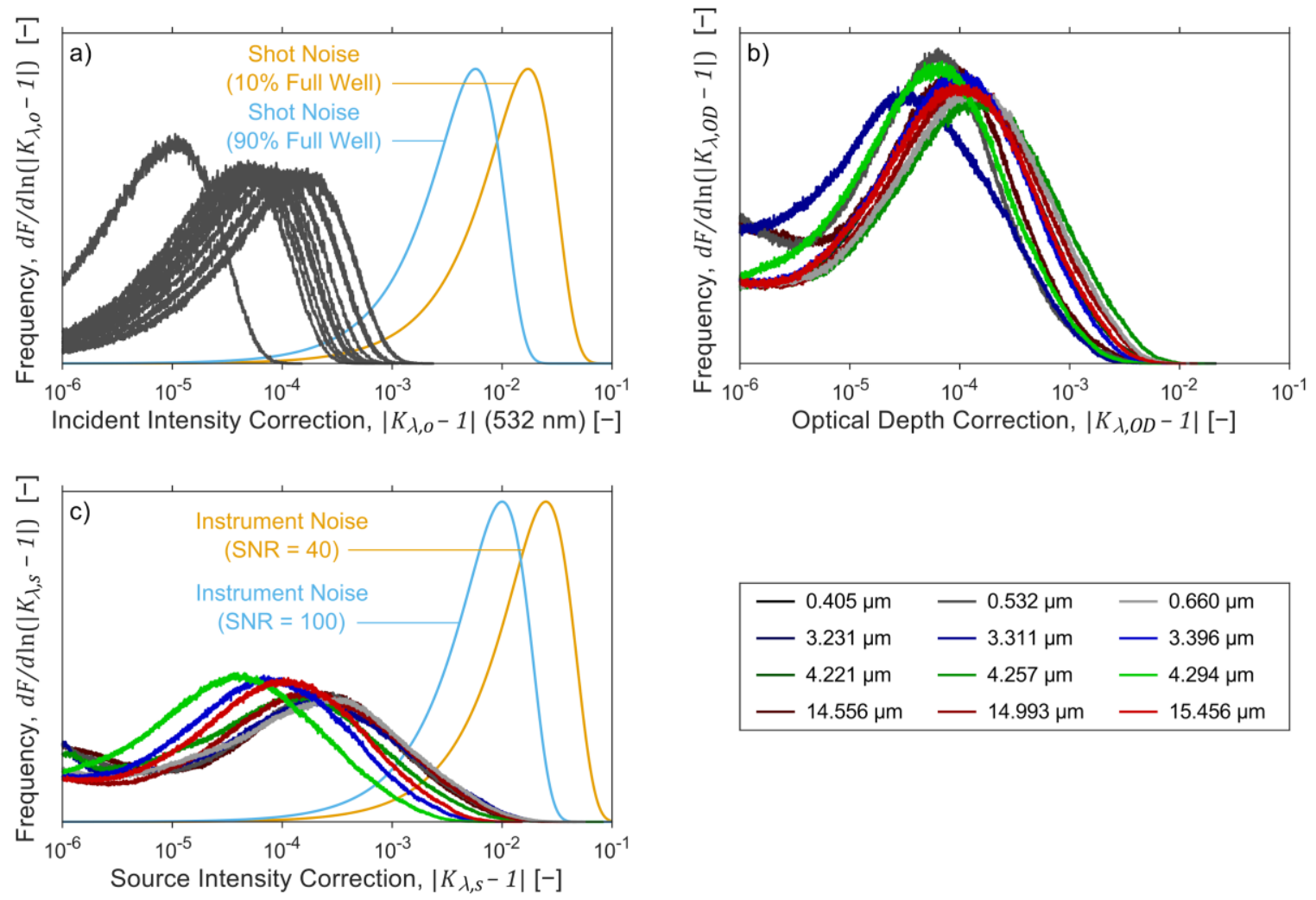

$$
\begin{array}{|lll}
\hline-0.405 \mu \mathrm{m} & -0.532 \mu \mathrm{m} & -0.660 \mu \mathrm{m} \\
-3.231 \mu \mathrm{m} & -3.311 \mu \mathrm{m} & -3.396 \mu \mathrm{m} \\
-4.221 \mu \mathrm{m} & -4.257 \mu \mathrm{m} & -4.294 \mu \mathrm{m} \\
-14.556 \mu \mathrm{m} & -14.993 \mu \mathrm{m} & -15.456 \mu \mathrm{m} \\
\hline
\end{array}
$$

Figure 3.7: Logarithmic frequency of non-dimensional correction parameters for steered light through turbulent flare plumes. (a) Absolute value of the deviation of

the incident intensity correction $\left(K_{\lambda, o}\right)$ from unity (grey curves) compared with example shot noise limits for a "low-noise" sky-LOSA camera (coloured curves). (b) Absolute value of the deviation of the optical depth correction $\left(K_{\lambda, O D}\right)$ from unity. (c) Absolute value of the deviation of the source intensity correction $(K, s)$ from unity compared with instrument noise for commercially available mid-wave hyperspectral imagers (Harley and Gross 2011; Gross et al. 2010b).

For context, Figure 3.7a also shows the equivalent distributions of Poisson/shot noise in the low-noise sky-LOSA camera for $10 \%$ and $90 \%$ of full well capacity. It is clear from this comparison that the effect of beam steering on the incident intensity for sky-LOSA is order(s) of magnitude less than the shot noise effect, which itself is negligible with respect to overall measurement uncertainty (Johnson et al. 2013). The present results suggest that 
the incident intensity correction is negligible and can be fixed at unity for the purposes of sky-LOSA and for other visible spectrum, transmissive measurements with skylight backgrounds.

In this analysis, the employed models of skylight intensity distribution (CIE 2003) are spatially smooth in the sense that they model the time-averaged luminance/intensity of skylight and do not permit consideration of instantaneous cloud structures. This suggests

that, for overcast and partly cloudy conditions, steep intensity gradients at the edges of cloud structures could amplify the incident intensity correction factor beyond the present observations. In the experience of the authors however, this is not likely to be practically important. Firstly, under partly cloudy conditions, imaging data is heuristically acquired when the control surface for emission rate analysis does not intersect cloud structures, such that the background is the clear component of the partly cloudy sky. As such, the presence of cloud structures does not practically affect $K_{\lambda, o}$ under partly cloudy conditions and the above results are representative. Under non-ideal overcast conditions, cloud edges may be well-defined and influence the correction parameter. Practically however, the larger challenge in this instance is the estimation of the background intensity $\left(I_{\lambda, \ell}^{o}\right)$ via spatial interpolation of the skylight intensity distribution behind the plume from the perspective of the camera. This limits the accuracy of transmissive techniques with skylight backgrounds under overcast conditions and suggests that such a measurement would likely be precluded before the effect of cloud structures on $K_{\lambda, o}$ would be significant.

\subsubsection{Optical Depth Correction, $K_{\lambda, O D}$}

Referring to Eq. (3.4), the optical depth (OD) correction parameter captures the difference in the path-integrated extinction of the curved and linear paths. The magnitude of $K_{\lambda, O D}$ 
includes both absorption and scattering effects due to gaseous and particulate components of the plume. In the context of remote optical measurement of combustion-generated pollutants, techniques can be parsed into two broad classifications: IR spectrum measurements of gaseous emissions and visible spectrum measurements of particulate emissions. In the former case, scattering effects from gases can be neglected and extinction by particulate is likewise negligible due to the $\lambda^{-1}$ the $\lambda^{-4}$ dependence of absorption and scattering from particulate matter, respectively. By contrast, in the latter case, extinction results from only absorption and scattering by particulate matter.

One additional challenge in quantifying the OD correction parameter arises from use of the small-angle limit of beam steering; specifically, the curved path $\left(c_{\lambda}\right)$ is not directly computed. The curved path was instead estimated by cumulative integration of the first-order Taylor series expansion over the $y$ - and $z$-coordinates. For example, the $y$ coordinate of the curved path was estimated as:

$$
y_{\lambda}(x)=y_{\ell}(x)+\int_{L}^{x} \theta_{\lambda}^{y}\left(x^{\prime}\right) d x^{\prime}
$$

where $y_{\ell}(x)$ corresponds to the straight path. With an equivalent approach used for the $z$ coordinate, points on the curved path were obtained, $\boldsymbol{r}=\left[\begin{array}{lll}x & y_{\lambda}(x) & z_{\lambda}(x)\end{array}\right]^{T}$. Computation of the local extinction coefficient or $\mathrm{CO}_{2}$ concentration (as a surrogate for local particulate extinction coefficient) on the linear path and curved path thus enabled the direct calculation of $K_{\lambda, O D}$ for a given optical axis via Eq. (3.4).

Logarithmic histograms of the OD correction parameter's absolute deviation from unity are shown in Figure 3.7b using all data for the 12 studied wavelengths. Across all 
simulations, the maximum deviation from unity was 0.0217 , with distribution modes of $\sim 10^{-4}$ or lower. These data suggest that in the extreme, the change in OD due to beam steering may approach the order of a percentage point, but it is generally orders of magnitude smaller. For context, consider the above-described sky-LOSA measurement technique. The OD is proportional to column density and the correction parameter thus represents a bias in the technique's quantification of the mass column density of flaregenerated soot. Typically however, measurement uncertainty driven by uncertainties in the optical properties of the particulate, are on the order of $-26 /+36 \%$ (Chapter 2, Conrad and Johnson 2017; Johnson et al. 2013). Treating the OD correction parameter as a beam steering-driven bias in the column density, its addition in quadrature with optical property uncertainties would have a trivial effect on the measurement technique, even in the most extreme case of $\left|K_{\lambda, O D}-1\right|=O\left(10^{-2}\right)$.

\subsubsection{Source Intensity Correction, $K_{\lambda, s}$}

The source intensity correction parameter considers the difference in the accumulated intensity source term in the RTE due to light being steered along a path different from the straight line. In general, the source term may include emission and inscattering components. Consider however the classification of remote optical measurements in Section 3.5.3. For visible spectrum measurements of particulate emissions, thermal emission is negligible due to the low temperature of cooled plumes, and for IR spectrum measurements of gaseous emissions, inscattering effects can be neglected. Consequently, it is again beneficial to separately assess the correction parameter for these two classes of measurement techniques. 
For IR spectrum measurements of gaseous emissions, the intensity source term is simply the blackbody spectral emission at the local plume temperature:

$$
I_{\lambda}^{S}(\boldsymbol{r})=I_{b \lambda}(T(\boldsymbol{r}))
$$

Thus, with knowledge of the path travelled, the source terms in the RTE can be directly calculated as required to compute the source intensity correction. Using Eq. (3.11) to compute the curved path from simulation data, the local absorption coefficient and blackbody intensity on the linear and curved paths were used to calculate $K_{\lambda, s}$ for a given optical axis via Eq. (3.5).

The source intensity correction parameter was computed for the same IR wavelengths and optical axes as those in Figure 3.6b. The logarithmic frequency of the correction's absolute deviation from unity are plotted in Figure 3.7c. Also plotted in the figure are the equivalent instrument noise distributions for two signal-to-noise ratios (SNRs), 40 and 100, corresponding to estimates for commercially available mid-wave hyperspectral imagers by Harley and Gross (2011) and Gross et al. (2010b), respectively. Like the incident intensity correction, the source intensity correction parameter tends to be overshadowed by instrument noise (up to and exceeding an order of magnitude) and can therefore be practically ignored for measurement techniques in the IR spectrum. One additional and important observation is that the observed intra- and inter-band variability in the total deflection angle (refer to Figure $3.6 \mathrm{~b}$ and surrounding discussion) does not seem to persist in the context of the source intensity correction. That is, there does not appear to be a consistent trend in $K_{\lambda, s}$ vs. $\theta_{\lambda}$ as a function of wavelength. This result implies that the observed spectral dispersion in the total deflection angle does not correspond to a consistently similar dispersion in the source intensity correction and that the source 
intensity correction can be reasonably fixed at unity across the entirety of the considered absorption bands.

For visible spectrum measurements of particulate emissions, the intensity source is due solely to inscattering. As shown in Section B.3, for all measurement conditions, the absolute deviation of the source intensity correction parameter from unity is upper-bounded by that of the OD correction parameter - i.e., $\left|K_{\lambda, s}-1\right| \leq\left|K_{\lambda, O D}-1\right|$. Since the latter was determined to be negligible even at its most extreme simulated magnitude, the analysis presented in Section B.3 suggests that the source intensity correction parameter for inscattering sources is likewise negligibly different than unity.

\subsubsection{Further Considerations - Beam Steering by Fine-Scale Turbulence}

Beam steering by fine-scale turbulence is challenging to fully consider in simulation work due to associated computational burden; this is especially true for remote optical measurements through combustion-generated plumes, where direct numerical simulation of turbulent scales down to the Kolmogorov limit is generally precluded. However, order of magnitude arguments can still yield valuable insights into the likely limits of their importance.

Referring to the governing differential equation for beam steering (Eq. (3.7)), the incremental deflection angle at a given position is a function of the relative gradient in the refractive index. Turbulence at smaller scales than the simulation data would thus tend to have an accentuated effect on local beam deflection. However, the isotropic nature of small scale turbulence would yield random Gaussian/white noise in the refractive index field, which will tend to average out when integrating over a LOS (as per Eq. (3.8)). Therefore, the effect on total beam deflection is muted. To garner insight into the 
magnitude of this turbulence effect, a MC analysis for light at $0.405 \mu \mathrm{m}$ was performed on the same optical axis as the analysis of Figure 3.5. As detailed in Section B.4, for this additional MC analysis, turbulence in the simulated refractive index field was introduced in the form of randomized fluctuations in plume temperature for varying degrees of grid refinement (representing turbulence length scale) and turbulence intensity. Results show that sub-grid turbulence tends to introduce a random walk in the travelled path of light about the path computed on the original grid. Consequently, added variability to beam steering components $\theta_{\lambda}^{y}$ and $\theta_{\lambda}^{z}$ is introduced, which follows a normal distribution without statistically changing the central tendency. Propagating this effect to the total deflection angle through summation in quadrature shows that the total deflection angle can be expected to follow a Rician distribution with a mean that is constrained to be greater than or equal to the total deflection angle computed on the original grid. That is, sub-grid turbulence introduces an upward bias in the total deflection.

Bounding the bias in the total deflection angle due to sub-grid turbulence is challenging since, unfortunately, the authors are unaware of published data on the Kolmogorov scale and turbulence intensity for flare plumes. An upper bound on the deflection (via a lower bound on the Kolmogorov scale of turbulence) has been estimated, however, using the viscosity of air at ambient conditions and allowing for eddy dissipation over a transit time of $\sim 1 \mathrm{~s}$ to the measurement control surface. These assumptions suggest a Kolmogorov scale on the order of $4 \mathrm{~mm}$ may be reasonable, however this is likely a lower bound since higher viscosities would be expected for a heated plume. Nevertheless, a scale of $4 \mathrm{~mm}$ would require grid resolution of $2 \mathrm{~mm}$ or less to capture the smallest eddies. As shown in Section B.4, at a grid resolution of $1.56 \mathrm{~mm}$ and $5 \%$ turbulence intensity, the total 
deflection angle is just $5 \%$ greater than that computed using the coarser, raw flare simulation grid. Moreover, considering the OD correction parameter as an example, added turbulence increases the correction parameter's average deviation from unity $\left(\left|K_{\lambda, O D}-1\right|\right)$ from $10^{-4.47}$ to just $10^{-4.08}$, suggesting that the effect of sub-grid turbulence is trivial and the present correction parameters are indeed negligible.

Although turbulence tends to increase variability in beam steering, there are also smoothing effects related to the finite nature of a detector's spatial and temporal resolution. With respect to spatial resolution, a detector (or pixel, in the case of an imaging detector) will accept light from a finite solid angle surrounding its nominal optical axis. Similarly, finite exposure times imply that detected intensity and the correction parameters are influenced by ray paths over a finite time interval. Thus, high spatial (through the detector's point spread function) and temporal (through finite exposure) frequencies in light intensity and the correction parameters would be smoothed to some degree in a practical measurement. This implies that the above analysis suggesting a small effect of isotropic sub-grid turbulence likely over-estimates reality. Therefore, the present results on the simulated grid is a good representation for what might be encountered in field experiments.

\subsection{Conclusions}

Simulations of beam steering along realistic optical axes intercepting atmospheric plumes of gas flares - representing an extreme case of beam steering due to elevated temperature and composition gradients near the flame - have shown that the total deflection angle for a ray of light traversing a plume is generally composed of a low-frequency, continuum-like component with added high-frequency spectral dispersion in the vicinity of IR line 
transitions. Over the 12 studied wavelengths, the observed maximum deflection angle was approximately $1.54 \mathrm{mrad}$. Wavelength-specific modes for the total deflection angle, however, were approximately one order of magnitude smaller. Finite spectral resolution of optical detectors suggests that the effect of beam steering on remote optical measurement techniques is practically driven by its low-frequency, continuum component. Consequently, simulation data at $0.405 \mu \mathrm{m}$, representing an upper-bound estimate of the continuum, enabled a probability distribution for the deflection angle of light. The resulting type II extreme value distribution permits modelling of the beam steering effect for use in measurement technique algorithms.

Three nondimensional correction parameters were derived from the radiative transfer equation and used to quantify the effect of beam steering on the incident intensity, optical depth, and source intensity. These multiplicative correction parameters were found to only slightly deviate from unity, with most-probable values within $1 \pm 10^{-4}$. Relative to typical instrument noise and measurement technique uncertainties, it was concluded that each of the correction parameters are practically equivalent to unity. A supplemental Monte Carlo analysis to provide insight into the effect of sub-grid turbulence not captured by the present flare simulations showed that consideration of fine-scale turbulence introduces variability and a slight upward bias to the beam steering effect. However, in a practical measurement this small added variability would most likely be reduced by the finite spatial and temporal resolution of optical detectors. Thus, the present analysis shows that beam steering can be justifiably ignored in remote optical measurements of atmospheric plumes, even in the extreme case of measuring combustion-generated pollutants in plumes of gas flares. 


\subsection{Acknowledgements}

This work was supported by the Natural Sciences and Engineering Research Council of Canada (NSERC) FlareNet Strategic Network (Grant \#479641), NSERC Discovery Research Grants (Grant \#06632 and 522658), and Natural Resources Canada (Project Manager, Michael Layer). We are grateful for the help of Prof. Philip Smith (University of Utah) in coordinating the sharing of high-fidelity LES data to enable this work. 


\section{Chapter 4 The Effect of Multiple Scattering on Optical Measurement of Soot Emissions in Atmospheric Plumes}

This chapter has been peer-reviewed and was published in the Journal of Quantitative Spectroscopy and Radiative Transfer. Only minor clarifications and formatting edits have been made for consistency throughout the thesis. It should be cited as:

Conrad, B.M., Thornock, J.N. and Johnson, M.R. (2020). The Effect of Multiple Scattering on Optical Measurement of Soot Emissions in Atmospheric Plumes. $J$. Quant. Spect. Rad. Tran., 254 (doi: 10.1016/j.jqsrt.2020.107220).

This article is included in its entirety as Chapter 4 with the corresponding supplemental material included as Appendix $C$. The article was co-authored by the thesis author, Bradley Conrad, Prof. Thornock (The University of Utah), and Mr. Conrad's thesis supervisor, Prof. Johnson. Mr. Conrad and Prof. Johnson conceptualized the research. Prof. Thornock executed the Large-Eddy Flare Simulations. Mr. Conrad developed the methodology and software and performed the formal analysis. Mr. Conrad wrote the first draft of the manuscript; editing and revision of the manuscript were conducted jointly.

\subsection{Abstract}

Measurements of soot/black carbon emissions via optical observations of atmospheric plume transmittance require a correction to account for bias in perceived plume brightness due to inscatter of ambient light. The ability to accurately correct for inscattering is hampered, however, by the potential for multiple scattering (MS) within the plume, which cannot be directly considered without detailed knowledge of the turbulent plume's structure. MS is thus oft-ignored within such measurement techniques, resulting in an inherent upward bias in calculated emissions. In this work, Monte Carlo "ray tracing" (MCRT) analyses for realistic lines-of-sight through large-eddy simulated, soot-laden 
atmospheric plumes of gas flares were used as case study data for analysis of MS effects. Through a reverse MCRT procedure, a remarkably simple yet accurate model was derived that relates the quantity of inscattered light under MS conditions to an estimate assuming single-scattering. Case study data from previous field measurements of gas flares using the sky-LOSA technique demonstrate that neglecting MS effects can bias reported soot emission rates by up to and exceeding one-quarter of typical measurement uncertainties. Coupling this model with an additional procedure to correct for minor model biases allows the complex influence of multiple scattering to be directly and accurately considered in optical measurements of soot emissions.

\subsection{Introduction}

Combustion-generated soot is an important atmospheric pollutant with severe negative effects on air quality and local public health (Ostro et al. 2015; Bell et al. 2014; Grahame et al. 2014) and the climate (Bond et al. 2013). Optical measurement of soot in atmospheric plumes from various sources is thus a vital, albeit challenging, scientific problem. For visible spectrum measurements of soot-laden atmospheric plumes, radiative transfer of ambient sky and sunlight into an optical detector is complicated by inscattering. Moreover, multiple scattering (MS) of ambient light within the plume can prohibit the ability to accurately quantify inscatter since prior knowledge of the spatial soot distribution, necessary to consider MS, is generally unavailable. MS effects can significantly complicate the interpretation of optical measurements and are often assumed to be negligible out of necessity. This paper seeks to quantify MS effects in optical measurements of soot-laden atmospheric plumes and develop a framework for correcting radiative transfer models that are based on the single-scattering assumption. Measurement 
of soot from the globally relevant problem of gas flaring is used as a detailed test case for analysis.

Gas flaring is a ubiquitous practice in the oil and gas industry where operators deem it infeasible to preserve extraneous gases for downstream processing, and gases are instead combusted on site in open-atmosphere flames. This practice can emit harmful pollutants including soot/black carbon directly to atmosphere. With global flared gas volumes typically exceeding 140 billion $\mathrm{m}^{3}$ per year as per satellite estimates (Elvidge et al. 2007, 2009, 2015), and an accentuated impact of soot on the sensitive Arctic climate (e.g., Jacobson 2004), there is significant concern over flare-generated soot emissions from authorities such as the Arctic council (Arctic Monitoring and Assessment Programme 2015), U.S. Environmental Protection Agency (U.S. EPA, 2012), and the World Bank (2015).

Sky-LOSA (line-of-sight attenuation using skylight) is a visible spectrum imaging technique enabling absolute soot emission rate quantification from gas flares. To date, skyLOSA has been deployed in 11 field measurement campaigns across the globe to yield 28 independent measurements of soot mass emission rate from 17 individual flares (Chapter 2, Conrad and Johnson 2017; Johnson et al. 2011, 2013). Most recently, efforts have been focused on coupling sky-LOSA with on-site measurement of flare gas flow rate and ex situ measurement of flare gas composition to compute soot yield in support of soot emission factor development (Chapter 2, Conrad and Johnson 2017). For lack of alternatives, current inventories and models generally rely on simple emission factor models for global flaring; however, laboratory and field observations have highlighted the sensitivity of the sooting propensity of similar flames to gas composition and aerodynamics (Mahmoud et 
al. 2018; Chapter 2, Conrad and Johnson 2017; McEwen and Johnson 2012; Ellzey et al. 1990; Becker and Liang 1982). Field data are thus sorely needed to improve emission factor models in support of international reporting obligations (United Nations Economic Commission for Europe 2012), inventories, and climate models.

In the sky-LOSA technique (Chapter 2, Conrad and Johnson 2017; Johnson et al. 2010, 2011, 2013), high framerate, two-dimensional, grayscale image data over a narrow bandwidth in the mid-visible spectrum $(531 \pm 20 \mathrm{~nm})$ are acquired of the atmospheric plume. These data are used to first compute the two-dimensional velocity field of the plume over the image plane via image correlation velocimetry. In parallel, bounded knowledge of optical and morphological properties of combustion-generated soot permits the quantification of soot mass column density (optical path-integrated mass concentration) over the image plane through consideration of radiative effects along each pixel's optical axis. The product of velocity and column density integrated over a control surface in the image plane yields the instantaneous soot mass emission rate, and Monte Carlo (MC) techniques are used to propagate uncertainties through the sky-LOSA algorithm (Johnson et al. 2013).

An important complexity of the sky-LOSA method is the need to account for plume brightening due to inscatter of ambient skylight and solar radiation into each pixel's optical axis. However, because ambient light may experience multiple scattering events before entering a pixel's optical axis, precise correction of this inscattering effect requires detailed knowledge of the spatial distribution of soot within the plume. For field measurements where prior knowledge of plume geometry does not exist, it is thus necessary to use the single-scattering assumption to model and correct the biasing effects of inscattered light. 
This enabling assumption reduces complex radiative transfer through the plume to a simpler case where inscattered light scatters only once on its path to the detector (e.g., Otterman 1978). To date, the validity of this single-scattering assumption has not been tested, and MS effects have not been considered in sky-LOSA MC analyses. To fully characterize sky-LOSA measurement uncertainties in support of method standardization, it is imperative to obtain a comprehensive understanding of the effects of MS on sky-LOSA measurements of soot emission rate.

This manuscript presents results from simulations to characterize, quantify, and ultimately correct the effects of MS on light transmission through soot-laden atmospheric plumes, using sky-LOSA measurement of flare-generated soot as a detailed case study. Section 4.3 provides a derivation of sky-LOSA theory and summarizes the influence of MS from a theoretical standpoint. Section 4.4 provides a summary of simulation efforts to quantify MS effects. Section 4.5 discusses results from these analyses and presents a simple MS correction model (Section 4.5.1). The utility of the model for sky-LOSA measurements is assessed, where anticipated bias and random/precision errors of the model are quantified (Section 4.5.2). The model is then applied in four case studies using previously analyzed sky-LOSA data (Chapter 2, Conrad and Johnson 2017) to investigate the effect of MS on sky-LOSA-calculated soot emission rates in the context of real-world data (Section 4.5.3). Finally, the potential feasibility of employing the derived MS correction model in similar measurement techniques for different particulate matter is discussed in Section 4.5.4. 


\subsection{Sky-LOSA Theory}

In the sky-LOSA measurement technique for flare soot emissions, a high-framerate, grayscale, scientific-CMOS camera (e.g., pco Edge 5.5) is positioned to obtain a time series of two-dimensional image data of the flare stack tip, flare flame, and portion of the sootladen atmospheric plume. From these image data, the temporally resolved soot mass emission rate is computed by calculating soot mass column density (line-of-sightintegrated soot mass concentration) and plume velocity over a control surface that traverses the plume within the image plane. This section briefly reviews the necessary theory to compute the soot mass emission rate using sky-LOSA.

A schematic of a plume measurement using the sky-LOSA technique is shown in Figure 4.1a. The pointing of a pixel's optical axis, or line-of-sight (LOS), is characterized by an inclination angle from the horizon, $\beta \in\left[0,90^{\circ}\right]$, while an arbitrary sky element (and the sun, given subscript "s") is defined by its azimuth angle relative to the detector, $\left\{\alpha, \alpha_{s}\right\} \in\left[0,360^{\circ}\right)$, and its zenith angle, $\left\{Z, Z_{s}\right\} \in\left[0,90^{\circ}\right]$, as shown. Scattering angles for the initial propagation directions of skylight and sunlight into the LOS are also shown in the figure, $\left\{\theta, \theta_{s}\right\} \in\left[0,180^{\circ}\right]$. To enable rigorous analysis of radiative effects along the LOS, a cartesian basis (shown in green) is affixed. The origin of the coordinate system is arbitrary but is selected as the intersection of the LOS with the plume. The $x$-dimension is positive towards the detector, while the $z$-dimension is positive and collinear with the general direction of plume propagation; the $y$-dimension completes the orthogonal basis. 

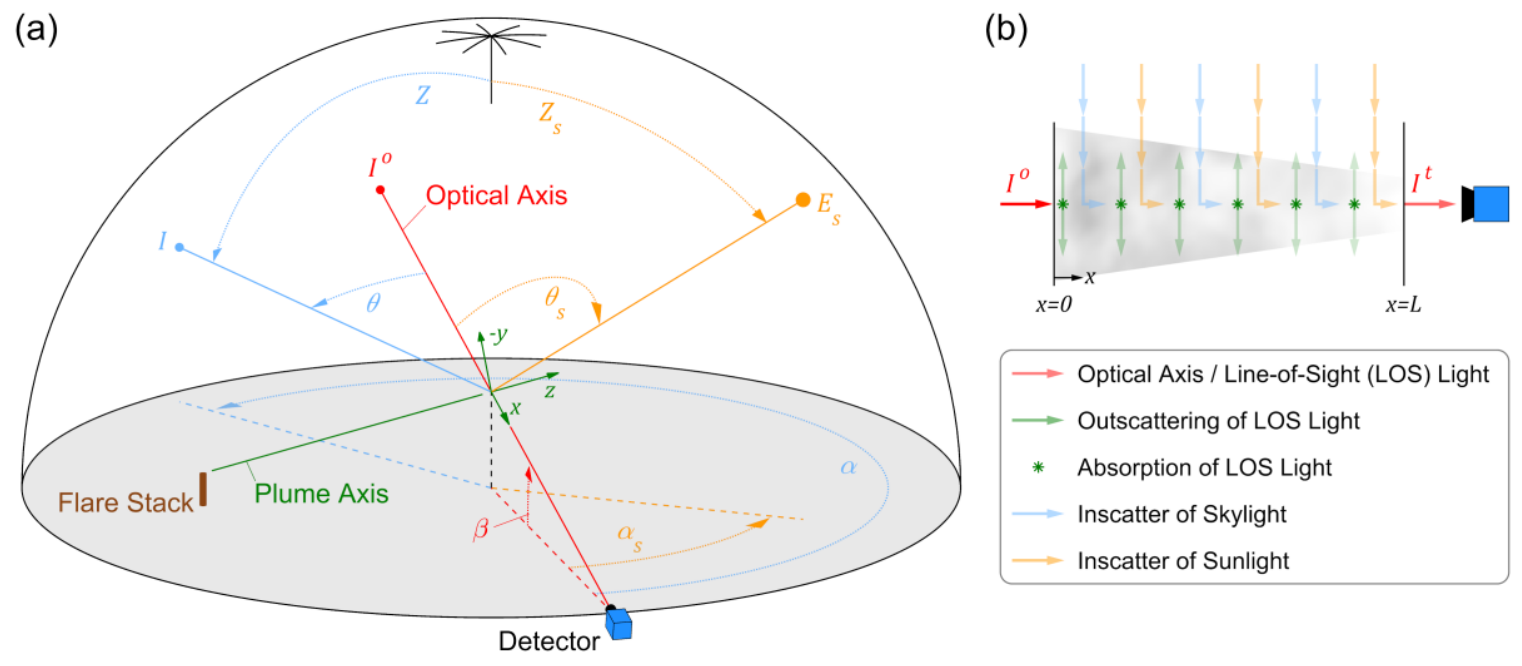

Figure 4.1: (a) Schematic of a plume measurement using the sky-LOSA technique beneath the hemispherical sky dome, (b) schematic of light transmission, absorption, outscattering, and inscattering of sky- and sunlight along the optical axis of the camera.

Radiometric observations are analyzed to compute the mass column density of soot along each LOS comprising a control surface for mass emission rate calculation. A representative schematic of these effects along one LOS traversing an arbitrary plume is shown in Figure 4.1b. In the figure, red arrows signify intensity [ $\mathrm{W} \mathrm{m}^{-2} \mathrm{sr}^{-1}$ ] (spectrally integrated over the employed optical filter) entering the plume (incident intensity, $I^{o}$ ) and exiting the plume towards the detector (transmitted intensity, $I^{t}$ ). Green arrows represent the scattering of light out of the optical axis (outscatter), green asterisks represent the absorption of light by the soot, and blue and orange arrows represent the scattering of skylight and sunlight into the optical axis (inscatter) by the soot particulate; where each of absorption, outscatter, and inscatter are functions of the position along the LOS. At visible spectrum wavelengths, the non-emitting form of the radiative transfer equation (RTE) for the cooled plume provides a mathematical description of these phenomena (adapted from e.g., Modest 2013): 


$$
I^{t}=I^{o} \exp \left(-\sigma_{e}^{m} \int_{-\infty}^{\infty} \rho_{s}(x) d x\right)+\int_{-\infty}^{\infty} \rho_{s}(x) J^{s, m}(x) \exp \left(-\sigma_{e}^{m} \int_{x}^{\infty} \rho_{s}\left(x^{\prime}\right) d x^{\prime}\right) d x
$$

where $\sigma_{e}^{m}$ is the polydisperse soot population's mean mass-normalized extinction crosssection $\left[\mathrm{m}^{2} \mathrm{~g}^{-1}\right], \rho_{s}(x)$ is the local mass concentration of soot $\left[\mathrm{g} \mathrm{m}^{-3}\right]$, and $J^{s, m}(x)$ is the local source radiant intensity due to inscattering of skylight and sunlight per unit mass of soot $\left[\mathrm{W} \mathrm{sr}^{-1} \mathrm{~g}^{-1}\right]$. In general, the infinite integration bounds need only encapsulate the flare plume.

The first term in Eq. (4.1) combines the incident light intensity with an exponential term that represents attenuation of light through the entire plume, following BeerLambert-Bouguer law (Howell et al. 2016). The second term in Eq. (4.1) is the accumulated intensity source via inscatter of skylight and sunlight at position $x$, which is then attenuated through the remainder of the plume towards the detector (hence, integration over $x^{\prime} \in[x, \infty]$ ). Using mean value theorem (e.g., Voxman and Goetschel Jr. 1981), a path-averaged source radiant intensity per unit mass $\left(\overline{J^{s, m}},\left[\mathrm{~W} \mathrm{sr}^{-1} \mathrm{~g}^{-1}\right]\right)$ can be introduced, yielding:

$$
I^{t}=I^{o} \underbrace{\exp \left(-\sigma_{e}^{m} \int_{-\infty}^{\infty} \rho_{s}(x) d x\right)}_{\tau^{*}}+\underbrace{\left(\frac{\overline{J^{s, m}}}{\sigma_{e}^{m}}\right)}_{\bar{I}^{s}} \underbrace{\int_{-\infty}^{\infty} \sigma_{e}^{m} \rho_{s}(x) \exp \left(-\sigma_{e}^{m} \int_{x}^{\infty} \rho_{s}\left(x^{\prime}\right) d x^{\prime}\right) d x}_{1-\tau^{*}}
$$

where $\sigma_{e}^{m} / \sigma_{e}^{m}=1$ has also been added to the second term and $\overline{I^{s}}=\overline{J^{s, m}} / \sigma_{e}^{m}$ represents a path-averaged inscattering source intensity $\left[\mathrm{W} \mathrm{m}^{-2} \mathrm{sr}^{-1}\right]$. If the exponential according to Beer-Lambert-Bouguer Law in the first term is defined as the idealized transmittance $\left(\tau^{*}\right.$, corresponding to the plume transmittance absent of inscattering sources) as indicated, it 
can be shown (derived in Section C.1) that the remaining integral in the second term is its complement. This enables isolation of the idealized transmittance:

$$
\tau^{*}=\frac{I^{t}-\overline{I^{S}}}{I^{o}-\overline{I^{S}}}=\frac{\tau_{o b s}-S}{1-S}
$$

where $\tau_{\text {obs }}=I^{t} / I^{o}$ is the observed/apparent transmittance of the plume $[-]$ and $S=\overline{I^{s}} / I^{o}$ is the relative path-averaged source intensity [-] - hereinafter referred to as the inscattering correction. Finally, by taking the negative logarithm of the idealized transmittance, the column density can be obtained:

$$
\rho_{s}^{\prime}=\int_{-\infty}^{\infty} \rho_{s}(x) d x=-\frac{\ln \tau^{*}}{\sigma_{e}^{m}}
$$

The instantaneous mass emission rate of soot $\left[\mathrm{g} \mathrm{s}^{-1}\right]$ over a user-defined control surface $(c)$ that crosses the plume within the image plane can then be computed by coupling the spatially resolved soot mass column density with the plume velocity:

$$
\dot{m}_{s}=\frac{1}{\sigma_{e}^{m}} \int_{C}\left(-\ln \tau^{*}\right) \boldsymbol{u} \cdot \boldsymbol{n} d s
$$

where $\boldsymbol{u}$ is the mass-concentration-weighted, path-averaged, two-dimensional plume velocity in the image plane $\left[\mathrm{m} \cdot \mathrm{s}^{-1}\right]$ computed using commercial image correlation velocimetry software (ICV; e.g., LaVision DaVis 8.3) and $\boldsymbol{n}$ is unit normal to the control surface.

The presented sky-LOSA theory shows that overall uncertainty in sky-LOSAcomputed soot mass emission rate is affected by ICV-computed plume velocity $(\boldsymbol{u})$, radiometric observations $\left(I^{t}\right.$ and $\left.I^{o}\right)$, and soot properties that influence $S$ and $\sigma_{e}^{m}$. These 
sources of uncertainty are propagated through the sky-LOSA algorithm using a Monte Carlo (MC) method as outlined in detail by Johnson et al. (2013). In the MC method, each contributing source of uncertainty is perturbed according to prior knowledge of its uncertainty and total measurement uncertainty is typically on the order of $-26 /+36 \%(95 \%$ confidence interval) (Chapter 2, Conrad and Johnson 2017; Johnson et al. 2013). SkyLOSA uncertainties are strongly driven by those of the fundamental soot properties required by sky-LOSA theory (Johnson et al. 2013).

Eight fundamental soot properties (describing the refractive index at the measurement wavelength, soot aggregate morphology and size distribution, and soot material density) are necessary for sky-LOSA calculations. Prior probability distributions of these soot properties, for use in the $\mathrm{MC}$ analysis, were derived via a rigorous literature review of buoyancy-driven turbulent diffusion flames by Johnson et al. (2013) and are summarized in Section C.2. In the sky-LOSA MC method, each MC draw requires random sampling of the fundamental properties, from which soot population-averaged optical properties are computed using Rayleigh-Debye-Gans theory for polydisperse fractal aggregates (RDG-PFA; e.g., Sorensen 2001). Within each MC draw, the use of population-averaged properties $\left(\sigma_{e}^{m}\right.$ and others that contribute to $\overline{I^{s}}$, as detailed in Section 4.3.1) implies that soot properties are spatially independent in the near-field of the cooled soot-laden plume, where reactions are assumed to have effectively halted; this assumption is justified by studies of overfire soot properties, such as Köylü and Faeth (1992). The implementation of RDG-PFA for the sky-LOSA algorithm (Johnson et al. 2013) uses the structure factor based on a Gaussian cut-off function for the monomer autocorrelation 
density and thus contains a confluent hypergeometric function in its solution (further details are available in Sorensen (2001) and references therein).

\subsubsection{Multiple Scattering}

Quantification of the inscattering correction within radiative transfer problems is exceedingly complex, requiring knowledge of the scattering element's phase function and the intensity of incident light resolved over $4 \pi$ sr at each location along the LOS. If prior knowledge of the scattering phase function and spatial distribution of scatterers are available, numerically exact inscattering can be computed via MC "ray tracing" (MCRT) methods for arbitrarily complex problems. Strictly speaking, MC "ray tracing" is a means of numerically solving the radiative transfer equation consistent with classical electromagnetics (Mishchenko 2014). Specifically, MCRT methods propagate packets of radiative energy along randomized straight paths ("rays") through a medium to obtain sufficient information permitting a numerical solution to the RTE (e.g., Wiscombe 2005). In practice however, the prior knowledge required to implement MCRT methods is seldom available, as is the case for remote, in-field measurements of flares using sky-LOSA. This necessitates use of the single-scattering assumption (1SA) to model the effect of inscattering within the technique's algorithm.

The premise of the 1SA is that inscattered skylight and sunlight result from a single initial scattering event that occurs at the intersection of the originating "ray" and the detector (pixel) LOS. Thus, the 1SA assumes skylight and sunlight are not attenuated before scattering into the LOS such that the local inscattering source intensity in the RTE

$\left(I^{S}(x)\right)$ can be replaced by a constant path-averaged source intensity $\overline{I_{1}^{S}}$ (where the subscript "1" denotes the use of the 1SA). By modelling the ground as a cold black surface, the 1SA 
allows the path-averaged source intensity to be estimated via Eq. (4.6) (which enables simple calculation of the inscattering correction via $\left.S_{1}=\overline{I_{1}^{S}} / I^{o}\right)$ :

$$
\begin{aligned}
\bar{I}_{1}^{s} & =\int_{2 \pi} I_{s k y}\left(\Omega_{s k y}, \Omega_{s} ; a\right) \frac{\omega(\boldsymbol{b})}{4 \pi} p\left(\Omega_{s k y}, \Omega ; \boldsymbol{b}\right)\left(1+f_{p o l}\left(\Omega_{s k y}, \Omega_{s}, \Omega\right)\right) d \Omega_{s k y} \\
& +E_{s}\left(\Omega_{s} ; a\right) \frac{\omega(\boldsymbol{b})}{4 \pi} p\left(\Omega_{s}, \Omega ; \boldsymbol{b}\right)
\end{aligned}
$$

where $I_{s k y}\left(\Omega_{s k y}, \Omega_{s} ; a\right)$ is the direction-dependent diffuse skylight intensity [ $\mathrm{W} \mathrm{m}^{-2} \mathrm{sr}^{-1}$ ], $\omega(\boldsymbol{b})$ is the soot population-averaged single-scattering albedo [-] (average scattering over average extinction cross-section), $p\left(\Omega^{\prime}, \Omega ; \boldsymbol{b}\right)$ is the scattering phase function of the soot population [ $\left.\mathrm{sr}^{-1}\right], f_{\text {pol }}\left(\Omega_{s k y}, \Omega_{s}, \Omega\right) \in[-1,1]$ is a function to consider the (de)polarizing effect of atmospheric light scattering [-] (derived in detail in Section C.3), and $E_{s}\left(\Omega_{s} ; a\right)$ is the ground-level solar normal irradiance $\left[\mathrm{W} \mathrm{m}^{-2}\right]$. In the sky-LOSA algorithm, directionally varying intensity of diffuse skylight $\left(I_{s k y}\left(\Omega_{s k y}, \Omega_{s} ; a\right)\right)$ is estimated using the fifteen standard models of the Commission Internationale de l'Eclairage (CIE, 2003). Each CIE sky model includes five constant coefficients defining the intensity distribution of the sky and its variability relative to the sun and the horizon. In this work, the scalar index $a \in\{1 \ldots 15\}$ is used to represent the specific CIE sky model that best represents the sky at the time of the sky-LOSA measurement. Vector $\boldsymbol{b}$ contains the fundamental soot properties required to compute soot population-averaged optical properties using RDG-PFA. Directions $\Omega_{s k y}, \Omega_{s}$, and $\Omega$ represent the propagation direction of skylight, sunlight, and light entering the detector along the LOS, respectively.

Use of the 1SA introduces an upward bias in the estimate of inscattering because unaccounted multiple scattering events further attenuate skylight and sunlight scattered 
into the optical axis. For example, consider a MC-randomized packet of radiative energy originating from the sun. During the packet's propagation through the plume, it might experience a number of attenuation "events" by soot. At each event, some fraction of the packet's energy is absorbed and the remaining fraction (in proportion to $\omega(\boldsymbol{b})$ ) is probabilistically scattered according to the scattering phase function. Any number of events might occur before some of the initial energy is possibly scattered into the detector LOS; hence, the photon packet experiences MS. Since energy is lost at each event, MS results in a reduction of inscattered energy; hence, the 1SA inherently overestimates inscattering. To accurately calculate the idealized transmittance, the inscattering correction must fully consider these multiple scattering events. A scalar MS correction parameter $\left(K_{m}\right)$ can be introduced to yield the true inscattering correction $\left(S \equiv S_{m}=\overline{I_{m}^{S}} / I^{o}=K_{m} S_{1}\right)$, such that:

$$
\tau^{*}=\frac{\tau_{o b s}-K_{m} S_{1}}{1-K_{m} S_{1}}
$$

where $K_{m} \in(0,1]$.

\subsubsection{Objective}

Without a detailed model of the MS correction parameter, the generalized sky-LOSA algorithm (Johnson et al. 2013) necessarily assumes $K_{m}=1$, such that inscattering - and, hence, mass emission rate - is biased high. The objective of this work is to quantify MS effects through detailed simulation of radiative transfer along lines-of-sight through turbulent plumes. This enables determination of the correction parameter $K_{m}$ for a range of realistic conditions. By comparing empirically calculated bias and precision uncertainties, the importance of MS relative to typical measurement uncertainties is 
revealed. Results also allow development of a surprisingly simple model to correct for MS in the chosen test case of sky-LOSA measurements of gas flares.

\subsection{Methodology}

Because prior knowledge of plume geometry is compulsory to quantify MS effects, a simulation approach was required for the present work. High-fidelity large-eddy simulations were performed for flares with design and operational conditions typical of Canada's upstream oil and gas industry to yield a broadly representative collection of flare plume geometry/spatial soot distribution data. These data were used to simulate a wide range of realistic optical axes along which soot column density could be computed in a remote measurement technique like sky-LOSA. Next, a reverse/backwards MCRT (rMCRT) algorithm was used to compute statistically representative sets of "ray" paths for optical axes intersecting a simulated plume, along which MC-simulated packet/bundles of radiative energy might travel into a detector. Finally, from simulated light propagation along the pre-computed paths, the path-averaged source intensity $\left(\overline{I_{m}^{s}}\right)$ was computed under MC-randomized ambient lighting while considering MS. This result was compared with

$\overline{I_{1}^{S}}$ computed using the 1SA to yield insights into the MS correction parameter $K_{m}$ over a broad range of realistic conditions.

\subsubsection{Flare Simulations}

The large-eddy simulations (LESs) of gas flares used in the present work were computed using Arches, a component within the Uintah Computational Framework Software Suite (e.g., Parker 2006; Parker et al. 2006; Davison De St. Germain et al. 2000), the details of which have been described previously (Chapter 3, Conrad et al. 2020a). Because these simulations did not include detailed soot modelling, the spatial distributions of soot 
concentration used in the MS simulations were linearly scaled from instantaneous gasphase species concentration and temperature fields. This was done leveraging the common assumption that gas- and solid-phase combustion products are spatially correlated within flame plumes. A similar assumption was recently used by Weyant et al. (2016) and Gvakharia et al. (2017), who assumed that carbon dioxide $\left(\mathrm{CO}_{2}\right)$, methane, and soot were spatially correlated up to altitudes $\sim 750 \mathrm{~m}$ above a flare stack, enabling estimates of soot yield from gas flares via aircraft-based sampling. While it is noted that gas- and solidphase species may not be perfectly correlated (e.g., Johnson et al. 2001), the present approach was sufficient for the purposes of considering the impact of plume geometry on MS effects even if the resulting soot fields may not be numerically precise in the same manner as simulated gas-phase concentrations. In particular, since chemically produced combustion products are inherently co-located relative to turbulent eddies of ambient air entrained into the near-field plume, scaled soot fields from $\mathrm{CO}_{2}$ concentrations would be expected to yield realistic turbulent fields representative of those encountered in the field. To further ensure that plume soot loadings were realistic in terms of magnitude, the linear scaling parameters used to obtain mass concentrations of soot from those of $\mathrm{CO}_{2}$ were deterministically calculated considering typical sky-LOSA observations from a range of field measurements (Chapter 2, Conrad and Johnson 2017; Johnson et al. 2013). Specifically, scaling parameters were selected to achieve realistic, minimum, idealized transmittances over modelled sky-LOSA control surfaces intersecting the simulated plumes. Finally, realistic optical axes through the plumes were selected from these control surfaces, which were themselves derived for randomized sky-LOSA camera positions and typical optics, as described in the authors' previous work (Chapter 3, Conrad et al. 2020a). 


\subsubsection{Radiative Transfer Simulations}

As noted above, a MC method was used to compute MS through the simulated soot-laden plumes. MC methods are employed in the field of radiative transfer to, for example, simulate view factors between complex surfaces or quantify radiative transfer from a source to a detector separated by an arbitrary environment (e.g., Modest 2013). In the case of the latter, the objective is to numerically solve the RTE by simulation of MC packets of radiative energy that are probabilistically released from radiative sources in the environment and propagated along straight paths ("rays") through the media while considering attenuation events that may occur during their travel. Information on the quantity/intensity of the MC packets that fall upon a detector provides a numerical estimate of radiative transfer from the source(s) to the detector. Unfortunately, optical detectors are collimated, such that a prohibitively small quantity of simulated MC packets are likely to fall upon the detector. To overcome this challenge, reciprocity of radiative transfer was leveraged and a reverse MCRT (rMCRT) simulation was instead performed (procedure summarized in Section C.4 and Figure C.4). In this rMCRT method, a set of statistically representative paths that $\mathrm{MC}$ packets might travel into the detector were computed by releasing $\mathrm{MC}$ packets in the reverse direction - i.e., outward from the detector along the LOS that represents the pinhole projection from the centre of a simulated pixel. Although this implies that each rMCRT simulation represents an infinitesimal point on the detector, the point spread function of diffraction-limited optics generally exceeds the size of a pixel such that these simulations are representative of the entire simulated pixel. Moreover, turbulent plume structures are generally much larger than a single pixel. The rMCRT procedure was especially useful in the present work as it parsed the cumbersome effort of simulating MS effects. That is, the rMCRT procedure was first performed for a given flare 
simulation (i.e., plume geometry) and LOS to obtain a statistically representative set of paths for the MC packets. Next, these pre-computed paths were used to quantify the pathaveraged source intensity $\left(\overline{I_{m}^{S}}\right)$ and, hence, the inscattering correction under MS conditions $\left(S_{m}\right)$ for an arbitrary ambient lighting condition, which, for sky-LOSA, refers to diffuse skylight intensity (and polarization state) resolved over the skydome and direct solar radiation. Critically, the pre-computed set of paths $\left(10^{6}\right.$ for each simulated plume/LOS in the present work) could therefore be bootstrapped in the sense that the inscattering correction could be quantified for a given plume and LOS for any number of ambient lighting conditions.

The availability of pre-computed and statistically representative paths for MC packets along a prescribed LOS and plume enabled the quantification of the inscattering correction $\left(S_{m}\right)$ under MS conditions via the forward propagation (from source to detector) of sky- and sunlight into the detector. A detailed description of this forward procedure is provided in Section C.5, leveraging in part the approach of Marchuk et al. (1980), as summarized by Gratiy et al. (2010). One important note is that, although Johnson et al. (2013) concluded that the effect of polarization is minimal with use of the 1SA in skyLOSA, the polarization of light might be important under MS conditions. This is a consequence of the sensitivity of the directional scattering of soot to the polarization of incident light relative to the scattering plane. As such, polarization effects in the forward light propagation stage of the simulation were considered using the Stokes-Mueller formalism (e.g., Goldstein 2003; Bohren and Huffman 1983) as further described in Section C.3. 


\subsection{Results}

\subsubsection{MS Correction Parameter Model}

The paths of MC packets generated in the rMCRT simulation procedure are inherently independent of the skylight intensity distribution, solar position, and solar irradiance (i.e., ambient lighting conditions). Thus, the effect of MS on radiative transfer through the plume can be quantified just from these reverse paths, which are sensitive to plume geometry, LOS, and soot properties. This enables a simple model for the MS correction parameter that is independent of ambient lighting conditions.

Prior to developing the MS correction parameter model, it is useful to clarify notation for the probabilistic nature of scattering by a particle or particle-laden plume. Firstly, consider an rMCRT analysis and let the scattering angle $(\theta)$ and scattering azimuth $(\phi)$ define the spherical coordinate system as shown in Figure 4.2. According to the present rMCRT method, a MC packet, originally released from the detector, will experience $n_{s} \geq$ 1 scattering events. After the final scattering event, its direction of propagation is described by $\theta$, the included angle between the detector optical axis and the direction of the exiting/outgoing vector, and $\phi$, the azimuth of the outgoing vector with respect to the vertical plane. These angles thus also represent the angle of the initial MC packet from the sky or sun reaching the plume in the forward direction. Under these definitions, let the mixed-variable joint density function $f_{\theta, \phi, n_{s}}\left(\theta, \phi, n_{s} ; \boldsymbol{b}\right)$ represent the probability that after $n_{s} \geq 1$ discrete scattering events by soot with properties $\boldsymbol{b}$, an exiting MC packet (in the reverse direction) travels in a direction defined by angles $\theta$ and $\phi$. After just one scattering event, the probability density that a scattered MC packet propagates in a specific direction is related to the particle's scattering phase function, $p(\theta, \boldsymbol{\phi} ; \boldsymbol{b})$, which is a function of the 
fundamental optical properties of the particle. Probabilistically, the scattering phase function, which defines the probability of the direction of scattered light relative to the direction of incident light, is proportional to the above-derived joint density function conditional on one scattering event; that is:

$$
f_{\theta, \phi \mid n_{s}}\left(\theta, \phi \mid n_{s}=1 ; \boldsymbol{b}\right) \equiv \frac{p(\theta, \phi ; \boldsymbol{b})}{4 \pi}
$$

where the factor $4 \pi$ is included to satisfy the law of total probability.

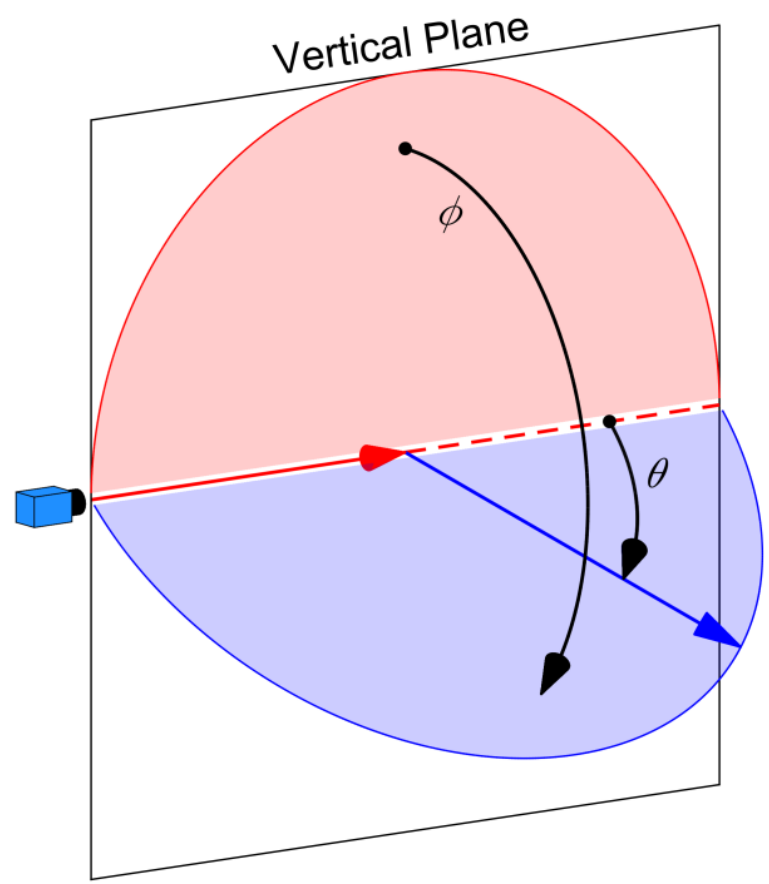

Figure 4.2: Spherical coordinate system for the development of the probabilistic definition of the scattering phase function and "energy distribution function". In an rMCRT analysis, where a MC packet is released from the detector, the angle $\theta$ represents the included angle between the detector LOS and the exiting/outgoing vector (after $n_{s} \geq 1$ total scattering events) and the azimuth $\phi$ represents the latter with respect to the vertical plane. These angles also represent the entry angle of a

MC packet from the sky or sun relative to the detector LOS in the forward direction.

While the above-described joint distribution $f_{\theta, \phi, n_{s}}\left(\theta, \phi, n_{s} ; \boldsymbol{b}\right)$ dictates the probability that an exiting MC packet exits in a given direction, it can also be used to 
consider the conservation of energy in an rMCRT analysis. Under general conditions (permitting MS), a fraction of energy, proportional to $1-\omega(\boldsymbol{b})$, is lost to absorption at each scattering event. Therefore, the quantity of energy escaping the plume diminishes for higher-order scattering events, since the relative energy of the MC packet after $n_{s}$ events is $\omega(\boldsymbol{b})^{n_{s}}$. With this in mind, the directional distribution of scattered energy (i.e., now accounting for lost energy due to absorption) can be obtained by weighting $f_{\theta, \phi, n_{s}}\left(\theta, \phi, n_{s} ; \boldsymbol{b}\right)$ with $\omega(\boldsymbol{b})^{n_{s}}$ and summing over $n_{s} \geq 1$. Let the resulting function be referred to as the "energy distribution function" $\left(p^{\omega}(\theta, \phi ; \boldsymbol{b})\right.$, where the superscript " $\omega$ " is employed):

$$
\begin{aligned}
p^{\omega}(\theta, \phi ; \boldsymbol{b}) & \equiv \sum_{n_{s} \geq 1} \omega(\boldsymbol{b})^{n_{s}} f_{\theta, \phi, n_{s}}\left(\theta, \phi, n_{s} ; \boldsymbol{b}\right) \\
& =\sum_{n_{s} \geq 1} \omega(\boldsymbol{b})^{n_{s}} f_{\theta, \phi \mid n_{s}}\left(\theta, \phi \mid n_{s} ; \boldsymbol{b}\right) P\left(n_{s}\right)
\end{aligned}
$$

where $P\left(n_{s}\right)$ represents the probability that a MC packet experiences $n_{s}$ events before escaping the plume. Importantly, by considering the loss of energy to absorption at each scattering event, $p^{\omega}(\theta, \phi ; \boldsymbol{b})$ does not consistently integrate to a specific value as does the scattering phase function. Rather, the integral of $p^{\omega}(\theta, \phi ; \boldsymbol{b})$ over the $4 \pi$ steradian sphere yields the total fraction of incident energy that escapes the plume. If only one scattering event is permitted - such that $P\left(n_{s}=1\right)=1$ and $P\left(n_{s}>1\right)=0$, as assumed in the 1SA then the single-scattering energy distribution function is:

$$
\begin{aligned}
p_{1}^{\omega}(\theta, \phi ; \boldsymbol{b}) & \equiv \omega(\boldsymbol{b}) f_{\theta, \phi \mid n_{s}}\left(\theta, \phi \mid n_{s}=1 ; \boldsymbol{b}\right) P\left(n_{s}=1\right) \\
& =\frac{\omega(\boldsymbol{b})}{4 \pi} p(\theta, \phi ; \boldsymbol{b})
\end{aligned}
$$


which integrates to $\omega(\boldsymbol{b})$.

Referring to Eq. (4.6), the path-averaged source intensity under the 1SA is simply the summation of incident ambient radiation weighted by $p_{1}^{\omega}(\theta, \phi ; b)$. Thus, the energy distribution function carries all plume- and particle-related information necessary to compute the inscattering correction $\left(S_{1}\right)$ for a given LOS under the 1SA. A corollary is that, under MS conditions, an equivalent energy distribution function must also exist that enables calculation of the true inscattering correction $\left(S \equiv S_{m}\right)$. This latter energy distribution function (computed via Eq. (4.9)) thus carries all plume- and particle-related information necessary to compute the inscattering correction under general MS conditions.

For a specific detector LOS intersecting a plume, and given a set of soot properties, the MS energy distribution function $\left(p_{m}^{\omega}(\theta, \phi ; \boldsymbol{b})\left[\mathrm{sr}^{-1}\right]\right)$ can be computed from the rMCRT-generated set of MC packet paths. Specifically, in the reverse direction, the $n^{\text {th }}$ path, which has $k_{n}$ scattering events, exits the plume in a known direction $\left(\theta_{n}, \phi_{n}\right)$ with a relative energy of $\omega(\boldsymbol{b})^{k_{n}}$. The MC-computed $p_{m}^{\omega}(\theta, \phi ; \boldsymbol{b})$ is then simply the relative energy-weighted histogram over the $\theta-\phi$ domain:

$$
p_{m}^{\omega}(\theta, \phi ; \boldsymbol{b}) \propto \sum_{\substack{\theta_{n} \in A \\ \phi_{n} \in B}} \omega(\boldsymbol{b})^{k_{n}}
$$

where $A=\left\{\theta_{n}:\left|\theta_{n}-\theta\right|<\Delta \theta\right\}, B=\left\{\phi_{n}:\left|\phi_{n}-\phi\right|<\Delta \phi\right\}$, and $\Delta \theta$ and $\Delta \phi$ are the halfwidths of the histogram bins. An example of a numerically calculated $p_{m}^{\omega}(\theta, \phi ; \boldsymbol{b})$ for one simulated LOS is shown in Figure 4.3a; this particular result is from an rMCRT analysis of a LOS intersecting a high-opacity plume with an idealized transmittance of $25 \%$. The image shows the function on a logarithmic scale against the scattering angle $(\theta)$ and 
azimuth $(\phi)$. There are two important observations. Firstly, $p_{m}^{\omega}(\theta, \phi ; \boldsymbol{b})$ is strongly asymmetric with a notable bias towards the forward scattering direction $\left(\theta=0^{\circ}\right)$, similar to $p_{1}^{\omega}(\theta, \phi ; \boldsymbol{b})$ computed using RDG-PFA. This implies that the vast majority of inscattered light is sourced from the region of sky near a detector's optical axis. The second observation is the influence of the plume in the figure, signified by the two regions with locally reduced magnitude. From these directions, ambient light is nearly collinear with the plume axis, and thus must travel through a larger optical depth to ultimately scatter into the detector along the optical axis. 


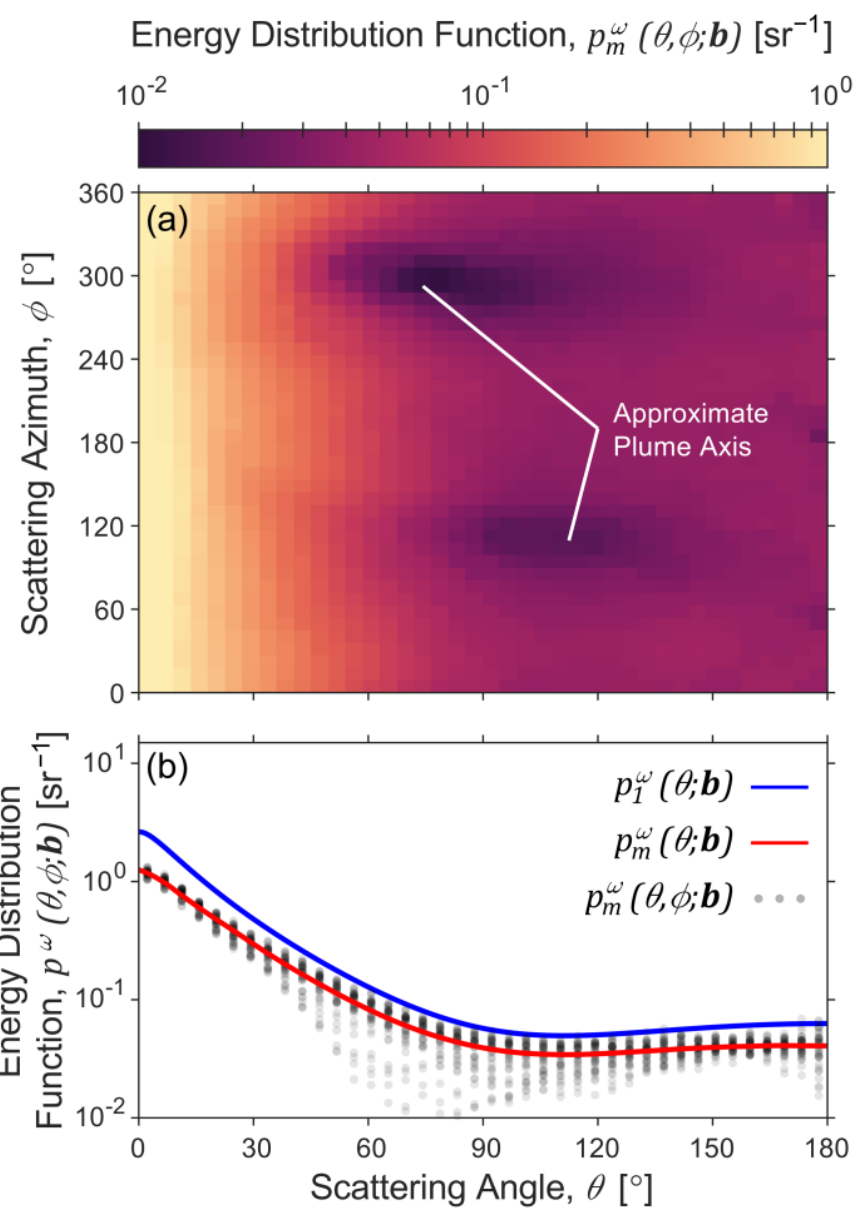

Figure 4.3: (a) Multiple-scattering energy distribution function $\left(p_{m}^{\omega}(\theta, \phi ; b)\right)$ plotted on a logarithmic scale, showing a strong bias toward forward scattering $\left(\theta=0^{\circ}\right)$. Labelled darker regions correspond to a local reduction in the function due to the presence of the plume where, from these directions, light must traverse a larger optical depth along the plume prior to scattering into the optical axis. (b) Singlescattering energy distribution function $\left(p_{1}^{\omega}(\theta ; b)\right.$, computed with RDG-PFA for the selected soot properties $), p_{m}^{\omega}(\theta, \phi ; b)$, and $p_{m}^{\omega}(\theta ; b)$ plotted against scattering angle. As expected, $p_{m}^{(\omega)}(\theta ; b)$ is upper-bounded by $p_{1}^{(\omega}(\theta ; b)$ illustrating the upward bias due to use of the 1SA.

As noted above, replacement of $p_{1}^{\omega}(\theta, \phi ; \boldsymbol{b})$ with $p_{m}^{\omega}(\theta, \phi ; \boldsymbol{b})$ in Eq. (4.6) enables calculation of the true inscattering correction under MS conditions. Practically though, $p_{m}^{\omega}(\theta, \phi ; \boldsymbol{b})$ defined over two dimensions has limited utility since it is a function of the LOS and the plume geometry. That is, spatiotemporal variability in plume geometry relative to a LOS makes it practically impossible to model the two-dimensional energy distribution function for use with field data. Noting the strong forward scattering however, 
the effect of variability in the $\phi$-coordinate can be muted by positioning the detector such that its axis and the plume axis are roughly orthogonal as shown in Figure 4.1a. If this is constrained to be true, then $p_{m}^{\omega}(\theta, \phi ; \boldsymbol{b})$ can be simplified by averaging over the $\phi$ coordinate, yielding $p_{m}^{\omega}(\theta ; \boldsymbol{b})$. Analogously to Eq. (4.11):

$$
p_{m}^{\omega}(\theta ; \boldsymbol{b}) \propto \sum_{\theta_{n} \in A} \omega(\boldsymbol{b})^{k_{n}}
$$

This loose constraint on the camera pointing is already heuristically imposed on sky-LOSA data acquisition to support computation of plume velocity with minimal uncertainty using image correlation velocimetry. Therefore, averaging over the $\phi$-coordinate as presented is reasonably justified for the sky-LOSA technique. However, if the orthogonality constraint is not satisfied, such that the plume axis approaches collinearity with the pixel's LOS, then local reductions in $p_{m}^{\omega}(\theta, \phi ; \boldsymbol{b})$ due to increased optical depth along the plume axis may approach the forward scattering direction where most inscattered light is sourced. In this extreme case, averaging of $p_{m}^{\omega}(\theta, \phi ; \boldsymbol{b})$ over the $\phi$-coordinate to yield $p_{m}^{\omega}(\theta ; \boldsymbol{b})$ may not be justified.

Figure $4.3 \mathrm{~b}$ shows a comparison of $p_{1}^{\omega}(\theta ; \boldsymbol{b})$ (via RDG-PFA), $p_{m}^{\omega}(\theta, \phi ; \boldsymbol{b})$, and $p_{m}^{\omega}(\theta ; \boldsymbol{b})$ for the same example plume, LOS, and soot properties. As expected, $p_{m}^{\omega}(\theta ; \boldsymbol{b})$ is upper bounded by $p_{1}^{\omega}(\theta ; \boldsymbol{b})$ across all scattering angles, which illustrates the inherent upward bias when using the 1SA to estimate inscatter. Note that the few values of $p_{m}^{\omega}(\theta, \phi ; \boldsymbol{b})$ that slightly exceed $p_{1}^{\omega}(\theta ; \boldsymbol{b})$ at large scattering angles do not violate the physical constraint that $p_{m}^{\omega}(\theta, \phi ; \boldsymbol{b}) \leq p_{1}^{\omega}(\theta, \phi ; \boldsymbol{b})$ but rather are attributable to noise resulting from the relatively small amount of data in these directions from the rMCRT analysis. 
While the above example shows that $p_{m}^{\omega}(\theta ; \boldsymbol{b})$ is sensitive to the geometry of the atmospheric plume relative to the LOS, it is also sensitive to plume soot loading. That is, the absolute magnitude of soot through which inscattered light must travel influences $p_{m}^{\omega}(\theta ; \boldsymbol{b})$ through the conditional distribution $f_{\theta, \phi \mid n_{s}}\left(\theta, \phi \mid n_{s} ; \boldsymbol{b}\right)$ and the distribution of the number of scattering events $\left(P\left(n_{s}\right)\right)$, where a more optically thick plume will create a positive shift in this latter distribution. Furthermore, soot properties also influence $p_{m}^{\omega}(\theta ; \boldsymbol{b})$ through their impact on the single-scattering albedo and scattering phase function. To glean insight into the relative influence of these factors on $p_{m}^{\omega}(\theta ; \boldsymbol{b})$, a preliminary MC analysis that independently perturbed soot properties was performed. In this analysis, 150 lines-of-sight corresponding to sky-LOSA control surface-minima were randomly selected from the modelled sky-LOSA control surfaces, and plumes were scaled to yield a control surface-minimum idealized transmittance of $0.25,0.50$, or 0.75 . Soot properties were varied according to the prior probability distributions defined by Johnson et al. (2013), such that each of the 150 rMCRT simulations employed different soot population-averaged optical properties. For each rMCRT simulation, the generated reverse paths enabled the development of $p_{m}^{\omega}(\theta ; \boldsymbol{b})$ and its comparison with $p_{1}^{\omega}(\theta ; \boldsymbol{b})$ calculated using RDG-PFA. Results of these analyses are summarized in Figure 4.4. 


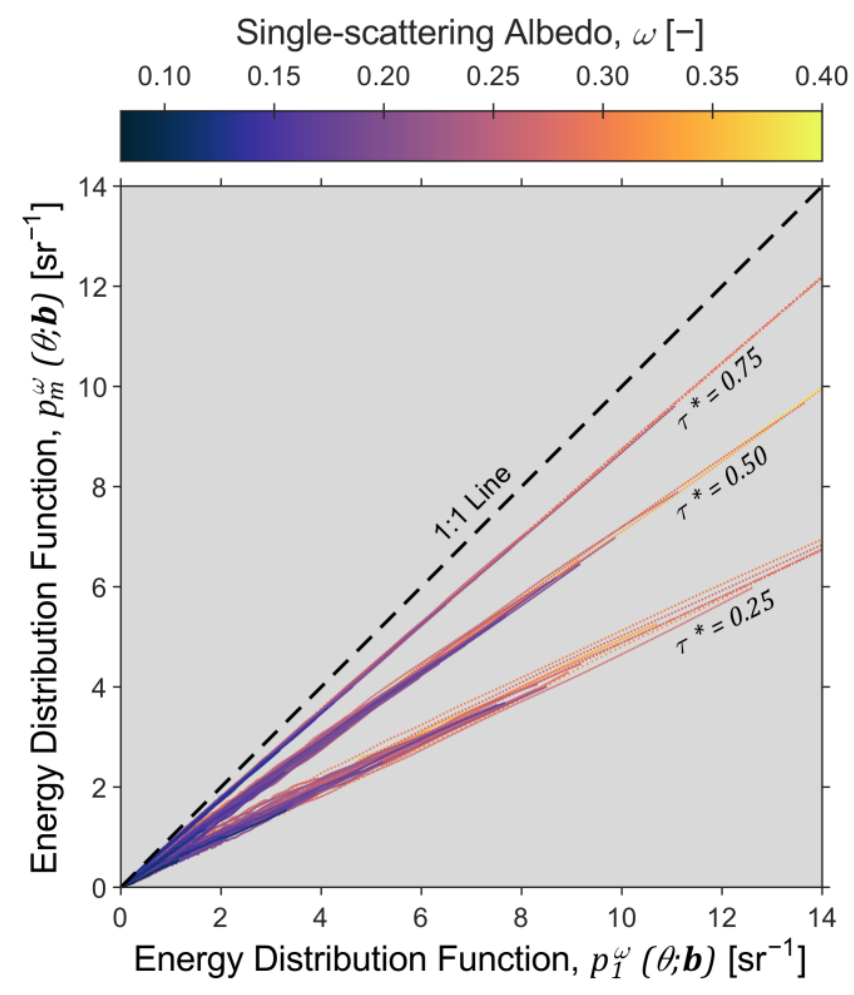

Figure 4.4: Comparison of $p_{1}^{\omega}(\theta ; b)$ and $p_{m}^{\omega}(\theta ; b)$ for example plume transmittances with variable soot properties. These data show that the difference between $p_{1}^{\omega}(\theta ; b)$ and $p_{m}^{\omega}(\theta ; b)$ is much more sensitive to plume transmittance than soot properties and that there is a high degree of linearity between the two functions.

The data of Figure 4.4 show high-linearity between the two energy distribution functions over the tested ranges of plume transmittance and soot properties. This observation suggests a minimal directional dependence between $p_{1}^{\omega}(\theta ; \boldsymbol{b})$ and $p_{m}^{\omega}(\theta ; \boldsymbol{b})$, even though a slight directional dependence can be observed on the logarithmic scale of Figure 4.3b. When plotted on a linear scale as in Figure 4.4, much of the functions are compressed into lower values (corresponding to higher scattering angles). However, higher values of the functions (corresponding to lower scattering angles/forward scattering) have more influence on inscattered energy; thus, it is important to analyze these data with a focus on the higher-energy domain. To this end, energy-weighted zero-intercept linear models were fit to the data of Figure 4.4 with minimal error $-R^{2}$ values averaging to 0.9970 
(median of 0.9991$)$ - quantitatively suggesting a highly linear relation between $p_{1}^{\omega}(\theta ; \boldsymbol{b})$ and $p_{m}^{\omega}(\theta ; \boldsymbol{b})$. This has an important implication for model development: if, for a given plume/LOS and set of soot properties, $p_{m}^{\omega}(\theta ; \boldsymbol{b})$ is linearly proportional to $p_{1}^{\omega}(\theta ; \boldsymbol{b})$ as suggested, then the former can be modelled as a scalar multiple of the latter. Recalling that $p_{m}^{\omega}(\theta ; \boldsymbol{b})$ can simply replace $p_{1}^{\omega}(\theta ; \boldsymbol{b})$ in Eq. (4.6) to enable calculation of the true inscattering correction, the slope of the relation is the MS correction parameter, $K_{m}$, by definition. Thus, the MS correction parameter effectively models $p_{m}^{\omega}(\theta ; \boldsymbol{b})$ as a scaled equivalent of $p_{1}^{\omega}(\theta ; \boldsymbol{b})$. The slope of each set of $\mathrm{MC}$ data (representing the MS correction parameter) is expectedly a strong function of the idealized transmittance since the probability of multiple scattering events increases with the optical depth of the plume. By contrast, Figure 4.4 shows that the relation between the slope and soot properties is less pronounced, implying that the idealized transmittance/plume loading is the dominant factor driving the MS correction parameter.

To enable a model for the MS correction parameter, a more comprehensive, fully randomized, $\mathrm{MC}$ procedure was performed. In this analysis, $10^{3}$ statistically representative sets of paths were computed using the rMCRT method for randomized plumes/lines-ofsight, using different randomly sampled soot properties in each rMCRT implementation. Sky-LOSA control surface-minimum idealized transmittances were randomized following a uniform distribution over 0.25 to 0.75 . As expected, the correlation between the MS correction parameter and the line transmittance was high $(0.980)$ while correlations with single-scattering albedo and asymmetry parameter $(g(\boldsymbol{b})$, which quantifies the peakedness/asymmetry of the scattering phase function; e.g., Bohren and Huffman (1983)) of the soot were negligible ( 0.048 and 0.039 , respectively). This implies that the MS 
correction parameter can be modelled as a function of the idealized transmittance alone i.e., $K_{m}=K_{m}\left(\tau^{*}\right)$. The computed MS correction parameters are plotted against the idealized transmittance for the $10^{3} \mathrm{MC}$ simulations in Figure 4.5, where symbol colour represents the single-scattering albedo.

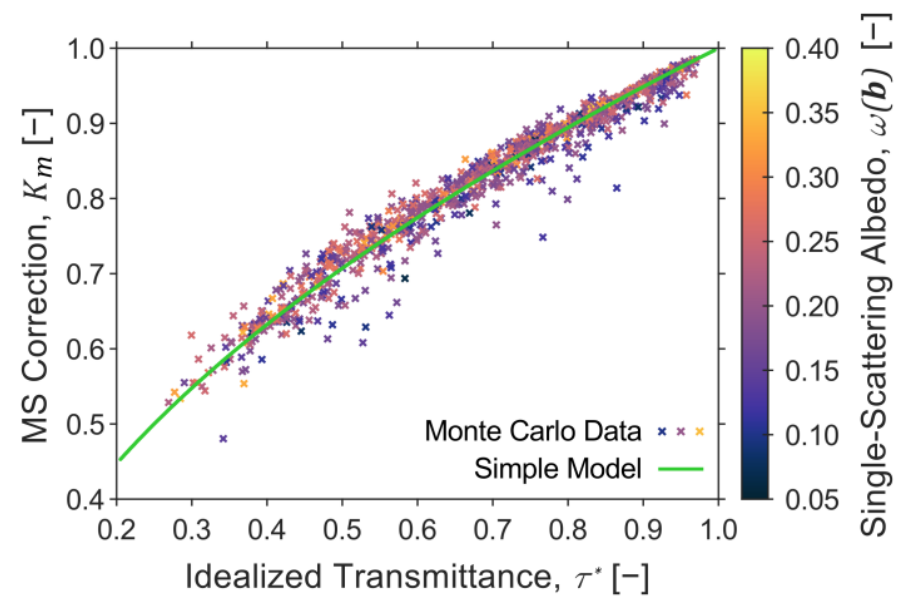

Figure 4.5: Monte Carlo-computed multiple scattering correction plotted as a function of idealized transmittance (representing plume loading) and coloured according to the soot population's single-scattering albedo. The correction parameter tends to increase with idealized transmittance and approaches unity as the plume becomes optically thin. A simple MS correction model $\left(K_{m}=\left(\tau^{*}\right)^{1 / 2}\right)$ is overlaid in green.

There is a monotonically increasing trend in the data, and the MS correction parameter approaches unity as does the idealized transmittance, since the effect of MS becomes diminished in the limit of an optically thin plume. A relative error-minimizing model was found to be a power law relation $K_{m}=\left(\tau^{*}\right)^{0.4986}$, which yielded a relative root mean squared error of $2.8259 \%$. A simple alternative model for the MS correction was instead defined, $K_{m}=\sqrt{\tau^{*}}$, shown in green in the figure. This simple model negligibly increases error in the fit (to $2.8266 \%$ ) and enables analytical calculation of idealized transmittance via Eq. (C.40) in Section C.6. The figure shows that there is some dispersion of the $\mathrm{MC}$ results about the simple model; however, residual error was poorly explained 
by soot properties (93.5\% of variance unexplained via linear regression in $\omega(\boldsymbol{b})$ and $g(\boldsymbol{b}))$. This highlights that model error apparent in Figure 4.5 is unlikely to be driven by soot properties, but rather by geometric variability in the turbulent soot-laden plume.

\subsubsection{Model Evaluation}

Although error in the simple model for the MS correction parameter $\left(K_{m}=\sqrt{\tau^{*}}\right)-$ derived solely using rMCRT data - is mostly due to the turbulent nature of the plume, additional error sources are relevant when using the model in the forward direction (to propagate ambient light into the detector). These additional sources of error include the effect of polarization and, for sky-LOSA, the directional distribution of ambient sky- and sunlight relative to the plume and LOS. Therefore, to enable an MS correction within the skyLOSA algorithm, it is critical to assess (correctable) bias errors and (uncorrectable/random) precision errors in calculated soot mass column density when using the present model. To this end, the fully randomized $10^{3}$ sets of rMCRT data used to derive the MS model were reversed to enable forward light propagation via the procedure described in Section C.5. Noting that the reverse paths are independent from ambient lighting conditions, the abovedescribed $10^{3}$ sets of rMCRT data were bootstrapped where, for each of the $10^{3}$ rMCRT data sets, $10^{3}$ randomized ambient lighting conditions were applied - randomly perturbing the relative position of the sun (constrained such that $\theta_{s}>30^{\circ}$ ) and the modelled skylight intensity distribution - to yield a total of $10^{6}$ simulations of the inscattering correction $\left(S_{m}\right)$ under MS conditions for realistic sky-LOSA positions. With these MC-computed inscattering corrections and known idealized transmittances of the plume $\left(\tau^{*}\right)$, plume transmittances that would be observed at the detector $\left(\tau_{o b s}\right)$ were quantified by inverting Eq. (4.3). Then, 1SA-estimated inscattering corrections $\left(S_{1}\right)$ were computed using Eq. 
(4.6) and the simple model for $K_{m}$ was used to estimate the idealized transmittances via solution of the implicit Eq. (4.7). This permitted comparison of model-estimated soot mass column density $\left(\widetilde{\rho_{s}^{\prime}}\right)$ against the known ground truth $\left(\rho_{s}^{\prime}\right)$ from LES simulations to enable bias correction and quantification of precision error when using the present model within sky-LOSA.

Figure 4.6a and Figure 4.6b plot the bias and precision error in soot mass column density computed using the MS correction parameter $\left(\widetilde{\rho_{s}^{\prime}}\right)$ against the ground truth from LES simulations $\left(\rho_{s}^{\prime}\right)$. Data are plotted for each of the 15 CIE sky models as a function of solar position $\left(\alpha_{s}\right.$ and $\left.Z_{s}\right)$ and were derived from $15 \times 15$ grids of bias and precision error in the $\alpha_{s}-Z_{s}$ domain, computed from the $\mathrm{MC}$ results. The bias errors in computed soot mass column density were consistently small (within $\pm 2.4 \%$ for all CIE sky models) but did show a directional dependency with solar position. Precision errors varied from 0.6 to 7.3\% and similarly showed directional dependency. As discussed in Section 4.5.2.1, the raw data from Figure 4.6 (which are included in a supplementary file as part of the paper's on-line supplemental information and discussed in Section C.7) can be used in conjunction with simple LOESS (locally estimated scatterplot smoothing) fits to correct for this minor bias as a function of solar position for each CIE sky model while considering the contribution of precision error in the sky-LOSA algorithm. 
(a)

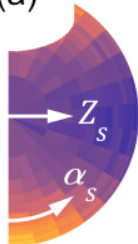

Sky 01

(I.1)

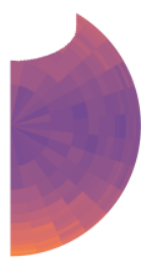

Sky 06

(III.2)

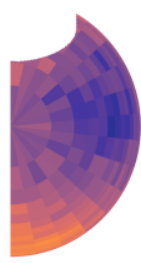

Sky 11

(IV.4)

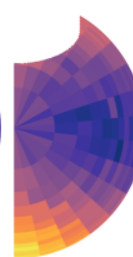

Sky 02

(I.2)

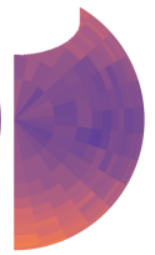

Sky 07

(III.3)

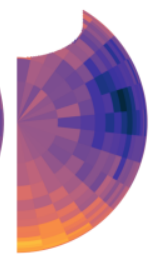

Sky 12

(V.4)

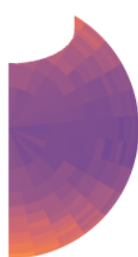

Sky 03

(II.1)

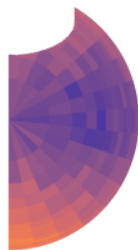

Sky 08

(III.4)

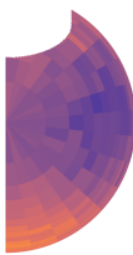

Sky 13

(V.5)

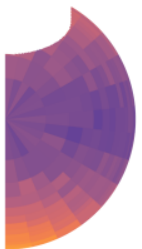

Sky 04

(II.2)

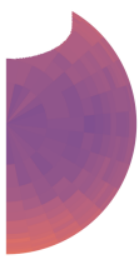

Sky 09

(IV.2)

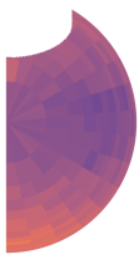

Sky 14

(VI.5)

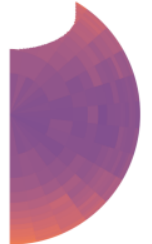

Sky 05

(III.1)

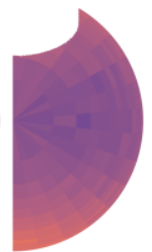

Sky 10

(IV.3)

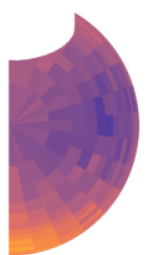

Sky 15

(VI.6)

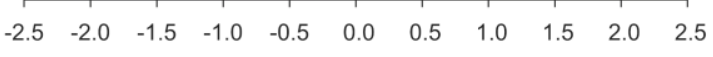

Bias Error in MS-Corrected Soot Mass Column Density [\%]

(b)
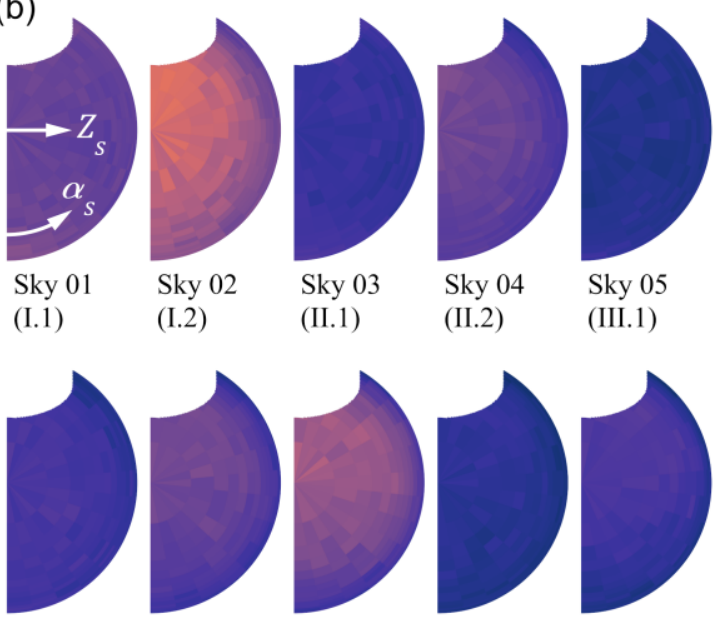

Sky 06 (III.2)
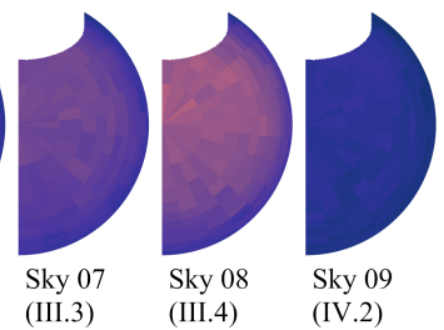

Sky 09 (IV.2)
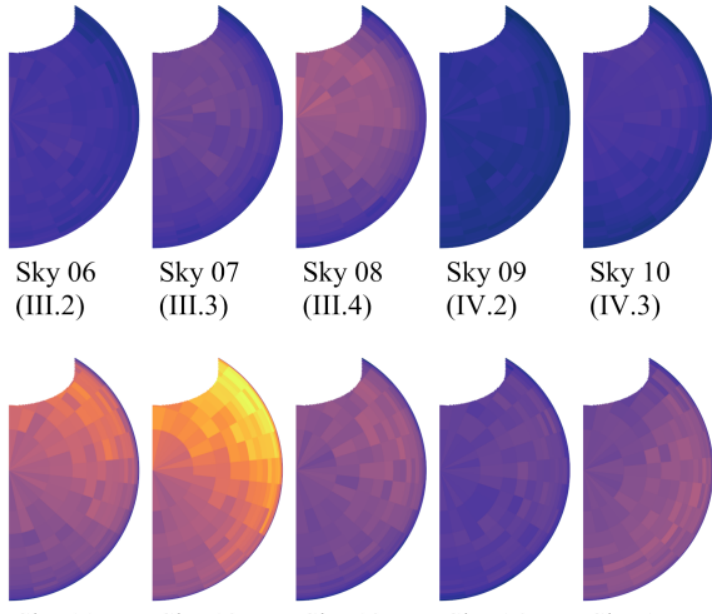

Sky 11

(IV.4)
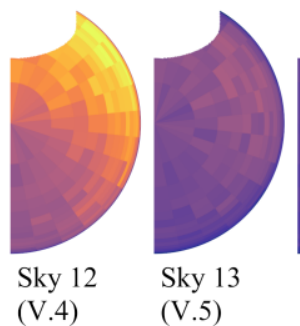

Sky 13

(V.5)

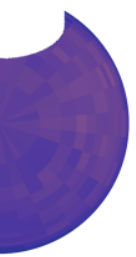

Sky 14

(VI.5)

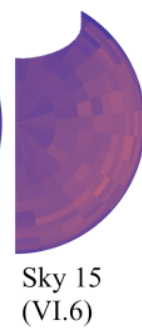

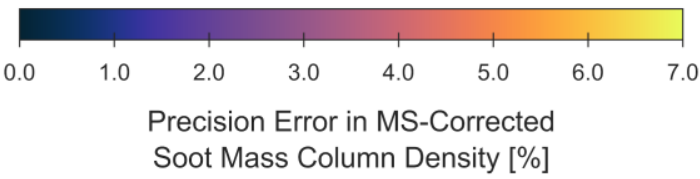

Figure 4.6: Bias (a) and precision (b) error in soot mass column density computed using the MS correction parameter against the ground truth from LES simulations

for each of the 15 CIE sky models as a function of solar position $\left(\alpha_{s}\right.$ and $\left.Z_{s}\right)$ as

indicated in the upper left-most panels. These data are used to derive biascorrection $\left(f\left(\alpha_{s}, Z_{s}, a\right)\right)$ and precision error $\left(\sigma\left(\alpha_{s}, Z_{s}, a\right)\right)$ functions for the sky-LOSA error model (Eq. (4.13)).

Error in the derived MS correction model $\left(K_{m}=\sqrt{\tau^{*}}\right)$ was attributed to the turbulent nature/inhomogeneity of the soot-laden plume - that is, noise about the MS model apparent in Figure 4.5 likely results from misrepresentation of the surrounding plume by the LOS idealized transmittance. For example, if the LOS transects the plume through a region of relatively low soot loading (such that $\tau^{*}$ is high relative to neighbouring lines-ofsight), then the simple model might over-estimate $K_{m}$ and thus under-estimate the effect of 
MS. Unfortunately, due to the lack of prior knowledge of the three-dimensional spatial soot loading, this effect is unpredictable in the context of a sky-LOSA field measurement. Since this source of error results from the rMCRT analysis alone, it will manifest as bias and/or precision error that is not dependent upon the solar position.

Consistent with previous analyses (Johnson et al. 2013), the effect of polarization in the computation of the 1SA-estimated inscattering correction $\left(S_{1}\right)$ was neglected by forcing $f_{p o l}=0$ in Eq. (4.6). By contrast, polarization was considered in the forward calculation of the true inscattering correction $\left(S_{m}\right)$ using the Stokes-Mueller formulism summarized in Section C.3. This approach implies that added error, engendered by the negligence of polarization in calculating $S_{1}$, is necessarily embedded in the present MS model. This was deemed acceptable since setting $f_{p o l}=0$ greatly simplifies the calculation of $S_{1}$ and is expected to yield negligible additional model error, based on the previously quantified effect of polarization (Johnson et al. 2013). Since the effect of polarization is dependent upon the specific collection of rMCRT-computed paths (i.e., plume geometry), in addition to solar position, solar position-dependent error due to the polarization effect contributes to both the bias and precision errors plotted in Figure 4.6.

The final source of error in the MS model is a consequence of the two-dimensional directional dependence of the MS energy distribution function. That is, the slight directional variations between $p_{1}^{\omega}(\theta ; \boldsymbol{b})$ and $p_{m}^{\omega}(\theta ; \boldsymbol{b})$ apparent on a logarithmic scale in Figure $4.3 \mathrm{~b}$ are treated as constant by the MS model (through $K_{m}=\sqrt{\tau^{*}}$ ). Thus, there exists some directionally dependent model error that is weighted by the directionally varying ambient lighting. For example, if the sun happens to be located at a position near the plume axis - where $p_{m}^{\omega}(\theta ; \boldsymbol{b})$ is likely to be over-estimated using the present model - 
then the sun component of the inscattering correction could also be over-estimated. This suggests that the positioning of the sun relative to the plume axis likely imposes additional bias and precision error on the model. While it is not practical to consider these errors based on the position of the sun relative to the plume axis, it is feasible to do so based strictly on the solar position relative to the detector pointing (LOS), since the latter is heuristically constrained closely orthogonal to the plume axis.

\subsubsection{Consideration of Residual Errors When Using the MS Correction Model with Sky-LOSA}

Residual bias and precision errors following application of the MS correction model $\left(K_{m}=\right.$ $\sqrt{\tau^{*}}$ ) are generally negligible (average bias and precision errors of $0.48 \%$ and $2.06 \%$ across all solar positions and sky models), but can become significant under certain conditions of ambient lighting. However, these solar position-dependent residual errors can be easily considered in the sky-LOSA algorithm through a simple error model for soot mass column density:

$$
\rho_{s}^{\prime}=\widetilde{\rho}_{s}^{\prime}\left(1+f\left(\alpha_{s}, Z_{s} ; a\right)\right)\left(1+\epsilon_{\rho}\right)
$$

This error model permits prediction of the ground truth soot mass column density $\left(\rho_{s}^{\prime}\right)$ using that computed with the MS correction parameter $\left(\widetilde{\rho_{s}^{\prime}}\right)$, a bias-correction function $\left(f\left(\alpha_{s}, Z_{s} ; a\right)\right)$, and a random error parameter, $\epsilon_{\rho} \sim \mathcal{N}\left(0, \sigma^{2}\left(\alpha_{s}, Z_{s} ; a\right)\right) . \quad$ The biascorrection function accounts for the directionally dependent mean relative error in $\widetilde{\rho_{s}^{\prime}}$ as a function of solar position for each of the $15 \mathrm{CIE}$ sky models, while $\epsilon_{\rho}$ accounts for precision error (modelled as Gaussian noise) about these mean values using the precision error function $\sigma\left(\alpha_{s}, Z_{s} ; a\right)$. Bias-correction and precision error functions were derived from the raw data of Figure 4.6 using simple LOESS fits. As demonstrated in the case 
studies below, the derived MS correction parameter, $K_{m}=\sqrt{\tau^{*}}$, and residual error functions $\left(f\left(\alpha_{s}, Z_{s} ; a\right)\right.$ and $\left.\sigma\left(\alpha_{s}, Z_{s} ; a\right)\right)$ provide a simple yet robust means to consider the otherwise complex effects of MS in sky-LOSA measurements.

\subsubsection{Sky-LOSA Case Studies}

The preceding analysis presents a simple model for considering MS effects when computing inscattering, which when applied to sky-LOSA can be further improved with a simple bias-correction function. The precision error function can then be used to include precision errors attributed to this MS approach within the sky-LOSA MC algorithm. The remaining analysis seeks to assess the implications for time-averaged soot emission rate measured using sky-LOSA, given that all analyses to date have necessarily ignored MS. Four comparative case studies were performed.

Representative sky-LOSA data were sourced from the study of Conrad and Johnson (Chapter 2, 2017), who performed measurements of soot emissions from flares in Mexico and Ecuador between 2012 and 2015. Four different flares, with plumes subject to overcast, partly cloudy, and clear skies, were selected for comparative analysis corresponding to flare identifiers O1, O3, and O4 measured in Ecuador in 2015, and flare V4 measured in Mexico in 2012. A one-minute subset of sky-LOSA data was analyzed for flares $\mathrm{O} 1, \mathrm{O} 3$, and $\mathrm{O} 4$ and the entire available data set of $51.14 \mathrm{~s}$ was analyzed for flare V4. Time-averaged soot emission rates for these flares spanned more than two orders of magnitude. Representative idealized transmittances (corresponding to the time-averaged minimum line transmittance over the sky-LOSA control surface) varied from 40.7 to $90.5 \%$. For each case, the time-averaged mass emission rate of soot was computed using the 1SA and again with the presented MS correction to investigate the effect of the latter 
on the time-averaged emission rate and its uncertainty. Results of these case studies are summarized in Table 4.1.

Table 4.1: Summary of the sky-LOSA-computed soot emission rates from four previously studied gas flares (Chapter 2, Conrad and Johnson 2017). Results using the single-scattering assumption (i.e., without MS correction) are compared with those computed using the present MS correction model without and with the consideration of residual errors using the error model of Eq. (4.13).

\begin{tabular}{|c|c|c|c|c|c|c|c|}
\hline \multirow{2}{*}{$\begin{array}{c}\text { Case } \\
\text { Study }\end{array}$} & \multirow{2}{*}{$\begin{array}{c}\text { CIE } \\
\text { Sky } \\
\text { Code }\end{array}$} & $\begin{array}{c}\text { Plume } \\
\text { Transmitt- } \\
\text { ance }\end{array}$ & $\begin{array}{c}\text { Without MS } \\
\text { Correction }\end{array}$ & $\begin{array}{c}\text { With MS } \\
\text { Correction }\end{array}$ & $\begin{array}{c}\text { With MS } \\
\text { Correction and } \\
\text { Error Model }\end{array}$ & $\begin{array}{c}\text { Change in } \\
\text { Mean } \\
\text { Emission } \\
\text { Rate }\end{array}$ & $\begin{array}{c}\text { Change in } \\
\text { Width } \\
\text { of 95\% CI }\end{array}$ \\
\hline O1 & II.1 & 0.905 & $\begin{array}{c}0.0131 \\
(-25.2 /+35.6 \%)\end{array}$ & $\begin{array}{c}0.0131 \\
(-25.2 /+35.7 \%)\end{array}$ & $\begin{array}{c}0.0131 \\
(-25.5 /+35.0 \%)\end{array}$ & $0.0 \%$ & $-0.3 \%$ \\
\hline O3 & II.2 & 0.495 & $\begin{array}{c}2.97 \\
(-26.2 /+32.3 \%)\end{array}$ & $\begin{array}{c}2.72 \\
(-24.9 /+35.2 \%)\end{array}$ & $\begin{array}{c}2.72 \\
(-24.8 /+35.5 \%)\end{array}$ & $-8.4 \%$ & $+1.8 \%$ \\
\hline O4 & V.5 & 0.787 & $\begin{array}{c}0.289 \\
(-26.0 /+37.2 \%)\end{array}$ & $\begin{array}{c}0.278 \\
(-25.3 /+36.3 \%)\end{array}$ & $\begin{array}{c}0.278 \\
(-25.1 /+36.6 \%)\end{array}$ & $-3.8 \%$ & $-1.5 \%$ \\
\hline V4 & IV.2 & 0.407 & $\begin{array}{c}7.36 \\
(-24.9 /+35.7 \%)\end{array}$ & $\begin{array}{c}6.92 \\
(-25.5 /+36.2 \%)\end{array}$ & $\begin{array}{c}6.90 \\
(-25.3 /+36.5 \%)\end{array}$ & $-6.3 \%$ & $+1.2 \%$ \\
\hline
\end{tabular}

a Time-average of the minimum line transmittance over the sky-LOSA control surface.

b Relative change in mean emission rate when using the MS correction with error model compared to the result without the MS correction.

c Absolute change in the relative $95 \%$ CI when using the MS correction with error model compared to the result without the MS correction.

In general, the MS correction reduced the calculated emission rate since MS serves to decrease total inscattering. The majority of the decrease in the emission rate resulted from the MS correction model $\left(K_{m}=\sqrt{\tau^{*}}\right)$, while consideration of residual errors via the procedure outlined in Section 4.5.2.1 had a comparably minor effect, perturbing only the third and higher significant digits. The observed reduction in emission rate was not consistently correlated with the representative plume transmittance. The lack of correlation between MS effects on plume opacity and emission rate is because the latter is a measure of flux, so that the effect of MS on the quantification of soot column density is weighted by the local plume velocity when performing the integration of Eq. (4.5). This highlights that plume opacity is not in itself a useful measure of the effect of MS on emission rate.

The table also shows that there is a varying effect on the width of the relative $95 \%$ confidence interval $(\mathrm{CI})$ of the mass emission rate when considering MS effects. 
Importantly, despite the inclusion of additional sources of error (i.e., bias and precision errors in the MS correction model), the width of the relative 95\% CI may actually be reduced when MS is considered. This is due to a reduction in the sensitivity of the idealized transmittance to the 1SA-estimated inscattering correction when MS is considered. Referring to Figure C.6, for realistic values of $\tau_{o b s}$ and $S_{1}$, variability in $S_{1}$ has a smaller effect on $\tau^{*}$ when using the MS correction model; alternatively stated, $\partial \tau^{*} / \partial S_{1}$ is reduced when the MS correction model is employed. In the cases of flare $\mathrm{O} 1$ and $\mathrm{O} 4$, use of the MS correction model actually reduces the width of the CI.

There is one additional and important consequence of the MS correction model that was observed during the execution of these case studies. Previously, Conrad and Johnson (Chapter 2, 2017) found that extreme values of $S_{1}$ computed during sky-LOSA MC analyses could yield non-physical results when using the 1SA. Specifically, when using Eq. (4.7) to compute $\tau^{*}$ and forcing $K_{m}=1$ (i.e., when using the 1SA), if $S_{1}>\tau_{o b s}$, then $\tau^{*} \notin(0,1]$ as physically required. By contrast, when using the MS correction model $\left(K_{m}=\right.$ $\left.\sqrt{\tau^{*}}\right), \tau^{*} \in(0,1]$ for all realistic values of $\tau_{o b s}$ and $S_{1}$. Consequently, even for extreme MC samples, the MS correction model always returns a physical result for $\tau^{*}$. This permits the use of MC variance reduction techniques that have been shown to significantly accelerate sky-LOSA processing (Chapter 2, Conrad and Johnson 2017).

Overall, these case studies suggest that the effect of MS on the sky-LOSAcomputed emission rate is generally much less than typical total uncertainties. The MS effect can be negligible as in case study $\mathrm{O} 1$, on the order of one-quarter of typical total uncertainties (as in case study O3), and likely higher under certain conditions with high 
plume opacity. Regardless, these results demonstrate the value in considering MS in the sky-LOSA algorithm using the easily implemented MS correction model.

\subsubsection{Further Considerations}

The present data show that the simple MS correction model has good utility for the purposes of sky-LOSA measurement of soot emissions. The question remains, however, whether the present or an equivalently simple model may have the same utility for optical measurement techniques of other particulate species where inscattering contributes to optical measurements.

Consider the derived form of the MS correction model, $K_{m}=\sqrt{\tau^{*}}$. Referring to the definition of the idealized plume transmittance, this is equivalent to $K_{m}=$ $\exp (-O D / 2)$, where $O D$ is the LOS optical depth of the plume [-]. Equivalently, since $\overline{I_{m}^{s}}=K_{m} \overline{I_{1}^{s}}$, the derived MS correction model implies that, on-average, simulated MC packets scattered into the optical axis must traverse an optical depth of one-half that of the LOS optical depth prior to inscattering. This is a remarkably simple explanation for the present model, but it is only likely to be reasonable for particulate matter with certain optical characteristics.

Combustion-generated soot particulate can reasonably be considered as a strong forward-scatterer and strong absorber within the visible spectrum. This has two important implications with respect to the present model. Firstly, according to the weighting $\omega(\boldsymbol{b})^{n_{s}}$ in the computation of the energy distribution function (Eq. (4.9)), the low albedo of soot implies that higher-order scattering events follow a decreasing geometric function in terms of their contribution to total inscattered energy. In fact, even for the most extreme simulations in the present study $\left(\tau^{*}=0.25\right.$, in the analysis of Figure 4.4$)$, where higher- 
order scattering events are most probable, the total contribution of the third- and higherorder scattering events was less than $0.5 \%$ of the total scattered energy. That is:

$$
\sum_{n_{s} \geq 3} \omega(\boldsymbol{b})^{n_{s}} P\left(n_{s}\right)<0.005 \sum_{n_{s} \geq 1} \omega(\boldsymbol{b})^{n_{s}} P\left(n_{s}\right)
$$

Second, on-average, directional scattering becomes more symmetric after successive scattering events. However, the above-noted effect of the low albedo implies that the originally high asymmetry of single-scattering by soot particulate is preserved to a degree following multiple scattering events by soot. This was observed in Figure 4.3b, where inscattered energy is heavily weighted in the forward direction for both $p_{1}^{\omega}(\theta ; \boldsymbol{b})$ and $p_{m}^{\omega}(\theta ; \boldsymbol{b})$. Thus, even under MS conditions, the majority of inscattered energy is sourced from the region near the LOS, such that the transmittance/optical depth of the LOS is a useful link between $\overline{I_{m}^{S}}$ and $\overline{I_{1}^{S}}$ as the present model suggests.

For particulate matter that is less absorbing than soot, the present model may become invalid since the weighting $\omega(\boldsymbol{b})^{n_{s}}$ decreases less rapidly for higher albedos. Thus, the relative contribution of higher-order scattering events to total inscattered energy will be larger for such particulate than for the case of soot. With this increased importance of higher-order scattering events, $p_{m}^{\omega}(\theta ; \boldsymbol{b})$ is likely to become more symmetric, regardless of the original asymmetry of the scattering phase function. In such a case, the LOS optical depth may become less indicative of the optical depth that simulated MC packets must traverse before inscattering. This same result is likely for small symmetric (Rayleigh) scatterers of any albedo. Consequently, measurement techniques for other, less-absorbing, particulate matter requiring an inscattering correction should consider analogous simulation efforts to quantify the effect of MS on technique accuracy. 


\subsection{Conclusions}

Monte Carlo "ray tracing" simulations of skylight and sunlight scattering through sootladen atmospheric plumes and into a detector along realistic lines-of-sight have shown that the complicated effects of multiple scattering can be accurately modelled for the purposes of optical measurement techniques of soot emissions. Despite the complex structure of turbulent atmospheric plumes, the effect of multiple scattering by soot can be reasonably modelled using a scalar multiple scattering correction parameter $K_{m}$ that relates the inscattered intensity under multiple scattering conditions with an estimate using the singlescattering assumption. Analyses have shown that the correction parameter is largely insensitive to soot properties, and can be treated as a remarkably simple yet accurate function of the idealized plume transmittance along the line-of-sight $\left(\tau^{*}\right), K_{m}=\sqrt{\tau^{*}}$. Use of this simple model brings a second significant advantage in eliminating non-physical results that can occasionally occur when using the single-scattering assumption to compute $\tau^{*}$ during the sky-LOSA Monte Carlo (MC) analysis of measurement uncertainties. This permits the use of MC variance reduction techniques that can significantly accelerate skyLOSA processing (Chapter 2, Conrad and Johnson 2017).

Using the sky-LOSA measurement of soot emissions from gas flares as a case study, simulations demonstrated that the simple multiple scattering model could be used to compute soot mass column densities with bias errors that were typically much less than $\pm 2.6 \%$. Furthermore, derived solar position-dependent bias-correction functions for standard sky models could remove this bias leaving residual $2 \sigma$ precision uncertainties on the order of $4.1 \%$ - approximately one-seventh of typical sky-LOSA measurement uncertainties. Previously published sky-LOSA data were recalculated using this new 
multiple scattering correction model, which as expected slightly reduced the calculated soot emission rate by zero to $8.4 \%$ for these case studies. Importantly, the approach allows multiple scattering to be considered with negligible effect on overall sky-LOSA measurement uncertainties.

\subsection{Acknowledgements}

This work was supported by the Natural Sciences and Engineering Research Council of Canada (NSERC) FlareNet Strategic Network (Grant \#479641), NSERC Discovery Research Grants (Grant \#06632 and 522658), and Natural Resources Canada (Project Manager, Michael Layer). We are grateful for the help of Prof. Philip Smith (University of Utah) in coordinating the sharing of high-fidelity LES data to enable this work. 


\section{Chapter 5 Mass Absorption Cross-Section of Flare- Generated Black Carbon: Variability, Predictive Model, and Implications}

This chapter has been peer-reviewed and was published in the journal Carbon. Only minor clarifications and formatting edits have been made for consistency throughout the thesis. It should be cited as:

Conrad, B.M. and Johnson, M.R. (2019). Mass Absorption Cross-Section of FlareGenerated Black Carbon: Variability, Predictive Model, and Implications. Carbon, 149:760-771 (doi: 10.1016/j.carbon.2019.04.086).

This article is included in its entirety as Chapter 5 with the corresponding supplemental material included as Appendix D. The article was co-authored by the thesis author, Mr. Conrad, and his thesis supervisor, Prof. Johnson. Mr. Conrad and Prof. Johnson conceptualized the research and developed the methodology. Mr. Conrad performed the investigation, validation, and formal analysis. Mr. Conrad wrote the first draft of the manuscript; editing and revision of the manuscript were conducted jointly.

\subsection{Abstract}

Global gas flaring is an important source of black carbon (BC) emissions with uncertain climate impacts. The link between atmospheric concentration and direct radiative forcing (DRF) by BC is its mass absorption cross-section (MAC). MAC data for flare-generated $\mathrm{BC}$ is lacking in the literature and the only known data conflicts with generally accepted $\mathrm{BC}$ MAC values, which are assumed to be source-independent. This paper presents the first measurements of $\mathrm{BC}$ MAC for large-scale flares, burning globally representative industry-relevant flare gas compositions in a controlled facility. BC MAC was calculated with quantified uncertainties using photoacoustic and thermal-optical instruments. Flaregenerated carbon was found to be primarily elemental in composition (typically $>92 \%$ ), 
and most probably externally mixed based on detailed analysis of attenuation vs. evolved carbon data and consideration of flare-specific mechanisms for organic carbon emissions. Flare BC MAC was generally larger than well-cited literature values and had statistically significant variations with fuel and operating conditions. Variability in BC MAC was wellpredicted by a novel phenomenological model based on flame radiative characteristics and relative $\mathrm{BC}$ production. The derived model consolidates previously unreconciled disparate data from different sources and suggests that flare BC MAC is likely $>1.3-2.0$ times standard values, implying an underestimation of DRF by flare-generated BC.

\subsection{Introduction}

Combustion-generated particulate black carbon $(\mathrm{BC})$ is an atmospheric pollutant composed of nano-scale carbon spherules that aggregate into fractal-like structures through diffusion-limited processes in hydrocarbon flames. BC may be the second-most important climate forcer after carbon dioxide (Bond et al. 2013; Ramanathan and Carmichael 2008; Sato et al. 2003; Jacobson 2001) due to its strong broadband absorption. The mass absorption cross-section (MAC) of $\mathrm{BC}$ defines the characteristic link between its atmospheric concentrations and climate impacts. MAC is a spectral quantity relating the absorptive efficiency of a particle per unit mass. When multiplied by the path-integrated mass concentration of a particle, MAC yields the observed absorption at a specific wavelength of light. Critically, direct radiative climate forcing therefore scales with the MAC of atmospheric particles (Kondo 2015; Bond and Bergstrom 2006).

One important source of atmospheric BC is gas flaring within the oil and gas industry. Gas flaring is the practice of combustion-based disposal of unwanted gases that are deemed uneconomical to preserve for market. Estimates based on satellite imagery 
suggest that global flared volumes typically exceed 140 billion $\mathrm{m}^{3}$ per year (Elvidge et al. $2007,2009,2015)$, approximately $90 \%$ of which is associated with upstream exploration, completion, and production (Elvidge et al. 2015). In the upstream industry, flares are almost exclusively simple vertical pipe flares, creating large-scale, buoyancy-driven, turbulent, diffusion flames in air at atmospheric pressure. Typical flared gases are mostly alkane hydrocarbons with small amounts of alkenes, naphthenes, aromatics, and hydrogen sulphide, and can be diluted significantly with nitrogen and carbon dioxide (Chapter 2, Conrad and Johnson 2017; Johnson and Coderre 2012). Climate impacts of BC are sensitive to the geographic location of the source, which precludes an accurate and consistent global warming potential for the pollutant (U.S. EPA 2012); however, it is known that $\mathrm{BC}$ emitted and deposited at northern latitudes can induce disproportionately strong warming through the reduction of snow and ice albedo (Flanner et al. 2007; Clarke and Noone 1985). For this reason, flaring activities in the Ural Federal District of Russia are particularly concerning, as wintertime emissions are efficiently transported over the Arctic (Law and Stohl 2007; Stohl 2006), likely contributing to the Arctic haze phenomenon (Huang et al. 2014, 2015) and ultimately depositing on the Arctic snow and ice pack.

Despite the magnitude and climatic importance of flare-generated $\mathrm{BC}$, targeted studies of flare-generated BC MAC do not currently exist. Recent work by Weyant et al. (2016), who performed estimates of flare BC emission factor in the Bakken region of North Dakota, were able to yield estimates of $\mathrm{BC}$ MAC as a secondary result of their work; however, uncertainties were large and the authors noted their results were much greater than generally accepted values in the literature. There remains a notable knowledge gap in 
flare-generated BC MAC and how it may differ from values of other important BC sources within the literature. This adds important uncertainty to climate models and impact assessments, especially in the Arctic.

This work presents the first targeted measurements of $\mathrm{BC}$ MAC from flames representative of gas flaring in the oil and gas industry. Experiments were performed on large-scale, buoyancy-driven, turbulent non-premixed flames burning a range of globally representative, industry-relevant flare gas compositions in a controlled facility in Ottawa, Canada. Diluted combustion products were captured and directed to a diagnostic instrument suite and then exhausted from the laboratory. Parallel thermal-optical and photoacoustic analyses were performed to obtain the carbon particulate mass concentration in the diluted products as well as the observed absorption at three wavelengths spanning the visible spectrum. These data enabled quantification of the spectral BC MAC specific to controlled flare gas compositions and flaring conditions. Instrumentation capabilities were robustly characterized via Monte Carlo analysis to provide results with carefully computed uncertainties. Observed variability of BC MAC is analyzed in the context of flaring conditions, enabling the creation of a predictive model of $\mathrm{BC} \mathrm{MAC}$ as a function of readily available flare parameters. Implications of the derived model on the climate impact of flare BC are discussed.

\subsection{Mass Absorption Cross-Section of Black Carbon}

A wide range of MAC data from myriad combustion sources exists within the literature. In the context of freshly-emitted $\mathrm{BC}$ however, which is the focus of the current study, the most widely-cited value is from the literature review of Bond and Bergstrom (2006), who suggest a consistent (over all relevant combustion sources) MAC for fresh $\mathrm{BC}$ of 
$7.5 \pm 1.2 \mathrm{~m}^{2} / \mathrm{g}(1 \sigma)$ at $550 \mathrm{~nm}$. Their data are sourced from reported measurements of diesel-and spark-ignition engines, ambient particulate, carbon black, and other combustion aerosols. Despite the assertion that a consistent MAC for all BC sources exists - justified on the notion that measurement uncertainties typically exceed the range of observed variations in MAC - Bond and Bergstrom (2006) also comment that the morphology and molecular structure of $\mathrm{BC}$ affects its optical properties. Regarding the latter, incipient $\mathrm{BC}$ particles that are amorphous in structure experience a transformation in nanostructure due to elevated temperatures within the flame. Amorphous carbon is graphitized when $\mathrm{sp}^{3}$ hybridized carbons become $\mathrm{sp}^{2}$-hybridized. This yields the familiar onion-like structure of spherical BC monomers (Bond and Bergstrom 2006) in which the fringes of graphene layers are visible in high-resolution electron microscopy (e.g., Buseck et al. 2014; Rinkenburger et al. 2017). Through conjugation of the carbon structure and associated delocalization of electrons, the creation of $\mathrm{sp}^{2}$-bonded carbons tends to increase broadband light absorption (Andreae and Gelencsér 2006). This effect implies an increase in MAC with a higher degree of graphitization; hence, it is logical to expect that the timetemperature history of $\mathrm{BC}$ affects its MAC. This may have an important implication for flare BC, where flames can be orders of magnitude larger than other combustion sources, particularly engine and laboratory flames. This hints at the potential for a different MAC of flare BC compared to Bond and Bergstrom's widely cited value.

It is worth noting that a range of recent research has sought to improve the understanding of $\mathrm{BC} \mathrm{MAC}$ with insights into the effect of atmospheric processing on $\mathrm{BC}$ MAC (e.g., Boucher et al. 2016; Gustafsson and Ramanathan 2016; Knox et al. 2009; Nordmann et al. 2013; Peng et al. 2016; You et al. 2016; Zanatta et al. 2018; Zhang et al. 
2016) and the relation of BC optical properties with particle morphology and composition (e.g., Dastanpour et al. 2017; Forestieri et al. 2018; Radney et al. 2014; Török et al. 2018). Additionally, the development, improvement, and inter-comparison of techniques to measure $\mathrm{BC}$ optical and morphological properties is another area of active research (e.g., Bescond et al. 2014; Giannoni et al. 2016; Lappi and Ristimäki 2017; Nicolosi et al. 2018; Panteliadis et al. 2015; Petzold et al. 2013a; Presler-Jur et al. 2017). Still, most contemporary studies tend to use Bond and Bergstrom's (2006) MAC value as a reference or baseline.

Rayleigh-Debye-Gans theory for Fractal Aggregates (RDG-FA, summarized rigorously by Sorensen (2001)) provides important insights into the spectral variation of MAC for freshly-emitted $\mathrm{BC}$ particles. Among other assumptions, if the $\mathrm{BC}$ aggregates are sufficiently open in structure (fractal dimension $<2.0$ (Berry and Percival 1986)), RDG-FA suggests that absorption by an aggregate is simply the sum of absorption by the monomers composing the aggregate (Dobbins and Megaridis 1991). At typical diagnostic wavelengths, fresh $\mathrm{BC}$ monomers generally exist within the small-particle limit known as the Rayleigh regime (see Section D.2), where the amplitude and phase of incident light is approximately uniform over the monomer. In this case, BC MAC is predicted by RDG-FA as:

$$
M A C_{B C, \lambda}=\frac{6 \pi}{\varrho_{B C}} \frac{E\left(m_{\lambda}\right)}{\lambda}
$$

where $\varrho_{B C}$ is the material density of $\mathrm{BC}\left[\mathrm{kg} / \mathrm{m}^{3}\right]$ and $E\left(m_{\lambda}\right)$ is the absorptive function of the spectral refractive index $\left(m_{\lambda}\right)[-]$. If the spectral refractive index is assumed to be constant, this formulation suggests that $\mathrm{BC}$ MAC should have an inverse wavelength 
dependence $\left(M A C_{B C, \lambda} \propto \lambda^{-1}\right)$ within the Rayleigh regime. However, the assumption of a spectrally constant refractive index is not necessarily true; moreover, at shorter wavelengths within the climate-critical spectrum of solar radiation, larger $\mathrm{BC}$ monomer sizes can exceed the nominal threshold for the Rayleigh regime such that an inverse wavelength dependence of MAC may not be observed. For these reasons, it is common instead to define a power-law relation for the spectral variation of MAC applicable over some spectrum:

$$
M A C_{B C, \lambda}=M A C_{B C, \lambda_{o}}\left(\frac{\lambda}{\lambda_{o}}\right)^{-A \AA \AA E}
$$

where $\lambda_{o}$ indicates a reference wavelength [m] and the dimensionless power-law exponent $(A \AA ̊ E)$ is known as the absorption Ångström exponent (Schuster et al. 2016; Ångström 1929), which, for BC, is generally close to unity based on the Rayleigh limit.

\subsection{Experimental Method}

\subsubsection{Flare Facility}

Experiments were conducted at the Carleton University Flare Facility (CUFF, Figure 5.1) in Ottawa, Canada, at which custom gas mixtures representative of gas flaring in the oil and gas industry can be created and combusted at volume flow rates of up to 400 SLPM (standard litres per minute, defined at $0^{\circ} \mathrm{C}$ and $1 \mathrm{~atm}$ ). Flare gas mixtures contained up to nine components from eleven different species including C1-C7 alkanes, $\mathrm{C} 2$ and $\mathrm{C} 3$ alkenes, nitrogen, and carbon dioxide $\left(\mathrm{CO}_{2}\right)$. Gas-phase species (as metered) were delivered from pressurized cylinders, re-warmed in a $\sim 27^{\circ} \mathrm{C}$ copper-coiled water bath following Joule-Thomson cooling through the pressure regulators, and then precisely flowcontrolled using thermal mass flow controllers (Brooks and Bronkhorst, various models). 
Liquid-phase species (as metered) were flow-controlled using Coriolis flow controllers (Bronkhorst, various models) and forwarded with methane carrier gas to a controlled evaporator and mixer (Bronkhorst, CEM W-202A). All combustibles were combined and forwarded to a vertically oriented flare stack $(38.1 \mathrm{~mm}, 50.8 \mathrm{~mm}$, or $76.2 \mathrm{~mm}$ internal diameter), creating buoyancy-driven, turbulent, diffusion flames as large as $\sim 3 \mathrm{~m}$ in length. Combustion products diluted with ambient air were captured in a hexagonal fume hood (3.1 m included diameter) and exhausted through a $40 \mathrm{~cm}$ diameter insulated duct. From the exhaust duct, diluted combustion products were extracted and forwarded through a flow-controlled dilution tunnel to the CUFF instrument suite. 


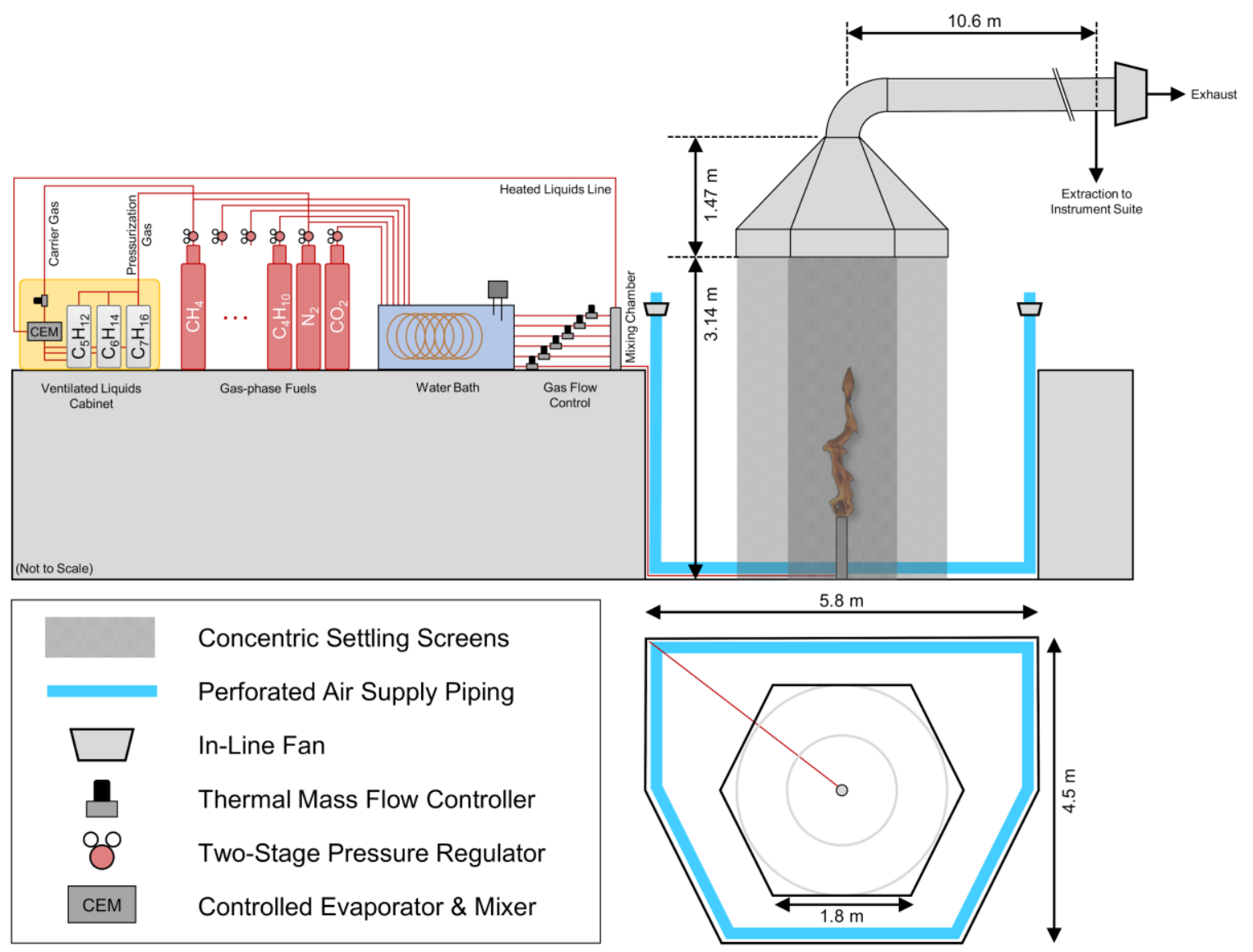

Figure 5.1: Schematic of the Carleton University Flare Facility (CUFF). Flare gas mixtures including $\mathrm{C} 1-\mathrm{C} 7$ alkanes, $\mathrm{C} 2$ and $\mathrm{C} 3$ alkenes, nitrogen, and carbon dioxide are mixed to desired volume fractions and sent to the flare stack. Combustion products and diluent air are captured in a $3.1 \mathrm{~m}$ diameter hexagonal hood and exhausted. Metered volumes of diluted combustion products are sampled from the duct (with optional further dilution) into the CUFF instrument suite.

\subsubsection{Aerosol Sampling and Dilution}

To ensure consistent samples between instruments, a custom aerosol sampling and dilution tunnel was used (Figure 5.2). Combustion products were drawn into the sampling tunnel through 1" nominal diameter stainless steel tubing by a $1 \mathrm{hp}$ vacuum pump. Pressurized, filtered dilution air was optionally introduced upstream of the sampling point to avoid overloading instrumentation in experiments with high concentrations of emitted particulate. This dilution air was injected at a controlled rate approximately 56 diameters upstream of the dilution tunnel using a mass flow controller (MFC, Bronkhorst model EL- 
FLOW Select) and allowed to fully mix with the sampled combustion products. Particulate measurement instruments (each equipped with an appropriately-sized vacuum pump) drew diluted samples from the centreline of the sampling tunnel via $1 / 4$ " stainless steel tubing and exhausted the samples upstream of an identical MFC which controlled the total flow rate of combustion products and dilution air through the sampling tunnel.

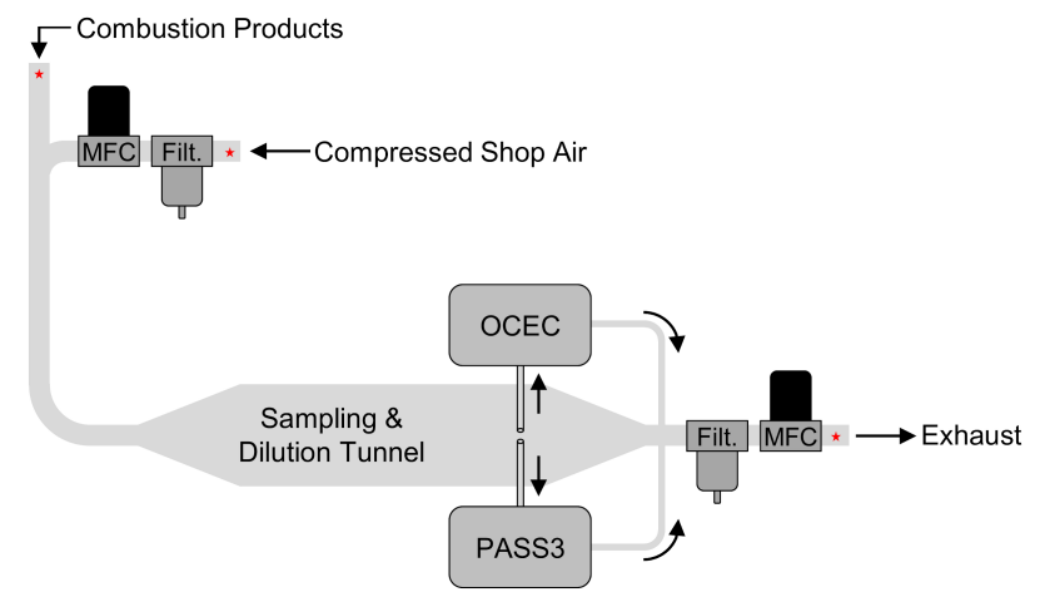

Figure 5.2: Schematic of the aerosol sampling and dilution tunnel. Combustion products are drawn into the tunnel with a metered rate of filtered dilution air (as necessary). Combustion products fully mix with metered dilution air prior to sampling by instrumentation, which returns sampled volumes to the downstream side of the dilution tunnel, just upstream of the mass flow controller (MFC) controlling total flow rate. Red stars in the figure correspond to sample locations for the quantification of dilution in the sampling tunnel.

The degree of dilution was measured to correct for background organic carbon. While not explicitly required for the calculation of $\mathrm{BC} \mathrm{MAC}$, this correction enables the analysis of the carbon content of co-emitted species from the studied flares and, ultimately, the operational-definition of $\mathrm{BC}$ in this study (see further discussion in Section 5.5.1). The dilution factor - i.e., the ratio of total exhaust to combustion product mass - is dependent upon the controlled flow rates and was quantified using a $\mathrm{CO}_{2}$-balance approach. An optical $\mathrm{CO}_{2}$ analyzer (LI-COR model LI-850) intermittently sampled from the three 
locations indicated by red stars in Figure 5.2, which were sufficient to calculate the dilution factor and associated uncertainties as further detailed in Section D.4.5.

\subsubsection{Flare Gas Compositions}

Flare gas compositions in this work are summarized in Table 5.1. These multi-component mixtures were derived from globally diverse field-measurements of flared gas in the oil and gas industry (see Section D.3). This experimental domain included 15 unique fuels: light, median, and heavy mixtures for production sites in Alberta, Canada (AB-L, -M, and -H); two mean compositions for the Bakken region (BK-1 (Brandt et al. 2016) and BK-2 (Wocken et al. 2013), respectively); four compositions from oil facilities in the Ecuadorean Amazon (Chapter 2, Conrad and Johnson 2017) (EC-O1 through EC-O4); a representative average of 20 offshore wells in the Norwegian North Sea (NS-A); two as-received compositions at gas processing plants in Russia's Khanty-Mansi Autonomous Okrug averaged over annual measurements from 2012-2017 (RU-G1 and RU-G2); and a representative composition of flared gas from three separation stages at an anonymous Russian oil well (Filippov 2013) using the weighting scheme of Huang et al. (2015) (RUO1). Also studied in this work were two additional compositions designed to probe the extremes of the composition domain: a crude propylene mixture (X-CP, $80 \%$ propylene and 20\% propane) employed in the seminal flare efficiency study of McDaniel (1983), and pure ethylene (X-C2). Although these mixtures would not be expected at upstream oil and gas production sites, they are relevant to emergency flares at downstream petrochemical facilities. 
Table 5.1: Summary of flare gas compositions in volume fraction (\%) studied in this experiment and their molecular weight (MW), volumetric higher heating value (HHVv), and molar carbon-hydrogen ratio (CHR). Bold values indicate the inclusion of alkenes (ethylene and propylene).

\begin{tabular}{|c|c|c|c|c|c|c|c|c|c|c|c|c|c|}
\hline Jurisdiction & Designator & C1 & $\mathrm{C2}$ & C3 & nC4 & iC5 & nC6 & nC7 & $\mathbf{N}_{2}$ & $\mathrm{CO}_{2}$ & $\begin{array}{c}\text { MW } \\
\text { [g/mol] }\end{array}$ & $\begin{array}{c}\mathrm{HHVV}_{\mathbf{V}} \\
{\left[\mathrm{MJ} / \mathrm{m}^{3}\right]}\end{array}$ & $\begin{array}{c}\text { CHR } \\
{[-]}\end{array}$ \\
\hline \multirow{3}{*}{$\begin{array}{l}\text { Alberta, } \\
\text { Canada }\end{array}$} & $\mathrm{AB}-\mathrm{L}^{\mathrm{a}}$ & 92.39 & 0.32 & 0.09 & 0.40 & 0.06 & 0.03 & 0.03 & 1.54 & 5.16 & 17.98 & 37.73 & 0.253 \\
\hline & $\mathrm{AB}-\mathrm{M}^{\mathrm{a}}$ & 86.36 & 6.83 & 2.36 & 0.97 & 0.33 & 0.13 & 0.18 & 1.62 & 1.22 & 19.03 & 43.81 & 0.271 \\
\hline & $\mathrm{AB}-\mathrm{H}^{\mathrm{a}}$ & 75.68 & 11.52 & 6.06 & 2.60 & 0.78 & 0.23 & 0.21 & 1.70 & 1.22 & 21.77 & 49.54 & 0.290 \\
\hline \multirow{2}{*}{$\begin{array}{l}\text { Bakken, } \\
\text { ND }\end{array}$} & $\mathrm{BK}-1^{\mathrm{b}}$ & 49.12 & 20.97 & 15.04 & 6.72 & 2.15 & 0.87 & 0.78 & 3.66 & 0.70 & 29.13 & 64.33 & 0.329 \\
\hline & $\mathrm{BK}-2^{\mathrm{c}}$ & 57.78 & 19.97 & 11.35 & 3.81 & 0.93 & 0.22 & 0.15 & 5.22 & 0.57 & 25.22 & 55.19 & 0.310 \\
\hline \multirow{4}{*}{$\begin{array}{l}\text { Orellana, } \\
\text { Ecuador }\end{array}$} & $\mathrm{EC}-\mathrm{O} 1^{\mathrm{d}}$ & 47.26 & 7.98 & 11.32 & 6.91 & 3.01 & 1.19 & 1.51 & 8.18 & 12.65 & 31.55 & 54.64 & 0.333 \\
\hline & $\mathrm{EC}-\mathrm{O} 2^{\mathrm{d}}$ & 58.89 & 7.80 & 9.29 & 5.19 & 2.18 & 0.89 & 0.81 & 5.75 & 9.20 & 27.71 & 51.54 & 0.315 \\
\hline & $\mathrm{EC}^{-O} 3^{\mathrm{d}}$ & 40.82 & 8.16 & 16.93 & 14.75 & 6.81 & 2.06 & 1.38 & 3.37 & 5.71 & 36.57 & 75.17 & 0.355 \\
\hline & $\mathrm{EC}^{-O} 4^{\mathrm{d}}$ & 55.22 & 7.63 & 11.65 & 8.79 & 3.95 & 1.37 & 0.94 & 4.12 & 6.31 & 30.31 & 60.92 & 0.331 \\
\hline North Sea & NS-A ${ }^{\mathrm{e}}$ & 82.48 & 8.09 & 4.06 & 1.53 & 0.40 & 0.16 & 0.15 & 1.43 & 1.70 & 20.07 & 45.65 & 0.278 \\
\hline \multirow{3}{*}{ Russia } & RU-G1f & 73.96 & 3.08 & 7.87 & 6.79 & 2.35 & 0.63 & 0.57 & 1.90 & 2.85 & 24.80 & 54.15 & 0.306 \\
\hline & RU-G $2^{f}$ & 77.05 & 5.29 & 8.24 & 4.28 & 1.24 & 0.30 & 0.27 & 1.56 & 1.76 & 22.72 & 51.07 & 0.295 \\
\hline & $\mathrm{RU}^{-O 1^{\mathrm{g}}}$ & 51.90 & 9.81 & 16.90 & 10.27 & 4.68 & 2.93 & 2.40 & 0.95 & 0.15 & 33.34 & 75.43 & 0.345 \\
\hline \multirow{2}{*}{ Experimental } & $\mathrm{X}-\mathrm{CP}^{\mathrm{h}}$ & 0 & 0 & 100 & 0 & 0 & 0 & 0 & 0 & 0 & 42.48 & 93.27 & 0.469 \\
\hline & $\mathrm{X}-\mathrm{C} 2^{\mathrm{i}}$ & 0 & 100 & 0 & 0 & 0 & 0 & 0 & 0 & 0 & 28.05 & 62.96 & 0.500 \\
\hline
\end{tabular}

a Light, median, and heavy flare gas compositions derived from 2016 Alberta Energy Regulator data.

Mean flare gas composition from $\sim 700$ reporting wells in the Bakken region (Brandt et al. 2016).

Assumed flare gas composition for the Bakken region based on seven reported wellhead samples (Wocken et al. 2013).

Measured gas composition at upstream facilities in Ecuador's Orellana province (Chapter 2, Conrad and Johnson 2017).

Average flare gas composition from 20 platforms in the Norwegian North Sea.

Gas composition as received at gas processing plants from two anonymous fields in Russia's Khanty-Mansi Autonomous Okrug, averaged over six years.

$\mathrm{g}$ Mean separation stage-weighted flare gas composition from an anonymous Russian oil well (Huang et al. 2015; Filippov 2013).

h "Crude propylene" composition from seminal experimental work (McDaniel 1983) cited in US EPA emission factors (U.S. EPA 2018) consisting of 80\% propylene, 20\% propane. Pure ethylene. 
Previous measurements of turbulent diffusion flames have shown that BC production is positively correlated with both the energy content of the fuel (represented by the volumetric higher heating value, $\mathrm{HHV}_{\mathrm{V}}$ ) (Chapter 2, Conrad and Johnson 2017; McEwen and Johnson 2012) and the fuel's degree of unsaturation (e.g., Glassman 1989), which can be parameterized with the molar carbon-hydrogen ratio, CHR). Both strongly influence the chemical pathways of soot formation and thus have potential to affect the nanostructure of emitted particulate that drives light absorption. Figure 5.3 plots the studied flare gas compositions on a $\mathrm{HHV}_{\mathrm{V}}-\mathrm{CHR}$ domain. In general, the industry-relevant compositions from around the globe appear to lie within a narrow region of this domain. However, typical gas chromatography standards used to quantify flare gas composition often do not resolve unsaturated species like alkenes (Curiale and Frolov 1998), such that the true spread could be broader. Nonetheless, the four samples from production sites in Ecuador (which used a high-resolution standard capable of resolving less-saturated components (Chapter 2, Conrad and Johnson 2017) and the additional outlying experimental compositions (alkene dominated X-CP and $\mathrm{X}-\mathrm{C} 2$ ) suggest possible bounds. 


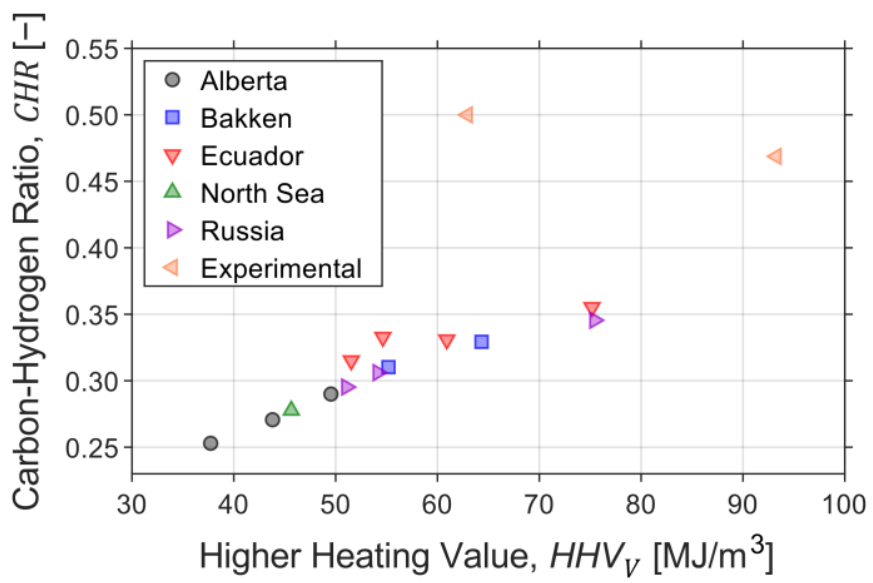

Figure 5.3: Studied flare gas compositions shown in the HHVv-CHR domain. Fuels from Ecuador are observed to have a modestly larger CHR than other fieldmeasured compositions due to the high-resolution analysis performed.

Experimental composition (X-CP at top right, and X-C2) deviate largely from fieldobserved compositions, offering an extreme perspective on the effect of fuel composition on flare BC MAC.

\subsubsection{Test Matrix}

As summarized in Table 5.2, 35 measurements were performed comprising 15 flare gas compositions from flare stacks of $38.1,50.8$, and $76.2 \mathrm{~mm}$ in internal diameter at volumetric flow rates of $96,156,216$, and 400 SLPM. The test matrix was designed to isolate the effects of flare gas composition on $\mathrm{BC}$ MAC at the nominal flaring condition of 156 SLPM from the $50.8 \mathrm{~mm}$ flare stack, and similarly to isolate the effects of flare aerodynamics using the median fuel from the Alberta, Canada jurisdiction (AB-M). Additionally, extreme conditions of flow rate and diameter (strongly impacting flame length and hence residence time) were performed for the RU-O1, AB-L, and AB-M compositions. Assessment of experimental repeatability was performed for the AB-M composition at the nominal condition where a total of six independent tests were performed. Combined data from these tests enabled evaluation of $\mathrm{BC} \mathrm{MAC}$ as a function of various fuel chemistry and flare aerodynamic parameters. 
Table 5.2: Test matrix of the current study indicating the 35 measurements performed. Analysis of experimental repeatability was executed for the AB-M composition at 156 SLPM using the $50.8 \mathrm{~mm}$ diameter stack where a total of six measurements were performed.

\begin{tabular}{|c|c|c|c|c|c|c|c|c|c|c|}
\hline $\begin{array}{c}\text { Stack Diameter } \\
\text { [mm] }\end{array}$ & \multicolumn{3}{|c|}{38.1} & \multicolumn{5}{c|}{50.8} & \multicolumn{3}{c|}{76.2} \\
\hline $\begin{array}{c}\text { Std. Flow Rate } \\
\text { [SLPM] }\end{array}$ & $\mathbf{9 6}$ & $\mathbf{1 5 6}$ & $\mathbf{2 1 6}$ & $\mathbf{9 6}$ & $\mathbf{1 5 6}$ & $\mathbf{2 1 6}$ & $\mathbf{4 0 0}$ & $\mathbf{9 6}$ & $\mathbf{1 5 6}$ & $\mathbf{2 1 6}$ \\
\hline AB-L & & $\mathrm{X}$ & & & $\mathrm{X}$ & & & & & X \\
\hline AB-M & $\mathrm{X}$ & $\mathrm{X}$ & $\mathrm{X}$ & $\mathrm{X}$ & $\mathrm{X}^{\mathrm{c}}$ & $\mathrm{X}$ & $\mathrm{X}$ & $\mathrm{X}$ & $\mathrm{X}$ & $\mathrm{X}$ \\
\hline RU-O1 & $\mathrm{X}$ & & & & $\mathrm{X}$ & & & & & X \\
\hline X-CP & & & & $\mathrm{X}$ & $\mathrm{X}$ & & & & & \\
\hline X-C2 & & & & $\mathrm{X}$ & $\mathrm{X}$ & & & & & \\
\hline All Other & & & & & X & & & & & \\
\hline
\end{tabular}

a Nominal average flare condition used to assess composition effects.

b AB-M composition used to assess flare aerodynamic effects.

c Condition/composition used to quantify experimental repeatability.

\subsubsection{MAC Determination and Data Reduction}

Wavelength-dependent $\mathrm{BC}$ MAC data were derived by combining measurements from two instruments operating in parallel. Firstly, a three-wavelength photoacoustic spectrometer (Droplet Measurement Technologies model PASS3) was used to quantify optical absorption coefficients at three wavelengths spanning the visible spectrum $-405,532$, and $870 \mathrm{~nm}$. Additionally, a semi-continuous thermal-optical organic/elemental carbon analyzer (OCEC, Sunset Laboratories model 4) was used to quantify the mass concentration of organic carbon (OC) and elemental carbon (EC) in the sample. For each condition, the OCEC instrument pulled a sample for up to 15 minutes after the flame had stabilized. A high-accuracy mass flow meter (Bronkhorst model EL-FLOW Prestige) was used to quantify the standard volume of the sample $\left(V_{o}\left[\mathrm{~m}^{3}\right]\right)$, which, when coupled with the carbon mass measured by the OCEC instrument $\left(m_{C}[\mathrm{~g}]\right)$, yielded a sample's mean carbon mass concentration. During the OCEC sampling period, the PASS3 instrument 
made time-resolved measurements of the absorption coefficient of the sample in its cell via the photoacoustic effect. Temperature and pressure data reported by the PASS3 were used to calculate the absorption coefficient on a standard volume basis, and the mean coefficient was computed over the OCEC sampling period, yielding the spectrally-resolved, timeaveraged, standard absorption coefficient $\left(\overline{\alpha_{\lambda, o}}\left[\mathrm{~m}^{-1}\right]\right)$. MAC of the fresh, flare-generated $\mathrm{BC}$ was then:

$$
M A C_{B C, \lambda}=\frac{\overline{\alpha_{\lambda, o}} V_{o}}{m_{C}}
$$

Measurement uncertainties were rigorously quantified using a comprehensive Monte Carlo (MC) analysis in which errors due to instrument calibration ( $\sim 4.5$ and $\sim 8.42 \%$ $(2 \sigma)$ on average for the PASS3 and OCEC, respectively) and overall experiment precision/repeatability $(\sim 8.7 \%)$ were propagated into the calculated BC MAC. Typical overall uncertainty in the measured BC MAC $(2 \sigma)$ was $\sim 13.0 \%$. A detailed description of employed calibration approaches and implementation of the MC analysis is provided in Section D.4.

\subsection{Results}

\subsubsection{Carbon Content}

Several different nomenclatures for light-absorbing carbonaceous particulate exist in the literature. These are broadly attributable to differences in measurement diagnostics; variations in particle nanostructure, morphology, solubility, and thermal stability; and distinctions in light attenuation properties (Petzold et al. 2013a). Throughout the literature, there is an unfortunate lack of consistency in the definition of $\mathrm{BC}$, hampering the comparability of studies (Bond et al. 2013). Consequently, it is prudent to define the 
specific definition of $\mathrm{BC}$ used in this work, based on characteristics of the experimental data.

Integral to the measurement of $\mathrm{BC}$ absorption is the understanding of the mixing state of co-emitted OC and EC - i.e., referring to Figure 2 in Section 3.4 of Bond and Bergstrom's (2006) review, whether OC and EC exist as an external mixture, heterogeneous internal mixture (coated particulate), or homogeneous internal mixture. In the current work, to avoid losses of particulate in the sampling system (especially sizedependent losses), a denuder or catalytic stripper was not employed. Thus, sampled carbonaceous combustion products consist of vapour-phase OC and aerosols (EC and potentially condensed OC) freshly emitted from the flame. The nature of the OC-EC mixing state was evaluated from analysis of the OCEC thermograms using attenuation versus evolved carbon plots (AVEC plots, conceived by Nicolosi et al. (2018)). The AVEC plots of all measurements showed a notable feature - laser attenuation through the OCEC filter was approximately constant during the evolution of OC (see Figure D.4 in Section D.4.4.1). Conceptually, this observation could be a consequence of one of two phenomena:

1) $\mathrm{OC}$ is internally mixed with $\mathrm{EC}$ and any increase in attenuation due to pyrolization of $\mathrm{OC}$ is exactly counteracted by a decrease in attenuation due to removal of OC that coats EC; or

2) OC is externally mixed in the samples and is composed of non-pyrolyzing species.

The latter phenomenon is most probable. Firstly, it is unlikely that the attenuation increase from pyrolization and attenuation decrease from OC coating removal (if present) would perfectly counteract during the inert phase of the thermal analysis, since the former is temperature-dependent and both are non-linear with coating mass (e.g., Nicolosi et al. 
2018; Bond et al. 2006). In contrast, previous observations of non-pyrolyzing organic species (e.g., Chow et al. 2001; Khan et al. 2012; Lappi and Ristimäki 2017; Schauer et al. 2003) exist and are largely attributed to the fact that some OC simply does not pyrolyze. In particular, Lappi and Ristimäki (2017) note that "when [particulate matter] is low in impurities, such as in distillates and hydrocarbon fuels ... pyrolysis is low or negligible" while Khan et al. (2012) suggest that some "OC compounds ... appear less amenable to charring" and specifically that "[polyaromatic hydrocarbons] ... do not pyrolyze much". Coupling these observations with the discussion below that measured OC is expected to mostly be unburned fuel (aliphatic, low molecular weight hydrocarbons), it is believed that OC and EC are most likely externally mixed within the present samples. This is also consistent with field observations (Schwarz et al. 2015) of flare plumes and previous electron microscopy images of BC from the CUFF (Kazemimanesh et al. 2019).

Since OC is negligibly absorbing, the above inference of the likely mixing state suggests that PASS3-measured absorption is due strictly to externally mixed EC particulate. Consequently, fresh $\mathrm{BC}$ in this work is defined as the strongly light absorbing, externally mixed EC, and the carbon mass used in Eq. (5.3) is therefore the elemental component. Based on the literature, the current results may thus be called "light absorbing carbon" (Bond and Bergstrom 2006), "soot carbon" (Andreae and Gelencsér 2006), or "equivalent BC" (Andreae and Gelencsér 2006), but nonetheless the current definition is consistent with BC properties noted by both Bond et al. (2013) and Petzold et al. (2013b).

The OCEC-measured carbon content for the flares in this study are shown in Figure 5.4a. EC to total carbon (TC) ratio (EC/TC) is plotted as a function of CHR with symbol colouring representing fuel flow rate and symbol shape representing stack diameter. These 
data show that EC/TC appears to be quite well-captured by the CHR of the fuel, where $\mathrm{EC} / \mathrm{TC}$ increases with degree of unsaturation (corresponding to higher CHRs). This is likely a consequence of the enhanced chemical pathways for pyrolysis of heavier fuels relative to lighter fuels - i.e., $\mathrm{BC}$ precursors are more abundantly present in flames of heavier fuels, such that carbon-based intermediate species are more likely to create $\mathrm{BC}$ rather than escape the flame as OC. Contrastingly, fuels with low carbon-hydrogen ratios, which inherently have a low sooting propensity, generally have a low EC/TC since BC precursors are less likely to be produced via the pyrolysis chemical mechanism.
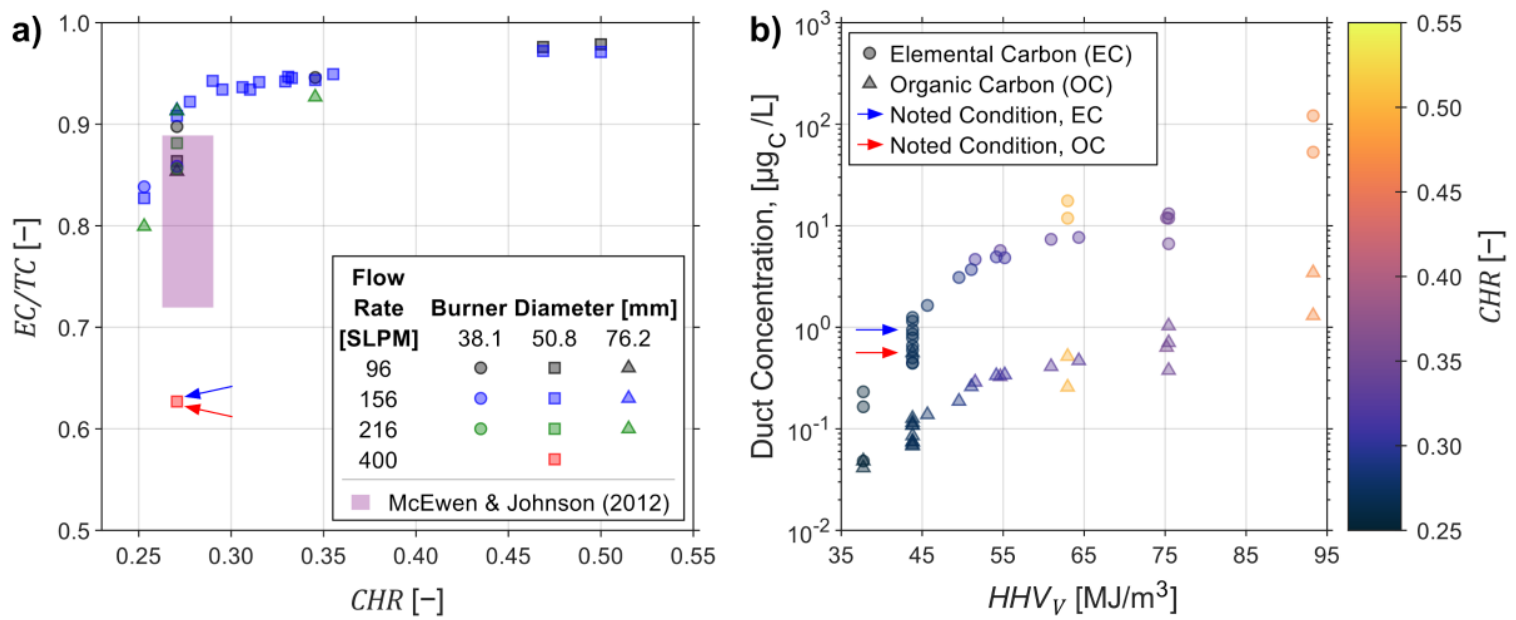

Figure 5.4: (a) Elemental carbon to total carbon (EC/TC) ratio in the flare combustion products where shaded area indicates previous estimates from McEwen

\& Johnson based on LII and gravimetric measurements (McEwen and Johnson 2012). (b) Raw concentrations of $E C$ and $O C$ in the collected product plume showing consistent trends of EC concentrations with flare gas heating value. Arrows identify the condition noted in text with higher relative OC likely attributable to the onset of flame liftoff.

Also of note in Figure 5.4a is that there appears to be a critical value of CHR below which EC/TC decreases quite rapidly. This is most likely a consequence of the onset of flame instability, near the point where velocity of the fuel jet at the stack exit is sufficient to initiate flame liftoff. In the classic model of Kalghatgi (1984), this effect is dependent 
on the premixed flame speed which tends to correlate well with adiabatic flame temperature $\left(T_{a d}\right)$ for a fixed family of hydrocarbons (e.g. alkanes). Importantly, over the range of fuels relevant to oil and gas flaring studied in this work, CHR is also highly correlated with $T_{a d}$ (correlation coefficient of $\sim 0.95$ ), such that CHR is a reasonable surrogate for flame speed over the experimental range of fuels. Thus, as the CHR reduces beyond approximately 0.28 , it is likely that the studied flares begin to become unstable, especially at higher stack exit velocities. At the onset of instability, local extinctions begin to occur at the base of the flame (Takahashi and Schmoll 1991), resulting in a reduction of combustion efficiency and an associated increase in unburned fuel emissions (i.e., externally mixed OC, which would correspond to a decreased EC/TC). The observed trend is remarkably similar to that seen by Pohl et al. (1986) in their study of flare combustion efficiency. They found that as the heating value of a flare gas (which Figure 5.3 shows is strongly correlated with CHR for the present fuels) was reduced beyond a critical stability limit, flare combustion efficiency dropped dramatically and emissions of unburnt fuel dramatically increased. Figure 8 in their work (plotting flare efficiency against flare gas heating value normalized by the minimum heating value required for stability) bears a striking resemblance to Figure $5.4 \mathrm{a}$ above.

One data point (AB-M fuel flared at 400 SLPM) on Figure 5.4a had a notably lower EC/TC fraction. As shown in Figure 5.4b where duct concentrations of EC and OC are plotted as a function of $\mathrm{HHV}_{\mathrm{V}}$, the EC concentrations for this point (indicated by the blue arrow) are similar to other values for the same fuel and fall along a consistent trend of EC with $\mathrm{HHV}_{\mathrm{v}}$. By contrast, the OC concentration is approximately 4.4-8.3 times higher than for lower flowrate conditions with the same fuel. Closer examination of this condition 
reveals that the Reynolds number $(13,000)$ exceeds the point of incipient flame liftoff as measured by Takahashi and Schmoll (1991) for burners of similar diameter ratios. Although the base of the flame was not visibly affected, the apparent onset of liftoff and corresponding flame instability could initiate a minor reduction of combustion efficiency and corresponding increase of OC emissions in the form of unburned fuel (Pohl et al. 1986) without affecting EC emissions. This further supports the assertion that measured OC is externally mixed.

The shaded region of Figure 5.4a shows the 95\% confidence interval of data from McEwen and Johnson (2012), who performed experiments of similar flames with generally smaller flow rates $(<95$ SLPM) that extended to smaller stack diameters $(12.7-76.2 \mathrm{~mm}$ ID). McEwen and Johnson (2012) estimated EC/TC via the comparison of EC mass (through laser-induced incandescence measurements) with TC mass measured gravimetrically. While there is limited overlap in the tested compositions with the present work, there remains good agreement with the current dataset based on the flare gas CHR.

Overall, the logarithmic scale in Figure 5.4b illustrates that emission magnitudes are disproportionately attributable to heavier fuels in agreement with previous laboratory (McEwen and Johnson 2012) and field (Chapter 2, Conrad and Johnson 2017) work; thus global emissions from flares would be expected to be dominated by EC (likely $>92 \%$ ). This important inference is again consistent with field-observations (Schwarz et al. 2015).

\subsubsection{Flare BC MAC Sensitivity}

The sensitivity of measured BC MAC to flare gas energy content and flaring conditions (i.e., aerodynamic parameters relating to the total volumetric flow rate and burner diameter) were quantified as summarized in Figure 5.5a and Figure 5.5b, respectively. In contrast to 
Bond and Bergstrom's (2006) observations, BC MAC is observed to be statistically sensitive to both flare gas composition and flaring conditions (see also discussion of Figure 5.5 below and Section D.4.6.2). For the independent perturbations in flare gas composition and condition over this range, the measured $\mathrm{BC}$ MAC varies by up to $23 \%$ and $19 \%$ (calculated as span / midrange), respectively. However, variation of MAC for full-scale flares in the field is expected to be even greater. Although the set of compositions in this study spans those expected within industry, stack diameters (Johnson et al. 2011) and gas flow rates (Chapter 2, Conrad and Johnson 2017) of flares in the field can exceed the tested facility-limited range by as much as one and two orders of magnitude, respectively. Thus, while variations in $\mathrm{BC}$ MAC with fuel composition and flaring conditions both have statistical significance, in the context of large industrial flares, flaring condition likely has a stronger practical significance as further discussed below.
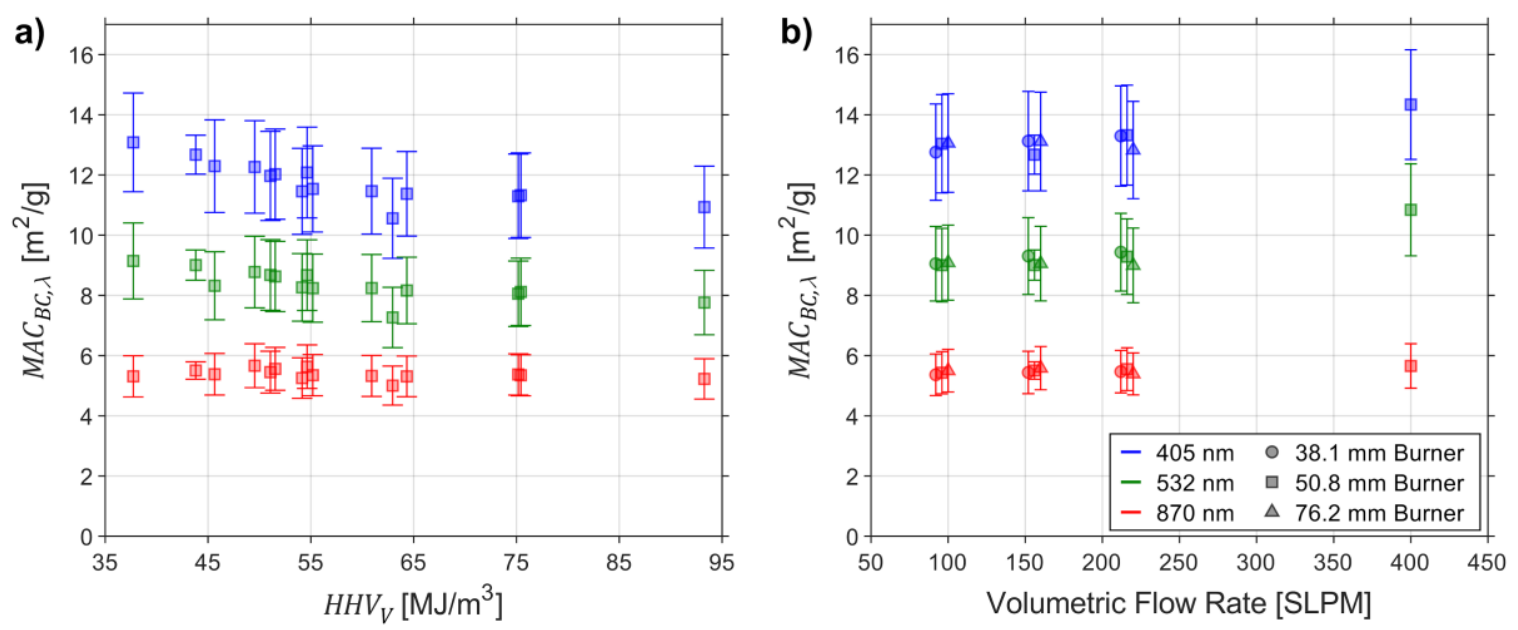

Figure 5.5: Sensitivity of BC MAC measurements to flare gas composition and flaring conditions. a) Sensitivity to composition, evaluated for the 15 compositions at the nominal average condition ( $50.8 \mathrm{~mm}$ burner and 156 SLPM) shown as a function of volumetric higher heating value. b) Sensitivity to flaring conditions, evaluated using the "median" Alberta composition (AB-M). Data at 96 and 216 SLPM in b) are slightly shifted ( \pm 4 SLPM) for visibility purposes. 
Experimental isolation of $\mathrm{BC}$ MAC sensitivity to specific fuel metrics is challenging since chemical properties tend to covary. However, expanding the discussion of the importance of time-temperature history on the nanostructure of $\mathrm{BC}$ particles, key parameters that could be expected to influence BC MAC include those impacting particle temperature (i.e., heat release rate, in-flame $\mathrm{BC}$ concentration, and radiative cooling) and residence time within the flame (i.e., flame length, stack exit velocity, and flow regime whether momentum- or buoyancy-driven). For example, the heat release rate is directly related to the energy content of the fuel, reported as a heating value. However, heating value is generally correlated with molecular weight (Pearson correlation coefficient of 0.946 for the flare gas compositions considered) and carbon-hydrogen ratio (0.752) and partially correlated with adiabatic flame temperature (0.598). Nevertheless, the correlation of observed BC MAC with pertinent and easily obtained flare composition and flow parameters was analyzed to gain insight into underlying drivers of observed MAC variation. Linear regression F-tests were performed (see Table D.9 for details) on the data in Figure 5.5 to assess potential correlations between flare metrics and $\mathrm{BC} \mathrm{MAC}-$ this hypothesis test is of the form:

$$
\begin{array}{ll}
H_{o}: & M A C_{B C, \lambda}=\beta_{0} \\
H_{a}: & M A C_{B C, \lambda}=\beta_{0}+x \beta_{1}
\end{array}
$$

where $\beta_{i}$ are constant coefficients and $x$ is some metric of interest. If the null hypothesis is rejected in favour of the alternative at a prescribed significance level, this implies that BC MAC trends in a statistically significant manner with the variable $x$. In summary, four fuel metrics (molecular weight, adiabatic flame temperature, higher heating value, and carbon-hydrogen ratio), two fundamental aerodynamic metrics (stack exit velocity and 
volumetric flow rate), and vertical flame length correlated statistically with BC MAC for at least one wavelength $(\mathrm{p}<0.05)$.

\subsubsection{Flare BC MAC Model}

The observed correlations between flare BC MAC and measurable flare parameters enables the development of a predictive model. A dimensionless scaling parameter $(\Lambda[-])$, derived from the statistically significant flare metrics presented above, was found to accurately predict observed flare $\mathrm{BC}$ MAC data. The derived scaling parameter is the reciprocal product of Becker and Liang's (1982) dimensionless thermal loading parameter and the fuel's carbon-hydrogen ratio:

$$
\Lambda=\frac{\dot{Q}_{t h}}{\sigma T_{a d}^{4} L_{v}^{2}} \cdot \frac{1}{C H R}
$$

where $\dot{Q}_{t h}\left(=\dot{V} H H V_{V}\right)$ is the theoretical heat release rate of the flame $[\mathrm{W}], \dot{V}$ is the volumetric flow rate of flare gas $\left[\mathrm{m}^{3} / \mathrm{s}\right]$, and $\sigma$ is the Stefan-Boltzmann constant $\left[\mathrm{W} \mathrm{m}{ }^{-2} \mathrm{~K}^{-4}\right]$. The adiabatic flame temperature $\left(T_{a d}[\mathrm{~K}]\right)$ is computed assuming a reference gas temperature of $25^{\circ} \mathrm{C}$ at the flare tip and the characteristic flame area is approximated as the square of the visible/vertical flame length $\left(L_{v}[\mathrm{~m}]\right)$ computed using the empirical correlation of Delichatsios (1993), which represents the time-averaged, luminous flame length of vertical jet flames. The term $\sigma T_{a d}^{4} L_{v}^{2}$ therefore approximates a characteristic radiative power [W] from an optically thick flame.

Figure 5.6a plots the current $\mathrm{BC}$ MAC data at the measurement wavelengths as a function of the scaling parameter. The error bars show the $95 \%$ confidence interval of the total uncertainty in measured MAC as calculated via the MC analysis (see Section D.4). Wavelength specific power-law models $\left(M A C_{B C, \lambda}=a_{\lambda} \Lambda^{b_{\lambda}}+c_{\lambda}\right)$ match the observed data 
with a good fit at low wavelengths ( $\mathrm{R}^{2}$ of 0.854 and 0.878 at 405 and $532 \mathrm{~nm}$, respectively). Towards the near infrared spectrum, the use of a power law model $\left(b_{\lambda} \neq 1\right)$ was not statistically justified $(p>0.05)$ and a linear model was instead employed $\left(\mathrm{R}^{2}\right.$ of 0.567 at $870 \mathrm{~nm}$ ). The empirical fit coefficients for each case are provided in Table 5.3 and the plotted 95\% confidence intervals (dark shading) and prediction intervals (light shading) illustrate the utility and predictive power of these models relative to the experimental uncertainties. 

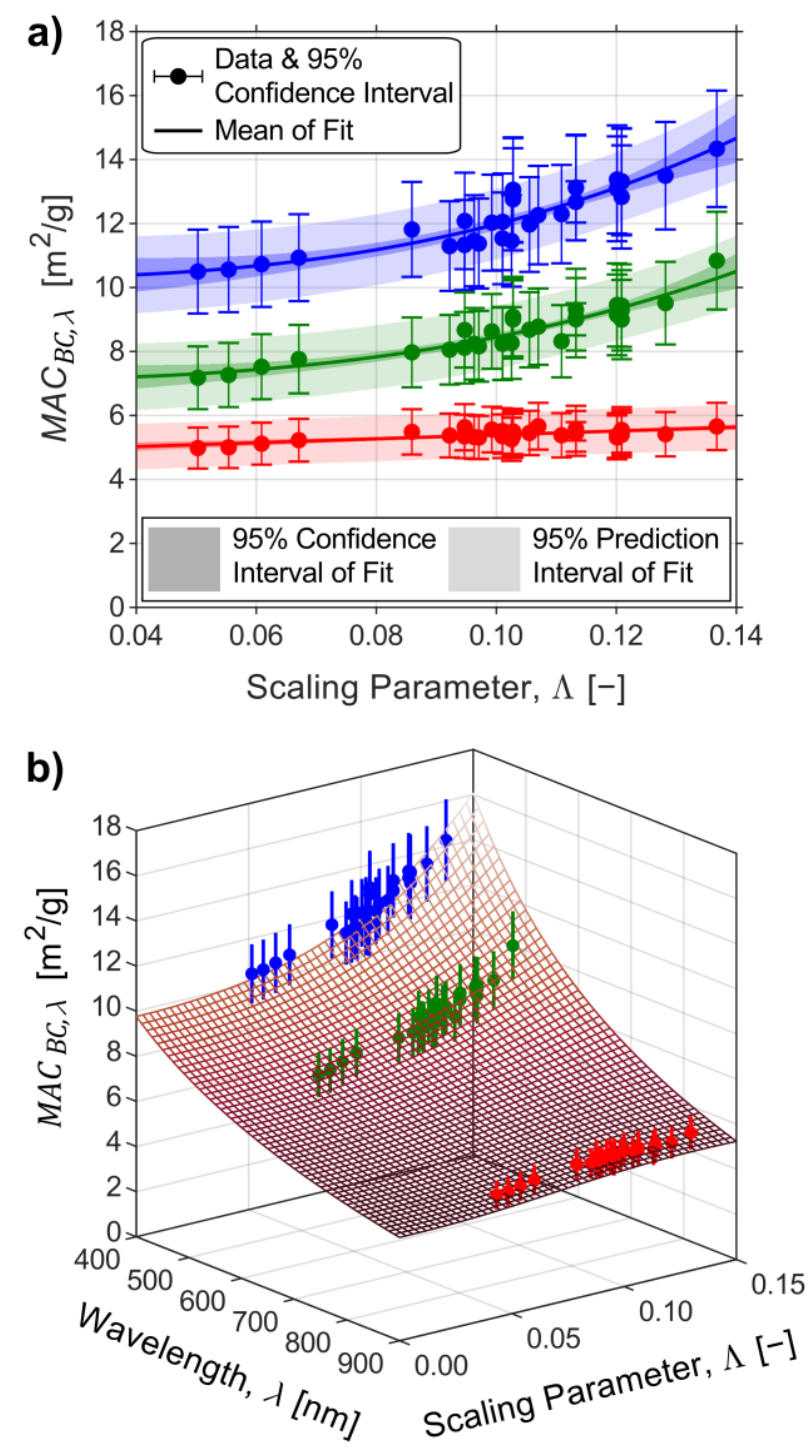

Figure 5.6: Proposed predictive correlation for wavelength dependent BC MAC. (a) Wavelength specific power-law predictive models (see Table 5.3) and corresponding 95\% confidence intervals (dark shading) and prediction intervals (light shading). (b)

Generalized model for BC MAC at varying wavelength.

Table 5.3: Empirical fit coefficients for the wavelength-dependent BC MAC models plotted in Figure 5.6a, at diagnostic wavelengths of the PASS3 instrument.

\begin{tabular}{|c|c|c|c|}
\hline \multirow{2}{*}{$\begin{array}{c}\text { Wavelength } \\
{[\mathbf{n m}]}\end{array}$} & \multicolumn{3}{|c|}{$\begin{array}{c}\text { Empirical Model Coefficients } \\
\boldsymbol{M} \boldsymbol{A} \boldsymbol{C}_{\boldsymbol{B} \boldsymbol{C}, \boldsymbol{\lambda}}=\boldsymbol{a}_{\boldsymbol{\lambda}} \boldsymbol{\Lambda}_{\boldsymbol{\lambda}}+\boldsymbol{c}_{\boldsymbol{\lambda}}\end{array}$} \\
\cline { 2 - 4 } & $\boldsymbol{a}_{\lambda}\left[\mathbf{m}^{\mathbf{2}} / \mathbf{g}\right]$ & $\boldsymbol{b}_{\lambda}[-]$ & $\boldsymbol{c}_{\boldsymbol{\lambda}}\left[\mathbf{m}^{\mathbf{2}} \mathbf{g}\right]$ \\
\hline 405 & 1100 & 2.81 & 10.3 \\
\hline 532 & 753 & 2.75 & 7.10 \\
\hline 870 & 6.09 & 1.00 & 4.78 \\
\hline
\end{tabular}


Figure 5.6b generalizes the model according to Eq. (5.2), where the entirety of the presented data is now modelled as a function of measurement wavelength and scaling parameter. Here, the reference $\mathrm{MAC}_{\mathrm{BC}, 550}$ is modelled with a power-law equation of $\Lambda$, as per Figure 5.6a and Table 5.3, and the AÅ is modelled as an offset exponential function of $\Lambda$. The figure shows the current data, again plotted with $95 \%$ confidence interval, and the derived BC MAC model plotted as a surface:

$$
M A C_{B C, \lambda}=M A C_{B C, 550}\left(\frac{\lambda_{[n m]}}{550 \mathrm{~nm}}\right)^{-A \AA \AA E}
$$

where:

$$
\begin{aligned}
M A C_{B C, 550} & =188 \Lambda^{2.17}+7.25 \\
A \AA ̊ E & =11.6 \exp \left(-\frac{0.487}{\Lambda}\right)+0.934
\end{aligned}
$$

The coefficient of determination of this general predictive model is 0.987 and the relative root mean squared error is $\sim 4.1 \%$. Correlation between the residuals and both $\lambda$ and $\Lambda$ is very low - less than 0.05 in magnitude in both cases - suggesting that error between the derived model and the data is largely unstructured, or random, and likely due to the precision of the CUFF experiment as a whole.

The fitted model is asymptotic as the scaling parameter approaches zero where, referring to Eq. (5.6), the $\mathrm{A} \AA \mathrm{E}$ converges to $\sim 0.934$ and $\mathrm{MAC}_{\mathrm{BC}, 550}$ to $\sim 7.25 \mathrm{~m}^{2} / \mathrm{g}$. Notably, this result is in very good agreement with existing literature, which tends to be dominated by studies on heavier fuels and/or smaller flames of laboratory burners and internal combustion engines - both factors corresponding to smaller values of $\Lambda$. For example, this value of $\mathrm{MAC}_{\mathrm{BC}, 550}$ is well within the uncertainties of Bond and Bergstrom's 
(2006) value of $7.5 \pm 1.2 \mathrm{~m}^{2} / \mathrm{g}(1 \sigma)$. Similarly, the observed A $\AA \mathrm{E}$ is bound by laboratory measurements of buoyancy-driven turbulent diffusion flames (e.g., $A \AA ̊ E=0.83 \pm 0.08$ (Köylü and Faeth 1996)) and the theoretical expectation for a spectrally-constant refractive index $(A \AA \AA E=1)$. As further discussed in Section D.2, the deviation of $\mathrm{A} \AA \mathrm{E}$ from unity at this limit likely implies a modest variation of refractive index over the measurement spectrum.

\subsection{Discussion}

Despite the agreement with the literature at low values of $\Lambda$, the present data do contradict the generally accepted notion that fresh $\mathrm{BC}$ MAC has a consistent value with respect to measurement uncertainties. At the extreme of a large scaling parameter, the AÅE and $\mathrm{MAC}_{\mathrm{BC}, 550}$ rapidly increase and at the largest experimental condition were 1.26 and $9.79 \mathrm{~m}^{2} / \mathrm{g}$, respectively; the latter of which is beyond the $97^{\text {th }}$ percentile of Bond and Bergstrom (2006). However, the present results and model potentially explain the observations of Weyant et al. (2016) in the Bakken region, where large values of flare BC MAC (averaging 22, 16, and $14 \mathrm{~m}^{2} / \mathrm{g}$ at 467, 530, and $\left.660 \mathrm{~nm}\right)$ and AAE $(1.2 \pm 0.8$, or $1.4 \pm 0.33$ excluding low absorption signals) were observed from field-operational flares. At the time, the authors could not reconcile their observations based on the existing literature. However, if the current model is extrapolated, Eq. (5.6) suggests that these MAC measurements are best described by a scaling parameter of 0.2343 . Applying Weyant et al.'s (2016) assumed fuel composition (BK-2; sourced from Wocken et al. (2013)), this corresponds to a full-scale flare volume flow of approximately 10,500 SLPM (see Section D.5.1). This flowrate is well within the range of published flare measurements in Ecuador (Chapter 2, Conrad and Johnson 2017) and recent measurements during oil and gas well 
completions in Alberta. The new flare BC MAC model thus bridges Weyant et al.'s (2016) field-measurement data of flares and the literature review of Bond and Bergstrom (2006).

The form of the proposed scaling parameter also provides insight into the mechanism of the observed variability in BC MAC. Consider the first term in Eq. (5.5). This ratio is the inverse of the optically-thick thermal loading parameter from Becker and Liang's (1982) study, which can be taken as a surrogate of the radiant cooling factor or radiant fraction of the flame. Consequently, as this first term increases, so does the relative fraction of chemically released energy that is not radiated away from the flame and instead heats material within the reaction zone. Consider next the second term in Eq. (5.5), the reciprocal of the CHR. There are two important interpretations of this term: 1) for aliphatic hydrocarbons of a given carbon number, CHR scales with the degree of unsaturation of the species; and 2) for the compositions of the present study, CHR is positively correlated with higher heating value. Since BC production is known to scale with both fuel heating value (Chapter 2, Conrad and Johnson 2017; McEwen and Johnson 2012) and the degree of unsaturation (Glassman 1989), the CHR can be interpreted in the present context as a surrogate for relative $\mathrm{BC}$ production or $\mathrm{BC}$ concentration within the flame. With this interpretation, the scaling parameter can be understood as a characteristic average radiative heating of $\mathrm{BC}$ particulate within the flame; i.e., heat absorption per BC. In this context, it is proposed that the scaling parameter captures the overall time-temperature history and perhaps the degree of graphitization (and hence nanostructure) of emitted BC particulate as explained below. 


\subsubsection{Potential Mechanism of BC MAC Variability via Changes in Nanostructure}

The present observations also provide insight into important material properties (nanostructure and morphology) of the emitted $\mathrm{BC}$ and enable an understanding of the likely mechanism of observed variability in BC MAC. Consider the light attenuation theory for fractal aggregates like fresh BC (i.e., RDG-FA). Despite well-documented accuracy limitations in the literature (e.g., Farias et al. 1995, 1996; Liu and Snelling, 2008), RDG-FA can be leveraged to examine the $\mathrm{BC}$ material and morphology implications of the present observations. First, suppose that three of the four main assumptions of RDG-FA theory are valid:

1) $\mathrm{BC}$ aggregates are comprised of monodisperse, spherical monomers;

2) $\mathrm{BC}$ aggregates are sufficiently open in structure, such that monomer-monomer shadowing is negligible; and

3) Monomers are in point contact.

As a thought experiment, allow the remaining assumption of RDG-FA theory (that monomers fall within the Rayleigh regime) to be relaxed such that the exact solution to Maxwell's equations for spheres (Mie theory (Born et al. 1999; Bohren and Huffman 1983; van de Hulst 1957)) can be used to directly quantify BC absorption with knowledge of monomer/primary particle diameter $\left(d_{p}\right)$ and the spectral refractive index $\left(m_{\lambda}\right)$. Following these assumptions, a MC analysis was performed to assess the absorption magnitude and $\mathrm{A} \AA \mathrm{E}$ as a function of monomer diameter and refractive index. Prior distributions used in the MC analysis were chosen as conservatively broad representations of typical combustion-generated $\mathrm{BC}: \quad d_{p} \sim \mathcal{U}(0 \mathrm{~nm}, 75 \mathrm{~nm})$ for monomer diameter and $\eta_{\lambda} \sim \mathcal{U}(1.5,2.0)$ and $\kappa_{\lambda} \sim \mathcal{U}(0.5,1.0)$ for the real and imaginary components of the 
refractive index, respectively, which are modelled as constant across the $405-870 \mathrm{~nm}$ spectrum. Results of this MC analysis are presented in Figure 5.7. Figure 5.7a shows the volume-normalized absorption cross-section at $550 \mathrm{~nm}\left(V A C_{B C, 550}=M A C_{B C, 550} \times \varrho_{B C}\right.$, units of $\left.\mu \mathrm{m}^{-1}\right)$ as a function of monomer diameter with symbol colour representing $E\left(m_{\lambda}\right)$, and Figure $5.7 \mathrm{~b}$ shows $\mathrm{A} \AA \mathrm{E}$ as a function of monomer diameter with symbol colour representing the absolute value of the complex refractive index.
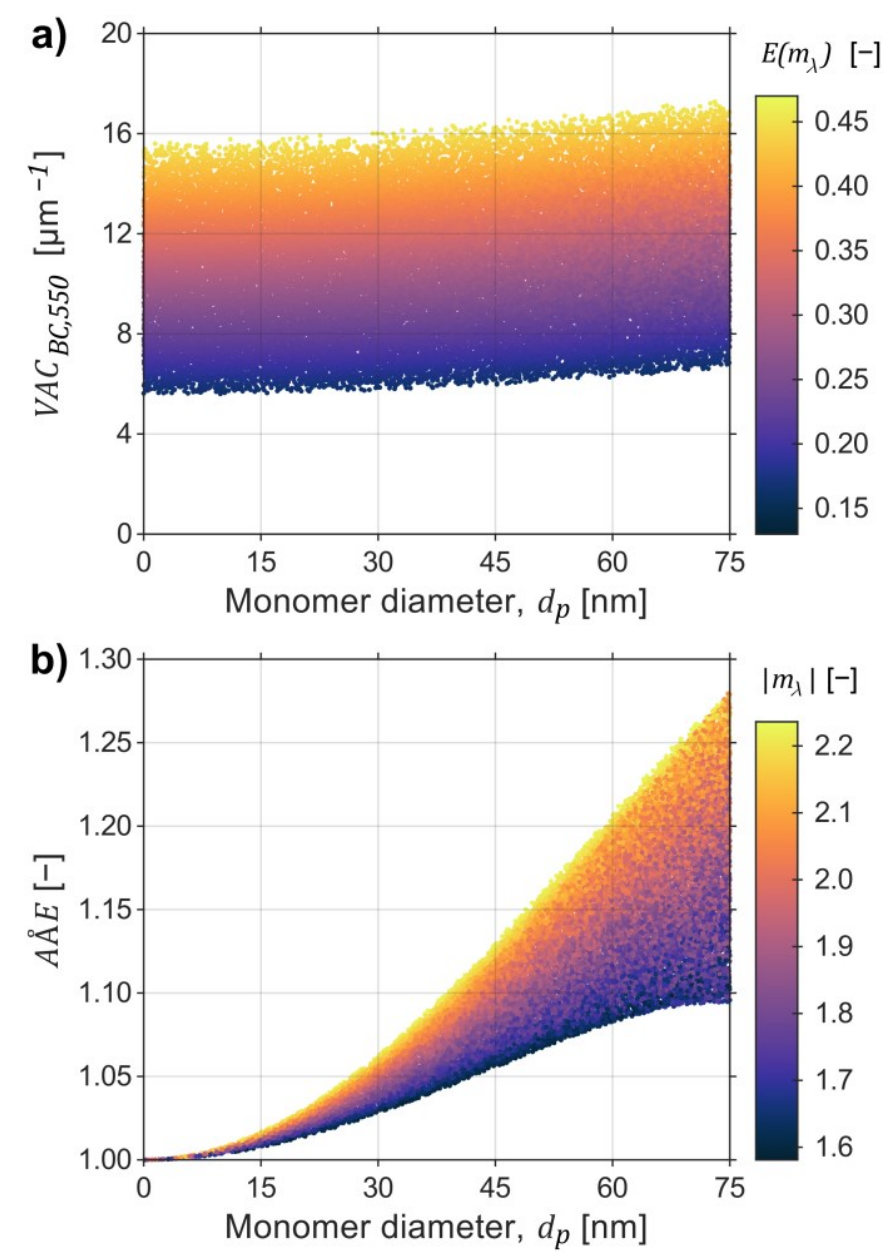

Figure 5.7: Absorption by BC calculated using Mie theory for a range of monomer diameters and refractive indices typical of combustion-generated BC. (a) Volumenormalized absorption cross-section at $550 \mathrm{~nm}$ vs. monomer diameter, coloured by absorption refractive index function and (b) AÅE vs. monomer diameter, coloured by absolute refractive index. 
A number of key observations in Figure 5.7 are noteworthy. Firstly, over the considered prior distributions, both absorption $\left(\mathrm{VAC}_{\mathrm{BC}, 550)}\right.$ and its spectral sensitivity (AÅE) increase with monomer diameter, with the latter effect being much larger than the former. Additionally, spectral sensitivity at fixed monomer diameter is positively correlated with refractive index magnitude and absorption is positively correlated with $E\left(m_{\lambda}\right)$ (as expected via Eq. (5.1)). Together, these observations have important implications for the current study: that is, the observed simultaneous increase in BC MAC and $\mathrm{AÅE}$ - corresponding to an increase in the MAC scaling parameter - likely suggests a simultaneous increase in monomer diameter, $E\left(m_{\lambda}\right)$, and the absolute value of the refractive index.

Consider now the effect on the refractive index. It can be shown using the functional form of $E\left(m_{\lambda}\right)$ that:

$$
\left\{\frac{\partial E\left(m_{\lambda}\right)}{\partial \eta_{\lambda}} \leq 0\right\} \cap\left\{\frac{\partial E\left(m_{\lambda}\right)}{\partial \kappa_{\lambda}} \geq 0\right\} \Leftrightarrow 3 \kappa_{\lambda}^{2} \leq 2-\eta_{\lambda}{ }^{2}+2 \sqrt{\eta_{\lambda}^{4}+2 \eta_{\lambda}^{2}+4}
$$

That is, should the righthand inequality be satisfied, only an independent increase in the imaginary component of the refractive index can yield a simultaneous increase in $E\left(m_{\lambda}\right)$ and the absolute value of the refractive index. Importantly, as shown in Figure D.2, this inequality is satisfied for all of the refractive index data tabulated by Bond and Bergstrom (Table 4 of Bond and Bergstrom (2006)) for light-absorbing carbon. Therefore, since an increase in the imaginary refractive index is consistent with graphitization of the particle (Bond and Bergstrom 2006), these current data suggest that under larger MAC scaling parameters, particles are more graphitized, are higher-absorbing, and have a higher spectral sensitivity. As previously noted, the present data also suggest that the scaling parameter is 
likely correlated with monomer diameter. This is consistent with recent observations of positive correlation between BC MAC and particle mass (Dastanpour et al. 2017; Radney et al. 2014). Furthermore, coupling these observations with the earlier work of Dastanpour and Rogak (2014), who observed correlation between intra-aggregate monomer size and the aggregate size itself (number of monomers per aggregate), the present data might suggest that as the scaling parameter increases, emitted $\mathrm{BC}$ also has a larger aggregate size.

\subsubsection{Limits to the Phenomenological Model}

While agreement between the present $\mathrm{BC}$ MAC model and the literature suggests reasonable generality of the model at low scaling parameters (outside of the flaring-specific context), the predicted monotonic increase of $\mathrm{BC}$ MAC with increasing $\Lambda$ is most likely bounded in practice. For turbulent diffusion flames, it is well-known that flame length does not increase indefinitely as the rate of combustion increases and the flame becomes

more momentum- (rather than buoyancy-) driven; in fact, flame length converges to a maximal value, the magnitude of which is a function of fuel stoichiometry and stack diameter alone (Delichatsios 1993). This is a consequence of improved mixing and air entrainment into the flame (as the momentum flux increases relative to the buoyant flux), which counteracts the intuitive increase in flame length with fuel flow rate (Turns 2012). This has important implications on the time-temperature history of $\mathrm{BC}$ particles and hence creates a likely bound on $\mathrm{BC}$ MAC of turbulent diffusion flames and flares at high flowrates. While flame residence time would be expected to decrease due to increasing momentum and a lack of corresponding increase in flame length, relative outward radiation (i.e., the radiative fraction) would also be expected to decrease (e.g., Delichatsios et al. 1992; Hu et al. 2014). Thus, it is hypothesized that as the combustion-regime moves 
towards momentum- rather than buoyancy-driven, the decrease in residence time would dominate, imposing a practical maximum on BC MAC. Further studies are required to assess this hypothesis and determine if a practical bound on flare BC MAC exists for large flares, and whether this bound is approached asymptotically or occurs at some identifiable transition in flaring condition.

Under field conditions, the presence of a crosswind could also affect flare BC MAC. Though not considered in the present experiments, the apparent agreement of Weyant et al.'s (2016) field estimates with the present model suggests that wind effects are not expected to be significant (at least for moderate wind speeds) - indeed, Weyant et al. (2016) measured wind speeds from 3.5-6.0 m/s, which they note did not correlate with BC emissions. This is consistent with wind tunnel measurements (Majeski et al. 2004; Johnson and Kostiuk 2000) that suggest low wind speeds mostly deflect the flame and alter its trajectory without significantly affecting its length. However, higher wind speeds do strongly affect flare combustion and flame length (Majeski et al. 2004; Johnson and Kostiuk 2000) and could be expected to influence BC MAC through enhanced mixing and the reduction of residence times. As the characteristic velocity of the flame becomes driven by the crosswind rather than buoyancy or momentum of the fuel jet itself, the aerodynamics of the flame are altered (Majeski et al. 2004). For example, increased shear layer vorticity with crosswind velocity (Majeski et al. 2004) and interactions between the fuel jet and wake vortices of the flare stack (Johnson and Kostiuk 2000) reduce flame length, provoke fragmentation of the flame, and can induce local extinctions due to aerodynamic strain of the flame. Although beyond the scope of the present experiments, it seems plausible that high crosswinds on average may result in reduced residence times, leading to the emission 
of less graphitized and therefore less absorbing $\mathrm{BC}$ than an equivalent vertical flame. Seeking insight from the model of Majeski et al. (2004) however, if the critical crosswind velocity required to decrease flame length is taken as an estimate of when $\mathrm{BC}$ formation might be notably affected, then a crosswind of $>70 \mathrm{~m} / \mathrm{s}$ would be required to influence BC MAC of Weyant et al.'s (2016) average flare. Consequently, it is anticipated that crosswind has little to negligible effect on BC MAC for typical flaring conditions.

\subsubsection{Implications for Climate Forcing of Flare BC}

Direct radiative climate forcing (DRF) by atmospheric particles is a function of numerous local parameters including atmospheric loading, spectral albedo of the ground, and cloud cover fraction (e.g., Chylek and Wong 1995). In terms of particle properties however, if the particle population is strongly forward-scattering and generally much more absorbing than scattering - like BC - then the forcing efficiency of the particle (DRF on a per-mass basis) scales linearly with its MAC.

Within the atmosphere, fresh BC (such as that measured at the CUFF and discussed by Bond and Bergstrom (2006)), ages as it becomes coated by secondary atmospheric material such as sulphate, nitrate, water, ammonium, and organic material (Gustafsson and Ramanathan 2016; Moffet and Prather 2009). When coated in this manner, the coating material induces an enhancement of absorption by the BC core (e.g., Andreae and Gelencsér 2006; Jacobson 2001; Peng et al. 2016), which tends to collapse towards a more compact rather than lacy morphology (e.g., Peng et al. 2016; Schnaiter et al. 2005). The magnitude of this absorption enhancement is an active research topic and there is some debate on likely bounds (e.g., Boucher et al. 2016; Peng et al. 2016; Zhang et al. 2016), but recently it has been shown to scale in a power-law relation with relative coating mass 
(Chakrabarty and Heinson 2018). Since the degree of coating is sensitive to local atmospheric composition (Gustafsson and Ramanathan 2016), these observations suggest that absorption enhancement is sensitive to the geographical history of the atmospheric $\mathrm{BC}$ particulate. Despite uncertainty in the absorption enhancement of a particular emission, the influence of atmospheric aging only serves to increase the effective MAC of emitted BC. In keeping with Gustafsson and Ramanathan (2016) who note that "it is imperative to better understand both $[\mathrm{MAC}]$ of the bare $\mathrm{BC}$ skeleton ... and to constrain the enhancement of $\mathrm{BC}$ absorption after mixing with non-BC aerosol components in the atmosphere", it would be prudent to perform controlled atmospheric aging studies on flare $\mathrm{BC}$ to ascertain its lifetime-integrated climate forcing potential.

Noting that the presently derived MAC model for fresh flare generated BC is lowerbounded by Bond and Bergstrom's (2006) highly-cited MAC value, the present results suggest at a minimum that the climate forcing efficiency of flare $\mathrm{BC}$ is currently underestimated. It is challenging however to quantify this underestimation, since the model predicts a power law increase in MAC with increasing volume flow as captured by the non-dimensional parameter $\Lambda$, and field-operational flares would generally exceed the current experimental range. Nonetheless, the measured MAC for the largest flare in this study was 30\% larger than Bond and Bergstrom's (2006) value, and a model prediction to match Weyant et al.'s (2016) field-measurement data suggests a discrepancy in BC forcing efficiency of a factor of two or more.

\subsection{Conclusions}

Fresh carbon emissions from gas flares are mostly elemental in nature (typically $>92 \%$ ) and likely exist as an external mixture of EC and OC. Light absorption by carbonaceous 
emissions from flares are therefore due to the elemental component, and EC is thus the appropriate mass-basis for flare BC. Flare BC MAC is variable and dependent upon flare gas composition and flaring conditions. Using a new phenomenological model that is a function of a novel dimensionless scaling parameter, observed variability in BC MAC is predictable with good accuracy over the tested conditions and fuels. This MAC scaling parameter is composed of easily-derived flare gas and flaring condition metrics and captures the radiation-driven heating of in-flame $\mathrm{BC}$ particulate; it is positively correlated with $\mathrm{BC}$ MAC and $\mathrm{AÅE}$, and Mie and RDG-FA theory calculations suggest that the scaling parameter also captures the $\mathrm{BC}$ nanostructure (degree of graphitization) and morphology. The derived phenomenological model bridges extreme observations of $\mathrm{BC}$ MAC from myriad combustion sources in the literature, including the only known measurement of BC MAC from a field-operational flare. Observed BC MAC variability, when taken into the context of in-field flares, suggests that direct radiative forcing by flare $\mathrm{BC}$ could be $>1.3-2.0$ times larger than current estimates.

\subsection{Acknowledgements}

This work was supported by the Natural Sciences and Engineering Research Council of Canada (NSERC) FlareNet Strategic Network (Grant \#479641), NSERC Discovery Research Grants (Grant \#06632 and 522658), and Natural Resources Canada (Project Manager, Michael Layer). We are grateful for the help of Prof. Catherine Lund Myhre (Norwegian Institute for Air Research) and Irina Isakova (Carbon Limits) in accessing additional flare gas composition data; Melina Jefferson for operating the flare during experiments; and Dr. Brian Crosland for designing the liquid fuels handling system used in our experiment. 


\section{Chapter 6 An Uncertainty-Based Protocol for the Setup and Measurement of Soot/Black Carbon Emissions from Gas Flares using Sky-LOSA}

This chapter has been submitted to the Journal of Atmospheric Measurement Techniques and is presently under peer-review. It should be cited as:

Conrad, B.M. and Johnson, M.R. (2020). An Uncertainty-Based Protocol for the Setup and Measurement of Soot/Black Carbon Emissions from Gas Flares using Sky-LOSA. Atm. Meas. Tech. [under review] (doi: 10.5194/amt-2020-255).

This article is included in its entirety as Chapter 6. The article was co-authored by the thesis author, Mr. Conrad, and his thesis supervisor, Prof. Johnson. Mr. Conrad and Prof. Johnson conceptualized the research. Mr. Conrad developed the methodology and software and performed the investigation, validation, and formal analysis. Mr. Conrad wrote the first draft of the manuscript; editing and revision of the manuscript were conducted jointly.

\subsection{Abstract}

Gas flaring is an important source of atmospheric soot/black carbon, especially in sensitive Arctic regions. However, emissions have traditionally been challenging to measure and remain poorly characterized, confounding international reporting requirements and adding uncertainty to climate models. The sky-LOSA optical measurement technique has emerged as a powerful means to quantify flare black carbon emissions in the field, but broader adoption has been hampered by the complexity of its deployment, where decisions during setup in the field can have profound, non-linear impacts on achievable measurement uncertainties. To address this challenge, this paper presents a prescriptive measurement protocol and associated open-source software tool that simplifies acquisition of sky-LOSA data in the field. Leveraging a comprehensive Monte Carlo-based General Uncertainty 
Analysis (GUA) to predict measurement uncertainties over the entire breadth of possible measurement conditions, general heuristics are identified to guide a sky-LOSA user toward optimal data collection. These are further extended in the open-source software utility, SetupSkyLOSA, which interprets the GUA results to provide detailed guidance for any specific combination of location, date/time, and flare, plume, and ambient conditions. Finally, a case study of a sky-LOSA measurement at an oil and gas facility in Mexico is used to demonstrate the utility of the software tool, where potentially small region(s) of optimal instrument setup are easily and quickly identified. It is hoped that this work will help increase the accessibility of the sky-LOSA technique and ultimately the availability of field measurement data for flare black carbon emissions.

\subsection{Introduction}

Gas flaring is a routine practice in the oil and gas industry in which producers and refiners burn excess or unwanted gases in open-atmosphere flames, typically from vertical pipe stacks. Flaring is generally preferable to the venting of gases to atmosphere because it reduces carbon dioxide $\left(\mathrm{CO}_{2}\right)$-equivalent emissions; however, flaring still emits potent climate-forcing pollutants directly to atmosphere (Johnson et al. 2001, 2011, 2013; Allen and Torres 2011; Pohl et al. 1986; McDaniel 1983). These pollutants have public health implications (e.g., Anenberg et al., 2012) and include unburnt hydrocarbons, volatile organic compounds, and particulate matter (U.S. EPA 2018). Soot particulate matter (commonly referred to as black carbon, BC) has been suggested by some to be the secondmost potent climate forcer after $\mathrm{CO}_{2}$ (Bond et al. 2013; Ramanathan and Carmichael 2008; Sato et al. 2003; Jacobson 2001). Annual flaring is estimated by satellite imagery to be $\sim 140$ billion $\mathrm{m}^{3}$ (Elvidge et al. 2007, 2009, 2015), making it one important source of global 
soot emissions. Although other industrial sectors dominate gas flaring in absolute soot emissions, the locations of flaring activities (particularly in Russia) likely have a disproportionate impact on the sensitive Arctic climate due to efficient transport pathways penetrating the Arctic air mass (e.g., Popovicheva et al., 2017; Stohl et al., 2006).

With the addition of $\mathrm{BC}$ to the Gothenburg protocol in 2012 (United Nations Economic Commission for Europe (UNECE), 2012), 34 countries are now legally bound to report, where data are available, soot/BC emissions under UNECE's Convention on Long-Range Transboundary Air Pollution, including the European Union, Russia, United States of America, and Canada. To attribute - and, hence, report and regulate - soot/BC emissions from various sources, emission factors that relate soot/BC emissions to a measure of industrial activity are required. Unfortunately, for gas flaring, commonly employed soot emission factors are crude single-valued parameters that link emitted soot mass to volume/mass of gas flared regardless of flare design, gas composition, or operating conditions. This contrasts with numerous studies that have observed a significant influence of flare gas composition and flame aerodynamics on soot emissions (Chapter 2, Conrad and Johnson 2017; McEwen and Johnson 2012; Becker and Liang 1982) and even soot properties (Trivanovic et al. 2020; Chapter 5, Conrad and Johnson 2019a). Further soot yield data are needed, particularly for real-world flares under field conditions, to develop and validate accurate flare soot/BC emission factor models.

At present, there are only two published methods for the quantitative measurement of soot emissions from individual in-field flares. One technique employs aircraft-based sampling of a flare plume (Gvakharia et al. 2017; Weyant et al. 2016), where measurements of soot, methane, and $\mathrm{CO}_{2}$ concentrations during transects through the plume are used to 
provide flare-specific estimates of soot yield, using assumed flare gas compositions. The second technique is a ground-based remote optical measurement called sky-LOSA (lineof-sight attenuation using skylight; Chapter 2, Conrad and Johnson, 2017; Johnson et al., 2010, 2011, 2013). Sky-LOSA quantifies time-resolved soot mass emission rates through analysis of highspeed image data. Parallel access to flare infrastructure permits simultaneous measurement of flare gas flow rate and gas sample extraction for off-site compositional analysis, which enables the direct calculation of soot yield for a targeted flare. To date, sky-LOSA has been deployed on 11 field measurement campaigns in Uzbekistan, Mexico, Ecuador, and Canada, providing 28 measurements of soot emissions from 17 unique flares (Chapter 2, Conrad and Johnson 2017; Johnson et al. 2011, 2013).

The key component of a sky-LOSA measurement is the quantification of plume soot loading using image data, via analysis of radiative transfer through the atmospheric flare plume at the measurement wavelength. For each acquired image, soot mass column density is resolved pixel-by-pixel over a control surface within the image plane to permit mass emission rate calculation. Uncertainties in sky-LOSA-calculated emission rate are computed under a Monte Carlo (MC) framework and are dominated by uncertainties that affect computation of soot mass column density. While these uncertainties are influenced by numerous parameters considered within the $\mathrm{MC}$ analysis, they are also sensitive to the positioning and pointing of the sky-LOSA camera relative to the horizon and sun. Consequently, a sky-LOSA user must position the camera according to several constraints, which may be heuristic but can also vary with uncontrollable measurement parameters. To make the measurement technique accessible to third parties, enabling an increase in flare soot emissions data, a standardized data acquisition protocol for sky-LOSA is required. 
This manuscript presents a general uncertainty analysis (GUA) for the sky-LOSA measurement technique and an accompanying open-source software tool that provides guidance to end-users in the acquisition of sky-LOSA data based on calculated uncertainties in soot mass column density. A summary of sky-LOSA theory, referring to derivations in previous works, is first provided in Section 6.3. The GUA methodology is summarized in Section 6.4, including special provisions necessary to reduce the computational burden of the MC-based approach (Section 6.4.1). Representative results from the MC GUA are shown in Section 6.5.1 and general heuristics for the acquisition of sky-LOSA data, including new observations based on MC GUA results are summarized in Sections 6.5.1.1 and 6.5.1.2. To provide case-by-case guidance, a new open-source software tool to calculate sky-LOSA measurement uncertainty is introduced in Section 6.5.2. Finally, in Section 6.5.3, the software tool is used in a case study that analyses optimal camera positioning for flare measurements at a gas refining and transport facility in Campeche, Mexico. This work enables a consistent approach for the selection of skyLOSA camera positioning and pointing to minimize measurement uncertainties, ultimately contributing to the standardization of the sky-LOSA measurement technique.

\subsection{Sky-LOSA Measurement}

The sky-LOSA theory was first derived in full by Johnson et al. (2013). Development of the theory begins with Figure 6.1, which shows an example sky-LOSA image from a measurement of a soot-laden flare plume in the Montney formation of Alberta, Canada. A highly linear, grayscale, scientific-CMOS camera (e.g., pco edge 5.5) is used to obtain upwards of ten minutes of high-speed image data of the flare and turbulent, soot-laden, atmospheric plume. Pseudo 16-bit images are acquired at framerates of $25-50 \mathrm{~Hz}$ with a 
narrow mid-visible bandpass filter $(531 \pm 20 \mathrm{~nm})$ to yield a scene of spectrally integrated light intensity.

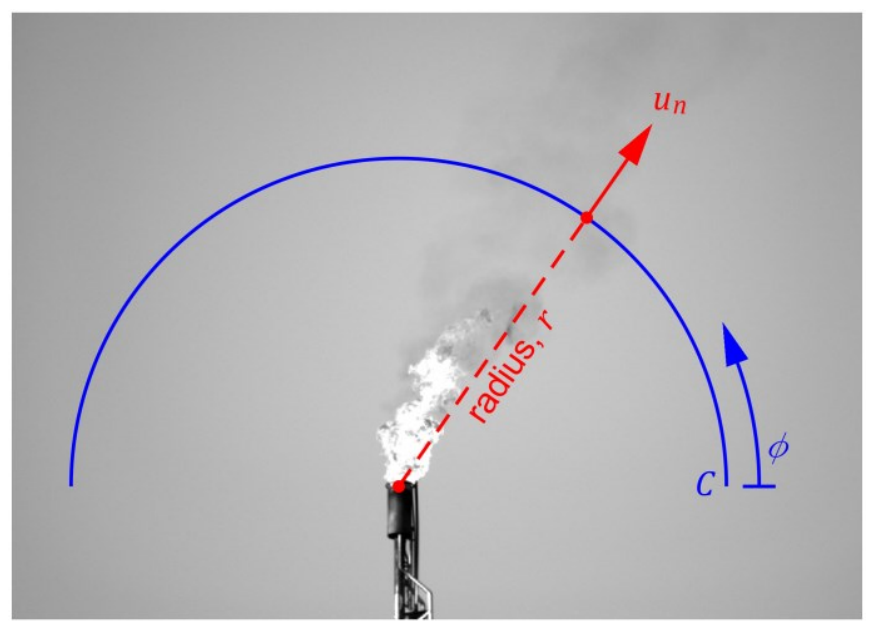

Figure 6.1: Sample sky-LOSA image of the flare and atmospheric plume, which is under slight crosswind in this example. A control surface $(C)$ is shown in blue, which is defined by its constant radius $(r)$ and the angle $\phi$. At each point on the control surface, the mass column density $\left(\rho_{s}^{\prime}(r, \phi)\right.$, not shown) is computed via careful consideration of radiative effects along the lines-of-sight (perpendicular to the image plane) that compose the control surface. Additionally, the path-averaged normal plume velocity $\left(u_{n}(r, \phi)\right)$ is computed via image correlation velocimetry. The instantaneous mass emission rate is computed by integrating the product of these over the control surface defined by $r d \phi$.

Overlaid in Figure 6.1 is an example control surface $(C)$ of specified radius $(r[\mathrm{~m}])$, through which the instantaneous mass emission rate of soot $\left(\dot{m}_{s}\left[\mathrm{~g} \mathrm{~s}^{-1}\right]\right)$ may be computed. For an arbitrary control surface in three dimensions, the instantaneous mass flux of soot through the surface is:

$$
\dot{m}_{s}=\iint_{A}\left(\rho_{s} \boldsymbol{u}_{3 D}\right) \cdot \boldsymbol{n} d A
$$

where $\rho_{s}$ is the mass concentration of soot $\left[\mathrm{g} \mathrm{m}^{-3}\right], \boldsymbol{u}_{3 D}$ is the three-dimensional local plume velocity vector $\left[\mathrm{m} \mathrm{s}^{-1}\right], \boldsymbol{n}$ is the unit vector locally normal to the control surface [-], and $d A$ is an infinitesimal area $\left[\mathrm{m}^{2}\right]$. For sky-LOSA, where three-dimensional data along 
a pixel's line-of-sight (LOS) are collapsed to two-dimensions through projection, an equivalent formulation for the instantaneous mass emission rate is:

$$
\dot{m}_{s}=\int_{C} \rho_{s}^{\prime}(r, \phi) u_{n}(r, \phi) r d \phi
$$

where $\rho_{s}^{\prime}(r, \phi)=\int \rho_{s}(r, \phi, x) d x$ is the soot mass column density along a LOS (where the $x$-dimension is orthogonal to the image plane) $\left[\mathrm{g} \mathrm{m}^{-2}\right]$ and $u_{n}(r, \phi)=$ $\left(\int \rho_{s}(r, \phi, x) \boldsymbol{u}_{3 D}(r, \phi, x)\right) \cdot \boldsymbol{n}(\phi) d x / \rho_{s}^{\prime}(r, \phi)$ represents the component of the mass concentration-weighted, LOS-averaged velocity of the plume $\left[\mathrm{m} \mathrm{s}^{-1}\right]$ that is normal to the control surface from the camera's perspective (as shown in the figure). Via Eq. (6.2), skyLOSA thus requires knowledge of three items to compute the emission rate: spatial scaling of the image plane to accurately quantify $r$, the velocity field of the plume within the image plane (yielding $u_{n}(r, \phi)$ ), and the soot mass column density resolved over the control surface. Spatial scaling of the image is obtained through use of a pinhole analogy for the sky-LOSA optics, coupled with a measurement of the distance to the flare stack tip by laser rangefinder (e.g., Laser Technology Inc. TruPulse 360R) (Johnson et al. 2013). Given that imaging is performed with a global shutter and at a sufficiently rapid framerate and exposure, the two-dimensional plume velocity field over the image plane is estimated via image correlation velocimetry (ICV) using a third party software suite such as LaVision DaVis 8.4 that includes a means of uncertainty quantification (Wieneke 2015). Finally, the novel enabling aspect of sky-LOSA is the use of bounded knowledge of soot optical properties from literature data to compute the soot mass column density with accurate uncertainties via radiometric observations and modelling of radiative transfer within the atmosphere and plume. 
Figure 6.2 shows an example positioning/pointing of the sky-LOSA camera and an optical axis/LOS within the surrounding skydome. For a given LOS, a cartesian coordinate system is defined where the positive $x$-direction is the path that light travels into the camera, the positive $z$-direction is the general direction of plume motion, and the $y$-dimension completes the orthogonal system. To model radiative transfer, there are three boundary conditions that must be considered. Firstly, the ground is treated as a cold, black surface and is thus ignored within the sky-LOSA algorithm. Secondly, the sky is modelled as a diffuse, polarized source concomitant with atmospheric scattering of solar radiation. The distribution of skylight intensity $\left(I_{s k y}\left(\alpha, \alpha_{s}, Z, Z_{s}, a\right)\left[\mathrm{W} \mathrm{m}^{-2} \mathrm{sr}^{-1}\right]\right)$ is considered using the standard models of the Commission Internationale de l'Eclairage (CIE, 2003), where the index $a \in\{1 \ldots 15\}$ indicates a specific CIE sky "type". The incident intensity of skylight for an optical axis $\left(I^{o}\left(\alpha_{s}, \beta, Z_{s}, a\right)\left[\mathrm{W} \mathrm{m}^{-2} \mathrm{sr}^{-1}\right]\right)$ is generally calculated by the interpolation of skylight intensity behind the plume from the perspective of the camera but, within a modelling framework, can be estimated from the CIE model for $I_{s k y}$. Finally, ground-level normal solar irradiance $\left(E_{s}\left[\mathrm{~W} \mathrm{~m}^{-2}\right]\right)$ is estimated using in-field, image-based measurements of the sun (Johnson et al. 2013) or modelled using the CIE models as described in Section 6.4.1.3. With this radiative transfer model, sky-LOSA-quantification of soot mass column density proceeds with the radiative transfer equation (RTE):

$$
I^{t}=I^{o} \exp \left(-\sigma_{e}^{m}(\boldsymbol{b}) \int_{-\infty}^{L} \rho_{s}(x) d x\right)+\int_{-\infty}^{L} J^{s, m}(x, \boldsymbol{b}) \rho_{s}(x) \exp \left(-\sigma_{e}^{m}(\boldsymbol{b}) \int_{x}^{L} \rho_{s}\left(x^{\prime}\right) d x^{\prime}\right) d x
$$

where $I^{t}$ is the measured "transmitted" intensity at the camera [W $\left.\mathrm{m}^{-2} \mathrm{sr}^{-1}\right], \sigma_{e}^{m}(\boldsymbol{b})$ is the mass-normalized extinction cross-section of soot $\left[\mathrm{m}^{2} \mathrm{~g}^{-1}\right]$ that is a function of eight soot properties (summarized in Section C.2) represented by the vector $\boldsymbol{b}, J^{s, m}(x, \boldsymbol{b})$ is a local 
source radiant intensity per unit mass $\left[\mathrm{W} \mathrm{sr}^{-1} \mathrm{~g}^{-1}\right]$ along the measurement path, and the sky-LOSA camera is located at $x=L$. Optical properties of soot are computed from the fundamental properties in $\boldsymbol{b}$ using Rayleigh-Debye-Gans theory for polydisperse fractal aggregates (RDG-PFA; e.g., Sorensen 2001).

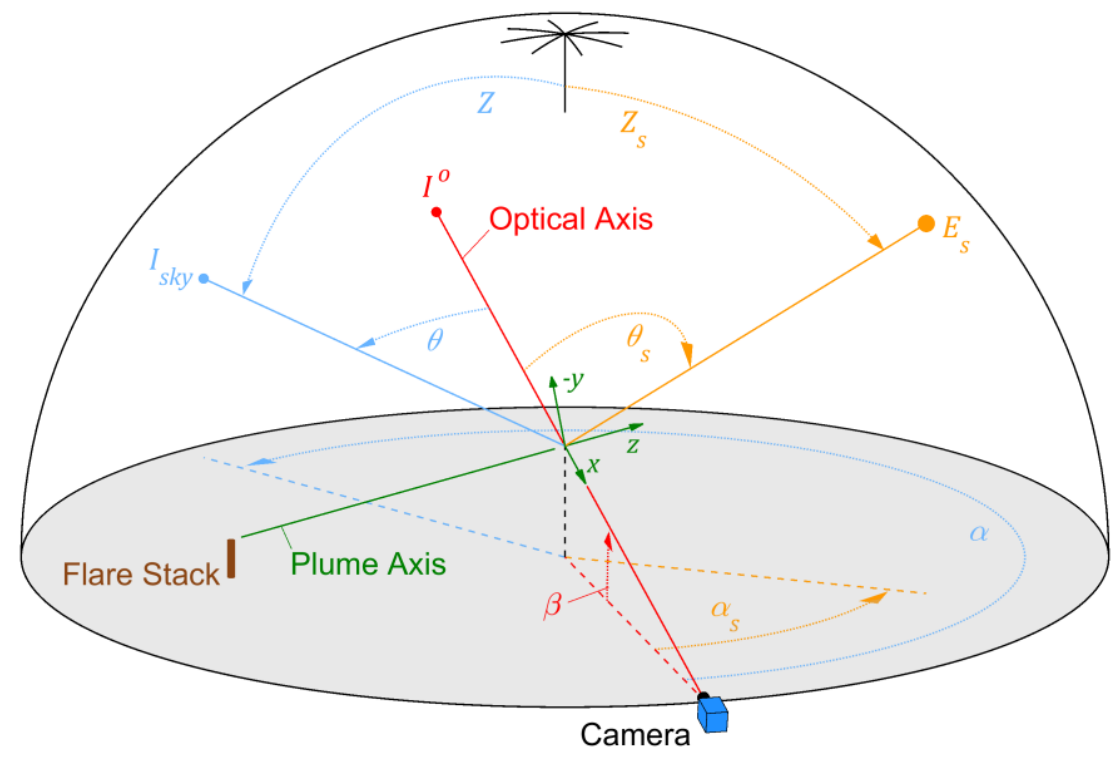

Figure 6.2: Schematic of a sky-LOSA measurement under the hemispherical sky dome showing the camera's optical axis relative to the horizon $(\beta)$, sun $\left(\alpha_{s}, \theta_{s}, Z_{s}\right)$, and example sky element $(\alpha, \theta, Z)$ (taken from Chapter 4 , Conrad et al. 2020b).

Using mean value theorem, a path-averaged source radiant intensity $\left(\overline{J^{s, m}}(\boldsymbol{b})\right.$ [W sr $\left.\mathrm{g}^{-1}\right]$ ) can be introduced to simplify the RTE:

$$
I^{t}=I^{o} \underbrace{\exp \left(-\sigma_{e}^{m}(\boldsymbol{b}) \int_{-\infty}^{L} \rho_{s}(x) d x\right)}_{\tau^{*}}+\underbrace{\frac{\overline{J^{s, m}}(\boldsymbol{b})}{\sigma_{e}^{m}(\boldsymbol{b})}}_{1-\tau^{*}} \underbrace{\int_{-\infty}^{L} \sigma_{e}^{m}(\boldsymbol{b}) \rho_{s}(x) \exp \left(-\sigma_{m}^{e}(\boldsymbol{b}) \int_{x}^{L} \rho_{s}\left(x^{\prime}\right) d x^{\prime}\right) d x}_{\bar{I}^{s}}
$$

where $\sigma_{e}^{m}(\boldsymbol{b}) / \sigma_{e}^{m}(\boldsymbol{b})=1$ has also been introduced to the source term. The resulting ratio in front of the second integral represents a path-averaged source intensity $\left(\overline{I^{s}}\left[\mathrm{~W} \mathrm{~m}{ }^{-2} \mathrm{sr}^{-1}\right]\right)$ and the exponential in the first term corresponds to the transmittance of the plume in the absence of radiative sources, defined as the idealized transmittance $\tau^{*}$ (Johnson et al. 
2013). It can be shown that the integral in the second term is equal to the complement of the idealized transmittance, permitting solution of the latter from the three intensities in Eq. (6.4):

$$
\tau^{*}=\frac{I^{t}-\overline{I^{S}}}{I^{o}-\bar{I}^{S}}=\frac{\tau_{o b s}-S}{1-S}
$$

where $\tau_{o b s}=I^{t} / I^{o}$ is the observed transmittance of the plume $[-]$ and $S=\bar{I}^{s} / I^{o}$ is a term that corrects for brightening of the plume by radiative sources [-]. Noting the definition of the idealized transmittance, the column density of soot is simply:

$$
\rho_{S}^{\prime}\left(\tau_{o b s}, S, \boldsymbol{b}\right)=\frac{-\ln \left(\frac{\tau_{o b s}-S}{1-S}\right)}{\sigma_{e}^{m}(\boldsymbol{b})}
$$

In general, the local source radiant intensity $\left(J^{s, m}(x, \boldsymbol{b})\right)$ is composed of thermal emission and inscattering components. For sky-LOSA however, where measurements are performed in the mid-visible spectrum and plume temperatures are near ambient, thermal emission negligibly contributes to the source term of the RTE (Chapter 3, Conrad et al. 2020a). By contrast, diffuse skylight and direct solar radiation can significantly augment the RTE via inscattering into the optical axis; hence, for sky-LOSA, $S$ represents an inscattering correction. Unfortunately, even if exact knowledge of skylight intensity distribution and solar radiation were available, it is not possible to fully account for the effect of inscattering without prior knowledge of the spatial distribution of soot within the plume. This is because multiple scattering events may occur during light's transmission into the camera. A recent simulation effort, however, has shown that complex multiple scattering effects can be accurately modelled to permit quantification of the inscattering 
correction (S) (refer to Chapter 4, Conrad et al. 2020b). This approach requires calculation of the inscattering correction using a single-scattering assumption (1SA, subscript "1"):

$$
\begin{aligned}
S_{1}\left(\alpha_{s}, \beta, Z_{s}, a, \boldsymbol{b}\right)= & \underbrace{\int_{0}^{2 \pi} \int_{0}^{\pi / 2} \frac{I_{s k y}\left(\alpha, \alpha_{s}, Z, Z_{s}, a\right)}{I^{o}\left(\alpha_{s}, \beta, Z_{s}, a\right)} \frac{\omega(\boldsymbol{b})}{4 \pi} p(\theta(\alpha, \beta, Z), \boldsymbol{b}) \sin Z d Z d \alpha}_{S_{1, s k y}\left(\alpha_{s}, \beta, Z_{s}, a, b\right)} \\
& +\underbrace{\frac{E_{s}\left(Z_{s}, a\right)}{I^{o}\left(\alpha_{s}, \beta, Z_{s}, a\right)} \frac{\omega(\boldsymbol{b})}{4 \pi} p\left(\theta_{s}\left(\alpha_{s}, \beta, Z_{s}\right), \boldsymbol{b}\right)}_{S_{1, \text { sun }}\left(\alpha_{s}, \beta, Z_{s}, a, \boldsymbol{b}\right)}
\end{aligned}
$$

where $\omega(\boldsymbol{b})$ is the single-scattering albedo of the polydisperse soot population $[-], p(\theta, \boldsymbol{b})$ is the scattering phase function of soot $\left[\mathrm{sr}^{-1}\right]$, and angles $\theta$ and $\theta_{s}$ represent the angles between the LOS and a region of sky or the sun as shown in Figure 6.2. The inscattering correction can be parsed into sky $\left(S_{1 . s k y}\right)$ and sun $\left(S_{1 . s u n}\right)$ components. Following Conrad et al. (Chapter 4, 2020b), the 1SA-estimated inscattering correction permits calculation of the idealized transmittance - i.e., $\tau^{*}=\tau^{*}\left(\tau_{o b s}, S_{1}\left(\alpha_{s}, \beta, Z_{s}, a, b\right)\right)$. Ultimately, this allows for calculation of the soot mass column density by:

$$
\rho_{s}^{\prime}\left(\alpha_{s}, \beta, Z_{s}, \tau_{o b s}, a, \boldsymbol{b}\right)=\frac{-\ln \tau^{*}\left(\tau_{o b s}, S_{1}\left(\alpha_{s}, \beta, Z_{s}, a, \boldsymbol{b}\right)\right)}{\sigma_{e}^{m}(\boldsymbol{b})}
$$

According to Eq. (6.8), sky-LOSA-computation of soot mass column density is a function of the position of the camera and sun, field-observed plume transmittance, skylight intensity distribution and solar irradiance (through $a$ ), and soot properties. In the sky-LOSA algorithm, uncertainty in soot mass column density is directly calculated by MC analysis over uncertain variables, which include soot properties and other intermediate parameters. This implies that uncertainty in sky-LOSA-computed soot mass column density is sensitive to $\alpha_{s}, \beta, Z_{s}, \tau_{o b s}$, and $a$. Of these five variables, only the pointing of 
the camera may be controlled by the user, through the selection of angles $\alpha_{s}$ and $\beta$. Critically, these user-selected angles are known to influence sky-LOSA measurement uncertainty and, in extreme cases, can even preclude computation of soot emission rate.

\subsubsection{Objective}

For end-users of sky-LOSA, the sensitivity of measurement uncertainty to camera pointing necessitates a standardized (and ideally simple) data acquisition protocol to optimize camera position and pointing under general conditions. This would allow a priori setup decisions to minimize or constrain uncertainties within reasonable limits. An acquisition protocol must therefore be constructed using quantitative knowledge of measurement uncertainty in sky-LOSA-computed soot mass column density. The objective of this work is to quantify, via a comprehensive general uncertainty analysis, the uncertainty in skyLOSA-computation of soot mass column density as a function of user-selectable and uncontrollable parameters under generalized conditions. These data permit the development of broad heuristics and, ultimately, an easy-to-use software tool to provide specific case-by-case constraints on camera position and pointing.

\subsection{General Uncertainty Analysis Methodology}

The goal of the present general uncertainty analysis (GUA) is to guide a sky-LOSA user in choosing a sky-LOSA camera position/pointing to minimize measurement uncertainties. The developed software tool can also be used to give an initial estimate of uncertainties in the measured soot emission rate ahead of a more detailed post-processing analysis. To provide generalized recommendations, the GUA quantifies measurement uncertainty in soot mass column density for a selected camera pointing and other independent variables via $\mathrm{MC}$ analysis over uncertain variables that include all relevant soot properties. This 
section describes the MC method used in the GUA and special considerations to make this work tractable.

\subsubsection{Special Considerations for MC Analysis}

\subsubsection{Expansion of the Scattering Phase Function}

For a given (modelled) skylight intensity distribution, measured/modelled solar irradiance, camera pointing, and set of soot properties, the 1SA-estimated inscattering correction $\left(S_{1}\right)$ can be directly calculated via Eq. (6.7). One significant challenge, and currently the timelimiting computation in sky-LOSA processing, is the calculation of the skylight component

$\left(S_{1, s k y}\right)$ via numerical integration over three dimensions: $\alpha, Z$, and $N$, where integration over the latter represents the aggregate size distribution of the polydisperse soot population. The complexity of this task is exacerbated by the scattering phase function $(\operatorname{SPF}, p(\theta, \boldsymbol{b}))$ of soot, which includes a computationally burdensome hypergeometric series in its solution (Sorensen 2001). For the present GUA, where the inscattering correction must be computed over a five-dimensional domain $\left(\alpha_{s}, \beta, Z_{s}, a, b\right)$, an alternative, more rapid means of computing the inscattering correction was required to avoid combinatorial explosion.

One such means is through a Fourier-Legendre expansion of the SPF. For an arbitrary set of MC-randomized soot properties $\boldsymbol{b}$, this procedure allows the SPF to be represented as:

$$
p(\theta, \boldsymbol{b})=\sum_{l=0}^{\infty} \Phi_{l}(\boldsymbol{b}) P_{l}(\cos \theta) \approx \sum_{l=0}^{L(\boldsymbol{b})} \Phi_{l}(\boldsymbol{b}) P_{l}(\cos \theta)
$$


where $P_{l}(\cos \theta)$ is the $l^{\text {th }}$-order Legendre polynomial $[-]$ and $\Phi_{l}(\boldsymbol{b})$ is the $l^{\text {th }}$-order Legendre coefficient $\left[\mathrm{sr}^{-1}\right]$ (hereinafter termed the $l^{\text {th }}$-order soot coefficient) for the set of soot properties $(\boldsymbol{b})$ computed via:

$$
\Phi_{l}(\boldsymbol{b})=\frac{2 l+1}{2} \int_{0}^{\pi} p(\theta, \boldsymbol{b}) P_{l}(\cos \theta) \sin \theta d \theta
$$

which can be accurately and efficiently computed via Gauss-Legendre quadrature (Schuster 2004). Since the soot coefficient decreases towards zero as the order $l$ approaches infinity, the infinite series expansion of the SPF can be truncated at a sufficiently large index $L(\boldsymbol{b})$ with negligible error.

\subsubsection{Sky and Sun Inscattering}

Introduction of the Fourier-Legendre-expanded SPF into the sky and sun components of the inscattering correction $\left(S_{1, \text { sky }}\right.$ and $S_{1, \text { sun }}$, Eq. (6.7)) yields:

$$
\begin{gathered}
S_{1, s k y}\left(\alpha_{s}, \beta, Z_{s}, a, \boldsymbol{b}\right)=\frac{\omega(\boldsymbol{b})}{4 \pi} \sum_{l=0}^{L(\boldsymbol{b})} \Phi_{l}(\boldsymbol{b}) \underbrace{\int_{0}^{2 \pi} \int_{0}^{\frac{\pi}{2}} \frac{I_{s k y}\left(\alpha, \alpha_{s}, Z, Z_{s}, a\right)}{I^{o}\left(\alpha_{s}, \beta, Z_{s}, a\right)} P_{l}(\cos \theta(\alpha, \beta, Z)) \sin Z d Z d \alpha}_{\Psi_{l, s k y}\left(\alpha_{s}, \beta, Z_{s}, a\right)} \\
S_{1, \text { sun }}\left(\alpha_{s}, \beta, Z_{s}, a, \boldsymbol{b}\right)=\frac{\omega(\boldsymbol{b})}{4 \pi} \sum_{l=0}^{L(\boldsymbol{b})} \Phi_{l}(\boldsymbol{b}) \underbrace{\frac{E_{S}\left(Z_{s}, a\right)}{I^{o}\left(\alpha_{s}, \beta, Z_{s}, a\right)} P_{l}\left(\cos \theta_{s}\left(\alpha_{s}, \beta, Z_{s}\right)\right)}_{\Psi_{l, \text { sun }}\left(\alpha_{s}, \beta, Z_{s}, a\right)}
\end{gathered}
$$

where $\Psi_{l, s k y}\left(\alpha_{s}, \beta, Z_{s}, a\right)$ and $\Psi_{l, \text { sun }}\left(\alpha_{s}, \beta, Z_{s}, a\right)$ are denoted as the sky and sun coefficients, respectively. Importantly, these equations show that use of the expanded SPF removes reference to soot properties from the integral in the computation of $S_{1, s k y}$, which vastly reduces computational burden. Furthermore, with this formulation, the soot 
coefficients (functions of $\boldsymbol{b}$ ) and sky/sun coefficients (functions of $\alpha_{s}, \beta, Z_{s}$, and $a$ ) do not share any independent variables and can therefore be independently pre-computed.

\subsubsection{Solar Irradiance}

As noted in Section 6.3, ground-level solar normal irradiance $-E_{S}\left(Z_{s}, a\right)$, usually measured via solar images taken in the field - can be modelled using the CIE skylight models. To accomplish this, the incident intensity-normalized solar normal irradiance is expanded:

$$
\frac{E_{s}\left(Z_{s}, a\right)}{I^{o}\left(\alpha_{s}, \beta, Z_{s}, a\right)}=\frac{E_{s}\left(Z_{s}, a\right)}{D_{h}\left(Z_{s}, a\right)} \frac{D_{h}\left(Z_{s}, a\right)}{I^{o}\left(\alpha_{s}, \beta, Z_{s}, a\right)}
$$

where $D_{h}\left(Z_{s}, a\right)$ is the diffuse horizontal irradiance, calculable for the CIE models via numerical integration of:

$$
D_{h}\left(Z_{s}, a\right)=\int_{0}^{2 \pi} \int_{0}^{\frac{\pi}{2}} I_{s k y}\left(\alpha, \alpha_{s}, Z, Z_{s}, a\right) \cos Z \sin Z d Z d \alpha
$$

which is independent of the value of $\alpha_{s}$.

The ratio of solar normal to diffuse horizontal irradiance is complex to quantify in a general sense as it is a function of atmospheric composition. However, for the purposes of the present GUA it is modelled as follows:

$$
\frac{E_{S}\left(Z_{s}, a\right)}{D_{h}\left(Z_{s}, a\right)}=\frac{E_{s h, o}\left(Z_{s}, a\right) \exp \left(-m\left(Z_{s}\right) \mu^{*}(m) T(a)\right)}{D_{h}\left(Z_{s}, a\right) \cos Z_{s}}
$$

The numerator of the righthand side is the ground-level solar horizontal irradiance, calculated as the product of the extra-terrestrial (ET, subscript "o") solar horizontal irradiance $\left(E_{s h, o}\left(Z_{s}, a\right)\right)$ with an exponential representing attenuation through the atmosphere. In computation of the latter, $m\left(Z_{s}\right)$ is the relative air mass quantifying the 
amount of air in the atmosphere at the solar zenith angle relative to the vertical direction, $\mu^{*}(m)$ is the ideal extinction for a clean atmosphere at a given relative air mass, and $T(a)$ is the model-dependent turbidity factor representing the number of clean atmospheres required to represent observations. In the denominator, the cosine is included to transform the numerator into the required ground-level solar normal irradiance. In the present work, typical values of the turbidity factor $(T(a))$ and irradiance ratio $D_{h}\left(Z_{s}, a\right) / E_{s h, o}\left(Z_{s}, a\right)$ are taken from Darula and Kittler (2002) and Kittler et al. (2012), while their recommended formulations for $m\left(Z_{s}\right)$ (Kasten and Young 1989) and $\mu^{*}(m)$ (Navvab et al. 1984) are employed:

$$
\begin{aligned}
& m\left(Z_{s}\right)=\left(\cos Z_{s}+0.50572\left(96.07995^{\circ}-Z_{s}\right)^{-1.6364}\right)^{-1} \\
& \mu^{*}(m)=(9.9+0.043 m)^{-1}
\end{aligned}
$$

To model some amount of unknown uncertainty due to the use of "typical" metrics listed in the literature, an additional randomized variable $\left(\xi_{E D}(a)\right)$ was introduced as a scalar multiplier to the sun component of the inscattering correction. For CIE sky models with an unobstructed sun (types 7-15), $\xi_{E D}(a) \sim \mathcal{U}(0.75,1.25)$ and for models with an obstructed sun (types 1-6), $\xi_{E D}(a) \sim \mathcal{U}(0,1.25)$. These prior distributions of $\xi_{E D}(a)$ were based on observations by Watanabe et al. (2016), who studied the "clearness index" (ground-level horizontal normalized by ET horizontal irradiance) over five years at 47 observation stations across Japan. They found that the relative variation in the clearness index was approximately $4.3 \%$ for skies with unobscured suns and 35\% for skies with obscured suns; corresponding variance-equivalent uniform distributions would have a range of $15 \%$ and $121 \%$, respectively. For skies with an unobscured sun, this range was expanded to $50 \%(0.75-1.25)$ to give a conservatively broad prior since measurement 
uncertainty can be quite sensitive to solar irradiance. By contrast, for skies with an obscured sun, where the solar irradiance has a small contribution to measurement uncertainty, the uniform distribution was only slightly widened to $125 \%(0-1.25)$.

\subsubsection{Truncation of the Expanded SPF}

For the prior probability distributions of soot properties derived by Johnson et al. (2013), the total order of soot coefficients required to represent the soot SPF according to the procedure of Schuster (2004) was typically $L(\boldsymbol{b})=76$ (median). In the most extreme case however, representing a strongly forward scattering particle (corresponding to large soot aggregate size), $L(\boldsymbol{b})$ reached 698 , suggesting that pre-computation of the sky and sun coefficients up to $\Psi_{698}$ would be necessary to compute the inscattering correction in the worst case. While calculation of the sun coefficients to this large order is generally trivial, calculation of the sky coefficients becomes increasingly cumbersome as the order gets larger. Importantly though, like the soot coefficients, the magnitude of the sky coefficients approaches zero as the order approaches infinity; therefore, the product of $\Phi_{l}(\boldsymbol{b})$ and $\Psi_{l, s k y}\left(\alpha_{s}, \beta, Z_{s}, \boldsymbol{a}\right)$ more-rapidly decreases in magnitude as $l$ increases than either component alone. This permits further truncation of the series for the calculation of $S_{1, s k y}$. Specifically, calculation of $S_{1, s k y}$ via Eq. (6.11) using $L(\boldsymbol{b})=200$ was consistently in close agreement with direct numerical integration via Eq. (6.7) - where, over $10^{6} \mathrm{MC}$ randomized sets of $\left(\alpha_{s}, \beta, Z_{s}, a, b\right)$, the median relative difference was just $2.3 \times 10^{-7}$. This implies that it is acceptable to impose that $\Psi_{l, s k y}\left(\alpha_{s}, \beta, Z_{s}, a\right)=0, \forall l>200$.

\subsubsection{Sky Model Groups}

The standard CIE sky models have found good utility in a variety of fields, from urban planning (e.g., Acosta et al., 2014) to building design (e.g., Wong, 2017); however, the 
models naturally suffer from directionally dependent error in skylight intensity. This is particularly true for overcast and partly cloudy skies since the models, which are smooth functions, do not capture steep gradients in skylight intensity due to cloud structures. Thus, there is some additional uncertainty in sky-LOSA-computed soot mass column density through use of a single selected CIE sky model in the MC method. To permit capture of CIE sky model error in the GUA, like skies were compiled into sky model "groups" that have similar properties but differing model coefficients and, hence, directional variability the derived sky groups $(a \in\{A \ldots D\})$ are summarized in Table 6.1. By randomly selecting the sky group's component sky models under the MC framework, uncertainty through use of the CIE models is propagated into sky-LOSA-computation of soot mass column density.

\section{Table 6.1: Sky model "groups" derived to propagate error in the CIE sky models through the sky-LOSA algorithm computing soot mass column density.}

\begin{tabular}{|c|c|c|}
\hline Sky Group & CIE Sky Types $(\boldsymbol{a})$ & Description \\
\hline A & $1-6$ & $\begin{array}{c}\text { Overcast and partly cloudy } \\
\text { skies, obscured sun }\end{array}$ \\
\hline B & $7-10$ & $\begin{array}{c}\text { Partly cloudy skies, } \\
\text { unobscured sun }\end{array}$ \\
\hline C & $11-15$ & Clear skies, all \\
\hline D & $11,13-15$ & Clear skies, polluted \\
\hline
\end{tabular}

Sky group A corresponds to overcast and partly cloudy conditions with an obscured sun where typical turbidity factors of the component skies $(T(a))$ are high. Sky group B represents partly cloudy conditions with an unobscured sun where turbidity factors of the component skies are moderate and hemispherical skylight is strong relative to ET solar radiation (high $D_{h}\left(Z_{s}, a\right) / E_{s h, o}\left(Z_{s}, a\right)$ ). Sky groups $\mathrm{C}$ and $\mathrm{D}$ capture the low-turbidity clear CIE sky models. Sky group C includes all five clear sky models, while sky group D excludes CIE sky type 12, the lowest turbidity (i.e., cleanest atmosphere) model. Sky group 
D was defined based on the notion that oil and gas activities can be relatively dense geographically. In this case, field experience suggests that local emissions from industrial infrastructure likely precludes CIE sky type 12 as a reasonable model, such that sky group D can be used for the case of clear skies in heavily industrial locales.

\subsubsection{Implementation}

Table 6.2 summarizes the independent, pre-computed, and randomized variables required to compute soot mass column density under the GUA MC framework. There are five independent variables that define the pointing of the camera relative to the sun $\left(\alpha_{s}, \beta, Z_{s}\right)$, the observed plume transmittance ( $\left.\tau_{o b s}\right)$, and the skylight intensity distribution (CIE sky type, $a$ ). Each MC draw randomly chooses soot properties $(\boldsymbol{b})$, the scalar multiplier to the sun component of inscattering $\left(\xi_{E D}(a)\right)$, and, for analysis of the sky groups A-D, one CIE sky model from the selected sky group. The $\mathrm{k}^{\text {th }}$ MC-estimate of the soot mass column density is obtained via:

$$
\rho_{s, k}^{\prime}\left(\alpha_{s}, \beta, Z_{s}, \tau_{o b s}, a, \boldsymbol{b}_{k}\right)=\frac{-\ln \tau^{*}\left(\tau_{o b s}, S_{1, k}\left(\alpha_{s}, \beta, Z_{s}, a, \boldsymbol{b}_{k}\right)\right)}{\sigma_{e}^{m}\left(\boldsymbol{b}_{k}\right)}
$$

where $\tau^{*}\left(\tau_{o b s}, S_{1, k}\left(\alpha_{s}, \beta, Z_{s}, a, \boldsymbol{b}_{k}\right)\right)$ is deterministically computed while considering multiple scattering effects, as described by Conrad et al. (Chapter 4, 2020b), and:

$$
S_{1, k}\left(\alpha_{s}, \beta, Z_{s}, a, \boldsymbol{b}_{k}\right)=\frac{\omega\left(\boldsymbol{b}_{k}\right)}{4 \pi} \sum_{l=0}^{L\left(\boldsymbol{b}_{k}\right)} \Phi_{l}\left(\boldsymbol{b}_{k}\right)\left(\Psi_{l, s k y}\left(\alpha_{s}, \beta, Z_{s}, a\right)+\xi_{E D, k}(a) \Psi_{l, s u n}\left(\alpha_{s}, \beta, Z_{s}, a\right)\right)
$$

and $a \equiv a_{k}$ for analysis of sky groups $\mathrm{A}-\mathrm{D}$. 
Table 6.2: Summary of independent, MC-randomized, and pre-computed variables in the GUA.

\begin{tabular}{|c|c|c|c|c|c|}
\hline $\begin{array}{l}\text { Variable } \\
\text { Group }\end{array}$ & Variable Name & Symbol & Unit & Source $^{a}$ & $\begin{array}{c}\text { GUA MC } \\
\text { Implementation }^{b}\end{array}$ \\
\hline \multirow{11}{*}{$\begin{array}{l}\text { Ambient } \\
\text { lighting }\end{array}$} & Sky model coefficients & $a$ & {$[-]$} & \multirow{3}{*}{ CIE models } & $\begin{array}{c}\text { Independent/ } \\
\text { MC-randomized }^{\mathrm{c}}\end{array}$ \\
\hline & Skylight intensity & $I\left(\alpha, \alpha_{s}, Z, Z_{s}, a\right)$ & {$\left[\mathrm{W} \mathrm{m}^{-2} \mathrm{sr}^{-1}\right]$} & & \multirow{9}{*}{$\begin{array}{l}\text { Pre-computation of sky } \\
\text { and sun coefficients }\end{array}$} \\
\hline & Incident skylight intensity & $I^{o}\left(\alpha_{s}, \beta, Z_{s}, a\right)$ & {$\left[\mathrm{W} \mathrm{m}{ }^{-2} \mathrm{sr}^{-1}\right]$} & & \\
\hline & Diffuse horizontal irradiance & $D_{h}\left(Z_{s}, a\right)$ & {$\left[\mathrm{W} \mathrm{m}^{-2}\right]$} & Eq. (6.14) & \\
\hline & $\begin{array}{c}\text { Diffuse-to-ET solar } \\
\text { horizontal irradiance ratio }\end{array}$ & $\frac{D_{h}\left(Z_{s}, a\right)}{E_{s h, o}\left(Z_{s}, a\right)}$ & {$[-]$} & \multirow[t]{2}{*}{$\begin{array}{l}\text { Typical } \\
\text { values }^{\mathrm{d}}\end{array}$} & \\
\hline & Turbidity factor & $T(a)$ & {$[-]$} & & \\
\hline & Relative air mass & $m\left(Z_{s}\right)$ & {$[-]$} & \multirow{2}{*}{ Eq. (6.16) } & \\
\hline & Ideal (clean atmosphere) extinction & $\mu^{*}(m)$ & {$[-]$} & & \\
\hline & Sky coefficients & $\Psi_{l, s k y}\left(\alpha_{s}, \beta, Z_{s}, a\right)$ & {$[-]$} & Eq. (6.11) & \\
\hline & Sun coefficients & $\Psi_{l, \text { sun }}\left(\alpha_{s}, \beta, Z_{s}, a\right)$ & {$[-]$} & Eq. (6.12) & \\
\hline & Irradiance ratio scaling & $\xi_{E D}(a)$ & {$[-]$} & $\begin{array}{l}\sim \mathcal{U}(0.00,1.25) \text { or } \\
\sim \mathcal{U}(0.75,1.25)^{\mathrm{e}}\end{array}$ & MC-randomized \\
\hline \multirow{5}{*}{$\begin{array}{c}\text { Soot } \\
\text { properties }\end{array}$} & Fundamental soot properties ${ }^{\mathrm{f}}$ & $\boldsymbol{b}$ & $\begin{array}{l}\text { Property- } \\
\text { dependent }^{\mathrm{f}}\end{array}$ & Prior distributions ${ }^{\mathrm{f}}$ & MC-randomized \\
\hline & Mass-normalized extinction cross-section & $\sigma_{e}^{m}(\boldsymbol{b})$ & {$\left[\mathrm{m}^{2} \mathrm{~g}^{-1}\right]$} & RDG-PFA & \multirow{4}{*}{$\begin{array}{l}\text { Pre-computation of } \\
\text { soot/Legendre } \\
\text { coefficients }\end{array}$} \\
\hline & Single-scattering albedo & $\omega(\boldsymbol{b})$ & {$[-]$} & RDG-PFA & \\
\hline & Scattering phase function & $p(\theta, \boldsymbol{b})$ & {$\left[\mathrm{sr}^{-1}\right]$} & RDG-PFA & \\
\hline & Soot/Legendre coefficients & $\Phi_{l}(\boldsymbol{b})$ & {$[-]$} & Eq. $(6.10)$ & \\
\hline Plume loading & Observed plume transmittance & $\tau_{\text {obs }}$ & {$[-]$} & --- & Independent \\
\hline
\end{tabular}


Table 6.2 (continued): Summary of independent, MC-randomized, and pre-computed variables in the GUA.

\begin{tabular}{|c|c|c|c|c|}
\hline $\begin{array}{c}\text { Variable } \\
\text { Group }\end{array}$ & Variable Name & Symbol & Unit $^{\text {Source }}$ & $\begin{array}{c}\text { GUA MC } \\
\text { Implementation }^{\mathbf{b}}\end{array}$ \\
\hline \multirow{3}{*}{ Camera pointing } & Relative solar azimuth & $\alpha_{s}$ & $\left.{ }^{\circ}\right]$ \\
\cline { 2 - 4 } & Camera inclination & $\beta$ & $\left.{ }^{\circ}\right]$ & --- \\
\cline { 2 - 4 } & Solar zenith & $Z_{s}$ & $\left.{ }^{\circ}\right]$ \\
\hline
\end{tabular}

a The means by which variables are randomly drawn or computed.

b The method by which a variable is considered in the GUA MC method. Given a set of "independent" and "MC-randomized" variables, pre-computed data $\left(\Psi_{l, s k y}\left(\alpha_{s}, \beta, Z_{s}, a\right), \Psi_{l, s u n}\left(\alpha_{s}, \beta, Z_{s}, a\right), \sigma_{e}^{m}(\boldsymbol{b})\right.$, and $\left.\Phi_{l}(\boldsymbol{b})\right)$ are obtained and used to calculate soot mass column density.

c Sky model coefficients are independent variables in the analysis of a single skylight intensity model $(a \in\{1 \ldots 15\})$ but, for the derived sky groups $(a \in$ $\{A \ldots D\})$, skylight intensity models are randomized following a discrete uniform distribution with support over the skylight intensity models included in the sky group.

d Typical values as listed for each CIE sky model as per the literature (Kittler et al. 2012; Darula and Kittler 2002).

e Author-selected distributions: $\mathcal{U}(0.00,1.25)$ for CIE sky type $1-6$ and $U(0.75,1.25)$ for CIE sky types $7-15$.

${ }^{\mathrm{f}}$ Prior probability distributions for soot properties $(\boldsymbol{b})$ are summarized in Section C.2 and include absorption function of the spectral refractive index, $E\left(m_{\lambda}\right)[-]$; scattering-to-absorption function of the spectral refractive index, $F\left(m_{\lambda}\right) / E\left(m_{\lambda}\right)$ [-]; soot primary particle diameter, $d_{p}$ [nm]; geometric mean aggregate size, $N_{g}[-]$; geometric standard deviation of aggregate size, $\sigma_{g}[-]$; fractal prefactor, $k_{f}[-]$; fractal (Hausdorff) dimension, $D_{f}[-]$; and soot material density, $\varrho_{s}\left[\mathrm{~kg} \mathrm{~m}^{-3}\right]$. 
In a standard MC analysis, the above procedure would be iterated upon $K$ times to yield a collection of soot mass column density estimates from which a distribution of soot mass column density could be computed. To accelerate MC procedures in this work, a MC variance reduction technique was employed - specifically, combined multiple Latin hypercube sampling (CM-LHS) summarized by Nakayama (2011). This variance reduction technique has been used previously for sky-LOSA (Chapter 2, Conrad and Johnson 2017) and was found to reduce computational burden by a factor of 2-3. For the GUA, $5 \times 10^{5}$ (500 sets of 1,000 Latin hypercube sampled data) CM-LHS MC draws were completed. The GUA MC approach permitted pre-computation of the soot coefficients, single-scattering albedo, and mass-normalized extinction cross-section for pre-drawn CM-LHS MC-randomized sets of soot properties $(\boldsymbol{b})$. Parallel pre-computation of the sky and sun coefficients was performed for each of the 15 CIE standard skylight intensity distributions and four sky groups over the angles $\alpha_{s}, \beta$, and $Z_{s}$ in increments of $2^{\circ}$, and for 18 observed transmittances from $0.25-0.99$. This amounted to execution of the CM-LHS MC analysis for almost $66 \times 10^{6}$ unique sky-LOSA conditions, permitting derivation of uncertainty statistics over the five independent variables listed in Table 6.2.

\subsubsection{Relative Uncertainty Metric}

To enable an objective comparison of sky-LOSA uncertainty as a function of the independent variables, a parameter describing the relative uncertainty of MC-computed soot mass column density was required. Since relative uncertainty is a comparison of data variability to central tendency, a natural metric is the coefficient of variation $(\mathrm{CV})$, which is the ratio of the standard deviation to the mean of a distribution or dataset. These momentbased statistics, however, are sensitive to outliers (e.g., Devore and Berk, 2012) or long- 
tailed/skewed distributions, causing some to suggest order statistics as more-robust metrics for variability and central tendency (e.g., Arachchige et al., 2019). For sky-LOSA, MCand time-averaged soot mass emission rates (moment-based statistic) have historically been reported alongside MC-computed 95\% confidence intervals (CIs, order statistic). Therefore, for consistency with past and future sky-LOSA measurements, a CV-estimator based on these statistics was used in this work. For variability, the width of the $95 \%$ CI scaled by that of a normal standard distribution $(\approx 3.92)$ was used as an estimator of the standard deviation, while the mean $(\overline{\boldsymbol{x}})$ was employed as the measure of central tendency. The CV-estimator for soot mass column density (or any MC-computed data $\boldsymbol{x}$ ) is thus:

$$
C V_{95}(\boldsymbol{x})=\frac{\mathcal{F}_{x}^{-1}(0.975)-\mathcal{F}_{x}^{-1}(0.025)}{3.92 \bar{x}}
$$

where subscript " 95 " signifies use of the $95 \%$ CI for variability and $\mathcal{F}_{\boldsymbol{x}}^{-1}(q)$ is the $q^{\text {th }}$ quantile of data vector $\boldsymbol{x}$.

\subsection{Results and Discussion}

\subsubsection{Representative Results}

Figure 6.3 shows relative uncertainty results at different camera pointings/positions for an example sky-LOSA measurement scenario of a flare with $90 \%$ observed plume transmittance. The selected solar zenith angle $\left(Z_{s}=32.8^{\circ}\right)$ represents the annual minimum for the Canadian city of Fort St. John, British Columbia, which is located in the Montney oil and gas-producing formation. $C V_{95}$ data are plotted for sky groups $\mathrm{A}-\mathrm{D}$ in Figure 6.3a-d, respectively, as a function of relative solar azimuth ( $\alpha_{s}$ in Figure 6.2) and camera inclination $(\beta)$; the position of the sun in these coordinates $\left(180^{\circ}, 57.2^{\circ}\right)$ is overlaid 
in each subfigure. Additionally, contours displaying the solar scattering angle $\left(\theta_{s}\right)$ are overlaid in Figure 6.3d.
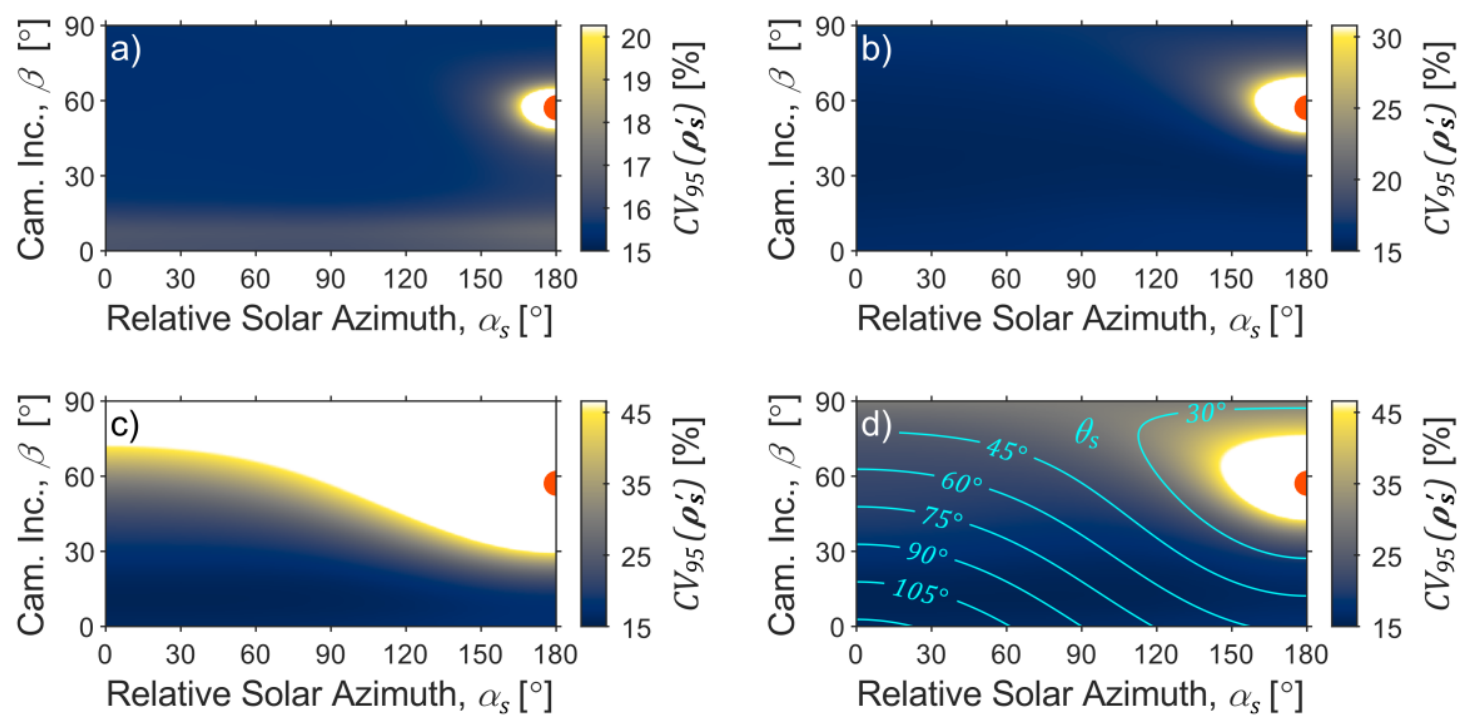

Figure 6.3: Example MC results at solar noon on the summer solstice in Fort St. John, British Columbia - solar zenith $\left(Z_{s}\right)$ of $32.8^{\circ}$ - for a plume of $90 \%$ observed plume transmittance $\left(\tau_{o b s}\right)$. Subplots $(\mathrm{a})-(\mathrm{d})$ show the relative uncertainty $\left(\mathrm{CV} \mathrm{V}_{95}\right)$ of soot mass column density as a function of relative solar azimuth $\left(\alpha_{s}\right)$ and camera inclination $(\beta)$ for sky groups $A-D$, respectively. Contour lines in subplot (d) show the scattering angle of sunlight into the sky-LOSA camera $\left(\theta_{s}\right)$.

There are two observable trends in the data of Figure 6.3 that persist through all measurement conditions. Firstly, the relative uncertainty is a strong function of the solar scattering angle, $\theta_{s}$. This is because of solar radiation's influence on $S_{1, \text { sky }}$ and $S_{1, \text { sun }}$. However, the rate at which relative uncertainty changes as a function of $\theta_{s}$ is sky model(as well as solar zenith- and plume transmittance-) dependent. This is partly a consequence of how $S_{1, s k y}$ varies with $\theta_{s}$, but is mostly due to variability in $S_{1, \text { sun }}$ with $\theta_{s}$. For example, the inscattering magnitude for sky group A is effectively due to $S_{1, s k y}$ alone due to high turbidity that strongly attenuates direct solar radiation while, for sky group $\mathrm{C}$, the sun's inscattering contribution $\left(S_{1, \text { sun }}\right)$ is significant due to the low turbidity of the sky. Thus, the results for sky group A largely represent the effect of $\theta_{s}$ on uncertainty through $S_{1, s k y}$ 
while the results for sky group $\mathrm{C}$ include an additional (and the most extreme) effect through $S_{1, \text { sun }}$. These observations imply that a constraint on $\theta_{S}$ is necessary, and that a stricter constraint is required for lower turbidity skies.

The mechanism for the decrease in relative uncertainty with increasing $\theta_{s}$ stems from the optical characteristics of soot particulate. Figure 6.4 shows statistics of the "energy distribution function" $(E D F=\omega(\boldsymbol{b}) p(\theta, \boldsymbol{b}) / 4 \pi)$ discussed in Chapter $4($ Conrad et al. 2020b), which describes how soot particulate directionally scatters light on an energybasis. The median and $C V_{95}$ of the EDF as a function of angle $\theta$ are shown for the range of the MC-sampled soot properties (b) used in the GUA. This is useful for the present discussion since the EDF contains all soot-dependent variables in the computation of $S_{1, \text { sun }}$ (see Eq. (6.7)). The figure shows that the EDF, and its influence on $S_{1, \text { sun }}$, is much larger and much more uncertain as $\theta_{s}$ decreases. The influence of these statistics on column density uncertainty depends on the relative intensity of sunlight - specifically $E_{s}\left(Z_{s}, a\right) / I^{o}\left(\alpha_{s}, \beta, Z_{s}, a\right)$ in Eq. (6.7). If this value is small (highly turbid skies), then variability in the EDF is less important, but if this value is large (low turbidity skies), then variability in the EDF can dominate relative uncertainty in soot mass column density. Interestingly, relative uncertainty in the EDF approaches a constant value towards $90^{\circ}$. This implies that, regardless of the ground-level intensity of the sun, the uncertainty of $S_{1, \text { sun }}$ becomes minimal at $\theta_{s} \widetilde{>} 90^{\circ}$ and is within $1 \%$ of this minimum for $\theta_{s} \widetilde{>} 60^{\circ}$ as indicated by the red line in Figure 6.4. 


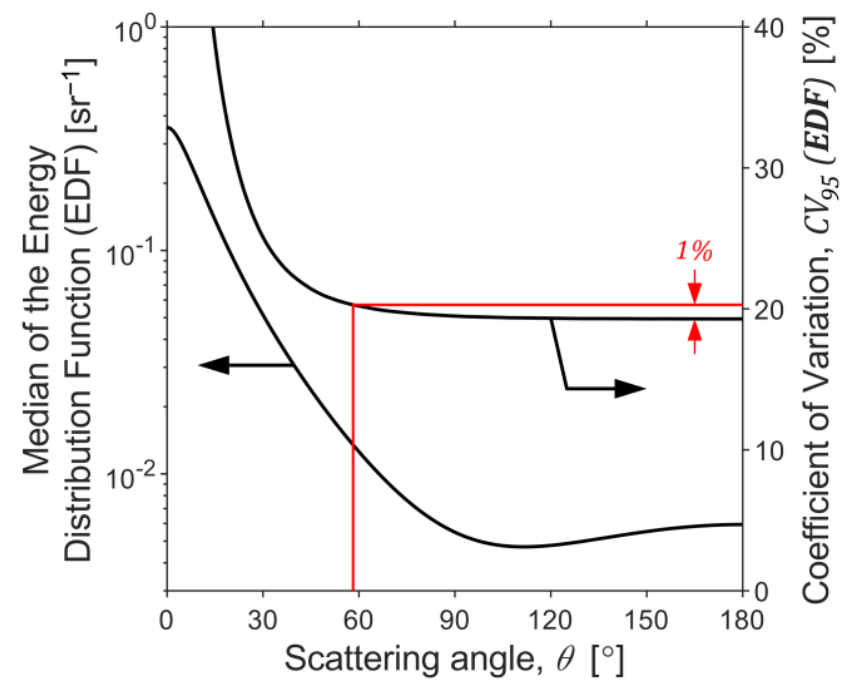

Figure 6.4: Central tendency (median; left logarithmic axis) and relative uncertainty ( $C V$ 95; right linear axis) of the "energy distribution function" $(E D F=\omega(b) p(\theta, b) / 4 \pi)$ that dictates the fraction of incident light energy scattered through angle $\theta$ by soot.

The magnitude and uncertainty in the EDF are much larger in the forward scattering direction (small $\theta$ ). As the scattering direction exceeds $\sim 90^{\circ}$ however, the relative uncertainty in the EDF approaches a constant minimum, implying that the relative uncertainty in $S_{1, \text { sun }}$ is minimized for $\theta_{s} \sim 90^{\circ}$. The red line shows that relative uncertainty is within $1 \%$ of the minimum if $\theta_{s}>60^{\circ}$.

The second trend in Figure 6.3 that is generally seen across all measurement conditions is the sensitivity of relative uncertainty to the camera inclination angle $(\beta)$. Referring to Figure 6.3 , much of the observed trend in $\beta$ can likely be attributed to the effect of $\theta_{s}$, since $\theta_{s}=\theta_{s}\left(\alpha_{s}, \beta, Z_{s}\right)$. However, $\beta$ still influences measurement uncertainty as can be observed in the region where the $\theta_{s}$ effect is small $\left(\theta_{s}>60^{\circ}\right)$. Figure $6.5 \mathrm{a}-\mathrm{e}$ show example trends in soot mass column density uncertainty as a function of $\beta$ for plume transmittances of 0.25 to 0.95 and a fixed solar azimuth of $\alpha_{s}=60^{\circ}$. These figures each plot $C V_{95}$ as a function of $\beta$, averaged over the range of $Z_{s}$ that ensures $\theta_{s}>60^{\circ}$. The trends are different for each sky model. Uncertainties for sky groups A and B tend to decrease as the camera inclines, while uncertainties for sky groups $\mathrm{C}$ and $\mathrm{D}$ increase. The severity of these trends increases with plume transmittance (effectively a reduction in the 
measured signal) and, as plume transmittance increases towards unity, local minima/maxima in uncertainties as a function of camera inclination may appear. The differing influence of $\beta$ between overcast/partly cloudy and clear sky models is largely due to the specific CIE sky models, which dictate the gradient in skylight intensity near the horizon and the sun. For clear, low-turbidity skies, intensity gradients are large such that small changes in camera pointing can yield significant changes in inscattered light. By contrast, for higher-turbidity skies, gradients are dampened, and the effect of camera pointing is small. 


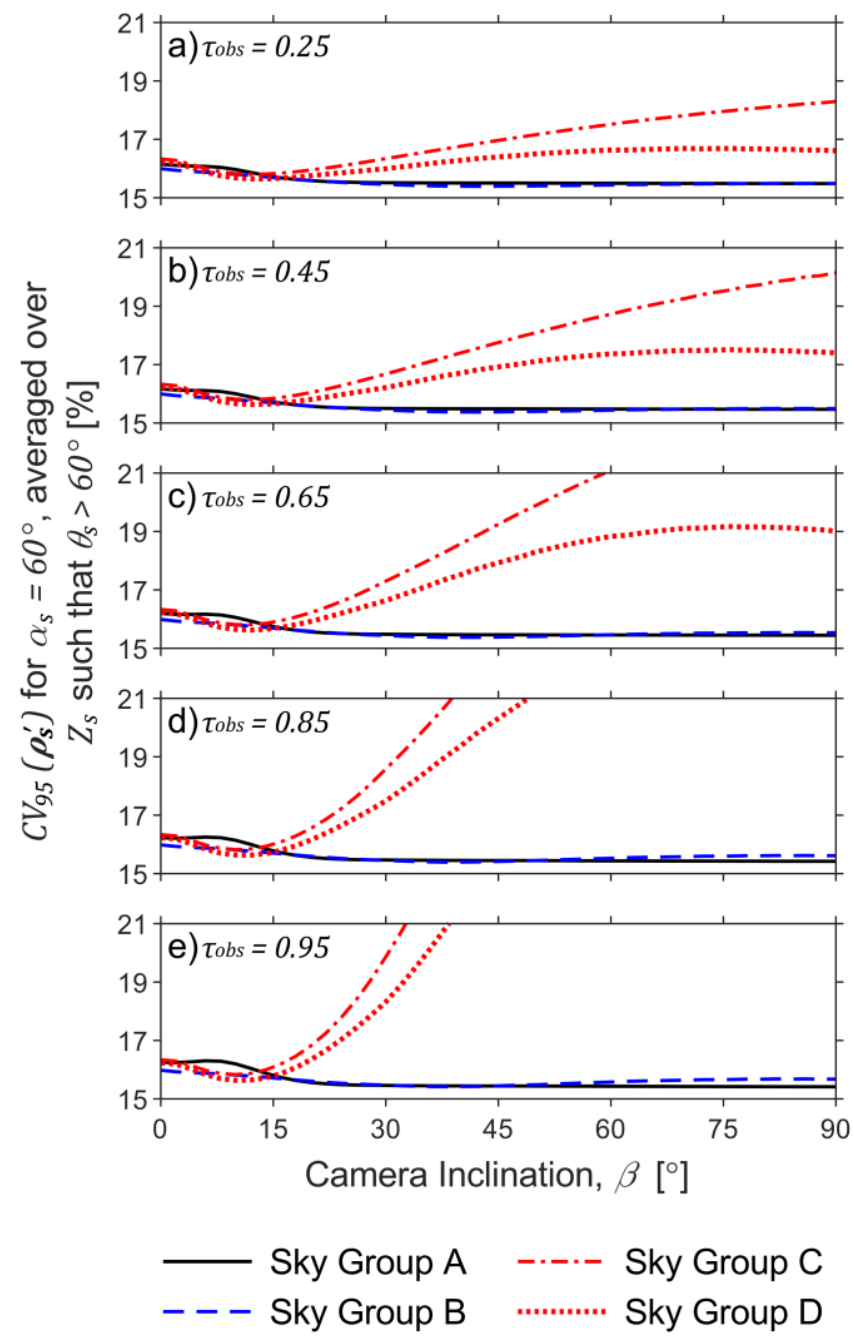

Figure 6.5: Percentage relative uncertainty in soot mass column density for a relative solar azimuth $\left(\alpha_{s}\right)$ of $60^{\circ}$ averaged over all solar zenith angles $\left(Z_{s}\right)$ as a function of camera inclination angle $(\beta)$. Data are plotted for each sky group for observed transmittances $\left(\tau_{o b s}\right)$ of 0.25 to 0.95 in subplots (a)-(e). For sky groups A and $B$ (representing overcast and partly cloudy skies), uncertainty can be slightly reduced by ensuring the camera inclination angle exceeds approximately $15^{\circ}$. For sky groups $C$ and $D$ (representing clear skies), minimal uncertainty is achieved at camera inclination of $9.75-12.50^{\circ}$, and uncertainty can drastically increase for camera inclination increase beyond $20^{\circ}$ - however, the effect becomes muted for more optically thick plumes (lower $\left.\tau_{o b s}\right)$. These observations support the general heuristic for clear skies that the camera inclination angle should kept below $\sim 20^{\circ}$, especially for lightly and moderately sooting flares.

Figure 6.5 also shows that the $C V_{95}$ of soot mass column density for sky group $\mathrm{C}$ tends to upper bound that of sky group $\mathrm{D}$. This observation holds under most combinations of the MC independent variables and is a result of the somewhat extreme nature of CIE sky 
model 12 (lowest turbidity / cleanest atmosphere), which is excluded in sky group D. When considered in sky group C however, CIE sky model 12 tends to increase both the variability and central tendency of soot mass column density, but the relative change in the former is larger - hence, inclusion of CIE sky model 12 typically increases relative uncertainty. This implies that sky group $\mathrm{C}$ reliably imposes the largest constraints on camera positioning and can therefore be used to conservatively locate sky-LOSA equipment under clear skies. However, if in a dense industrial area, where the clearest sky model is not relevant, the less-constraining sky group D can be instead used as noted in Section 6.4.1.5.

\subsubsection{Camera Pointing Heuristics}

Upon arrival at a measurement facility, the sky-LOSA user's first task is to determine the position of the sky-LOSA camera for data acquisition. This important decision can be made by considering viable camera pointings from GUA MC data through constraints on $\beta$ and $\theta_{s}$. That is, for a user-identified sky group and plume transmittance and given the position of the sun, the sky-LOSA camera's pointing can be constrained based on a desired threshold in relative uncertainty, $C V_{95}$. Table 6.3 provides an example of such constraints. The table lists bounds on the camera inclination angle $(\beta)$ and solar scattering angle $\left(\theta_{s}\right)$ for each sky group, given the plume transmittance; where bounds were computed by determining where $C V_{95} \leq 17 \%$ for all values of $Z_{s} \in\left[0,90^{\circ}\right]$, such that the results are independent of solar zenith. 
Table 6.3: Summary of constraints regarding camera pointing relative to the horizon and sun as a function of plume transmittance $\left(\tau_{o b s}\right)$. Compliance with these heuristics ensures that uncertainty in sky-LOSA-computed soot mass column density is low $\left(C V_{95} \leq 17 \%\right)$, regardless of solar zenith angle $\left(Z_{s}\right)$. (N.C. $=$ no constraint).

\begin{tabular}{|c|c|c|}
\hline $\begin{array}{c}\text { Sky } \\
\text { Group }\end{array}$ & $\begin{array}{c}\text { Camera Inclination } \\
\text { Angle, } \boldsymbol{\beta}\end{array}$ & $\begin{array}{c}\text { Solar Scattering } \\
\text { Angle, } \boldsymbol{\theta}_{\boldsymbol{s}}\end{array}$ \\
\hline \multicolumn{3}{|c|}{ Plume Transmittance $\left(\tau_{\text {obs }}\right) \leq 0.45$} \\
\hline A & N.C. & $\theta_{s} \geq 13^{\circ}$ \\
\hline B & N.C. & $\theta_{s} \geq 23^{\circ}$ \\
\hline C & $\beta \leq 20^{\circ}$ & $\theta_{s} \geq 79^{\circ}$ \\
\hline D & $\beta \leq 25^{\circ}$ & $\theta_{s} \geq 54^{\circ}$ \\
\hline \multicolumn{3}{|c|}{ Plume Transmittance $\left(\tau_{\text {obs }}\right) \leq 0.70$} \\
\hline A & N.C. & $\theta_{s} \geq 15^{\circ}$ \\
\hline B & N.C. & $\theta_{s} \geq 29^{\circ}$ \\
\hline C & $\beta \leq 16^{\circ}$ & $\theta_{s} \geq 83^{\circ}$ \\
\hline D & $\beta \leq 19^{\circ}$ & $\theta_{s} \geq 59^{\circ}$ \\
\hline \multicolumn{3}{|c|}{ Plume Transmittance $\left(\tau_{\text {obs }}\right)>0.70$} \\
\hline A & $\beta \geq 10^{\circ}$ & $\theta_{s} \geq 17^{\circ}$ \\
\hline B & N.C. & $\theta_{s} \geq 35^{\circ}$ \\
\hline C & $\beta \leq 13^{\circ}$ & $\theta_{s} \geq 84^{\circ}$ \\
\hline D & $\beta \leq 17^{\circ}$ & $\theta_{s} \geq 62^{\circ}$ \\
\hline
\end{tabular}

There are two additional considerations regarding the pointing of the sky-LOSA camera. Firstly, to compute the observed transmittance of the plume $\left(\tau_{o b s}\right)$, the sky-LOSA algorithm requires the incident intensity of skylight $\left(I^{o}\right)$ at the distal side of the plume from the perspective of the camera. This is generally obtained with minimal error via the interpolation of skylight intensity within the image plane as detailed by Johnson et al. (2010). However, if intensity gradients of the sky are large as in some overcast conditions, errors in the estimation of incident intensity by interpolation can become significant. This highlights that a sky-LOSA user must strive to point the camera such that spatial variations of the background skylight intensity are minimal - for example, under partly cloudy 
conditions, the camera should be pointed to avoid the presence of cloud structures behind the plume. Secondly, the pointing of the sky-LOSA camera relative to the propagation of the plume must also be considered. Under quiescent conditions, buoyancy-driven flare plumes will propagate vertically away from the flare stack; however, under sufficiently strong crosswinds, the flame and plume can bend over and propagate horizontally, parallel to the wind direction. In this latter case, if the plume propagates towards or away from the sky-LOSA camera, plume structures within the turbulent shear layer can overlap or become smeared from the perspective of the camera. Therefore, it is best to position the sky-LOSA camera such that it points orthogonally to the wind direction, which minimizes out-ofimage plane motion of the plume and yields the best data for ICV analyses.

It should be noted that uncertainties in the calculation of the incident intensity and mass concentration-weighted, LOS-averaged plume velocities are not included within the present GUA. Firstly, uncertainties in $I^{o}$ via the reconstruction of skylight behind the plume from the camera's perspective are sensitive to the specific sky conditions and thus cannot be estimated in a generalized manner. Specifically, Johnson et al. (2010) showed that accuracy in the reconstruction of skylight intensity can be easily and quantitatively evaluated by attempting the reconstruction of skylight intensity on a set of reference sky images acquired under the same conditions as the sky-LOSA data. Second, it is challenging to quantify the veracity of the assumption that ICV-calculated velocities represent the mass concentration-weighted, LOS-averaged plume velocity required by the sky-LOSA theory. This has been previously addressed by Johnson et al. (2011), who justified the use of ICV for the purposes of sky-LOSA via qualitative arguments; moreover, the present sky-LOSA algorithm quantifies uncertainties in ICV-computed velocities using 
the method of Wieneke (2015). Regardless of the impact of these uncertainties on skyLOSA-computed soot mass emission rate, they are generally overwhelmed by uncertainties in the fundamental soot properties (Johnson et al. 2013), which are indeed considered in this GUA of soot mass column density.

\subsubsection{Further Camera Heuristics}

Following selection of a permissible sky-LOSA camera position, the imaging optics must be chosen. Prime (fixed focal length) lenses are employed in the sky-LOSA technique to avoid ambiguity in optical magnification and, hence, spatial scaling of the image. The most appropriate prime lens for the studied flare is one that maintains the entirety of the flare flame well within the image during the data acquisition period. This helps to ensure that a control surface within the image plane that transects the plume and encloses the flame can be derived, as shown in Figure 6.1. For a flame that is relatively unsteady -i.e., moving with the wind - it is suggested to keep the flare flame approximately one-quarter of the smallest image dimension. By contrast, if the flame is steady, a flame length of approximately one-third of the smallest image dimension should be targeted. For the skyLOSA camera used by the authors (minimum sensor dimension of $\sim 14 \mathrm{~mm}$ ), a good ruleof-thumb is that the appropriate focal length $(f,[\mathrm{~mm}])$ will be on the order of:

$$
f[\mathrm{~mm}] \approx \frac{4.1}{\cos \beta} \frac{H}{L_{f}}
$$

where $H$ is the horizontal stand-off distance from the flare stack [m] and $L_{f}$ is the length of the flare flame $[\mathrm{m}]$. However, use of a prime lens necessitates a trade-off between lens focal length, horizontal stand-off distance, and size of the flame within the image. 
With an appropriate lens selected, the user must then choose imaging parameters that influence the exposure and focus of the image. The objective is to obtain an image that maximizes the digital signal while minimizing exposure time and ensuring the flame is in focus. In the authors' experience, this can be obtained with a lens aperture close to full-open (typically f-number $\leq 5.6$ ) and an exposure time less than $\sim 2 \mathrm{~ms}$. Prior to acquiring the image data, the flame and flare stack should be brought into focus. The user can then obtain sky-LOSA data for the desired duration; it is recommended, however, that a minimum dataset of ten minutes be obtained to permit good convergence of the timeaveraged soot emission rate.

\subsubsection{Open-source Software Tool for Simpler Sky-LOSA Setup - SetupSkyLOSA}

While the camera pointing heuristics presented in Table 6.3 can be used to ensure that $C V_{95} \leq 17 \%$ for the listed plume transmittances regardless of solar zenith angle, this simplified set of constraints is also necessarily overly conservative and excludes specific combinations of inputs that might produce similar or better uncertainties in different scenarios. An even better approach would be to use the wealth of computed GUA data to provide camera position and pointing constraints on a case-by-case basis. This is made possible using a new open-source software tool, SetupSkyLOSA (Conrad 2020), that was developed as part of this work using the presented GUA MC data. This MATLAB-based application enables a sky-LOSA user to probe statistics of soot mass column density (such as $C V_{95}$ ) for their specific measurement conditions. The key output of the software tool is an image of the desired soot mass column density statistic plotted as a function of absolute camera pointing. The software tool is briefly described in this section and employed in a case study in Section 6.5.3. 
Figure 6.6 shows a flowchart describing the SetupSkyLOSA software's main procedure. For a user-inputted location and time, the software first determines the current position of the sun using an integrated solar position calculator - a MATLAB implementation of the National Renewable Energy Laboratory's (NREL's) Solar Position Algorithm (SPA) (Reda and Andreas 2008). The SPA returns the solar zenith $\left(Z_{s}\right)$ and absolute bearing of the sun $\left(\alpha_{s N}\right.$, where the subscript " $\mathrm{N}$ " implies the absolute bearing measured clockwise from true North) at the current time and over the measurement date. The user also inputs the index ( $a$ ) of the most appropriate CIE sky model/group, an estimate of the observed plume transmittance at the sky-LOSA measurement wavelength $\left(\tau_{o b s}\right)$, and the desired statistic of soot mass column density $(\eta)$. With these inputs, SetupSkyLOSA then loads the GUA MC data for the selected sky model/group and interpolates for the desired statistic using the current solar zenith and estimated plume transmittance. 


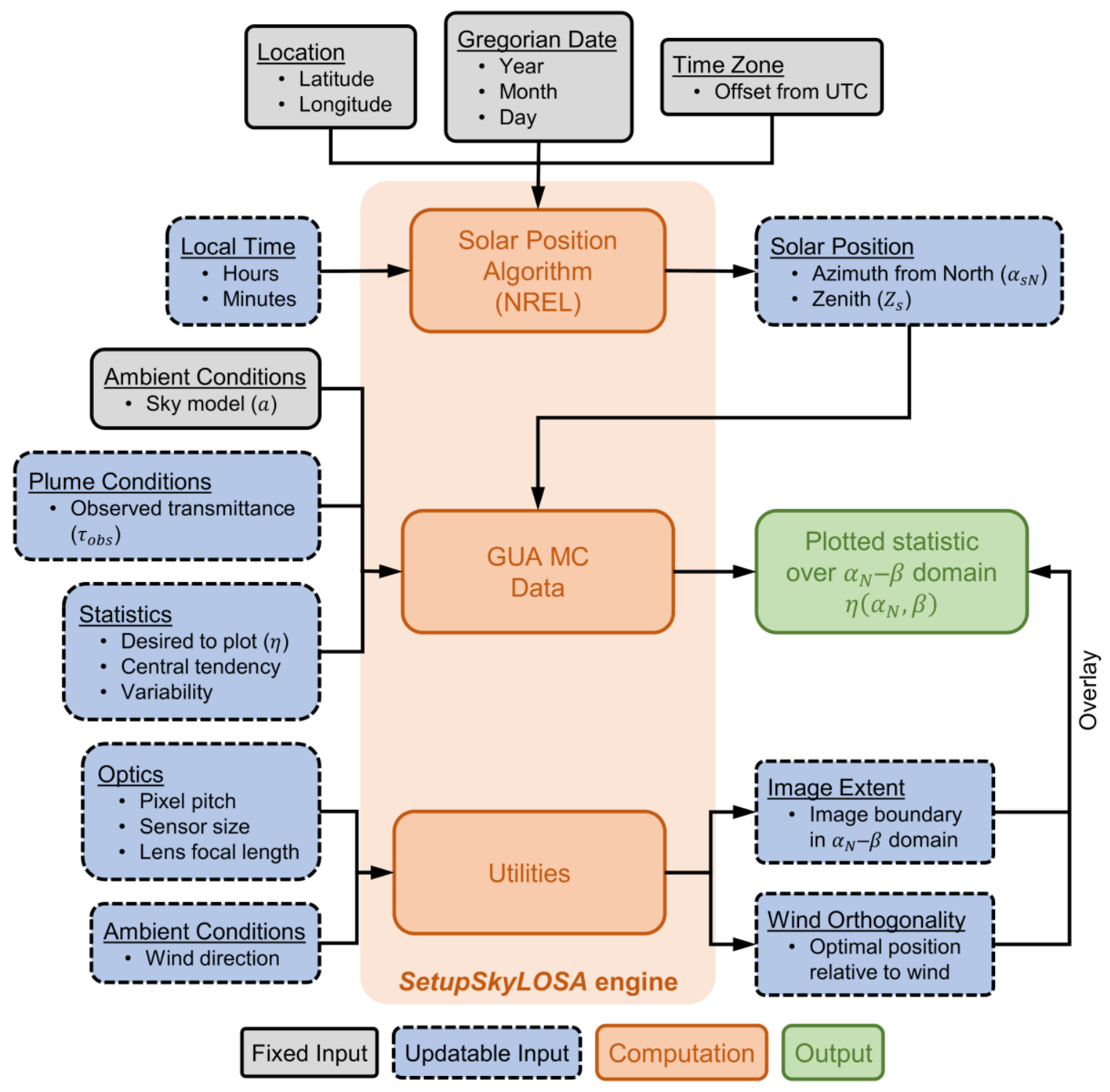

Figure 6.6: Flowchart of the main procedure of the SetupSkyLOSA software tool. The user provides the location, Gregorian date, time zone, and local time, which are used to compute the corresponding solar position using the Solar Position Algorithm of the National Renewable Energy Laboratory (NREL, Reda and Andreas, 2008).

Then, with data on ambient conditions and observed plume transmittance, the software tool plots the desired statistic of soot mass column density over the $\alpha_{N-\beta}$ domain. Additional utilities include the overlay of the image sensor and optimal positioning relative to the wind on the plotted statistic, in addition to the "maximizer" and "positioner" utilities, which are not shown in the figure. The latter two utilities permit a user to identify acceptable camera positions/pointings over a measurement period and output this data in . $\mathrm{kml}$ format for use with mapping software such as Google Earth. 
At this point, the software has computed $\eta\left(\alpha_{s}, \beta\right)$ for the user's current set of independent variables $\left(Z_{s}, \tau_{o b s}, a\right)$. However, rather than plotting $\eta\left(\alpha_{s}, \beta\right)-$ i.e., using the relative bearing - the software uses the known absolute bearing of the sun $\left(\alpha_{S N}\right.$, computed by the SPA) to plot $\eta\left(\alpha_{N}, \beta\right)$. That is, the requested statistic is plotted as a function of absolute camera bearing and inclination, which together define the camera pointing. The user can then easily determine an acceptable camera pointing using a laser rangefinder (for inclination) and a compass (corrected to true North), laser rangefinder, or GPS device (for absolute bearing).

SetupSkyLOSA also includes several added utilities to support optimal positioning and pointing of the sky-LOSA camera. Firstly, using the same pinhole camera model that enables spatial scaling of the image, the software tool can optionally overlay the approximate extent of the image sensor in the $\alpha_{N}-\beta$ domain, based on sensor dimensions, employed optics, and the pointing of the centre of the image. This helps a user ensure that the entirety of the image frame - including the eventual control surface used for emission rate calculation - has reasonable levels of measurement uncertainty, given a user-selected lens of known focal length. To support ideal velocity calculation, the software can also overlay camera pointings $\left(\alpha_{N}\right)$ that are closely orthogonal to the wind. This follows the heuristic discussed in Section 6.5.1.1. The software shows the optimal range of camera pointing as orthogonal to the wind $\pm 18.2^{\circ}$, which corresponds to $5 \%$ out-of-image plane motion $\left(\cos ^{-1}(0.05)\right)$. Two additional utilities are not shown in Figure 6.6. The "maximizer" utility computes the maximum of a chosen relative uncertainty statistic over a user-defined period. This tool allows a user to seek camera pointings that yield satisfactory uncertainties as the sun moves during the anticipated duration of the sky-LOSA 
measurement. The "positioner" utility takes the plotted relative uncertainty statistic as a function of camera pointing and provides region(s) where the sky-LOSA camera may be positioned relative to the flare stack given the flare stack height, maximum horizontal stand-off distance from the flare, and relative uncertainty threshold. The user can optionally print these permissible regions to a. $\mathrm{kml}$ file for use in mapping software like Google Earth. These latter two utilities are employed in the following case study.

\subsubsection{Case Study - Atasta Facility}

The utility of the novel software tool, SetupSkyLOSA, is shown in this section via a case study of a sky-LOSA measurement at a real oil and gas facility. The Atasta Gas Processing and Transport Centre (Centro de Proceso y Transporte de Gas Atasta) is a midstream oil and gas facility near Atasta, in the Mexican state of Campeche. The facility is under the jurisdiction of Petróleos Mexicanos and receives sour gas and condensates from the Cantarell offshore oil field for processing and transport to the national market. As shown in Figure 6.7a, the Atasta facility is located $35 \mathrm{~km}$ West of Ciudad (Cd) del Carmen and approximately $5 \mathrm{~km}$ South of the shore of the Bay of Campeche. The facility occupies approximately $1 \mathrm{~km}^{2}$, with most infrastructure in the Southeast corner of the site as visible in Figure 6.7b. Flaring activities include multiple pit-style and vertical stack flares, which are in the Northwest corner of the site. 

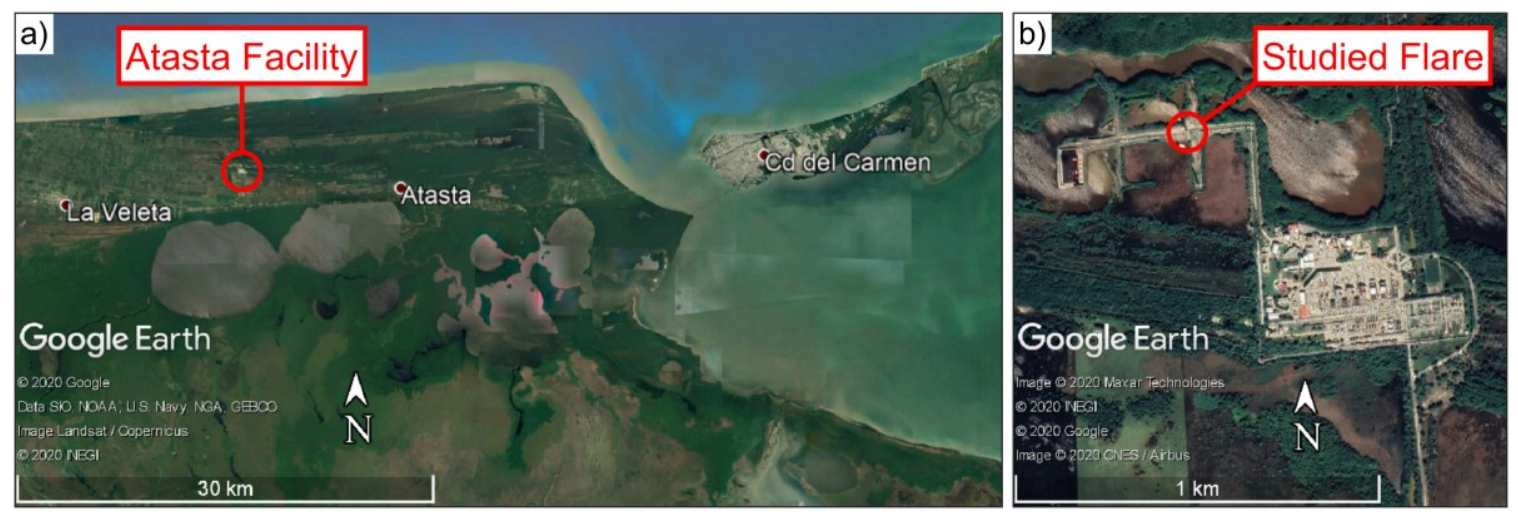

Figure 6.7: (a) Location of the Atasta Gas Processing and Transport Centre in the Mexican state of Campeche, $35 \mathrm{~km}$ West of Ciudad (Cd) del Carmen and approximately $5 \mathrm{~km}$ South of the Bay of Campeche. (b) Location of the flare that is the focus of the case study located in the Northwest corner of the site amongst other flaring infrastructure.

For this case study, a sky-LOSA measurement of soot emissions from the central flare stack at the Atasta station was considered as indicated in Figure $6.7 \mathrm{~b}$, the base of which is located at $18^{\circ} 38^{\prime} 41.46^{\prime \prime} \mathrm{N}$ and $92^{\circ} 10^{\prime} 08.59^{\prime \prime} \mathrm{W}$. The following example measurement details are assumed:

1. The sky-LOSA measurements occur on May 13, 2021, which is the date of that year that the sun most closely reaches the solar zenith $\left(Z_{s}=0^{\circ}\right)$.

2. Predicted sky conditions are uncertain and may change between overcast and fully clear conditions throughout the day.

3. Wind speed is predicted to be low and the flare is strongly buoyant.

4. The flare stack is $30 \mathrm{~m}$ in height, and the horizontal stand-off distance of the skyLOSA camera is limited to $250 \mathrm{~m}$ or less due to available optics.

5. The flare is lightly sooting, with an observed transmittance of approximately $90 \%$ $\left(\tau_{o b s} \approx 0.90\right)$. 
Assumption \#2 implies that sky-LOSA data acquisition may occur under skies represented by any of sky groups A-D. Furthermore, assumption \#3 suggests that the soot-laden flare plume propagates vertically from the flare stack and, therefore, the constraint on camera position with respect to wind direction is unimportant. The sky-LOSA user wishes to obtain sky-LOSA data with minimal measurement uncertainty, while also avoiding relocation of the sky-LOSA camera throughout the day, if possible.

Given the known GPS coordinates of the flare stack, measurement date, and the approximate plume transmittance, SetupSkyLOSA can be used to constrain sky-LOSA camera pointing for any sky condition and time of the day based on the $C V_{95}$ of soot mass column density. Since the user wishes to avoid re-location of the sky-LOSA camera, camera position and pointing should be constrained using the relative uncertainty maximized over the day. The "maximizer" utility of SetupSkyLOSA permits this calculation for each of the sky groups; results are shown in Figure 6.8a-d for sky groups A-D. Noting the differing colour scales in the four figures, there is significant variability in sky-LOSA uncertainties for each of the sky groups, as in Figure 6.3. For further context, the path of the sun over the measurement date is overlaid in Figure $6.8 \mathrm{a}-\mathrm{d}$ in addition to a contour of $C V_{95}=16.5 \%$, which is within $1 \%$ of the best attainable uncertainty for these conditions.

Using the uncertainty data in Figure $6.8 \mathrm{a}-\mathrm{d}$, the "positioner" utility of SetupSkyLOSA can be employed to highlight where the sky-LOSA camera may be positioned relative to the flare stack. This was performed for each of the sky groups using the uncertainty threshold of $16.5 \%$. Permissible camera positions were output in.$k m l$ format by the "positioner" utility and are overlaid on a map of the Atasta facility in Figure 
6.8e and are quite different for each of the sky groups. Permissible camera positions for sky group B exist beyond a small region near the stack tip, while those for sky group A are within an annular region surrounding the flare stack - since the lower limit on the camera inclination angle in Figure 6.8a imposes a maximum permissible stand-off distance. Sky group D contains two permissible regions - one to the South and one to the North of the flare stack - while the most-constrained sky group $\mathrm{C}$ has one relatively small region to the North of the flare stack. Recalling assumption $\# 2$, that predicted sky conditions were uncertain, the sky-LOSA user should ideally position the camera at the intersection of the sky group-dependent permissible regions. This small area is outlined in black in the figure, is $\sim 136 \mathrm{~m}$ due North of the flare stack and is just $604 \mathrm{~m}^{2}$ in size $(\sim 0.31 \%$ of the $250 \mathrm{~m}$ radius region). It is apparent in Figure 6.8e that this ideal position intersects a clearing in the treed area where the sky-LOSA camera should be positioned for the specific conditions of this case study. 

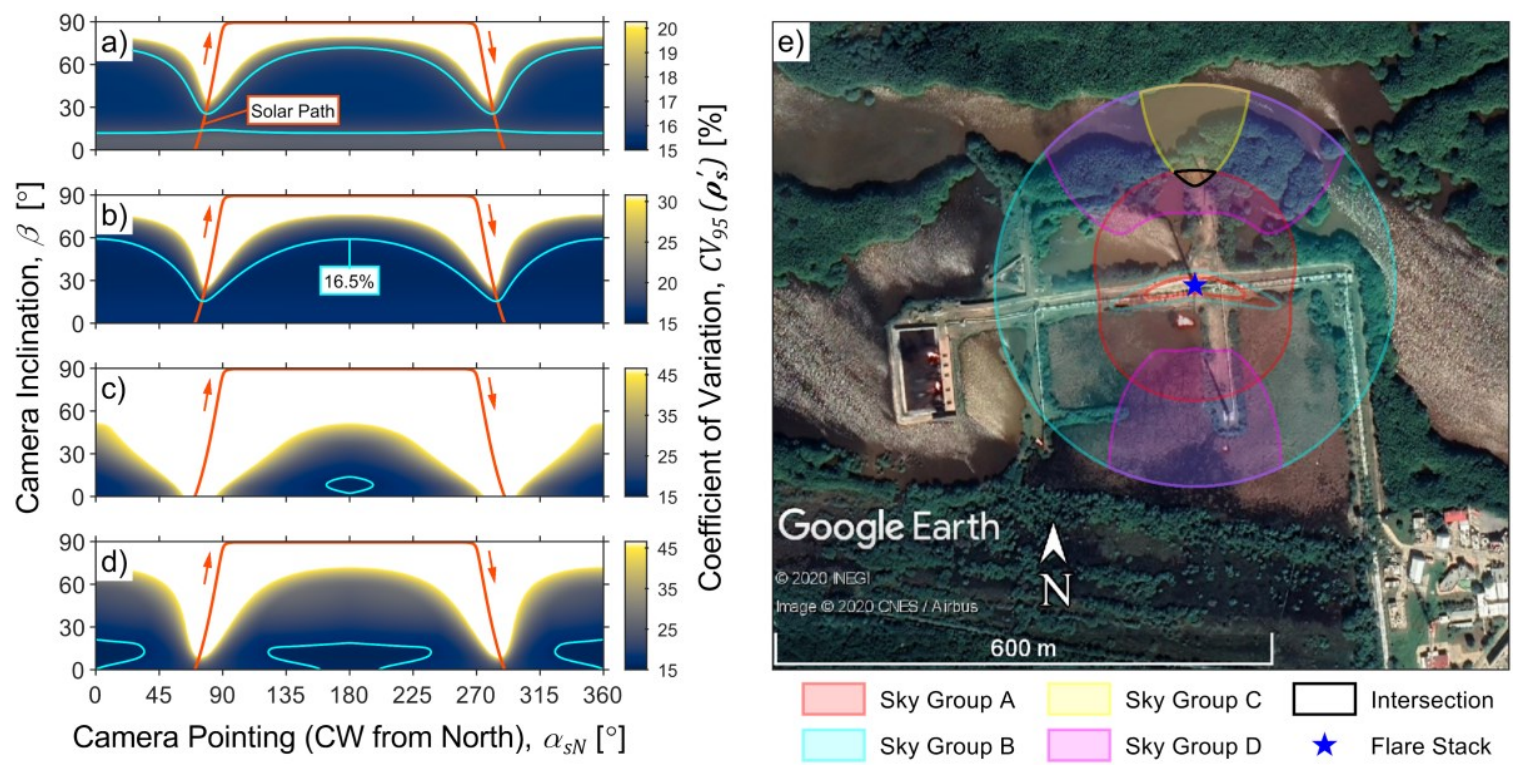

Figure 6.8: (a-d) Relative uncertainty $\left(C V_{95}\right)$ in soot mass column density as a function of camera inclination $(\beta)$ and pointing $\left(\alpha_{s, N}\right)$, maximized over the measurement day for sky groups $A-D$, respectively, given an observed plume transmittance $\left(\tau_{o b s}\right)$ of 0.90 . Overlaid in the figures is the path of the sun over the day (which approximately reaches $Z_{s}=0^{\circ}$ at 13:05 Central Daylight Time) in addition to a contour of $C V_{95}=16.5 \%$. (e) Permissible regions for sky-LOSA camera positioning relative to the studied flare stack for sky groups $A-D$. The black line shows the intersection of these regions which is $a \sim 600 \mathrm{~m}^{2}$ that the skyLOSA camera can be positioned to minimize measurement uncertainty, regardless of sky conditions.

This case study shows the utility of the SetupSkyLOSA software tool. The tool quickly provides resolved measurement uncertainty data from the GUA that would otherwise require millions of $\mathrm{MC}$ analyses to compute. These uncertainty data enable optimal sky-LOSA camera positioning and pointing and also represent a first-order estimate of soot emission rate uncertainties that are computed in post-processing. Together with the additional utilities and general camera heuristics, this software tool permits a skyLOSA user to obtain optimal sky-LOSA data that minimizes measurement uncertainties under generalized conditions. 


\subsection{Conclusions}

A comprehensive Monte Carlo-based general uncertainty analysis (GUA) has been used to develop heuristics constraining the pointing/positioning of sky-LOSA equipment for measurement of soot/black carbon emissions from gas flares. The GUA identifies generalized constraints based on predicted measurement uncertainties in soot mass column density, computed using sky-LOSA. The results show that equipment setup constraints can be classified based on the conditions of the sky, relative positioning of the sun, and inclination angle of the camera. With additional heuristics on camera optics and imaging parameters, the presented results provide generalized guidance to greatly simplify acquisition of optimal sky-LOSA data in the context of complex, non-linear measurement uncertainties. These are further extended in the open-source software utility, SetupSkyLOSA, which interprets the GUA results to provide detailed guidance for any specific combination of location, date/time, and flare, plume, and ambient conditions. Furthermore, software-displayed soot mass column density statistics provide the user with a first-order estimate of measurement uncertainty in soot/black carbon emission rate that otherwise is only computable during post-processing. The case study using SetupSkyLOSA to identify optimal equipment setup at a real oil and gas facility in Mexico demonstrates the utility of this new software tool, which as an open-source application can hopefully facilitate broader use of the sky-LOSA technique and ultimately help increase the knowledge base of soot/black carbon emissions from gas flares.

\subsection{Data Availability}

The developed software tool and associated data are available online as open-source and build distributions (Conrad 2020). 


\subsection{Acknowledgements}

This work was supported by the Natural Sciences and Engineering Research Council of Canada (NSERC) FlareNet Strategic Network (Grant \#479641), NSERC Discovery Research Grants (Grant \#06632 and 522658), and Natural Resources Canada (Project Manager, Michael Layer). We are grateful to Dr. Brian Crosland (Natural Resources Canada) for supplying computational resources. 


\section{Chapter 7 Conclusions and Recommendations}

\subsection{Conclusions}

This thesis makes important contributions to the study of flare-generated soot/black carbon (BC) emissions and properties and prepares an emergent optical diagnostic, sky-LOSA (line-of-sight attenuation using skylight), for standardization. The presented work sought to meet four broad objectives: use of the existing sky-LOSA measurement technique to quantify BC yields from in-field flares and develop an improved flare BC emission factor model; refinement of the sky-LOSA algorithm to consider previously ignored radiative transfer considerations; experimental studies investigating wavelength-dependent flare $\mathrm{BC}$ optical properties for comparison with other $\mathrm{BC}$ sources; and completion of a general uncertainty analysis supporting the development of a setup and measurement protocol for sky-LOSA that enables consistent acquisition of optimal data, the accelerated deployment of the technique, and, ultimately, standardization.

To improve upon the existing limited knowledge base regarding $\mathrm{BC}$ emissions from in-field gas flares, data from sky-LOSA measurement campaigns in Mexico and Ecuador were analyzed to obtain a collection of absolute $\mathrm{BC}$ emission rate data and the first direct measurements of $\mathrm{BC}$ yield from in-field flares. Flare $\mathrm{BC}$ emission rates measured using sky-LOSA span more than four orders of magnitude from $3 \mathrm{mg} / \mathrm{s}$ to $53.7 \mathrm{~g} / \mathrm{s}$. These data support the notion that flare $\mathrm{BC}$ emissions are subject to the common challenge of "superemitters" - where a small proportion of flares contribute disproportionately to global emissions. Parallel measurements of flare gas flow rate and extractive sampling for compositional analyses enabled the direct calculation of $\mathrm{BC}$ yield from four of the studied

flares in Ecuador. Like previous laboratory studies, measured BC yields were found to 
scale with flare gas energy content, albeit over a much larger range of heating values. These new observations enabled an extended emission factor model using data that more closely bounds actual flare gas compositions encountered in the field. The extended model highlights that commonly used yet overly simplistic, single-valued emission factors could underestimate global flare $\mathrm{BC}$ emissions by almost a factor of two.

To quantify line-of-sight (LOS) BC mass column density from plume observations, sky-LOSA considers radiative transfer along optical axes intersecting the plume including inscattering of sky and sunlight into the LOS. To date, this complex radiative transfer has been considered using a simplified model where refractive index gradient-driven beam steering and multiple scattering are both ignored. Omission of these effects can, however, impose unidentified quantities of bias and precision error on sky-LOSA calculations. This thesis presents numerical studies of available large-eddy simulated gas flares to address the effects of beam steering and multiple scattering on sky-LOSA. Beam steering effects through cooled atmospheric plumes were found to be negligible for spectral bands commonly used by optical diagnostics for particulate matter, methane, and carbon dioxide. By contrast, multiple scattering effects expectedly imposed a negative bias on sky-LOSAcomputed $\mathrm{BC}$ emission rates, which was found to be as large as one-quarter of typical measurement uncertainties. A simple model to correct for multiple scattering was derived and introduced (along with the consideration of residual bias and precision errors) into the sky-LOSA algorithm. Case study analyses showed that the easily implemented model accurately corrects for multiple scattering within the sky-LOSA algorithm with negligible changes to overall measurement uncertainty. 
The literature contains myriad and often disparate measurements of optical properties of soot/BC from a variety of combustion sources. One optical property critical to climate forcing metrics and numerous optical diagnostics of $\mathrm{BC}$ is the mass-normalized absorption cross-section (MAC). For flare $\mathrm{BC}$, the representativeness of MAC data available in the literature is questionable since these data were generally obtained from small-scale flames within combustion regimes not representative of in-field flares while burning fuels vastly different than typical flare gases. Moreover, the solitary field measurement data of flare BC MAC were anomalously large relative to generally accepted values in the literature. Thus, to examine BC MAC for gas flares specifically, studies of large-scale flames at the Carleton University flare facility were performed. Flares spanning compositions representative of the upstream oil and gas industry were flared at rates approaching those that might be encountered in the field. Flare BC MAC was observed to be statistically sensitive to numerous flare metrics that describe flame aerodynamics and fuel chemistry. A phenomenological MAC model was derived using a novel dimensionless scaling parameter that is thought to capture the time-temperature history of $\mathrm{BC}$ within flare flames. Over the breadth of tested fuels and conditions, the derived model suggests that larger in-flame residence times may result in a more-graphitized BC nanostructure, which would enhance the absorbing efficiency per unit mass (i.e., MAC) of flare-generated BC, as observed in the experiments. Importantly, this new model reconciles the anomalously large field-measured flare BC MAC data with generally accepted values from experiments on small flames burning heavy fuels.

Finally, in support of standardization of sky-LOSA, a comprehensive general uncertainty analysis (GUA) for the technique was executed using the updated algorithm 
that includes the correction of multiple scattering effects. Uncertainties in sky-LOSAcomputed soot/BC mass column densities were computed over a five-dimensional domain of independent variables using a Monte Carlo method with a variance reduction technique. To avoid combinatorial explosion, the computational procedure for soot/BC mass column density was reformulated to permit independent pre-computation of the effects of soot properties and sky/sun conditions. These GUA data allowed the quantification of measurement uncertainties as a function of plume and ambient conditions as well as userselected positioning/pointing of sky-LOSA equipment, ultimately enabling a sky-LOSA setup and measurement protocol. An open-source software tool was developed as an interface between a sky-LOSA user and the GUA data to provide case-specific guidance for the acquisition of optimal sky-LOSA data under generalized conditions. By greatly simplifying critical data acquisition decisions by the user, it is hoped that this new software tool will expand the accessibility of the sky-LOSA technique and, ultimately, the availability of flare $\mathrm{BC}$ emissions data to support inventories, regulations, climate modelling, and emissions mitigation efforts.

\subsection{Recommendations for Future Work}

The research presented in this thesis prepares the sky-LOSA measurement technique for standardization with an authority such as the United States Environmental Protection Agency (EPA). All relevant sources of measurement uncertainty have been quantified and are now included in the technique's refined computational algorithm. Furthermore, the measurement protocol described in Chapter 6 provides a standardized approach to acquire sky-LOSA data of consistently high quality. Ultimately, these efforts have brought skyLOSA to maturity, enabling increased deployment in the field to support rapid 
development of emission factor models and emissions inventories. The most-immediate next task is the submission of the technique to the EPA for consideration as a measurement standard.

The novel results presented in Chapter 5 highlight the potential uniqueness of flaregenerated soot/BC MAC relative to other sources. The findings suggest that the timetemperature history of $\mathrm{BC}$ in large flare flames results in a nanostructure with higher graphitization and thus stronger absorption per unit mass. Furthermore, these data imply that flare $\mathrm{BC}$ might also differ from other sources in terms of aggregate morphology. While variability in $\mathrm{BC}$ properties is not detrimental to the sky-LOSA measurement technique since reported emissions of equivalent $\mathrm{BC}$ are based on specified property distributions (see Section 1.1.5) - these observations demonstrate the need for further study of BC emitted from large-scale flames like flares. Since laboratory measurements tend to be flame size-limited, efforts should be made to implement measurements of MAC from infield flares. It would be beneficial to acquire parallel extractive samples of $\mathrm{BC}$ for highresolution electron microscopy, Raman spectroscopy, and/or X-ray diffraction studies of $\mathrm{BC}$ morphology and nanostructure. By expanding on the work presented in this thesis, these data could provide further insight into the climate effects of gas flares and inform future iterations of the sky-LOSA technique, including the subsequently recommended future work.

For optimally acquired sky-LOSA data, measurement uncertainties in soot/BC emission rate are modest but are constrained by limited knowledge of the properties of emitted $\mathrm{BC}$ particulate, mainly through the absorption function of the refractive index, $E\left(m_{\lambda}\right)$. This implies that efforts to simultaneously measure $E\left(m_{\lambda}\right)$ from $\mathrm{BC}$ aggregates in 
the flare plume during sky-LOSA data acquisition could reduce sky-LOSA uncertainties. Unfortunately, however, gravimetric/optical techniques usually employed to quantify $E\left(m_{\lambda}\right)$ would be challenging to implement in the field. One alternative means to reduce measurement uncertainties could be via the simultaneous acquisition of sky-LOSA data at multiple wavelengths spanning the visible spectrum. This is because optical interactions of light with fine particulate matter like $\mathrm{BC}$ are known to vary in predictable manners as a function of wavelength. Specifically, under the Rayleigh approximation, absorption is inversely related to measurement wavelength $\left(\lambda^{-1}\right)$ while scattering is inversely related to measurement wavelength raised to the fourth power $\left(\lambda^{-4}\right)$. Thus, multispectral sky-LOSA data could improve the ability to better constrain inscattering by distinguishing absorption from scattering effects. Moreover, multispectral sky-LOSA data could also improve the estimation of skylight intensity obscured by the plume, since spectral skylight intensity (resulting from Rayleigh scattering by the atmosphere) also varies predictably.

Quantifying the effect of inscattering is a significant challenge in the sky-LOSA method, requiring the integration of direction-dependent scattering over $4 \pi$ steradians for each LOS (refer to Eq. (6.7)). A potential means to avoid the need to correct for inscattering is to perform sky-LOSA-like measurements in the infrared spectrum where, due to its $\lambda^{-4}$ spectral variability, scattering is negligible. In contrast with the standard sky-LOSA method, the radiative transfer equation for such an approach instead includes an emissive source term. This formulation thus trades the need to consider directiondependent inscattering for temperature- and concentration-dependent thermal emission along each LOS. Although this presents a different challenge, requiring an inverse model for the LOS-variation of temperature and concentration, it is possible that measurement 
uncertainties could be reduced. This is partly because, under the assumptions of RDG-FA, the fractal nature of soot (as dictated by the fractal prefactor and dimension that are perturbed in sky-LOSA's Monte Carlo algorithm) does not affect soot's absorption/emission characteristics. Additionally, recent research suggests that simple inverse models for temperature and concentration can yield good estimates of column density using multi/hyperspectral image data. This potential diagnostic should be considered as an alternative to sky-LOSA; following the development of the theory, the large-eddy flare simulations used in this thesis could be leveraged to assess the benefits of this alternative technique in the context of measurement uncertainties.

Most importantly, the refined sky-LOSA measurement technique presented in this thesis - as well as future iterations and other similar techniques - should be maximally deployed to enhance the current understanding of $\mathrm{BC}$ emissions from gas flares and increase the knowledge base of flare $\mathrm{BC}$ effects on the Arctic and global climates. These data, sorely needed by climate modellers and policymakers alike, could be used to quantitatively assess and direct emissions mitigation efforts and ultimately help delay Earth's crossing of critical temperature thresholds. 


\section{References}

Acosta, I., Navarro, J., and Sendra, J.J. (2014). Lighting design in courtyards: Predictive method of daylight factors under overcast sky conditions. Renew. Energy, 71:243254.

Alberta Energy Regulator (2020). Alberta Directive 060: Upstream Petroleum Industry Flaring, Incinerating, and Venting. Edmonton, AB.

Allen, D.T. and Torres, V.M. (2011). TCEQ 2010 Flare study final report (No. PGA No. 582-8-862-45-FY09-04). Texas Commission on Environmental Quality (TCEQ), Austin, TX.

Amann, M., Bertok, I., Borken-Kleefeld, J., Cofala, J., Heyes, C., Höglund-Isaksson, L., Klimont, Z., Nguyen, B., Posch, M., Rafaj, P., Sandler, R., Schöpp, W., Wagner, F., and Winiwarter, W. (2011). Cost-effective control of air quality and greenhouse gases in Europe: Modeling and policy applications. Environ. Model. Softw., 26(12):14891501.

Andreae, M.O. and Gelencsér, A. (2006). Black carbon or brown carbon? The nature of light-absorbing carbonaceous aerosols. Atmos. Chem. Phys., 6(10):3131-3148.

Anenberg, S.C., Schwartz, J., Shindell, D., Amann, M., Faluvegi, G., Klimont, Z., Janssens-Maenhout, G., Pozzoli, L., Van Dingenen, R., Vignati, E., Emberson, L., Muller, N.Z., West, J.J., Williams, M., Demkine, V., Hicks, W.K., Kuylenstierna, J., Raes, F., and Ramanathan, V. (2012). Global Air Quality and Health Co-benefits of Mitigating Near-Term Climate Change through Methane and Black Carbon Emission Controls. Environ. Health Perspect., 120(6):831-839.

Ångström, A. (1929). On the Atmospheric Transmission of Sun Radiation and on Dust in the Air. Geogr. Ann., 11(2):156-166.

Arachchige, C.N.P.G., Prendergast, L.A., and Staudte, R.G. (2020). Robust analogs to the coefficient of variation. J. Appl. Stat., 1-23.

Arctic Monitoring and Assessment Programme (2015). AMAP Assessment 2015: Black Carbon and Ozone as Arctic Climate Forcers. Oslo, Norway.

Arnott, W.P., Moosmüller, H., Rogers, C.F., Jin, T., and Bruch, R. (1999). Photoacoustic spectrometer for measuring light absorption by aerosol: Instrument description. Atmos. Environ., 33(17):2845-2852.

ASTM (2014). D6729-14: Standard Test Method for Determination of Indiviual Components in Spark Ignition Engine Fuels by 100 Metre Capillary High Resolution Gas Chromatography. West Conshohocken, PA.

Basagaña, X., Jacquemin, B., Karanasiou, A., Ostro, B., Querol, X., Agis, D., Alessandrini, E., Alguacil, J., Artiñano, B., Catrambone, M., de la Rosa, J.D., Díaz, J., Faustini, A., Ferrari, S., Forastiere, F., Katsouyanni, K., Linares, C., Perrino, C., Ranzi, A., Ricciardelli, I., Samoli, E., Zauli-Sajani, S., Sunyer, J., and Stafoggia, M. (2015). Short-term effects of particulate matter constituents on daily hospitalizations 
and mortality in five South-European cities: Results from the MED-PARTICLES project. Environ. Int., 75:151-158.

Becker, H.A. and Liang, D. (1982). Total emission of soot and thermal radiation by free turbulent diffusion flames. Combust. Flame, 44(1-3):305-318.

Bell, M.L., Ebisu, K., Leaderer, B.P., Gent, J.F., Lee, H.J., Koutrakis, P., Wang, Y., Dominici, F., and Peng, R.D. (2014). Associations of PM ${ }_{2.5}$ Constituents and Sources with Hospital Admissions: Analysis of Four Counties in Connecticut and Massachusetts (USA) for Persons $\geq 65$ Years of Age. Environ. Health Perspect., 122(2):138-144.

Berry, M. V. and Percival, I.C. (1986). Optics of fractal clusters such as smoke. Opt. Acta (Lond)., 33(5):577-591.

Bescond, A., Yon, J., Ouf, F.X., Ferry, D., Delhaye, D., Gaffié, D., Coppalle, A., and Rozé, C. (2014). Automated Determination of Aggregate Primary Particle Size Distribution by TEM Image Analysis: Application to Soot. Aerosol Sci. Technol., 48(8):831-841.

Bohren, C.F. and Huffman, D.R. (1983). Absorption and Scattering of Light by Small Particles. Wiley, New York.

Bond, T.C. and Bergstrom, R.W. (2006). Light Absorption by Carbonaceous Particles: An Investigative Review. Aerosol Sci. Technol., 40(1):27-67.

Bond, T.C., Doherty, S.J., Fahey, D.W., Forster, P.M., Berntsen, T.K., DeAngelo, B.J., Flanner, M.G., Ghan, S., Kärcher, B., Koch, D., Kinne, S., Kondo, Y., Quinn, P.K., Sarofim, M.C., Schultz, M.G., Schulz, M., Venkataraman, C., Zhang, H., Zhang, S., Bellouin, N., Guttikunda, S.K., Hopke, P.K., Jacobson, M.Z., Kaiser, J.W., Klimont, Z., Lohmann, U., Schwarz, J.P., Shindell, D.T., Storelvmo, T., Warren, S.G., and Zender, C.S. (2013). Bounding the role of black carbon in the climate system: A scientific assessment. J. Geophys. Res. Atmos., 118(11):5380-5552.

Bond, T.C., Habib, G., and Bergstrom, R.W. (2006). Limitations in the enhancement of visible light absorption due to mixing state. J. Geophys. Res., 111(D20):D20211.

Born, M., Wolf, E., and Bhatia, A.B. (1999). Principles of Optics, 7th ed. Cambridge University Press, Cambridge, UK.

Boucher, O., Balkanski, Y., Hodnebrog, Ø., Myhre, C.L., Myhre, G., Quaas, J., Samset, B.H., Schutgens, N., Stier, P., and Wang, R. (2016). Jury is still out on the radiative forcing by black carbon. Proc. Natl. Acad. Sci., 113(35):E5092-E5093.

Bowerman, N.H.A., Frame, D.J., Huntingford, C., Lowe, J.A., Smith, S.M., and Allen, M.R. (2013). The role of short-lived climate pollutants in meeting temperature goals. Nat. Clim. Chang., 3(12):1021-1024.

Brandt, A.R., Yeskoo, T., McNally, M.S., Vafi, K., Yeh, S., Cai, H., and Wang, M.Q. (2016). Energy intensity and greenhouse gas emissions from tight oil production in the Bakken formation. Energy \& Fuels, 30(11):9613-9621. 
Buseck, P.R., Adachi, K., Gelencsér, A., Tompa, É., and Pósfai, M. (2014). Ns-Soot: A Material-Based Term for Strongly Light-Absorbing Carbonaceous Particles. Aerosol Sci. Technol., 48(7):777-788.

Cai, J. and Sorensen, C.M. (1994). Diffusion of fractal aggregates in the free molecular regime. Phys. Rev. E, 50(5):3397-3400.

Calel, R. and Mahdavi, P. (2020). Opinion: The unintended consequences of antiflaring policies - and measures for mitigation. Proc. Natl. Acad. Sci., 117(23):12503-12507.

Cameron, A.W., Tavoularis, S., and Johnson, M.R. (2011). Flow visualization of lowmomentum elevated jets in cross-flows. J. Flow Vis. Image Process., 18(2):137-164.

CAPP (2007). A recommended approach to completing the National Pollutant Release Inventory (NPRI) for the upstream oil and gas industry (No. 2007-0009). Canadian Association of Petroleum Producers (CAPP).

CCAC (2020). Measuring \& Minimizing Mineral Methane Emissions and Reducing Black Carbon through Flaring (Mineral Methane Initiative). Available at https://www.ccacoalition.org/en/initiatives/oil-gas (Accessed 18 July 2020).

Chakrabarty, R.K. and Heinson, W.R. (2018). Scaling Laws for Light Absorption Enhancement Due to Nonrefractory Coating of Atmospheric Black Carbon Aerosol. Phys. Rev. Lett., 121(21):218701.

Chang, H. and Charalampopoulos, T.T. (1990). Determination of the Wavelength Dependence of Refractive Indices of Flame Soot. Proc. R. Soc. A Math. Phys. Eng. Sci., 430(1880):577-591.

Choi, M.Y., Mulholland, G.W., Hamins, A., and Kashiwagi, T. (1995). Comparisons of the soot volume fraction using gravimetric and light extinction techniques. Combust. Flame, 102(1-2):161-169.

Chow, J.C., Watson, J.G., Crow, D., Lowenthal, D.H., and Merrifield, T. (2001). Comparison of IMPROVE and NIOSH Carbon Measurements. Aerosol Sci. Technol., 34(1):23-34.

Chow, J.C., Watson, J.G., Pritchett, L.C., Pierson, W.R., Frazier, C.A., and Purcell, R.G. (1993). The DRI thermal/optical reflectance carbon analysis system: description, evaluation and applications in U.S. Air quality studies. Atmos. Environ. Part A. Gen. Top., 27(8):1185-1201.

Chylek, P. and Wong, J. (1995). Effect of absorbing aerosols on global radiation budget. Geophys. Res. Lett., 22(8):929-931.

CIE (2003). Spatial Distribution of Daylight - CIE Standard General Sky (No. CIE S 011/E:2003; ISO 15469:2003(E)). Commission Internationale de l'Eclairage, Vienna, Austria.

CIE (1990). 2-degree spectral luminous efficiency for photopic vision. Vienna, Austria.

Clarke, A.D. and Noone, K.J. (1985). Soot in the Arctic snowpack: a cause for perturbations in radiative transfer. Atmos. Environ., 19(12):2045-2053. 
Clean Air Strategic Alliance (2004). Gas Flaring and Venting in Alberta: Report and Recommendations for the Upstream Petroleum Industry. Edmonton, AB.

Coderre, A.R., Thomson, K.A., Snelling, D.R., and Johnson, M.R. (2011). SpectrallyResolved Light Absorption Properties of Cooled Soot from a Methane Flame. Appl. Phys. B, 104(1):175-188.

Conrad, B.M. (2020). SetupSkyLOSA: A MATLAB Tool to support Acquisition of SkyLOSA Data (v1.0).

Conrad, B.M. and Johnson, M.R. (2019a). Mass absorption cross-section of flaregenerated black carbon: Variability, predictive model, and implications. Carbon N. Y., 149:760-771.

Conrad, B.M. and Johnson, M.R. (2019b). Split point analysis and uncertainty quantification of thermal-optical organic/elemental carbon measurements. J. Vis. Exp., (151).

Conrad, B.M. and Johnson, M.R. (2017). Field measurements of black carbon yields from gas flaring. Environ. Sci. Technol., 51(3):1893-1900.

Conrad, B.M. and Johnson, M.R. (2015). Uncertainties in Overcast Sky Reconstruction for Open-Path Attenuation Measurements., in Air \& Waste Management Association (AWMA) 108th Annual Conference, June 22-25 (ID \#74), Raleigh, NC.

Conrad, B.M., Thornock, J.N., and Johnson, M.R. (2020a). Beam steering effects on remote optical measurements of pollutant emissions in heated plumes and flares. $J$. Quant. Spectrosc. Radiat. Transf., 254.

Conrad, B.M., Thornock, J.N., and Johnson, M.R. (2020b). The effect of multiple scattering on optical measurement of soot emissions in atmospheric plumes. J. Quant. Spectrosc. Radiat. Transf., 254:107220.

Corbin, D.J. (2014). Methodology and experiments to determine soot and NOx yields from a vertical lab-scale flare burning alkane-mixtures and ethylene (M.A.Sc. Thesis). Carleton University.

Crosland, B.M., Johnson, M.R., and Thomson, K.A. (2011). Analysis of uncertainties in instantaneous soot volume fraction measurements using two-dimensional, autocompensating, laser-induced incandescence (2D-AC-LII). Appl. Phys. B Lasers Opt., 102(1):173-183.

Crosland, B.M., Thomson, K.A., and Johnson, M.R. (2015). Simultaneous instantaneous measurements of soot volume fraction, primary particle diameter, and aggregate size in turbulent buoyant diffusion flames. Proc. Combust. Inst., 35(2):1851-1859.

Crosland, B.M., Thomson, K.A., and Johnson, M.R. (2013). Instantaneous in-flame measurement of soot volume fraction, primary particle diameter, and aggregate radius of gyration via auto-compensating laser-induced incandescence and two-angle elastic light scattering. Appl. Phys. B Lasers Opt., 112(3):381-393.

Curiale, J.A. and Frolov, E.B. (1998). Occurrence and origin of olefins in crude oils. A critical review. Org. Geochem., 29(1-3-3 pt 1):397-408. 
Cushing, L.J., Vavra-Musser, K., Chau, K., Franklin, M., and Johnston, J.E. (2020). Flaring from Unconventional Oil and Gas Development and Birth Outcomes in the Eagle Ford Shale in South Texas. Environ. Health Perspect., 128(7):077003.

Dahlberg, A.R. (2010). All-sky polarization imager deployment at Mauna Loa observatory, Hawaii (M.Sc.). Montana State University.

Dalzell, W.H., Williams, G.C., and Hottel, H.C. (1970). A light-scattering method for soot concentration measurements. Combust. Flame, 14:161-170.

Darula, S. and Kittler, R. (2002). Cie general sky standard defining luminance distributions. Bratislava, Slovakia.

Dastanpour, R., Momenimovahed, A., Thomson, K., Olfert, J.S., and Rogak, S.N. (2017). Variation of the optical properties of soot as a function of particle mass. Carbon N. Y., 124:201-211.

Dastanpour, R. and Rogak, S.N. (2014). Observations of a Correlation Between Primary Particle and Aggregate Size for Soot Particles. Aerosol Sci. Technol., 48(10):10431049.

Davison De St. Germain, J., McCorquodale, J., Parker, S.G., and Johnson, C.R. (2000). Uintah: A massively parallel problem solving environment. Proc. IEEE Int. Symp. High Perform. Distrib. Comput., 33-41.

De Iuliis, S., Cignoli, F., Benecchi, S., and Zizak, G. (1998). Determination of soot parameters by a two-angle scattering-extinction technique in an ethylene diffusion flame. Appl. Opt., 37(33):7865-74.

Delichatsios, M.A. (1993). Transition from momentum to buoyancy-controlled turbulent jet diffusion flames and flame height relationships. Combust. Flame, 92(4):349-364.

Delichatsios, M.A., Orloff, L., and Delichatsios, M.M. (1992). The effects of fuel sooting tendency and the flow on flame radiation in luminous turbulent jet flames. Combust. Sci. Technol., 84(1):199-215.

Devore, J.L. and Berk, K.N. (2012). Modern Mathematical Statistics with Applications, Second. ed, Springer Texts in Statistics. Springer New York, New York, NY.

Dobbins, R.A. and Megaridis, C.M. (1991). Absorption and scattering of light by polydisperse aggregates. Appl. Opt., 30(33):4747-4754.

ECCC (2020). Canada's Black Carbon Inventory Report (2013-2018). Gatineau, QC.

Edner, H., Fredriksson, K., Sunesson, A., Svanberg, S., Unéus, L., and Wendt, W. (1987). Mobile remote sensing system for atmospheric monitoring. Appl. Opt., 26(19):4330.

Ellzey, J.L., Berbee, J.G., Tay, Z.F., and Foster, D.E. (1990). Total soot yield from a propane diffusion flame in cross-flow. Combust. Sci. Technol., 71(1-3):41-52.

Elvidge, C.D., Baugh, K.E., Tuttle, B.T., Howard, A.T., Pack, D.W., Milesi, C., and Erwin, E.H. (2007). A Twelve Year Record of National and Global Gas Flaring Volumes Estimated Using Satellite Data: Final Report to the World Bank. 
Elvidge, C.D., Bazilian, M.D., Zhizhin, M., Ghosh, T., Baugh, K., and Hsu, F.-C. (2018). The potential role of natural gas flaring in meeting greenhouse gas mitigation targets. Energy Strateg. Rev., 20:156-162.

Elvidge, C.D., Zhizhin, M., Baugh, K., Hsu, F., and Ghosh, T. (2015). Methods for global survey of natural gas flaring from visible infrared imaging radiometer suite data. Energies, 9(1):14.

Elvidge, C.D., Ziskin, D., Baugh, K.E., Tuttle, B.T., Ghosh, T., Pack, D.W., Erwin, E.H., and Zhizhin, M. (2009). A Fifteen Year Record of Global Natural Gas Flaring Derived from Satellite Data. Energies, 2(3):595-622.

Environment and Climate Change Canada (2016). Historical Climate Data: Edmonton International Airport.

Everest, D.A., Driscoll, J.F., Dahm, W.J.A., and Feikema, D.A. (1995). Images of the two-dimensional field and temperature gradients to quantify mixing rates within a non-premixed turbulent jet flame. Combust. Flame, 101(1-2):58-68.

Faeth, G.M. and Köylü, Ü.Ö. (1995). Soot morphology and optical properties in nonpremixed turbulent flame environments. Combust. Sci. Technol., 108(4-6):207229.

Farias, T.L., Carvalho, M.G., Köylü, Ü.Ö., and Faeth, G.M. (1995). Computational evaluation of approximate Rayleigh-Debye-Gans/fractal-aggregate theory for the absorption and scattering properties of soot. J. Heat Transfer, 117(1):152-159.

Farias, T.L., Köylü, Ü.Ö., and Carvalho, M.G. (1996). Range of validity of the RayleighDebye-Gans theory for optics of fractal aggregates. Appl. Opt., 35:6560-6567.

Filippov, A. (2013). Chemical composition of associated gas. Neft. Mag., 22-26.

Flanner, M.G., Zender, C.S., Randerson, J.T., and Rasch, P.J. (2007). Present-day climate forcing and response from black carbon in snow. J. Geophys. Res., 112(D11):D11202.

Forestieri, S.D., Helgestad, T.M., Lambe, A.T., Renbaum-Wolff, L., Lack, D.A., Massoli, P., Cross, E.S., Dubey, M.K., Mazzoleni, C., Olfert, J.S., Sedlacek III, A.J., Freedman, A., Davidovits, P., Onasch, T.B., and Cappa, C.D. (2018). Measurement and modeling of the multiwavelength optical properties of uncoated flame-generated soot. Atmos. Chem. Phys., 18(16):12141-12159.

Fung, K. (1990). Particulate Carbon Speciation by $\mathrm{MnO}_{2}$ Oxidation. Aerosol Sci. Technol., 12(1):122-127.

Gardiner, W.C., Hidaka, Y., and Tanzawa, T. (1981). Refractivity of combustion gases. Combust. Flame, 40(C):213-219.

Gasser, T., Peters, G.P., Fuglestvedt, J.S., Collins, W.J., Shindell, D.T., and Ciais, P. (2017). Accounting for the climate-carbon feedback in emission metrics. Earth Syst. Dyn., 8(2):235-253.

Giannoni, M., Calzolai, G., Chiari, M., Cincinelli, A., Lucarelli, F., Martellini, T., and Nava, S. (2016). A comparison between thermal-optical transmittance elemental 
carbon measured by different protocols in $\mathrm{PM}_{2.5}$ samples. Sci. Total Environ., 571:195-205.

Gini, C. (1912). Variabilità e mutabilità: contributo allo studio delle distribuzioni e delle relazioni statistiche. Tipographi di Paolo Cuppini, Bologna, Italy.

Glassman, I. (1998). Sooting laminar diffusion flames: Effect of dilution, additives, pressure, and microgravity. Proc. Combust. Inst., 27(1):1589-1596.

Glassman, I. (1989). Soot formation in combustion processes. Symp. Combust., Springer Series in Chemical Physics, 22(1):295-311.

Goldstein, D.H. (2003). Polarized Light, 2nd ed. Marcel Dekker, New York, NY.

Gollahalli, S.R., Brzustowski, T.A., and Sullivan, M.H.F. (1975). Characteristics of a turbulent propane diffusion flame in a cross-Wind. Trans. CSME, 3(4):205-214.

Gordon, I.E., Rothman, L.S., Hill, C., Kochanov, R.V., Tan, Y., Bernath, P.F., Birk, M., Boudon, V., Campargue, A., Chance, K.V., Drouin, B.J., Flaud, J.-M., Gamache, R.R., Hodges, J.T., Jacquemart, D., Perevalov, V.I., Perrin, A., Shine, K.P., Smith, M.-A.H., Tennyson, J., Toon, G.C., Tran, H., Tyuterev, V.G., Barbe, A., Császár, A.G., Devi, V.M., Furtenbacher, T., Harrison, J.J., Hartmann, J.-M., Jolly, A., Johnson, T.J., Karman, T., Kleiner, I., Kyuberis, A.A., Loos, J., Lyulin, O.M., Massie, S.T., Mikhailenko, S.N., Moazzen-Ahmadi, N., Müller, H.S.P., Naumenko, O.V., Nikitin, A.V., Polyansky, O.L., Rey, M., Rotger, M., Sharpe, S.W., Sung, K., Starikova, E., Tashkun, S.A., Auwera, J. Vander, Wagner, G., Wilzewski, J., Wcisło, P., Yu, S., and Zak, E.J. (2017). The HITRAN2016 molecular spectroscopic database. J. Quant. Spectrosc. Radiat. Transf., 203:3-69.

Gordon, S. and McBride, B. (1996). Computer program for calculation of complex chemical equilibrium compositions and applications. NASA Reference Publication 1311.

Grahame, T.J., Klemm, R., and Schlesinger, R.B. (2014). Public health and components of particulate matter: The changing assessment of black carbon. J. Air Waste Manage. Assoc., 64(6):620-660.

Gratiy, S.L., Levin, D.A., and Walker, A.C. (2010). Rassvet: Backward Monte Carlo radiative transfer in spherical-shell planetary atmospheres. Icarus, 206(1):366-379.

Greenberg, P.S. and Ku, J.C. (1997). Soot volume fraction imaging. Appl. Opt., 36(22):5514-5522.

Gross, K.C., Bradley, K.C., and Perram, G.P. (2010a). Remote identification and quantification of industrial smokestack effluents via imaging Fourier-transform spectroscopy. Environ. Sci. Technol., 44(24):9390-9397.

Gross, K.C., Tremblay, P., Bradley, K.C., Chamberland, M., Farley, V., and Perram, G.P. (2010b). Instrument calibration and lineshape modeling for ultraspectral imagery measurements of industrial smokestack emissions., in Algorithms and Technologies for Multispectral, Hyperspectral, and Ultraspectral Imagery XVI, S.S. Shen, P.E. Lewis, eds, p. 769516. 
Guilkey, J., Harman, T., Luitjens, J., Schmidt, J., Thornock, J., de St. Germain, J.D., Shankar, S., Peterson, J., Brownlee, C., Reid, C., Saad, T., Beckvermit, J., Humphrey, A., and Peterson, B. (2017). Uintah User Guide: Version 2.1.

Gülder, Ö.L. (1995). Effects of oxygen on soot formation in methane, propane, and nButane diffusion flames. Combust. Flame, 101(3):302-310.

Gülder, Ö.L. and Snelling, D.R. (1993). Influence of nitrogen dilution and flame temperature on soot formation in diffusion flames. Combust. Flame, 92(1-2):115-124.

Gustafsson, Ö. and Ramanathan, V. (2016). Convergence on climate warming by black carbon aerosols. Proc. Natl. Acad. Sci., 113(16):4243-4245.

Gvakharia, A., Kort, E.A., Brandt, A.R., Peischl, J., Ryerson, T.B., Schwarz, J.P., Smith, M.L., and Sweeney, C. (2017). Methane, black carbon, and ethane emissions from natural gas flares in the Bakken Shale, North Dakota. Environ. Sci. Technol., 51(9):5317-5325.

Harley, J. and Gross, K. (2011). Remote quantification of smokestack effluent mass flow rates using imaging fourier transform spectrometry. Proc. SPIE, 8018:1-13.

Harley, J.L., Rankin, B.A., Blunck, D.L., Gore, J.P., and Gross, K.C. (2014). Imaging Fourier-transform spectrometer measurements of a turbulent nonpremixed jet flame. Opt. Lett., 39(8):2350.

Howell, J.R., Siegel, R., and Mengüç, M.P. (2016). Thermal Radiation Heat Transfer, 6th ed. CRC Press, Boca Raton, FL.

Hu, L., Wang, Q., Delichatsios, M.A., Lu, S., and Tang, F. (2014). Flame radiation fraction behaviors of sooty buoyant turbulent jet diffusion flames in reduced- and normal atmospheric pressures and a global correlation with Reynolds number. Fuel, 116:781-786.

Huang, K., Fu, J.S., Hodson, E.L., Dong, X., Cresko, J., Prikhodko, V.Y., Storey, J.M., and Cheng, M.D. (2014). Identification of missing anthropogenic emission sources in Russia: Implication for modeling Arctic haze. Aerosol Air Qual. Res., 14(7):17991811.

Huang, K., Fu, J.S., Prikhodko, V.Y., Storey, J.M., Romanov, A., Hodson, E.L., Cresko, J., Morozova, I., Ignatieva, Y., and Cabaniss, J. (2015). Russian anthropogenic black carbon: emission reconstruction and arctic black carbon simulation. J. Geophys. Res. Atmos., 120(21):11,306-11,333.

IPCC (2013). Climate Change 2013: The Physical Science Basis. Working Group I Contribution to the Fifth Assessment Report of the Intergovernmental Panel on Climate Change. Cambridge University Press, New York, NY.

IPCC (2006). Guidelines for National Greenhouse Gas Inventories - Chapter 4: Fugitive Emissions. Intergovernmental Panel on Climate Change (IPCC), Hayama Japan.

IPIECA (2014). Understanding short-lived climate forcers. London, UK. 
Iyer, S.S., Litzinger, T.A., Lee, S.-Y., and Santoro, R.J. (2007). Determination of soot scattering coefficient from extinction and three-angle scattering in a laminar diffusion flame. Combust. Flame, 149(1-2):206-216.

Jacobson, M.Z. (2010). Short-term effects of controlling fossil-fuel soot, biofuel soot and gases, and methane on climate, Arctic ice, and air pollution health. J. Geophys. Res., 115(D14209):1-24.

Jacobson, M.Z. (2004). Climate response of fossil fuel and biofuel soot, accounting for soot's feedback to snow and sea ice albedo and emissivity. J. Geophys. Res., 109(D21):1-15.

Jacobson, M.Z. (2001). Strong radiative heating due to the mixing state of black carbon in atmospheric aerosols. Nature, 409(6821):695-697.

Jhanwar, B.L. and Meath, W.J. (1982). Dipole oscillator strength distributions, sums, and dispersion energy coefficients for $\mathrm{CO}$ and $\mathrm{CO}_{2}$. Chem. Phys., 67(2):185-199.

Johnson, M.R. and Coderre, A.R. (2012). Compositions and greenhouse gas emission factors of flared and vented gas in the western Canadian sedimentary basin. J. Air Waste Manage. Assoc., 62(9):992-1002.

Johnson, M.R., Devillers, R.W., and Thomson, K.A. (2013). A generalized sky-LOSA method to quantify soot/black carbon emission rates in atmospheric plumes of gas flares. Aerosol Sci. Technol., 47(9):1017-1029.

Johnson, M.R., Devillers, R.W., and Thomson, K.A. (2011). Quantitative field measurement of soot emission from a large gas flare using sky-LOSA. Environ. Sci. Technol., 45(1):345-350.

Johnson, M.R., Devillers, R.W., Yang, C., and Thomson, K.A. (2010). Sky-Scattered solar radiation based plume transmissivity measurement to quantify soot emissions from flares. Environ. Sci. Technol., 44(21):8196-8202.

Johnson, M.R. and Kostiuk, L.W. (2002). Visualization of the fuel stripping mechanism for wake-stabilized diffusion flames in a crossflow., in IUTAM Symposium on Turbulent Mixing and Combustion, Fluid Mechanics and Its Applications, A. Pollard, S. Candel, eds, Springer Netherlands, Dordrecht, pp. 295-303.

Johnson, M.R. and Kostiuk, L.W. (2000). Efficiencies of low-momentum jet diffusion flames in crosswinds. Combust. Flame, 123(1-2):189-200.

Johnson, M.R. and Tyner, D.R. (2019). A black carbon inventory for gas flaring in Alberta's upstream oil and gas sector. Ottawa, ON.

Johnson, M.R., Wilson, D.J., and Kostiuk, L.W. (2001). A fuel stripping mechanism for wake-stabilized jet diffusion flames in crossflow. Combust. Sci. Technol., 169(1):155174.

Kalghatgi, G.T. (1984). Lift-off heights and visible lengths of vertical turbulent jet diffusion flames in still air. Combust. Sci. Technol., 41(1-2):17-29.

Kasten, F. and Young, A.T. (1989). Revised optical air mass tables and approximation formula. Appl. Opt., 28(22):4735-4738. 
Kazemimanesh, M., Dastanpour, R., Baldelli, A., Moallemi, A., Thomson, K.A., Jefferson, M.A., Johnson, M.R., Rogak, S.N., and Olfert, J.S. (2019). Size, effective density, morphology, and nano-structure of soot particles generated from buoyant turbulent diffusion flames. J. Aerosol Sci., 132:22-31.

Khan, B., Hays, M.D., Geron, C., and Jetter, J. (2012). Differences in the OC/EC Ratios that Characterize Ambient and Source Aerosols due to Thermal-Optical Analysis. Aerosol Sci. Technol., 46(2):127-137.

Kim, S.-Y., Dutton, S.J., Sheppard, L., Hannigan, M.P., Miller, S.L., Milford, J.B., Peel, J.L., and Vedal, S. (2015). The short-term association of selected components of fine particulate matter and mortality in the Denver Aerosol Sources and Health (DASH) study. Environ. Heal., 14(1):49.

Kittler, R., Kocifaj, M., and Darula, S. (2012). Daylight Science and Daylighting Technology. Springer New York, New York, NY.

Knox, A., Evans, G.J., Brook, J.R., Yao, X., Jeong, C.-H., Godri, K.J., Sabaliauskas, K., and Slowik, J.G. (2009). Mass Absorption Cross-Section of Ambient Black Carbon Aerosol in Relation to Chemical Age. Aerosol Sci. Technol., 43(6):522-532.

Köhler, M., Geigle, K.-P., Meier, W., Crosland, B.M., Thomson, K.A., and Smallwood, G.J. (2011). Sooting turbulent jet flame: characterization and quantitative soot measurements. Appl. Phys. B, 104(2):409-425.

Kondo, Y. (2015). Effects of Black Carbon on Climate: Advances in Measurement and Modeling. Monogr. Environ. Earth Planets, 3(1):1-85.

Kopp, R.E. and Mauzerall, D.L. (2010). Assessing the climatic benefits of black carbon mitigation. Proc. Natl. Acad. Sci. U. S. A., 107(26):11703-11708.

Köylü, Ü.Ö. and Faeth, G.M. (1996). Spectral extinction coefficients of soot aggregates from turbulent diffusion flames. J. Heat Transfer, 118(2):415-421.

Köylü, Ü.Ö. and Faeth, G.M. (1994). Optical-properties of soot in buoyant laminar diffusion flames. J. Heat Transfer, 116(4):971-979.

Köylü, Ü.Ö. and Faeth, G.M. (1992). Structure of overfire soot in buoyant turbulent diffusion flames at long residence times. Combust. Flame, 89(2):140-156.

Köylü, Ü.Ö., Faeth, G.M., Farias, T.L., and Carvalho, M.G. (1995). Fractal and projected structure properties of soot aggregates. Combust. Flame, 100(4):621-633.

Kwiatkowski, D., Phillips, P.C.B., Schmidt, P., and Shin, Y. (1992). Testing the null hypothesis of stationarity against the alternative of a unit root. J. Econom., 54(13):159-178.

Lappi, M.K. and Ristimäki, J.M. (2017). Evaluation of thermal optical analysis method of elemental carbon for marine fuel exhaust. J. Air Waste Manage. Assoc., 67(12):12981318.

Law, K.S. and Stohl, A. (2007). Arctic air pollution: origins and impacts. Science, 315(5818):1537-1540. 
Li, Y., Henze, D.K., Jack, D., Henderson, B.H., and Kinney, P.L. (2016). Assessing public health burden associated with exposure to ambient black carbon in the United States. Sci. Total Environ., 539:515-525.

Liu, F. and Snelling, D.R. (2008). Evaluation of the Accuracy of the RDG Approximation for the Absorption and Scattering Properties of Fractal Aggregates of Flame-Generated Soot., in 40th Thermophysics Conference, American Institute for Aeronautics and Astronautics, Seattle, pp. 1-13.

Luben, T.J., Nichols, J.L., Dutton, S.J., Kirrane, E., Owens, E.O., Datko-Williams, L., Madden, M., and Sacks, J.D. (2017). A systematic review of cardiovascular emergency department visits, hospital admissions and mortality associated with ambient black carbon. Environ. Int., 107(January):154-162.

Mahmoud, S.M., Nathan, G.J., Alwahabi, Z.T., Sun, Z.W., Medwell, P.R., and Dally, B.B. (2018). The effect of exit Reynolds number on soot volume fraction in turbulent non-premixed jet flames. Combust. Flame, 187:42-51.

Majeski, A.J., Wilson, D.J., and Kostiuk, L.W. (2004). Predicting the length of lowmomentum jet diffusion flames in crossflow. Combust. Sci. Technol., 176(12):20012025.

Marchuk, G.I., Nazaraliev, G.A., Darbinjan, R.A., Kargin, B.A., and Elepov, B.S. (1980). The Monte Carlo Methods in Atmospheric Optics, 1st ed. Springer-Verlag Berlin Heidelberg, New York, NY.

Margoliash, D.J. and Meath, W.J. (1978). Pseudospectral dipole oscillator strength distributions and some related two body interaction coefficients for $\mathrm{H}, \mathrm{He}, \mathrm{Li}, \mathrm{N}, \mathrm{O}$, $\mathrm{H}_{2}, \mathrm{~N}_{2}, \mathrm{O}_{2}, \mathrm{NO}, \mathrm{N}_{2} \mathrm{O}, \mathrm{H}_{2} \mathrm{O}, \mathrm{NH}_{3}$, and $\mathrm{CH}_{4}$. J. Chem. Phys., 68(4):1426-1431.

Mathar, R.J. (2007). Refractive index of humid air in the infrared: model fits. J. Opt. A Pure Appl. Opt., 9(5):470-476.

Mathar, R.J. (2004). Calculated refractivity of water vapor and moist air in the atmospheric window at $10 \mu \mathrm{m}$. Appl. Opt., 43(4):928.

Maxwell Garnett, J.C. (1904). Colours in metal glasses and in metallic films. Philos. Trans. R. Soc. London. Ser. A, Contain. Pap. a Math. or Phys. Character, 203(359371):385-420.

McDaniel, M. (1983). Flare efficiency study (No. EPA-600/2-83-052). United Stated Environmental Protection Agency, Research Triangle Park, NC.

McEwen, J.D.N. and Johnson, M.R. (2012). Black carbon particulate matter emission factors for buoyancy-driven associated gas flares. J. Air Waste Manage. Assoc., 62(3):307-321.

Mehr, P. (2020). Experimental Modelling of Black Carbon Emissions from Gas Flares in the Oil and Gas Sector. Carleton University.

Mellqvist, J., Samuelsson, J., Johansson, J., Rivera, C., Lefer, B., Alvarez, S., and Jolly, J. (2010). Measurements of industrial emissions of alkenes in Texas using the solar occultation flux method. J. Geophys. Res., 115:D00F17. 
Mie, G. (1908). Beiträge zur Optik trüber Medien, speziell kolloidaler Metallösungen. Ann. Phys., 330(3):377-445.

Mishchenko, M.I. (2014). Electromagnetic Scattering by Particles and Particle Groups. Cambridge University Press, New York, NY.

Mishchenko, M.I., Travis, L.D., and Lacis, A.A. (2002). Scattering, absorption, and emission of light by small particles. Cambridge University Press, Cambridge, UK.

Modest, M.F. (2013). Radiative Heat Transfer, Third Edit. ed. Elsevier Inc., Oxford, UK.

Moffet, R.C. and Prather, K.A. (2009). In-situ measurements of the mixing state and optical properties of soot with implications for radiative forcing estimates. Proc. Natl. Acad. Sci., 106(29):11872-11877.

Moore, D.T. (1975). Ray tracing in gradient-index media. J. Opt. Soc. Am., 65(4):451.

Moran, M.J. and Shapiro, H.N. (2006). Fundamentals of Engineering Thermodynamics, 5 th editio. ed. John Wiley \& Sons, Chichester, UK.

Nakayama, M.K. (2011). Asymptotically Valid Confidence Intervals for Quantiles and Values-at-Risk When Applying Latin Hypercube Sampling. Int. J. Adv. Syst. Meas., 4(1):86-94.

Navvab, M., Karayel, M., Ne'eman, E., and Selkowitz, S. (1984). Analysis of atmospheric turbidity for daylight calculations. Energy Build., 6(3):293-303.

Nicolosi, E.M.G., Quincey, P., Font, A., and Fuller, G.W. (2018). Light attenuation versus evolved carbon (AVEC) - A new way to look at elemental and organic carbon analysis. Atmos. Environ., 175:145-153.

NOAA (2020). Visible Infrared Imaging Radiometer Suite (VIIRS): Global Flare Data.

Nordmann, S., Birmili, W., Weinhold, K., Müller, K., Spindler, G., and Wiedensohler, A. (2013). Measurements of the mass absorption cross section of atmospheric soot particles using Raman spectroscopy. J. Geophys. Res. Atmos., 118(21):12,075-12,085.

Oltmann, H., Reimann, J., and Will, S. (2010). Wide-angle light scattering (WALS) for soot aggregate characterization. Combust. Flame, 157(3):516-522.

Ostro, B., Tobias, A., Karanasiou, A., Samoli, E., Querol, X., Rodopoulou, S., Basagaña, X., Eleftheriadis, K., Diapouli, E., Vratolis, S., Jacquemin, B., Katsouyanni, K., Sunyer, J., Forastiere, F., and Stafoggia, M. (2015). The risks of acute exposure to black carbon in Southern Europe: results from the MED-PARTICLES project. Occup. Environ. Med., 72(2):123-129.

Otterman, J. (1978). Single-scattering solution for radiative transfer through a turbid atmosphere. Appl. Opt., 17(21):3431.

Panteliadis, P., Hafkenscheid, T., Cary, B., Diapouli, E., Fischer, A., Favez, O., Quincey, P., Viana, M., Hitzenberger, R., Vecchi, R., Saraga, D., Sciare, J., Jaffrezo, J.L., John, A., Schwarz, J., Giannoni, M., Novak, J., Karanasiou, A., Fermo, P., and Maenhaut, W. (2015). ECOC comparison exercise with identical thermal protocols after temperature offset correction - Instrument diagnostics by in-depth evaluation of operational parameters. Atmos. Meas. Tech., 8(2):779-792. 
Parker, S.G. (2006). A component-based architecture for parallel multi-physics PDE simulation. Futur. Gener. Comput. Syst., 22(1-2):204-216.

Parker, S.G., Guilkey, J., and Harman, T. (2006). A component-based parallel infrastructure for the simulation of fluid-structure interaction. Eng. Comput., 22(34):277-292.

Peng, J., Hu, M., Guo, S., Du, Z., Zheng, Jing, Shang, D., Levy Zamora, M., Zeng, L., Shao, M., Wu, Y.-S., Zheng, Jun, Wang, Y., Glen, C.R., Collins, D.R., Molina, M.J., and Zhang, R. (2016). Markedly enhanced absorption and direct radiative forcing of black carbon under polluted urban environments. Proc. Natl. Acad. Sci., 113(16):4266-4271.

Petzold, A., Ogren, J.A., Fiebig, M., Laj, P., Li, S.-M., Baltensperger, U., Holzer-Popp, T., Kinne, S., Pappalardo, G., Sugimoto, N., Wehrli, C., Wiedensohler, A., and Zhang, X.-Y. (2013a). Recommendations for reporting "black carbon" measurements. Atmos. Chem. Phys., 13(16):8365-8379.

Petzold, A., Onasch, T., Kebabian, P., and Freedman, A. (2013b). Intercomparison of a Cavity Attenuated Phase Shift-based extinction monitor (CAPS PMex) with an integrating nephelometer and a filter-based absorption monitor. Atmos. Meas. Tech., 6(5):1141-1151.

PFC Energy (2007). Using Russia’s Associated Gas: Prepared for the Global Gas Flaring Reduction Partnership and the World Bank.

Pierson, H.O. (1993). Handbook of Carbon, Graphite, Diamond, and Fullerenes: Properties, Processing, and Applications. Noyes Publications, Park Ridge, NJ.

Platt, U. and Stutz, J. (2008). Differential Optical Absorption Spectroscopy: Principles \& Applications. Springer-Verlag, Berlin/Heidelberg, Germany.

Pohl, J.H., Tichenor, B.A., Lee, J., and Payne, R. (1986). Combustion Efficiency of Flares. Combust. Sci. Technol., 50(4-6):217-231.

Popovicheva, O., Timofeev, M., Persiantseva, N., Jefferson, M.A., Johnson, M., Rogak, S.N., and Baldelli, A. (2019). Microstructure and Chemical Composition of Particles from Small-scale Gas Flaring. Aerosol Air Qual. Res., 19(10):2205-2221.

Popovicheva, O.B., Evangeliou, N., Eleftheriadis, K., Kalogridis, A.C., Sitnikov, N., Eckhardt, S., and Stohl, A. (2017). Black Carbon Sources Constrained by Observations in the Russian High Arctic. Environ. Sci. Technol., 51(7):3871-3879.

Poudenx, P., Howell, L.W., Wilson, D.J., and Kostiuk, L.W. (2004). Downstream Similarity of Thermal Structure in Plumes From Jet Diffusion Flames in a Crossflow. Combust. Sci. Technol., 176(3):409-435.

Presler-Jur, P., Doraiswamy, P., Hammond, O., and Rice, J. (2017). An evaluation of mass absorption cross-section for optical carbon analysis on Teflon filter media. J. Air Waste Manage. Assoc., 67(11):1213-1228.

Pust, N.J. and Shaw, J.A. (2012). Wavelength dependence of the degree of polarization in cloud-free skies: simulations of real environments. Opt. Express, 20(14):15559-68. 
Radney, J.G., You, R., Ma, X., Conny, J.M., Zachariah, M.R., Hodges, J.T., and Zangmeister, C.D. (2014). Dependence of Soot Optical Properties on Particle Morphology: Measurements and Model Comparisons. Environ. Sci. Technol., 48(6):3169-3176.

Raga, G.B., Vallack, H., Kuylenstierna, J., Dore, C., Claxton, R., Shindell, D., Foltescu, V., Cong, H., and Borgford-Parnell, N. (2018). Addressing black carbon emission inventories.

Ramanathan, V. and Carmichael, G. (2008). Global and regional climate changes due to black carbon. Nat. Geosci., 1(4):221-227.

Reda, I. and Andreas, A. (2008). Solar Position Algorithm for Solar Radiation Applications (Revised), National Renewable Energy Laboratory Nrel/Tp-560-34302. Golden, CO (United States).

Rinkenburger, A., Toriyama, T., Yasuda, K., and Niessner, R. (2017). Catalytic Effect of Potassium Compounds in Soot Oxidation. ChemCatChem, 9(18):3513-3525.

Saskatchewan Ministry of Energy and Resources (2020). Directive PNG036: Venting and Flaring Requirements. Regina, SK.

Sato, M., Hansen, J., Koch, D., Lacis, A., Ruedy, R., Dubovik, O., Holben, B., Chin, M., and Novakov, T. (2003). Global atmospheric black carbon inferred from AERONET. Proc. Natl. Acad. Sci., 100(11):6319-6324.

Savary, S., Gagnon, J.-P., Gross, K.C., Tremblay, P., Chamberland, M., and Farley, V. (2011). Standoff identification and quantification of flare emissions using infrared hyperspectral imaging. Adv. Environ. Chem. Biol. Sens. Technol. VIII, 8024:80240T.

Schauer, J.J., Mader, B.T., DeMinter, J.T., Heidemann, G., Bae, M.S., Seinfeld, J.H., Flagan, R.C., Cary, R.A., Smith, D., Huebert, B.J., Bertram, T., Howell, S., Kline, J.T., Quinn, P., Bates, T., Turpin, B., Lim, H.J., Yu, J.Z., Yang, H., and Keywood, M.D. (2003). ACE-Asia Intercomparison of a Thermal-Optical Method for the Determination of Particle-Phase Organic and Elemental Carbon. Environ. Sci. Technol., 37(5):993-1001.

Schnaiter, M., Linke, C., Möhler, O., Naumann, K.H., Saathoff, H., Wagner, R., Schurath, U., and Wehner, B. (2005). Absorption amplification of black carbon internally mixed with secondary organic aerosol. J. Geophys. Res. D Atmos., 110(19):1-11.

Schulz, M., Textor, C., Kinne, S., Balkanski, Y., Bauer, S., Berntsen, T., Berglen, T., Boucher, O., Dentener, F., Guibert, S., Isaksen, I.S.A., Iversen, T., Koch, D., Kirkevåg, A., Liu, X., Montanaro, V., Myhre, G., Penner, J.E., Pitari, G., Reddy, S., Seland, Ø., Stier, P., and Takemura, T. (2006). Radiative forcing by aerosols as derived from the AeroCom present-day and pre-industrial simulations. Atmos. Chem. Phys. Discuss., 6(3):5095-5136.

Schuster, G.L. (2004). Inferring the Specific Absorption and Concentration of Black Carbon From Aeronet Aerosol Retrievals. Pennsylvania State University. 
Schuster, G.L., Dubovik, O., Arola, A., Eck, T.F., and Holben, B.N. (2016). Remote sensing of soot carbon - Part 2: Understanding the absorption Ångström exponent. Atmos. Chem. Phys., 16(3):1587-1602.

Schwarz, J.P., Holloway, J.S., Katich, J.M., McKeen, S., Kort, E.A., Smith, M.L., Ryerson, T.B., Sweeney, C., and Peischl, J. (2015). Black carbon emissions from the Bakken oil and gas development region. Environ. Sci. Technol. Lett., 2(10):281-285.

Seymour, S.P. (2019). Spectroscopic Measurements of Path-Averaged Species Correlations in Turbulent Flare Plumes. Carleton University.

Snelling, D.R., Thomson, K.A., Smallwood, G.J., and Gülder, Ö.L. (1999). Twodimensional imaging of soot volume fraction in laminar diffusion flames. Appl. Opt., 38(12):2478-2485.

Sorensen, C.M. (2001). Light Scattering by Fractal Aggregates: A Review. Aerosol Sci. Technol., 35(2):648-687.

Standards Council of Canada (1999). CAN/CGSB-3.0 No. 14.3-99 Methods of testing petroleum and associated products standard test method for the identification of hydrocarbon components in automotive gasoline using gas chromatography.

Statistics Canada (2020). Table 25-10-0055-01: Supply and Disposition of Natural Gas, Monthly (data in thousands) (x 1,000).

Stevens, S.S. (1957). On the psychophysical law. Psychol. Rev., 64(3):153-181.

Stohl, A. (2006). Characteristics of atmospheric transport into the Arctic troposphere. $J$. Geophys. Res., 111(D11):D11306.

Stohl, A., Berg, T., Burkhart, J.F., Fjæraa, A.M., Forster, C., Herber, A., Hov, Ø., Lunder, C., McMillan, W.W., Oltmans, S., Shiobara, M., Simpson, D., Solberg, S., Stebel, K., Ström, J., Tørseth, K., Treffeisen, R., Virkkunen, K., and Yttri, K.E. (2006). Arctic smoke - record high air pollution levels in the European Arctic due to agricultural fires in Eastern Europe. Atmos. Chem. Phys. Discuss., 6(5):9655-9722.

Stohl, A., Klimont, Z., Eckhardt, S., Kupiainen, K., Shevchenko, V.P., Kopeikin, V.M., and Novigatsky, A.N. (2013). Black carbon in the Arctic: the underestimated role of gas flaring and residential combustion emissions. Atmos. Chem. Phys., 13(17):88338855 .

Subramanian, R., Khlystov, A., and Robinson, A. (2006). Effect of peak inert-mode temperature on elemental carbon measured using thermal-optical analysis. Aerosol Sci. Technol., 40(10):763-780.

Subramanian, R., Kok, G.L., Baumgardner, D., Clarke, A., Shinozuka, Y., Campos, T.L., Heizer, C.G., Stephens, B.B., de Foy, B., Voss, P.B., and Zaveri, R.A. (2010). Black carbon over Mexico: the effect of atmospheric transport on mixing state, mass absorption cross-section, and BC/CO ratios. Atmos. Chem. Phys., 10(1):219-237.

Takahashi, F. and Schmoll, W.J. (1991). Lifting criteria of jet diffusion flames. Symp. Combust., 23(1):677-683. 
Textor, C., Schulz, M., Guibert, S., Kinne, S., Balkanski, Y., Bauer, S., Berntsen, T., Berglen, T., Boucher, O., Chin, M., Dentener, F., Diehl, T., Easter, R., Feichter, H., Fillmore, D., Ghan, S., Ginoux, P., Gong, S., Grini, A., Hendricks, J., Horowitz, L., Huang, P., Isaksen, I., Iversen, I., Kloster, S., Koch, D., Kirkevåg, A., Kristjansson, J.E., Krol, M., Lauer, A., Lamarque, J.F., Liu, X., Montanaro, V., Myhre, G., Penner, J., Pitari, G., Reddy, S., Seland, Ø., Stier, P., Takemura, T., and Tie, X. (2006). Analysis and quantification of the diversities of aerosol life cycles within AeroCom. Atmos. Chem. Phys., 6(7):1777-1813.

Thomson, K.A., Johnson, M.R., Snelling, D.R., and Smallwood, G.J. (2008). Diffuselight two-dimensional line-of-sight attenuation for soot concentration measurements. Appl. Opt., 47(5):694-703.

Török, S., Malmborg, V.B., Simonsson, J., Eriksson, A., Martinsson, J., Mannazhi, M., Pagels, J., and Bengtsson, P.-E. (2018). Investigation of the absorption Ångström exponent and its relation to physicochemical properties for mini-CAST soot. Aerosol Sci. Technol., 52(7):757-767.

Trivanovic, U., Sipkens, T.A., Kazemimanesh, M., Baldelli, A., Jefferson, A.M., Conrad, B.M., Johnson, M.R., Corbin, J.C., Olfert, J.S., and Rogak, S.N. (2020). Morphology and size of soot from gas flares as a function of fuel and water addition. Fuel, 279:110 .

Turns, S.R. (2012). An Introduction to Combustion: Concepts and Applications, 3rd ed. McGraw-Hill, New York, NY.

Tyner, D.R. and Johnson, M.R. (2018). A Techno-Economic Analysis of Methane Mitigation Potential from Reported Venting at Oil Production Sites in Alberta. Environ. Sci. Technol., 52(21):12877-12885.

U.S. EPA (2018). AP 42 - Compilation of Air Pollutant Emission Factors, Volume I, Section 13.5 Industrial Flares. Research Triangle Park, NC.

U.S. EPA (2012). Report to Congress on Black Carbon (No. EPA-450/R-12-001). United States Environmental Protection Agency (U.S. EPA), Research Triangle Park, NC.

U.S. EPA (1995). AP 42 - compilation of air pollutant emission factors, volume I, 5 th ed. - section 13.5 industrial flares (No. AP-42). United States Environmental Protection Agency (U.S. EPA), Research Triangle Park, NC.

U.S. EPA (1991). Data from landfill gas flare, Confidential Report No. ERC-55. United States Environmental Protection Agency (U.S. EPA).

U.S. EPA (1974). Visual Determination of the Opacity of Emissions from Stationary Sources. Code of Federal Regulations, Title 40, Part 60, Appendix A-4, Method 9, United States of America.

United Nations Economic Commission for Europe (2012). 1999 Protocol to Abate Acidification, Eutrophication and Ground-level ozone to the Convention on Longrange Transboundary Air Pollution (No. ECE/EB. AIR/114).

van de Hulst, H.C. (1957). Light Scattering by Small Particles. Dover publications. 
Voxman, W.L. and Goetschel Jr., R.H. (1981). Advanced Calculus: An Introduction to Modern Analysis. Marcel Dekker, New York, NY.

Watanabe, T., Takamatsu, T., and Nakajima, T.Y. (2016). Evaluation of Variation in Surface Solar Irradiance and Clustering of Observation Stations in Japan. J. Appl. Meteorol. Climatol., 55(10):2165-2180.

Weinberg, F. (1996). On the use of light sheet methods in the presence of refraction by flames. Symp. Combust., 26(1):939-944.

Weinberg, F.J. (1963). Optics of Flames. Butterworths, London, UK.

Weyant, C.L., Shepson, P.B., Subramanian, R., Cambaliza, M.O.L.L., Heimburger, A., Mccabe, D., Baum, E., Stirm, B.H., and Bond, T.C. (2016). Black carbon emissions from associated natural gas flaring. Environ. Sci. Technol., 50(4):2075-2081.

Wieneke, B. (2015). PIV uncertainty quantification from correlation statistics. Meas. Sci. Technol., 26(7):074002.

Will, S., Schraml, S., and Leipertz, A. (1996). Comprehensive two-dimensional soot diagnostics based on laser-induced incandescence (LII)., in Proceedings of the 26th International Symposium on Combustion., Symposium (International) on Combustion, Napoli, Italy, pp. 2277-2284.

Wiscombe, W.J. (2005). Scales, Tools and Reminiscences., in 3D Radiative Transfer in Cloudy Atmospheres, A. Marshak, A. Davis, eds, Springer-Verlag, Berlin/Heidelberg, pp. 3-92.

Witten, T.A. and Sander, L.M. (1983). Diffusion-limited aggregation. Phys. Rev. B, 27(9):5686-5697.

Wocken, C.A., Stevens, B.G., Almlie, J.C., and Schlasner, S.M. (2013). End-Use Technology Study - An Assessment of Alternative Uses for Associated Gas.

Wong, I.L. (2017). A review of daylighting design and implementation in buildings. Renew. Sustain. Energy Rev., 74:959-968.

World Bank (2015). Zero Routine Flaring by 2030 Initiative. Available at http://www.worldbank.org/en/programs/zero-routine-flaring-by-2030 (Accessed 23 February 2018).

You, R., Radney, J.G., Zachariah, M.R., and Zangmeister, C.D. (2016). Measured Wavelength-Dependent Absorption Enhancement of Internally Mixed Black Carbon with Absorbing and Nonabsorbing Materials. Environ. Sci. Technol., 50(15):79827990 .

Zanatta, M., Laj, P., Gysel, M., Baltensperger, U., Vratolis, S., Eleftheriadis, K., Kondo, Y., Dubuisson, P., Winiarek, V., Kazadzis, S., Tunved, P., and Jacobi, H.-W. (2018). Effects of mixing state on optical and radiative properties of black carbon in the European Arctic. Atmos. Chem. Phys., 18(19):14037-14057.

Zeng, Y., Morris, J., and Dombrowski, M. (2016). Validation of a new method for measuring and continuously monitoring the efficiency of industrial flares. J. Air Waste Manage. Assoc., 66(1):76-86. 
Zerbs, J., Geigle, K.P., Lammel, O., Hader, J., Stirn, R., Hadef, R., and Meier, W. (2009). The influence of wavelength in extinction measurements and beam steering in laserinduced incandescence measurements in sooting flames. Appl. Phys. B Lasers Opt., 96(4):683-694.

Zhang, R., Peng, J., Wang, Y., and Hu, M. (2016). Reply to Boucher et al.: Rate and timescale of black carbon aging regulate direct radiative forcing. Proc. Natl. Acad. Sci., 113(35):E5094-E5095. 


\section{Appendix A Supplemental Information for Field Measurements of Black Carbon Yields from Gas Flaring}

\section{A.1 Details of the Measured Flares}

\section{A.1.1 Measured Flares in Veracruz, Mexico}

The measured flares in the state of Veracruz, Mexico were located at two downstream facilities under the jurisdiction of Petróleos Mexicanos (PEMEX) located outside Coatzacoalcos and Minatitlán. Flares were not pre-selected for measurement, instead PEMEX personnel provided access to the two facilities and all flares within the site were available to be measured. Figure A.1a shows the locations of Coatzacoalcos and Minatitlán within Veracruz near the southernmost point of the Bay of Campeche, and Figure A.1b shows the locations of the measured flares (labelled V1-V5 as in the manuscript) with respect to Coatzacoalcos and Minatitlán. Black carbon (BC) emission measurements on these flares were performed using sky-LOSA during December 15-17, 2012. 

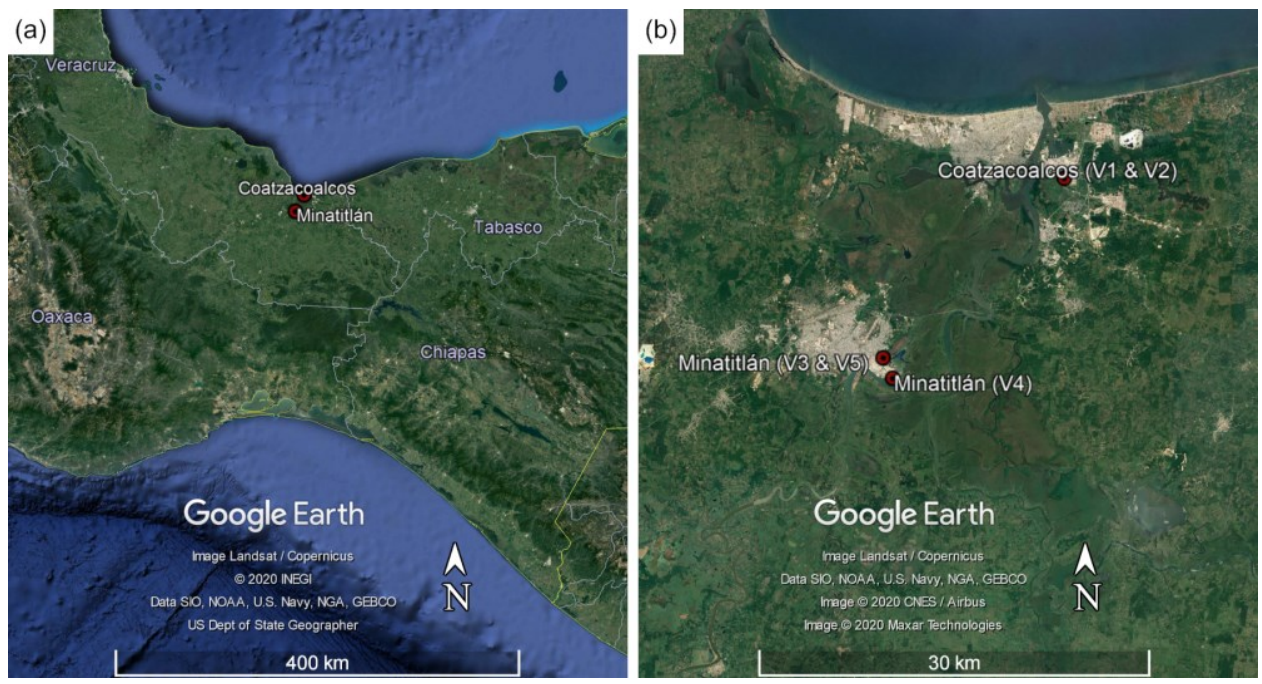

Figure A.1: The locations of measured flares in Veracruz, Mexico. (a) The location of Coatzacoalcos and Minatitlán within the state of Veracruz and (b) The location of measured flares with respect to Coatzacoalcos and Minatitlán.

As shown in Figure A.2, flares V1, V3, and V5 were pit flares, Flare V2 was an enclosed steam-assisted ground flare with a $\sim 12-\mathrm{m}$ diameter vertical-axis cylindrical enclosure, and Flare V4 was a large, $\sim 1$-m diameter, $\sim 85-\mathrm{m}$ tall steam-assisted vertical stack flare. Flares V2, V3, and V5 appeared to be operating steadily during the measurement campaign. 

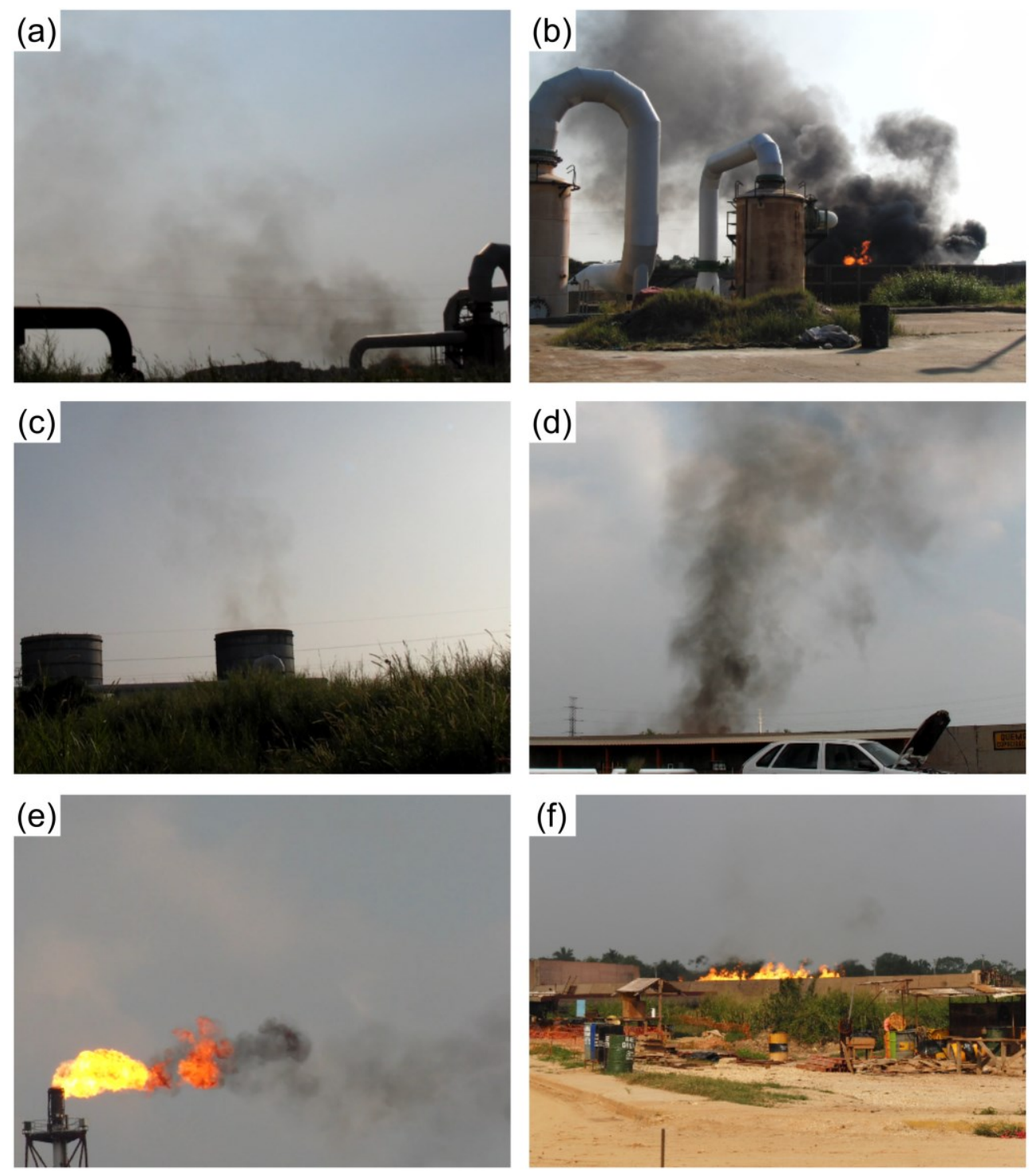

Figure A.2: Sample colour images of the five measured flares in Veracruz, Mexico. (a) Flare V1 (pit flare) showing how visible plume appeared upon arrival; (b) Flare V1 shown as large, 24 hour duration flaring event was beginning (flame is visible in bottom right of image); (c) Flare V2 (enclosed steam-assisted ground flare); (d) Flare V3 (pit flare); (e) Flare V4 (steam-assisted vertical stack flare); and (f) Flare V5 (pit flare).

Flare V4 was noted to be experiencing control issues attributed to its steam-assist system. This caused slow oscillations in the size of the flame and visible magnitude of particulate emissions. At the extremes of these large oscillations, the flame could intermittently be visibly extinguished for several seconds (leading to qualitatively large 
gaseous hydrocarbon emissions as visible through an infrared camera) before returning to a sustained (approximately 30 -s to 2-minute duration) operation in which significant visible smoke was apparent. $\mathrm{BC}$ emission rate measurements were performed during one comparatively steady period between large extinction events.

Upon arrival on site for measurements on December 15, 2012, Flare V1 appeared as in Figure A.2a. However, beginning shortly thereafter, Flare V1 experienced a very significant, approximately 24-hour duration event, during which the size of the flame and visible emissions were dramatically increased. Shortly after onset, the BC emissions from Flare V1 rapidly increased to the extent that direct solar radiation could not penetrate the plume, as illustrated in Figure A.3a (note that the sun was positioned behind the plume in the top-left of the image), such that sky-LOSA measurements were not possible. SkyLOSA measurements of Flare V1 were successfully performed from a different location on the following day as the large flaring event was coming to an end and the associated $\mathrm{BC}$ emissions were in decline. Three independent sky-LOSA measurements were performed over an eleven-minute period. Figure A.3b-d shows images of this flare during sky-LOSA measurements at 12:40, 12:45, and 12:51 local time on December 16, 2012. Figure A.3a (broadband camera image) was taken from the original setup location during near-peak BC emissions at 14:57 local time the day prior. Based on the observed optical attenuation by the plume in the acquired raw sky-LOSA data as compared to other data processed to date, it is speculated that peak $\mathrm{BC}$ emissions from Flare V1 could have been an order of magnitude greater than those measured the following day. 

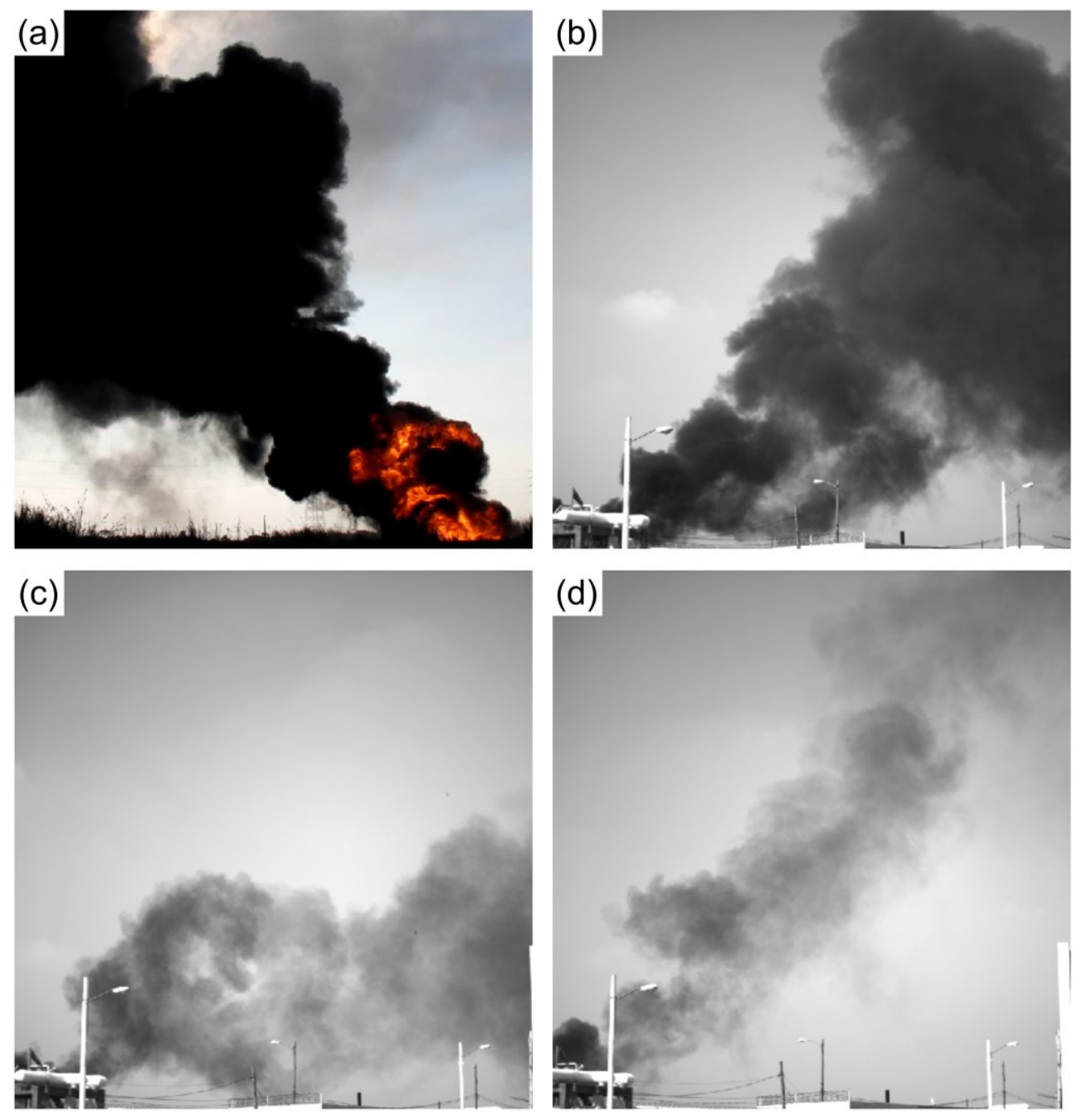

Figure A.3: Sample images of Flare V1 at various times during 24-hour duration large-volume flaring event. (a) Broadband image of the flare shortly after start of the large flaring event, taken from approximately $158 \mathrm{~m} \mathrm{NE}$ of the flare pit. The sun is behind the plume in the top-left corner of the image and is completely blocked by the plume. Given that the optical density of the visibly accessible plume region from this location was sufficient to fully attenuate direct solar radiation, sky-LOSA measurements were unfortunately not possible. (b-d) Grayscale images taken from a different set-up location ( $281 \mathrm{~m}$ SE of the flare pit) approximately 22 hours later as the large-scale flaring event ended. Three independent sky-LOSA measurements were completed over an eleven-minute period and sample images $(b-d)$ are selected from each of these measurements. From these images, it is apparent that the black carbon emissions were rapidly dropping alongside a field-observed decrease in audible flare gas flow rate and visible flame size. 


\section{A.1.2 Measured Flares in Orellana, Ecuador}

$\mathrm{BC}$ emissions from a total of four flares in Ecuador were measured using sky-LOSA in two separate measurement campaigns during June 2-5, 2014 and October 26-29, 2015. The measured flares were situated at four sites in Petroamazonas' Auca field, located $25-40 \mathrm{~km}$ SSE of Puerto Francisco de Orellana (Coca), Ecuador and were selected in consultation with Petroamazonas personnel and the World Bank Global Gas Flaring Reduction partnership because they were consistently operational and represented a range of scales in the Auca field. Figure A.4a shows the general location of Coca within Ecuador and with respect to the capital Quito, and Figure A.4b shows the location of the measurement sites with respect to Coca. The measured flares are numbered in the manuscript as $\mathrm{O} 1-\mathrm{O} 4$ as shown in Figure A.4b. Flare $\mathrm{O} 4$ was not measured during the 2014 campaign.
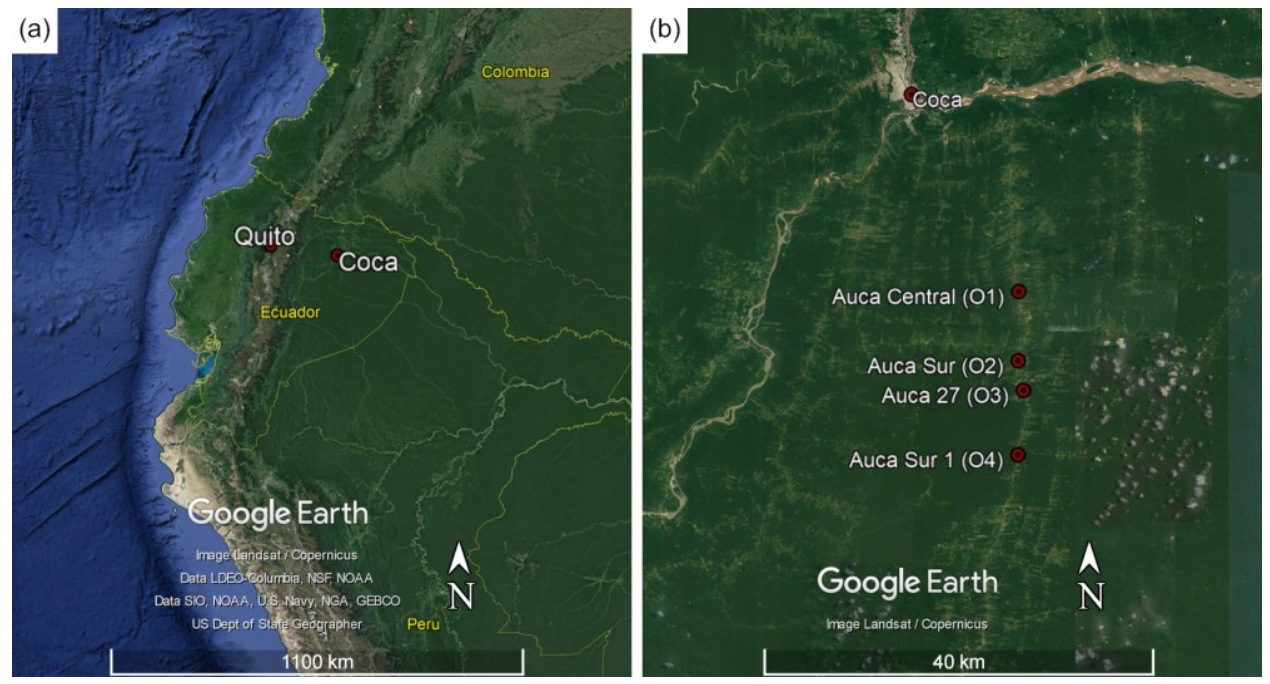

Figure A.4: (a) The location of Ecuador's capital, Quito, and Coca within Ecuador. (b) The location of measurements sites with respect to Coca.

As shown in Figure A.5, all of the measured flares within the Auca field were simple vertical stack flares (pipe flares), each fed by one to four flare lines. These flares were associated with upstream oil production activities in Petroamazonas' Auca field. During the 2015 measurement campaign, flare gas flow rate measurements were performed 
on, and extractive gas samples collected from, all flare lines feeding the measured flares to support quantitative $\mathrm{BC}$ yield calculations. Table A.1 summarizes the number and dimensions of the lines feeding each flare.
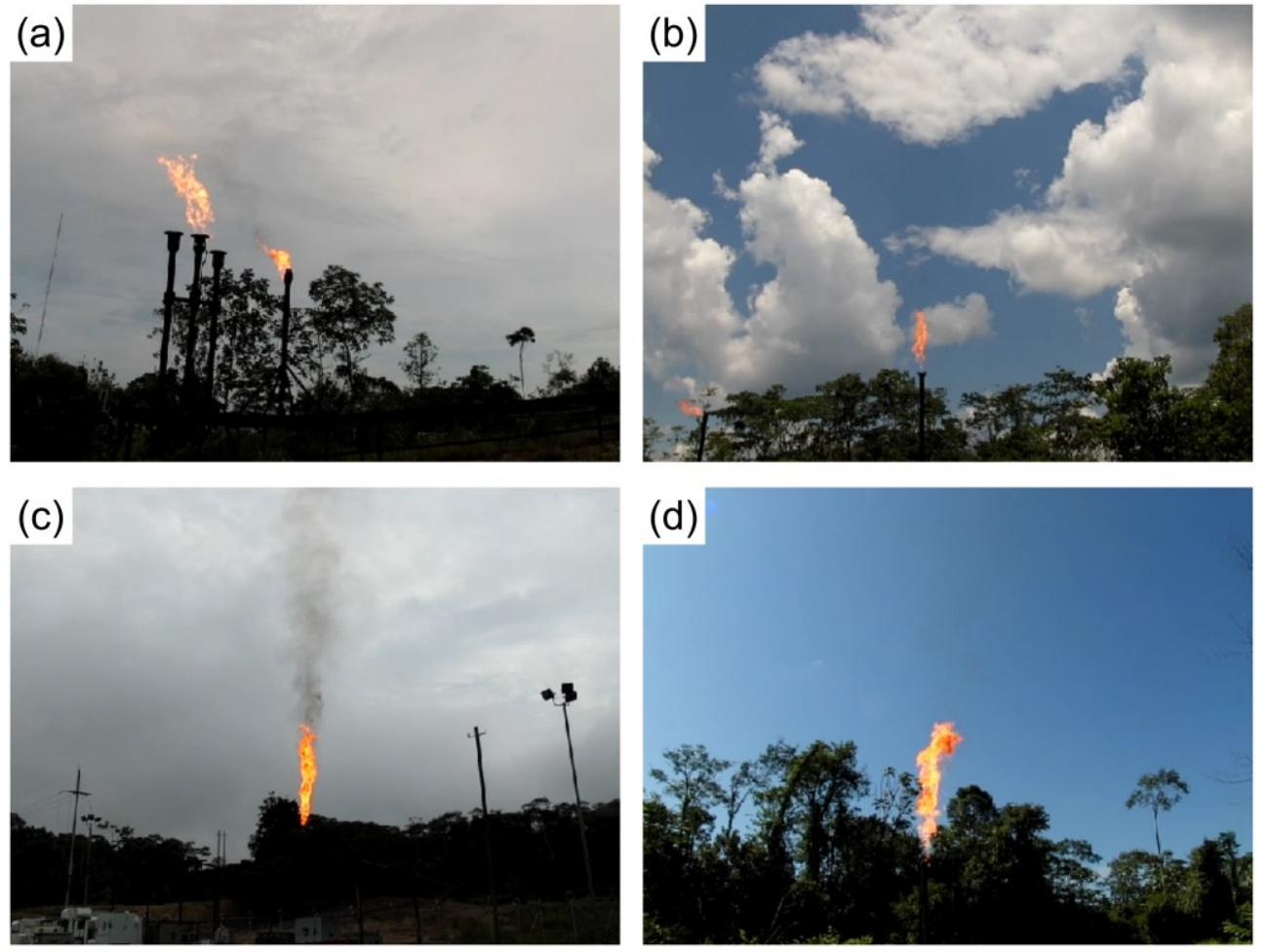

Figure A.5: Sample colour images of the measured flares in Petroamazonas' Auca field near Coca, Ecuador. (a) Flare $\mathrm{O1}$ (measured flare is on the right); (b) Flare $\mathrm{O2}$ (measured flare is on the right); (c) Flare O3; and (d) Flare O4.

As noted in the manuscript, Flare $\mathrm{O} 1$ employed a nozzle arrangement at the base of the flare stack presumably intended to promote air-entrainment into the flare stream prior to combustion. As shown in Figure A.6, the line feeding this flare terminated in a verticalaxis converging nozzle, which was offset vertically from the stack to enable the entrainment of ambient air into the flare gas stream. This design causes aeration and further dilution of the flare gas, resulting in the reduction of $\mathrm{BC}$ emissions from the turbulent flame. 
Table A.1: The dimensions of flare lines feeding the measured flares in Orellana, Ecuador.

\begin{tabular}{|c|c|c|c|}
\hline Location & Line \# & $\begin{array}{c}\text { Nominal Diameter } \\
\text { [in] }\end{array}$ & $\begin{array}{c}\text { Internal Radius } \\
\text { [cm] }\end{array}$ \\
\hline Auca Central (O1) & L1 & 10 & 12.67 \\
\hline \multirow{2}{*}{ Auca Sur (O2) } & L1 & 8 & 10.09 \\
& L2 & 12 & 15.10 \\
\hline \multirow{3}{*}{ Auca 27 (O3) } & L1 & 4 & 5.08 \\
& L2 & 6 & 7.66 \\
& L3 & 4 & 5.08 \\
\hline \multirow{2}{*}{ Luca Sur 1 (O4) } & L1 & 6 & 7.66 \\
\hline
\end{tabular}

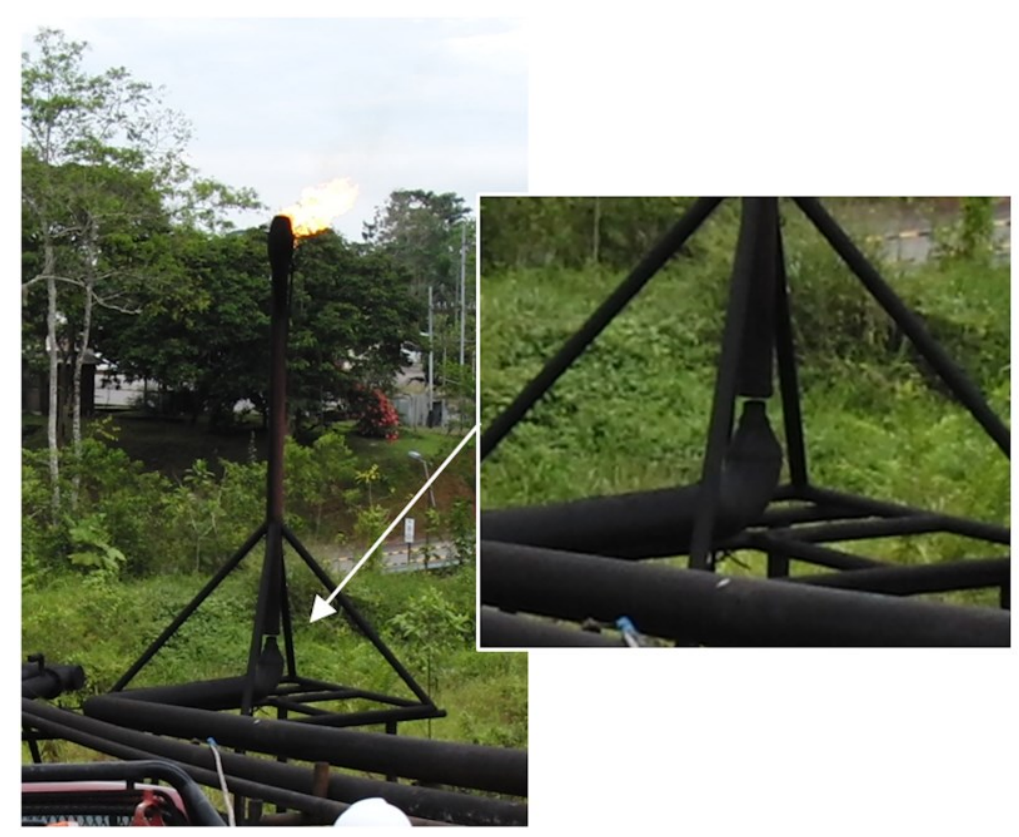

Figure A.6: Image showing the nozzle arrangement at the base of Flare 01 used to induce partial premixing of air into the flare gas prior to combustion.

\section{A.2 Sky-LOSA Measurement of BC Emission Rate}

All sky-LOSA image sets in the present study were acquired at a $50 \mathrm{~Hz}$ framerate using hardware similar to that described by Johnson et al. (2013) apart from the software/algorithmic enhancements and hardware upgrades, as discussed in the manuscript and this Appendix. Datasets acquired in Veracruz, Mexico were RAM-limited and sensor cropping was therefore employed to maximize image acquisition length. Hardware 
upgrades enabling longer sky-LOSA image acquisitions were implemented in 2014 such that all datasets acquired in Orellana, Ecuador were not RAM-limited, allowing for multiple full-sensor $(2160(\mathrm{H}) \times 2560(\mathrm{~W}))$ ten-minute image acquisitions for each flare. As in Johnson et al. (2013), plume images were acquired with a $40 \mathrm{~nm}$ bandpass filter centred at $531 \mathrm{~nm}$; further neutral density filtering with a cumulative broadband transmittance as low as $10^{-5}$ was employed when capturing images of the sun to quantify relative solar irradiance, where necessary.

\section{A.2.1 Sky-LOSA Data Acquired in Veracruz, Mexico}

Table A.2 summarizes the location, date, time, and length of sky-LOSA image sets acquired in Veracruz, Mexico as well as laser-measured distance of the flare from the camera and the pixel resolution of acquired images (which was reduced to increase the length of image acquisition due to RAM-limitations of the image acquisition hardware).

Table A.2: Summary of acquired sky-LOSA image data in Veracruz, Mexico (images were acquired on a cropped sensor to maximize measurement duration).

\begin{tabular}{|c|c|c|c|c|c|c|}
\hline Flare & $\begin{array}{c}\text { GPS } \\
\text { Coordinates }\end{array}$ & Date & $\begin{array}{l}\text { Local Time } \\
\text { at Start of } \\
\text { Acquisition }\end{array}$ & $\begin{array}{c}\text { Length of } \\
\text { Acquisition } \\
\text { [s] }\end{array}$ & $\begin{array}{c}\text { Pixel } \\
\text { Resolution } \\
(\mathbf{H} \times \mathbf{W})\end{array}$ & $\begin{array}{l}\text { Distance } \\
\text { to Flare } \\
{[\mathrm{m}]}\end{array}$ \\
\hline \multirow{3}{*}{ V1 } & \multirow{3}{*}{$\begin{array}{l}18^{\circ} 06^{\prime} 34.37^{\prime \prime} \mathrm{N} \\
94^{\circ} 23^{\prime} 43.47^{\prime \prime} \mathrm{W}\end{array}$} & \multirow{3}{*}{ 16-DEC-2012 } & $12: 40$ & 42.10 & $2080 \times 2160$ & \multirow{3}{*}{261.1} \\
\hline & & & $12: 45$ & 42.10 & $2080 \times 2160$ & \\
\hline & & & $12: 51$ & 42.10 & $2080 \times 2160$ & \\
\hline V2 & $\begin{array}{rll}18^{\circ} & 06^{\prime} & 29.88^{\prime \prime N} \\
94^{\circ} & 23^{\prime} & 41.37^{\prime \prime} \mathrm{W}\end{array}$ & 16-DEC-2012 & $15: 52$ & 51.02 & $1472 \times 2560$ & 189.0 \\
\hline V3 & $\begin{array}{l}17^{\circ} 58^{\prime} 58.26^{\prime \prime} \mathrm{N} \\
94^{\circ} 31^{\prime} 36.45^{\prime \prime} \mathrm{W}\end{array}$ & 17-DEC-2012 & $13: 14$ & 51.02 & $1472 \times 2560$ & 95.0 \\
\hline V4 & $\begin{array}{rr}17^{\circ} 58^{\prime} & 16.46^{\prime \prime} \mathrm{N} \\
94^{\circ} 31^{\prime} 09.25^{\prime \prime} \mathrm{W}\end{array}$ & 17-DEC-2012 & $16: 59$ & 51.14 & $1472 \times 2560$ & 458.0 \\
\hline V5 & $\begin{array}{l}17^{\circ} 59^{\prime} 16.72^{\prime \prime} \mathrm{N} \\
94^{\circ} 31^{\prime} 31.66^{\prime \prime} \mathrm{W}\end{array}$ & 17-DEC-2012 & $14: 16$ & 49.70 & $1472 \times 2560$ & 216.5 \\
\hline
\end{tabular}

\section{A.2.2 Sky-LOSA Data Acquired in Orellana, Ecuador}

Table A.3 summarizes the location, date, time, and length of sky-LOSA image sets acquired in Orellana, Ecuador, along with the laser-measured distance from the camera to 
the flare tip for each measurement. As noted in the footnotes of Table A.3, the 2015 measurement of Flare $\mathrm{O} 2$ was of reduced length with respect to the other measurements in Ecuador due to the thermal throttling of image acquisition hardware from intense ambient heat (resulting in dropped frames towards the end of the acquisition).

Table A.3: Summary of acquired sky-LOSA image data in Orellana, Ecuador (all images employed the full-sensor of the sCMOS sky-LOSA camera).

\begin{tabular}{|c|c|c|c|c|c|c|}
\hline Flare & $\begin{array}{c}\text { GPS } \\
\text { Coordinates }\end{array}$ & Date & $\begin{array}{l}\text { Local Time } \\
\text { at Start of } \\
\text { Acquisition }\end{array}$ & $\begin{array}{c}\text { Length of } \\
\text { Acquisition } \\
{[\mathbf{s}]}\end{array}$ & $\begin{array}{c}\text { Pixel } \\
\text { Resolution } \\
(\mathbf{H} \times \mathbf{W})\end{array}$ & $\begin{array}{c}\text { Distance } \\
\text { to Flare } \\
{[\mathrm{m}]}\end{array}$ \\
\hline \multirow{2}{*}{$\mathrm{O} 1$} & \multirow{2}{*}{$\begin{array}{c}00^{\circ} 38^{\prime} 36.65^{\prime \prime} \mathrm{S} \\
76^{\circ} 53^{\prime} 06.89^{\prime \prime} \mathrm{W}\end{array}$} & 02-JUN-2014 & $17: 22: 10$ & 600.00 & $2160 \times 2560$ & 24.6 \\
\hline & & 26-ОCТ-2015 & 17:17:03 & 600.00 & $2160 \times 2560$ & 21.8 \\
\hline \multirow{2}{*}{$\mathrm{O} 2$} & \multirow{2}{*}{$\begin{array}{c}00^{\circ} 42^{\prime} 25.66^{\prime \prime} \mathrm{S} \\
76^{\circ} 53^{\prime} 09.01^{\prime \prime} \mathrm{W}\end{array}$} & 03-JUN-2014 & $08: 45: 39$ & 600.00 & $2160 \times 2560$ & 61.0 \\
\hline & & 28-OCT-2015 & $10: 19: 36$ & $345.04^{\mathrm{a}}$ & $2160 \times 2560$ & 48.0 \\
\hline \multirow{2}{*}{$\mathrm{O} 3$} & \multirow{2}{*}{$\begin{array}{c}00^{\circ} 44^{\prime} 03.38^{\prime \prime} \mathrm{S} \\
76^{\circ} 52^{\prime} 51.78^{\prime \prime} \mathrm{W}\end{array}$} & 04-JUN-2014 & 08:17:02 & 600.00 & $2160 \times 2560$ & 175.0 \\
\hline & & 27-OCT-2015 & $08: 06: 15$ & 600.00 & $2160 \times 2560$ & 96.0 \\
\hline $\mathrm{O} 4$ & $\begin{array}{c}00^{\circ} 47^{\prime} 36.56^{\prime \prime S} \\
76^{\circ} 53^{\prime} 09.82^{\prime \prime} \mathrm{W}\end{array}$ & 27-OCT-2015 & $17: 26: 56$ & 600.00 & $2160 \times 2560$ & 43.0 \\
\hline
\end{tabular}

${ }^{a}$ Acquisition length was reduced due to thermal throttling of hardware resulting in dropped frames at the end of the acquisition.

\section{A.3 Sky-LOSA Emission Rate Uncertainty Calculations}

\section{A.3.1 CM-LHS Monte Carlo Method}

The most recent sky-LOSA algorithms use a Combined Multiple Latin Hypercube Sampled (CM-LHS) Monte Carlo (MC) method, as described by Nakayama (2011), to compute mean $\mathrm{BC}$ emission rates and associated uncertainties. The CM-LHS MC method employs the stratified Latin hypercube sampling technique to enable satisfactory bounds on emission rate statistics with fewer calculations than an equivalent standard MC method. The CM-LHS MC method requires $m$ groups of $t$ sample variables drawn using the Latin hypercube sampling technique, which provides $m t$ estimates of the mean $\mathrm{BC}$ emission rate using the sky-LOSA algorithms that can then be used to compute required emission rate statistics. 
For a sky-LOSA measurement using the CM-LHS MC method, the estimator of a flare's mean $\mathrm{BC}$ emission rate using the $i^{\text {th }}$ Latin hypercube sampled draw from the $j^{\text {th }}$ sample group is the collection of calculated instantaneous $\mathrm{BC}$ emission rates, averaged over all acquired image frames $(K)$ :

$$
\dot{m}_{B C, i, j}=\frac{\varrho_{B C, i, j} \lambda}{6 \pi\left(1+\rho_{s a, i, j}\right) E\left(m_{\lambda}\right)_{i, j}} \frac{1}{K} \sum_{k=1}^{K} \int u_{n, i, j, k}\left[-\ln \tau_{i, j, k}^{*}\right] d y
$$

where the summation indicates the time-averaging of $K$ frames acquired during the skyLOSA measurement and integration is performed along the control surface in the image plane. For a standard MC method, the mean $\mathrm{BC}$ emission rate for the $i^{\text {th }} \mathrm{MC}$ draw is similarly calculated, where:

$$
\dot{m}_{B C, i}=\frac{\varrho_{B C, i} \lambda}{6 \pi\left(1+\rho_{s a, i}\right) E\left(m_{\lambda}\right)_{i}} \frac{1}{K} \sum_{k=1}^{K} \int u_{n, i, k}\left[-\ln \tau_{i, k}^{*}\right] d y
$$

\section{A.3.2 Assessment of Uncertainty Associated with Sky Reconstructions}

Reconstruction of background skylight intensity was performed on a measurement-bymeasurement basis. It has been shown that, under clear sky conditions, skylight intensity behind the plume can be obtained via interpolation with negligible contribution to emission rate uncertainty (Johnson et al. 2013). In the present work however, seven of the fourteen measurements were performed under cloudy sky conditions (V3, V4, and V5 in 2012; O2 and $\mathrm{O} 3$ in 2014; and $\mathrm{O} 1$ and $\mathrm{O} 3$ in 2015). In these cases, deviation between the directions of plume motion and cloud motion can be exploited to artificially transport cloud structures behind the plume with knowledge of the background sky velocity field (Conrad and Johnson 2015). Alternatively, oscillating plume motion can enable the intermittent capture 
of regions of background skylight intensity, following which regions of unexposed sky can be obtained via interpolation, with some uncertainty. The former technique was used for the 2014 measurement of the $\mathrm{O} 3$ flare, while the latter technique was necessarily used for the remaining measurements under cloudy conditions. Potential additional uncertainties in the mean $\mathrm{BC}$ emission rates resulting from the reconstruction of cloudy/overcast background skies for all seven cases were considered. Sky conditions during the V3, V4, and O2 (2014) measurements were sufficiently flat, such that no additional uncertainty due to interpolation across the plume was expected. For Flare V5, Flare O3 (in 2014 and 2015), and Flare O1 in 2015 however, additional uncertainties due to skylight intensity reconstruction were quantified by assessing the sensitivity of calculated $\mathrm{BC}$ emission rates to reconstruction algorithm parameters. Ultimately, the computed $95 \%$ CIs describing the time-averaged BC emission rates for Flare V5, Flare O3 (in 2014 and 2015), and Flare O1 in 2015 were conservatively broadened in each direction by, $22 \%, 4 \%$, and $10 \%$, respectively, while holding the means constant.

\section{A.3.3 Derivation of Time-Averaged BC Emission Rate Probability Distribution}

To support the calculation of $\mathrm{BC}$ yield via secondary $\mathrm{MC}$ analyses, probability distributions of time-averaged $\mathrm{BC}$ emission rates were developed for the Orellana, Ecuador measurements in 2015 using statistics obtained from the employed MC methods. Discrete inverse cumulative distribution functions (ICDFs) were developed for each flare by obtaining percentile estimates of the time-averaged $\mathrm{BC}$ emission rate distribution from $0.5 \%$ to $99.5 \%$ in increments of $0.5 \%$ using the MC results. Subsequently, standard ICDFs were fit to the calculated percentiles, where it was found that the Generalized Extreme Value (GEV) distribution fit well with the experimental data $\left(R^{2}>0.99982\right.$ in all cases). 
The probability distribution function and ICDF of the GEV distribution have the following forms when the shape parameter is nonzero:

$$
\begin{gathered}
f\left(\dot{m}_{B C}\right)=\frac{1}{\sigma}\left(1+\xi\left(\frac{\dot{m}_{B C}-\mu}{\sigma}\right)\right)^{-1-\frac{1}{\xi}} \exp \left\{-\left(1+\xi\left(\frac{\dot{m}_{B C}-\mu}{\sigma}\right)\right)^{-1 / \xi}\right\} \\
\mathcal{F}_{\dot{m}_{B C}}^{-1}(q)=\mu+\frac{\sigma}{\xi}\left((-\ln q)^{-\xi}-1\right)
\end{gathered}
$$

where $\mu, \sigma$, and $\xi$ are the location, scale, and shape parameters, respectively, and quantile $q \in[0,1]$ represents the quantile. In all cases, the fitted shape parameter was less than zero, indicating that the best-fitting GEV distribution corresponds to a type III extreme value (reverse/negative Weibull) distribution.

\section{A.3.4 Handling of Non-Physical Results during MC Draws}

Occasionally during a sky-LOSA MC uncertainty analysis, randomly drawn BC aggregate properties can produce non-physical calculated values for the idealized line-of-sight plume transmittance (i.e., $\tau^{*} \leq 0$ ) when the experimental transmittance is low. The resulting nonphysical idealized transmittance may imply that the specific randomly drawn $\mathrm{BC}$ aggregate properties are infeasible or, alternatively, could imply that multiple-scattering phenomena are occurring within the plume. In a sky-LOSA MC implementation, these non-physical results preclude the calculation of $\mathrm{BC}$ emission rate for a specific $\mathrm{MC}$ draw at a specific time step (image frame). For a CM-LHS MC method, the occurrence of even one nonphysical result (from a single draw) at a given time step renders the derivation of $\mathrm{BC}$ emission rate statistics at that time step impossible. This can be accommodated by employing one of two techniques. Firstly, if temporal gaps in $\mathrm{BC}$ emission rate data due to non-physical results are small with respect to the rate of change in instantaneous $\mathrm{BC}$ 


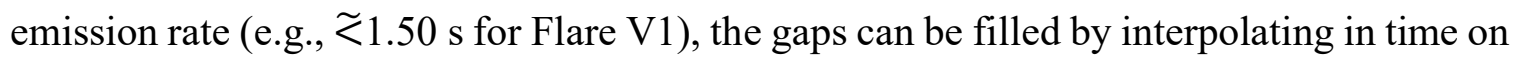
a draw-by-draw basis. However, for larger temporal gaps in the $\mathrm{BC}$ emission rate data, it would be imprudent to interpolate missing data since the stochastic nature of the instantaneous emission rate could lead to error in the calculation of the time-average. In such cases, a standard MC method can instead be employed as BC emission rate statistics can still be calculated by simply omitting MC draws yielding larger gaps of non-physical results. For any small gaps, the MC data can be interpolated as with the CM-LHS method. Since non-physical MC calculations correspond to highly optically dense plume structures (yielding low experimental transmissivities), the instantaneous $\mathrm{BC}$ emission rates at these time steps would be expected to be greater than neighbouring time steps. Consequently, the use of a bounded interpolation algorithm (such as linear interpolation) in the first technique, and the omission of non-physical data (correlated with larger estimates of integrated line-of-sight $\mathrm{BC}$ concentrations) in the second technique would both provide conservatively low estimates of the emission rate based on the $\mathrm{BC}$ aggregate property distributions employed.

A standard MC method was used for three of the sky-LOSA measurements in the current dataset to compute the uncertainties in the time-averaged BC emission rate: the first measurement of Flare V1 (i.e., V1a), the measurement of Flare V2, and the 2015 measurement of Flare O3. For flares V2 and O3 (2015), minimal interpolation and omission of MC draws were required, suggesting that any potential underestimation in emission rates for these flares is likely negligible. For V1a however, almost $25 \%$ of MC draws were omitted due to non-physical results, making the potential for negative bias (i.e. underestimation in emission rate) difficult to quantify. One approach to quantify this 
negative bias is to compute comparable mean $\mathrm{BC}$ emission rates using average $\mathrm{BC}$ aggregate optical properties computed from MC draws. When MC draws yielding nonphysically sensible data are excluded from this averaging procedure, the mean $\mathrm{BC}$ emission rate was $3.3 \%$ less than that computed when these draws are included. As such, it is suggested that the presented mean $\mathrm{BC}$ emission rate for V1a may underestimate the actual emission rate by $\sim 3.3 \%$, implying that this result is indeed conservative.

The second measurement of Flare V1 (i.e., V1b) was observed to have a long period $(6.50 \mathrm{~s})$ at the beginning of the measurement where plume transmittance was quite low, such that a significant proportion of MC draws yielded non-physical results. To avoid extrapolation of data, this period of time was disregarded in the sky-LOSA analysis. As this period would be associated with increased BC concentrations in the plume, the mean $\mathrm{BC}$ emission rate during the $\mathrm{V} 1 \mathrm{~b}$ measurement is assumed to be conservative. For the remaining portion of the $\mathrm{V} 1 \mathrm{~b}$ measurement and the other ten flares constituting the current dataset, linear interpolation of minor temporal gaps in the data was employed with the CM-LHS MC technique. The maximum temporal gap was eight frames $(0.16 \mathrm{~s})$ long and MC data from six of these ten flare measurements did not produce any non-physical results. Computed $\mathrm{BC}$ emission rates from all flares varied by less than $0.03 \%$ when using a cubic spline interpolation algorithm rather than a linear algorithm, implying that the selected allowable temporal gaps were sufficiently small to avoid any significant error due to the interpolation of data. 


\section{A.3.5 Black Carbon Emission Rate Distributions and Observations}

Table A.4 summarizes the current set of $\mathrm{BC}$ emission rate measurements from flares in Veracruz, Mexico and Orellana, Ecuador with 95\% CIs calculated via the above described MC methodology.

Table A.4: Summary of flare-specific mean BC emission rate measurements.

\begin{tabular}{|c|c|cc|c|c|}
\hline \multirow{2}{*}{ Flare } & \multirow{2}{*}{ Year } & \multicolumn{2}{|c|}{$\begin{array}{c}\text { Mean BC Emission Rate } \\
\text { [g/s] (95\% CI) }\end{array}$} & $\begin{array}{c}\text { Length of } \\
\text { useful data } \\
\text { [s] }\end{array}$ & Skewness \\
\hline V1a & \multirow{3}{*}{$2012^{\mathrm{a}}$} & 53.7 & $(-42.8 /+70.5 \%)$ & 42.10 & 0.448 \\
\cline { 3 - 6 } & & 14.4 & $(-42.3 /+70.5 \%)$ & 35.60 & 0.459 \\
\cline { 1 - 5 } V1b & 12.5 & $(-42.1 /+69.6 \%)$ & 42.10 & 0.972 \\
\hline V1c & $2012^{\mathrm{b}}$ & 1.53 & $(-33.8 /+58.7 \%)$ & 51.02 & -0.021 \\
\hline V2 & 2012 & 5.08 & $(-25.2 /+34.2 \%)$ & 51.02 & 0.503 \\
\hline V3 & 2012 & 7.33 & $(-25.0 /+35.8 \%)$ & 51.14 & 0.019 \\
\hline V4 & $2012^{\mathrm{c}}$ & 0.311 & $(-48.3 /+58.5 \%)$ & 49.70 & 1.006 \\
\hline V5 & 2014 & 0.026 & $(-25.3 /+36.3 \%)$ & 600.00 & 0.774 \\
\hline \multirow{2}{*}{ O1 } & $2015^{\mathrm{c}}$ & 0.014 & $(-35.0 /+45.8 \%)$ & 589.96 & 1.260 \\
\hline \multirow{2}{*}{ O2 } & 2014 & 0.051 & $(-25.3 /+35.9 \%)$ & 600.00 & 1.623 \\
\cline { 2 - 6 } & 2015 & 0.003 & $(-26.1 /+36.9 \%)$ & 165.06 & 3.208 \\
\hline \multirow{2}{*}{ O3 } & $2014^{\mathrm{c}}$ & 2.29 & $(-29.2 /+40.1 \%)$ & 600.00 & 1.842 \\
\cline { 2 - 6 } & $2015^{\mathrm{c}}$ & 2.60 & $(-28.7 /+38.7 \%)$ & 600.00 & 1.322 \\
\hline O4 & 2015 & 0.23 & $(-25.4 /+35.7 \%)$ & 600.00 & 1.107 \\
\hline
\end{tabular}

a Increased uncertainty resulting from uncertain spatial scaling due to the direction of plume propagation

b Increased uncertainty due to a low scattering angle of incident solar radiation

c Increased uncertainty due to background skylight intensity reconstruction under overcast/cloudy conditions

Instantaneous $\mathrm{BC}$ emission rates were highly stochastic, exhibiting non-stationary behaviour and, in all cases except Flare V2, positive skewness. As an example, Figure A.7a shows the mean $\mathrm{BC}$ emission rate over $1 \mathrm{~s}$ intervals for the ten-minute measurement of Flare $\mathrm{O} 3$ in 2015. Figure A.7b presents a $5 \mathrm{~s}$ subset of these data with instantaneous BC emission rates and computed $95 \%$ CIs plotted at the full framerate of $50 \mathrm{~Hz}$. Variations in the $\mathrm{BC}$ emission rate on a large timescale (Figure A.7a) indicate the non-stationarity of the emissions, while fluctuations on a short timescale (Figure A.7b) relate to turbulent motion of the atmospheric plume and are associated with coherent visible structures in the plume. 


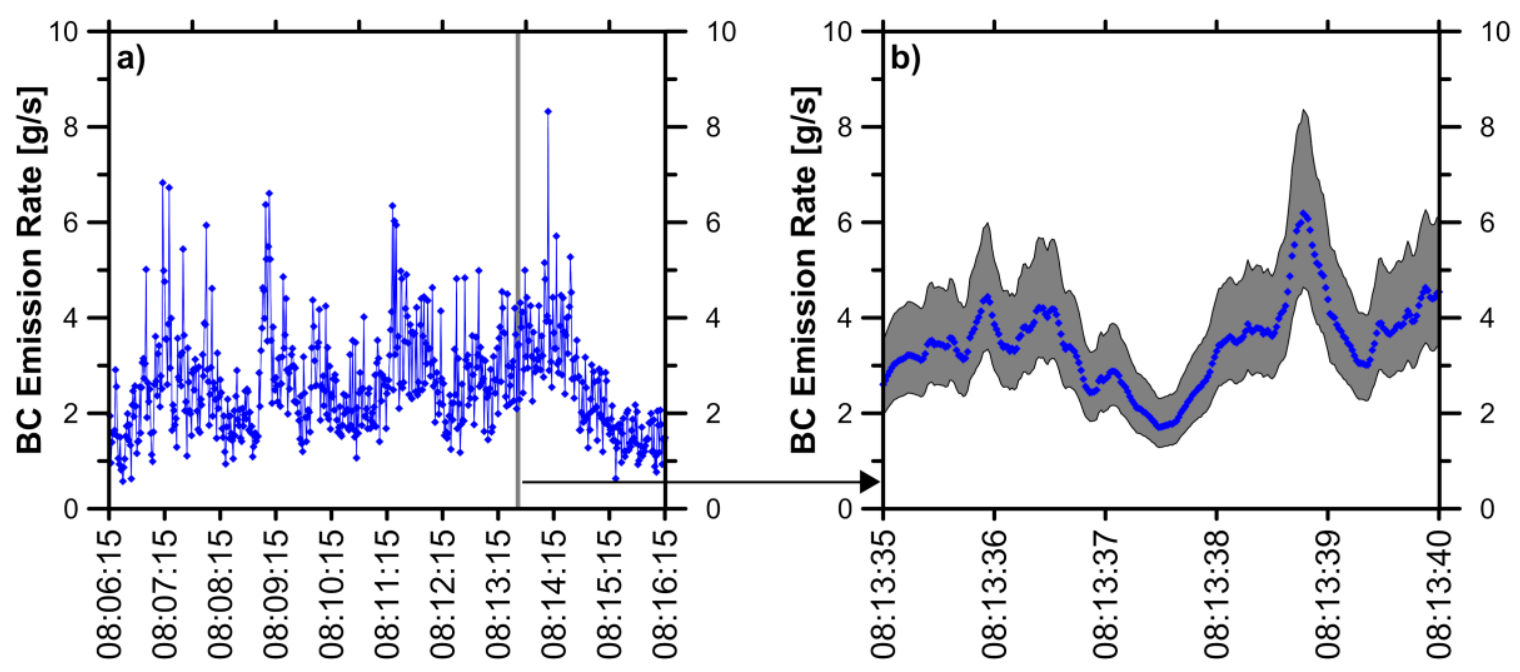

Figure A.7: (a) Mean BC emission rate data over $1 \mathrm{~s}$ intervals (mean from MC results) for Flare $\mathrm{O3}$ (2015) over the ten-minute analysis period alongside, (b) a $5 \mathrm{~s}$ subset of these data plotted at the data acquisition rate $(50 \mathrm{~Hz})$ with grey shading showing the $95 \%$ CIs computed using the described MC methodology. The stochastic nature of the instantaneous $\mathrm{BC}$ emission rate is clearly visible, where oscillations in the data indicate a highly turbulent atmospheric plume.

Figure A.8 shows the cumulative distribution functions of the mean-normalized instantaneous $\mathrm{BC}$ emission rates from all flares in the current dataset. Shown in red is the highly non-stationary and strongly skewed data from the measurement of Flare O2 in 2015. Plotted in green is the cumulative distribution function (CDF) for Flare V2, which was the only dataset with a negative skewness. The generally positively skewed nature of the instantaneous $\mathrm{BC}$ emission rate data suggests that time-averaged $\mathrm{BC}$ emissions are heavily weighted by short bursts of optically dense structures in the plume containing relatively larger quantities of BC. As positive skewness could lead to erroneous estimations of central tendency, these data suggest that future measurements of $\mathrm{BC}$ emissions from gas flaring should strive to maximize measurement duration as much as reasonably possible. 


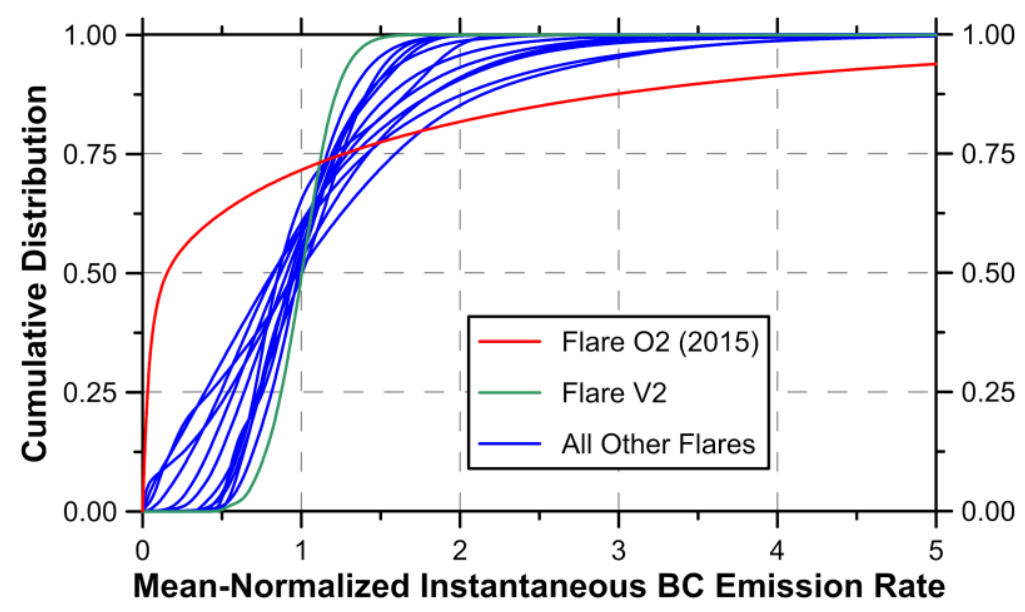

Figure A.8: Cumulative distribution function of instantaneous BC emission rates from each of the measured flares in the current data set. Except for Flare V2 (green), all flares exhibited a positive skewness (as quantified in Table A.4). The measured instantaneous BC emission rates of Flare $\mathrm{O} 2$ in 2015 (shown in red) were highly non-stationary as well as strongly-skewed such that $10 \%$ of the instantaneous data were responsible for $56 \%$ of total emissions during the measurement.

\section{A.4 Flare Gas Composition Measurements}

Table A.5 shows summary details of the measured chemical compositions of combusted flare gas for the four flares in Ecuador based on extractive samples collected in October 2015. The presented composition data is separated into volume fractions of alkanes (simple and branched); cycloalkanes; alkenes and cycloalkenes; aromatics; other combustibles; and diluents. It should be noted that additional species with measured volume fractions below the detection limits of the employed method (CAN/CGSB-3.0 No. 14.3-99) were omitted from Table A.5. 
Table A.5: Semi-detailed chemical composition (\% Volume) of combined flare gas from all lines feeding each measured flare. (LDL $=$ Lower Detection Limit).

\begin{tabular}{|c|c|c|c|c|c|c|c|c|c|}
\hline \multicolumn{2}{|c|}{ Components } & \multicolumn{2}{|c|}{ Flare 01} & \multicolumn{2}{|c|}{ Flare O2 } & \multicolumn{2}{|c|}{ Flare $\mathbf{0 3}$} & \multicolumn{2}{|c|}{ Flare 04} \\
\hline & $\begin{array}{c}\text { Carbon } \\
\#\end{array}$ & Simple & Branched & Simple & Branched & Simple & Branched & Simple & Branched \\
\hline Alkanes & $\begin{array}{l}\mathrm{C} 1 \\
\mathrm{C} 2 \\
\mathrm{C} 3 \\
\mathrm{C} 4 \\
\mathrm{C} 5 \\
\mathrm{C} 6 \\
\mathrm{C} 7 \\
\mathrm{C} 8 \\
\mathrm{C} 9 \\
\end{array}$ & $\begin{array}{c}47.21 \\
7.97 \\
11.31 \\
4.44 \\
1.35 \\
0.39 \\
0.10 \\
0.01 \\
0.01 \\
\end{array}$ & $\begin{array}{l}--- \\
--- \\
--- \\
2.46 \\
1.65 \\
0.80 \\
0.23 \\
0.14 \\
0.03 \\
\end{array}$ & $\begin{array}{c}58.81 \\
7.79 \\
9.29 \\
3.24 \\
0.95 \\
0.30 \\
0.08 \\
0.02 \\
<\mathrm{LDL} \\
\end{array}$ & $\begin{array}{c}--- \\
--- \\
--- \\
1.95 \\
1.22 \\
0.59 \\
0.17 \\
0.05 \\
<\text { LDL } \\
\end{array}$ & $\begin{array}{c}40.71 \\
8.14 \\
16.89 \\
9.81 \\
2.98 \\
0.66 \\
0.12 \\
0.02 \\
<\text { LDL } \\
\end{array}$ & $\begin{array}{c}--- \\
--- \\
--- \\
4.91 \\
3.81 \\
1.40 \\
0.29 \\
0.06 \\
<\text { LDL } \\
\end{array}$ & $\begin{array}{c}55.11 \\
7.62 \\
11.64 \\
5.21 \\
1.50 \\
0.38 \\
0.07 \\
0.01 \\
<\text { LDL } \\
\end{array}$ & $\begin{array}{c}--- \\
--- \\
--- \\
3.57 \\
2.45 \\
0.99 \\
0.21 \\
0.04 \\
<\text { LDL } \\
\end{array}$ \\
\hline $\begin{array}{l}\text { Cyclo- } \\
\text { alkanes }\end{array}$ & $\begin{array}{l}\mathrm{C} 5 \\
\mathrm{C} 6 \\
\mathrm{C} 7 \\
\mathrm{C} 8 \\
\mathrm{C} 9 \\
\end{array}$ & & & & $\begin{array}{l}5 \\
19 \\
6 \\
65 \\
1 \\
\end{array}$ & $\begin{array}{r}0 \\
0 \\
0 \\
0 \\
<1 \\
\end{array}$ & $\begin{array}{l}19 \\
52 \\
30 \\
04 \\
\mathrm{DL} \\
\end{array}$ & $\begin{array}{r}0 \\
0 \\
0 \\
0 \\
<1 \\
\end{array}$ & $\begin{array}{l}12 \\
32 \\
19 \\
05 \\
\mathrm{DL} \\
\end{array}$ \\
\hline $\begin{array}{c}\text { Alkenes } \\
\text { and Cyclo- } \\
\text { alkenes }\end{array}$ & $\begin{array}{l}\mathrm{C} 4 \\
\mathrm{C} 5 \\
\mathrm{C} 6 \\
\mathrm{C} 7 \\
\mathrm{C} 8 \\
\mathrm{C} 9 \\
\end{array}$ & & $\begin{array}{l}2 \\
\mathrm{DL} \\
06 \\
26 \\
94 \\
98 \\
\end{array}$ & $\begin{array}{r}0 \\
<\mathrm{I} \\
<\mathrm{I} \\
0 \\
0 \\
0 \\
\end{array}$ & $\begin{array}{l}2 \\
\mathrm{DL} \\
\mathrm{DL} \\
03 \\
01 \\
01 \\
\end{array}$ & $\begin{array}{r}<1 \\
<1 \\
<1 \\
0 \\
0 \\
<1\end{array}$ & $\begin{array}{l}\mathrm{DL} \\
\mathrm{DL} \\
\mathrm{DL} \\
02 \\
02 \\
\mathrm{DL}\end{array}$ & & $\begin{array}{l}\mathrm{DL} \\
\mathrm{DL} \\
\mathrm{DL} \\
03 \\
02 \\
01 \\
\end{array}$ \\
\hline Aromatics & $\begin{array}{c}\text { Benzene } \\
\text { Toluene } \\
\end{array}$ & & & & 1 & & $\begin{array}{l}02 \\
03 \\
\end{array}$ & & \\
\hline $\begin{array}{c}\text { Other } \\
\text { Combust- } \\
\text { ibles }\end{array}$ & $\begin{array}{c}\mathrm{H}_{2} \\
\mathrm{H}_{2} \mathrm{~S}\end{array}$ & & $\begin{array}{l}01 \\
\mathrm{DL}\end{array}$ & & $\begin{array}{l}03 \\
\mathrm{DL}\end{array}$ & & $\begin{array}{l}\mathrm{DL} \\
\mathrm{DL}\end{array}$ & & $\begin{array}{l}04 \\
\text { DL }\end{array}$ \\
\hline Diluents & $\begin{array}{c}\mathrm{He} \\
\mathrm{N}_{2} \\
\mathrm{CO}_{2}\end{array}$ & & $\begin{array}{l}01 \\
17 \\
64\end{array}$ & & $\begin{array}{l}91 \\
74 \\
19\end{array}$ & & $\begin{array}{l}01 \\
35 \\
69\end{array}$ & & $\begin{array}{l}01 \\
11 \\
30\end{array}$ \\
\hline
\end{tabular}

Uncertainties in flare gas composition and associated metrics were assessed with a standard MC method. Each analyte detected in quantities above the detection threshold was prescribed a normal distribution; the distribution means were made equal to the reported results and the standard deviations were calculated using the root-mean-square of repeatability and reproducibility estimates from an equivalent standard, ASTM D6729 (ASTM 2014). Analytes not referenced in ASTM D6729 (ASTM 2014) were given normal distributions with a coefficient of variance of $10 \%$. Composition data presented in Table A.5 are the mean results from the employed MC method. 


\section{A.5 Calculation of Flare Gas Flow Rate and Uncertainty}

Two techniques were employed to measure flare gas flow rate in support of $\mathrm{BC}$ emission factor calculations for the 2015 measurements in Orellana, Ecuador: an optical velocity measurement technique and a tracer-dilution technique, as described in the manuscript. The governing equations of each technique are derived here.

\section{A.5.1 Optical Velocity Measurement}

A power-law representation of the mean turbulent velocity profile in a duct of circular cross-section (with axisymmetric flow implied), was assumed:

$$
u(r)=u_{C L}\left(1-\frac{r}{R}\right)^{\frac{1}{n}}
$$

where $u_{C L}$ is the centreline velocity $\left[\mathrm{m} \cdot \mathrm{s}^{-1}\right], r$ is the radial distance from the centreline $[\mathrm{m}]$, $R$ is the internal radius of the duct [m], and $n$ is the power-law exponent [-]. The volumetric flow rate of flare gas, $\dot{V}\left[\mathrm{~m}^{3} \mathrm{~s}^{-1}\right]$, can be obtained via integration over the crosssection of the flare line:

$$
\dot{V}=\int_{0}^{2 \pi} \int_{0}^{R} u_{(r)} r d r d \theta=\frac{2 n^{2} \pi R^{2}}{(n+1)(2 n+1)} u_{C L}
$$

where $\theta$ is the angular coordinate [rad] of the cylindrical coordinate system defining the pipe flow.

A centreline deviation parameter, $\delta \in[0, R)[\mathrm{m}]$, is introduced to enable the backcalculation of the centreline (maximum) velocity from a measured velocity at some radius using the power-law turbulent mean velocity profile: 


$$
\dot{V}=\frac{2 n^{2} \pi R^{2}}{(n+1)(2 n+1)} \frac{u_{m}}{\left(1-\frac{\delta}{R}\right)^{\frac{1}{n}}}
$$

where $u_{m}$ is the measured velocity $\left[\mathrm{m} \cdot \mathrm{s}^{-1}\right]$ at $r=\delta$.

To account for minor pipeline fouling observed during field measurements (assumed to be axisymmetric), a non-dimensional fouling parameter, $\varepsilon \in[0,1]$, where $R=$ $R_{o}(1-\varepsilon$ ), is introduced (where $\varepsilon=0$ implies an unobstructed flow and $\varepsilon=1$ implies a fully occluded pipe):

$$
\dot{V}=\frac{2 n^{2} \pi R_{o}^{2}(1-\varepsilon)^{2}}{(n+1)(2 n+1)} \frac{u_{m}}{\left(1-\frac{\delta}{R_{o}(1-\varepsilon)}\right)^{\frac{1}{n}}}
$$

where $R_{o}$ is the nominal internal radius [m] of the flare line.

From the ideal gas relation, this measure of volumetric flow rate can be transformed into standardized units $\left(\dot{V}_{o}\right.$ in $\left.\left[\mathrm{m}^{3} \mathrm{~s}^{-1}\right]\right)$ with the ratios of measured and standard process pressure and temperature. Furthermore, the time-averaged measurement of velocity can be introduced to yield an estimate of the mean standard volumetric flow rate during the measurement:

$$
\overline{\dot{V}}_{o}=\left(\frac{P_{m}}{P_{o}}\right)\left(\frac{T_{o}}{T_{m}}\right) \frac{2 n^{2} \pi R_{o}^{2}(1-\varepsilon)^{2}}{(n+1)(2 n+1)} \frac{\overline{u_{m}}}{\left(1-\frac{\delta}{R_{o}(1-\varepsilon)}\right)^{\frac{1}{n}}}
$$

where $P_{m}$ and $T_{m}$ are the process pressure $[\mathrm{Pa}]$ and temperature $[\mathrm{K}]$ and $P_{o}$ and $T_{o}$ are the standard pressure and temperature defined for the current study $\left(1 \mathrm{~atm}\right.$ and $\left.15^{\circ} \mathrm{C}\right)$. 
Uncertainty in the flare gas flow rate using the centreline velocity measurement technique was evaluated in the present study using a standard MC method. Probability distributions for input parameters to the MC method are presented in Table A.6.

\section{Table A.6: Summary of input parameter distributions for the Monte Carlo method used to compute the flare gas flow rate and associated uncertainty using optical velocity measurements.}

\begin{tabular}{|c|c|c|c|c|}
\hline Input Parameter & Distribution & $\begin{array}{c}\text { Location } \\
\boldsymbol{\mu}\end{array}$ & $\begin{array}{c}\text { Scale } \\
\boldsymbol{\sigma}\end{array}$ & $\begin{array}{c}\text { Source of } \\
\text { Uncertainty }\end{array}$ \\
\hline $\begin{array}{c}\text { Process Pressure, } \\
P_{m}\end{array}$ & Normal & $P_{m}$ & $172.37[\mathrm{~Pa}]$ & Instrumentation \\
\hline $\begin{array}{c}\text { Process Temperature, } \\
T_{m}\end{array}$ & Normal & $T_{m}$ & $0.15+0.0025 T_{\left({ }^{\circ} \mathrm{C}\right)}[\mathrm{K}]$ & Instrumentation \\
\hline $\begin{array}{c}\text { Mean Measured Velocity, } \\
\overline{u_{m}}\end{array}$ & Normal & $\frac{\sum_{i=1}^{p} u_{i}}{p}$ & $\sqrt{\frac{s_{u_{m}}^{2}}{p}+\frac{\sum_{i=1}^{p} s_{u_{m, i}}^{2}}{p}}$ & $\begin{array}{c}\text { Central Limit } \\
\text { Theorem and } \\
\text { Instrumentation }\end{array}$ \\
\hline $\begin{array}{c}\text { Fouling Parameter, } \\
\varepsilon\end{array}$ & $\begin{array}{c}\text { Folded } \\
\text { Normal }\end{array}$ & 0 & 0.0510 & Arbitrary $^{\mathrm{b}}$ \\
\hline $\begin{array}{c}\text { Centreline Deviation, } \\
\delta\end{array}$ & $\begin{array}{c}\text { Folded } \\
\text { Normal }\end{array}$ & 0 & $6.48[\mathrm{~mm}]$ & Arbitrary $^{\mathrm{c}}$ \\
\hline $\begin{array}{c}\text { Power-law Exponent, } \\
n\end{array}$ & Normal & 7 & 0.5 & Heuristic $^{\mathrm{d}}$ \\
\hline
\end{tabular}

a Standard deviation of the mean is treated as the root sum square of the standard error of the mean and the root mean square of measurement standard deviations due to instrumentation error. Standard deviation of each measurement is taken as the root mean square of reported instrument accuracy and repeatability, where applicable:

$$
\begin{gathered}
\text { Optical Flow Meter: } s_{u_{m, i}}^{2}=\left\{\begin{array}{lc}
3.250 \times 10^{-4} u_{m, i}^{2}, & u_{m, i} \leq 1 \frac{m}{s} \\
0.906 \times 10^{-4} u_{m, i}^{2}, & 1 \frac{m}{s}<u_{m, i}<100 \frac{m}{s}
\end{array}\right\} \\
\text { Vane Wheel Anemometer: } s_{u_{m, i}}^{2}=\left\{\left(0.1 \frac{m}{s}+0.005 u_{m, i}\right)^{2}\right\}
\end{gathered}
$$

Implies that the $95^{\text {th }}$-percentile of pipeline fouling is $10 \%$ of the nominal radius. Implies that the $95^{\text {th }}$-percentile of the measurement location is $12.7 \mathrm{~mm}\left(1 / 2^{\prime \prime}\right)$ from the centreline.

d The mean value for the power-law exponent is based on heuristics within the literature. The scale of the distribution implies that the $95 \% \mathrm{CI}$ of the power-law exponent is $7 \pm 1$, which is considered to be conservative for the moderate Reynolds numbers observed.

\section{A.5.2 Tracer-Dilution Measurement}

The tracer-dilution measurement technique entails the injection of a stable tracer gas into the flare gas stream at a known rate. Following sufficient mixing of the tracer with background flare gas, a sample of the mixture is extracted and tracer concentration is measured. A molar balance of tracer species on a control volume containing the injection 
and sampling points ultimately provides the flare gas flow rate. Derivation begins with the molar balances of the tracer and bulk gases over this control volume for a non-reacting flow:

$$
\begin{aligned}
\dot{n}_{t o t} X_{t o t} & =\dot{n}_{i n j} X_{i n j}+\dot{n}_{b g} X_{b g} \\
\dot{n}_{t o t} & =\dot{n}_{i n j}+\dot{n}_{b g}
\end{aligned}
$$

where $\dot{n}_{i}$ represents the molar flow rate of some species or mixture $\left[\mathrm{kmol} \mathrm{s}^{-1}\right], X_{i}$ represents the volume fraction of the tracer at location $i[-]$, the subscript inj represents the injected species (tracer and impurities), the subscript $b g$ represents the background (upstream) flare gas, and the subscript tot represents the mixture of background flare gas and injected species. Introducing bulk molar continuity into the tracer gas molar continuity and re-arranging, the total molar flow rate of flared gas during a tracer-dilution measurement is:

$$
\dot{n}_{t o t}=\dot{n}_{i n j}\left\{\frac{X_{i n j}-X_{b g}}{X_{t o t}-X_{b g}}\right\}
$$

The molar flow rate of injected tracer species was controlled using the calibrated mass flow controller (MFC) specified in the manuscript, was logged in real-time as a standard volumetric flow rate, and can be recovered using the defined standard state of the $\operatorname{MFC}\left(1\right.$ atm and $\left.0^{\circ} \mathrm{C}\right)$ :

$$
\dot{n}_{i n j}=\frac{\dot{V}_{i n j}}{\Gamma} \frac{P_{o}}{R_{u} T_{o, M F C}}
$$

where $\dot{V}_{i n j}$ is the injected volumetric flow rate using the MFC [SLPM]; $R_{u}$ is the universal gas constant; $P_{o}$ is the standard pressure; $T_{o, M F C}$ is the standard temperature for the MFC 
$[\mathrm{K}]$; and $\Gamma$ is a conversion factor $\left(60,000 \mathrm{~L} \mathrm{~s} \mathrm{~min}^{-1} \mathrm{~m}^{-3}\right)$ used to bring the injected volumetric flow rate from SLPM into base SI units.

The mean standard volumetric flow rate of flare gas during a tracer dilution measurement $\left(\overline{\hat{V}}_{o}\right.$ in $\left[\mathrm{m}^{3} \mathrm{~s}^{-1}\right]$ at the standard temperature defined in this study, $\left.T_{o}\right)$ can be obtained by combining equations (11) and (12) with the ideal gas law:

$$
\overline{\dot{V}}_{o}=\left(\frac{T_{o}}{T_{o, M F C}}\right)\left(\frac{{\overline{V_{l n j}}}_{\Gamma}}{\Gamma}\right)\left(\frac{X_{i n j}-\overline{X_{b g}}}{\overline{X_{t o t}}-\overline{X_{b g}}}\right)
$$

Uncertainty in the flare gas flow rate using the tracer-dilution technique was evaluated using a standard MC method. Probability distributions for input parameters to the MC method are shown in Table A.7.

Table A.7: Summary of input parameter distributions for the Monte Carlo method used to compute the flare gas flow rate and associated uncertainty using the tracerdilution technique.

\begin{tabular}{|c|c|c|c|c|}
\hline Input Parameter & Distribution & $\begin{array}{c}\text { Location } \\
\qquad \mu\end{array}$ & $\begin{array}{c}\text { Scale } \\
\sigma\end{array}$ & $\begin{array}{l}\text { Source of } \\
\text { Uncertainty }\end{array}$ \\
\hline $\begin{array}{l}\text { Volumetric Flow Rate of } \\
\text { Injected Species, }{\overline{\dot{V}_{l n}}}\end{array}$ & Normal & $\frac{\sum_{i}^{p} \dot{V}_{i n j, i}}{p}$ & $\sqrt{\frac{s_{\dot{V}_{i n j}}^{2}}{p}+\frac{\sum_{i=1}^{p} s_{\dot{V}_{i n j, i}}^{2}}{p}}$ & $\begin{array}{l}\text { Central Limit } \\
\text { Theorem and } \\
\text { Instrumentation }^{\mathrm{a}}\end{array}$ \\
\hline $\begin{array}{l}\text { Impurity of Injected Tracer } \\
\text { Gas, }\left(1-X_{i n j}\right)\end{array}$ & $\begin{array}{l}\text { Folded } \\
\text { Normal }\end{array}$ & $\begin{array}{c}1-X_{i n j}= \\
0.01\end{array}$ & 0.00608 & Arbitrary ${ }^{b}$ \\
\hline $\begin{array}{l}\text { Measured Volume Fraction } \\
\left(\overline{X_{m}}\right) \text { of Tracer Species }\end{array}$ & Normal & $\frac{\sum_{i=1}^{p} \overline{X_{m, l}}}{p}$ & $\sqrt{\frac{s_{X_{m}}^{2}}{p}+\frac{\sum_{i=1}^{p} s_{X_{m, i}}^{2}}{p}}$ & $\begin{array}{l}\text { Central Limit } \\
\text { Theorem and } \\
\text { Instrumentation }^{\mathrm{c}}\end{array}$ \\
\hline
\end{tabular}

Standard deviation of the mean is treated as the root sum square of the standard error of the mean and the root mean square of measurement standard deviations due to instrumentation error. Standard deviation of each measurement is taken as the root mean square of reported instrument accuracy and repeatability:

$$
S_{\dot{V}_{i n j, i}}^{2}=\left\{\begin{array}{cc}
13 \times 10^{-6} \dot{V}_{i n j, i}^{2} & , \quad 1 S L P M \leq \dot{V}_{i n j, i} \leq 5 S L P M \\
12.5 \times 10^{-6}+0.5 \times 10^{-6} \dot{V}_{i n j, i}^{2} & , \\
\dot{V}_{i n j, i}<1 S L P M
\end{array}\right\}
$$

Selection of scale parameter provides $95 \%$ confidence that the purity of the injected tracer is within $1 \%$ of specified.

Standard deviation of the mean is treated as the root sum square of the standard error of the mean and the root mean square of measurement standard deviations due to instrumentation error. Standard deviation of each measurement based on reported total instrument uncertainty:

$$
s_{X_{m, i}}^{2}=25 \times 10^{-6} X_{m, i}^{2}
$$




\section{A.6 Calculation of Black Carbon Yield}

Table A. 8 shows the $\mathrm{BC}$ yield results for each flare during the 2015 measurement campaign in Ecuador along with calculated $95 \%$ CIs. These data were calculated using a standard MC method as further described below. Based on the observed non-stationarity of the data acquired at Flare $\mathrm{O} 2$, it is suggested that this flare is atypical and the computed $\mathrm{BC}$ yield may be considered an outlier relative to all other flares measured to date.

Table A.8: Mean and 95\% confidence intervals for the mean $\mathrm{BC}$ emission rate, mean standard volumetric flow rate, and mean BC yield of the flares in Orellana, Ecuador measured during the $\mathbf{2 0 1 5}$ measurement campaign.

\begin{tabular}{|c|c|c|c|c|}
\hline Variable & Flare 01 & Flare $\mathrm{O} 2$ & Flare $\mathbf{O 3}$ & Flare 04 \\
\hline $\begin{array}{c}\text { Mean BC Emission } \\
\text { Rate } \\
(95 \% \mathrm{CI}),[\mathrm{g} / \mathrm{s}]\end{array}$ & $\begin{array}{c}0.014 \\
(-35.0 /+45.8 \%)\end{array}$ & $\begin{array}{c}0.003 \\
(-26.1 /+36.9 \%)\end{array}$ & $\begin{array}{c}2.596 \\
(-28.7 /+38.7 \%)\end{array}$ & $\begin{array}{c}0.225 \\
(-25.4 /+35.7 \%)\end{array}$ \\
\hline $\begin{array}{c}\text { Mean Standard } \\
\text { Volumetric Flow Rate } \\
(95 \% \mathrm{CI}),\left[\mathrm{m}^{3} / \mathrm{s}\right]\end{array}$ & $\begin{array}{c}0.010 \\
(-1.6 /+1.7 \%)\end{array}$ & $\begin{array}{c}0.118 \\
(-9.2 /+14.6 \%)\end{array}$ & $\begin{array}{c}0.678 \\
(-2.7 /+3.5 \%)\end{array}$ & $\begin{array}{c}0.109 \\
( \pm 2.7 \%)\end{array}$ \\
\hline $\begin{array}{c}\text { Mean BC Yield (95\% } \\
\text { CI), }\left[\mathrm{g} / \mathrm{m}^{3}\right]\end{array}$ & $\begin{array}{c}1.34 \\
(-35.1 /+45.9 \%) \\
\end{array}$ & $\begin{array}{c}0.03 \\
\left(7 \times 10^{-5}-0.212\right)\end{array}$ & $\begin{array}{c}3.85 \\
(-28.6 /+38.8 \%) \\
\end{array}$ & $\begin{array}{c}2.09 \\
(-25.3 /+35.5 \%) \\
\end{array}$ \\
\hline
\end{tabular}

A single $\mathrm{MC}$ iteration included a randomly drawn time-averaged $\mathrm{BC}$ emission rate (from the computed GEV distributions) and randomly and independently computed timeaveraged flare gas flow rates from each relevant flare line; with these data, Eq. (A.14) was used to compute the $i^{\text {th }}$ estimator of a flare's BC yield:

$$
Y_{B C, i}\left[\frac{\mathrm{g}_{\mathrm{BC}}}{\mathrm{m}_{\mathrm{FG}}^{3}}\right]=\left(\dot{m}_{B C, i}\left[\frac{\mathrm{g}_{\mathrm{BC}}}{\mathrm{s}}\right]\right) \cdot\left(\sum_{l \in L} \dot{V}_{o, l, l}\left[\frac{\mathrm{m}_{\mathrm{FG}}^{3}}{\mathrm{~s}}\right]\right)^{-1}
$$

where $L$ indicates the set of flare lines feeding the measured flare. BC yield statistics were then obtained from the collection of MC-computed estimators. A more conservative implementation of the standard MC method was employed to account for the non- 
stationarity of Flare $\mathrm{O} 2$. For each $\mathrm{MC}$ draw in the analysis of this flare, flare gas flow rate and $\mathrm{BC}$ emission rate estimates were sampled from the collection of instantaneous data as opposed to the derived distributions describing the time-averages. This technique was adopted to provide a more conservative interval on the $\mathrm{BC}$ yield of this flare, where highly non-stationary instantaneous $\mathrm{BC}$ emissions and instantaneous flow rate measurements were observed.

\section{A.7 Estimation of a Representative Global Flare Gas Heating Value}

As mentioned in the manuscript, it is challenging to derive a representative heating value for flare gas across the globe. However, available flare gas composition data in the literature (Huang et al. 2015; Wocken et al. 2013; Johnson and Coderre 2012) and from this study can be coupled with estimates of country-specific flared volumes from satellite imagery (Elvidge et al. 2015) to preliminarily obtain a globally representative heating value based on the four jurisdictions discussed in the manuscript. Shown in Table A.9 are the flared volumes and assumed representative heating values for Canada, USA, Russia, and Ecuador. A volume-weighted average heating value of these four jurisdictions, representing $23.2 \%$ of global flaring, is $67.23 \mathrm{MJ} / \mathrm{m}^{3}$. More conservatively, if the simple arithmetic average of these four jurisdictions $\left(57.86 \mathrm{MJ} / \mathrm{m}^{3}\right)$ is assumed to apply to the remaining $76.8 \%$ of global flaring, then in combination with the volume-weighted estimate for the four countries with sufficient data, a representative global heating value could be $60.03 \mathrm{MJ} / \mathrm{m}^{3}$. 
Table A.9: Country-specific flared volumes and representative heating values for Canada, USA, Russia and Ecuador. Flared volumes are sourced from 2012 satellite imagery data analyzed by Elvidge et al. (2015).

\begin{tabular}{|c|c|c|c|}
\hline Jurisdiction & $\begin{array}{c}\text { 2012 Flared } \\
\text { Volume }\left[\mathbf{1 0}^{\mathbf{6}} \mathbf{~ m}^{\mathbf{3}} \mathbf{]}\right.\end{array}$ & $\begin{array}{c}\text { Representative } \\
\text { Heating Value } \\
{\left[\mathbf{M J} / \mathbf{m}^{\mathbf{3}} \mathbf{]}\right.}\end{array}$ & Source \\
\hline Canada & 0.783 & 38.236 & $\begin{array}{c}\text { Johnson and Coderre (2012) } \\
\text { Alberta Data }\end{array}$ \\
\hline USA & 6.900 & 55.11 & $\begin{array}{c}\text { Wocken et al. (2013) } \\
\text { Bakken Region Data }\end{array}$ \\
\hline Russia & 24.961 & 71.5 & $\begin{array}{c}\text { Huang et al. (2015)- } \\
\text { Recomputed at the present } \\
\text { standard conditions }\end{array}$ \\
\hline Ecuador & 0.553 & 66.6 & $\begin{array}{c}\text { Volume-weighted average of } \\
\text { data in this study }\end{array}$ \\
\hline
\end{tabular}




\section{Appendix B Supplemental Information for Beam Steering Effects on Remote Optical Measurements of Pollutant Emissions in Heated Plumes and Flares}

\section{B.1 Computation of the Complex Refractive Index for Multi-Component Gas-Phase Plumes}

Note: This section does not appear in the published paper but is included here for additional background at the request of the thesis examiners.

\section{B.1.1 Visible Spectrum}

Within the visible spectrum, the imaginary component of the complex refractive index $\left(\kappa_{\lambda}\right)$ for the studied gas-phase plumes is assumed to be negligibly different from zero, which permits rapid computation of the spectrally resolved real component of the refractive index $\left(\eta_{\lambda}\right)$. This section summarizes the implemented procedure to compute the real refractive index of the gas-phase plumes within the visible spectrum following the approach outlined by Gardiner et al. (1981). A detailed discussion and derivation of the underlying model is available in the text of Weinberg (1963).

Consider an ideal gas mixture of $I$ species and let the spectral real refractive index of component $i \in\{1 \ldots I\}$ be $\eta_{\lambda, i}[-]$. The Lorentz-Lorenz molar refractivity $\left(R_{L L}\left[\mathrm{~m}^{3}\right.\right.$ $\left.\mathrm{kmol}^{-1}\right]$ ) of component $i$ at wavelength $\lambda$ is (e.g., Weinberg 1963):

$$
R_{L L, i}(\lambda)=\frac{\eta_{\lambda, i}^{2}-1}{\eta_{\lambda, i}^{2}+2} \cdot \frac{R_{u} T}{P}
$$


where $R_{u}$ is the universal gas constant $\left[\mathrm{J} \mathrm{kmol}^{-1} \mathrm{~K}^{-1}\right], T$ is the mixture temperature $[\mathrm{K}]$, and $P$ is the pressure of the mixture $[\mathrm{Pa}]$. For gases far from the critical point (i.e., ideal gases) where real refractive indices are close to unity, the molar refractivity of a gas species can be treated as constant and the molar refractivity of the mixture $\left(\bar{R}_{L L}\right)$ is simply the volume-weighted sum of the components (Weinberg 1963):

$$
\bar{R}_{L L}(\lambda)=\frac{\eta_{\lambda}^{2}-1}{\eta_{\lambda}^{2}+2} \cdot \frac{R_{u} T}{P}=\sum_{i=1}^{I} X_{i} R_{L L, i}
$$

where $X_{i}$ is the volume fraction of component $i$ within the mixture [-]. Eq. (B.2) can then be inverted to yield the spectral real refractive index of the mixture:

$$
\eta_{\lambda}=\sqrt{\frac{\frac{R_{u} T}{P}+2 \bar{R}_{L L}(\lambda)}{\frac{R_{u} T}{P}-\bar{R}_{L L}(\lambda)}}
$$

In this work, the spectrally resolved molar refractivities of $\mathrm{CO}_{2}, \mathrm{CO}, \mathrm{CH}_{4}, \mathrm{H}_{2} \mathrm{O}, \mathrm{N}_{2}$, and $\mathrm{O}_{2}$ were obtained from the dispersion formulae for $\eta_{\lambda, i}$ of Gardiner et al. (1981) from which the component- and mixture-level molar refractivities and, hence, $\eta_{\lambda}$ was computed.

\section{B.1.2 Infrared Spectrum}

Within the infrared spectrum, the spectrally resolved complex refractive index is obtained via the HITRAN (High Resolution Transmission; Gordon et al. 2017) database of spectroscopic parameters for electromagnetic line transitions. As in Section B.1.1, this section summarizes the procedure that was used to compute the spectrally resolved complex refractive index of a mixture in the infrared spectrum; a detailed description of the underlying physics is available in Mathar (2007) and references therein. 
The complex refractive index of a mixture $\left(m_{\lambda}[-]\right)$ is a function of the mixture's macroscopic electrical susceptibility $(\bar{\chi}[-])$ via (e.g., Mathar 2007):

$$
m_{\lambda}^{2}=1+\bar{\chi}(\lambda)
$$

The mixture's susceptibility can be computed from that of its components $\left(\chi_{i}[-]\right)$ using the Clausius-Mossotti/Lorentz-Lorenz formula:

$$
\bar{\chi}(\lambda)=\frac{\sum \chi_{i}(\lambda)}{1-\frac{1}{3} \sum \chi_{i}(\lambda)}
$$

Following Mathar (2007), the necessary $\chi_{i} \forall i \in\{1 \ldots I\}$ were computed within the angular frequency domain $\left(\omega=2 \pi c / \lambda\left[\mathrm{rad} \mathrm{s}^{-1}\right]\right.$, where $c$ is the speed of light in vacuum $\left.\left[\mathrm{m} \mathrm{s}^{-1}\right]\right)$ by summation over atomic and molecular line transitions for the $i^{\text {th }}$ component:

$$
\chi_{i}(\omega)=2 \frac{P X_{i}}{R_{u} T} c^{2} \sum_{l} \frac{S_{i l}}{\omega_{0, i l}} \phi\left(\omega ; \omega_{0, i l}, \gamma_{i l}\right)
$$

Here, $S_{i l}, \omega_{0, i l}$, and $\gamma_{i l}$ are the intensities $\left[\mathrm{m} \mathrm{kmol}^{-1}\right]$, centre frequencies $\left[\mathrm{rad} \mathrm{s}{ }^{-1}\right]$, and halfwidths at half-maximum $\left[\mathrm{rad} \mathrm{s}^{-1}\right]$ of the transitions for the species $i$. The function $\phi\left(\omega ; \omega_{0, i l}, \gamma_{i l}\right)$ is the complex Lorentzian lineshape $\left[\mathrm{s} \mathrm{rad}^{-1}\right]$ given by:

$$
\phi\left(\omega ; \omega_{0, i l}, \gamma_{i l}\right)=\frac{1}{\omega+\omega_{0, i l}+j \gamma_{i l}}-\frac{1}{\omega-\omega_{0, i l}+j \gamma_{i l}}
$$

where $j=\sqrt{-1}$ is the imaginary unit. As noted by Mathar (2004), when using this framework the HITRAN spectroscopic database must by augmented to account for the absorption spectrum at high excitation energies and the photoionization continuum. This was accomplished by introducing ten pseudo-oscillators (i.e., ten additional line transitions in Eq. (B.6)) for each component species, which, in this work, were sourced from Jhanwar 
and Meath (1982) for $\mathrm{CO}_{2}$ and $\mathrm{CO}$ and Margoliash and Meath (1978) for $\mathrm{CH}_{4}, \mathrm{H}_{2} \mathrm{O}, \mathrm{N}_{2}$, and $\mathrm{O}_{2}$.

\section{B.2 The Effect of Soot Particulate on Beam Steering in Flare Plumes}

In this work, spatiotemporally resolved concentrations of combustion products from largeeddy simulations of gas flares are used to quantify the effect of beam steering on remote optical diagnostics of heated plumes. As noted in the manuscript, these simulations did not employ a soot model so reported combustion products therefore did not include concentrations of soot particulate. Through an example, this section addresses the potential impact of excluding soot particulate from the present analyses by estimating the typical contribution of soot to beam steering through flare plumes.

To estimate the effect of soot particulate on beam steering, one timestep in simulation I was randomly selected for further analysis. A scalar field of soot volume fraction (SVF) was constructed for this simulation data by scaling reported carbon dioxide concentrations. The scalar multiplier was selected to achieve a maximum in-flame SVF of $10 \mathrm{ppm}$, which upper-bounds observations of in-flame SVF in ethylene/air flames of 27-8 ppm (Crosland et al. 2013; Köhler et al. 2011; Thomson et al. 2008). This represents an extreme sooting propensity relative to typical methane-based flare gas compositions (e.g., Chapter 5, Conrad and Johnson 2019a); for example, a study of turbulent, nonpremixed, methane/air flames observed peak in-flame SVFs in the tens of ppbs (Crosland et al. 2015) which are likely more representative. Nevertheless, using $10 \mathrm{ppm}$ as a conservatively high estimate for in-flame SVF, the Maxwell Garnett effective medium approximation (Maxwell Garnett 1904) was then used to estimate the refractive index field

of the soot-laden plume at each of the 12 studied wavelengths, given the known refractive 
index field of the gaseous medium and an assumed spectral refractive index for soot, where the dispersion relations of Chang and Charalampopoulos (1990) were used for the latter. Finally, the total deflection angle with the addition of soot in the plume was computed $\left(\theta_{\lambda, s}\right.$ [rad]) and compared to the total deflection angle with soot ignored (as in the manuscript, $\left.\theta_{\lambda}[\mathrm{rad}]\right)$

Figure B.1a shows $\theta_{\lambda}$ in units of mrad at a wavelength of $15.456 \mu \mathrm{m}$ for an orthogonal projection through the domain of simulation I at the randomly selected timestep. The total deflection angle is highest in the flame region (near the flare stack exit) and predictably decreases as the plume propagates away from the flame where gas temperatures decline towards ambient and combustion products disperse. Figure B.1b shows the absolute change in the total deflection angle $\left(\Delta \theta_{\lambda, s}=\left|\theta_{\lambda, s}-\theta_{\lambda}\right|\right)$ with the consideration of soot particulate as described above. Plotted in $\mu \mathrm{rad}$, this figure shows that deflection attributable to the presence of soot is negligible compared to the total deflection angle. 

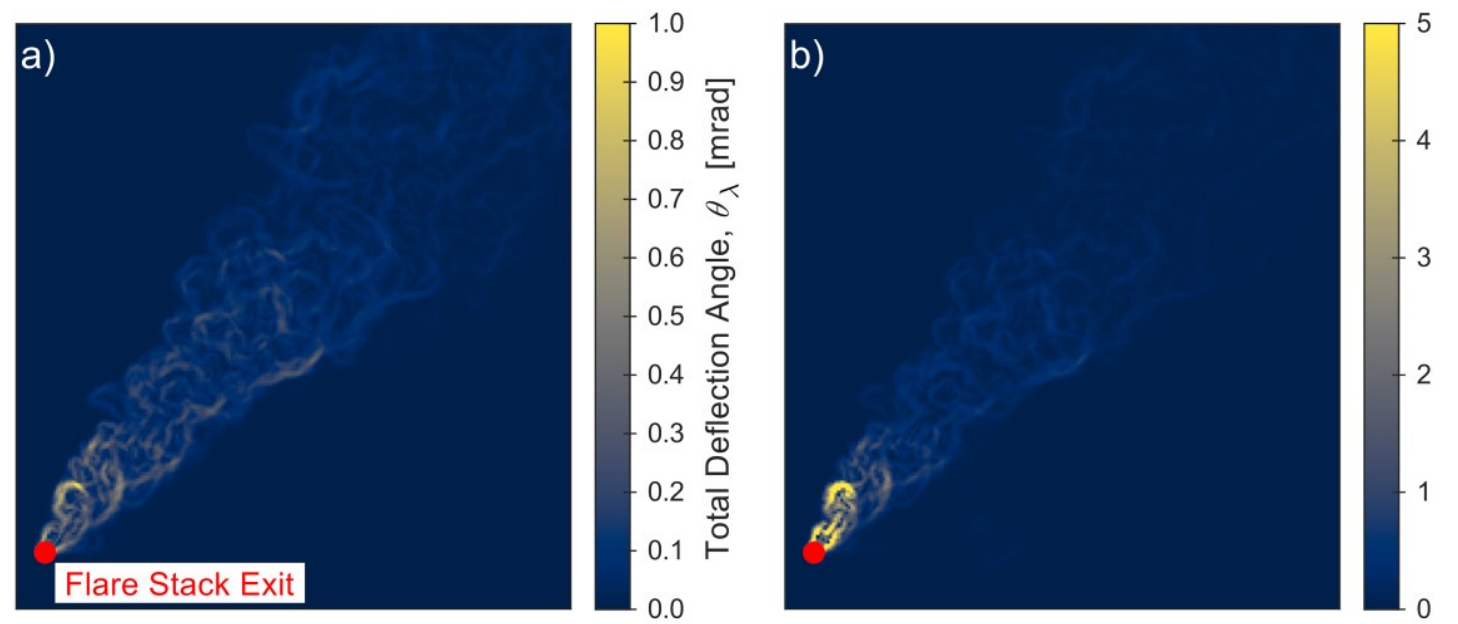

Figure B.1: Example results of total deflection angle at $15.456 \mu \mathrm{m}$ for lines-of-sight intersecting the flare plume from simulation $I$. a) Nominal total deflection angle (in mrad) while ignoring the presence of soot particulate and b) the change in the total deflection angle (in $\mu \mathrm{rad}$ ) due to the inclusion of soot particulate, where soot particulate is modelled by scaling carbon dioxide concentrations to yield a simulation-maximum in-flame soot volume fraction of $10 \mathrm{ppm}$. This example shows the negligible effect of soot particulate on beam steering through flare plumes.

In the cooled plume region of this example analysis, where diagnostics like skyLOSA are performed, the change in the total deflection angle due to the presence of soot is even smaller, on the order of $1 \mu \mathrm{rad}$ or less. Moreover, as noted above, typical flare gas compositions are usually much lighter than the studied ethylene flames used to estimate the peak in-flame SVF. Based on Crosland et al.'s (2015) measurements of SVFs in a turbulent, non-premixed, methane/air flame, if a presumably more reasonable peak SVF of $\sim 50 \mathrm{ppb}$ is used in this analysis, typical changes in the total deflection angle due to the presence of soot would be $\sim 35 \mathrm{nrad}$ or less. This is more than four orders of magnitude less than the nominal result without the presence of soot. This analysis conclusively shows that omission of fine soot particulate in the present beam steering analyses is justified. 


\section{B.3 Source Intensity Correction for Inscattering Sources}

Note: There is a mathematical error in the supplemental information of the published paper, which has been corrected in this section. Specifically, Eq. (B.10) originally contained an extraneous factor $\left(K_{\lambda, O D}\right)$ in the denominator of the rightmost term. Removal of this extraneous factor simplifies the subsequent math and strengthens the implications. As shown in Eq. (B.12), it is now concluded that the absolute deviation from unity of the source correction for inscattering sources is upper-bounded by that of the optical depth correction across the entire $K_{\lambda, O D}-\tau_{\lambda, \ell}$ domain, and can thus be considered negligible in all cases.

For visible spectrum measurements of particulate emissions, in which the intensity source is due solely to inscattering, the local, spectral source intensity is:

$$
I_{\lambda}^{S}(\boldsymbol{r})=\frac{\omega_{\lambda}}{4 \pi} \int_{4 \pi} I_{\lambda}\left(\boldsymbol{r}, \Omega^{\prime}\right) p_{\lambda}\left(\Omega^{\prime}, \Omega\right) d \Omega^{\prime}
$$

where $\omega_{\lambda}$ is the spectral single-scattering albedo of the particles $[-], I_{\lambda\left(r, \Omega^{\prime}\right)}$ is the incident spectral intensity at position $\boldsymbol{r}$ from direction $\Omega^{\prime}\left[\mathrm{W} \mathrm{m} \mathrm{m}^{-2} \mu \mathrm{m}^{-1} \mathrm{sr}^{-1}\right.$, and $p_{\lambda}\left(\Omega^{\prime}, \Omega\right)$ is the spectral scattering phase function for incident $\left(\Omega^{\prime}\right)$ and outgoing $(\Omega)$ directions $\left[\mathrm{sr}^{-1}\right]$, where the outgoing direction is tangent to the local direction of light propagation. Through integration over $4 \pi \mathrm{sr}$, the inscattering source intensity is not strictly a local phenomenon; rather, it is a function of the entirety of the surrounding plume. Unfortunately, this makes it challenging to quantify the effect of beam steering on the inscattered source intensity and, hence, the source intensity correction parameter, $K_{\lambda, s}$.

To enable an estimate of the source intensity correction parameter for inscattering

sources, consider the introduction of a path-averaged source intensity $\left(\bar{I}_{\lambda, i}^{s}\right.$, where the 
subscript " $i$ " represents the linear or curved path). The source intensity correction parameter is thus:

$$
K_{\lambda, s}=\frac{\bar{I}_{\lambda, c}^{s}}{\bar{I}_{\lambda, \ell}^{s}} \frac{\int_{c_{\lambda}} \mu_{\lambda}(\boldsymbol{r}) \exp \left(-\int_{c_{\lambda}^{\prime}(\boldsymbol{r})} \mu_{\lambda}(\boldsymbol{r}) d s^{\prime}\right) d s}{\int_{0}^{\infty} \mu_{\lambda}(x) \exp \left(-\int_{x}^{\infty} \mu_{\lambda}\left(x^{\prime}\right) d x^{\prime}\right) d x}
$$

Defining the "idealized line transmittance" as the transmittance along a line-of-sight (LOS) intersecting the plume, absent of any intensity sources - i.e., $\tau_{\lambda, \ell}=\exp \left(-\int_{0}^{\infty} \mu_{\lambda}(x) d x\right)-$ it can be shown that:

$$
K_{\lambda, s}=\frac{\bar{I}_{\lambda, c}^{s}}{\bar{I}_{\lambda, \ell}^{s}} \frac{1-\tau_{\lambda, \ell}^{K_{\lambda, O D}}}{1-\tau_{\lambda, \ell}}
$$

where $K_{\lambda, O D}$ is the optical depth (OD) correction parameter [-]. Since the inscattering source intensity is not strictly a local effect and the curved and linear paths differ only slightly in general (with respect to the scale of the plume), the path-averaged source intensities should be approximately equivalent - i.e., $\bar{I}_{\lambda, c}^{s} \approx \bar{I}_{\lambda, \ell}^{s}$. Leveraging this assumption, the source intensity correction parameter for inscattering sources may thus be approximated as a function of the idealized line transmittance and OD correction parameter alone:

$$
K_{\lambda, s} \approx \frac{1-\tau_{\lambda, \ell}^{K_{\lambda, O D}}}{1-\tau_{\lambda, \ell}}
$$

As shown by Eq. (B.12), the absolute deviation from unity of the source correction parameter is therefore upper-bounded by that of the optical depth correction across the entire $K_{\lambda, O D}-\tau_{\lambda, \ell}$ domain: 


$$
\frac{\left|K_{\lambda, s}-1\right|}{\left|K_{\lambda, O D}-1\right|} \approx \frac{\tau_{\lambda, \ell}^{K_{\lambda, O D}}-\tau_{\lambda, \ell}}{\left(1-K_{\lambda, O D}\right)\left(1-\tau_{\lambda, \ell}\right)} \leq 1
$$

where the equality holds in the optically thin $\operatorname{limit}\left(\tau_{\lambda, \ell}=1\right)$ or the extreme case of $K_{\lambda, O D}=$ 0. Since the OD correction parameter was generally determined to be negligible, it therefore follows that the source intensity correction parameter for inscattering sources is similarly negligible.

\section{B.4 The Effect of Sub-Grid Turbulence}

Referring to Eq. (3.7) and Figure 3.4, the incremental deflection in a two-dimensional plane (e.g., the $x-y$ plane for light propagating in the $x$-direction), under the small-angle limit, is:

$$
\frac{d \theta_{\lambda}^{y}}{d x}=\frac{1}{\eta_{\lambda}} \frac{\partial \eta_{\lambda}}{\partial y}
$$

where $\eta_{\lambda}$ is the local, real refractive index [-]. This equation shows that, locally, beam steering is a gradient-driven effect, which is then integrated over an optical axis to yield a total deflection angle within the two-dimensional plane (Eq. (3.8)). An important question is whether sub-grid turbulence in the present simulations could significantly affect the incremental deflection, since random isotropic turbulence would tend to increase the derivative on the righthand side of Eq. (B.13). By contrast however, integration over an optical axis to compute a total deflection has an averaging effect that would subsequently dampen the effect of turbulence on the total deflection.

To understand the potential influence of sub-grid turbulence, a Monte Carlo (MC) analysis was performed. The first objective of the MC analysis was to provide realistic sub-grid turbulence for coarse simulation data in the form of turbulent fluctuations in temperature. Firstly, the structured computational grid from the large-eddy flare 
simulations was refined using linear interpolation for the mean temperature field $(\bar{T}(\boldsymbol{r})$ $[\mathrm{K}])$. Next, the local fluctuating component of the temperature was characterized with a root-mean-square fluctuation according to:

$$
T_{r m s}^{\prime}(\boldsymbol{r})=I_{T}\left(\bar{T}(\boldsymbol{r})-T_{\infty}\right)
$$

where $I_{T}$ is a measure of turbulence intensity [-] and $T_{\infty}$ is the simulated ambient temperature $(298.15 \mathrm{~K})$. This enabled the provision of sub-grid turbulence at position $\boldsymbol{r}$ via:

$$
T(\boldsymbol{r})=\bar{T}(\boldsymbol{r})+\xi_{\mathcal{N}} T_{r m s}^{\prime}(\boldsymbol{r})
$$

where $\xi_{\mathcal{N}}$ is a MC-randomized variable from the standard normal distribution [-]. With a turbulent temperature field defined, the same beam steering analysis procedure employed in the manuscript was used to compute the deflection components and total deflection angle under turbulent conditions.

This MC analysis was performed using the specific LOS shown in Figure 3.2 and used for the analysis presented in Figure 3.5 at a wavelength of $0.405 \mu \mathrm{m}$, representing a conservatively high estimate of the continuum-like component of observed beam deflections. Flare simulation data were first refined by a factor of $2^{m}(m \in\{1 \ldots 7\})$ to yield grid resolutions from $0.05 \mathrm{~m}$ (raw flare simulation data) down to sub-mm resolution of $0.391 \mathrm{~mm}$. Turbulence intensities of up to $10 \%$ were considered. For each specific degree of grid refinement and turbulence intensity, $10^{5} \mathrm{MC}$ simulations of beam steering with added turbulence were performed.

Beam deflection components $\theta_{\lambda}^{y}$ and $\theta_{\lambda}^{z}$ were found to be normally distributed about the baseline case (coarse, raw simulation data), such that the effect of isotropic sub- 
grid turbulence was to impose a random walk in the path of light about the baseline path. Coefficients of variation of the deflection components increased approximately linearly with turbulence intensity and exponentially with the degree of grid refinement, $m$. That is, added turbulence tended to have a relatively nominal effect on the central tendency of beam deflection components relative to the baseline, but variance about the baseline was sensitive to turbulence intensity, and even more sensitive to grid refinement/turbulence scale.

Summing the beam deflection components in quadrature to yield the total deflection can be expected to yield a Rician distribution (quadrature summation of two uncorrelated, normally distributed, random variables). Figure B.2a shows Rician cumulative distributions fitted to MC data of the total deflection angle for all simulated degrees of grid refinement, and turbulence intensities of 5\% and 10\%. These distributions illustrate that the effect of added turbulence was mainly to cause variance in the deflection angle about that computed on a coarser grid (shown by the dotted red line). Importantly however, the total deflection angle also increased slightly from the baseline when added turbulence was applied. The mean increase in the total deflection angle (representing bias due to turbulence) is plotted in Figure B.2b as a function of grid resolution for four simulated turbulence intensities. For a conservatively small Kolmogorov scale on the order of $4 \mathrm{~mm}$ - requiring a grid resolution of $2 \mathrm{~mm}$ (shown by the dotted blue line) to be resolved - and a realistic turbulence intensity of $5 \%$, upward bias in the mean total deflection angle due to turbulence was approximately $5 \%$. In the most extreme simulation of grid resolution $0.391 \mathrm{~mm}$ (capturing scales of $\sim 0.8 \mathrm{~mm}-$ i.e., almost one order of magnitude less than 
what might be expected) and turbulence intensity of $10 \%$, the average total deflection was estimated as $84 \%$ greater than the baseline.

To assess the degree that this upward bias in deflection persists into the turbulence effect on the correction parameters, the MC analysis was extended to compute the OD correction parameter under varying turbulent conditions as in Section 3.5.2. Figure B.2c shows the cumulative distribution of the OD correction parameter conditional on the turbulence intensity and grid resolution, as compared to the baseline (coarse, raw computational grid) identified by the dotted red line. The effect of added turbulence is to increase the variability in the OD correction parameter in the same manner as the deflection angle. As plotted however, the change in the central tendency of the correction parameter appears modest. The average absolute deviation of the correction parameter from unity $\left(\left|K_{\lambda, O D}-1\right|\right)$ from the MC analysis is shown in Figure B.2d, and remained negligible over all simulated turbulence scale and intensities. In the most extreme simulation, the deviation increased to $10^{-3.49}$ from the baseline of $10^{-4.47}$, while at a Kolmogorov scale of $4 \mathrm{~mm}$ and realistic turbulence intensity of 5\%, sub-grid turbulence increased the deviation to just $10^{-4.08}$. Considering the negligibility of the OD correction parameter's deviation from unity under the present $\mathrm{MC}$ simulations, in addition to the dampening of the turbulence effect through finite spatial and temporal resolution of optical detectors (see Section 3.5.4), the conclusions within the manuscript should not change based on the results of this MC analysis. That is, corrections due to the effect of beam steering can be considered negligible. 

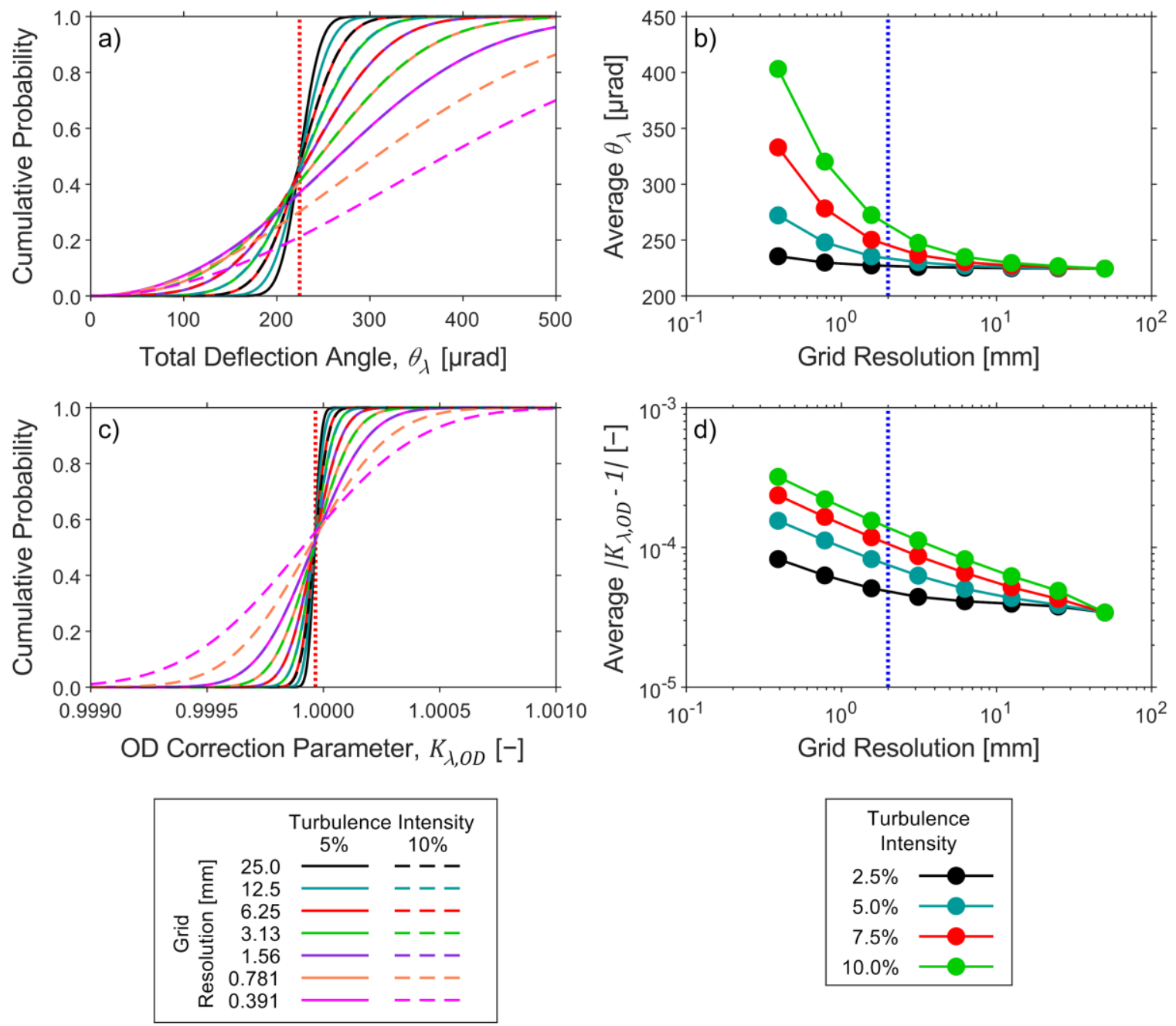

Figure B.2: MC-derived cumulative distribution of the total deflection angle (a) and average total deflection angle (b) for added turbulence at listed values of grid resolution and turbulence intensity. Cumulative distribution of the OD correction parameter (c) and average of the parameter's deviation from unity (d) for added turbulence as in $(a, b)$. Vertical red lines $(a, c)$ correspond to the nominal result on the coarse, raw computational grid and vertical blue lines $(b, d)$ correspond to the required grid resolution to resolve turbulence of $4 \mathrm{~mm}$ in scale. 


\section{Appendix C Supplemental Information for The Effect of Multiple Scattering on Optical Measurement of Soot Emissions in Atmospheric Plumes}

\section{C.1 Simplification of Radiative Transfer using the Idealized Transmittance}

Eq. (C.1) (taken from Eq. (4.2)) shows the source component of the radiative transfer equation (RTE) for an arbitrary line-of-sight (LOS) transecting a soot-laden plume like that shown in Figure 4.1b:

$$
\overline{\frac{J^{s, m}}{\sigma_{e}^{m}}} \int_{-\infty}^{\infty} \sigma_{e}^{m} \rho_{s}(x) \exp \left(-\sigma_{e}^{m} \int_{x}^{\infty} \rho_{s}\left(x^{\prime}\right) d x^{\prime}\right) d x
$$

$\overline{J^{s, m}}$ is the LOS path-averaged source radiant intensity due to inscattering of skylight and sunlight per unit mass of soot $\left[\mathrm{W} \mathrm{sr}^{-1} \mathrm{~g}^{-1}\right], \rho_{s}$ is the local mass concentration of soot $\left[\mathrm{g} \mathrm{m}^{-3}\right], \sigma_{e}^{m}$ is the spatially invariant average mass-normalized extinction cross-section of the soot population $\left[\mathrm{m}^{2} \mathrm{~g}^{-1}\right]$, and the ratio $\sigma_{e}^{m} / \sigma_{e}^{m}=1$ is added to simplify the subsequent derivation. Let the function:

$$
h(x)=\int_{x}^{\infty} \rho_{s}\left(x^{\prime}\right) d x^{\prime}
$$

such that $h(\infty) \equiv 0$ and $h(-\infty) \equiv \rho_{s}^{\prime}$, the soot mass column density along the LOS [ $\left.\mathrm{g} \mathrm{m}^{-2}\right]$. Eq. (C.1) is then equivalent to: 


$$
\overline{\bar{J}_{e}^{s, m}} \int_{-\infty}^{\infty} \sigma_{e}^{m} \rho_{s}(x) \exp \left(-\sigma_{e}^{m} h(x)\right) d x
$$

Consider integration by substitution using the new variable $w=\sigma_{e}^{m} h(x)$. By the first fundamental theorem of calculus:

$$
w^{\prime}=\frac{d}{d x}\left(\sigma_{e}^{m} \int_{x}^{\infty} \rho_{s}\left(x^{\prime}\right) d x^{\prime}\right)=-\sigma_{e}^{m} \rho_{s}(x) \stackrel{\text { giving }}{\longrightarrow} d w=-\sigma_{e}^{m} \rho_{s}^{\prime} d x
$$

Introducing the substitution into Eq. (C.3) gives:

$$
\frac{\overline{J^{s, m}}}{\sigma_{e}^{m}} \int_{\sigma_{e}^{m} \rho_{s}^{\prime}}^{0}-\exp (-w) d w
$$

Solving the integral, this becomes:

$$
\frac{\overline{J^{s, m}}}{\sigma_{e}^{m}}\left[1-\exp \left(-\sigma_{e}^{m} \rho_{s}^{\prime}\right)\right]
$$

The exponential term is defined as the idealized transmittance, $\tau^{*}=\exp \left(-\sigma_{e}^{m} \rho_{s}^{\prime}\right)$. Thus, the source component of the RTE can be constructed as presented in Eq. (4.2), i.e.:

$$
\overline{I^{s}}\left(1-\tau^{*}\right)
$$

where $\overline{I^{s}}=\overline{J^{s, m}} / \sigma_{e}^{m}$ represents a LOS path-averaged inscattering source intensity $\left[\mathrm{W} \mathrm{m}^{-2} \mathrm{sr}^{-1}\right]$.

\section{C.2 Prior Distributions of Fundamental Soot Properties}

Uncertain soot properties are perturbed within the Monte Carlo (MC) framework according to literature-derived prior distributions developed by Johnson et al. (2013) (source references available in Table 2 of Johnson et al. (2013)). These distributions are replicated 
for convenience in Table C.1. It should be noted that soot aggregate size ( $N$, the number of spherical primary particles per aggregate) is modelled to follow a lognormal distribution:

$$
f_{N}(N)=\frac{1}{N \ln \sigma_{g} \sqrt{2 \pi}} \exp \left(-\frac{\left(\ln N-\ln N_{g}\right)^{2}}{2 \ln ^{2} \sigma_{g}}\right)
$$

where $N_{g}$ and $\sigma_{g}$ are the geometric mean and standard deviation, respectively.

Table C.1: Prior distributions of fundamental soot properties derived by Johnson et al. (2013).

\begin{tabular}{|c|c|c|c|}
\hline Variable & Symbol & Unit & Distribution $^{\mathbf{a}}$ \\
\hline $\begin{array}{c}\text { Absorption function of the complex } \\
\text { refractive index }\end{array}$ & $E\left(m_{\lambda}\right)$ & {$[-]$} & $\mathcal{N}(0.332,0.0439)$ \\
\hline $\begin{array}{c}\text { Scatter-to-absorption functions of } \\
\text { the complex refractive index }\end{array}$ & $\frac{F\left(m_{\lambda}\right)}{E\left(m_{\lambda}\right)}$ & {$[-]$} & $\mathcal{N}(0.901,0.128)$ \\
\hline Primary particle diameter & $d_{p}$ & {$[\mathrm{~nm}]$} & $\mathcal{N}(36.0,5.81)$ \\
\hline $\begin{array}{c}\text { Aggregate size } \\
\text { (geometric mean) }\end{array}$ & $N_{g}$ & {$[-]$} & $\mathcal{U}(30,300)$ \\
\hline $\begin{array}{c}\text { Aggregate size } \\
\text { (geometric standard deviation) }\end{array}$ & $\sigma_{g}$ & {$[-]$} & $\mathcal{N}(2.74,0.499)$ \\
\hline Fractal prefactor & $k_{f}$ & {$[-]$} & $\mathcal{N}(8.145,0.451)$ \\
\hline Fractal dimension & $D_{f}$ & {$[-]$} & $\mathcal{N}(1.74,0.0574)$ \\
\hline Material density & $\varrho_{s}$ & {$\left[\mathrm{~kg} \mathrm{~m}^{-3}\right]$} & $\mathcal{N}(1860,120)$ \\
\hline
\end{tabular}

${ }^{\text {a }} \mathcal{N}(\mu, \sigma)$ : Normal distribution with mean $\mu$ and standard deviation $\sigma ; \mathcal{U}(a, b)$ : Uniform distribution from $a$ to $b$.

\section{C.3 Inscattering of Polarized Light}

The purpose of this section is to derive the intensity of soot-scattered skylight of some known polarization state into a line-of-sight (LOS) axis while allowing for multiple scattering (MS) within a plume. The polarization state of light through optical components and due to scattering processes can be tracked using the Stokes-Mueller formalism 
(Goldstein 2003) as discussed in Section C.3.2. Relevant background nomenclature for light polarization are first presented in Section C.3.1.

\section{C.3.1 Polarization Nomenclature}

To define the polarization state of light propagating in the arbitrary $n$-direction, it is common to first define a principal coordinate system relative to the direction of propagation as shown in Figure C.1. For linearly polarized light propagating in the $n$-direction ( $\boldsymbol{n}$, out of the page), two orthogonal axis pairs ( $v-h$ and $a-b)$ are defined, where the $a-b$ axis pair is righthand rotated $45^{\circ}$ about the direction of propagation, relative to the $v-h$ pair. The polarization of light (with intensity proportional to $|\boldsymbol{E}|^{2}$, where $\boldsymbol{E}$ is the electric field vector $[\mathrm{V} / \mathrm{m}])$ is defined through its angle relative to the $v$-axis $(\psi)$.

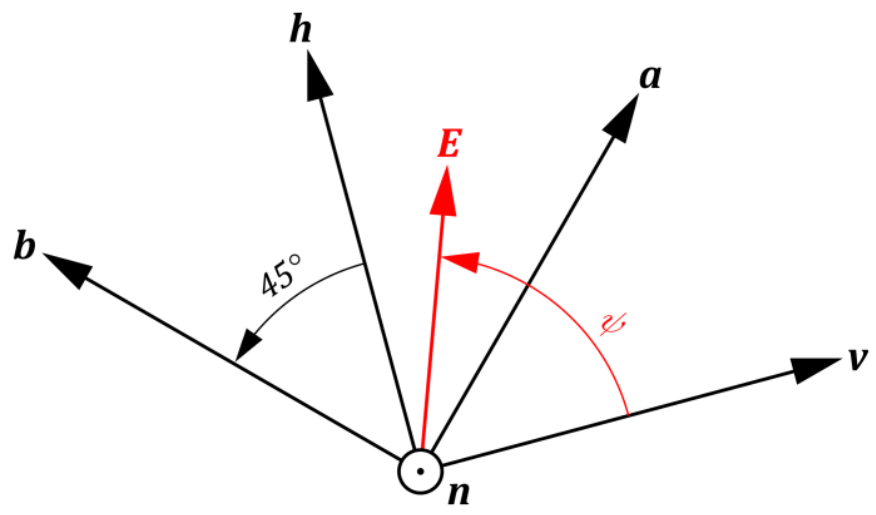

Figure C.1: Coordinate system used to describe the polarization state of light. Propagation of light is in the $n$-direction $(n=v \times h=a \times b$, out of the page).

While the orientation of the reference polarization axes $(v-h)$ about the direction of propagation are arbitrary for stand-alone light, it is beneficial to define these axes with reference to incident and outgoing propagation directions when analyzing a scattering event. Consider a scattering event where incident light (subscript "i") travelling along the incident propagation direction $\left(\boldsymbol{n}_{i}\right)$ scatters through a scattering angle of $\theta$ such that outgoing light (subscript "o") travels along the outgoing propagation direction $\left(\boldsymbol{n}_{o}\right)$. The 
scattering plane of this specific scattering event is defined as the plane containing both the incident and outgoing propagation directions. By convention, both the incident and outgoing $v-h$ axes are defined with respect to this scattering plane, where the $h$-axes are located within the scattering plane and the $v$-axes are equivalent and represent the normal of the scattering plane. The notation $\boldsymbol{x}_{y, z}$ is used to identify axes, where $\boldsymbol{x}$ represents the $h$ - or $v$-axis of the propagation direction $\boldsymbol{n}_{y}$ with respect to the propagation direction $\boldsymbol{n}_{z}$. An example of $v-h$ axis pairs for a scattering event using this notation is shown in Figure C.2. The coordinate system axes $\left(\boldsymbol{v}_{i, o}, \boldsymbol{v}_{o, i}, \boldsymbol{h}_{i, o}, \boldsymbol{h}_{o, i}\right)$ are computed from the propagation vectors $\boldsymbol{n}_{i}$ and $\boldsymbol{n}_{o}$ as summarized in Eq. (C.1).

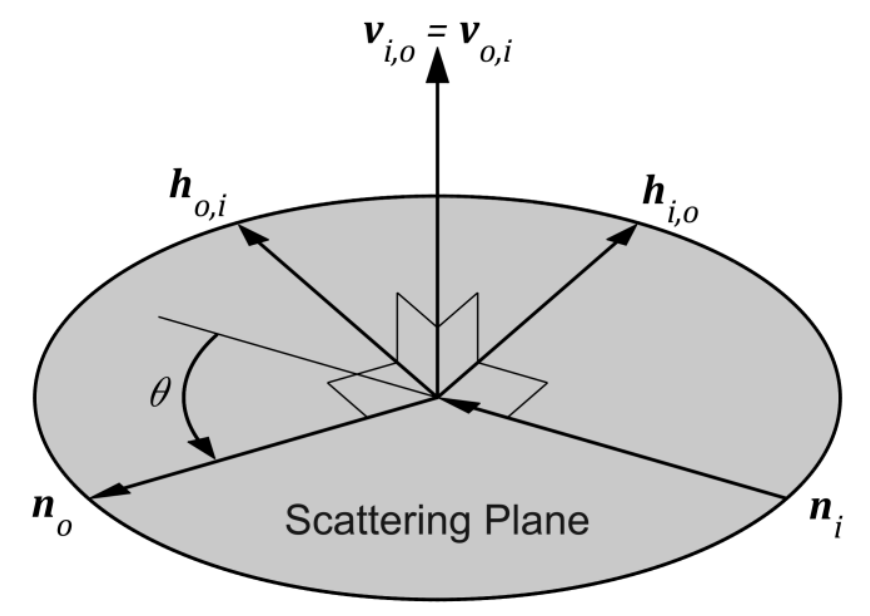

Figure C.2: Definition of the scattering plane, scattering angle $(\theta)$, and polarization coordinate system for a scattering event with incident and outgoing vectors indicated by subscripts "i" and "o", respectively.

$$
\begin{aligned}
\boldsymbol{v}=\boldsymbol{v}_{i, o}=\boldsymbol{v}_{o, i} & =\frac{\boldsymbol{n}_{i} \times \boldsymbol{n}_{o}}{\left|\boldsymbol{n}_{i} \times \boldsymbol{n}_{o}\right|} \\
\boldsymbol{h}_{i, o} & =\boldsymbol{n}_{i} \times \boldsymbol{v}_{i, o} \\
\boldsymbol{h}_{o, i} & =\boldsymbol{n}_{o} \times \boldsymbol{v}_{o, i}
\end{aligned}
$$




\section{C.3.2 Stokes Mueller Formalism and the Stokes Vector}

Under the Stokes-Mueller formalism, the intensity and polarization of light is captured in terms of its polarization axes by a real-valued $4 \times 1$ Stokes vector $(\boldsymbol{q})$, as shown in Eq. (C.10). In this work, an intensity-based Stokes vector is employed, where: the first element of the vector is equivalent to the intensity of the light; the second and third elements represent the degree of linear polarization with respect to the $v-h$ and $a-b$ axis pairs, respectively; and the fourth element represents the degree of circular polarization, which is ignored throughout this work. The subscript " $\mathrm{i}, \mathrm{o}$ " of the Stokes vector matches that of the defined $v-h$ coordinate system for a scattering event.

$$
\boldsymbol{q}_{i, o}=\left[\begin{array}{c}
I \\
I_{h}-I_{v} \\
I_{a}-I_{b} \\
0
\end{array}\right]=I\left[\begin{array}{c}
1 \\
\cos 2 \psi \\
\sin 2 \psi \\
0
\end{array}\right]
$$

The Stokes vector of light outgoing from a scattering event can be computed from that of the incident light by multiplication with the real $4 \times 4$ Mueller Matrix, M, that represents the specific scattering phenomena.

$$
\boldsymbol{q}_{o, i}=\mathbf{M} \boldsymbol{q}_{i, o}=\left[\begin{array}{llll}
m_{11} & m_{12} & m_{13} & m_{14} \\
m_{21} & m_{22} & m_{23} & m_{24} \\
m_{31} & m_{32} & m_{33} & m_{34} \\
m_{41} & m_{42} & m_{43} & m_{44}
\end{array}\right]^{\boldsymbol{q}_{i, o}}
$$

For small particles within the Rayleigh regime and soot particles analyzed using the assumptions of the Rayleigh-Debye-Gans (RDG) approximation, symmetry in scattering permits simplification of the Mueller matrix as a function of only three unique elements (e.g., Bohren and Huffman 1983). Furthermore, for scattering by large populations of soot aggregates, the RDG approximation imposes a simplification of the scattering phase function; that is, scattering is a function of the included angle $(\theta)$ between the incident and 
outgoing vectors only. The simplified form of the Mueller matrix for scattering by the atmosphere and soot-laden plume used in this work is:

$$
\mathbf{M}(\theta)=\left[\begin{array}{cccc}
m_{11}(\theta) & m_{12}(\theta) & 0 & 0 \\
m_{12}(\theta) & m_{11}(\theta) & 0 & 0 \\
0 & 0 & m_{33}(\theta) & 0 \\
0 & 0 & 0 & m_{33}(\theta)
\end{array}\right]
$$

\section{C.3.3 Intensity of Skylight Single-Scattered by Soot}

The objective of this section is to quantify the intensity of single-scattered skylight by soot (representing the 1SA) at an observer on the ground as a function of sky azimuth $(\alpha)$, sky zenith $(Z)$, solar azimuth $\left(\alpha_{s}\right)$, solar zenith $\left(Z_{s}\right)$, and camera inclination $(\beta)$, as defined in Figure 4.1a.

Skylight is the result of diffuse scattering of incident solar radiation by Earth's atmosphere. While these scattering events are numerous, such that skylight is the result of multiple scattering, skylight polarization is modelled in sky-LOSA using a simple approach known as the Rayleigh sky model. In this model, the polarization state of skylight at a ground-based detector is modelled as that which would be observed due to single-scattering by small aerosols (Rayleigh scatterers) within a non-absorbing atmosphere. Depolarization effects that inherently occur due to multiple scattering within the atmosphere are thus not directly modelled but are rather considered using a scaling variable applied to the entire sky dome as presented below. Additionally, the intensity of ground-observed skylight is not directly considered by modelling the composition of the atmosphere but is instead modelled using well-known standard distributions (CIE 2003). The intensity and polarization state of ground-detected soot-scattered skylight can then be entirely described via the Stokes-Mueller formalism as sky- then soot-scattered solar radiation. 
A simplified schematic of the measurement of single-soot-scattered skylight is shown for an arbitrary sky element in Figure C.3. Solar radiation enters the atmosphere along the propagation direction $\boldsymbol{n}_{0}=\boldsymbol{n}_{0}\left(\alpha_{s}, Z_{s}\right)$. Solar radiation of initial intensity $I_{0}$ is diffusely scattered by atmospheric aerosols towards the soot-laden plume through scattering angle $\chi \in[0, \pi)$. The atmosphere-scattered solar radiation (skylight) then travels a distance of $c$ along propagation direction $\boldsymbol{n}_{1}=\boldsymbol{n}_{1}(\alpha, Z)$ corresponding to the sky element. Skylight, of intensity $I_{1}$, is then scattered by soot towards a ground-based detector through scattering angle $\theta \in[0, \pi)$. The soot-scattered skylight then travels along the direction of propagation $\boldsymbol{n}_{2}=\boldsymbol{n}_{2}\left(0, Z_{d}\right)$ (where subscript "d" signifies the detector and $\left.Z_{d}=90^{\circ}-\beta\right)$ to the detector where an intensity of $I_{2}$ is measured. 


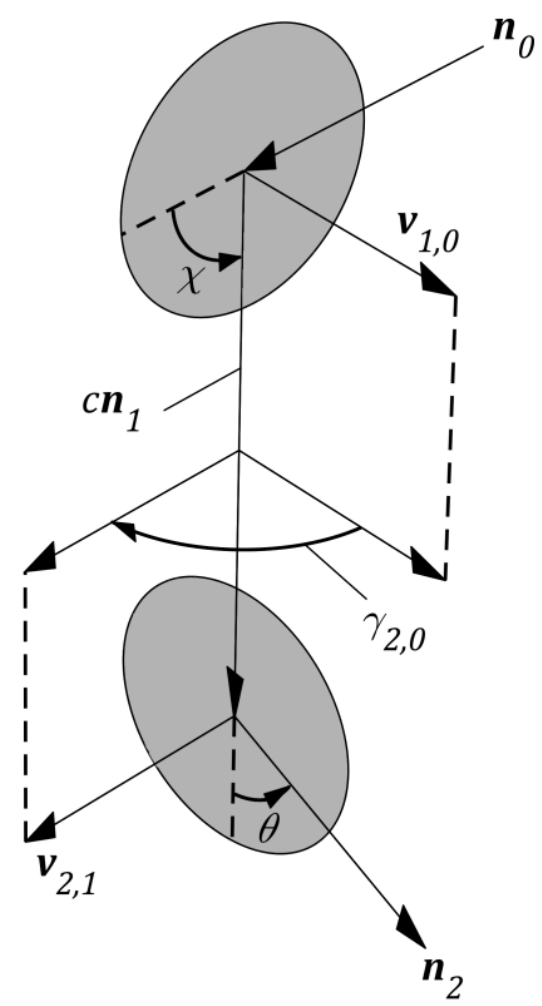

Figure C.3: Simplified schematic of single-soot scattered skylight using the Rayleigh sky model. Solar radiation propagates along $n_{0}$ and is scattered by atmospheric aerosol through angle $\chi$ in the direction of $n_{1}$. Diffusely scattered solar radiation (skylight) travels a distance of $c$ towards the plume and is scattered by soot through angle $\theta$ in the direction $n_{2}$, towards the detector. Also shown in the figure are the scattering planes and $\mathbf{v}$-axes for the scattering events. The angle $\gamma_{2,0}$ is the angle of rotation necessary to change the reference of the skylight's Stokes vector (i.e., from $s_{1,0}$ to $s_{1,2)}$.

To derive the single-soot-scattered skylight intensity at the sky-LOSA camera, the Stokes-Mueller formalism is employed. The Stokes vector of solar radiation at the location of the first scattering event is:

$$
\boldsymbol{q}_{0,1}=I_{0}\left[\begin{array}{l}
1 \\
0 \\
0 \\
0
\end{array}\right]
$$

where the bottom three elements of the Stokes vector are zero since sunlight is inherently unpolarized. The Stokes vector of the sky-scattered solar radiation (i.e., skylight) is 
computed by application of the Mueller matrix of the atmospheric scatterers. Recalling that the Rayleigh sky model is employed, the Mueller matrix takes the form of a Rayleigh scatterer (adapted from Bohren and Huffman 1983):

$$
\begin{aligned}
\boldsymbol{q}_{1,0}=\mathbf{M}_{R A Y}(\chi) \boldsymbol{q}_{0,1} & =\frac{\omega_{a t m}}{4 \pi} p_{R A Y}(\chi)\left[\begin{array}{cccc}
1 & -\delta(\chi) & 0 & 0 \\
-\delta(\chi) & 1 & 0 & 0 \\
0 & 0 & \zeta(\chi) & 0 \\
0 & 0 & 0 & \zeta(\chi)
\end{array}\right]^{\boldsymbol{q}_{0,1}} \\
& =\frac{\omega_{a t m}}{4 \pi} p_{R A Y}(\chi) \overline{\mathbf{M}}(\chi) \boldsymbol{q}_{0,1}
\end{aligned}
$$

where $\omega_{\text {atm }}$ is the single-scattering albedo of representative atmospheric aerosols, $p_{R A Y}(\chi)$ is the scattering phase function for Rayleigh particles, $\delta(\chi)$ and $\zeta(\chi)$ are:

$$
\delta(\chi)=\frac{1-\cos ^{2} \chi}{1+\cos ^{2} \chi} \quad \zeta(\chi)=\frac{2 \cos \chi}{1+\cos ^{2} \chi}
$$

with $\delta(\chi)$ representing the degree of linear polarization of incident unpolarized light scattered by a Rayleigh particle, and $\overline{\mathbf{M}}(\chi)$ is hereinafter referred to as the normalized Mueller matrix (i.e., where $m_{11}=1$ ). It follows that the Stokes vector of Rayleighscattered skylight is:

$$
\boldsymbol{q}_{1,0}=I_{0} \frac{\omega_{a t m}}{4 \pi} p_{R A Y}(\chi)\left[\begin{array}{c}
1 \\
-\delta(\chi) \\
0 \\
0
\end{array}\right]
$$

This formulation assumes accurate knowledge of extra-terrestrial solar intensity $\left(I_{0}\right)$ and the optical properties of atmospheric particles (through $\omega_{s k y}$ and $\mathrm{p}_{\mathrm{RAY}}(\chi)$ ). Furthermore, as skylight travels towards a ground-based observer, its intensity may be reduced due to attenuation by absorbing gases and aerosols. These mechanisms are not 
considered in in sky-LOSA, as they are dependent on spatial concentrations of optically active gases and aerosols in the atmosphere and are therefore not known a priori. Consequently, the intensity of sky-scattered sunlight at ground-level is instead modelled. Noting that only a relative light distribution is necessary for sky-LOSA, standard relative luminance distributions developed by the Commission Internationale de l'Eclairage (CIE, 2003) are employed in the sky-LOSA algorithm. For a ground-based observer, the skylight intensity can be modelled as:

$$
I_{1}=I_{0} \frac{\omega_{a t m}}{4 \pi} p_{R A Y}(\chi)=I_{s k y}\left(\alpha, Z, \alpha_{s}, Z_{s} ; a\right)
$$

where $I_{s k y}\left(\alpha, Z, \alpha_{s}, Z_{s} ; a\right)$ represents the relative intensity at the ground for a sky element defined by $\{\alpha, Z\}$ and a sun position of $\left\{\alpha_{S}, Z_{s}\right\}$, for the CIE sky model defined by index $a$.

One additional practical consideration must be made to appropriately model $\boldsymbol{q}_{1,0}$. Within the atmosphere, sky-scattered solar radiation may become depolarized through multiple scattering events and reflections from the ground (e.g., Pust and Shaw 2012). This complex depolarization is spectrally dependent and is a function of the solar zenith angle $\left(Z_{S}\right)$, bidirectional reflectance function of the ground, and sky conditions (Pust and Shaw 2012). To bound depolarization of skylight by these complex mechanisms, a simple scaling variable $(\hat{\delta} \in[0,1])$ is introduced, which linearly scales the degree of linear polarization of the sky and limits its maximum value. The Stokes vector $\boldsymbol{q}_{1,0}$ is then:

$$
\boldsymbol{q}_{1,0}=I_{s k y}\left(\alpha, Z, \alpha_{s}, Z_{s} ; a\right)\left[\begin{array}{c}
1 \\
-\hat{\delta} \delta_{(\chi)} \\
0 \\
0
\end{array}\right]
$$


To consider the scattering of skylight by soot, the Stokes vector $\boldsymbol{q}_{1,0}$ must first be framed with respect to the scattering plane defined by incident skylight and outgoing sootscattered skylight, yielding $\boldsymbol{q}_{1,2}$. A rotation matrix $(\mathbf{R}(\gamma))$ is required to adjust the frame of reference from "1,0" to "1,2" (Goldstein 2003; Mishchenko et al. 2002; Bohren and Huffman 1983). The desired rotation is righthand about the direction of propagation (here, $\left.\boldsymbol{n}_{1}\right)$ from the $v$-axis of the preceding scattering event $\left(\boldsymbol{v}_{1,0}\right)$ to that of the succeeding $\left(\boldsymbol{v}_{2,1}\right)$ which defines angle $\gamma_{2,0}$ shown in Figure C.3.

$$
\boldsymbol{q}_{1,2}=\mathbf{R}\left(\gamma_{2,0}\right) \boldsymbol{q}_{1,0}=\left[\begin{array}{cccc}
1 & 0 & 0 & 0 \\
0 & \cos 2 \gamma_{2,0} & -\sin 2 \gamma_{2,0} & 0 \\
0 & \sin 2 \gamma_{2,0} & \cos 2 \gamma_{2,0} & 0 \\
0 & 0 & 0 & 1
\end{array}\right]^{\boldsymbol{q}_{1,0}}
$$

where $\gamma_{2,0} \in[0,2 \pi)$. Propagating the matrix multiplication:

$$
\boldsymbol{q}_{1,2}=I_{s k y}\left(\alpha, Z, \alpha_{s}, Z_{s} ; a\right)\left[\begin{array}{c}
1 \\
-\hat{\delta} \delta(\chi) \cos 2 \gamma_{2,0} \\
-\hat{\delta} \delta(\chi) \sin 2 \gamma_{2,0} \\
0
\end{array}\right]
$$

Next, the Mueller matrix for soot-scattering is applied. In the present work, the Mueller matrix is developed for the population-averaged soot particle using RDG-PFA theory. This has a similar form as that for Rayleigh scatterers:

$$
\mathbf{M}_{R D G}(\theta ; \boldsymbol{b})=\frac{\omega(\boldsymbol{b})}{4 \pi} p(\theta ; \boldsymbol{b}) \overline{\mathbf{M}}(\theta)
$$

where $\omega(\boldsymbol{b})$ is the single-scattering albedo of the polydisperse soot population (computed using RDG-PFA given soot properties contained in vector $\boldsymbol{b})$ and $p(\theta ; \boldsymbol{b})$ is the population-averaged scattering phase function computed with RDG-PFA. This gives the 
Stokes vector of soot-scattered skylight at the ground-based detector $\left(\boldsymbol{q}_{d}\right)$, located at direction $\boldsymbol{n}_{2}$ from the soot-scattering event:

$$
\begin{aligned}
\boldsymbol{q}_{d}=\boldsymbol{q}_{2,1} & =\mathbf{M}_{R D G}(\theta ; \boldsymbol{b}) \boldsymbol{q}_{1,2} \\
& =I_{s k y}\left(\alpha, Z, \alpha_{s}, Z_{s} ; a\right) \frac{\omega(\boldsymbol{b})}{4 \pi} p(\theta ; \boldsymbol{b})\left[\begin{array}{c}
1+\hat{\delta} \delta(\chi) \delta(\theta) \cos 2 \gamma_{2,0} \\
-\delta(\theta)-\hat{\delta} \delta(\chi) \cos 2 \gamma_{2,0} \\
-\hat{\delta} \delta(\chi) \zeta(\theta) \sin 2 \gamma_{2,0} \\
0
\end{array}\right]
\end{aligned}
$$

Finally, the intensity of soot-scattered skylight at the detector, for a given region of sky defined by $\{\alpha, Z\}$, is equivalent to the first component of the Stokes vector $\boldsymbol{q}_{2,1}$ :

$$
I_{d}=I_{s k y}\left(\alpha, Z, \alpha_{s}, Z_{s} ; a\right) \frac{\omega(\boldsymbol{b})}{4 \pi} p(\theta ; \boldsymbol{b})(1+\underbrace{\hat{\delta} \delta(\chi) \delta(\theta) \cos 2 \gamma_{2,0}}_{f_{p o l}\left(\alpha, Z, \alpha_{s} Z_{s}, \beta\right)})
$$

where $f_{p o l}\left(\alpha, Z, \alpha_{s} Z_{s}, \beta\right)$ represents the correction to the detector-measured intensity due to the effect of polarization.

Derivation of the polarization correction function $\left(f_{p o l}\left(\alpha, Z, \alpha_{s} Z_{s}, \beta\right)\right)$ is shown for completion, but previous efforts have indicated that polarization has a negligible effect on sky-LOSA-computed soot mass emission rates (Johnson et al. 2013). The main reason for this observation is that the skylight intensity distribution has an opposite dependence on the scattering angle $\chi$ than the polarization correction function. That is, through $\delta(\chi)$, the polarization correction function is small in the vicinity of the sun, but the skylight intensity is maximal in this same region. This serves to dampen the effect of polarization on the inscattered skylight intensity. Furthermore, realistic values of the scaling parameter $\hat{\delta}$ (maximum degree of linear polarization) are typically much less than unity, especially 
under overcast and partly cloudy conditions. Indeed, in the extreme case of high polarization, clear conditions at the Mauna Loa observatory, measurements of $\hat{\delta}$ in the mid visible spectrum are typically on the order of 0.60 to 0.75 (Dahlberg 2010). For these reasons, polarization effects can be justifiably ignored when computing the inscattering correction $\left(S_{1}\right)$ by forcing the polarization correction function to zero, as done in the manuscript. Importantly, through the bias-correction procedure outlined in Sections 4.5.2 and 4.5.2.1, any minor bias resulting from ignoring polarization is captured in the skyLOSA error model and now considered in the sky-LOSA algorithm.

\section{C.3.4 Intensity of Skylight Multiple-Scattered by Soot}

For the more general case of multiple soot-scattering events, the Stokes-Mueller formalism can be implemented with repeated matrix multiplications of the necessary rotation and Mueller matrices corresponding to each of $K$ scattering events. Letting $\theta_{k}$ represent the scattering angle of the $\mathrm{k}^{\text {th }}$ soot-scattering event, the Stokes vector for multiple sootscattered skylight at the detector is:

$$
\begin{aligned}
\boldsymbol{q}_{d} & \equiv \boldsymbol{q}_{K+1, K} \\
& =\mathbf{M}_{R D G}\left(\theta_{K} ; \boldsymbol{b}\right) \mathbf{R}\left(\gamma_{K+1, K-1}\right) \ldots \mathbf{M}_{R D G}\left(\theta_{2} ; \boldsymbol{b}\right) \mathbf{R}\left(\gamma_{3,1}\right) \mathbf{M}_{R D G}\left(\theta_{1} ; \boldsymbol{b}\right) \mathbf{R}\left(\gamma_{2,0}\right) \boldsymbol{q}_{1,0}
\end{aligned}
$$

where (as above), the first element of the Stokes vector $\boldsymbol{q}_{d}$ represents the intensity of multiple soot-scattered skylight at the detector.

\section{C.4 Reverse Monte Carlo "Ray Tracing” Procedure}

A detailed flowchart outlining the procedure of the present reverse Monte Carlo "ray tracing" (rMCRT) method is shown in Figure C.4. The position of the detector and reverse direction of light propagation for an optical axis are defined in Cartesian space $\left(\boldsymbol{r}_{k}\right.$ and $\boldsymbol{s}_{k}$ 
where the index $k$ is initially set to zero, "step 1"). For the specific position, direction, and soot concentration field, the transmittance of the plume $(\tau)$ is computed along the zeroindexed $\operatorname{LOS}\left(\tau_{0}\left(\boldsymbol{r}_{0}, \boldsymbol{s}_{0}\right)\right.$, “step 2"). Next, a MC packet/bundle of radiative energy, initiated from the detector, is propagated towards the plume along the optical axis, where a minimum of one attenuation event is forced to occur. The location of this first attenuation event is determined by selecting a random propagation transmittance, $\xi_{\tau} \sim$ $\mathcal{U}\left(\tau_{0}\left(\boldsymbol{r}_{0}, \boldsymbol{s}_{0}\right), 1\right)$, and finding the distance of travel, $x$, that satisfies (adapted from Modest (2013) and (Howell et al. (2016)):

$$
-\frac{\ln \xi_{\tau}}{\sigma_{e}^{m}}=\int_{0}^{x} \rho_{s}\left(\boldsymbol{r}_{0}+x^{\prime} \boldsymbol{s}_{0}\right) d x^{\prime}
$$

which represents the location of the first attenuation event, $\boldsymbol{r}_{1}=\boldsymbol{r}_{0}+x \boldsymbol{s}_{0}$ ("step 3") and does not require any assumptions on the spatial distribution of soot concentration. In forward MCRT, the intensity of the MC packet would be reduced to account for the absorption of light at the attenuation event; however, since the rMCRT method is used only to calculate representative ray paths, there is no need to consider energy conservation or polarization in the rMCRT procedure. Thus, the path is continued by obtaining a random scattering angle $\left(\xi_{\theta}\right)$ from the soot population's azimuthally uniform scattering phase function and a random scattering azimuth $\left(\xi_{\phi} \sim \mathcal{U}\left(0,360^{\circ}\right)\right.$, about $\left.\boldsymbol{s}_{0}\right)$ to yield the next propagation direction, $\boldsymbol{s}_{k+1}=\boldsymbol{s}_{1}$ ("step 4"). At this point the index $k$ is incremented and the plume transmittance for the updated position/direction is computed ("step 2"). A random propagation transmittance is again acquired but now from the standard uniform distribution, $\xi_{\tau} \sim \mathcal{U}(0,1)$, (i.e., not forcing the occurrence of an additional event) and the procedure is continued. This algorithmic loop is only exited when the ray path escapes the 
plume - i.e., if $\xi_{\tau}<\tau_{k}\left(\boldsymbol{r}_{k}, \boldsymbol{s}_{k}\right)$ - or a significantly large number of scattering events has occurred. This latter option to exit the loop is not shown in the figure but is justified through the notion that the remaining energy of a MC packet after $k$ scattering events is equivalent to $\omega(\boldsymbol{b})^{k}$, where $\omega(\boldsymbol{b})$ is quite small for soot (approximately $20 \%$ on average). In this work, a maximum of ten scattering events were permitted. The derived set of statistically representative paths are then fully defined by the computed position and direction vectors, which are stored for later analysis ("step 5"). 


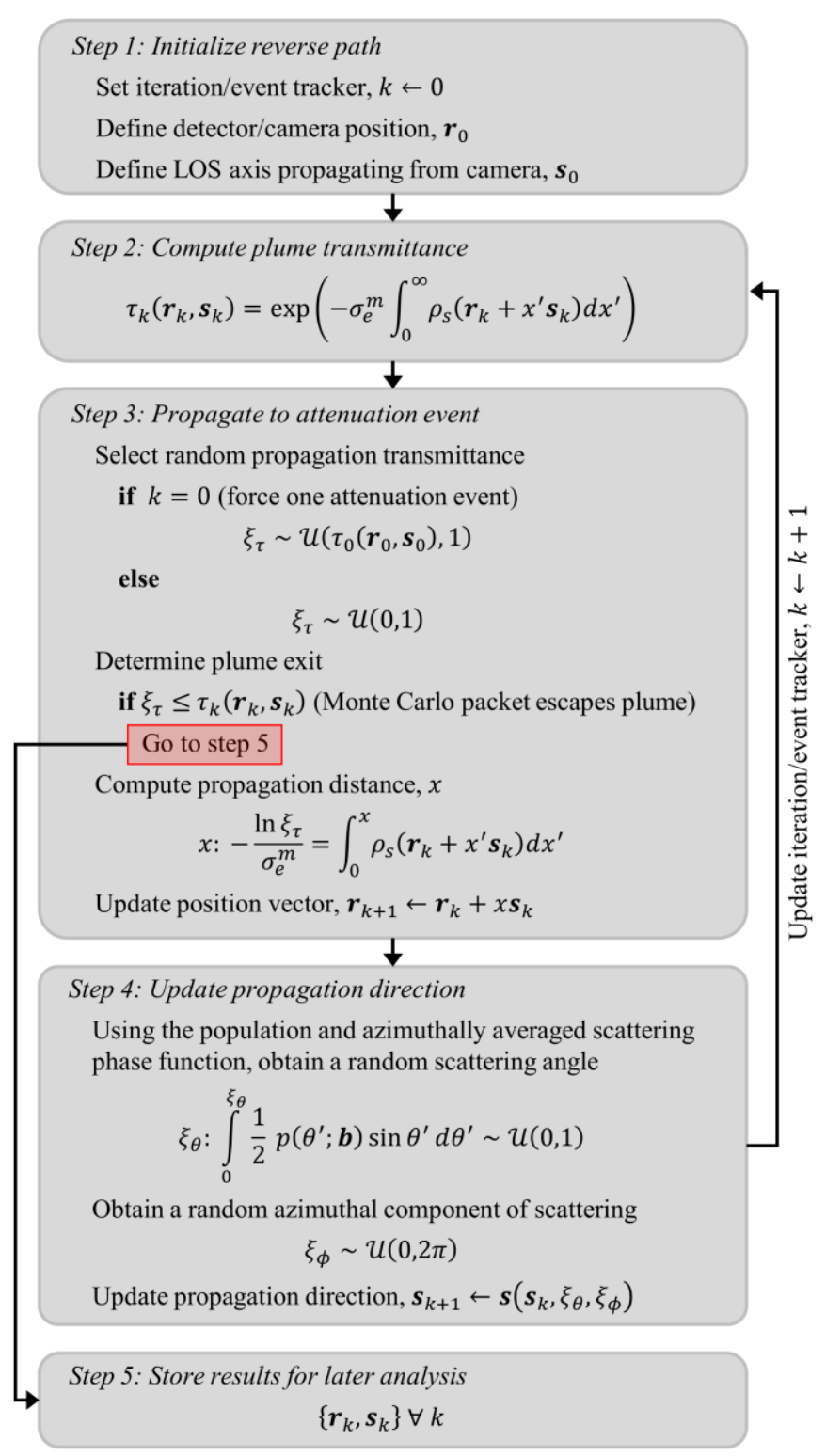

Figure C.4: Flowchart describing the reverse Monte Carlo "ray tracing" (rMCRT) procedure. For an a given detector position, line-of-sight, and plume, the rMCRT procedure provides a statistically representative set of paths that ambient light might travel into the detector.

\section{C.5 Forward Propagation of Light along rMCRT-Computed Paths}

To assess the simple MS model derived in the manuscript, a procedure entailing the forward propagation of sky- and sunlight along rMCRT-computed paths was required. As described in the manuscript, this procedure enabled the comparison of the model-estimated soot column density with the known ground truth for randomized ambient light conditions 
to ultimately derive a bias correction for the model and quantify random/precision error. The key output required from the forward propagation of light along the rMCRT-computed paths was the path-averaged source intensity under MS conditions $\left(\overline{I_{m}^{s}}\right)$, which permits calculation of the inscattering source term, $S_{m}$. The procedure for this forward propagation of light is summarized in this section.

For a given Monte Carlo (MC)-randomized solar position, solar radiation is propagated in the forward direction (from sun to detector) along the rMCRT pre-computed paths. To enable description of this technique, reverse paths are "reversed" again by reindexing each ray path as shown by example in Figure C.5. Re-indexing is performed for the $\mathrm{n}^{\text {th }}$ path such that sunlight and skylight travel along vectors $\boldsymbol{s}_{0}$ and $\boldsymbol{s}_{1}^{n}$, respectively. Scattering events are indexed $k \in\left\{1 \ldots K_{n}\right\}$ and are located at position vectors $\boldsymbol{r}_{k}^{n}$. Under these definitions, the propagation vector $\boldsymbol{s}_{k}^{n}$ culminates at the $\mathrm{k}^{\text {th }}$ soot-scattering event $\left(\boldsymbol{r}_{k}^{n}\right)$, and scattering angles at each event are $\theta_{k}^{n}=\cos ^{-1}\left(\boldsymbol{s}_{k}^{n} \cdot \boldsymbol{s}_{k+1}^{n}\right)$. 


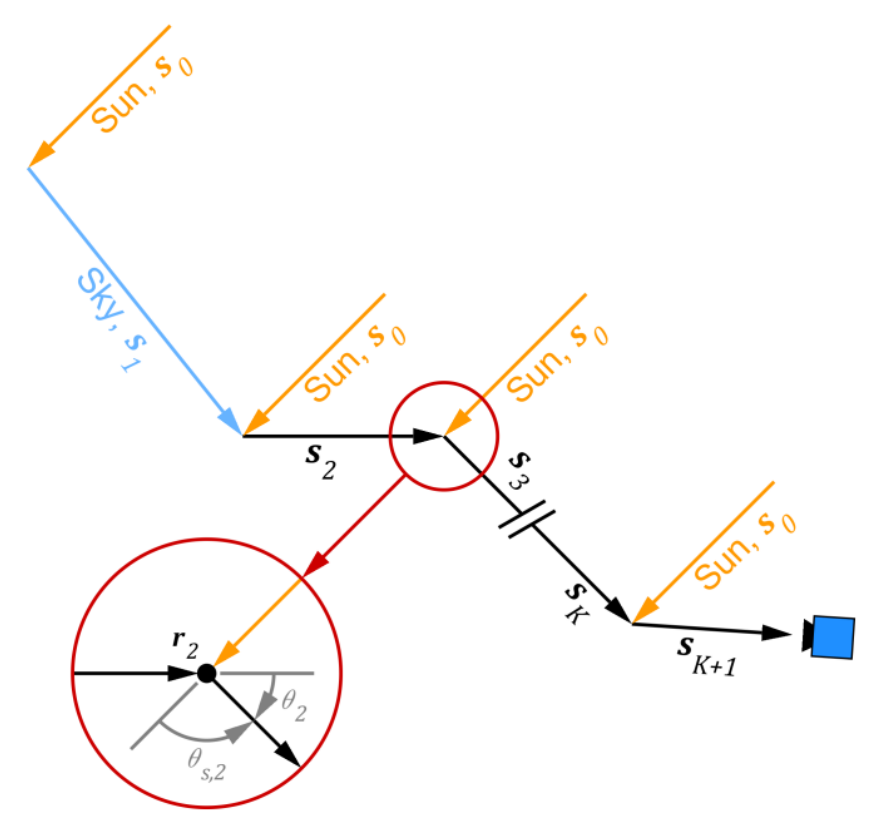

Figure C.5: Notation for the forward modelling of sunlight and skylight along a "reversed" rMCRT-computed path. Sub- and superscripts "n" are omitted for readability.

\section{C.5.1 Skylight Component}

For a prescribed skylight intensity distribution (such as the CIE skylight intensity models (CIE 2003) used in the present work), relative solar position, and MC packet path, the first task is the computation of the Stokes vector for diffuse skylight intensity propagating along $\boldsymbol{s}_{1}^{n}-$ which is denoted $\boldsymbol{q}_{1,0}^{n}$ according to Eq. (C.10), where $\chi=\cos ^{-1}\left(\boldsymbol{s}_{0} \cdot \boldsymbol{s}_{1}^{n}\right)$. A MC packet is initiated with intensity and polarization state according to $\boldsymbol{q}_{1,0}^{n}$ and is launched in the direction $\boldsymbol{s}_{1}^{n}$ towards $\boldsymbol{r}_{1}^{n}$. At the first scattering event $\left(\boldsymbol{r}_{1}^{n}\right)$, a rotation matrix $\left(\mathbf{R}\left(\gamma_{2,0}^{n}\right)\right)$ is applied to yield $\boldsymbol{q}_{1,2}^{n}$. Next, a normalized Mueller matrix $\left(\overline{\mathbf{M}}\left(\theta_{1}^{n}\right)\right)$ is applied and the intensity of the MC packet is deprecated by $\omega(\boldsymbol{b})$, to consider the absorption of a fraction of energy at the event. The outgoing Stokes vector is then:

$$
\boldsymbol{q}_{2,1}^{n}=\omega(\boldsymbol{b}) \overline{\mathbf{M}}\left(\theta_{1}^{n}\right) \mathbf{R}\left(\gamma_{2,0}^{n}\right) \boldsymbol{q}_{1,0}^{n}
$$


The normalized Mueller Matrix is employed since the probabilistic nature of scattering (defined by $p(\theta ; \boldsymbol{b}) / 4 \pi$ was inherently considered in the rMCRT-computation of statistically representative paths. Propagating through successive scattering events (with $K_{n}$ in total), the Stokes vector at the detector is obtained:

$$
\boldsymbol{q}_{K_{n}+1, K_{n}}^{n}=\omega(\boldsymbol{b})^{K_{n}} \overline{\mathbf{M}}\left(\theta_{K_{n}}^{n}\right) \mathbf{R}\left(\gamma_{K_{n}+1, K_{n}-1}^{n}\right) \ldots \overline{\mathbf{M}}\left(\theta_{1}^{n}\right) \mathbf{R}\left(\gamma_{2,0}^{n}\right) \boldsymbol{q}_{1,0}^{n}
$$

and the measured skylight source intensity for path " $\mathrm{n}$ " is:

$$
I_{s k y}^{s, n}=\boldsymbol{q}_{K_{n}+1, K_{n}(1)}^{n}
$$

where the subscript "(1)" indicates the first element of the Stokes vector. Finally, the pathaveraged source intensity is simply the average over all rMCRT-computed paths:

$$
\overline{I_{m, s k y}^{S}}=\frac{1}{N} \sum_{n=1}^{N} I_{s k y}^{s, n}
$$

\section{C.5.2 Sunlight Component}

The sunlight component of the inscattering source intensity has an additional challenge in its quantification since both the detector and the source are collimated. Consequently, paths generated in the rMCRT procedure are prohibitively unlikely to escape the plume in the exact direction of the sun and an alternative model of forward propagation is thus required. This work employs a procedure similar to the "local estimate" approach proposed by Marchuk et al. (1980) and summarized by Gratiy et al. (2010), adapted to consider the effect of polarization. The main tenet of this procedure is the notion that sunlight could theoretically enter a pre-computed path at any scattering event. That is, considering the example path shown in Figure C.5, sunlight is modelled as being able to enter the pre- 
computed path at the scattering events $j \in\left\{1 \ldots K_{n}\right\}$ and, for each of these possibilities, the Stokes-Mueller formalism permits quantification of the detector-measured intensity. Introducing the superscript " $\mathrm{j}$ " to indicate the scattering event at which sunlight enters the path, the Stokes vector at the detector is:

$$
\begin{aligned}
\boldsymbol{q}_{K_{n}+1, K_{n}}^{j, n}= & \omega(\boldsymbol{b})^{K_{n}-j} \overline{\mathbf{M}}\left(\theta_{K_{n}}^{n}\right) \mathbf{R}\left(\gamma_{K_{n}+1, K_{n}-1}^{n}\right) \ldots \\
& \ldots \overline{\mathbf{M}}\left(\theta_{j+2}^{n}\right) \mathbf{R}\left(\gamma_{j+3, j+1}^{n}\right) \overline{\mathbf{M}}\left(\theta_{j+1}^{n}\right) \mathbf{R}\left(\gamma_{j+1,0}^{n}\right) \boldsymbol{q}_{j+1,0}^{n}
\end{aligned}
$$

In this case, computation of the Stokes vector is quite similar to that of the sky component shown in Eq. (C.27); however, the deprecation of energy due to absorption considers a reduced number of scattering events (i.e., $\omega(\boldsymbol{b})^{K_{n}-j}$ rather than $\left.\omega(\boldsymbol{b})^{K_{n}}\right)$ and the initial Stokes vector is referenced to the $(j+1)^{\text {th }}$ direction (i.e., $\boldsymbol{q}_{j+1,0}^{n}$ rather than $\boldsymbol{q}_{1,0}^{n}$ ), hence referring to soot-scattered sunlight rather than sky-scattered sunlight. This initial Stokes vector is not modelled in the same manner as sky-scattered solar radiation (i.e., skylight Eq. (C.10)), but is instead quantified directly. The Stokes vector of solar radiation after scattering at the $\mathrm{j}^{\text {th }}$ scattering event is:

$$
\boldsymbol{q}_{j+1,0}^{n}=\mathbf{M}_{R D G}\left(\theta_{s, j}^{n} ; \boldsymbol{b}\right)\left(\tau\left(\boldsymbol{r}_{j}^{n},-\boldsymbol{s}_{0}\right) \boldsymbol{q}_{0, j+1}^{n}\right)
$$

where $\mathbf{M}_{\mathrm{RDG}}\left(\theta_{s, j}^{n} ; \boldsymbol{b}\right)$ is the Mueller matrix accounting for the scatter of solar radiation into the path at the $j^{\text {th }}$ scattering event, $\tau\left(\boldsymbol{r}_{j}^{n},-\boldsymbol{s}_{0}\right)$ is the transmittance of the plume between the $\mathrm{j}^{\text {th }}$ scattering event and the sun, and $\boldsymbol{q}_{0, j+1}^{n}$ is the Stokes vector of solar radiation incident upon the scattering event. Expanding Eq. (C.31): 


$$
\boldsymbol{q}_{j+1,0}^{n}=E_{S}\left(Z_{s} ; a\right) \tau\left(\boldsymbol{r}_{j}^{n},-\boldsymbol{s}_{0}\right) \frac{\omega(\boldsymbol{b})}{4 \pi} p\left(\theta_{s, j}^{n} ; \boldsymbol{b}\right)\left[\begin{array}{c}
1 \\
-\delta\left(\theta_{s, j}^{n}\right) \\
0 \\
0
\end{array}\right]
$$

The total detector-measured intensity of inscattered sunlight for the $\mathrm{n}^{\text {th }}$ path is obtained by summing the first component of the Stokes vector $\left(\boldsymbol{q}_{K_{n}+1, K_{n}}^{j, n}\right)$ over index $j$.

$$
I_{\text {sun }}^{s, n}=\sum_{j=1}^{K_{n}} \boldsymbol{q}_{K_{n}+1, K_{n}(1)}^{j, n}
$$

Finally, identically to the skylight component, the path-averaged source intensity for sunlight is simply the average over all rMCRT-computed paths:

$$
\overline{I_{m, \text { sun }}^{S}}=\frac{1}{N} \sum_{n=1}^{N} I_{\text {sun }}^{s, n}
$$

From which the total path-averaged source intensity is obtained:

$$
\overline{I_{m}^{S}}=\overline{I_{m, s k y}^{S}}+\overline{I_{m, s u n}^{S}}
$$

\section{C.6 Solution of Multiple Scattering-Corrected Transmittance Equation}

This section provides the analytical solution for the idealized transmittance $\left(\tau^{*}\right)$ via solution of Eq. (4.7):

$$
\tau^{*}=\frac{\tau_{o b s}-K_{m} S_{1}}{1-K_{m} S_{1}}
$$

where the present work derives a simple model for the MS correction parameter, $K_{m}=$ $\sqrt{\tau^{*}}$. This results in an implicit equation, that can be transformed to a cubic equation and solved using standard methods. 
The solution begins by introducing the MS model into Eq. (C.36) and defining $x \equiv$ $\sqrt{\tau^{*}}$, resulting in the following cubic equation:

$$
S_{1} x^{3}-x^{2}-S_{1} x+\tau_{o b s}=0
$$

for which the discriminant is non-negative for $\tau_{o b s} \in[0,1]$, such that there exist three real roots.

Disregarding the trivial case where $S_{1}=0$ (yielding $\tau^{*}=\tau_{o b s}$ ), the solution to this cubic can be obtained by first defining parameters:

$$
\begin{aligned}
& p=-\frac{1+3 S_{1}^{2}}{3 S_{1}^{2}} \\
& q=\frac{\tau_{o b s}}{S_{1}}-\frac{1}{3 S_{1}}-\frac{2}{27 S_{1}^{3}}
\end{aligned}
$$

Then, performing Vieta's substitution, the roots of Eq. (C.37) can be obtained in trigonometric form:

$$
x_{k}=2 \sqrt{-\frac{p}{3}} \cos \left[\frac{1}{3} \operatorname{acos}\left(\frac{3 q}{2 p} \sqrt{\frac{-3}{p}}\right)-k \frac{2 \pi}{3}\right]+\frac{1}{3 S_{1}}, \forall k \in\{0,1,2\}
$$

Finally, the root using $k=1$ satisfies the physical constraints that $\tau^{*} \in[0,1]$ and $\tau_{o b s} \geq$ $\tau^{*}:$

$$
\tau^{*}=\left(2 \sqrt{-\frac{p}{3}} \cos \left[\frac{1}{3} \operatorname{acos}\left(\frac{3 q}{2 p} \sqrt{\frac{-3}{p}}\right)-\frac{2 \pi}{3}\right]+\frac{1}{3 S_{1}}\right)^{2}
$$


The idealized transmittance using the simple MS and 1SA are plotted in Figure C.6a and $\mathrm{b}$ as a function of the observed transmittance for a range of 1SA-estimated inscattering source terms, $S_{1}$.
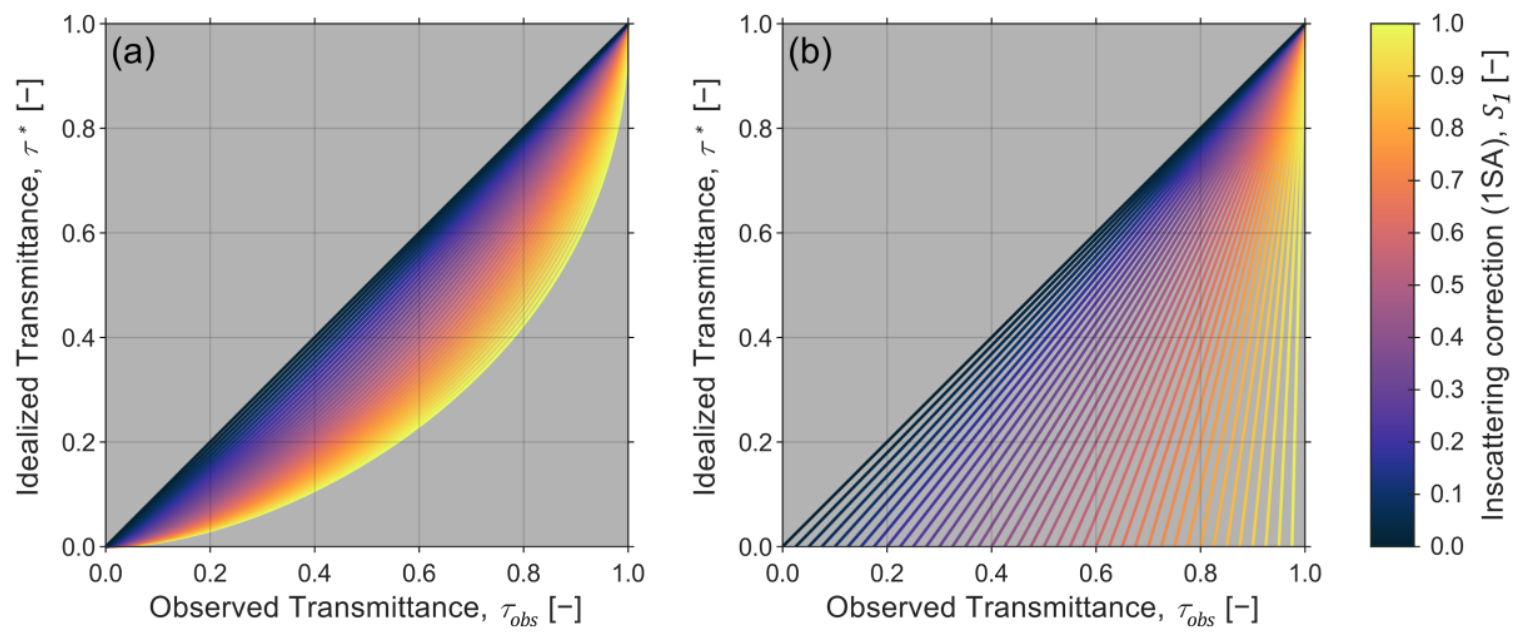

Figure C.6: (a) Idealized transmittance as a function of the observed transmittance for a range of 1SA-estimated inscattering source terms with MS correction using the present simple model $K_{m}=\left(\tau^{*}\right)^{1 / 2}$. (b) Same as (a) but assuming $K_{m}=1$ as in skyLOSA analyses to date (Chapter 2, Conrad and Johnson 2017; Johnson et al. 2013).

\section{C.7 Supplementary Data: Raw Errors in MS Correction Model}

Supplementary file "Raw_Bias_and_Precision_Errors.xlsx" contains the raw bias and precision error data for each CIE sky model as plotted in Figure 4.6a and Figure 4.6b. As discussed in Section 4.5.2.1, these data permit derivation of the bias-correction and precision error functions to consider residual errors when using the MS correction model with sky-LOSA. For the case study results summarized in Table 4.1, these data were fit using a LOESS local regression (implemented in MATLAB with a quadratic polynomial and span of 15\%) and used to calculate the bias correction and precision error for the chosen sky model and solar angle. 


\section{Appendix D Supplemental Information for Mass Absorption Cross-Section of Flare- Generated Black Carbon: Variability, Predictive Model, and Implications}

\section{D.1 Flaring Facility}

The present measurements were performed at the Carleton University Flare Facility (CUFF) in Ottawa, Canada. As detailed in the manuscript, this is a large-scale facility capable of creating flames of up to $\sim 3 \mathrm{~m}$ burning fuel mixtures of up to nine components including gas- and liquid-phase fuels. To provide context for the schematic of the CUFF (Figure 5.1) example photographs of the facility are provided in Figure D.1. 

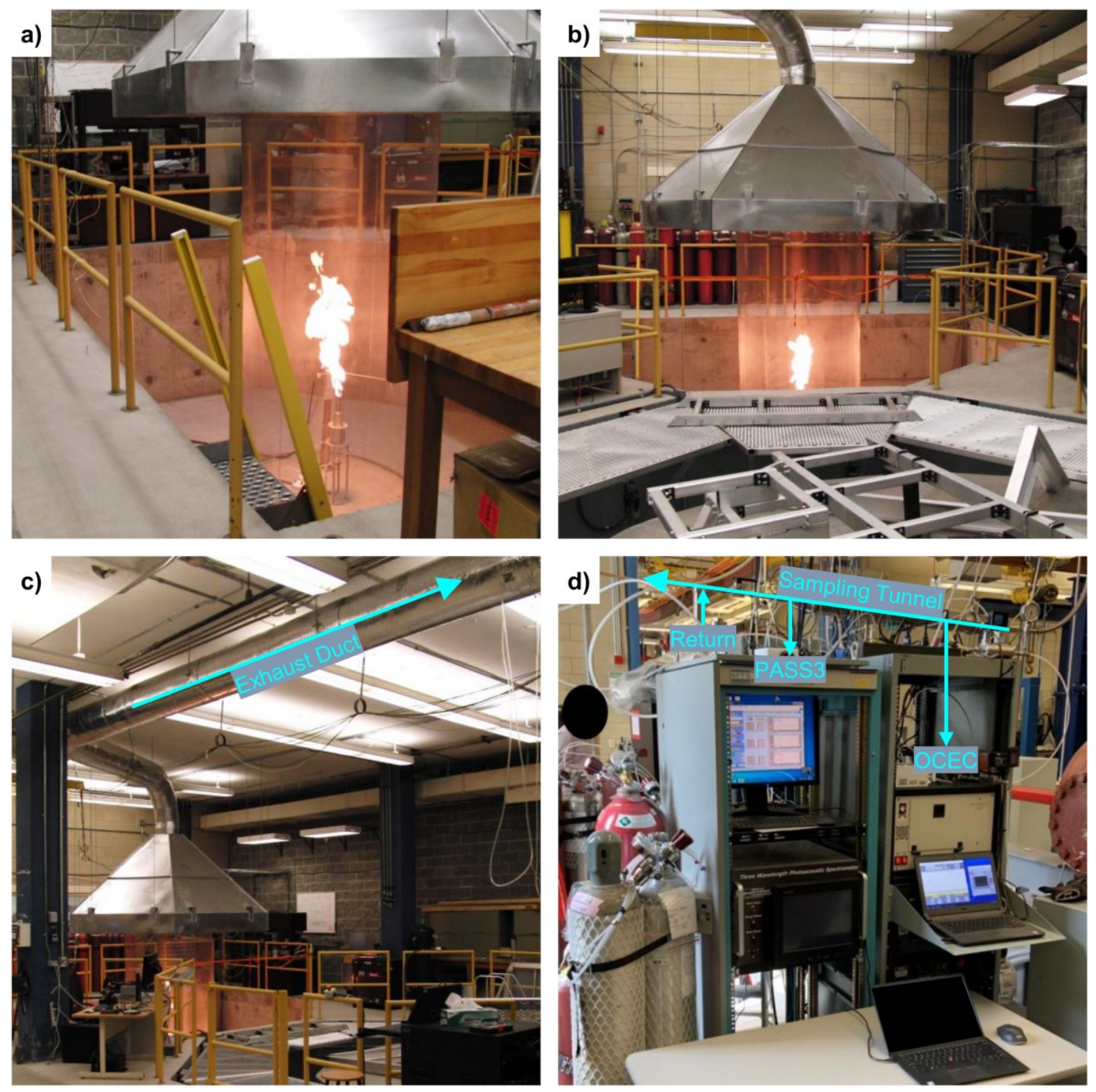

Figure D.1: Carleton University Flare Facility (CUFF). (a, b) An operational flare of $\sim 1.5 \mathrm{~m}$ in length located in the recessed flooring of the facility (outer concentric settling screen removed for visibility). (c) Broader perspective of the facility showing the $40 \mathrm{~cm}$ diameter insulated duct used to transfer emissions to the diagnostic instruments and exhaust combustion products from the laboratory.

(d) Instrumentation suite for the calculation BC MAC (PASS3 and OCEC instruments) showing the location of the sampling tunnel and a high-level plumbing schematic.

Figure D.1a and Figure D.1b show an operational flare of $\sim 1.5 \mathrm{~m}$ in length housed in a section of recessed floor in the laboratory (for visibility, one of the concentric settling screens has been removed). Visible on the right side of Figure D.1b is the control station 
for the facility and located behind the flare and large fume hood is the bank of pressurized cylinders for gas-phase fuels used to create the flare gas mixtures. Figure D.1c shows a broader perspective of the facility, including a portion of the $40 \mathrm{~cm}$ diameter insulated duct from which emissions are sampled by diagnostic equipment and ultimately exhausted from the laboratory. Lastly, shown in Figure D.1d are the instrument racks housing the required diagnostic instruments - the three-wavelength photoacoustic soot spectrometer (PASS3) and thermal-optical organic/elemental carbon analyzer (OCEC) - and showing the location of the dilution tunnel (refer to Figure 5.2) and high-level plumbing schematic.

\section{D.2 Light Absorption by Black Carbon Aggregates}

Combustion-generated black carbon (BC) particulate is composed of aggregates of nanoscale carbon spherules. BC aggregates are defined as being mass fractal-like in structure, which enables a power-law relation describing aggregate size and morphology

(e.g., Sorensen 2001):

$$
N=k_{f}\left(\frac{R_{g}}{d_{p}}\right)^{D_{f}}
$$

where $N$ is the number of spherical monomers in a BC aggregate [-], $k_{f}$ is the fractal prefactor [-], $R_{g}$ is the aggregate radius of gyration [m], $d_{p}$ is the diameter of the monodisperse monomers (or, primary particles) [m], and $D_{f}$ is the fractal (or, Hausdorff) dimension [-]. Within the flame, monomers aggregate together through a diffusion-limited process driven by three-dimensional Brownian motion, in which case a fractal dimension of order 1.8 is typically observed (Sorensen 2001). If the fractal dimension is less than approximately 2.0 (e.g., Berry and Percival 1986), the fractal aggregate is considered to be 
"open" in structure. This enables one of the key driving assumptions of Rayleigh-Debye-Gans optical theory for Fractal Aggregates (RDG-FA); that is, all monomers are subject to the same incident light - or, the effect of shadowing of one monomer by another is negligible. So long as necking between monomers - also known as sintering - is negligible (i.e., monomers are in point contact), the total absorption by a $\mathrm{BC}$ aggregate can be represented simply as the algebraic sum of the absorption by each (assumed identical) monomer. The challenge of quantifying light absorption by structurally complex aggregates is therefore reduced to the quantification of light absorption by the monodisperse, spherical monomers that comprise the aggregate.

An exact solution to the interaction of monochromatic light with spherical particles was first published by Gustav Mie (1908). Commonly called "Mie theory," the exact solution solves the vector wave equation in spherical coordinates with appropriate boundary conditions at the particle's centre and surface. The solution is beyond the scope of this document but is obtained through separation of variables yielding an infinite series in the radial (Ricatti-Bessel functions), polar (Legendre polynomials), and azimuthal (sine and cosine) coordinates (e.g., Bohren and Huffman 1983; Born et al. 1999; van de Hulst 1957). In the limit of a small sphere, the first term of the series is frequently sufficient for characterizing optical cross-sections. This truncated, small-sphere result is known as "Rayleigh theory," which is equivalent to representing the sphere as an electric dipole. To justify the use of Rayleigh theory, the external electric field (incident light) and internal electric field (within the particle) must be reasonably modelled as homogeneous (Mishchenko et al. 2002). The former constrains the physical size of the particle relative 
to the wavelength of incident light, and the latter constrains the penetration velocity into the particle (van de Hulst 1957); mathematically, the constraints are typically of the form:

$$
\begin{aligned}
x & \ll 1 \\
\left|m_{\lambda} x\right| & \ll 1
\end{aligned}
$$

where $x=\pi d_{p} / \lambda$ is the size parameter of the sphere [-] and $m_{\lambda}=\eta_{\lambda}+i \kappa_{\lambda}$ is the complex refractive index of the sphere (relative to the suspending medium) $[-]$.

For typical diagnostic wavelengths, observed monomer diameters of combustiongenerated BC aggregates generally fall within the Rayleigh regime. Thus, in RDG-FA, the Rayleigh-limit of Mie theory is employed when quantifying optical interactions of light with a monomer. For absorption, this yields:

$$
M A C_{B C, \lambda}=\frac{6 \pi}{\varrho_{B C}} \frac{E\left(m_{\lambda}\right)}{\lambda}=\frac{6 \pi}{\varrho_{B C}} \frac{1}{\lambda} \operatorname{Im}\left(\frac{m_{\lambda}^{2}-1}{m_{\lambda}^{2}+2}\right)
$$

where $\varrho_{B C}$ is the material density of BC $\left[\mathrm{g} \mathrm{m}^{-3}\right], E\left(m_{\lambda}\right)$ is the absorptive refractive index function [-] defined on the right side of Eq. (D.3), and $\lambda$ is the measurement wavelength [m]. If it is assumed that the index of refraction is constant over a given spectrum, then this equation suggests an inverse wavelength-dependence of BC massnormalized absorption cross-section (MAC) - given that the monomer is within the Rayleigh regime over the entire spectrum. In practice, it is not generally assumed that the complex refractive index is constant over a given spectrum. Furthermore, at the smallwavelength extreme of the climate-critical solar spectrum $(\sim 400 \mathrm{~nm})$, it is difficult to justify the use of Rayleigh theory for BC monomers as per Eq. (D.2). It is therefore common to apply a power-law fit to MAC data over some spectrum: 


$$
M A C_{B C, \lambda}=M A C_{B C, \lambda_{o}}\left(\frac{\lambda}{\lambda_{o}}\right)^{-A \AA E}
$$

where $\lambda_{o}$ is some reference wavelength [nm] and AÅE is the absorption Ångström exponent [-], which is close to unity for monomers within the Rayleigh regime.

Although the RDG-FA model has accuracy limitations that are well-documented in the literature (e.g., Farias et al. 1995, 1996; Liu and Snelling 2008), the RDG-FA framework can still be used to examine the effect of monomer size and refractive index on the magnitude and spectral sensitivity of $\mathrm{BC}$ aggregate absorption. If the following RDG-FA assumptions are assumed to be valid:

1) $\mathrm{BC}$ aggregates are comprised of monodisperse, spherical monomers;

2) the fractal dimension is such that monomer-monomer shadowing is negligible; and

3) monomers are in point contact,

the remaining assumption of RDG-FA that monomers fall within the Rayleigh regime can be removed, and Mie theory used instead. This approach provides insight into BC aggregate absorption magnitude and spectral sensitivity as the monomer size is varied beyond the Rayleigh limit.

As presented in Figure 5.7 and associated discussion, an analysis of $\mathrm{BC}$ absorption was performed under the above approach using a Monte Carlo (MC) method. The data demonstrated that total absorption and the spectral sensitivity of absorption are both sensitive to monomer diameter and the refractive index. In particular for a specific range of the complex refractive index where the inequality in Eq. (D.5) is satisfied, the 
observations of the present work suggest a positive correlation between the MAC scaling parameter and graphitization of the particle.

$$
3 \kappa_{\lambda}^{2} \leq 2-\eta_{\lambda}^{2}+2 \sqrt{\eta_{\lambda}^{4}+2 \eta_{\lambda}^{2}+4}
$$

Figure D.2 plots the summary refractive index data of light-absorbing carbon from Table 4 of Bond and Bergstrom (2006). Importantly, the data clearly show that typical refractive indices for light absorbing carbon satisfy the above inequality.
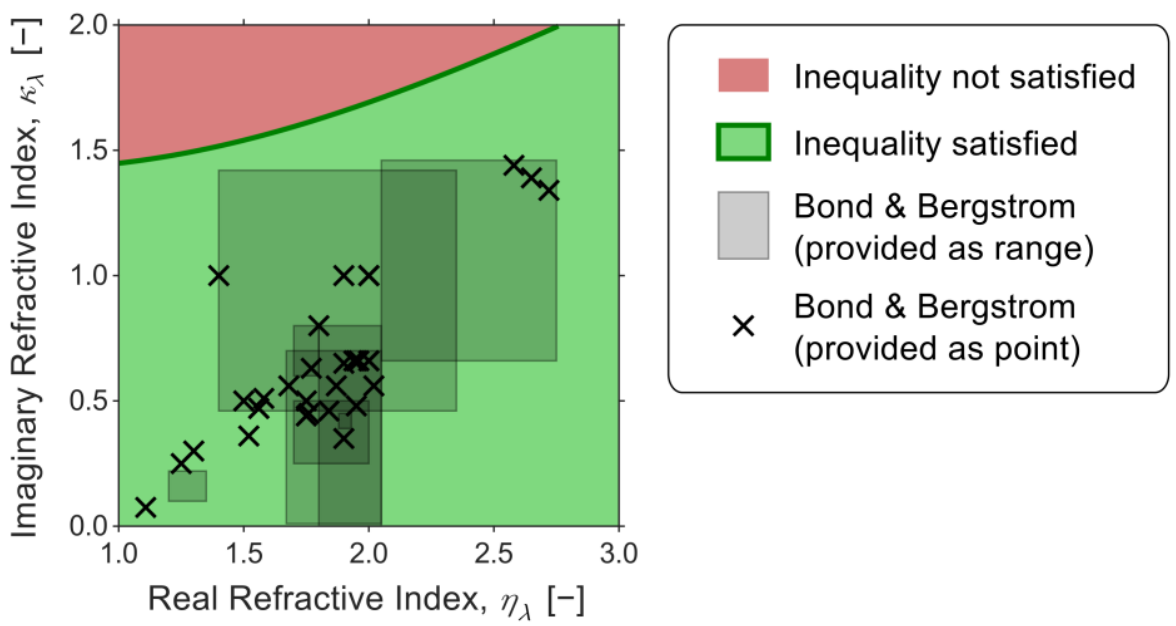

Figure D.2: Refractive indices of light-absorbing carbon (as summarized in Table 4 of Bond and Bergstrom (2006) with respect to the inequality in Eq. (D.5). When the inequality is satisfied (green region), an independent increase in the imaginary refractive index (consistent with graphitization) yields simultaneous increases in the absorption refractive index function and absolute refractive index, which is suggested to occur by the observations of the current study.

To the authors' knowledge, data or studies do not exist on the potential variation of $\mathrm{BC}$ material density with refractive index or degree of graphitization. Thus, the data in Figure 5.7a are plotted in terms of the volume-normalized cross-section. It is challenging to speculate the degree to which $\mathrm{BC}$ material density may change with graphitization and the corresponding transformation of $\mathrm{sp}^{3}$ - to $\mathrm{sp}^{2}$-bonded carbons. Considering the $\mathrm{sp}^{3}$ and $\mathrm{sp}^{2}$ limits of a carbon lattice (cubic diamond and hexagonal graphite), there may be 
opposing effects of graphitization on $\mathrm{BC}$ material density: $\mathrm{sp}^{2}$ bonds within the layers of hexagonal graphite are shorter $(0.141 \mathrm{~nm})$ than $\mathrm{sp}^{3}$ bonds in cubic diamond $(0.154 \mathrm{~nm})$ (Pierson 1993) and could be expected to increase density; while weak van der Waals bonding between the layers of hexagonal graphite yields large interlayer spacing $(0.335 \mathrm{~nm})$ that could be expected to decrease density. Further detailed study of this matter is beyond the scope of this work. Nonetheless, if cubic diamond (density $3.51 \mathrm{~g} / \mathrm{cm}^{3}$ (Pierson 1993)) and hexagonal graphite (density $2.26 \mathrm{~g} / \mathrm{cm}^{3}$ (Pierson 1993)) are considered as surrogates for incipient and highly-graphitized BC particles, respectively, it might be argued that density decreases with $\mathrm{BC}$ graphitization. If true, since $\mathrm{VAC}$ and $\mathrm{MAC}$ are linked by $\mathrm{BC}$ material density $\left(V A C=M A C \times \varrho_{B C}\right)$, the trend in $\mathrm{VAC}_{\mathrm{BC}, 550}$ in manuscript Figure 5.7a may underestimate the true variation in MAC with monomer diameter and refractive index.

Figure $5.7 \mathrm{~b}$ illustrates that the variation in absorption spectral sensitivity with index of refraction is diminished at small monomer diameters as Mie theory converges toward Rayleigh theory and the MC-calculated Å̊E approaches unity. In the phenomenological model presented in the manuscript however, the value of AÅ converges to approximately 0.93 at low scaling parameters, which is outside of the range of results in the MC analysis. To observe an Å̊E of less than unity, the absorption refractive index function must increase with decreasing wavelength. This behaviour cannot be captured by the employed $\mathrm{MC}$ as indices of refractions are assumed to be constant over the analysis spectrum. 


\section{D.3 Derivation of Reduced Flare Gas Compositions for Experimental Work}

The studied flare gas compositions were derived from globally diverse measured compositions of flare gas. Although available gas composition data for a few flares include highly-resolved saturated and unsaturated isomers (e.g., Chapter 2, Conrad and Johnson 2017), reported compositions in industry are generally crude in their resolution, where only inert species and saturated compounds (at times grouped by their number of carbon atoms) are reported (e.g., Curiale and Frolov 1998). Thus, with the exception of the experimental ethylene (X-C2) and propylene (X-CP) mixtures, flare gas compositions in the experiments were represented using alkane species that were derived as follows:

1) If the original reported composition included non-hydrocarbon combustibles (i.e., $\mathrm{H}_{2}$ and $\mathrm{H}_{2} \mathrm{~S}$, which had combined mole fractions of $<0.20 \%$ in all cases), these were added to the reported $\mathrm{CH}_{4}$ fraction.

$$
X_{C_{4}}+X_{H_{2}}+X_{H_{2} S} \mapsto X_{C_{4}}
$$

2) If the original reported composition included helium, this mole fraction $(<0.03 \%$ in all cases) was allocated in equal parts to $\mathrm{N}_{2}$ and $\mathrm{CO}_{2}$.

$$
\begin{aligned}
X_{N_{2}}+X_{\mathrm{He}} / 2 & \mapsto X_{N_{2}} \\
X_{\mathrm{CO}_{2}}+X_{\mathrm{He}} / 2 & \mapsto X_{\mathrm{CO}_{2}}
\end{aligned}
$$

3) If the original reported composition resolved alkane isomers, these were combined into the appropriate alkane group. For example:

$$
X_{i C 4}+X_{n C 4} \mapsto X_{C 4}
$$


4) If the original reported composition resolved alkanes beyond $\mathrm{C} 7, \mathrm{C} 7$ volume fraction was perturbed to match the higher heating value of the original reported composition.

5) Otherwise, if the original reported composition resolved alkanes to C6 only, the reported $\mathrm{C}^{+}$volume fraction was split into $\mathrm{C} 6$ and $\mathrm{C} 7$ based on the average ratio (denoted $K$ ) of $\mathrm{C} 6$ to $\mathrm{C} 6+\mathrm{C} 7$ observed in higher-resolution composition data $(K=$ 52.7\%). This approach yielded a marginal change in higher heating value but supplied a realistic quantity of C7 in the flare gas surrogate.

$$
\begin{gathered}
(1-K) X_{C 6} \mapsto X_{C 7} \\
K X_{C 6} \mapsto X_{C 6}
\end{gathered}
$$

Original reported flare gas compositions are listed in Table D.1 alongside the derived surrogates used at the CUFF. 
Table D.1: Original reported flare gas compositions and CUFF surrogates shown as volume fraction in \%. NR = not resolved by analysis or reported by source.

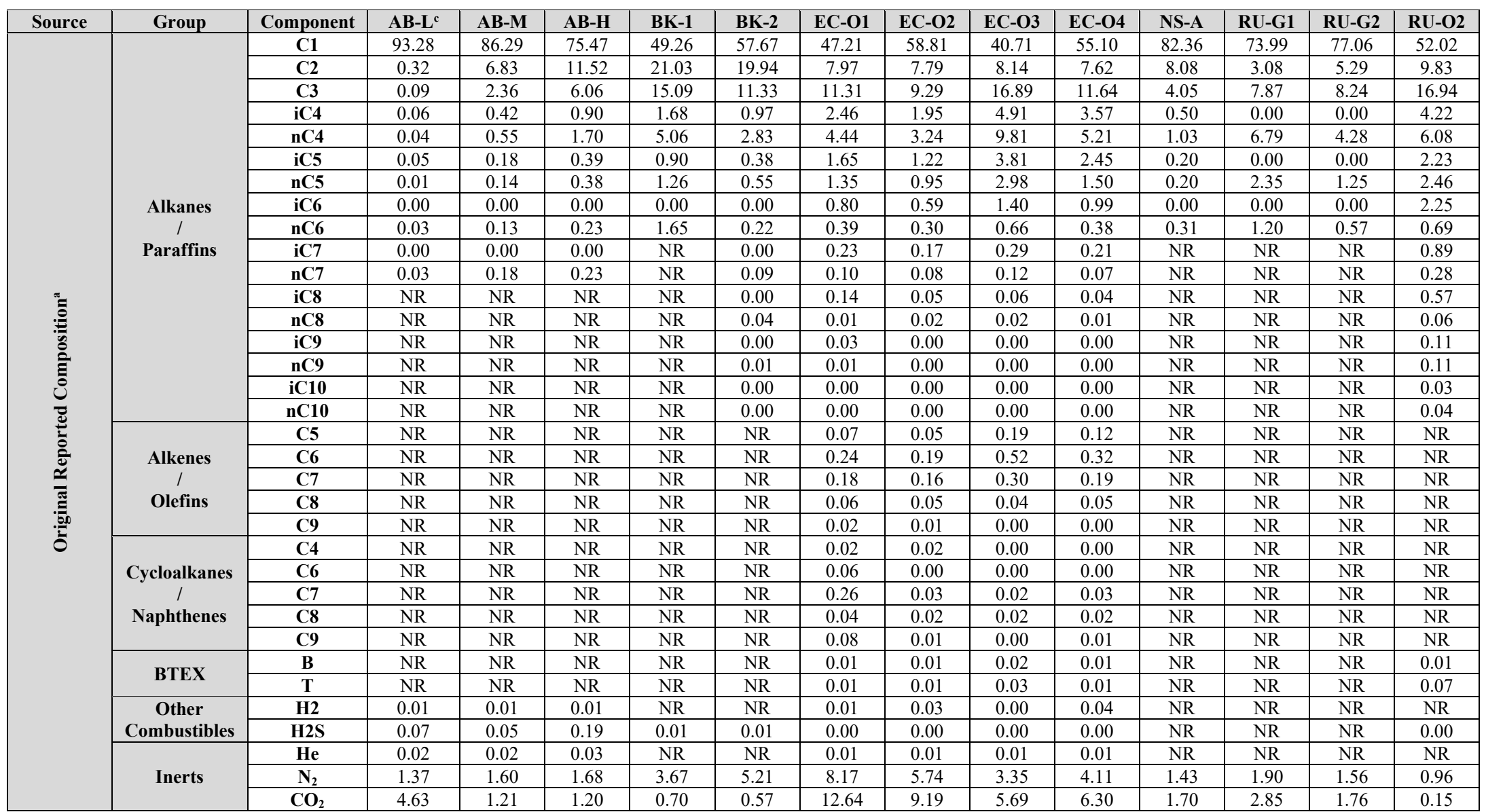


Table D.1 (continued): Original reported flare gas compositions and CUFF surrogates shown as volume fraction in \%. NR= not resolved by analysis or reported by source.

\begin{tabular}{|c|c|c|c|c|c|c|c|c|c|c|c|c|c|c|c|}
\hline \multirow{9}{*}{ 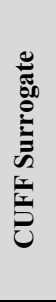 } & \multirow{7}{*}{$\begin{array}{c}\text { Alkanes } \\
/ \\
\text { Paraffins }^{\mathbf{b}}\end{array}$} & C1 & 92.39 & 86.36 & 75.68 & 49.12 & $\mathbf{5 7 . 7 8}$ & 47.26 & 58.89 & 40.82 & 55.22 & 82.48 & 73.96 & 77.05 & 51.90 \\
\hline & & $\mathrm{C} 2$ & 0.32 & \begin{tabular}{|l|}
6.83 \\
\end{tabular} & 11.52 & 20.97 & 19.97 & $\begin{array}{l}7.98 \\
\end{array}$ & $\begin{array}{l}7.80 \\
\end{array}$ & 8.16 & 7.63 & 8.09 & 3.08 & 5.29 & 9.81 \\
\hline & & C3 & 0.09 & 2.36 & 6.06 & 15.04 & 11.35 & 11.32 & 9.29 & 16.93 & 11.65 & 4.06 & 7.87 & 8.24 & 16.90 \\
\hline & & nC4 & 0.40 & 0.97 & 2.60 & 6.72 & 3.81 & 6.91 & 5.19 & 14.75 & 8.79 & 1.53 & 6.79 & 4.28 & 10.27 \\
\hline & & iC5 & 0.06 & \begin{tabular}{|l|l|}
0.33 \\
\end{tabular} & $\begin{array}{l}0.78 \\
\end{array}$ & 2.15 & 0.93 & 3.01 & 2.18 & 6.81 & 3.95 & 0.40 & 2.35 & 1.24 & 4.68 \\
\hline & & nC6 & $\mathbf{0 . 0 3}$ & 0.13 & 0.23 & 0.87 & 0.22 & 1.19 & 0.89 & 2.06 & 1.37 & 0.16 & 0.63 & 0.30 & 2.93 \\
\hline & & nC7 & $\begin{array}{l}0.03 \\
\end{array}$ & 0.18 & 0.21 & $\begin{array}{l}0.78 \\
\end{array}$ & 0.15 & 1.51 & $\begin{array}{l}0.81 \\
\end{array}$ & $\begin{array}{l}1.38 \\
\end{array}$ & 0.94 & 0.15 & 0.57 & 0.27 & 2.40 \\
\hline & \multirow{2}{*}{ Inerts } & $\mathbf{N}_{2}$ & 1.54 & 1.62 & 1.70 & 3.66 & 5.22 & 8.18 & 5.75 & 3.37 & 4.12 & 1.43 & 1.90 & 1.56 & 0.95 \\
\hline & & $\mathrm{CO}_{2}$ & 5.16 & 1.22 & 1.22 & $\begin{array}{l}0.70 \\
\end{array}$ & 0.57 & 12.65 & 9.20 & 5.71 & \begin{tabular}{|l|l|}
6.31 \\
\end{tabular} & 1.7 & 2.85 & 1.76 & 0.15 \\
\hline
\end{tabular}

${ }^{a}$ Additional components below detection limits are not shown.

${ }^{b}$ Specific isomers of C4-C7 alkanes selected based on availability.

Additional Butane ( $\mathrm{nC} 4)$ introduced due to experimental limitations. Further inerts $\left(\mathrm{N}_{2}\right.$ and $\left.\mathrm{CO}_{2}\right)$ added to achieve original heating value with added volumes removed from $\mathrm{C} 1$. 


\section{D.4 Consideration of Uncertainty via Monte Carlo Analysis}

\section{D.4.1 PASS3 Instrument Calibration}

The PASS3 instrument simultaneously measures absorption at three wavelengths (405, 532, and $870 \mathrm{~nm}$ ) using photoacoustic spectroscopy (e.g., Arnott et al. 1999). Briefly, to quantify absorption at a specified wavelength, a sample volume is illuminated by a laser modulated at a frequency near the acoustic resonant frequency of the cell containing the sample volume. Absorption of incident laser light by absorbing material (gases and aerosols) in the sample causes local heating and, hence, expansion of the gases within the cell. The resulting acoustic pressure wave is, by design, near the resonant frequency of the cell resulting in amplification of the acoustic signal, which is detected by a microphone. The absorption coefficient of the cell (in units of $\mathrm{m}^{-1}$ ) is quantified using the measured acoustic signal with knowledge of the dimensions and acoustic characteristics of the cell; gas (air) properties within the cell; and incident intensity (power) of laser light. Importantly, for instrument calibration and data reduction, the spectral absorption coefficient $\left(\alpha_{\lambda}\right)$ is directly related to the measured acoustic signal $\left(P_{a}\right)$ and inversely related to the incident spectral laser power $\left(P_{\lambda, L}\right)$.

$$
\alpha_{\lambda} \propto \frac{P_{a}}{P_{\lambda, L}}
$$

To measure absorption coefficient, the PASS3 instrument therefore includes two sets of components that are calibrated by the user:

1) Photodiodes that measure spectral laser powers after traversing the measurement cell. The calibration constants are in units of $\mathrm{mW} / \mathrm{V}$. 
2) A microphone that measures the acoustic pressure at the resonant frequency of the measurement cell, as necessary to compute the absorption coefficient. The calibration constant is in units of $\mathrm{mV} / \mathrm{Pa}$.

A schematic of the PASS3's optical cell is provided in Figure D.3 for reference in this section.

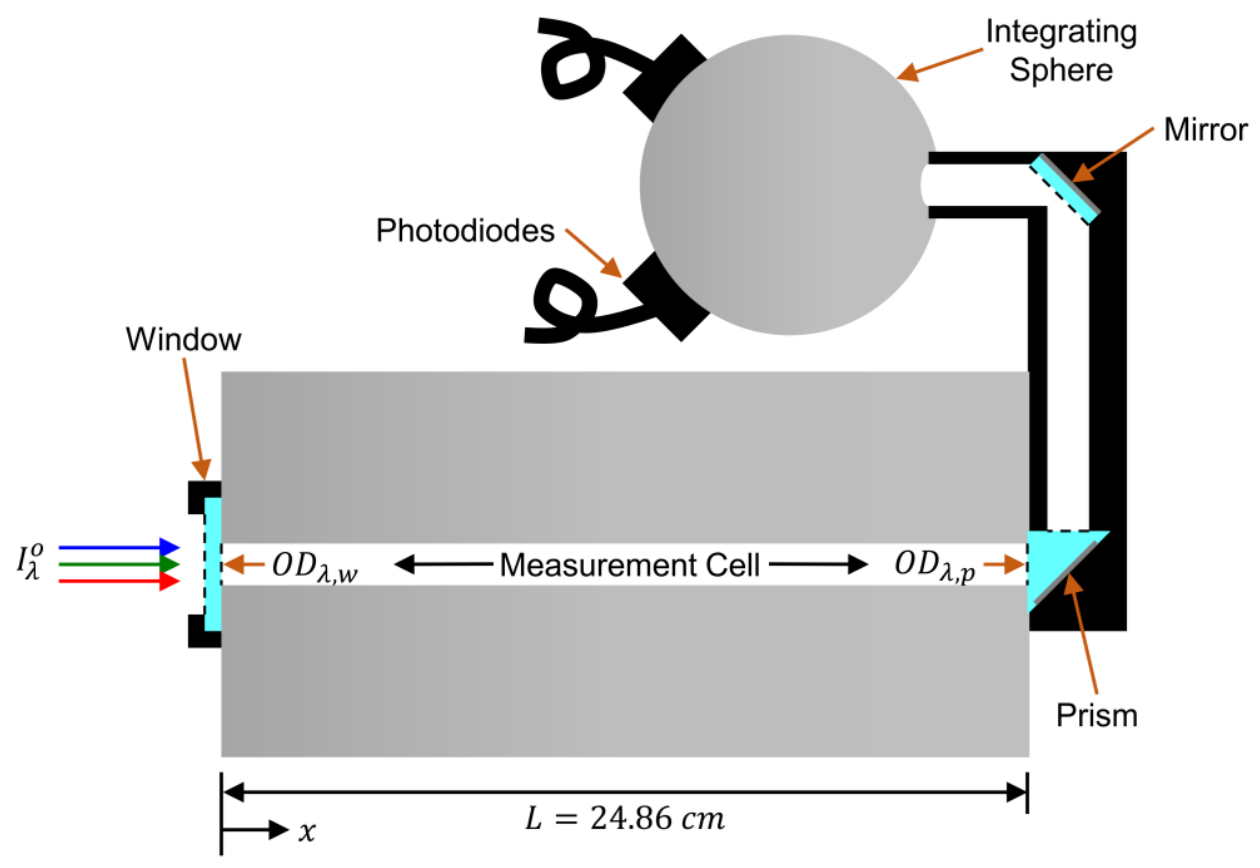

Figure D.3: Schematic of the optical path through the PASS3 measurement cell. Laser power is attenuated at the window, through the $\mathrm{BC}$-laden cell, and at the prism prior to detection by the photodiodes. Attenuation at the mirror and within the integrating sphere are constant over time and the photodiodes are calibrated considering this attenuation.

Calibration of the photodiodes first requires an external measurement of laser intensity / power. Optical components adjacent to the measurement cell are removed from the instrument and cleaned to remove residue from prior fouling of the optics. The optical window (optically upstream of the cell) is re-installed and an externally calibrated thermal laser power meter (TLPM, Ophir model 10A-P) is placed in the position of the prism (optically downstream of the cell). Each laser is independently energized for a fixed period 
(three minutes, to allow high-frequency startup transients to stabilize) and the measured laser power at the end of the period is logged. Next, the TLPM is removed, and the prism and photodiodes are reinstalled. Each laser is again independently energized for a fixed period that is $30 \mathrm{~s}$ longer than that used with the TLPM (three minutes and thirty seconds). Software-logged laser power from the last minute of the energized period yields statistics on the mean photodiode-measured power $\left(\overline{P_{\lambda, P D}}\right)$. Comparisons of the TLPM-measured and instrument-reported mean laser powers yield corrections to the calibration constants of the photodiodes.

The PASS3's microphone is calibrated using an external nitrogen dioxide $\left(\mathrm{NO}_{2}\right)$ standard, which is strongly absorbing at the measurement wavelength of $532 \mathrm{~nm}$. The $532 \mathrm{~nm}$ laser is energized and allowed to become stable, following which a zeroing procedure is performed while sampling filtered ambient air. Following zeroing, $\mathrm{NO}_{2}$ from a pressurized vessel of known concentration $\left(\mathrm{X}_{\mathrm{NO}_{2}}\right)$ is flowed through the measurement cell for a period of minutes. Throughout this time, acoustic zeros are performed, updating the resonance frequency of the cell as humidity in the cell reduces due to injection of the dry $\mathrm{NO}_{2}$ standard. Once the relative humidity in the cell decreases below a nominal threshold (taken as $2 \%$ ), with $\mathrm{NO}_{2}$ still flowing, instrument-reported data are logged for one minute yielding statistics on the mean absorption $\left(\overline{\alpha_{\lambda}}\right)$, cell pressure $(\bar{P})$, and cell temperature $(\bar{T})$. Comparison of observed absorption (corrected to standard conditions) with expected absorption by $\mathrm{NO}_{2}$ over the spectrum of the $532 \mathrm{~nm}$ laser yields a correction to the existing microphone calibration constant.

Uncertainty in the calibration of the PASS3 instrument is performed under a Monte Carlo (MC) framework. Table D.2 summarizes the MC input parameters contributing to 
calibration uncertainty, the modelled uncertainty of each parameter, and the method of implementation within the MC analysis.

Table D.2: Input parameter distributions for the Monte Carlo analysis of PASS3 calibration uncertainty. Also shown are the sources of the modelled distributions and the means of implementation within the Monte Carlo method.

\begin{tabular}{|c|c|c|c|c|}
\hline $\begin{array}{l}\text { Instrument } \\
\text { Component }\end{array}$ & $\begin{array}{l}\text { Uncertain } \\
\text { Parameter }\end{array}$ & $\begin{array}{c}\text { Modelled } \\
\text { Distribution }^{a}\end{array}$ & Source $^{b}$ & $\begin{array}{c}\text { Monte Carlo } \\
\text { Implementation }\end{array}$ \\
\hline \multirow{2}{*}{ Photodiodes } & $\begin{array}{l}\text { Laser power } \\
\text { meter }\end{array}$ & $\mathcal{N}(1,1.68 \%)[-]$ & Instrumentation & Relative bias \\
\hline & $\begin{array}{c}\text { Photodiode } \\
\text { measurement }\end{array}$ & $\overline{P_{\lambda, P D}} \sim \mathcal{N}\left(\bar{x}, s_{\bar{x}}\right)[\mathrm{W}]$ & CLT & $\begin{array}{l}\text { Measurement } \\
\text { data }\end{array}$ \\
\hline \multirow{7}{*}{ Microphone } & $\begin{array}{c}\mathrm{NO}_{2} \\
\text { concentration }\end{array}$ & $X_{\mathrm{NO}_{2}} \sim \mathcal{N}(470,4.7)[-]$ & $\begin{array}{c}\text { Reported } \\
\text { concentration }\end{array}$ & Absolute bias \\
\hline & $\begin{array}{l}\text { Measured } \\
\text { absorption }\end{array}$ & $\overline{\alpha_{\lambda}} \sim \mathcal{N}\left(\bar{x}, s_{\bar{x}}\right)\left[\mathrm{m}^{-1}\right]$ & CLT & $\begin{array}{c}\text { Measurement } \\
\text { data }\end{array}$ \\
\hline & $\begin{array}{l}\text { Cell pressure } \\
\text { measurement }\end{array}$ & $\bar{P} \sim \mathcal{N}\left(\bar{x}, s_{\bar{x}}\right)[\mathrm{Pa}]$ & CLT & $\begin{array}{c}\text { Measurement } \\
\text { data }\end{array}$ \\
\hline & $\begin{array}{c}\text { Cell pressure } \\
\text { device }\end{array}$ & $\mathcal{N}(1,0.125 \%)[-]$ & Instrumentation $^{\mathrm{c}}$ & Relative bias \\
\hline & $\begin{array}{c}\text { Cell temperature } \\
\text { measurement }\end{array}$ & $\bar{T} \sim \mathcal{N}\left(\bar{x}, s_{\bar{x}}\right)[\mathrm{K}]$ & CLT & $\begin{array}{c}\text { Measurement } \\
\text { data }\end{array}$ \\
\hline & \multirow{2}{*}{$\begin{array}{c}\text { Cell temperature } \\
\text { device }\end{array}$} & $\mathcal{N}\left(1, T_{\left({ }^{\circ} \mathrm{C}\right)} \times 0.1 \%\right)[-]$ & \multirow{2}{*}{ Instrumentation $^{\mathrm{d}}$} & Relative bias \\
\hline & & $\mathcal{N}(0,0.075)[\mathrm{K}]$ & & Absolute bias \\
\hline
\end{tabular}

Typical uncertainty in the calibration corrections and uncertainty due to repeatability of the calibrations are shown in Table D.3. Also shown in the table is the total calibration uncertainty for absorption measurements by the PASS3, which considers both calibration constant and repeatability errors summed in quadrature. 
Table D.3: Typical uncertainties in the calibration of the PASS3 photodiodes and microphone. Repeatability of the calibration procedures are shown alongside the total uncertainty for the PASS3 measurement of absorption.

\begin{tabular}{|c|c|c|c|c|c|}
\hline \multirow{2}{*}{$\begin{array}{c}\text { Wavelength, } \\
\lambda(\mathbf{n m})\end{array}$} & \multicolumn{2}{|c|}{ Photodiodes } & \multicolumn{2}{|c|}{ Microphone } & \multirow{2}{*}{$\begin{array}{c}\text { Total Absorption } \\
\text { Calibration } \\
\text { Uncertainty }\end{array}$} \\
\hline & $\begin{array}{c}\text { Calibration } \\
\text { Correction }\end{array}$ & Repeatability & $\begin{array}{l}\text { Calibration } \\
\text { Correction }\end{array}$ & Repeatability & \\
\hline 405 & \multirow{3}{*}{$3.35 \%$} & $1.81 \%$ & \multirow{3}{*}{$2.43 \%$} & \multirow{3}{*}{$0.71 \%$} & $4.57 \%$ \\
\hline 532 & & $2.05 \%$ & & & $4.68 \%$ \\
\hline 870 & & $0.86 \%$ & & & $4.29 \%$ \\
\hline
\end{tabular}

\section{D.4.1.1 Employed Calibrations}

The manufacturer of the PASS3 instrument (Droplet Measurement Technologies), suggests that calibrations be performed bi-monthly. It is unclear whether this recommendation implies twice-monthly or every two months; however, to ensure accurate calibration during experiments, the standard procedure at the Carleton University Flare Facility (CUFF) requires calibration whenever the instrument optics are removed and cleaned. For the present study, photodiode calibrations were performed daily during measurements. Microphone calibrations were performed during and immediately following the measurement campaign, and a distribution of these calibrations was used in the MC uncertainty calculations. It has been observed by CUFF operators that both the photodiode (if proper optics cleaning procedures are followed) and microphone calibration constants are quite constant in time and are highly repeatable (as summarized in Table D.3).

\section{D.4.2 PASS3 Data Reduction}

\section{D.4.2.1 Optical Fouling Correction}

Experience has shown that the optical components attached to the PASS3's measurement cell (i.e., the window and prism) can become fouled with particulate matter over the course of many experiments, such that the laser power measured by the instrument's photodiodes 
may not represent the instantaneous laser power within the measurement cell as required for the determination of absorption. Referring to the simplified schematic in Figure D.3, laser power (discussed here as laser intensity) is attenuated at the optical window, $x=0$; throughout the measurement cell due to sample absorption and scattering, $x \in(0, L)$; and at the prism, $x=L$. Attenuation at the angular mirror and within the integrating sphere are constant in time and are corrected using the above-described calibration of the photodiodes.

The laser spectral intensity $\left(I_{\lambda}\right)$ as a function of position within the measurement cell is:

$$
I_{\lambda}(x)=I_{\lambda}^{o} \exp \left(-O D_{\lambda, w} H(x)-\mu_{\lambda, L} x-O D_{\lambda, p} H(x-L)\right)
$$

where $I_{\lambda}^{o}$ is the incident spectral intensity, $\mu_{\lambda, L}$ is the homogeneous spectral extinction coefficient throughout the cell $\left[\mathrm{m}^{-1}\right], O D_{\lambda, i}$ is the spectral optical depth [-] of the window $(w)$ or prism $(p)$, and $H(x)$ represents the Heaviside (unit) step function. Thus, the pathaveraged laser spectral intensity subject on the measurement sample, and hence creating the photoacoustic signal, is:

$$
\bar{I}_{\lambda}^{c}=\frac{1}{L} \int_{0^{+}}^{L^{-}} I_{\lambda}(x) d x=\frac{I_{\lambda}^{o} \exp \left(-O D_{\lambda, w}\right)}{\mu_{\lambda, L} L}\left(1-\exp \left(-\mu_{\lambda, L} L\right)\right)
$$

For typical BC-loading, the extinction coefficient is sufficiently small such that $\exp \left(-\mu_{\lambda, L} L\right) \approx 1-\mu_{\lambda, L} L$ (to within $0.1 \%$ in the most heavily-loaded case), giving:

$$
\bar{I}_{\lambda}^{c}=I_{\lambda}^{o} \exp \left(-O D_{\lambda, w}\right)
$$

In the same manner (ignoring attenuation by the sample itself), the intensity at the photodiode detectors can be estimated as: 


$$
I_{\lambda, P D}^{c}=I_{\lambda}\left(L^{+}\right)=I_{\lambda}^{o} \exp \left(-\left(O D_{\lambda, w}+O D_{\lambda, p}\right)\right)
$$

Referring to Eq. (D.6), fouling of the optical prism implies an under-estimation of laser power within the cell and a corresponding over-estimation of absorption. A spectrally dependent, multiplicative, fouling correction parameter to the measured absorption coefficient $[-]$ can then be defined:

$$
K_{\lambda, F}=\frac{I_{\lambda, P D}^{c}}{\bar{I}_{\lambda}^{c}}=\exp \left(-O D_{\lambda, p}\right)
$$

Unfortunately, knowledge of $O D_{\lambda, p}$ is not readily available during experiments. However, it can be modelled based on the total fouling, which can be quantified with measurement data. Introducing the fraction of total fouling that occurs on the prism, $\zeta_{\lambda}=O D_{\lambda, p} / O D_{\lambda} \in$ $[0,1]$ where the total fouling $O D_{\lambda}=O D_{\lambda, w}+O D_{\lambda, p}=\ln \left(I_{\lambda}^{o} / I_{\lambda, P D}^{c}\right)$, provides an alternate form of the fouling correction parameter:

$$
K_{\lambda, F}=\exp \left(-\zeta_{\lambda} O D_{\lambda}\right)
$$

Ideally, if accurate knowledge of the instantaneous incident laser intensity were available, this could be coupled with the calibrated, measured intensity during an instrument zero to obtain total fouling. Unfortunately, emitted laser intensity drifts over time due to power supply variability as well as variations in the temperature of controlling electronics. Consequently, an alternative approach is needed to quantify total fouling.

Letting superscripts indicate a specific measurement on a given day (indexed from one) and subscripts "1" and "2" indicate the zeroing period before and after that measurement, operational procedures at the CUFF enable three key observations: 
1) PASS3 optical components are cleaned and the instrument is calibrated daily, suggesting that total fouling is nominally zero (or, at a minimum, corrected by calibration) at the beginning of the day's first measurement:

$$
O D_{\lambda, 1}^{1}=0
$$

2) Between measurements, the instrument is forced to sample filtered shop air, suggesting that total fouling at the end of measurement $n$ is equal to total fouling at the beginning of measurement $n+1$ :

$$
O D_{\lambda, 2}^{n}=O D_{\lambda, 1}^{n+1}
$$

3) Laser drift occurs at timescales much longer than measurement periods, such that the change in total fouling during measurement $n$ can be obtained from measured laser power during pre- and post-measurement zeroing periods:

$$
O D_{\lambda, 2}^{n}=O D_{\lambda, 1}^{n}+\ln \left(\frac{I_{\lambda, P D, 1}^{c, n}}{I_{\lambda, P D, 2}^{c, n}}\right)
$$

Error and uncertainty due to optical fouling are quantified under a MC framework. For each MC draw, random estimates of $O D_{\lambda, 1}^{n}$ and $O D_{\lambda, 2}^{n}$ are obtained from distributions based on pre- and post-measurement zeroing data. Linear interpolation in time (assuming the rate of fouling is constant during a measurement) yields a $\mathrm{MC}$-estimate of $O D_{\lambda}$ at any time during the measurement. By conservatively modelling the fraction of total fouling at the prism $\left(\zeta_{\lambda}\right)$ with a standard uniform distribution $(\mathcal{U}(0,1))$, a MC-estimated and timeresolved fouling correction parameter can be obtained. 


\section{D.4.2.2 Monte Carlo Computation of Absorption Coefficients}

Raw PASS3-measured absorption data are reported in local conditions (absorption crosssection per unit local volume), which can be corrected to standard conditions with temperature and pressure data provided by the instrument. Like the calibration of the PASS3 instrument, spectral absorption coefficients in standard volume units were computed using a MC analysis. Table D.4 summarizes the MC input parameters contributing to absorption uncertainty, the modelled uncertainty of each parameter, and the method of implementation within the MC analysis. Typical uncertainties in the computation of spectral absorption coefficients at standard conditions are shown in Table D.5.

Table D.4: Input parameter distributions for the Monte Carlo analysis of PASS3 calibration uncertainty. Also shown are the sources of the modelled distributions and the means of implementation within the Monte Carlo method.

\begin{tabular}{|c|c|c|c|c|}
\hline & $\begin{array}{l}\text { Uncertain } \\
\text { Parameter }\end{array}$ & $\begin{array}{c}\text { Modelled } \\
\text { Distribution }^{\mathrm{a}}\end{array}$ & Source $^{b}$ & $\begin{array}{c}\text { Monte Carlo } \\
\text { Implementation }\end{array}$ \\
\hline \multirow{3}{*}{$\begin{array}{l}\text { Optical } \\
\text { Fouling }\end{array}$} & $\begin{array}{l}\text { Pre-measurement } \\
\text { total fouling }\end{array}$ & $\overline{O D_{\lambda, 1}^{n}} \sim \mathcal{N}\left(\bar{x}, s_{\bar{x}}\right)[-]$ & CLT & $\begin{array}{l}\text { Measurement } \\
\text { data }\end{array}$ \\
\hline & $\begin{array}{l}\text { Post-measurement } \\
\text { total fouling }\end{array}$ & $\overline{O D_{\lambda, 2}^{n}} \sim \mathcal{N}\left(\bar{x}, s_{\bar{x}}\right)[-]$ & CLT & $\begin{array}{l}\text { Measurement } \\
\text { data }\end{array}$ \\
\hline & $\begin{array}{l}\text { Prism fouling } \\
\text { fraction }\end{array}$ & $\zeta_{\lambda} \sim \mathcal{U}(0,1)[-]$ & CLT & Randomized \\
\hline \multirow{6}{*}{ Microphone } & $\begin{array}{l}\text { Measured } \\
\text { absorption }\end{array}$ & $\overline{\alpha_{\lambda}} \sim \mathcal{N}\left(\bar{x}, s_{\bar{x}}\right)\left[\mathrm{m}^{-1}\right]$ & CLT & $\begin{array}{c}\text { Measurement } \\
\text { data }\end{array}$ \\
\hline & $\begin{array}{l}\text { Cell pressure } \\
\text { measurement }\end{array}$ & $\bar{P} \sim \mathcal{N}\left(\bar{x}, s_{\bar{x}}\right)[\mathrm{Pa}]$ & CLT & $\begin{array}{c}\text { Measurement } \\
\text { data }\end{array}$ \\
\hline & $\begin{array}{c}\text { Cell pressure } \\
\text { device }\end{array}$ & $\mathcal{N}(1,0.125 \%)[-]$ & Instrumentation $^{\mathrm{c}}$ & Relative bias \\
\hline & $\begin{array}{c}\text { Cell temperature } \\
\text { measurement }\end{array}$ & $\bar{T} \sim \mathcal{N}\left(\bar{x}, s_{\bar{x}}\right)[\mathrm{K}]$ & CLT & $\begin{array}{c}\text { Measurement } \\
\text { data } \\
\end{array}$ \\
\hline & \multirow{2}{*}{$\begin{array}{l}\text { Cell temperature } \\
\text { device }\end{array}$} & $\mathcal{N}\left(1, T_{\left[{ }^{\circ} \mathrm{C}\right]} \times 0.1 \%\right)[-]$ & \multirow{2}{*}{ Instrumentation $^{\mathrm{d}}$} & Relative bias \\
\hline & & $\mathcal{N}(0,0.075)[\mathrm{K}]$ & & Absolute bias \\
\hline
\end{tabular}


Table D.5: Typical uncertainties in the PASS3-measurement of spectral absorption coefficient at standard conditions.

\begin{tabular}{|c|c|c|c|}
\hline $\begin{array}{c}\text { Wavelength, } \\
\lambda[\mathbf{n m}]\end{array}$ & $\begin{array}{c}\text { Calibration } \\
\text { Uncertainty }\end{array}$ & $\begin{array}{c}\text { Measurement } \\
\text { Uncertainty } \\
\text { (Maximum) }\end{array}$ & $\begin{array}{c}\text { Total Absorption } \\
\text { Uncertainty } \\
\text { (Maximum) }\end{array}$ \\
\hline 405 & $4.57 \%$ & $0.62 \%$ & $4.62 \%$ \\
\hline 532 & $4.68 \%$ & $3.34 \%$ & $5.75 \%$ \\
\hline 870 & $4.29 \%$ & $0.76 \%$ & $4.36 \%$ \\
\hline
\end{tabular}

\section{D.4.3 OCEC Instrument Calibration}

Calibration of the thermal-optical organic/elemental carbon analyzer (OCEC) is summarized in a separate manuscript (Conrad and Johnson 2019b) but key relevant concepts pertaining to the procedure and computation of uncertainties are summarized here.

The OCEC instrument quantifies organic carbon (OC) and elemental carbon (EC) in a sample volume of particle-laden gas. During the experiment, a sample volume is pulled through the instrument, where particulate matter is deposited onto, and gas-phase organics are adsorbed by, a quartz filter at the entrance of the analysis oven. Following acquisition of the sample, the instrument is purged, and the analysis phase begins. The quartz filter is first heated through a prescribed temperature protocol in an inert helium atmosphere (He-phase). During the heating procedure, a fraction of the carbonaceous species is pyrolyzed from the filter. The gaseous exhaust is forwarded to a Manganese Oxide $\left(\mathrm{MnO}_{2}\right)$ oven that converts pyrolyzed species to carbon dioxide, the concentration of which is in turn measured by a non-dispersive infrared (NDIR) detector. Following the He-phase, the sample is again heated through a prescribed temperature protocol in an oxidizing atmosphere (Ox-phase). In the presence of oxygen, refractive carbonaceous species remaining on the quartz filter are oxidized and then forwarded through the $\mathrm{MnO}_{2}$ 
oven and NDIR detector in the same manner. At the termination of the Ox-phase, a "calibration loop" is executed. This final phase entails the injection of a known (fixed) quantity of carbon in the form of a prescribed methane-helium mixture $\left(\mathrm{CH}_{4}\right.$-phase). The injected mixture is oxidized in the $\mathrm{MnO}_{2}$ oven and the resulting carbon dioxide is measured by the NDIR detector as in the He- and Ox-phases.

The $\mathrm{CH}_{4}$-phase of the OCEC analysis is employed as a drift correction to the NDIR detector or, put differently, acts as a pseudo-calibration of the NDIR detector's response to $\mathrm{CO}_{2}$. During the $\mathrm{CH}_{4}$-phase, the integrated NDIR signal (in digital units) corresponds to the total mass of injected carbon in the methane-nitrogen mixture, yielding a digital unitto-carbon mass calibration factor. This calibration factor - quantified at the termination of each OCEC analysis - is used to correlate the integrated NDIR signal during the He- and Ox-phases with evolved carbon mass. Importantly, beyond the natural drift and uncertainty of the NDIR detector's response, the injected carbon mass during the $\mathrm{CH}_{4}$-loop is uncertain and must be calibrated itself.

Calibration/quantification of injected carbon mass during the $\mathrm{CH}_{4}$-loop is accomplished using an external sucrose standard. A precisely known mass of carbon is introduced to the OCEC instrument by deposition of a prescribed volume of an aqueous sucrose solution onto the quartz filter, using a calibrated pipette. Subsequent implementation of the above described analysis protocol yields a single datum of: 1) known carbon mass in sucrose, 2) integrated NDIR signal from sucrose evolution, and 3) integrated NDIR signal from $\mathrm{CH}_{4}$-loop. Repetition of this sucrose procedure with different carbon masses yields sufficient data to perform a linear regression of carbon mass in sucrose (1, above) vs. integrated NDIR signal from sucrose evolution (2, above). Using 
this regression, the carbon mass corresponding to the mean of the integrated NDIR signals from all the $\mathrm{CH}_{4}$-loops ( 3 , above) yields an estimate of the carbon mass that is introduced during the $\mathrm{CH}_{4}$-loop.

Calibration uncertainties are rigorously computed using a MC method (Conrad and Johnson 2019b). Listed below are the seven sources of error that propagate through the calibration procedure:

- Mass fraction of sucrose in the aqueous sucrose solution:

1) Uncertainty in mass of distilled water in solution (scale bias)

2) Uncertainty in mass of sucrose in solution (scale bias)

- Partial density of carbon in the aqueous sucrose solution:

3) Uncertainty in temperature of sucrose solution (modelled ambient temperature)

- Volume of sucrose solution introduced into the instrument:

4) Uncertainty in deposited volume via reported pipette bias and precision

5) Human pipetting error (inter-operator bias error)

6) Human pipetting error (intra-operator precision error)

- NDIR detector response:

7) Bias in NDIR signal

Repeatability of the calibration procedure was estimated using the coefficient of variance of 10 calibrations performed over a 12-day period in February 2018 - this is considered a conservatively high estimate of repeatability since the underlying calibration 
might not be expected to be constant over 12 consecutive days. Total calibration uncertainty is then calculated as the sum-in-quadrature of a specific calibration (via MC consideration of the above sources of error) and this measurement of repeatability. Typical calibration uncertainties for the OCEC instrument during the measurement campaign are presented in Table D.6.

Table D.6: Typical uncertainty in the calibration of the OCEC instrument.

\begin{tabular}{|c|c|c|}
\hline $\begin{array}{c}\text { Calibration } \\
\text { Constant }\end{array}$ & Repeatability & $\begin{array}{c}\text { Total Calibration } \\
\text { Uncertainty }\end{array}$ \\
\hline $2.47 \%$ & $7.90 \%$ & $8.28 \%$ \\
\hline
\end{tabular}

\section{D.4.4 OCEC Data Reduction}

Quantification of evolved OC and EC from the OCEC instrument requires a detailed examination of analysis thermograms, which contain time-resolved NDIR and oven (filter) temperature data among other useful data during the analysis. The procedure employed in the current work is summarized elsewhere (Conrad and Johnson 2019b) but key relevant concepts pertaining to the procedure, interpretation of the results, and computation of uncertainties are summarized here.

When OC pyrolyzes during the He-phase of the temperature protocol, a portion of it chars on rather than being released from the filter, oxidized in the $\mathrm{MnO}_{2}$ oven, and detected by the NDIR. In theory, this charred refractory OC (termed pyrolyzed carbon, PC) remains on the filter until the Ox-phase, and is thus oxidized at the same time as EC. Consequently, labelling all evolved carbon during the He-phase as OC and evolved carbon during the Ox-phase as EC tends to incorrectly estimate the true fraction (or, split) of OC vs. EC. Conversely, premature evolution of $\mathrm{PC}$ and $\mathrm{EC}$ during the He-phase is a common 
occurrence in OCEC instruments, typically resulting from use of a high peak He-phase temperature (present in the employed NIOSH (National Institute of Occupational Safety and Health)-5040 protocol (e.g., Subramanian et al. 2006) and/or the presence of metal oxides in the sample (e.g., Chow et al. 2001; Fung 1990). Due to this ambiguity in the OCEC data, a critical factor in the interpretation of OCEC results is the determination of the split point between OC and EC. In this specific work, uncertainty in the location of the split point is also important to the uncertainty of measured EC mass and, hence, MAC.

The Sunset Laboratories semi-continuous OCEC instrument employed at the CUFF uses an optical transmission approach to aid determination of the split point. A $632.8 \mathrm{~nm}$ laser is shined through the quartz filter during the thermal analysis and its power following attenuation by the filter is detected by a photodetector. By assuming that $\mathrm{OC}$ is not optically active at the wavelength (i.e., OC negligibly absorbs), and PC shares the same optical properties as EC, the split point can be estimated. The premise is to first measure the attenuated laser power at the start of the analysis. As OC evolves (partly into PC), the attenuation of the laser is expected to be enhanced such that the photodetector signal drops. As the Ox-phase is entered and EC/PC are co-evolved, attenuation is reduced, and the photodetector signal begins to increase. The split point is then defined as the instance in time when the measured laser power returns to its initial value. While straightforward in concept, implementation of this approach is subject to several challenges:

1) Incident laser power is affected by oven temperature. Accordingly, laser attenuation must be corrected for the instantaneous, instrument-measured oven temperature.

2) $\mathrm{OC}$ that is internally-mixed with $\mathrm{EC}$ is effectively absorbing as it enhances absorption by the EC core (e.g., Andreae and Gelencsér 2006). Therefore, if 
evolved OC were originally internally mixed, an overall reduction in attenuation could be observed as OC evolves, despite the occurrence of charring.

3) The notion that PC and EC are optically equivalent has not, to the authors' knowledge, been tested for the studied combustion conditions; as such, this assumption may be invalid.

Consequently, it is important to note that OCEC-measured carbon masses, EC and OC, are "operationally-defined" in that they are dependent on the specific analysis protocol employed and procedure used to determine the split point (Nicolosi et al. 2018; Petzold et al. 2013a; Subramanian et al. 2010).

\section{D.4.4.1 Split Point}

In this work, the split point was determined by examination of the laser attenuation and evolved carbon via attenuation vs. evolved carbon (AVEC) plots (Nicolosi et al. 2018). These plots are invaluable as they provide a clear representation of phenomena during the OCEC analysis relevant to determination of the split point.

With computed laser attenuation and cumulative evolved carbon mass data, AVEC plots for the analyses in this study were created (Conrad and Johnson 2019b). Shown in Figure D.4 is an example AVEC plot from a measurement in the current work, specifically for the analysis of the BK-2 fuel combusted from the $50.8 \mathrm{~mm}$ burner at 156 SLPM. Cumulative evolved carbon (per quartz filter area) is on the horizontal axis and the concurrent measurement of temperature-corrected laser attenuation is on the vertical axis. Each point in the figure corresponds to a moment in time (reported by the instrument at $1 \mathrm{~Hz}$ frequency) with the colour of the point representing the measured temperature at the front oven (near the quartz filter). It is important to note that neither axis is linear with 
analysis time, as evolution of carbon from the filter is an unsteady process. Furthermore, it should be noted that there is a delay in the NDIR signal relative to that of the laser, as evolved carbon must transit the $\mathrm{MnO}_{2}$ oven before reaching the NDIR detector - this transit time was measured by the manufacturer as $5 \mathrm{~s}$ for the OCEC instrument and is accounted for in the AVEC plot.

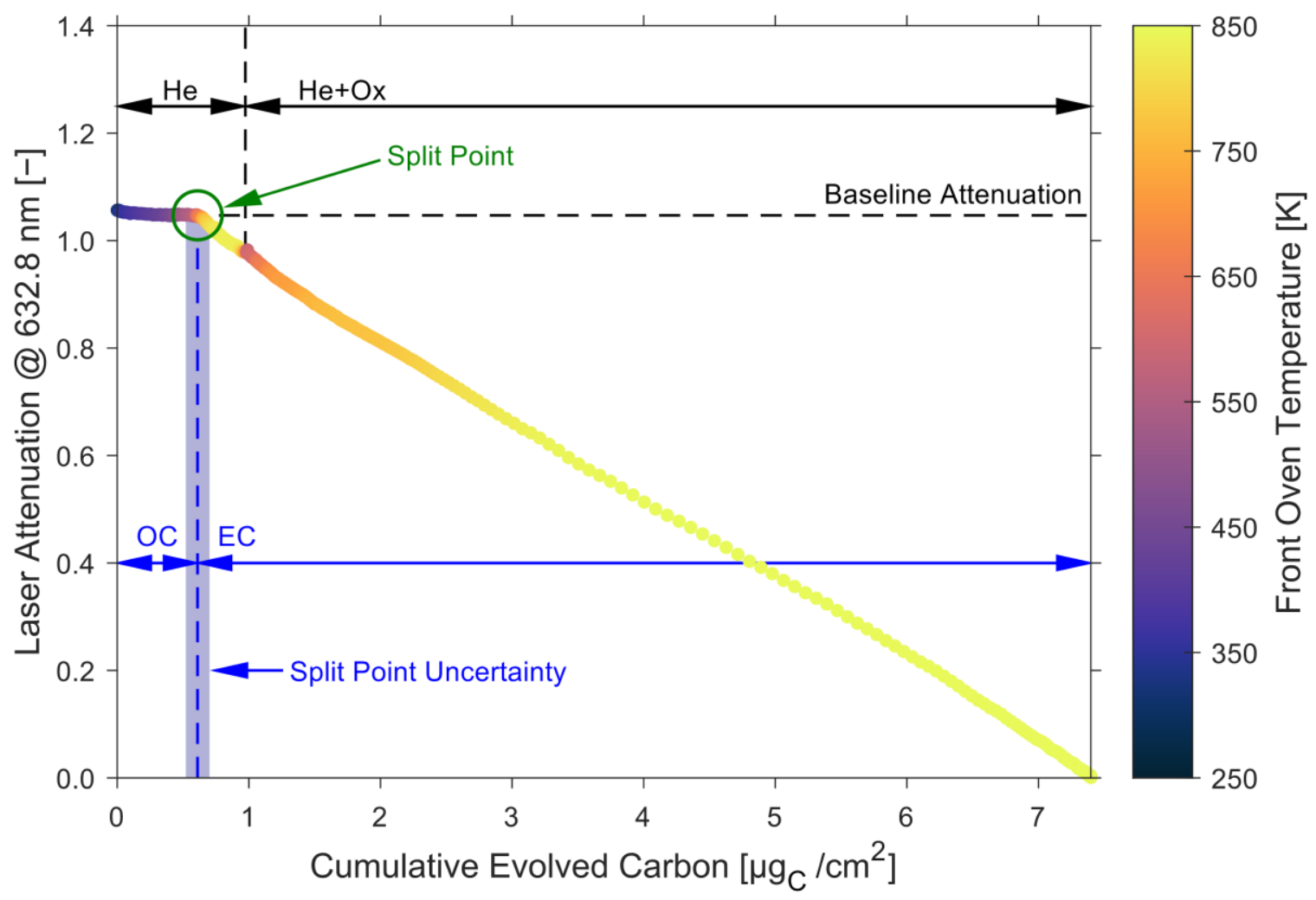

Figure D.4: An example AVEC plot showing laser attenuation at $632.8 \mathrm{~nm}$ against cumulative evolved carbon from the quartz filter. Colouring of the points represents the instantaneous measurement of front oven (filter) temperature. The inflection in the curve (shown in the green circle) indicates the split point of the analysis.

The AVEC plot formulation provides significant insight into the nature of carbonaceous species captured on and evolved from the OCEC filter. As shown in the example figure, there are two distinct stages in the analyses of this study: a constantattenuation stage followed by a linearly decreasing-attenuation stage. The near-constant 
attenuation stage occupies most of the He-phase of the OCEC analysis, during which only $\mathrm{OC}$ is expected to evolve from the filter. Typically, the evolution of $\mathrm{OC}$ is associated with an increase in laser attenuation due to the in-situ pyrolization of $\mathrm{OC}$ on the filter as discussed above - it is discussed in the manuscript that the absence of an increase in attenuation in this example suggests that measured $\mathrm{OC}$ is most likely externally mixed with $\mathrm{EC}$ and that the former is non-pyrolyzing.

The linearly decreasing stage in the example AVEC plot occupies a portion of the He-phase and the entirety of the Ox-phase of the OCEC protocol. While only OC is expected to evolve during the inert phase, it is suggested that this is indeed EC. This notion is based on the common occurrence of premature evolution of EC during the inert phase, as discussed above. Based on this and the discussion of OC-EC mixing state, the split point between the evolution of OC and EC is quite clearly defined in the present work, it is the inflection in the AVEC plot data indicated by the green circle in Figure D.4.

In this work, uncertainties in the quantification of OC and EC are computed under a MC framework (Conrad and Johnson 2019b). Main sources of uncertainty perturbed in the $\mathrm{MC}$ analysis are the calibration constant of the instrument and the estimated split point (as shown by the blue shaded region in Figure D.4). Typical contributions of these sources to uncertainty in OCEC-measured carbon mass are shown in Table D.7. For the AVEC plots in this work (refer to the example shown in Figure D.4), the MC analysis indicates that uncertainty in the $\mathrm{OC} / \mathrm{EC}$ mass is dominated by calibration uncertainty, rather than the split point. This is particularly true for EC, which dominates the total carbon mass, and is relatively insensitive to split point uncertainty in the $\mathrm{MC}$ analysis - indeed, mean uncertainty in EC mass due to estimation of the split point was just $1.90 \%$, despite the $2 \sigma$ 
confidence interval of computed split points being wide (as low as $45 \mathrm{~s}$ and as high as $226 \mathrm{~s})$.

Table D.7: Median uncertainties in OCEC-measured carbon mass.

\begin{tabular}{|c|c|c|c|}
\hline $\begin{array}{c}\text { Carbon } \\
\text { Type }\end{array}$ & $\begin{array}{c}\text { Carbon Mass } \\
\text { Uncertainty due to } \\
\text { Split Point }\end{array}$ & $\begin{array}{c}\text { Carbon Mass } \\
\text { Uncertainty due to } \\
\text { Calibration }\end{array}$ & $\begin{array}{c}\text { Total Carbon Mass } \\
\text { Uncertainty }\end{array}$ \\
\hline OC & $16.4 \%$ & \multirow{2}{*}{$8.28 \%$} & $18.3 \%$ \\
\hline EC & $2.03 \%$ & & $8.42 \%$ \\
\hline
\end{tabular}

\section{D.4.4.2 Sample Volume}

The volume of diluted combustion products sampled by the OCEC instrument was measured in this study using an external thermal mass flow meter (MFM, Bronkhorst ELFLOW Prestige). Sample volume was quantified using trapezoidal integration of timeresolved volume flow rate data. Precision $(0.2 \%$ of reading $)$ and bias $(0.5 \%$ of reading plus $0.1 \%$ of full scale - corresponding to $8 \mathrm{cc} / \mathrm{min}$ ) errors in the MFM-reported flow rate are randomly applied to the raw flow rate data in a $\mathrm{MC}$ framework prior to integration in time, ultimately yielding MC data of total sampled volume. Precision error of the MFM is effectively integrated out of the total sampled volume such that uncertainty in the total volume is a function of the above-noted bias errors and the set sampling flow rate of the instruments. Uncertainty in sampled volume therefore ranged from $1.30 \%$ at $\sim 1 \mathrm{ls} / \mathrm{min}$ ("litres standard" per minute as used by the MFM manufacturer - corresponding to conditions of $20^{\circ} \mathrm{C}$ and $\left.1 \mathrm{~atm}\right)$ to $0.60 \%$ at $\sim 8 \mathrm{ls} / \mathrm{min}$.

\section{D.4.5 Dilution Factor}

To avoid overloading the instruments during measurements at the CUFF, sampled combustion products are occasionally diluted. Dilution requirements depend upon the $\mathrm{BC}$ 
production efficiency of the flared gas and flaring conditions and knowledge of the quantity of dilution is used to correct for background/ambient OC measured by the thermal-optical instrument. Ambient $\mathrm{OC}$ is necessary only for the determination of the elemental-to-total carbon ratio; it is not required for the determination of MAC, which is based on the OCECmeasured EC.

Referring to Figure 5.2, dilution of the sampled combustion products is accomplished by injecting a metered rate of filtered shop air into the sampling tunnel. Product mixture fraction - i.e., the fraction of measured volume sourced from the combustion products - is quantified in this work using a species-continuity method. An optical $\mathrm{CO}_{2}$ analyzer (LI-COR model LI-850) employing pressure-corrected, temperaturestabilized infrared detection is used to quantify $\mathrm{CO}_{2}$ concentrations in the combustion product duct $\left(X_{d u c}\right)$, filtered dilution air $\left(X_{\text {air }}\right)$, and diluted sample $\left(X_{\text {sam }}\right)$, allowing for the computation of mixture fraction:

$$
f=\frac{X_{\text {sam }}-X_{\text {air }}}{X_{d u c}-X_{a i r}}
$$

Throughout the study, sampling from each location is performed for a period of two minutes during a measurement. Uncertainty in the dilution factor is computed with a MC analysis, where the distribution of the mean for each $X_{i}$ is employed in Eq. (D.16). Uncertainty in the computed dilution factor was found to generally scale with the magnitude of dilution; throughout the measurement campaign, the uncertainty ranged from $1.29-12.13 \%$, with a median of $2.61 \%$. 


\section{D.4.6 Mass Absorption Cross-Section Calculation and Analysis}

Spectral mass absorption cross-section $\left(M A C_{B C, \lambda}\right)$ is obtained quite simply as the ratio of measurement-average spectral absorption coefficient at standard conditions $\left(\overline{\alpha_{\lambda}^{o}}\right)$ to mass concentration of $\mathrm{EC}\left(\rho_{E C}\right)$ :

$$
M A C_{B C, \lambda}=\frac{\overline{\alpha_{\lambda}^{o}}}{\rho_{E C}}=\frac{\overline{\alpha_{\lambda}^{o}}}{\left(\frac{m_{E C}}{V_{o}}\right)}
$$

where $\rho_{E C}$ is the ratio of EC mass $\left(m_{E C}\right)$ to sampled volume at standard conditions $\left(V_{o}\right)$. Uncertainty in $M A C_{B C, \lambda}$ is quantified using a $\mathrm{MC}$ analysis, which propagates uncertainties in $\overline{\alpha_{\lambda}^{o}}$ (as per Section D.4.2.2), $m_{E C}$ (as per Section D.4.4 and Conrad and Johnson (2019b)), and $V_{o}$ (as per Section D.4.4.2).

\section{D.4.6.1 Repeatability}

Repeatability of MAC (i.e., precision of the flare experiment as a whole) was estimated using the coefficient of variation of six repeats of the nominal "average" case - AB-M composition from the $50.8 \mathrm{~mm}$ burner at 156 SLPM. Repeatability is summed in quadrature with the uncertainty of $M A C_{B C, \lambda}$ acquired from the $\mathrm{MC}$ results using Eq. (D.17) to obtain total uncertainty for a specific measurement. Summarized in Table D.8 are the median uncertainty metrics for the three studied wavelengths.

Table D.8: Typical (median) uncertainties in the calculation of MAC at the measurement wavelengths.

\begin{tabular}{|c|c|c|c|}
\hline $\begin{array}{c}\text { Laser Wavelength, } \\
\boldsymbol{\lambda} \text { [nm] }\end{array}$ & $\begin{array}{c}\text { Total } \\
\text { Measurement } \\
\text { Uncertainty }\end{array}$ & Repeatability & Total Uncertainty \\
\hline 405 & $9.67 \%$ & $7.90 \%$ & $12.49 \%$ \\
\hline 532 & $9.92 \%$ & $9.39 \%$ & $13.66 \%$ \\
\hline 870 & $9.53 \%$ & $8.60 \%$ & $12.84 \%$ \\
\hline
\end{tabular}




\section{D.4.6.2 Trends in MAC vs. Flare Metrics}

Adding to the discussion in Section 5.5.2, the test matrix enables the assessment of trends (or, sensitivity) in MAC as a function of flare metrics. To this end, linear regression Ftests are performed on the computed MAC data within the MC procedure. Computed $\mathrm{p}$ values of these hypothesis tests are shown in Table D.9, where metrics that are statistically significant $(\mathrm{p}<0.05)$ are highlighted in bold.

Table D.9: Sensitivity of observed variation in flare BC MAC to specific flare metrics. Trends with fuel chemistry metrics computed at the nominal flaring condition of 156 SLPM from $50.8 \mathrm{~mm}$ burner, while trends with geometry and flow metrics computed using tests with the AB-M composition. p-values represent the statistical significance of a linear model versus a constant model. Bolded values represent statistically significant trends $(p<0.05)$.

\begin{tabular}{|c|c|c|c|c|}
\hline \multirow{4}{*}{$\begin{array}{c}\text { Metric } \\
\text { Type }\end{array}$} & Metric Name & \multicolumn{3}{|c|}{ p-value } \\
\cline { 2 - 5 } & & $\mathbf{4 0 5} \mathbf{~ n m}$ & $\mathbf{5 3 2} \mathbf{~ n m}$ & $\mathbf{8 7 0} \mathbf{~ n m}$ \\
\hline \multirow{4}{*}{$\begin{array}{c}\text { Fuel } \\
\text { Chemistry }\end{array}$} & Molecular Weight, $M[\mathrm{~g} / \mathrm{mol}]$ & $<\mathbf{0 . 0 0 1}$ & $\mathbf{0 . 0 0 3}$ & 0.147 \\
\cline { 2 - 5 } & Adiabatic Flame Temperature, $T_{a d}[\mathrm{~K}]$ & $<\mathbf{0 . 0 0 1}$ & $<\mathbf{0 . 0 0 1}$ & $\mathbf{0 . 0 0 1}$ \\
\cline { 2 - 5 } & Volumetric Higher Heating Value, $H H V_{V}\left[\mathrm{MJ} / \mathrm{m}^{3}\right]$ & $<\mathbf{0 . 0 0 1}$ & $<\mathbf{0 . 0 0 1}$ & 0.061 \\
\cline { 2 - 5 } & Carbon-to-hydrogen ratio, $C H R[-]$ & $<\mathbf{0 . 0 0 1}$ & $<\mathbf{0 . 0 0 1}$ & $<\mathbf{0 . 0 0 1}$ \\
\cline { 2 - 5 } & Stoichiometric Mixture Fraction, $f_{s}[-]$ & 0.416 & 0.526 & 0.833 \\
\hline \multirow{4}{*}{$\begin{array}{c}\text { Geometry } \\
\text { \& Flow }\end{array}$} & Flare stack Diameter, $d_{s}[\mathrm{~m}]$ & 0.981 & 0.628 & 0.586 \\
\cline { 2 - 5 } & Flare stack exit Velocity, $u_{e}[\mathrm{~m} / \mathrm{s}]$ & 0.055 & $\mathbf{0 . 0 1 7}$ & 0.450 \\
\cline { 2 - 5 } & Standard Volumetric Flow Rate, $\dot{V}_{o}\left[\mathrm{~m}^{3} / \mathrm{s}\right]$ & $\mathbf{0 . 0 1 1}$ & $\mathbf{0 . 0 0 2}$ & 0.063 \\
\cline { 2 - 5 } & Visible/vertical Flame Length ${ }^{\mathrm{a}}, L_{v}[\mathrm{~m}]$ & $\mathbf{0 . 0 3 3}$ & $\mathbf{0 . 0 1 2}$ & 0.070 \\
\hline
\end{tabular}

${ }^{a}$ Computed using the empirical relation of Delichatsios (Delichatsios 1993)

\section{D.5 Scaling Parameter Expansion}

As presented in the manuscript, the dimensionless scaling parameter used to develop the predictive model of flare BC MAC is the reciprocal product of Becker and Liang's (1982) thermal loading parameter and the fuel CHR:

$$
\Lambda=\frac{\dot{Q}_{t h}}{\sigma T_{a d}^{4} L_{v}^{2}} \cdot \frac{1}{C H R}
$$


where $\dot{Q}_{t h}\left(=\dot{V}_{o} H H V_{V}\right)$ is the theoretical heat release rate from the flame [W], $\dot{V}_{o}$ is the standard volumetric flow rate of flare gas $\left[\mathrm{m}^{3} / \mathrm{s}\right], H H V_{V}$ is the volumetric higher heating value (quantified at standard conditions of 1 atm and $0^{\circ} \mathrm{C}$ as employed in the manuscript) of the flare gas $\left[\mathrm{MJ} / \mathrm{m}^{3}\right], \sigma$ is the Stefan-Boltzmann constant $\left[\mathrm{W} \mathrm{m}^{-2} \mathrm{~K}^{-4}\right], T_{a d}$ is the adiabatic flame temperature $[\mathrm{K}]$, and $L_{v}$ is a characteristic flame length $\left[\mathrm{m}^{2}\right]$. The adiabatic flame temperature is estimated for the CUFF's flare gas surrogates from a reference temperature of $25^{\circ} \mathrm{C}$ using NASA's (National Aeronautics and Space Administration's) Chemical Equilibrium with Applications (CEA) program (Gordon and McBride 1996).

The characteristic flame length of the flare is estimated using the empirical correlation of Delichatsios (1993):

$$
L_{v}=\frac{d_{s}}{f_{s}} \sqrt{\frac{\varrho_{e}}{\varrho_{\infty}}} \frac{13.5 F r_{f}^{2 / 5}}{\left(1+0.07 F r_{f}^{2}\right)^{1 / 5}}
$$

where $\varrho$ represents gas density $\left[\mathrm{kg} / \mathrm{m}^{3}\right]$ at the stack exit ("e") and ambient ("ळ"), and $F r_{f}$ represents the fire Froude number, a dimensionless parameter quantifying the momentumto-buoyancy characteristics of the flame. If the fire Froude number is small, suggesting a strongly buoyant flame, the characteristic flame length is simplified to:

$$
L_{v}=\frac{d_{s}}{f_{s}} \sqrt{\frac{\varrho_{e}}{\varrho_{\infty}}} 13.5 F r_{f}^{2 / 5}
$$

Expanding the fire Froude number, it can be shown that:

$$
L_{v}=13.5\left(\frac{\varrho_{e}}{\varrho_{\infty}} \frac{4 \dot{V}_{o}}{\pi f_{s}} \frac{T_{e}}{T_{o}}\right)^{\frac{2}{5}}\left(\frac{T_{\infty}}{\Delta T g}\right)^{\frac{1}{5}}
$$


where subscript "o" indicates the chosen standard conditions, $\Delta T$ is the adiabatic temperature rise of the flame $[\mathrm{K}], T_{e}$ is the gas temperature at the stack exit $[\mathrm{K}], T_{\infty}$ is the ambient temperature $[\mathrm{K}]$, and $g$ is the gravitational constant $\left[\mathrm{m} \mathrm{s}^{-2}\right]$. Introducing this flame length relation into the scaling parameter (Eq. (D.18)) and simplifying yields:

$$
\Lambda=\frac{H H V_{V}}{13.5^{2} \sigma T_{a d}^{4} C H R}\left(\frac{\pi f_{s}}{4} \frac{\varrho_{\infty}}{\varrho_{o}} \sqrt{\frac{\Delta T g}{T_{\infty}}}\right)^{\frac{4}{5}} \dot{V}_{o}^{\frac{1}{5}}
$$

\section{D.5.1 Model-Inferred Flare Flow Rate from MAC Measurements of Weyant et al.}

Referenced in the manuscript is the only known data of flare BC MAC from fieldoperational flares. Weyant et al. (2016), who performed estimates of BC emission factors from flares in the Bakken region of North Dakota using aircraft-based sampling techniques, were able to report estimates of flare $\mathrm{BC} \mathrm{MAC}$ as a secondary outcome from their measurements. The average of their flare BC MAC measurements was 22, 16 , and $14 \mathrm{~m}^{2} / \mathrm{g}$ at 467, 530, and $660 \mathrm{~nm}$, respectively. Using Eq. (5.6), these average BC MAC data yield a best-fitting scaling parameter of 0.2343 .

The formulation of the scaling parameter presented in Eq. (D.22) can be inverted to estimate flare gas flow rate with knowledge of flare gas composition, initial flare gas temperature, and ambient temperature:

$$
\dot{V}_{o}=\left(\frac{4}{\pi f_{s}} \frac{\varrho_{o}}{\varrho_{\infty}} \sqrt{\frac{T_{\infty}}{\Delta T g}}\right)^{4}\left(\frac{13.5^{2} \Lambda \sigma T_{a d}^{4} C H R}{H H V_{V}}\right)^{5}
$$

To estimate BC emission factors in their work, Weyant et al. (2016) assumed a representative "average" composition for the Bakken region (Wocken et al. 2013). Using 
the CUFF surrogate for this composition data, and assuming an ambient temperature of $25^{\circ} \mathrm{C}$ and molecular weight of $28.85 \mathrm{~g} / \mathrm{mol}$, the derived $\mathrm{BC}$ MAC model suggests an "average" flare gas flow rate of approximately 10,500 SLPM as summarized in Table D.10. In the experience of the authors, this flare gas flow rate is certainly reasonable for upstream oil and gas flares as it is within range of measurements performed in Ecuador (Chapter 2, Conrad and Johnson 2017) and recent unpublished measurements during an oil well completion in Alberta.

Table D.10: Summary of data necessary for the estimate of flare gas flow rate for the average MAC results of Weyant et al.'s (2016) study in the Bakken region of North Dakota.

\begin{tabular}{|c|c|c|}
\hline & Property & Value \\
\hline \multirow{4}{*}{$\begin{array}{c}\text { Flare Gas } \\
\text { Composition }\end{array}$} & Stoichiometric mixture fraction, $f_{S}$ & 0.06193 \\
\cline { 2 - 3 } & Adiabatic flame temperature, $T_{a d}$ & $2242.3 \mathrm{~K}$ \\
\cline { 2 - 3 } & Adiabatic temperature rise, $\Delta T$ & $1944.2 \mathrm{~K}$ \\
\cline { 2 - 3 } & Carbon-Hydrogen Ratio, $C H R$ & 0.3102 \\
\cline { 2 - 3 } & Higher heating value, $H H V_{V}$ & $55.19 \mathrm{MJ} / \mathrm{m}^{3}$ \\
\cline { 2 - 3 } & Standard density, $\varrho_{o}$ & $1.125 \mathrm{~kg} / \mathrm{m}^{3}$ \\
\hline \multirow{2}{*}{$\begin{array}{c}\text { Ambient } \\
\text { Conditions }\end{array}$} & Density, $\varrho_{\infty}$ & $1.179 \mathrm{~kg} / \mathrm{m}^{3}$ \\
\cline { 2 - 3 } Required MAC & Temperature, $T_{\infty}$ & $298.15 \mathrm{~K}$ \\
\hline Estimate & Scaling parameter, $\Lambda$ & 0.2343 \\
\hline
\end{tabular}

